\title{
On Synthetic Jet Actuation for Aerodynamic Load Control
}

\section{5}




\section{On Synthetic Jet Actuation for Aerodynamic Load Control}

H. de Vries 
On Synthetic Jet Actuation for Aerodynamic Load Control H. de Vries

Thesis University of Twente, Enschede, The Netherlands With ref. - With summary in Dutch.

ISBN 978-90-365-0006-7

Printed by: Gildeprint Drukkerijen - The Netherlands

Copyright (c) 2013 by H. de Vries 


\section{ON SYNTHETIC JET ACTUATION FOR}

AERODYNAMIC LOAD CONTROL

\section{PROEFSCHRIFT}

ter verkrijging van

de graad van doctor aan de Universiteit Twente, op gezag van de rector magnificus, prof. dr. H. Brinksma, volgens besluit van het College voor Promoties in het openbaar te verdedigen op vrijdag 5 juli 2013 om 12.45 uur

door

Hein de Vries

geboren op 27 februari 1980

te Lemsterland 
Dit proefschrift is goedgekeurd door de promotoren:

prof. dr. ir. H.W.M. Hoeijmakers

prof. dr.ir. A. Hirschberg

en de assistent-promotor:

dr. ir. E.T.A. van der Weide 


\section{SUMMARY}

A major goal for the wind energy industry is the reduction of the cost of energy. This drives the design towards increasingly larger wind turbines. The technology of smart rotor control is expected to allow wind turbines to increase even further in size. Smart rotor control consists of a sequence of local load control mechanisms, distributed along the span of a wind turbine blade, which are operated individually based on local sensory information. This technology has the potential to reduce fatigue inducing variations in blade loads.

Fatigue loads are induced by cyclic effects, such as wind shear, gravity and yaw misalignment, or by stochastic effects, such as turbulence in the upstream flow. Alleviation of the fatigue loads reduces the structural requirements of multiple components of a wind turbine, which allows for relatively lighter structures and less maintenance. It is expected that this will decrease the cost of energy, due to a combination of increased energy production and relatively lower capital and maintenance costs of a large but lighter wind turbine equipped with smart rotor control.

The aerodynamic effect needed for smart rotor control is 'local pitch control', which aims for changes in the local aerodynamic characteristics, mainly the lift coefficient $\left(c_{l}\right)$, over the range of angles of attack $(\alpha)$ in the linear $c_{l}(\alpha)$-regime. This aerodynamic effect can also be employed for other turbomachinery and in wing aerodynamics in the field of aeronautics. Potential options for local pitch control are trailing edge flaps, micro-tabs (small deployable Gurney flaps) and blade morphing. Active fluidic control close to the trailing edge by means of jets is an alternative option.

In the present research, synthetic jets have been investigated as a potential option for local pitch control. Synthetic jets are generated by repeated ingestion and subsequent ejection of air, into and out of a cavity below the surface of the blade, respectively, through holes or slits in the surface of this blade. This oscillatory ejection/ingestion is caused by a vibrating wall inside the cavity, such as a piston or a piezoceramic composite diaphragm. The technology of synthetic jets can be used for boundary layer separation control, as well as pitch control.

In the present thesis, a multi-purpose computational method has been developed for the simulation of unsteady compressible viscous flows. This method is, in principle, able to address the relevant characteristic flow effects associated with synthetic jet actuation. The computational method solves the unsteady Reynolds-averaged Navier-Stokes (URANS) equations for unsteady compressible viscous flow, together with the equation(s) of a linear eddy-viscosity turbulence model. The equations are discretized on unstructured computational grids, employing the Finite Volume method on cell-centered control volumes. The discretization is nominally of second order accuracy, both in space and time. 
An exception is the discretization of the convective flux in the equation(s) of the turbulence model, for which a first-order accurate scheme is employed. An implicit time integration method is used, in which the nonlinear equations resulting from the discretization are solved by iteration in pseudo-time. Within each pseudo-time step, the linearized equations are solved by means of Block Symmetric Gauß-Seidel iteration, accelerated by an agglomeration-based algebraic multigrid method (for the URANS equations). The equations corresponding to the turbulence model are solved loosely coupled to the URANS equations. Additional computational acceleration is achieved through parallelization by means of domain decomposition. The implementation of the mathematical models has been verified by means of several verification cases.

In the developed computational method, time-dependent inflow/outflow boundary conditions have been included that enable the simulation of flows with synthetic jets. For a two-dimensional flow configuration, i.e. the flow around a non-rotating airfoil, the computational costs can be kept within reasonable bounds, such that it has been possible to perform a parameter study of synthetic jet actuation for this configuration. In this parameter study, a synthetic jet has been placed close to the trailing edge of a NACA0018 airfoil, and its effect on the lift and drag coefficients of the airfoil has been studied.

The involved parameters can be separated into main flow parameters, geometric parameters and actuation parameters. The parameters that have been varied in the present investigation are the angle of attack of the airfoil, $\alpha$, the chordwise location of the slit of the synthetic jet, $x_{j} / c$, the dimensionless actuation frequency, $F^{+}=f c / U_{\infty}$, and the momentum coefficient, $c_{\mu}$, which is a measure of the momentum of the jet during the ejection phase of the actuation cycle. Here, $f$ is the actuation frequency, $c$ is the chord length of the airfoil and $U_{\infty}$ is the free-stream velocity.

Simulations of high-frequency synthetic jet actuation show that the inclusion in the computational domain of the slit and (part of) the cavity of the synthetic jet is essential. It has turned out to be necessary to simultaneously compute the exterior flow and the flow inside the slit and (part of) the cavity to accurately describe all the observed timedependent characteristics of a synthetic jet. The possible alternative of prescribing the flow at the exit of the slit by means of a surface boundary condition turned out to be a challenge. Furthermore, compressibility effects cannot be neglected, as large fluctuations of the density across the exit of the slit are observed at high actuation frequencies and amplitudes. At these conditions, the action of the synthetic jet also generates large acoustic pressure fluctuations. Solving the equations for compressible flow yields the correct wave speed of these pressure waves.

For relatively low actuation frequencies $\left(F^{+}<1\right)$, we have been able to compare computational results for the time-averaged pressure distribution on the surface of the airfoil to experimentally obtained surface pressure measurements. The computational method generally predicts well the effect of low-frequency actuation, but the effect is overpredicted in comparison with the effect observed in the experiments. It is suspected that due to the actuation being performed over only a part of the span in the experiments, three-dimensional flow effects occur that are not represented in the two-dimensional flow 
configuration considered in the numerical simulations.

At high actuation frequencies $\left(F^{+} \geq 1\right)$, vortices are generated, which are subsequently convected with the flow along the surface of the airfoil. When these vortices are generated in rapid succession $\left(F^{+} \geq 10\right)$, they can cause a displacement of the main flow, effectively forming an obstruction. This displacement can influence the direction of the flow at the trailing edge, i.e. the circulation around the airfoil, which is associated with a change of the lift and drag coefficients.

It has been established that the performance in terms of the change in the lift coefficient increases with increasing momentum coefficient of the jet, a location of the slit closer to the trailing edge, i.e. higher $x_{j} / c$, and decreasing angle of attack, $\alpha$, for a slit on the lower side of the airfoil. Furthermore, the accompanying time-averaged change in the drag coefficient is generally lower than for a trailing edge flap or a micro-tab. The aerodynamic response time to high-frequency synthetic jet actuation reduces for locations of the slit closer to the trailing edge. For $x_{j} / c=0.95,50 \%$ of the final time-averaged change in the lift coefficient is obtained within a time span of $c / U_{\infty}$.

However, for reasonable values of the momentum coefficient, the response of the lift and drag coefficients in time is associated with relatively large fluctuations superposed on the time-averaged changes of these coefficients. This is a result of the pressure fluctuations generated by the action of the synthetic jet and is associated with noise emission.

Only high-frequency synthetic jet actuation $\left(F^{+} \geq 10\right)$ is a feasible option for smart rotor control, since it is expected that the frequency of the fluctuations of the lift and drag coefficients is then high enough to prevent the excitation of important structural modes of the blades and other components of the wind turbine.

A combination of the studied parameters has been found that yields a time-averaged change in the lift coefficient close to the performance deemed necessary for smart rotor control. However, the associated power consumption appears to be prohibitive. Further study on the other parameters is needed to find a less power intensive combination of the parameters involved in high-frequency synthetic jet actuation. Also, a combination of synthetic jet actuation with other control methods can be considered. 



\section{SAMENVATTING}

Een belangrijk doel voor de windenergie-industrie is het verlagen van de kosten van energieproductie, hetgeen blijkt te leiden tot het ontwerpen van steeds grotere windturbines. De verwachting is dat smart rotor control een verdere toename van de grootte mogelijk maakt. Smart rotor control bestaat uit een opeenvolging van lokale regelmechanieken, verdeeld over (een deel van) de spanwijdte van een windturbineblad, die individueel worden bediend op basis van informatie van lokale sensoren. Deze technologie heeft de potentie om vermoeiingsbelastingen op de bladen van windturbines te verminderen.

Vermoeiingsbelastingen worden geïnduceerd door cyclische effecten, zoals windschering, zwaartekracht en scheefstand van de rotor, of door stochastische effecten, zoals turbulentie in de stroming voor de bladen. Een verlaging van de vermoeiingsbelastingen leidt tot lagere structurele eisen voor meerdere componenten van een wind turbine, resulterend in relatief lichtere constructies en minder onderhoud. Verwacht wordt dat dit de energiekosten verlaagt, door een combinatie van verhoogde energieproductie en relatief lagere kapitaal- en onderhoudskosten van een grote maar lichte windturbine uitgerust met smart rotor control.

Het aërodynamische effect dat nodig is voor smart rotor control is 'lokale bladhoek regeling', dat gericht is op veranderingen in de lokale aërodynamische eigenschappen, vooral de liftcoëfficiënt $\left(c_{l}\right)$, over het bereik van invalshoeken $(\alpha)$ in het lineare $c_{l}(\alpha)$-regime. Dit mechanisme kan ook worden gebruikt voor andere roterende stromingsmachines en in de vleugel-aërodynamica. Mogelijke opties voor 'lokale bladhoek regeling' zijn kleppen aan de achterrand van het blad, micro-tabs (kleine regelbare Gurney-kleppen) en bladvervorming. Een alternatief is actieve stromingsbeïnvloeding nabij de achterrand door middel van jets (vrije stralen).

Dit onderzoek heeft synthetic jets onderzocht als één van de mogelijke opties voor 'lokale bladhoek regeling'. Synthetic jets ontstaan door lucht herhaaldelijk achtereenvolgens in een holte in het blad te zuigen en uit te blazen, door gaten of sleuven in het oppervlak van het blad of bladdeel. Dit aanzuig/uitblaas effect wordt veroorzaakt door een vibrerende wand in de holte, zoals een zuiger of een piëzokeramisch membraan. Deze technologie kan worden gebruikt voor zowel het beïnvloeden van grenslagen, als het beïnvloeden van de bladhoek via het veranderen van de effectieve welving van het blad.

In dit onderzoek is een rekenmethode ontwikkeld voor de simulatie van instationaire, compressibele, viskeuze stromingen, die voor meerdere doeleinden gebruikt kan worden. Deze methode kan, in principe, de relevante karakteristieke stromingseffecten van synthetic jets representeren in de numerieke stromingsoplossing. De rekenmethode lost de instationaire Reynolds-gemiddelde Navier-Stokesvergelijkingen op, samen met de vergelijking(en) van een lineair eddy-viscositeits turbulentiemodel. De vergelijkingen worden 
gediscretiseerd op ongestructureerde rekenroosters, gebruik makend van de eindige volume methode op celgecentreerde controle-volumes. De discretisatie heeft een nominale tweede-orde nauwkeurigheid, zowel ruimtelijk als in de tijd. Een uitzondering is de discretisatie van de convectieve flux in de vergelijking(en) van het turbulentiemodel, waarvoor een eerste-orde nauwkeurig discretisatieschema wordt toegepast. Er wordt gebruik gemaakt van een impliciete tijdsintegratie, waarin de niet-lineare vergelijkingen resulterend van de discretisatie iteratief worden opgelost in pseudo-tijd. Binnen elke pseudo-tijdstap worden de gelineariseerde vergelijkingen opgelost door middel van blok-symmetrische Gauß-Seidel iteratie, versneld door een op agglomeratie gebaseerde algebraische multigrid methode (voor de stromingsvergelijkingen). De vergelijkingen die corresponderen met het turbulentiemodel worden ontkoppeld van de stromingsvergelijkingen opgelost. Extra versnelling van de rekenmethode wordt bereikt door middel van parallelisatie via domein decompositie. De implementatie van de wiskundige modellen is geverifieerd aan de hand van een aantal verificatietesten.

In de rekenmethode zijn tijdsafhankelijke instroom/uitstroom randvoorwaarden opgenomen die het mogelijk maken stromingen met synthetic jets te simuleren. Voor een twee-dimensionale stromingsconfiguratie, namelijk de stroming langs een niet-roterend vleugelprofiel, kan de rekentijd binnen redelijke grenzen worden gehouden. Dit heeft het mogelijk gemaakt om een parameterstudie uit te voeren van de beïnvloeding van de stroming door synthetic jets. In deze parameterstudie is voor een synthetic jet, gepositioneerd nabij de achterrand van een NACA0018, het effect onderzocht op de lift- en weerstandscoëfficiënten van het profiel.

De parameters die in dit probleem een rol spelen kunnen worden onderverdeeld in parameters die betrekking hebben op de hoofdstroming, geometrische parameters en actuatieparameters. De parameters die in dit onderzoek zijn gevarieerd zijn de invalshoek van het vleugelprofiel, $\alpha$, de locatie van de sleuf langs de koorde, $x_{j} / c$, de dimensieloze actuatiefrequentie, $F^{+}=f c / U_{\infty}$, en de impulscoëfficiënt, $c_{\mu}$, welke een maat is voor de impuls van de synthetic jet tijdens het uitblazen. Hierbij is $f$ de actuatiefrequentie, $c$ de koordelengte van het vleugelprofiel en $U_{\infty}$ de vrije-stroomsnelheid.

Uit simulaties met hoogfrequente synthetic jets blijkt dat het essentieel is de sleuf en (een deel van) de holte van de synthetic jet op te nemen in het rekendomein. Voor de nauwkeurige representatie van de effecten van de synthetic jet is het noodzakelijk gebleken om de externe stroming en de stroming in de sleuf en de holte gelijktijdig op te lossen. Het mogelijke alternatief om de stroming voor te schrijven als randvoorwaarde aan de uitgang van de sleuf is een uitdaging gebleken. Bovendien blijkt dat de compressibiliteit van het medium niet kan worden verwaarloosd, aangezien bij hoge frequenties en amplitudes grote fluctuaties in de dichtheid van het medium zijn waargenomen bij de uitgang van de sleuf. In deze omstandigheden genereert de actie van de synthetic jet ook grote drukfluctuaties. Het oplossen van de vergelijkingen voor compressibele stromingen resulteert in de juiste voortplantingssnelheid van deze drukgolven.

Voor relatief lage actuatiefrequenties $\left(F^{+}<1\right)$ was het mogelijk om rekenresultaten voor de tijdsgemiddelde drukverdeling op het oppervlak van het vleugelprofiel te vergelijken met experimenteel verkregen resultaten. De rekenmethode voorspelt in het algemeen het 
effect van laagfrequente actuatie goed, maar het effect wordt overschat in vergelijking met het effect waargenomen in de experimenten. Een vermoedelijke reden is dat de actuatie in de experimenten wordt uitgevoerd over slechts een deel van de spanwijdte van het vleugelprofiel. Driedimensionale stromingseffecten die optreden in de experimenten zijn niet gerepresenteerd in de numerieke simulaties voor de stroming om de tweedimensionale configuratie.

Bij hoge actuatiefrequenties $\left(F^{+} \geq 1\right)$ worden wervels gegenereerd, die vervolgens met de stroming langs het oppervlak van het profiel worden meegenomen. Wanneer deze wervels snel achter elkaar worden gegenereerd $\left(F^{+} \geq 10\right)$, kunnen ze leiden tot een verplaatsing van de hoofdstroom van het oppervlak af. Deze verplaatsing kan de richting van de stroming aan de achterrand en daarmee de circulatie rond het vleugelprofiel beïnvloeden, wat gepaard gaat met een verandering van de lift- en weerstandscoëfficiënten.

Vastgesteld is dat de prestatie, in termen van de verandering van de liftcoëfficiënt, toeneemt met toenemende impulscoëfficiënt, een locatie van de sleuf dichter bij de achterrand, dus hogere $x_{j} / c$, en dalende invalshoek, $\alpha$, voor een sleuf aan de onderzijde van het vleugelprofiel. Bovendien is de bijbehorende tijdsgemiddelde verandering van de weerstandscoëfficiënt in het algemeen lager dan voor een klep aan de achterrand of een micro-tab. De aërodynamische reactietijd bij hoogfrequente actuatie vermindert naarmate de sleuf dichter bij de achterrand wordt geplaatst. Voor $x_{j} / c=0,95$, wordt $50 \%$ van de uiteindelijke tijdsgemiddelde verandering van de liftcoëfficiënt verkregen binnen een tijd van $c / U_{\infty}$.

Voor redelijke waarden van de impulscoëfficiënt wordt de responsie van de lift- en weerstandscoëfficiënten gekarakteriseerd door relatief grote fluctuaties gesuperponeerd op de tijdsgemiddelde veranderingen van deze coëfficiënten. Dit is het gevolg van de drukfluctuaties die ontstaan door de actie van de synthetic jet. Deze drukfluctuaties zijn ook geassocieerd met geluidsemissie.

Alleen hoogfrequente actuatie met synthetic jets $\left(F^{+} \geq 10\right)$ is een haalbare optie voor smart rotor control. De verwachting is dat de frequentie van de fluctuaties van de lift- en weerstandscoëfficiënten dan hoog genoeg is om de excitatie van belangrijke structurele resonantie-modes van de bladen en andere componenten van de windturbine te voorkomen.

$\mathrm{Er}$ is een combinatie van de onderzochte parameters gevonden die een tijdsgemiddelde verandering van de liftcoëfficiënt levert die dichtbij de voor smart rotor control benodigde prestaties zit. Het bijbehorende energieverbruik lijkt echter te hoog om dit in werkelijkheid toe te kunnen passen. Verder onderzoek naar andere combinaties van parameters is nodig om minder energie-intensieve configuraties te vinden. Ook kan een combinatie van synthetic jets en andere opties voor 'lokale bladhoek regeling' worden overwogen. 



\section{Contents}

Summary iii

Samenvatting vii

1 Introduction 1

1.1 Background, Goal and Approach . . . . . . . . . . . . . . . . . . . . . . 1

1.2 Thesis Outline . . . . . . . . . . . . . . . . . . . . . . . 3

2 Smart Rotor Control for HAWTs 5

2.1 Horizontal Axis Wind Turbines . . . . . . . . . . . . . . . . . . . . 5

2.2 Aerodynamics of Horizontal Axis Wind Turbines . . . . . . . . . . . . . . 9

2.2.1 Actuator Disk Model . . . . . . . . . . . . . . . . . . . . . . . 10

2.2.2 Blade Element Momentum Model . . . . . . . . . . . . . . . . . 11

2.3 Airfoil Characteristics . . . . . . . . . . . . . . . . . . . . . . . . . . . . . 14

2.3.1 Steady Aerodynamics ... . . . . . . . . . . . . . . . . . . . . . . . . . . . . . . . . . . . 15

2.3.2 Unsteady Aerodynamics . . . . . . . . . . . . . . . . . . . . 15

2.4 Aerodynamic Control . . . . . . . . . . . . . . . . . . . . . . . . 17

2.5 Smart Rotor Control . . . . . . . . . . . . . . . . . . . . . . . . . . . . . 19

2.5 .1 Requirements ... . . . . . . . . . . . . . . . . . . . . 19

2.5 .2 Potential Options for Smart Rotor Control . . . . . . . . . . . . . . 22

2.6 Synthetic Jets . . . . . . . . . . . . . . . . . . . . . . . . . . . 27

2.6 .1 Principle . . . . . . . . . . . . . . . . . . . . . . . . . . 27

2.6 .2 Parameters . . . . . . . . . . . . . . . . . . . . . . . . . 29

2.6.3 Separation Control versus Pitch Control . . . . . . . . . . . . . . 30

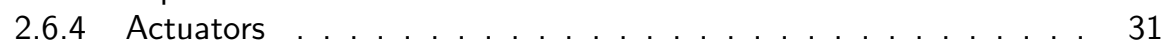

2.7 Concluding Remarks . . . . . . . . . . . . . . . . . . . . . . . . . . . 32

3 Mathematical Modeling 35

3.1 Inertial Frame of Reference . . . . . . . . . . . . . . . . . . . . . . . . 35

3.1 .1 Conservation of Mass . . . . . . . . . . . . . . . . . . . . . . . . . . . . . . . . . . . . . . . . . . 36

3.1 .2 Conservation of Momentum . . . . . . . . . . . . . . . . . . 36

3.1 .3 Conservation of Total Energy . . . . . . . . . . . . . . . . . . . . . 37

3.1 .4 Navier-Stokes Equations . . . . . . . . . . . . . . . . . . . . . . . 37

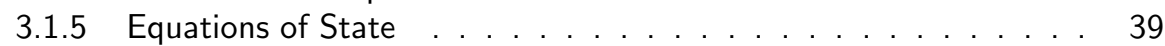

3.1 .6 Constitutive relations . . . . . . . . . . . . . . . . . . . . . . . . 40

3.2 Non-Inertial Frame of Reference . . . . . . . . . . . . . . . . . . . 41

3.2.1 Transformation to Rotating Frame of Reference . . . . . . . . . . 41 
3.2.2 Navier-Stokes equations in Rotating Frame of Reference . . . . 42

3.3 Turbulence Modeling . . . . . . . . . . . . . . . . . . . . . . 43

3.3 .1 Scales of Motion . . . . . . . . . . . . . . . . . . 43

3.3.2 Turbulence Modeling Approach . . . . . . . . . . . . . . . 46

3.3 .3 Reynolds- and Favre Averaging . . . . . . . . . . . . . 46

3.3 .4 Derivation of URANS Equations . . . . . . . . . . . . . 48

3.3 .5 Closure Approximations . . . . . . . . . . . . . . . . . . . . 52

3.3 .6 Summary of Closed URANS Equations . . . . . . . . . . . . 54

3.3.7 URANS Equations in a Rotational Frame of Reference . . . . . . 55

3.4 Eddv-Viscosity Turbulence Models . . . . . . . . . . . . . . . 56

3.4.1 General Aspects of Eddy-Viscosity Turbulence Models . . . . . . . 56

3.4.2 Spalart-Allmaras One-Equation Turbulence Model . . . . . . . . . 59

3.4.3 Shear Stress Transport Two-Equation Turbulence Model . . . . . 61

3.4.4 Turbulence Model Equations in Integral Conservation Form . . . . 63

3.5 Boundary Conditions . . . . . . . . . . . . . . . . . 64

3.5.1 Physical Boundary Conditions . . . . . . . . . . . . . . 64

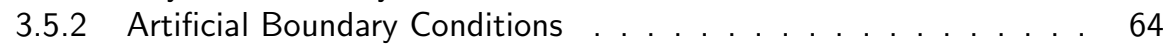

3.5.3 Boundary Conditions for Synthetic Jet Actuation . . . . . . . . 66

4 Numerical Solution of the Mathematical Models $\quad 71$

4.1 Introduction . . . . . . . . . . . . . . . . . . . . . . 71

4.1.1 Aspects of Computational Grids . . . . . . . . . . . . . 72

4.1 .2 Aspects of Spatial Discretization . . . . . . . . . . . . . 72

4.1 .3 Aspects of Temporal Integration . . . . . . . . . . . . . 75

4.1.4 Aspects of Computational Time . . . . . . . . . . . . 77

4.1 .5 Outlook . . . . . . . . . . . . . . . . . . . . 78

4.2 Spatial Discretization . . . . . . . . . . . . . . . . . . . . 78

4.2 .1 Finite Volume Formulation . . . . . . . . . . . . . . . . 78

4.2 .2 Central Convective Flux Discretization . . . . . . . . . . . . 80

4.2 .3 Upwind Convective Flux Discretization . . . . . . . . . . . . . 82

4.2.4 MUSCL-type Solution Reconstruction Technique . . . . . . . . . 90

4.2 .5 Computation of Gradients . . . . . . . . . . . . . . . . . . . 93

4.2 .6 Central Viscous Flux Discretization . . . . . . . . . . . . . . . 94

4.3 Spatial Discretization of the Turbulence Model Equations . . . . . . . . . 96

4.3.1 Spatial Discretization of the SST Turbulence Model . . . . . . . . 96

4.3.2 Spatial Discretization of the SA Turbulence Model . . . . . . . . 97

4.3.3 Semi-Discretized Turbulence Model Equations . . . . . . . . . . . 97

4.4 Time Integration . . . . . . . . . . . . . . . . . . . . . . . . . . . . . . . . . . 989

4.4 .1 Dual-Time Stepping Approach . . . . . . . . . . . . . . . . 98

4.4 .2 Steady Flow Solutions . . . . . . . . . . . . . . . . . . . . . . 101

4.4 .3 Initial Conditions . . . . . . . . . . . . . . . . . . . . . . 101

$4.4 .4 \quad$ Approximate Flux Jacobian Matrix . . . . . . . . . . . . . . . . 101

4.4 .5 Flux Jacobian of URANS Equations . . . . . . . . . . . 103

4.4 .6 Flux Jacobian of Turbulence Model Equations . . . . . . . . . 106

4.5 Iterative Solution Method . . . . . . . . . . . . . . . . . . . . . . . . 109 
4.5 .1 Loosely Coupled Iteration Method ... . . . . . . . . . . . . 109

4.5 .2 Block Symmetric Gauß-Seidel Iteration . . . . . . . . . . . . . . 110

4.5 .3 Pseudo-time Step Size . . . . . . . . . . . . . . . . . . . . 111

4.6 Convergence Acceleration Techniques . . . . . . . . . . . . . . 112

4.6.1 Agglomeration-Based Algebraic Multigrid Method . . . . . . . . 112

4.6 .2 Domain Decomposition . . . . . . . . . . . . . . . . . . . . . 121

4.7 Numerical Treatment of Boundary Conditions . . . . . . . . . . . . . 122

4.7.1 Physical Boundary: Adiabatic Solid Wall . . . . . . . . . . . . . 124

$4.7 .2 \quad$ Artificial Boundaries . . . . . . . . . . . . . . . . . . . 125

4.7 .3 Synthetic Jet Actuation . . . . . . . . . . . . . . . . . 130

5 Verification and Validation 133

5.1 Introduction . . . . . . . . . . . . . . . . . . . . . 133

5.2 Verification . . . . . . . . . . . . . . . . . . . . . . 135

5.2 .1 Laminar Boundary Layer on Flat Plate . . . . . . . . . . . . 135

5.2 .2 Turbulent Boundary Laver on Flat Plate . . . . . . . . . . . 141

5.2 .3 Turbulent Flow around NACA0012 Airfoil . . . . . . . . . . . 146

5.2 .4 Time-dependent flow around circular cylinder . . . . . . . . . . 152

5.2 .5 Conclusions . . . . . . . . . . . . . . . . . . . . . . 161

5.3 Validation . . . . . . . . . . . . . . . . . . . . . . . . . 162

5.3 .1 General Remarks . . . . . . . . . . . . . . . . . . . 162

5.3.2 Validation of Flows with Boundary Laver Separation Control . . . 164

6 Pitch Control using SJ Actuation 183

6.1 Introduction . . . . . . . . . . . . . . . . . . . . . . . 183

6.1 .1 Problem Description . . . . . . . . . . . . . . . . . . . . 183

6.1 .2 Outlook . . . . . . . . . . . . . . . . . . . . . . . . . 184

6.2 Dimensional Analysis . . . . . . . . . . . . . . . . . . . . . 185

6.2.1 Physically Independent Variables . . . . . . . . . . . . . . 185

6.2 .2 Dimensionless Parameters . . . . . . . . . . . . . . . . . . . . . . 187

6.2 .3 Subset of Parameters for Study . . . . . . . . . . . . . . . . . 187

6.3 Experimental Approach . . . . . . . . . . . . . . . . . . . . . . . 188

$6.3 .1 \quad$ Introduction . . . . . . . . . . . . . . . . . . . 188

6.3 .2 Wind-Tunnel Model . . . . . . . . . . . . . . . . . . . . . . 188

6.3 .3 Actuators ... . . . . . . . . . . . . . . . . . . . . . . 190

6.3 .4 Measurement Equipment . . . . . . . . . . . . . . . . . . . . 190

6.3 .5 Wind Tunnell. . . . . . . . . . . . . . . . . . . . . . . . . . . . 194

6.3 .6 Measurement Procedure . . . . . . . . . . . . . . . . 195

6.4 Computational Approach . . . . . . . . . . . . . . . . . . . . . 196

6.4 .1 Computational Domain and Boundary Conditions . . . . . . . . 196

6.4 .2 Computational Grids . . . . . . . . . . . . . . . . . . . . . . 197

6.4.3 Discretization and Turbulence Model . . . . . . . . . . . . . . . 199

6.5 Baseline Results . . . . . . . . . . . . . . . . . . . . . . . 200

6.5 .1 Effect of Boundary Laver Tripping . . . . . . . . . . . . . 202

6.5 .2 Validation of RANS Method . . . . . . . . . . . . . . . 205 
6.5 .3 Conclusions . . . . . . . . . . . . . . . . . . . . . . . 210

6.6 Performance of Synthetic Jet Actuation System . . . . . . . . . . . . 211

6.7 Results for Low-Frequency Synthetic Jet Actuation . . . . . . . . . . . . 214

6.7 .1 Peak Eiection Velocity ... . . . . . . . . . . . . . . . . 214

6.7 .2 Time-averaged Pressure Distribution . . . . . . . . . . . 215

6.7 .3 Time-Dependent Lift and Drag Force . . . . . . . . . . . 217

6.7 .4 Time-Dependent Flow Field . . . . . . . . . . . . . . . . . . 221

6.7 .5 Discussion . . . . . . . . . . . . . . . . . . . . . . . . 224

6.8 Results for High-Frequency Synthetic Jet Actuation . . . . . . . . . . . . 226

6.8 .1 Timestep Refinement . . . . . . . . . . . . . . . . . . . . . 227

6.8 .2 Performance of Synthetic Jets _. . . . . . . . . . . . . 229

6.8.3 Characteristic Flow Phenomena and Aerodynamic Performance. . . 235

6.8 .4 Effect of Momentum Coefficient . . . . . . . . . . . . . . . 247

6.8 .5 Effect of Dimensionless Actuation Frequency . . . . . . . . . 250

$6.8 .6 \quad$ Effect of Slit Location . . . . . . . . . . . . . . . . . . . . . 255

6.8 .7 Effect of Angle of Attack . . . . . . . . . . . . . . . . . 262

6.8 .8 Discussion . . . . . . . . . . . . . . . . . . . . . . . 270

7 Conclusions and Recommendations 273

7.1 Conclusions . . . . . . . . . . . . . . . . . . . . . . . . . . . 273

7.2 Recommendations . . . . . . . . . . . . . . . . . . . . . . 278

\begin{tabular}{ll}
\hline Bibliography & 281
\end{tabular}

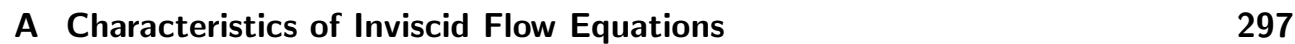

A.1 Eigenvalues and Eigenvectors . . . . . . . . . . . . . . . . . . . . . 298

A.1.1 Problem Definition . . . . . . . . . . . . . . . . 298

A.1.2 Transformation to Primitive Variables . . . . . . . . . . . . . 299

A.1.3 Eigenvalues of Convective Flux Jacobian . . . . . . . . . . . 300

A.1.4 Eigenvectors of the Convective Flux Jacobian . . . . . . . . . . 301

A.2 Wave Propagation Along Characteristics . . . . . . . . . . . . . 303

\begin{tabular}{ll}
\hline B Convective Flux Jacobian Matrix & 307
\end{tabular}

\begin{tabular}{ll}
\hline C Complex-Step Gradient Approximation & 309
\end{tabular}

\begin{tabular}{ll}
\hline C Computation of Aerodynamic Forces & 311
\end{tabular}

\begin{tabular}{ll}
\hline Acknowledgements & 313
\end{tabular}

\begin{tabular}{ll}
\hline About the Author & 315
\end{tabular} 


\section{Chapter 1}

\section{INTRODUCTION}

\subsection{Background, Goal and Approach}

This thesis deals with a specific challenge that occurs during the design and operation of large wind turbines, more specifically horizontal axis wind turbines (HAWTs). This challenge involves the reduction of fatigue-inducing load variations on wind turbine blades by means of local, rapid changes in the aerodynamic characteristics of the blades. Changes in the aerodynamic characteristics are associated with changes in the magnitude and direction of the aerodynamic forces on the blades. The technology to accomplish this goal, sometimes referred to as 'smart rotor control', is under active investigation, and several potential options have been proposed. In the present work we define the aerodynamic effect needed for smart rotor control as 'local pitch control'.

In the present research, synthetic jet actuation has been investigated as one of the potential options for local pitch control. This is a flow-control technology that involves the repeated ingestion and subsequent ejection of air into and out of a cavity, respectively, through holes or slits in the surface of the blade or blade section. A synthetic jet is therefore generated using the available working fluid, i.e. an external source is not needed. Although there is no net mass addition to the flow system, there is a net addition of momentum. By carefully choosing the geometry of the cavity and the holes or slits, and by choosing an optimal actuation frequency and momentum of the jets, the additional momentum can be used to influence the main flow around the blade section. For example, it can be used to promote transition from laminar to turbulent flow, and delay or promote flow separation along the surface.

Although the background of the present research is the aerodynamics of wind turbine blades, the technology of synthetic jets is not limited to this field. Other turbomachinery (compressors, pumps, propellors) could also benefit from this flow control technology. Additional areas include aeronautics (airplane wings) or duct systems (inlets, diffusors).

For the accurate simulation of flows with synthetic jet actuation for different applications, it is important to consider the corresponding flow conditions. These conditions effectively dictate the minimum level of complexity of the employed flow model and also the maximum level of complexity that can be handled with the present computational resources. First of all, synthetic jet actuation is inherently unsteady. Therefore, it is important to simulate the flows time-accurately. Important dimensionless coefficients that 
can be used to assess other important flow aspects such as compressibility, viscosity and turbulence are the Mach number, M, and the Reynolds number, Re.

The Mach number is the ratio of the main flow velocity to the speed of sound in the flow, and essentially describes the importance of compressibility effects. In low Mach number flows, $\mathrm{M} \lesssim 0.3$, compressibility effects can be assumed negligible. For the flow about wind turbine blades, the Mach number is indeed low. However, in the other applications mentioned above, the range of Mach numbers is not restricted to lower values. Furthermore, compressibility effects can also become important in low Mach number flows with synthetic jet actuation, because the jet velocity can become higher than the main flow velocity. Additionally, the action of the synthetic jet can generate pressure waves. Taking compressibility into account yields the correct wave speed of these acoustic waves.

The Reynolds number is the ratio of the inertial forces in the flow to the viscous forces in the flow, and always includes a characteristic length scale. It describes the importance of fluid viscosity on this relevant length scale. In the applications considered here, the Reynolds number of the main flow is usually high. For airplane wings or the tips of wind turbine blades, the Reynolds number based on a characteristic chord length is of the order of $\operatorname{Re}_{c}=\mathcal{O}\left(10^{6}\right)$ or higher. Although it may be tempting to neglect viscous effects for flows at these high Reynolds numbers, there is always a small region along solid surfaces in the flow, the boundary layer region, in which viscous effects are essential. This is especially true in case of flow separation. Since synthetic jet actuation directly influences the boundary layer region and flow separation, viscous effects can not be neglected. However, flows at high Reynolds numbers are also associated with turbulence, i.e. highly chaotic behavior due to nonlinear inertial forces. This is associated with a large range of spatial and temporal scales. With the present computational resources it is not feasible to resolve the entire range of scales. This means that the effect of turbulence must be modeled.

Due to these considerations, in the present research a multi-purpose computational method has been developed that solves the unsteady Reynolds-averaged Navier-Stokes (URANS) equations for compressible, viscous flow. Two different linear eddy-viscosity turbulence models have been selected, which model the effect of turbulence on the mean flow. Furthermore, boundary conditions have been included that enable the simulation of flows with synthetic jet actuation.

The computational method has subsequently been used in a parameter study of synthetic jet actuation for a two-dimensional airfoil configuration, complemented with experiments carried out in a wind tunnel. The reason for considering two-dimensional flow simulations is the necessary high spatial resolution in the region of the jets and the wake of the airfoil. This in combination with the temporal resolution, leads to very high computational costs. Two-dimensional flow simulations limit the computational costs, allowing a parameter study to be carried out.

This thesis gives an introduction to wind turbine aerodynamics and smart rotor control, describes the employed flow models and the computational method, and presents the results of the parameter study of synthetic jet actuation. In particular, the effects on pitch control of the actuation frequency, actuation amplitude, and location of the synthetic jet 
are considered.

\subsection{Thesis Outline}

In chapter 2, a brief overview is presented of modern horizontal axis wind turbines and the necessity to reduce blade load variations. Then, an introduction into wind turbine aerodynamics is given, followed by the present state-of-the-art of load control mechanisms, and the options for smart rotor control. The chapter ends with a discussion of the technology of synthetic jets.

In chapter 3 the mathematical description of unsteady, compressible, viscous flows is presented, i.e. the Navier-Stokes equations. Then the necessity of turbulence modeling is explained in more detail and the derivation of the unsteady Reynolds-Averaged NavierStokes (URANS) equations is given. Closure approximations are presented and two eddyviscosity turbulence models are selected. The chapter ends with a description of the necessary boundary conditions and the approach taken to model synthetic jet actuation.

In chapter 4, a detailed description is given of how the mathematical models are solved numerically. The combination of the mathematical models and their numerical solution procedure is called the computational method.

In chapter 5, the correct implementation of the mathematical models is verified. Furthermore, the degree to which the computational method is able to accurately simulate the physics of real flows is investigated and discussed. For this purpose, benchmark experiments from the literature on boundary layer separation control by means of synthetic jet actuation are used. This is known as validation.

Chapter [deals with the problem of synthetic jet actuation on a two-dimensional airfoil configuration for pitch control. The involved parameters are identified, the experimental setup is presented, and the results of a parameter study are presented.

The thesis ends with chapter 7 in which the conclusions of the present research are formulated and recommendations for further study are given. 



\section{Chapter 2}

\section{Smart Rotor Control For Horizontal Axis Wind Turbines}

\subsection{Horizontal Axis Wind Turbines}

A wind turbine allows for the conversion of wind power to electric power, via the mechanical power of a rotating shaft. The wind passing the blades of the wind turbine induces aerodynamic forces on these blades. These forces (primarily the lift component) are responsible for the torque on the shaft, and thereby the useful extraction of power from the wind, and for an axial force, i.e. the thrust.

The blades are connected to the hub, which are jointly called the rotor. Most modern HAWTs have a three-bladed, pitch-regulated, upwind-directed rotor, which is connected to a drive train that drives a generator. The drive train, generator and other additional systems are fixed to a mainframe and are located inside the nacelle. The nacelle is located on top of the tower, which is fixed to a foundation. Figure 2.1 shows a sketch of this configuration.

A wind turbine relies on a control system to successfully produce electric power. Its purpose is to satisfy multiple requirements in a balanced fashion. The three most important requirements are, see Manwell et al. [1]:

1. setting upper bounds on the torque and power experienced by the drive train,

2. maximizing the fatigue life of the rotor, drive train and other structural components in the presence of changes in wind direction and speed, turbulence, as well as startstop cycles of the wind turbine,

3. maximizing the energy production.

The first requirement is dealt with by modifying the torque generated by the rotor (aerodynamic torque control) and/or modifying the resistance of the generator (generator torque control). This depends on the mean wind velocity, and the envelope of operating wind speeds, associated with a minimum (cut-in), normal operation (rated) and maximum (cut-out) wind speed.

The second requirement is associated with loads, i.e. forces and moments, and variations in loads, i.e. fatigue loads. Besides aerodynamic loads, there are loads due to 


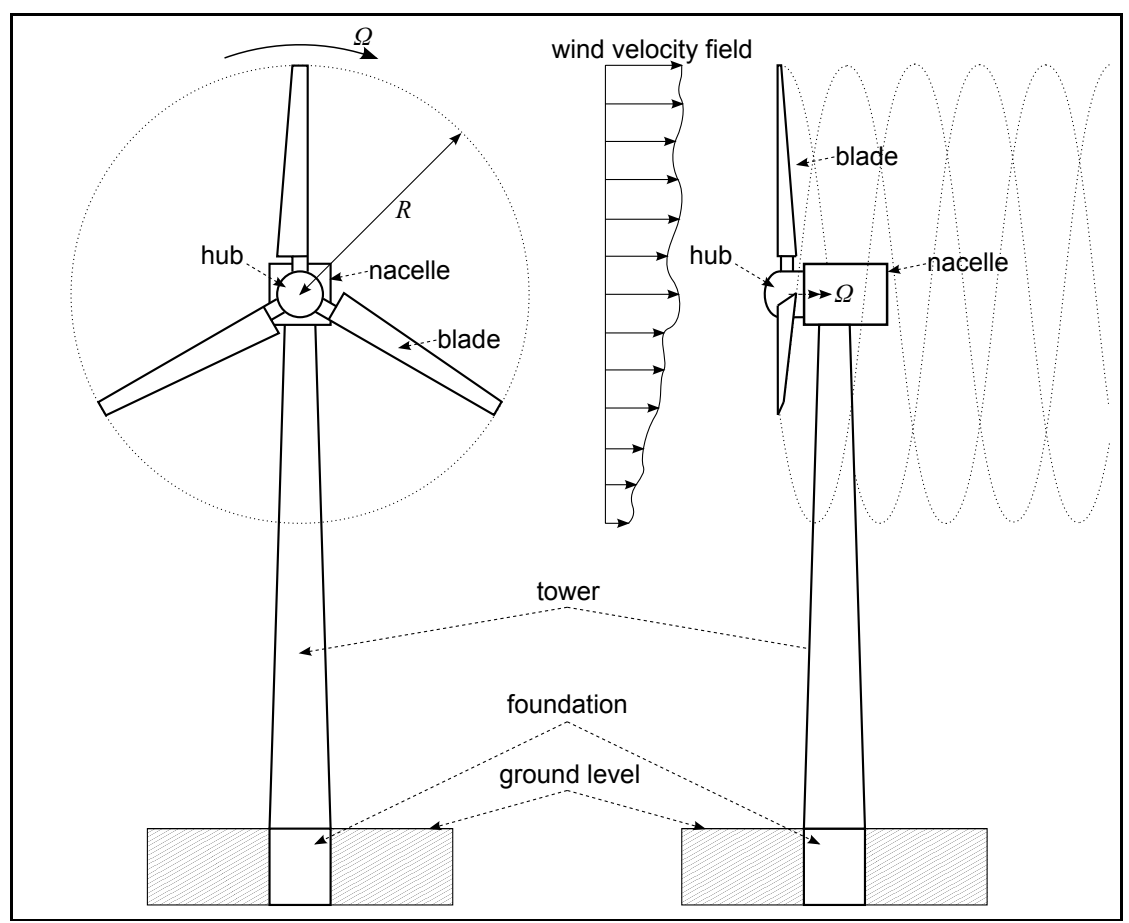

Figure 2.1: Sketch of three-bladed upwind-directed horizontal axis wind turbine, frontal view (left) and side view (right) with an impression of a wind velocity field and the helical structure of the tip vortices.

gravity and due to inertia, i.e. accelerations. Note that control actions may also cause variations in loads. The wind turbine must withstand certain ultimate loads, which can occur in extreme circumstances, but also a certain spectrum of load variations, which occur at normal operating conditions. Large modern wind turbines are known as 'fatigue critical machines', i.e. the design of many components is driven by the load variations they endure [2]. This is due to a unique load spectrum and the large number of load cycles during the lifetime of a wind turbine, which is between 20 and 30 years. For a list of different load cases and wind conditions that must be considered during design, see the International Electrotechnical Commission's standard IEC 61400-1 [3]. This standard prescribes cases for normal operation, including turbulence, as well as cases at extreme conditions such as extreme gusts, operation in the wake of another turbine, start/stop sequences and parking conditions in combination with extreme wind velocities.

Note that load variations can excite natural frequencies of wind turbine components, such as the blades, tower and drive train. Each natural frequency is associated with a characteristic mode shape. For a wind turbine blade, these modes can be [1] flapwise modes, i.e. bending motions out of the rotor plane, edgewise modes, i.e. bending motions 
in the rotor plane, also known as lead-lag motions, and torsional modes, i.e. motions about the pitch axis. Furthermore, when the frequency ratio between a flapwise mode and a torsional mode is close to unity $\sqrt{1}$, a self-sustained oscillation (flutter) can arise, see for example Hansen [4]. The components of a wind turbine are designed such that no natural frequencies are excited under normal operating conditions, or that these frequencies are actively or passively damped in case they are excited.

The third operating requirement, see page 5, is used once a particular wind turbine design is in operation. During the design and optimization stage of a wind turbine, however, maximizing the energy production (for a given wind climate) is no longer used as a criterion, but rather the minimization of the cost of energy, see Snel [5] for example. Wind turbines designed with this criterion have blades that may have less aerodynamic efficiency, but possess an increased manufacturability and a decreased load spectrum, which reduces the amount of materials needed and increases the reliability and life span of the whole wind turbine. So, ultimately, a less efficient blade design can still lead to lower cost of energy.

Costs can be divided in capital costs, operation and maintenance costs, and financing costs [1. A typical breakdown of capital costs is shown in figure 2.2 for a reference 1.5 $\mathrm{MW}$ onshore wind turbine (a) and a reference 3.6 MW offshore wind turbine (b), see Tegen et al. [6]. In this case, the total capital costs are approximately $1600 € / \mathrm{kW}$ for the onshore and $4200 € / \mathrm{kW}$ for the offshore wind turbine (price level 2010). Figure 2.2 shows that for an onshore wind turbine, the turbine itself has the largest contribution to the capital costs, which is directly related to the amount of material needed. For an offshore wind turbine, the contributions of the support structure (foundation) and installation costs also become a major part of the total capital costs.

For more than 30 years, large reductions in capital costs have been achieved, which are coupled with increases in turbine performance through more advanced turbine components and larger turbines (rotor size and tower height) [7]. Note that rotor power scales with $R^{2}$, i.e. the square of the rotor radius. The relatively large contributions of the support structure and the installation to the capital costs of offshore wind turbines do not depend strongly on the size of the rotor [5], which leads to an even more pronounced trend towards larger rotors.

Examples of very large turbines that are currently being installed are the Siemens SWT-6.0-154, a 6.0 MW offshore wind turbine with a rotor diameter of $154 \mathrm{~m}$, and the Enercon E-126, a 7.5 MW onshore wind turbine with a rotor diameter of $126 \mathrm{~m}$.

The downside of naively increasing the size of a given rotor is the increase in weight, which is scaled with the rotor radius cubed, $R^{3}$. In case the stresses are kept constant during upscaling, self-weight effects lead to an exponent even higher higher than 3 , see Sieros et al. [8]. This will have a major impact on the capital costs of many wind turbine components. A way to avoid this problem is the development of new advanced control mechanisms that are able to decrease the (amplitude of) fatigue loads on the blades. This reduces the structural requirements for the blades and allows for larger rotors that are relatively lighter. Furthermore, a reduction of the fatigue loads will reduce the structural

\footnotetext{
${ }^{1}$ The second flapwise mode is usually interacting with the first torsion mode [4].
} 


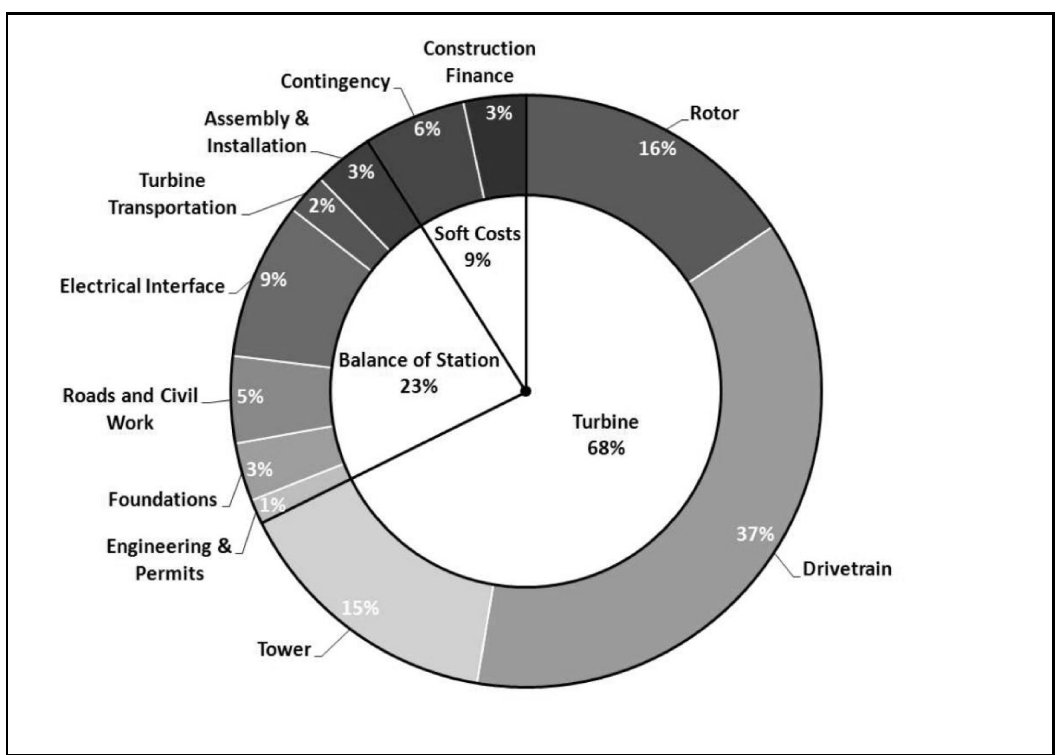

(a) 1.5 MW onshore wind turbine

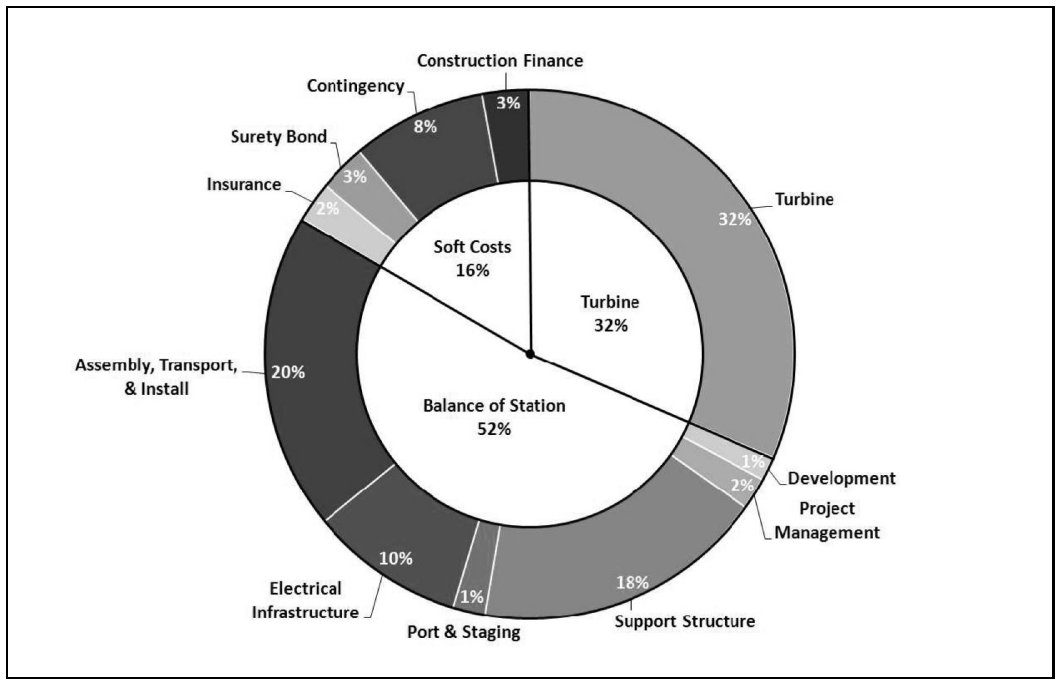

(b) $3.6 \mathrm{MW}$ offshore wind turbine

Figure 2.2: Typical breakdown of wind turbine capital costs, from Tegen et al. [6].

requirements of other components of the wind turbine, such as the drive train and the tower, and increase the reliability and reduce the maintenance of the wind turbine as well, see Barlas \& Van Kuik [9]. The combination of increased energy production and 
the relatively lower capital and maintenance costs will therefore decrease the cost of wind energy production.

For the present investigation it is important to note that the natural frequencies of wind turbine blades approximately scale with the inverse of the rotor radius, i.e. $R^{-1}$, see Manwell et al.[1]. When the tip speed ratio, see section 2.2 is held constant, the angular velocity of the rotor also scales as $R^{-1}$, such that the tendency to excite a resonance frequency is independent of the rotor size.

These considerations show that the design and optimization of wind turbines is a complex multi-disciplinary problem, involving aerodynamics and structural dynamics, electrodynamics and control, material science, wind climate conditions and economics. Some aerodynamic aspects are presented in the following section.

\subsection{Aerodynamics of Horizontal Axis Wind Turbines}

The main dimensionless coefficients characterizing the global aerodynamic operation of a wind turbine are the power coefficient $C_{P}$ and the thrust coefficient, $C_{T}$, see for example Sørensen [10]. They are defined as:

$$
\begin{aligned}
C_{P} & =\frac{P}{\frac{1}{2} \rho U_{0}^{3} A}, \\
C_{T} & =\frac{T}{\frac{1}{2} \rho U_{0}^{2} A},
\end{aligned}
$$

where $P$ is the extracted power by the rotor, $T$ is the axial force (thrust) on the rotor, $\rho$ is the density of the air, $A$ is the rotor area and $U_{0}$ is the average wind speed upstream of the rotor.

Since the extracted power equals the integral of the tangential force on the blades times the local tangential velocity, the velocity of the blades is also important. It is usually expressed in terms of the tip speed ratio, $\lambda$, which is a dimensionless parameter defined as

$$
\lambda=\frac{\Omega R}{U_{0}}
$$

where $\Omega$ is the angular velocity of the rotor and $R$ is the radius of the rotor. Large modern wind turbines have a typical tip speed ratio between 6 and 8 [1]. Note that for the same amount of extracted power, the necessary (tangential) forces on the blades and therefore the torque produced by the rotor are smaller for higher tip speed ratios. This means that lighter constructions can be employed. However, the disadvantage of higher tip speed ratios is the increased noise emission. The sound power scales with at least the fifth power of the effective inflow velocity, see Oerlemans [11] for example.

The wake of a wind turbine blade is characterized by a vortical shear layer or vortex sheet, with concentrated vortices at the root and tip of the blades. An impression of the tip vortices is shown in figure 2.1. Usually a distinction is made between trailed and shed vorticity [5]. Trailed vorticity is directed into the wake, normal to the trailing edge of 
the blade, and is related to the distribution of the aerodynamic forces along the blade in spanwise direction. Shed vorticity is directed along the blade in spanwise direction and is related to changes of the aerodynamic forces in time, see also subsection 2.3.2.

\subsubsection{Actuator Disk Model}

A model by Betz [12] can be employed to estimate $C_{P}$ and $C_{T}$ under simplified circumstances 2 . It is based on one-dimensional integral conservation laws of mass and axial momentum applied to a streamtube enclosing the rotor, in which the rotor is represented by an actuator disk, see figure 2.3 . It follows that

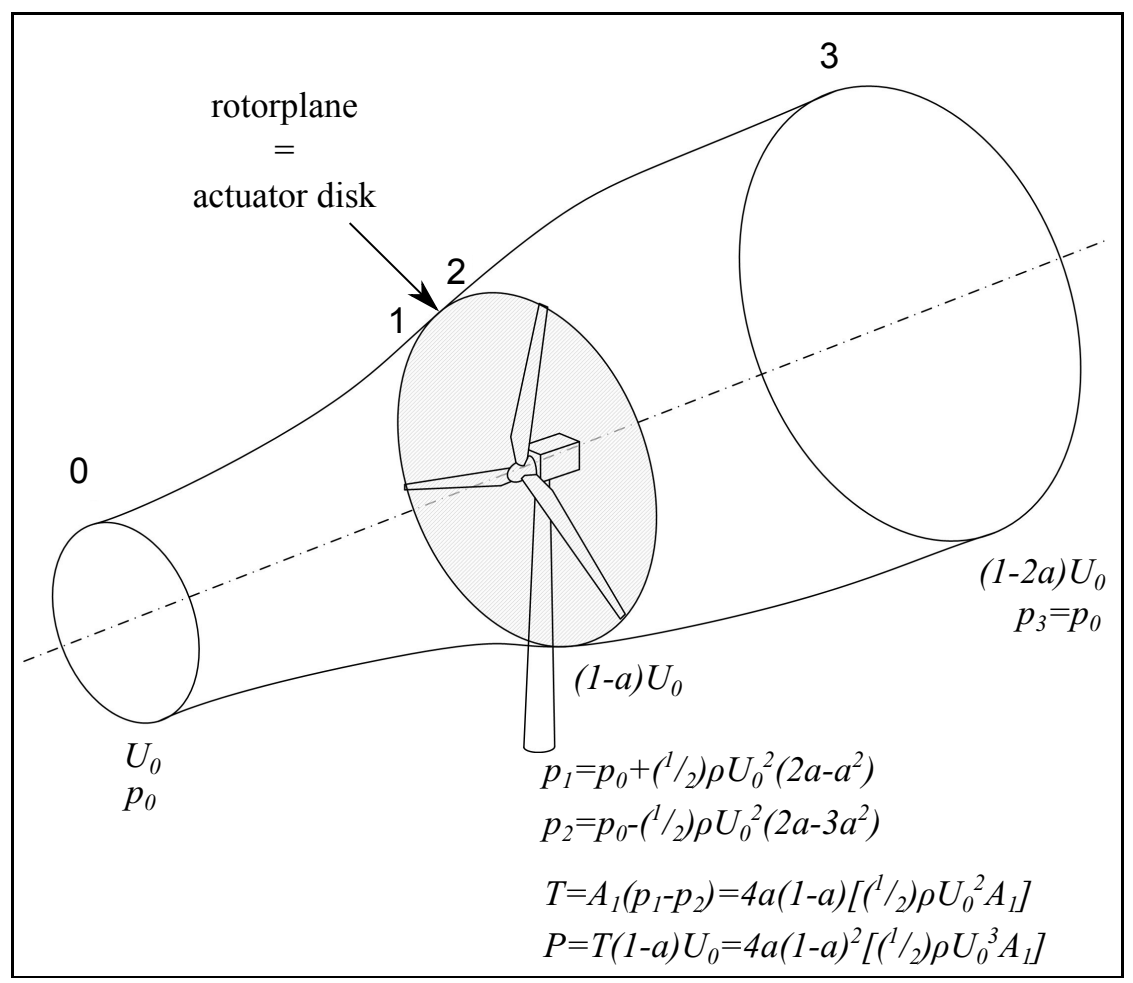

Figure 2.3: Sketch of streamtube passing actuator disk, with results for axial velocities and pressures from actuator disk model. The parameter $a$ is the axial induction factor.

\footnotetext{
${ }^{2}$ The simplifications are [1]: uniform, incompressible, steady, inviscid flow; infinite number of blades, uniform thrust over the actuator disc; non-rotating wake. Furthermore, it is assumed that there is no net axial pressure force due to the pressure distribution on the external streamtube and that there are no external radial forces on the flow [13].
} 


$$
\begin{aligned}
& C_{P}=4 a(1-a)^{2}, \\
& C_{T}=4 a(1-a),
\end{aligned}
$$

where $a=1-\frac{u_{a x}}{U_{0}}$ is the axial induction factor, with $u_{a x}$ the axial velocity in the rotor plane. From these relations, it can be determined that the maximum amount of extracted power from the wind occurs when the velocity of the air in the rotor plane is $\frac{2}{3} U_{0}$, i.e. when $a=1 / 3$, and equals $C_{P}=16 / 27 \approx 0.593$, i.e. less than $60 \%$ of the total available amount of power. This is known as the Betz limit. For this ideal case the thrust coefficient equals $C_{T}=8 / 9 \approx 0.889$. Furthermore, it can be shown that when the power coefficient is reduced by $1 \%$ from its theoretical maximum, the thrust coefficient reduces by $5 \%$ [5]. Since reduced thrust leads to lower structural requirements and thereby lower capital costs, it shows that the minimization of the cost of energy does not equal the maximization of energy production. Note that the Betz limit is valid under ideal circumstances, i.e. the maximum amount of extracted power is lower due to losses induced by the rotation of the wake, a finite number of blades, and drag. However, the inclusion of other neglected effects can also slightly increase the maximum amount of extracted power above the Betz limit, as discussed by Van Kuik [14] for example.

\subsubsection{Blade Element Momentum Model}

An engineering method that allows for rapid computations of the dynamic behavior of a wind turbine under different load and wind conditions is the Blade Element Momentum (BEM) method. It is based on:

- one-dimensional integral conservation laws (mass, axial momentum and angular momentum) applied to annular slices at radial position $r$ with width $d r$ of a streamtube passing the actuator disk, see Glauert [15] and figure 2.4. The result can be expressed as the combined axial forces $f_{a x}$ and tangential forces $f_{\text {tan }}$ (per unit span) on the blades in each annular slice of the rotor as a function of the local axial induction factor $a(r)=1-u_{a x}(r) / U_{0}$, the local angular induction factor $a^{\prime}(r)=u_{\text {tan }}(r) / \Omega r$, and the local speed ratio, $\lambda_{r}=\Omega r / U_{0}=\lambda r / R$. Here, $u_{a x}$ is the axial velocity in the rotor plane, $U_{0}$ the axial velocity far upstream of the rotor, $u_{t a n}$ the induced tangential or azimuthal velocity in the rotorplane and $\Omega$ the angular velocity of the rotor. The tangential force per unit span, $f_{t a n}$, causes a moment about the rotational axis of the rotor, i.e. the torque.

- blade element theory, which considers aerodynamic forces on blade sections or elements at radial position $r$, with width $d r$, due to two-dimensional flow passing each blade section, i.e. without influence of the rest of the blade. This also gives expressions for the previously mentioned axial and tangential force on the blades in each annular slice of the rotor, see figure 2.4

- several (semi-)empirical corrections that account for three-dimensional flow effects (such as stall delay due to radial flow, observed on inboard blade sections), dynamic stall, tip-loss effects (important for a finite number of blades instead of the infinite 
number of blades with which the actuator disk model is associated), turbulent wake state effects (for heavily loaded rotors) and effects of yaw misalignment. See Snel [13] for a discussion on this subject.

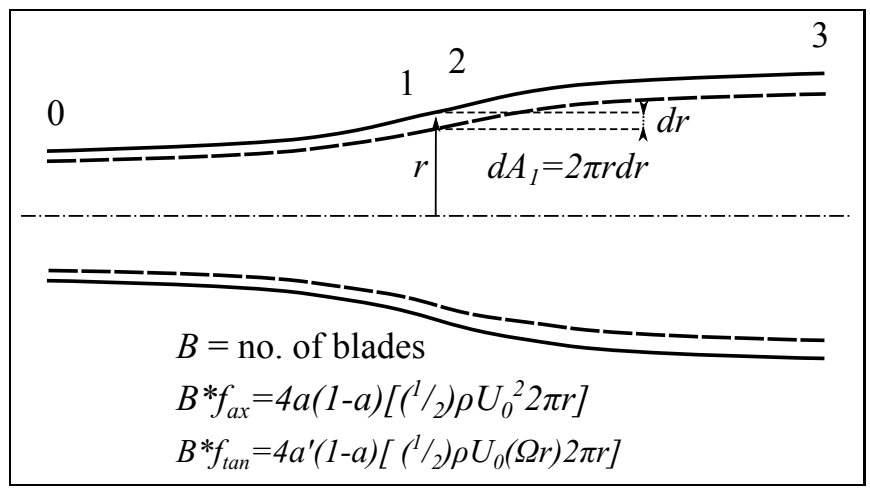

Figure 2.4: Cross-section of annular streamtube passing actuator disk, with results for axial and tangential forces per unit span from blade element momentum model.

Figure 2.5 shows a blade element, which has a certain cross-sectional shape and chord length, an inflow angle, $\varphi$, and an effective inflow velocity, $U_{\text {eff }}$. The inflow angle is the angle between the effective inflow velocity and the rotor plane. It equals the sum of the angle of attack, $\alpha$, and the total pitch angle, $\theta_{p t}$. The latter consists of the global pitch angle of the blade, $\theta_{p}$, and the local twist angle, $\theta_{t w}$. The effective inflow velocity depends on the wind speed far upstream of the rotor, $U_{0}$, the local speed ratio, $\lambda_{r}$, and the induced axial and tangential velocities.

The induced axial and tangential velocities, $a U_{0}$ and $a^{\prime} \Omega r$, respectively, are due to the presence of trailing vorticity in the wake downstream of the wind turbine. A positive $a$ causes a reduction in the axial velocity, whereas a positive $a^{\prime}$ causes an increase in the tangential (azimuthal) velocity.

As shown in figure 2.5] the resulting aerodynamic force (per unit span) on the blade element, $f$, can either be resolved in an axial and a tangential component, $f_{a x}$ and $f_{\text {tan }}$, respectively, or in a lift and drag component, $l$ and $d$, respectively. The lift force is perpendicular to the effective inflow velocity and the drag is parallel to the effective inflow velocity.

The two-dimensional (sectional) aerodynamic performance of a blade element, used in the blade element theory, is determined from experiments or pre-computed flow simulations (look-up tables). For more information on sectional aerodynamic performance, see section 2.3 .

Since the BEM method is very fast in computing the aerodynamic loads, it can be coupled to a structural solver for the rotor and other components of the wind turbine. Furthermore, a stochastic wind simulator and control algorithms that adjust settings of the control mechanisms can be included. The simplest structural model of a wind turbine 


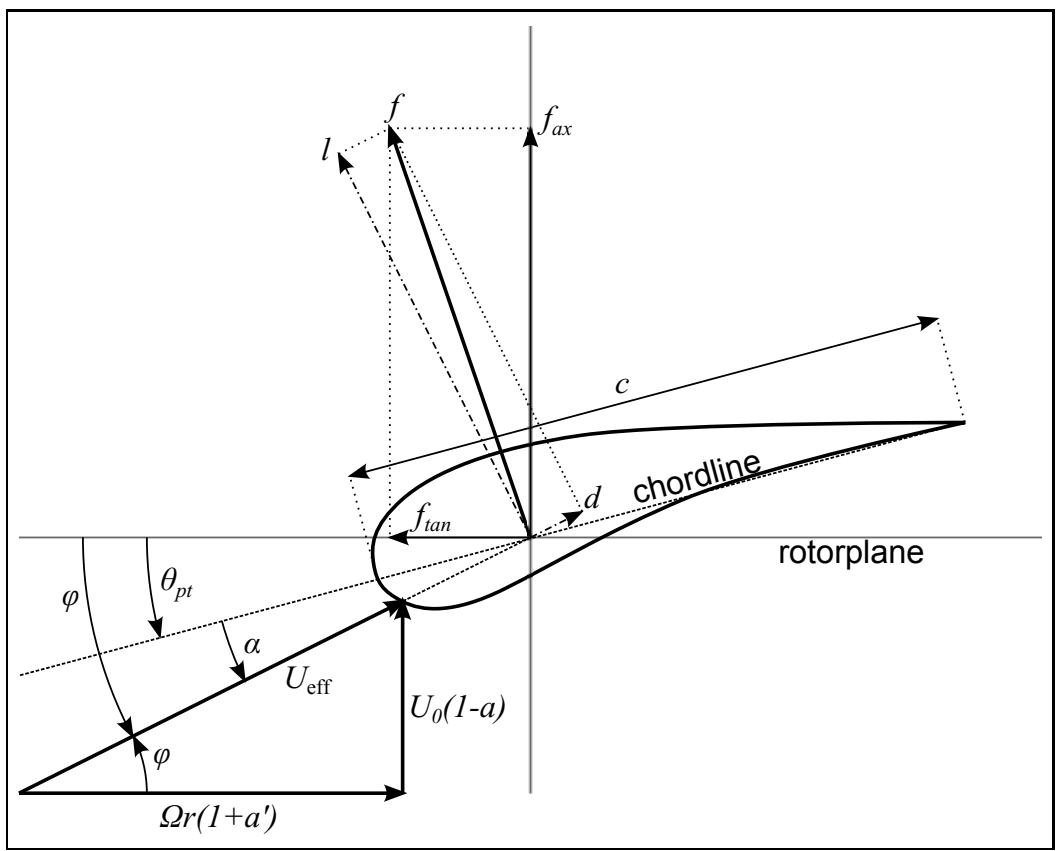

Figure 2.5: Blade element with chord length $c$ at radial position $r$, with relative inflow velocity $U_{r e l}(r)$, relative inflow angle $\varphi(r)$, and aerodynamic force $f(r)$.

blade is a rigid beam connected to the hub by an ideal spring-hinge. More advanced models divide the turbine into several rigid and flexible bodies. Flexible bodies are modeled either using beam theory, with structural couplings between flapwise bending, edgewise bending and torsion included, or through finite element analysis.

The resulting aeroelastic method is known to have deficiencies, see for example Schepers et al. [16]. This reference presents the results of a verification and validation exercise for European wind turbine design codes, in which load predictions from these codes were compared mutually and with measurements, for different operating conditions. The results show a spread in predicted blade fatigue loads up to $+/-15 \%$, in mean blade loads up to $+/-5 \%$ or $+/-10 \%$ in some cases, and in loads on other components up to $+/-20 \%$ or $+/-30 \%$ in some cases. Furthermore, the differences between calculated and measured mean blade loads are also of the order of 5 to $10 \%$ and between calculated and measured loads on other components of the order of 10 to $40 \%$.

Despite these uncertainties, the BEM method is frequently used during the design and certification of wind turbines, due to its simplicity and computational efficiency. However, there is an ongoing shift towards employing more sophisticated aerodynamic models to provide airfoil data and to give more insight in the physics of the flow around rotating blades. These models range from lifting-line wake models to models that solve some filtered form of the Navier-Stokes equations, using either generalized actuator disc 
hypotheses or full rotor simulations [10].

\subsection{Airfoil Characteristics}

\subsubsection{Steady Aerodynamics}

It is common to express the sectional aerodynamic performance in three non-dimensional coefficients, which are a function of angle of attack, $\alpha$, and Reynolds number, $\operatorname{Re}_{c}=$ $\frac{\rho_{\infty} U_{\infty} c}{\mu}$. The Reynolds number is the ratio of inertial forces to viscous forces. The coefficients are defined as

- lift coefficient:

$$
c_{l}=\frac{l}{\frac{1}{2} \rho_{\infty} U_{\infty}^{2} c}
$$

- drag coefficient:

$$
c_{d}=\frac{d}{\frac{1}{2} \rho_{\infty} U_{\infty}{ }^{2} c},
$$

- pitching moment coefficient:

$$
c_{m}=\frac{m}{\frac{1}{2} \rho_{\infty} U_{\infty}{ }^{2} c^{2}},
$$

where $l$ and $d$ are the lift and drag force per unit span, respectively, $m$ is the moment per unit span (about the quarter chord point for example), $\rho_{\infty}$ is the free-stream density of the flow, $U_{\infty}$ is the free-stream velocity of the flow, and $c$ is the chord length of the blade section. When these coefficients are used in the BEM method, $U_{\infty}$ should be interpreted as $U_{\text {eff }}$.

Typical behavior of the lift and drag coefficient can be seen in figure 2.6, for the NREL S809 airfoil, as measured in the low-turbulence wind tunnel of the Delft University of Technology, see Somers [17]. Here, $c_{l}$ and $c_{d}$ are plotted as a function of $\alpha$ for two different Reynolds numbers. For angles of attack in the linear $c_{l}(\alpha)$-regime, an increase of the angle of attack is associated with an increase of the lift force on the airfoil. Stall occurs at higher angles of attack, when the flow over the upper (suction) side of the airfoil separates. It is associated with a considerable decrease in the lift over drag ratio, $l / d$, in a time-averaged sense, due to some loss of lift and a strong increase in drag. Since stall is a highly unsteady flow phenomenon, both the lift and the drag force fluctuate, however.

From thin airfoil theory, see for example Anderson [18], valid for thin airfoils in steady, incompressible, inviscid, irrotational flows, it follows that the slope of the curve for the lift coefficient equals

$$
\frac{d c_{l}}{d \alpha}=2 \pi \text { per radian } \approx 0.11 \text { per degree. }
$$




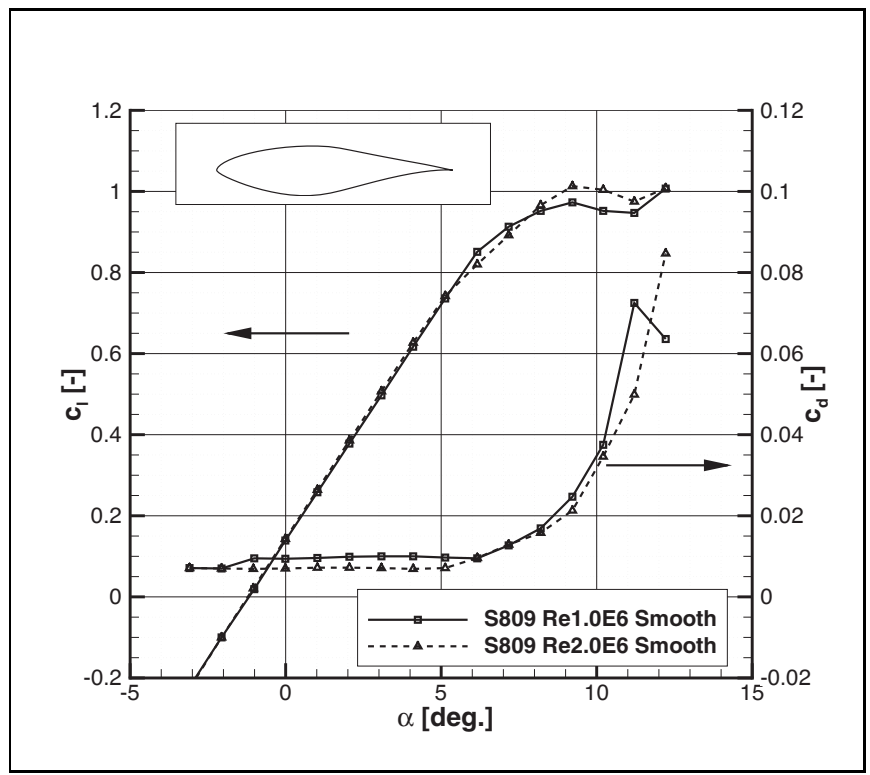

Figure 2.6: Lift coefficient, $c_{l}$, and drag coefficient, $c_{d}$, as function of the angle of attack, $\alpha$, for the NREL S809 airfoil [17].

It can be seen in figure 2.6, that the slope of the lift curve for the S809 airfoil closely follows the theoretical value in the linear regime, even though its relative thickness is $21 \%$. For this airfoil, the ratio of lift to $\mathrm{drag}, l / d$, is maximal at approximately $\alpha=6^{\circ}$. Below this angle of attack, the drag is approximately constant, whereas it increases above this angle of attack.

\subsubsection{Unsteady Aerodynamics}

Airfoil characteristics as shown in figure 2.6 are valid for steady flows only, however. When the angle of attack suddenly increases, which can occur on a rotating wind turbine blade due to a sudden increase of the wind speed, $U_{0}$, for example, the change in the aerodynamic force is not instantaneous, but a function of the dimensionless convective time $U_{\infty} t / c$. The effect is caused by the shedding of a so-called starting vortex. This vorticity is directed in spanwise direction. For a positive change in the angle of attack, $\Delta \alpha$, the starting vortex creates a downwash that partly counteracts the sudden increase in the angle of attack. This means that the total increase in lift, for example, is not immediately attained. As the starting vortex convects away from the airfoil, its effect on the lift diminishes in time and the steady state increase in lift is attained asymptotically.

\footnotetext{
${ }^{3}$ This is because the ignored effects of thickness, which causes an increase in the slope, and viscosity, which causes a decrease in the slope, appear to cancel each other to some extent for many airfoils.
} 
A model for this effect, based on potential flow theory, has been developed by Wagner [19], see also Leishman [20]. It describes the effect of the starting vortex on an impulsively started flat plate in inviscid, incompressible flow by the Wagner function, here denoted as $\phi_{w}(t)$. The indicial response can be written in terms of a step change in the angle of attack, $\Delta \alpha$, at time $t=t_{0}$, as

$$
c_{l}(t)=c_{l}\left(t_{0}\right)+2 \pi \Delta \alpha \phi_{w}\left(t-t_{0}\right) .
$$

The Wagner function states that the instantaneous change in lift, directly following the step change in the angle of attack, is equal to half the steady state value. Since the Wagner function is not in a convenient analytic form, an approximation by Jones [21] is often used, which agrees with the exact solution of the Wagner function to an accuracy within $1 \%$ :

$$
\phi_{w}(t)=\left\{\begin{array}{cc}
0 & \text { for } t<0 \\
1-A_{1} e^{-2 b_{1}\left(U_{\infty} t / c\right)}-A_{2} e^{-2 b_{2}\left(U_{\infty} t / c\right)} & \text { for } t \geq 0
\end{array},\right.
$$

with $A_{1}=0.165, A_{2}=0.335, b_{1}=0.0455, b_{2}=0.3$. Note that the effect of arbitrary unsteady motion can be modeled as a superposition of many small impulsive changes in angle of attack.

Figure 2.7 shows equation (2.11) plotted as a function of the convective time $U_{\infty} t / c$. It can be seen that $\phi_{w} \rightarrow 1$ for $U_{\infty} t / c \rightarrow \infty$ and that $95 \%$ of the total response is

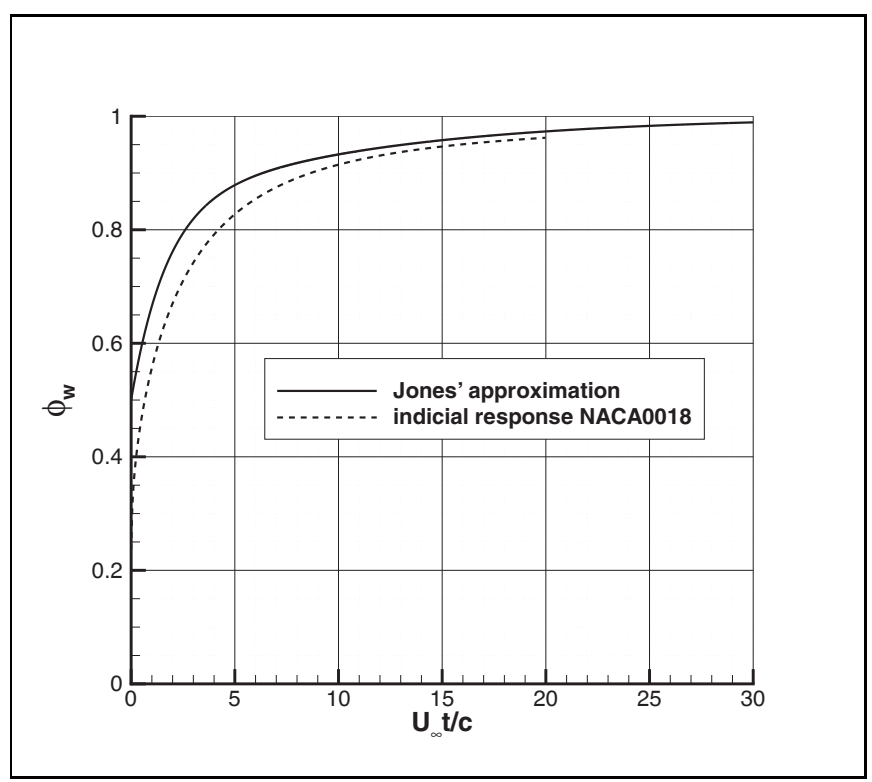

Figure 2.7: Jones' approximation to Wagner function for indicial response of flat plate and indicial response of NACA0018 airfoil, as a function of convective time $U_{\infty} t / c$. 
obtained within $t=15 c / U_{\infty}$.

It was observed by Chow \& Huang [22], Gaunaa [23] and Gaunaa, Bergami \& Heinz [24] that airfoil thickness (and thickness distribution) influences the indicial response: thicker airfoils have a slower response and a lower value of the instantaneous change in lift. This is shown in figure 2.7 with a dashed line4, for the NACA0018 airfoil (symmetric, $18 \%$ thickness). This response has been computed with a potential flow method using panels with constant-vortex distributions, constant-source distributions and twodimensional point vortices in the wake [23].

To get an idea of the convective timescales, $c / U_{\text {eff, }}$, for a typical large wind turbine blade, we can consider the NREL 5MW reference offshore wind turbine [25]. This turbine has a rotor radius of $R=63 \mathrm{~m}$ and a rated rotor speed of 12.1 rotations per minute, i.e. $\Omega=1.27 \mathrm{rad} / \mathrm{s}$. The rated wind speed is $11.4 \mathrm{~m} / \mathrm{s}$, such that the tip speed ratio equals $\lambda=7$. The chord length at the tip of the blades is $c_{\text {tip }}=1.419 \mathrm{~m}$, which leads to a convective timescale of $c / U_{\text {eff }} \approx 0.02 \mathrm{~s}$. The longest chord length is $c_{\mathrm{R} / 4}=4.652 \mathrm{~m}$ at $r=0.25 R$, which leads to a convective timescale of $c / U_{\text {eff }} \approx 0.2 \mathrm{~s}$.

Note that on a rotating wind turbine blade, an additional unsteady behavior occurs, usually denoted by the term 'dynamic inflow'. It is associated with a time delay in the change in the induced velocities due to changes in the trailed vorticity in the wake, see Snel \& Schepers [26]. A negative change in pitch angle, for example, increases the angle of attack, but also increases the induced velocities after some time. The initial increase in the angle of attack is then partly counteracted. The time delay is associated with the wake traveling a distance of 2 to 4 rotor diameters downstream of the rotor, see Schepers [27]. Thus, the time scale is approximately 4 to 8 times $R / U_{0}$. For the NREL reference wind turbine, this is of the order of $20 \mathrm{~s}$ to $40 \mathrm{~s}$ at the rated wind speed. Therefore, the timescale of the dynamic inflow effects is much larger compared to the timescale of the effects of shed vorticity.

\subsection{Aerodynamic Control}

To satisfy the operating requirements, control systems are needed that obtain information from sensors and act on this information by adjusting the settings of several components and control mechanisms. Those that influence the aerodynamic properties of the wind turbine include:

- a yawing mechanism that enables the nacelle to rotate with respect to the tower, such that the rotor can be directed normal to the average wind direction,

- pitching mechanisms between the hub and each blade that enables the blades to rotate around their main axis (pitch), such that the angle of attack of the blades to the effective inflow velocity can be adjusted and thereby the magnitude and direction of the aerodynamic forces.

\footnotetext{
${ }^{4}$ Data obtained from M. Gaunaa through private communication.
} 
The pitching mechanisms can be operated in different ways [1]. The following concepts can be used additively, with each layer increasing the complexity:

- The basic concept is collective pitch control, in which all blades have the same pitch angle that can vary in time. This is used for power regulation and also allows for reducing load variations due to changes in average wind speed.

- Secondly, in cyclic pitch control, the pitch angle of each blade is a predefined function of the azimuth angle. This allows for the reduction of load variations of a cyclic nature, usually an integer load variation per revolution denoted by ' $P$ '. For example, wind shear causes a 'once per revolution' or $1 \mathrm{P}$ load variation on a blade. Therefore, the drive train, nacelle and tower of a three-bladed wind turbine experience a 3P load variation due to wind shear. Usually, higher harmonics of these frequencies are also present. Other cyclic load variations are caused by the weight of the blades, i.e. gravity, yaw misalignment and hydrodynamic interaction upon blade passage in front of the tower.

- Finally, in individual pitch control, the pitch angle of each blade is set independently based on additional sensors, which allows for the reduction of stochastic load variations, see Bossanyi [28]. A source of stochastic load variations is turbulence in the air passing the blades of the wind turbine.

In a comparison between these three concepts, see Larsen et al [29], it was shown that the reduction of flapwise fatigue loads at the root of the blades can be reduced by $15 \%$ when using cyclic pitch control and by $28 \%$ when using individual pitch control.

Individual pitch control is the present state of the art way to reduce blade load variations. However, the bandwidth of the blade pitch actuators is too narrow to be able to deal with higher-than $1 \mathrm{P}$ load variations. Typically, the maximum attainable pitch speed of a large blade is approximately $8 \%$ s. Moreover, continuous use of the pitching mechanisms close to their limitations can lead to excessive wear of these mechanisms. Furthermore, as rotor sizes increase with respect to the typical sizes of turbulent eddies, the importance of turbulent wind speed variations across the rotor disc increases [28. Pitching the entire blade becomes therefore less practical.

To get an idea of the limits of the blade pitching mechanisms, we can again consider the NREL 5MW offshore wind turbine [25]. The rated rotor speed of 12.1 rotations per minute leads to a $1 \mathrm{P}$ frequency of $0.2 \mathrm{~Hz}$. Focusing on this frequency alone, we further assume a sinusoidal time-dependent pitch angle, $\theta_{p}=\Theta \sin (2 \pi f t)$, with pitch amplitude $\Theta$ and pitch frequency $f$. The maximum pitch speed therefore equals $2 \pi f \Theta$. Using a value of $8^{\circ} / \mathrm{s}$ yields a maximum attainable change in pitch angle for $1 \mathrm{P}$ load alleviation of approximately $\pm 6.4^{\circ}$. The typical maximum attainable change in lift coefficient in attached flow is then approximately $\Delta c_{l} \approx \pm 0.7$.

A potentially more effective way to deal with turbulent wind speed variations would be a sequence of local load control mechanisms, distributed along the span of the wind turbine blade. When operated individually, i.e. acting on local sensory information, they are able to change the local aerodynamic properties of the blades. Such a combination of 
control mechanisms is referred to as 'smart rotor control'. This technology is discussed in the next section.

\subsection{Smart Rotor Control}

Control techniques can be categorized in passive and active techniques. Passive techniques for fatigue load alleviation on wind turbine blades can be based on the coupling of elastic deformation modes by careful selection of composite fiber orientation. For example, in bend-twist coupling, increased bending of the blade due to increased wind speed induces an extra twist of the blade (along the pitch axis), such that the increase in the angle of attack and lift force is limited [30, 31]. In tension-twist coupling, the same is achieved when blades are elongated due to centrifugal forces.

Passive techniques do not require sensors and controllers and are therefore often inherently simpler than active techniques. Furthermore, passive control techniques tend to be lighter, less expensive to design and manufacture, and easier to maintain [32]. However, they can also not be 'turned off' and could have negative effects in some operating conditions. Furthermore, active control techniques have more flexibility than passive control techniques in dealing with unsteady flow behavior. In the remainder of this chapter, we will focus on active aerodynamic control techniques.

Smart rotor control is an active research field throughout the world. For example, this topic was included in the work package 'Smart Rotor Blades and Rotor Control' in the recent European Union funded project called 'UPWIND 5, which investigated the necessary technology for very large wind turbines (8-10 MW). Furthermore, a special issue of the journal 'Wind Energy' on smart blades has been published in 2010 [33. The following subsections describe the requirements and the potential options for smart rotor control. It is mainly based on results and conclusions from other studies and will facilitate the assessment of synthetic jet actuation, the topic of the present research, later on in this thesis.

\subsubsection{Requirements}

In terms of aerodynamic performance, novel smart rotor control techniques should be able to rapidly generate changes in the local aerodynamic force, most importantly the lift component. These changes should be generated over a range of angles of attack in the linear $c_{l}(\alpha)$-regime. This is because the outboard blade sections of a pitch regulated wind turbine operate in attached flow conditions. Due to the large distance to the blade root, generating changes in the lift force at the outboard blade sections will have a large impact on the fatigue loads. Figure 2.8 shows a schematic representation of the required effect of smart rotor control. Since changes in the lift coefficient are associated with changes in the (effective) angle of attack or pitch angle, we define the aerodynamic effect needed

\footnotetext{
${ }^{5}$ See www.upwind.eu for more information.
} 


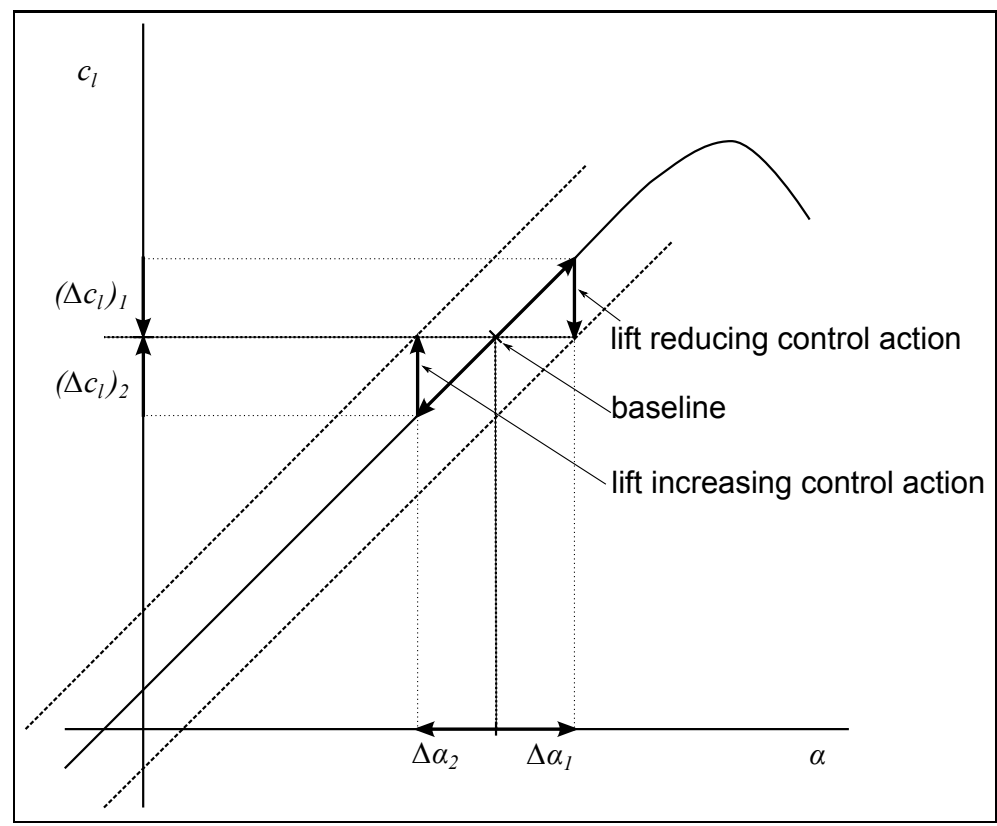

Figure 2.8: Lift coefficient, $c_{l}$, as a function of angle of attack, $\alpha$, with schematic representation of required effect of smart rotor control. A positive $\Delta \alpha$ requires a lift reducing control action, whereas a negative $\Delta \alpha$ requires a lift increasing control action.

for smart rotor control as 'local pitch control'. Smart rotor control, or load control, can therefore be defined as the control of fatigue loads by means of local pitch control.

Techniques that are able to delay the onset of stall could also be used to alleviate fatigue loads, see Maldonado et al. [34], since stall is a highly unsteady flow phenomenon associated with fluctuating aerodynamic forces and a large pressure-induced drag component. Although stall delay reduces fluctuations and the drag component of the aerodynamic forces at high angles of attack, it also increases the lift component. This causes an increase in the average bending moment at the root of the blade. These type of control mechanisms are therefore better suited to increase the performance of a wind turbine blade at inboard blade sections, close to the root of the blade, where higher angles of attack are common and where an increased lift component has less impact on the root bending moment. Furthermore, for the present goal of fatigue load alleviation, these techniques are expected to be less effective.

The UPWIND project adopted the NREL 5MW offshore wind turbine [25] as a reference turbine. For this turbine, which uses collective pitch control, a BEM-based investigation into the angles of attack encountered by the blades and a fatigue load analysis was performed to obtain more insight into the requirements of smart rotor control, see Barlas [35]. This analysis included three representative average wind speeds, covering 
below-rated $(8 \mathrm{~m} / \mathrm{s})$, rated $(11.4 \mathrm{~m} / \mathrm{s})$, and above-rated $(18 \mathrm{~m} / \mathrm{s})$ conditions, medium turbulence characteristics [3], and two yaw misalignment angles $\left(15^{\circ}\right.$ and $\left.35^{\circ}\right)$. The investigation focused on the outboard part of the blades, in particular on three blade sections located at $r / R=0.77, r / R=0.89$ and $r / R=0.98$. At these spanwise locations normally attached flow is present and smart rotor control will have the largest impact due to the large distance to the blade root. From a statistical analysis of the range of disturbances encountered by these blade sections the following information can be extracted [35]:

- The maximum fluctuations in the angle of attack, $\alpha$, and the lift coefficient, $c_{l}$, occur at $r / R=0.77$ for the above-rated wind condition, despite the activation of the pitching mechanisms with collective pitch control. They are:

- $\Delta \alpha \approx \pm 3.9^{\circ}$ and $\Delta c_{l} \approx \pm 0.47$ for zero yaw angle,

- $\Delta \alpha \approx \pm 4.5^{\circ}$ and $\Delta c_{l} \approx \pm 0.54$ for $15^{\circ}$ yaw angle and

- $\Delta \alpha \approx \pm 6.5^{\circ}$ and $\Delta c_{l} \approx \pm 0.73$ for $35^{\circ}$ yaw angle.

At rated wind speed and zero yaw angle, the maximum fluctuations are smaller:

- $\Delta \alpha \approx \pm 2.8^{\circ}$ and $\Delta c_{l} \approx \pm 0.34$ for $r / R=0.77$,

- $\Delta \alpha \approx \pm 2.5^{\circ}$ and $\Delta c_{l} \approx \pm 0.30$ for $r / R=0.89$ and

- $\Delta \alpha \approx \pm 2.3^{\circ}$ and $\Delta c_{l} \approx \pm 0.27$ for $r / R=0.98$.

Note that the maximum fluctuations in the lift coefficient correspond well to the maximum fluctuations in the angle of attack in the light of thin airfoil theory, since $\Delta c_{l} / \Delta \alpha \in\left[0.112 /^{\circ}, 0.121 /^{\circ}\right]$, which is close to the theoretical value of $0.11 /^{\circ}$.

- The standard deviation of the angle of the attack increases with increasing wind speed and yaw angle. The maximum observed standard deviation is $2.3^{\circ}$. At rated wind speed and zero yaw angle, the standard deviation is between $0.71^{\circ}$ and $0.94^{\circ}$.

- The standard deviation of the lift coefficient ranges from 0.1 for $r / R=0.98$ at zero yaw angle and low wind speed to 0.3 for $r / R=0.77$ at $35^{\circ}$ yaw angle and above-rated wind speed.

From these results, it can be concluded that for the reference wind turbine with collective pitch control, additional effective changes in the angle of attack of the order of $\Delta \alpha= \pm 3^{\circ}$ can drastically reduce fatigue loads, at least for ideal circumstances (rated wind speed and zero yaw angle). To obtain similar reductions for less ideal circumstances, effective changes in the angle of attack that are at least twice as large are needed.

A spectral analysis of the variations in the angle of attack for $r / R=0.77$ shows that frequencies up to approximately $6 \mathrm{~Hz}$ are present. The $1 \mathrm{P}$ fluctuations $(\approx 0.2 \mathrm{~Hz})$ are dominant and the higher harmonics have smaller amplitudes in all investigated cases.

Barlas [35] also conducted a BEM-based fatigue load analysis for these cases, which yields useful information on the contribution of the frequencies to fatigue. The analysis 
focused on the flapwise root bending moment at the root of the blades $(r=1.5 \mathrm{~m})$. The analysis shows that the contribution of high frequencies (above 1P) to the fatigue damage is on the same level as the contribution of low (below 1P) frequencies. So, the low frequency loads have the highest amplitude, but the high-frequency loads yield comparable fatigue loading due to the higher number of cycles.

This means that ideally, the smart rotor control technique should be able to generate effective changes in the angle of attack over the whole range of frequencies, up to $f=6$ $\mathrm{Hz}$, which is associated with a response time far below $1 / f \approx 0.167 \mathrm{~s}$. In subsection 2.3 .2 it was shown that $95 \%$ of the aerodynamic response to a step change in the angle of attack is reached in approximately $t=15 \mathrm{c} / U_{\text {eff }}$. At the blade tip, this approximately equals $0.3 \mathrm{~s}$, whereas at $r / R=0.77$, where the chord length is approximately $c=3 \mathrm{~m}$, the aerodynamic response time is approximately $0.7 \mathrm{~s}$. This means that to fully counteract all disturbances up to the maximum required frequency of $6 \mathrm{~Hz}$, the smart rotor control mechanisms should be able to reach effective changes in the angle of attack much higher than necessary for steady conditions. Otherwise, fatigue loads can still be reduced but not entirely prevented.

The actual control authority depends, of course, on the type of sensory information and the total response time of the control technique. The total response time does not only include the aerodynamic response time, but also the time needed for the controller to process and act on sensory information and the 'deployment time' of the actuators. With respect to the type of sensory information, it would be most useful to be able to sense the incoming disturbances before their arrival. Instead of pressure measurements close to the leading edge of the blades or strain measurements on the blades, Light Detection and Ranging (LIDAR) could be used to measure the relative velocities (in the line of sight) ahead of the blades. This technique uses the Doppler shift for light backscattered from natural aerosols in the atmosphere, see Harris et al. [36] and Dunne et al. [37] for example.

\subsubsection{Potential Options for Smart Rotor Control}

Several investigations have been made into the most promising options for smart rotor control. Extensive overviews can be found in Barlas \& Van Kuik [38], Johnson et al. [39, 40], and Pechlivanoglou [41], for example. These investigations have evaluated the potential options based on criteria such as: effectiveness (range of angles of attack), maximum performance (amplitude, speed, frequency), simplicity, costs (weight, power consumption, capital costs), maintenance and reliability.

The following options have been found most promising:

- trailing edge flaps, which can be rigid (ailerons) or flexible (continuous deformable trailing edge), see option no. 1 in figure 2.9 .

- micro-tabs, which are small $(\mathcal{O}(0.01 c))$ deployable Gurney flaps located close to the trailing edge, on both sides of the blade section, see option no. 2 in figure 2.9

- blade morphing (camber control), see option no. 3 in figure 2.9. 


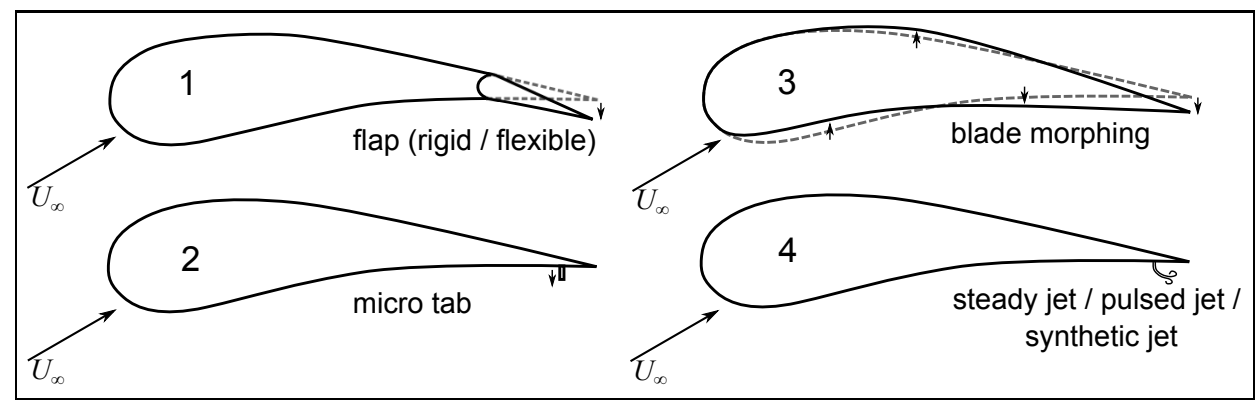

Figure 2.9: Sketch of potential options for smart rotor control. For each option, the lift increasing configuration is shown.

These options are based on changing the shape of the blade section, such that camber is added on the lower or upper side of the blade section. Camber is an asymmetry of the airfoil shape with respect to the chord line. The flaps create actual camber close to the trailing edge, whereas micro-tabs generate 'effective camber' at this location (as seen by the flow passing the blade section). This can be seen in figure 2.10 [42]. Blade morphing can introduce camber anywhere along the camber line, not only close to the trailing edge. Addition of camber close to the trailing edge on the lower side of the blade section, for example, forces the passing flow downwards, which is associated with an increase in the lift and drag force. Forcing the flow upwards also increases drag, but now the lift force decreases. The different pressure distribution on the surface of the blade section is the main contributor to the changes in lift and drag.

Aerodynamically, the flexible trailing edge flap seems the most efficient option. Computational studies by Troldborg [43] show that compared to a rigid flap, a flexible trailing

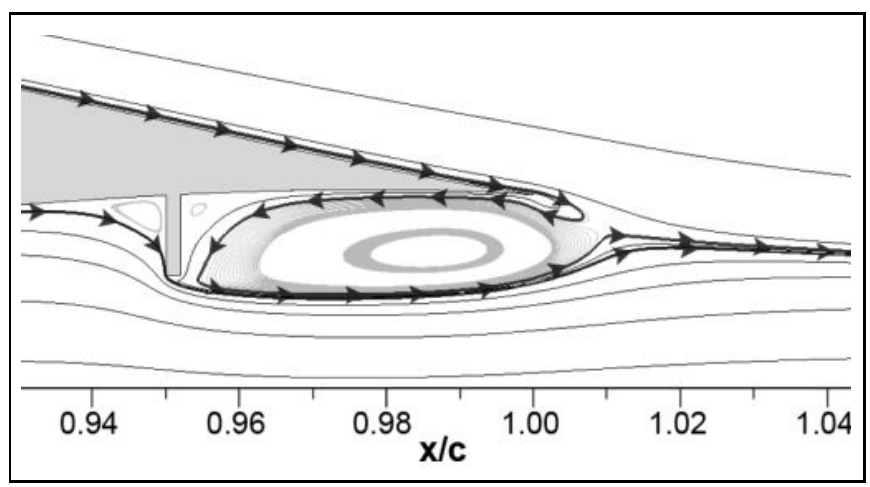

Figure 2.10: Characteristic flow pattern, indicated by streamlines, induced by micro-tab located at chordwise location of $0.95 \mathrm{c}$, from Blaylock et al. [42]. 
edge flap reduces flow separation and thereby reduces the drag and noise generation.

A comparative study, see Bæk et al. [44], focusing on the rigid flap and micro-tab options shows that a rigid flap with length of $0.1 \mathrm{c}$ and deflection of $8^{\circ}$ has the same ultimate change in lift coefficient as a fully deployed micro-tab with a height of $0.01 c$, located at a chordwise location of $0.95 \mathrm{c}$. However, the response of the lift coefficient in time is different, see figure 2.11. Whereas the flap yields a monotonous increase in lift, the micro-tab initially yields a decrease in lift, before the lift builds up. Thus, the aerodynamic delay of the micro-tab is larger, which reduces the potential for fatigue load alleviation. The change in lift coefficient, $\Delta c_{l}$, in steady flow conditions is between \pm 0.2 and \pm 0.25 over a large range of angles of attack. The response of the drag coefficient for the rigid flap and micro-tab shows an initial increase of approximately $0.015-0.02$. Subsequently, the drag is gradually reduced again until the increase in drag becomes $\Delta c_{d} \approx 0.003$ for the micro-tab and between $\Delta c_{d} \approx 0.001$ at low angles of attack and $\Delta c_{d} \approx 0.003$ at high angles of attack for the rigid flap.

The initial reverse lift peak induced by the the micro-tab has also been observed by Chow \& Van Dam [45]. They show that a micro-flap, which is a Gurney flap located at the trailing edge that can be deployed by rotation rather than translation, is a better option since it does not feature this behavior. The faster response for the micro-flap is only present during the initial deployment phase, however. Post-deployment, the temporal responses of the two devices are essentially identical. This is due to the convection of

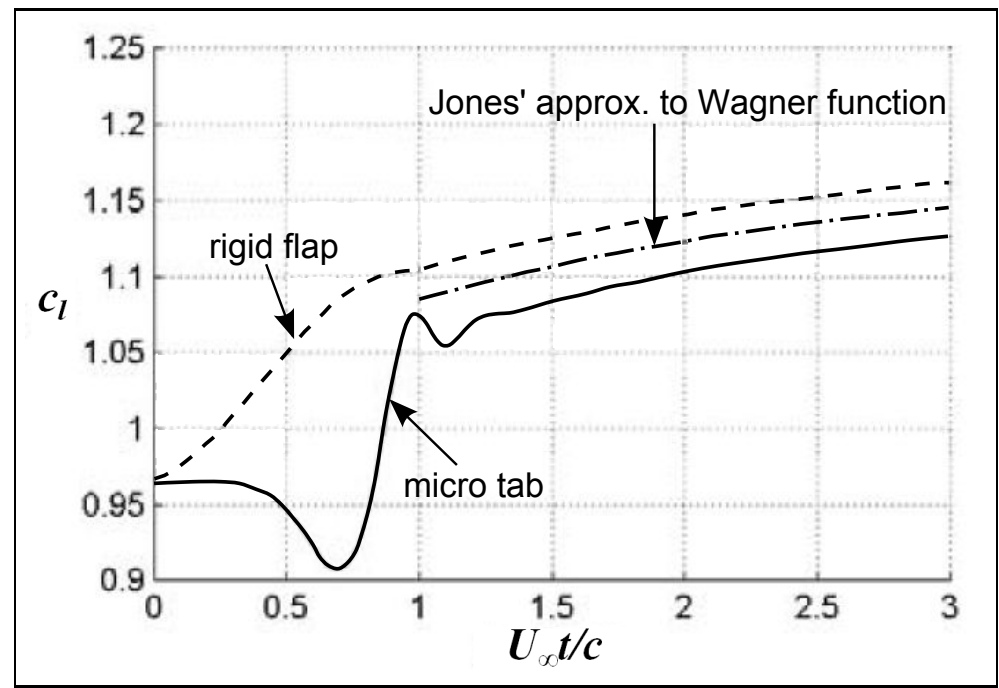

Figure 2.11: Characteristic behavior of lift coefficient, $c_{l}$, as a function of dimensionless time, $U_{\infty} t / c$, for a rigid trailing edge flap (dashed line) and micro-tab (solid line), using a dimensionless deployment time of 1 . Jones' approximation to the Wagner function is shown with a dash-dotted line. From Bæk et al. [44]. 
the associated starting vortex into the wake, see subsection 2.3.2 which is the same for any device. Both devices reach approximately $90 \%$ of the asymptotic change in lift at $U_{\infty} t / c \approx 9$.

Besides the aforementioned options, there is also the potential option of active fluidic control by means of jets, see option no. 4 in figure 2.9. These jets can be steady jets, pulsed jets, or synthetic jets. In a study related to the present research by Boeije et al. [46], it is shown that steady jets from spanwise slits with a width of $0.00606 c$ and average velocity of the order of the free-stream velocity, $U_{\text {jet }}=1.2 U_{\infty}$, ejected perpendicular to the surface of a blade section at a chordwise location of $0.88 c$, yield changes in the lift coefficient of $\Delta c_{l} \approx \pm 0.4$ over a wide range of angles of attack. These changes can be positive or negative, depending on whether the jets are located on the lower (pressure) side or upper (suction) side of the blade section, respectively. The effect of a steady jet on the lower side is shown in figure 2.12. In comparison with the baseline case without active jets, the slope of the lift coefficient, $d c_{l} / d \alpha$, is slightly higher for the case with active jets.

Figure 2.13 shows computational results of the flow in the region of the trailing edge with and without the steady jet. The instantaneous streamlines superposed on a contour

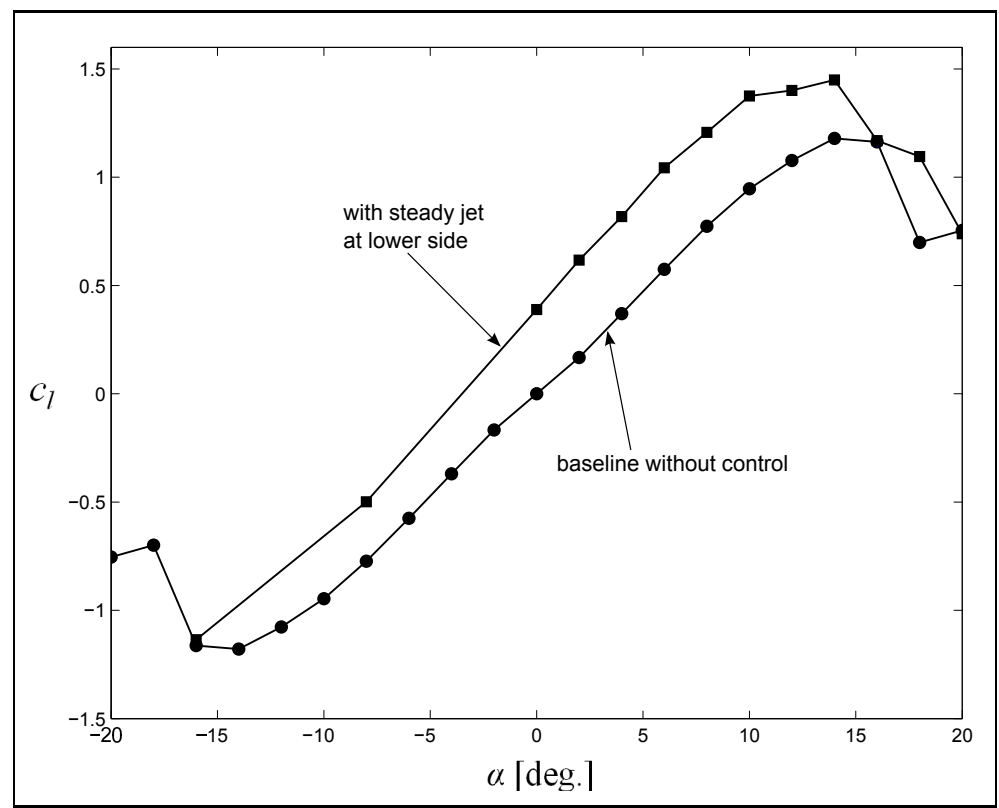

Figure 2.12: Lift coefficient, $c_{l}$, as a function of angle of attack, $\alpha$, for NACA0018 airfoil at $\operatorname{Re}_{c}=6.6 \cdot 10^{5}$, with and without steady jet. Jet has with width of $0.00606 \mathrm{c}$ and average velocity of $U_{j e t}=1.2 U_{\infty}$, ejected perpendicular to the surface at a chordwise location of $0.88 \mathrm{c}$. From Boeije et al. [46]. 


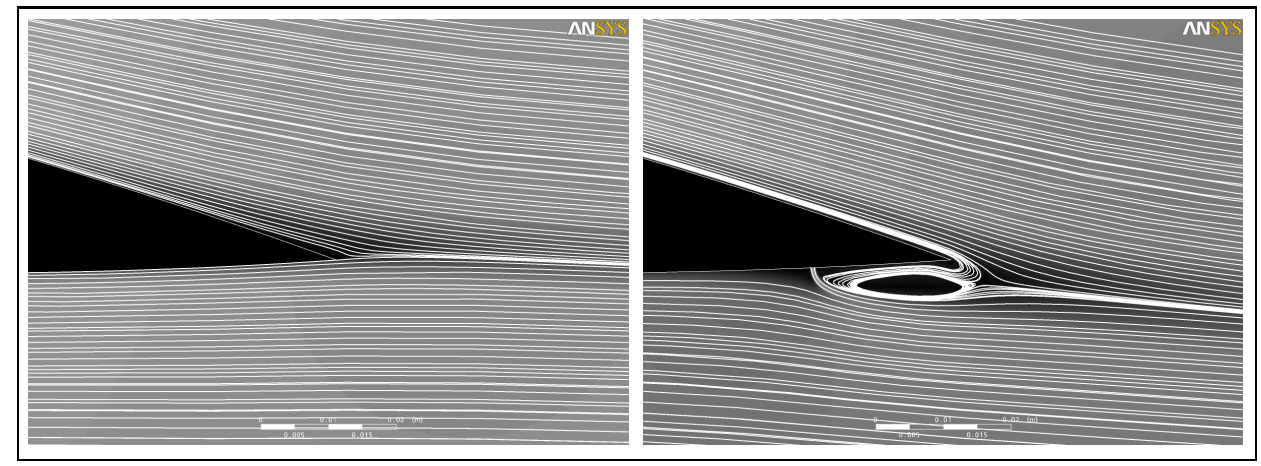

Figure 2.13: Streamlines superimposed on contour plot of Mach number (darker regions indicate lower velocities), near trailing edge of NACA0018 airfoil for $\operatorname{Re}_{c}=6.6 \cdot 10^{5}$ and $\alpha=8^{\circ}$. Left: baseline without control. Right: steady jet with a width of $0.00606 \mathrm{c}$ and average velocity of $U_{j e t}=1.2 U_{\infty}$, ejected perpendicular to the surface at a chordwise location of $0.88 c$. From Boeije et al. [46].

plot of the Mach number field (darker regions indicate lower velocities) indicate that the jet generates a recirculation area between the location of the jet and the trailing edge, which effectively causes an increase of the circulation of the airfoil: the angle at which the flow leaves the trailing edge changes from parallel to the lower surface to parallel to the upper surface. It appears that the jet entrains low-momentum fluid from the upper side of the airfoil. It is also clear that the flow stagnates just upstream of the jet, forcing the flow along the lower side of the airfoil downward. Downstream of the trailing edge the injected fluid merges with the boundary layers from the upper and lower surface.

In a computational study by Blaylock et al. [42] for the same configuration as used by Boeije and co-workers, the added momentum by the steady jet is varied. It is shown that a reduction of the added momentum to approximately $20 \%$ of the original value still yields a change in the lift coefficient of $\Delta c_{l} \approx \pm 0.25$. Below this value, the change in lift rapidly reduces, whereas it increases almost linearly above this value.

Blaylock et al. [42] have also performed a comparative study of the application of steady jets and micro-tabs. They show that both options affect the flow in the vicinity of the trailing edge in a similar way and therefore yield very similar aerodynamic effects. Furthermore, in case the final change in lift for both options are the same, the steady jets produce slightly less drag in comparison with the micro-tabs and also yield less initial adverse lift effects.

For the steady and pulsed jets, an external supply of fluid is needed in combination with additional piping systems. The external supply of fluid can be a compressor, located in the hub, for example. However, it is also feasible to use the rotating wind turbine blades as centrifugal pump, to pump air from an inlet near the root of the blades towards the tip, where it is ejected. In this way, steady jet velocities up to $U_{j e t}=\Omega R$ may be 
reached without additional compression 6 .

In the case of the synthetic jets, no external supply of air or additional piping systems are needed, since they are formed by ingestion and ejection of the controlled flow itself. Inspired by the results of steady jets, the present research investigates the potential use for pitch control of synthetic jet actuation near the trailing edge of a blade section. It is expected that synthetic jets can also cause effective changes in camber, in a time-averaged manner. The results of this investigation are presented in chapter 6 In the next section, more detailed information about synthetic jets is given.

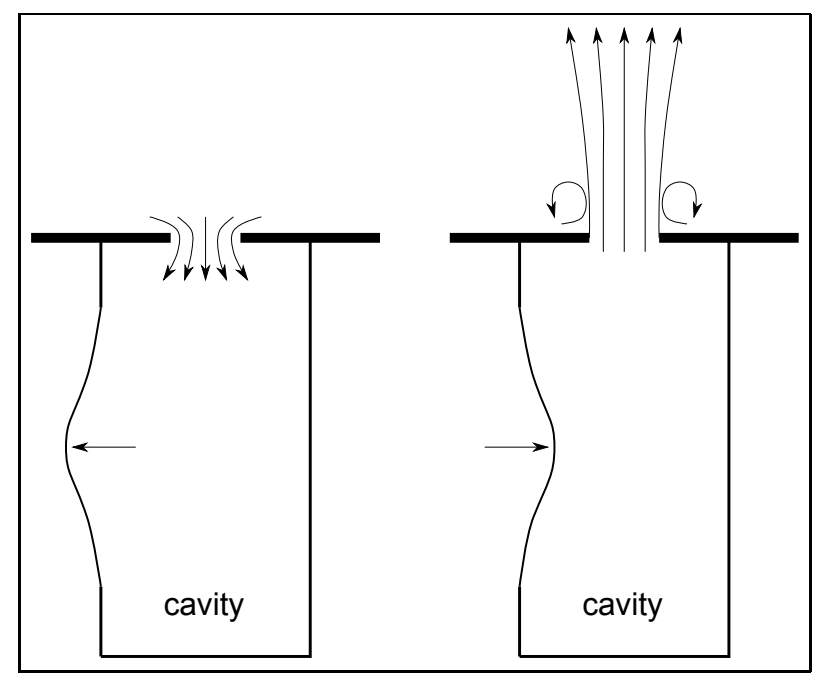

Figure 2.14: Schematic representation of a synthetic jet during ingestion (left) and ejection (right).

\subsection{Synthetic Jets}

\subsubsection{Principle}

A schematic representation of a synthetic jet actuator is shown in figure 2.14. It consists of a cavity with a vibrating wall and an orifice that connects the cavity with the outside world. The vibration of the cavity wall causes the repeated ingestion and ejection of fluid into and out of the cavity. The orifice can be a circular hole or a rectangular slit with a certain aspect ratio (length to width ratio). Furthermore, the ratio of width (or diameter) to depth of the slit (or circular hole) is also an important geometric parameter.

An excellent overview of work related to synthetic jets is given by Glezer \& Amitay [47]. Without a cross-flow, during ingestion, the synthetic jet draws fluid from all sides

${ }^{6}$ G. G. M. Zwart, personal communication. 


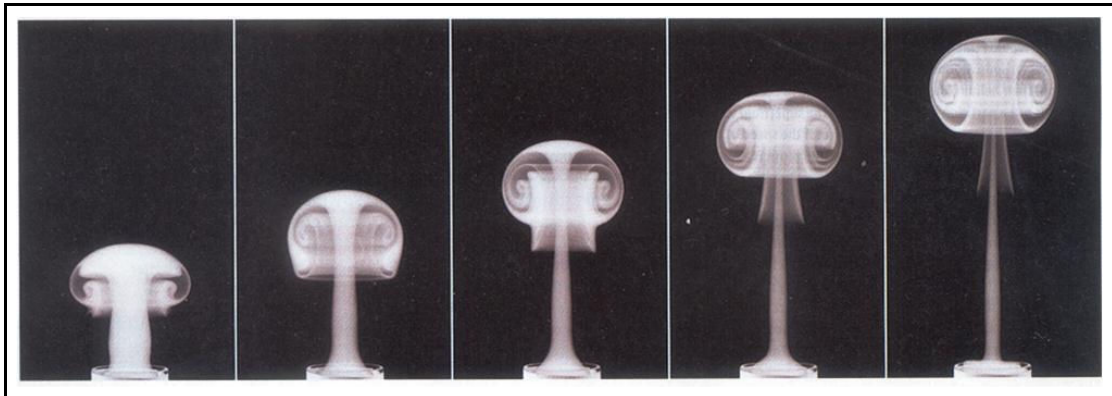

Figure 2.15: Evolution of a ring vortex generated by a synthetic jet, obtained from http://freshscience.org. $a u / ? p=1541$.

around the orifice, i.e. like a sink. During ejection, however, a jet is formed because of flow separating at the outer edge of the orifice 7 . In this way, a vortex ring is formed that is subsequently advected away from the orifice under its own self-induced velocity, see figure 2.15 for an example of this effect. This means that the flow generated by a synthetic jet is dominated by the time-periodic formation, advection, and interactions of discrete vortical structures (line-vortex pairs or vortex rings), which ultimately become turbulent, slow down, and lose their coherence. In the far field, synthetic jets bear much resemblance to continuous jets in that the time-averaged self-similar velocity profiles are identical, see Smith \& Swift [48]. However, in the near field, the vortex pairs or rings cause an increased entrainment of fluid in comparison with a continuous jet, such that synthetic jets grow more rapidly.

For non-circular orifices, the aspect ratio of the orifice may influence the out-of-plane distortion of the vortices and the streamwise advection and evolution, see Quinn [49].

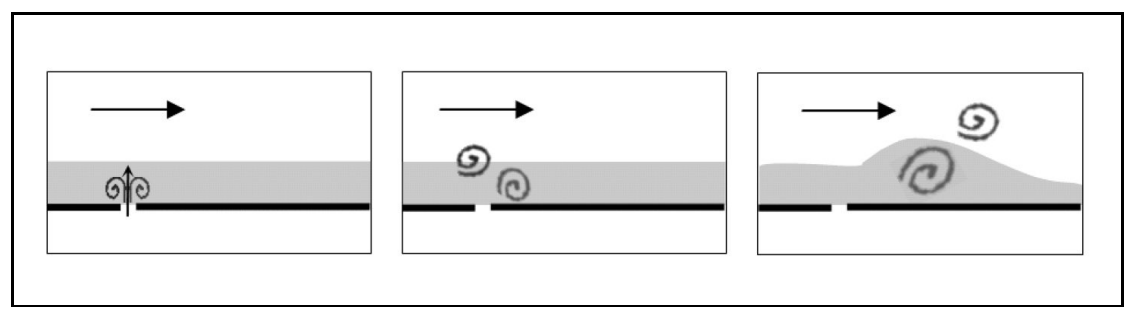

Figure 2.16: Sketch of evolution of a vortex pair in presence of a cross-flow, from Brozozowski \& Glezer [50].

In the presence of a cross-flow, the line-vortex pairs or vortex rings interact with the boundary layer along the surface such that they are tilted. For a two-dimensional situation,

\footnotetext{
${ }^{7}$ Separation can also take place at the inner edges of the orifice, thereby reducing the effective width of the orifice because of the vena contracta.
} 
i.e. for line-vortex pairs, this interaction is sketched in figure 2.16 [50]. The clockwise vortex effectively thickens the boundary layer, i.e. causes additional displacement of the cross-flow, whereas the counter-clockwise vortex is lifted higher into the cross-flow where it is accelerated due to the higher main flow velocity. Furthermore, due to the ingestion of opposite-sense vorticity from the upstream boundary layer, the counter-clockwise vortex weakens much faster than the clockwise vortex.

\subsubsection{Parameters}

During the operation of a synthetic jet, one can prescribe the frequency and the amplitude of the actuator. For a given actuation input, the performance of the synthetic jet can be maximized when the actuator and cavity are driven at a coupled resonance that depends on both the cavity flow (and geometry) and the structural characteristics of the actuator [47].

Usually, two parameters are used to describe the operation of a synthetic jet in aerodynamic applications: the dimensionless actuation frequency, $F^{+}$, and the momentum coefficient, $c_{\mu}$ :

- The dimensionless actuation frequency relates the period of the actuation cycle, $T_{j}=1 / f_{j}$, with a convection time over a characteristic distance, e.g. $L / U_{\infty}$. For separation control, see subsection 2.6.3 it is common to use the chordwise distance from the orifice to the end of the separated flow region. However, in the present work, the chord length is used. The dimensionless actuation frequency is therefore defined as

$$
F^{+}=\frac{f_{j} c}{U_{\infty}}
$$

where $f_{j}$ is the actuation frequency $(\mathrm{Hz}), c$ is the chord length and $U_{\infty}$ is the free-stream velocity.

- The momentum coefficient is a measure of the relative momentum addition by the synthetic jet. In this thesis, for two-dimensional cases the following definition is used:

$$
c_{\mu}=\frac{\bar{\rho}_{j} \bar{U}_{j}^{2} w_{j}}{\rho_{\infty} U_{\infty}^{2} c},
$$

where $\bar{\rho}_{j}$ and $\bar{U}_{j}$ are the average density and velocity of the jet (across the slit and during the ejection part of the cycle), respectively, and $w_{j}$ is the width of the slit.

In the literature, different definitions for the momentum coefficient $c_{\mu}$ can be found. There are definitions that use a different characteristic velocity of the jet. Examples are the peak velocity at the centerline [51], or the root-mean-square value of the velocity at the centerline [52]. Additionally, a momentum coefficient directly based on time-averaging the added momentum during the ejection part of the cycle is also used [53]. Furthermore, some authors use the free-stream dynamic pressure, $q_{\infty}=\frac{1}{2} \rho_{\infty} U_{\infty}^{2}$, to make the added momentum dimensionless, which introduces an additional factor of 2 in equation 2.13 
Besides the actuation parameters, the effectiveness of synthetic jets depends on the cross-flow, or its boundary layer, which in the present aerodynamic applications is characterized by a Reynolds number, $\mathrm{Re}_{c}$, and the Mach number, $\mathrm{M}_{\infty}$. They are defined as

$$
\begin{array}{r}
\operatorname{Re}_{c}=\frac{\rho_{\infty} U_{\infty} c}{\mu_{\infty}} \\
\mathrm{M}_{\infty}=\frac{U_{\infty}}{c_{\infty}^{*}},
\end{array}
$$

where $\rho_{\infty}$ is the free-stream density, $\mu_{\infty}$ is the dynamic viscosity of the free-stream and $c_{\infty}^{*}$ is the free-stream speed of sound.

\subsubsection{Separation Control versus Pitch Control}

In applications of synthetic jet actuation, a distinction can be made between boundary layer separation control and pitch control. The former can be used for delay of stall to higher angles of attack, whereas the latter is associated with changes in the lift coefficient over a relatively wide range of angles of attack.

In the feasibility studies of fatigue load reduction for wind turbines, the option of synthetic jet actuation is usually not deemed a useful option. This is due to this technology often being used for the purpose of boundary layer separation control. A recent example of this for wind turbine blades is the experimental study by Maldonado [34], in which synthetic jet actuation is applied on the suction side of a blade at a chordwise location of $0.25 c$, i.e. close to the leading edge. The jets originate from rectangular orifices, oriented such that they are aligned in pairs at an angle of $20^{\circ}$ to the flow. Similar to conventional vortex generators, such a configuration leads to the formation of counter-rotating streamwise vortices. These vortices cause transfer of high momentum fluid towards the wall. The effect of synthetic jet actuation in these cases is an increase of the stall angle of attack. For smaller angles of attack, the effects are minimal.

For boundary layer separation control, an array of synthetic jets that causes smallamplitude perturbations through small-sized holes or slits can be very effective in leveraging flow instabilities and generating vortical structures that increase turbulent mixing of the outer flow with the boundary layer. Small-scale forcing is an easier goal for many actuators, which explains the reason why synthetic jet actuation has been investigated mostly for boundary layer control.

Note that steady jets can also be used for boundary layer separation control, either by using an array of holes or slits, or by directly adding momentum into the boundary layer by jets directed tangentially to the surface. However, periodic excitation using pulsed jets or synthetic jets is more energy efficient, see Greenblatt \& Wygnanski [54].

The characteristic time scale of the synthetic jet actuation is important for its effectiveness, see for example Seifert et al. [55] and Amitay \& Glezer [53]. For separation control, the actuation time scale can be of the order of the convective time scale, $c / U_{\infty}$, which couples to the instability of the separating shear layer and the wake. This results in 
a deflection of the separating shear layer towards the surface of the airfoil and is associated with an increase in the time-averaged lift. The corresponding dimensionless actuation frequency, $F^{+}=f_{j} c / U_{\infty}$, is therefore $F^{+}=\mathcal{O}(1)$. The time scale of actuation can also be one order of magnitude smaller than the convective time scale, i.e. $F^{+}=\mathcal{O}(10)$. This is associated with 'trapped vorticity' that can locally increase the apparent camber of the airfoil such that a favorable pressure gradient is obtained, which can delay or prevent flow separation. Depending on the location of the synthetic jets, this mechanism can be used for separation control [53, 56] as well as for pitch control.

For pitch control, synthetic jets have mainly been used to control a region with vorticity near the trailing edge, which has been created by other means, such as a wedge shaped obstacle [57, 58, 59] or a Gurney flap [60]. An other example is described by Lopez et al. [61], who performed numerical simulations for a NACA4415 airfoil with a modified trailing edge in which synthetic jets actuators are integrated, see figure 2.17. These jets are directed tangential to the upper and lower surface and manipulate the vortical flow regions formed close to the trailing edge. This modifies the angle at which the flow leaves the trailing edge, which affects the circulation of the airfoil and thereby the pressure distribution, leading to changes in the aerodynamic properties of the airfoil. In this case, the obtained change in the lift coefficient is $\Delta c_{l} \approx 0.06$, which is somewhat low in comparison with those observed in research on trailing edge flaps, micro-tabs and steady jets.

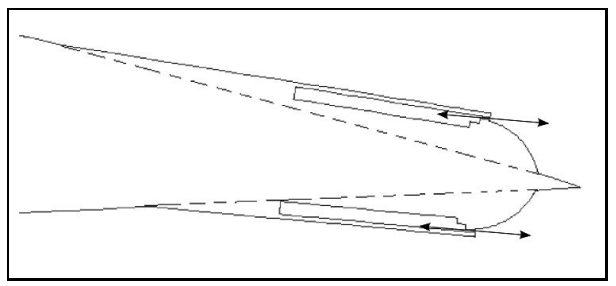

Figure 2.17: Modified NACA4415 airfoil with integrated synthetic jet actuators, directed tangentially to the upper and lower surface, from [61].

Introducing a rigid object that cannot be retracted in the flow, for the purpose of flow control, increases drag. Therefore, in the present research, see chapter 6 , the option of controlling a region with vorticity created by rigid objects is not chosen. Instead, we have investigated the possibility of synthetic jets that are directed perpendicular to the surface, in order to influence the main flow much like the micro-tab does. However, it is expected that such a synthetic-jet configuration might consume more energy, since it forces the flow on a larger scale.

\subsubsection{Actuators}

For the vibrating wall inside the cavity different actuators can be chosen. Actuators can be either based on piezoceramic composite diaphragms [53, 34], voice-coils (speakers) 
[53, 62], or pistons [51].

Piezoelectric actuators are able to operate at high frequencies (e.g. up to $1800 \mathrm{~Hz}$, but the velocity amplitudes that can be obtained are generally low, due to the small displacements of the diaphragm.

Speakers are able to generate higher velocity amplitudes, but only on the lower end of the frequency spectrum, say up to $100 \mathrm{~Hz}$. This has also been observed in the experiments performed for the present research, in which synthetic jets based on speakers have been used, see chapter 6 .

Pistons can generate high velocity amplitudes. Their actuation frequency depends on the driver mechanism, but they often operate in the low-frequency spectrum. Piston driven actuators have the advantage that the velocity amplitude can be decoupled from the actuation frequency.

In general, not many reasonably sized actuators are capable of reaching large enough velocity amplitudes across a reasonable range of frequencies. There is often a tradeoff between maximum volume displacement and bandwidth, as mentioned by Cattafesta \& Sheplak [63], for example in the design of synthetic jets based on clamped circular piezoceramic composite diaphragms, see Papila et al. [64]. The development of actuators with increased performance is therefore an important issue that has to be resolved, before the technology can actually be used for pitch control.

\subsection{Concluding Remarks}

With the ongoing trend towards larger wind turbines, the need for distributed load control mechanisms, i.e. smart rotor control, becomes increasingly more important. The aerodynamic effect needed for smart rotor control is 'local pitch control', which is associated with changes in the aerodynamic forces on a blade element equivalent to changes in local angle of attack. Effective changes in the angle of attack on the outboard part of the blades, i.e. close to the tip, will have the most impact on the reduction of fatigue loads due to the large distance to the root of the blade. The changes in the local aerodynamic forces, most importantly the lift component, should be generated over a range of angles of attack in the linear $c_{l}(\alpha)$-regime, since the outboard blade sections of a pitch regulated wind turbine operate in attached flow conditions.

From the work of Barlas [38] on the NREL reference offshore wind turbine, it can be concluded that smart rotor control should be able to generate additional effective changes in the angle of attack of the order of $\Delta \alpha \approx \pm 3^{\circ}$. However, to fully counteract all disturbances in the important range of frequencies, much higher effective changes in the angle of attack are needed, since the aerodynamic response time to these changes is larger than the period of the highest frequencies involved. If these large effective changes can not be realized, fatigue loads can still be reduced but not entirely prevented.

Besides the options for local pitch control currently investigated in other projects (trailing edge flaps, micro-tabs and blade morphing), there is also the potential option of active fluidic control by means of jets. This technology uses less vulnerable moving parts than the above mentioned options. Steady jets, directed normal to the surface and 
located close to the trailing edge of a blade section, have shown promising results for the purpose of pitch control. However, they require additional piping systems inside the blades. Furthermore, an external supply of compressed air may be needed.

The potential option of synthetic jets does not have these negative aspects, since it involves the ingestion and ejection of the controlled flow itself. The flow generated by a synthetic jet is dominated by the time-periodic formation, advection and interactions of vortical structures. For a given location and geometry of the cavity and slit or hole of a synthetic jet, the parameters that influence the operation are the dimensionless actuation frequency, $F^{+}$, and the momentum coefficient, $c_{\mu}$. Furthermore, the Reynolds number, $\operatorname{Re}_{c}$, and the Mach number, $\mathrm{M}_{\infty}$, also influence the performance since these numbers determine the cross-flow encountered by the jets.

The technology of synthetic jets is most frequently investigated for the purpose of boundary layer separation control. However, in the present research it is investigated for the purpose of pitch control. It is expected that synthetic jet actuation through a long spanwise slit close to the trailing edge can cause effective changes in camber, although in a time-averaged manner. The results of a parameter study of synthetic jet actuation for pitch control is presented in chapter 6. Especially, the effect of $F^{+}, c_{\mu}$ and the location of the slit is investigated. The present research focuses on the aerodynamic aspects of this technology, but acknowledges the need for more research into synthetic jet actuators with increased performance. 



\section{Chapter 3}

\section{Mathematical Modeling}

This chapter introduces the Navier-Stokes equations, the mathematical model of compressible, viscous flow. This set of equations describes the conservation of mass, momentum, and energy.

In the first section, the conservation equations are derived with respect to an inertial frame. In section 3.2 the conservation equations are derived for a non-inertial frame: a frame connected to a single rotating object, e.g. a wind turbine blade, rotating at constant angular velocity $\underline{\Omega}$. In the latter formulation, the control volumes are considered to be undeformable and fixed to the rotating frame. Section 3.3 discusses the necessity of turbulence modeling and the so-called Unsteady Reynolds Averaged Navier-Stokes (URANS) equations are derived. To resolve the closure problem associated with these equations, several eddy-viscosity turbulence models, based on the Boussinesq assumption, are introduced in section 3.4. Finally, section 3.5 describes the boundary conditions needed for the mathematical models. This includes the approach taken to model synthetic jet actuation.

\subsection{Conservation Equations in an Inertial Frame of Ref- erence}

In this derivation, the Eulerian approach is followed, with spatial coordinates $\underline{x}$ and time $t$. The conservation equations for mass, momentum and energy are formulated for an open system or control volume. At a certain instance in time, the arbitrary control volume is denoted by $V(t)$, which is bounded by a closed, permeable boundary $A(t)=\partial V$ with the unit outward normal vector $\underline{n}(\underline{x}, t)$.

The Reynolds Transport Theorem is used to obtain the conservation equations for the control volume. The used symbols are presented in table 3.1. The Reynolds Transport Theorem states that the total time rate of change of an extensive property $\Phi(t)$ for a system, i.e. mass, momentum or energy, equals the time rate of change of this property in the control volume plus the net rate of convection of this property crossing the control volume boundary:

$$
\frac{d \Phi}{d t}=\frac{\partial}{\partial t} \iiint_{V(t)} \rho \phi d V+\iint_{A(t)} \rho \phi\left(\underline{u}-\underline{u}_{A}\right) \cdot \underline{n} d A,
$$


where $\phi(\underline{x}, t)$ is the related intensive property. The total rate of change of $\Phi$ can be due to a source of $\rho \phi$ inside the control volume, denoted by $\mathcal{V}_{\phi}$, or due to a non-convective flux of $\rho \phi$ at the surface of the control volume, denoted by $\underline{\mathcal{S}}_{\phi} \cdot \underline{n}$ :

$$
\frac{\partial}{\partial t} \iiint_{V(t)} \rho \phi d V+\iint_{A(t)} \rho \phi\left(\underline{u}-\underline{u}_{A}\right) \cdot \underline{n} d A=\iiint_{V(t)} \mathcal{V}_{\phi} d V-\iint_{A(t)} \underline{\mathcal{S}}_{\phi} \cdot \underline{n} d A
$$

\begin{tabular}{|c|c|}
\hline$\rho(\underline{x}, t)$ & density \\
\hline$\underline{u}(\underline{x}, t)$ & flow velocity \\
\hline$\underline{u}_{A}(\underline{x}, t)$ & velocity of the control-volume surface \\
\hline$p(\underline{x}, t)$ & pressure \\
\hline$\underline{\underline{\tau}}(\underline{x}, t)$ & viscous stress tensor \\
\hline$f_{b}(\underline{x}, t)$ & specific body force vector \\
\hline$E(\underline{x}, t)$ & specific total energy \\
\hline$e(\underline{x}, t)$ & specific internal energy \\
\hline$q(\underline{x}, t)$ & heat flux vector \\
\hline
\end{tabular}

Table 3.1: Symbols used in the derivation of the Navier-Stokes equations.

\subsubsection{Conservation of Mass}

The application of the Reynolds Transport Theorem yields the following conservation equation for mass within control volume $V(t)$ :

$$
\frac{\partial}{\partial t} \iiint_{V(t)} \rho d V+\iint_{A(t)} \rho\left(\underline{u}-\underline{u}_{A}\right) \cdot \underline{n} d A=0 \text {. }
$$

Here it is assumed that there are no sources or sinks of mass within $V(t)$.

\subsubsection{Conservation of Momentum}

Application of Newton's second law to control volume $V(t)$ yields that the rate of change of momentum $\underline{P}$ equals the sum of forces applied to $V(t)$. The forces can consist of volumetric forcefields, $\underline{f}_{b}$, and surface stresses, described by a stress vector $\underline{\underline{\sigma}} \underline{n}$ acting on $A(t)$, with $\underline{\underline{\sigma}}$ the stress tensor. The volumetric forcefields are body forces such as gravity, whereas the stresses are effects due to pressure (working in the direction opposite to the external normal) and due to viscosity, described by the viscous stress tensor $\underline{\tau}$ :

$$
\underline{\underline{\sigma}}=-p \underline{\underline{I}}+\underline{\underline{\tau}}
$$

where $\underline{\underline{I}}$ is the $3 \times 3$ identity matrix. 
Application of the Reynolds Transport Theorem yields the conservation equation for momentum within control volume $V(t)$ :

$$
\frac{\partial}{\partial t} \iiint_{V(t)} \rho \underline{u} d V+\iint_{A(t)} \rho \underline{u}\left(\underline{u}-\underline{u}_{A}\right) \cdot \underline{n} d A=\iint_{A(t)}(-p \underline{\underline{I}}+\underline{\tau}) \underline{n} d A+\iiint_{V(t)} \rho \underline{f} b d V .
$$

For the kind of flows the numerical method will be applied to, the role of gravity is small compared to the effects of pressure and viscous stresses. Therefore, the effects of gravity (and other force fields) will be neglected, i.e. $\underline{f}_{b}=\underline{0}$.

\subsubsection{Conservation of Total Energy}

From the first law of thermodynamics applied to control volume $V(t)$, it follows that the total rate of change of energy in $V(t)$ equals the sum of the rate at which work is applied to the system, and the rate at which heat is supplied to the system.

The total energy contained in $V(t)$ equals the sum of internal energy, kinetic energy, potential energy, etc. For aerodynamic applications, the first two are dominant. Therefore, the specific total energy equals

$$
E=e+\frac{1}{2}|\underline{u}|^{2}
$$

The rate of work applied to the system equals the inner product of the forces acting on system and the velocity of the fluid. As before in the momentum equation, only pressure and viscous stresses are considered, i.e. $\underline{f}_{b}=\underline{0}$. Furthermore, the only contribution included in the rate at which heat is supplied to the system is the heat flux due to thermal conduction $(\underline{q} \cdot \underline{n})$, i.e. volumetric sources of heat are not considered.

Application of the Reynolds Transport Theorem then yields the following conservation equation for total energy:

$$
\frac{\partial}{\partial t} \iiint_{V(t)} \rho E d V+\iint_{A(t)} \rho E\left(\underline{u}-\underline{u}_{A}\right) \cdot \underline{n} d A=\iint_{A(t)}[(-p \underline{I}+\underline{\underline{\tau}}) \underline{u}] \cdot \underline{n} d A-\iint_{A(t)} \underline{q} \cdot \underline{n} d A .
$$

\subsubsection{Navier-Stokes Equations}

\section{Integral Formulation}

The five conservation equations derived in the preceding subsections can be jointly written as:

$$
\frac{\partial}{\partial t} \iiint_{V(t)} \boldsymbol{U} d V+\iint_{A(t)}\left(\underline{\boldsymbol{F}}^{c}-\boldsymbol{U}_{A}\right) \cdot \underline{n} d A-\iint_{A(t)} \underline{\boldsymbol{F}}^{v} \cdot \underline{n} d A=0,
$$

where $\boldsymbol{U}(\underline{x}, t)$ is the column vector with conserved variables:

$$
\boldsymbol{U}=\left[\begin{array}{c}
\rho \\
\rho \underline{u} \\
\rho E
\end{array}\right]
$$


Furthermore, $\underline{\boldsymbol{F}}^{c}(\underline{x}, t)$ and $\underline{\boldsymbol{F}}^{v}(\underline{x}, t)$ are the convective and viscous flux vector,

$$
\underline{\boldsymbol{F}}^{c}=\left[\begin{array}{c}
\rho \underline{u} \\
\rho \underline{u} \underline{u}+p \underline{I} \\
\rho H \underline{u}
\end{array}\right], \quad \underline{\boldsymbol{F}}^{v}=\left[\begin{array}{c}
0 \\
\underline{\tau} \\
\underline{\underline{u}} \underline{\underline{u}}-\underline{q}
\end{array}\right],
$$

respectively. $H(\underline{x}, t)$ is the specific total enthalpy defined as $H \equiv E+p / \rho$.

The integral equations can also be written using the index notation 1 and the Einstein summation convention as:

$$
\begin{aligned}
& \frac{\partial}{\partial t} \iiint_{V(t)} \boldsymbol{U} d V+\iint_{A(t)}\left(\boldsymbol{F}_{j}^{c}-\boldsymbol{U}_{A_{j}}\right) n_{j} d A-\iint_{A(t)} \boldsymbol{F}_{j}^{v} n_{j} d A=0, \\
& \boldsymbol{U}=\left[\begin{array}{c}
\rho \\
\rho u_{i} \\
\rho E
\end{array}\right], \quad \boldsymbol{F}_{j}^{c}=\left[\begin{array}{c}
\rho u_{j} \\
\rho u_{i} u_{j}+p \delta_{i j} \\
\rho H u_{j}
\end{array}\right], \quad \boldsymbol{F}_{j}^{v}=\left[\begin{array}{c}
0 \\
\tau_{i j} \\
u_{i} \tau_{i j}-q_{j}
\end{array}\right],
\end{aligned}
$$

where $\delta_{i j}$ is the Kronecker delta.

\section{Partial Differential Formulation}

The Navier-Stokes equations can also be written in partial differential form. Applying Leibniz' rule to the first term yields

$$
\frac{\partial}{\partial t} \iiint_{V(t)} \boldsymbol{U} d V=\iiint_{V(t)} \frac{\partial \boldsymbol{U}}{\partial t} d V+\iint_{A(t)} \boldsymbol{U}\left(\underline{u}_{A} \cdot \underline{n}\right) d A .
$$

All surface integrals can subsequently be written as volume integrals using Gauß' divergence theorem. The control volume is arbitrary, so each of the integral conservation equations reduces to the integrand of the volume integral having to be zero. The result is the system of partial differential equations:

$$
\frac{\partial}{\partial t} \boldsymbol{U}+\underline{\nabla} \cdot\left(\underline{\boldsymbol{F}}^{c}-\underline{\boldsymbol{F}}^{v}\right)=0
$$

or equivalently using index notation:

$$
\frac{\partial}{\partial t} \boldsymbol{U}+\frac{\partial}{\partial x_{j}}\left(\boldsymbol{F}_{j}^{c}-\boldsymbol{F}_{j}^{v}\right)=0 .
$$

These equations describe the flow of any uniform, homogenous fluid that can be considered a continuum. However, these equations need to be closed by additional relations, presented in the next subsections. Unfortunately, the additional relations will not be general but specific for the fluid considered. Therefore, they are specifically aimed at the flows observed in the applications at hand: flows of air at moderate pressures and temperatures.

\footnotetext{
${ }^{1}$ Here, the column vectors $\boldsymbol{U}, \underline{F}^{c}$ and $\underline{F}^{v}$ do not obtain the index $i$ when they are written in index notation. It is understood that the momentum equation still has the free index $i$.
} 


\subsubsection{Equations of State}

First, the thermodynamic variables need to be related to each other through algebraic equations called the equations of state. The thermodynamic variables are: density $\rho$, pressure $p$, specific internal energy $e$, and temperature $T$. Furthermore, $\rho, p$ and $e$ can be combined in the specific enthalpy $h$ :

$$
h=e+\frac{p}{\rho} .
$$

The Thermodynamic State Principle states that for a simple compressible system 2, of a fluid of fixed composition, the thermodynamic state is fully described by two independent thermodynamic variables [65. For example, when $\rho$ and $e$ are chosen as the independent variables, the remaining variables follow from

$$
p=p(\rho, e), \quad T=T(\rho, e), \quad h=h(\rho, e) .
$$

Here we assume that air is a calorically perfect gas, which is valid for temperatures between approximately $250 \mathrm{~K}$ and $800 \mathrm{~K}$ [65]. A perfect gas obeys the ideal gas law, which reads

$$
p=\rho R T,
$$

where $R$ is the specific gas constant 3 . Furthermore, the specific heats, the internal energy and the specific enthalpy of a thermally perfect gas are a function of temperature only, i.e.

$$
\begin{aligned}
d e & =\left(\frac{\partial e}{\partial T}\right)_{v} d T=C_{v}(T) d T, \\
d h & =\left(\frac{\partial h}{\partial T}\right)_{p} d T=C_{p}(T) d T,
\end{aligned}
$$

where $C_{v}$ is the specific heat at constant volume, and $C_{p}$ is the specific heat at constant pressure. With these relations, it follows from equation (3.18) that the specific gas constant is given by

$$
R=C_{p}-C_{v}
$$

For a calorically perfect gas, the specific heats, $C_{v}$ and $C_{p}$, are constant, i.e.

$$
e=C_{v} T, \quad h=C_{p} T .
$$

Equation (3.18) can therefore be written as

$$
p=(\gamma-1) \rho e,
$$

\footnotetext{
${ }^{2}$ In a simple compressible system, electrical, magnetic, gravitational, motion, and surface effects are absent or negligible.

${ }^{3}$ The specific gas constant $R$ is related to the universal gas constant, $R=\mathcal{R} / \mathcal{M}$, with $\mathcal{R}=8314.3$ $\mathrm{J} /(\mathrm{kmol} \cdot \mathrm{K})$ and $\mathcal{M}$ is the molecular weight in $\mathrm{kg} / \mathrm{kmol}$.
} 
where $\gamma$ is the ratio of specific heats, $\gamma=\frac{C_{p}}{C_{v}}$. Additionally, we can write

$$
\begin{aligned}
& T=\frac{\gamma-1}{R} e, \\
& h=\gamma e,
\end{aligned}
$$

such that all thermodynamic variables are now expressed in terms of $\rho$ and $e$.

The specific gas constant for air is $R=287.05 \mathrm{~J} /(\mathrm{kg} \cdot \mathrm{K})$, whereas its ratio of specific heats is $\gamma=1.4$.

\subsubsection{Constitutive relations}

The viscous stress tensor $\underline{\underline{\tau}}$ and heat flux vector $\underline{q}$ need to be defined.

\section{Viscous Stresses: Newtonian Fluids}

For the flow of air it is common to assume Newtonian behavior, i.e. the viscous stress is linearly related to the strain rate:

$$
\underline{\underline{\tau}}=2 \mu \underline{\underline{s}}+\lambda(\underline{\nabla} \cdot \underline{u}) \underline{\underline{I}}
$$

where $\underline{\underline{s}}$ is the instantaneous strain rate tensor defined as

$$
\underline{\underline{s}}=\frac{1}{2}\left(\underline{\nabla} \underline{u}+(\underline{\nabla} \underline{u})^{T}\right)
$$

$\mu$ is the molecular dynamic viscosity coefficient, and $\lambda$ the second coefficient of viscosity. This can also be written in index notation as

$$
\tau_{i j}=2 \mu s_{i j}+\lambda s_{k k} \delta_{i j}, \quad s_{i j}=\frac{1}{2}\left(\frac{\partial u_{i}}{\partial x_{j}}+\frac{\partial u_{j}}{\partial x_{i}}\right) .
$$

According to Stokes' hypothesis, the trace of the viscous stress tensor should be zero, i.e. $(2 \mu+3 \lambda) \underline{\nabla} \cdot \underline{u}=0$. This assumption means that the mean normal stress, $\frac{1}{3} \sigma_{i i}$, and the thermodynamic pressure cancel each other, or that the mechanical pressure and thermodynamic pressure are equal [66]. Therefore, the second viscosity coefficient is equal to $\lambda=-\frac{2}{3} \mu$.

The molecular dynamic viscosity coefficient depends on temperature and follows from the semi-empirical relation by Sutherland [67]:

$$
\mu(T)=\mu_{0}\left(\frac{T}{T_{0}}\right)^{3 / 2} \frac{T_{0}+S}{T+S},
$$

where $S$ is Sutherland's constant, and $\mu_{0}$ and $T_{0}$ are reference values. For air the following values are used:

$$
S=110.4 \mathrm{~K}, \mu_{0}=1.716 \cdot 10^{-5} \mathrm{~Pa} \cdot \mathrm{s}, T_{0}=273.15 \mathrm{~K} .
$$




\section{Heat conduction: Fourier's Law}

Heat conduction is modeled using Fourier's law:

$$
\underline{q}=-\kappa(\underline{\nabla} T)
$$

where $\kappa$ is the thermal conduction coefficient. Using the equations of state, equation (3.29) can be rewritten in terms of the specific internal energy, e. Furthermore, we can introduce the Prandtl number, $\mathrm{Pr}$, which is the ratio of the molecular diffusivity of momentum (kinematic viscosity, $\nu=\mu / \rho)$ to the molecular diffusivity of heat $\left(\kappa / \rho C_{p}\right)$, i.e.

$$
\operatorname{Pr}=\frac{\nu}{\alpha}=\frac{C_{p} \mu}{\kappa}
$$

Fourier's law can therefore be rewritten as

$$
\underline{q}=-\frac{\gamma \mu}{\operatorname{Pr}}(\underline{\nabla} e)
$$

Since $\gamma$ and $\operatorname{Pr}$ are (approximately) constant, this formulation gives an implicit temperature dependency of the conductivity coefficient through the use of Sutherland's law for the viscosity coefficient. For air, $\operatorname{Pr}=0.72$.

\subsection{Conservation Equations in a Non-Inertial, Rotating Frame of Reference}

For simulation of flows around steadily rotating wind turbine blades, it is advantageous to transform the conservation laws from an inertial frame of reference to a non-inertial frame of reference: a frame rotating with constant angular velocity $\underline{\Omega}$. In the following derivation, the control volumes are considered to be fixed to the rotating frame and undeformable. The approach taken to derive the equations is to start with the equations in the inertial frame of reference and introduce transformations to the rotating frame of reference.

Although vectors can be defined with respect to the inertial system as well as with respect to the rotating system, the components of all vectors can be expressed using the basis vectors of the rotating system, i.e. $\underline{f}=f_{i} \underline{e}_{i}$. A vector defined with respect to the inertial system is an absolute vector, whereas a vector defined with respect to the rotating frame of reference is a relative vector.

\subsubsection{Transformation to Rotating Frame of Reference}

If we let the unit basis vectors $\underline{e}_{i}$ rotate with constant angular velocity $|\underline{\Omega}|$ around axis $\underline{\Omega} /|\underline{\Omega}|$, then the following relation holds for the unit vectors of the rotating system:

$$
\frac{d \underline{e}_{i}}{d t}=\underline{\Omega} \times \underline{e}_{i}
$$


This means that the time derivative of a vector $\underline{f}(t)$ equals:

$$
\left(\frac{d \underline{f}(t)}{d t}\right)_{\mathrm{abs}}=\frac{d f_{i}}{d t} \underline{e}_{i}+f_{i} \frac{d \underline{e}_{i}}{d t}=\left(\frac{d \underline{f}(t)}{d t}\right)_{\mathrm{rot}}+\underline{\Omega} \times \underline{f}(t),
$$

where the subscripts 'abs' and 'rot' mean 'as observed in the inertial frame of reference' and 'as observed in the rotating frame of reference', respectively.

The material derivative of a vector $\underline{f}(\underline{x}, t)$ can also be derived in the same way:

$$
\left(\frac{D \underline{f}(\underline{x}, t)}{D t}\right)_{\mathrm{abs}}=\left(\frac{D \underline{f}(\underline{x}, t)}{D t}\right)_{\mathrm{rot}}+\underline{\Omega} \times \underline{f}(\underline{x}, t),
$$

or

$$
\begin{aligned}
& \left(\frac{\partial \underline{f}(\underline{x}, t)}{\partial t}\right)_{\mathrm{abs}}+\underline{u}_{\mathrm{abs}}(\underline{x}, t) \cdot \underline{\nabla} \underline{f}(\underline{x}, t) \\
& \quad\left(\frac{\partial \underline{f}(\underline{x}, t)}{\partial t}\right)_{\mathrm{rot}}+\underline{u}_{\mathrm{rot}}(\underline{x}, t) \cdot \underline{\nabla} \underline{f}(\underline{x}, t)+\underline{\Omega} \times \underline{f}(\underline{x}, t) \\
& \Leftrightarrow \\
& \left(\frac{\partial \underline{f}(\underline{x}, t)}{\partial t}\right)_{\mathrm{abs}}=\left(\frac{\partial \underline{f}(\underline{x}, t)}{\partial t}\right)_{\mathrm{rot}}-\underline{u}_{c . s .} \cdot \underline{\nabla} \underline{f}(\underline{x}, t)+\underline{\Omega} \times \underline{f}(\underline{x}, t),
\end{aligned}
$$

where $\underline{u}_{c . s .}=\underline{u}_{\mathrm{abs}}-\underline{u}_{\mathrm{rot}}=\underline{\Omega} \times \underline{x}$ is the velocity of the rotating coordinate system. Then this can be rewritten as

$$
\left(\frac{\partial \underline{f}}{\partial t}\right)_{\mathrm{abs}}=\left(\frac{\partial \underline{f}}{\partial t}\right)_{\mathrm{rot}}-\underline{\nabla} \cdot\left(\underline{f} \underline{u}_{c . s .}\right)+\underline{\Omega} \times \underline{f},
$$

since

$$
\underline{\nabla} \cdot\left(\underline{f}_{c . s .}\right)=\underline{u}_{c . s .} \cdot \underline{\nabla} \underline{f}+\underline{f} \underbrace{\left(\underline{\nabla} \cdot \underline{u}_{c . s .}\right)}_{=0} .
$$

For the transformation of the material derivative of a scalar $\phi(\underline{x}, t)$, the following is obtained:

$$
\begin{aligned}
\left(\frac{D \phi}{D t}\right)_{\mathrm{abs}} & =\left(\frac{D \phi}{D t}\right)_{\mathrm{rot}} \\
& \Leftrightarrow \\
\left(\frac{\partial \phi}{\partial t}\right)_{\mathrm{abs}} & =\left(\frac{\partial \phi}{\partial t}\right)_{\mathrm{rot}}-\underline{\nabla} \cdot\left(\phi \underline{u}_{c . s .}\right)
\end{aligned}
$$

\subsubsection{Navier-Stokes equations in Rotating Frame of Reference}

When the transformation relations of the preceding subsection are introduced in the conservation equations of mass, momentum and energy, the following equations are obtained, 
in terms of absolute quantities:

$$
\frac{\partial}{\partial t} \iiint_{V(t)} \rho d V+\iint_{A(t)} \rho\left(\underline{u}-\underline{u}_{c . s .}\right) \cdot \underline{n} d A=0,
$$

where $\underline{u}=\underline{u}_{\text {abs }}$, i.e. the absolute velocity vector,

$$
\frac{\partial}{\partial t} \iiint_{V(t)} \rho \underline{u} d V+\iint_{A(t)} \rho \underline{u}\left(\underline{u}-\underline{u}_{c . s .}\right) \cdot \underline{n} d A=\iint_{A(t)}(-p \underline{\underline{I}}+\underline{\underline{\tau}}) \underline{n} d A-\iint_{V(t)}(\underline{\Omega} \times(\rho \underline{u})) d V,
$$

where the last volume integral in equation (3.39) describes the centrifugal and Coriolis effects due to the rotation, and

$$
\begin{aligned}
\frac{\partial}{\partial t} \iiint_{V(t)} \rho E d V+\iint_{A(t)} \rho E\left(\underline{u}-\underline{u}_{c . s .}\right) \cdot \underline{n} d A= \\
\qquad \iint_{A(t)}(-p \underline{\underline{I}}+\underline{\underline{\tau}}) \underline{u} \cdot \underline{n} d A-\iint_{V(t)}(\underbrace{(\Omega \times(\rho \underline{u})) \cdot \underline{u}}_{=0}) d V-\iint_{A(t)} \underline{q} \cdot \underline{n} d S .
\end{aligned}
$$

Note that the centrifugal and Coriolis effects do not affect the conservation of total energy, because these forces do not perform any work.

Summarizing, the complete system of conservation equations in the rotational frame, in terms of absolute quantities, reads:

$$
\frac{\partial}{\partial t} \iiint_{V(t)} \boldsymbol{U} d V+\iint_{A(t)}\left(\underline{\boldsymbol{F}}^{c}-\boldsymbol{U} \underline{u}_{c . s}\right) \cdot \underline{n} d A=\iint_{A(t)} \underline{\boldsymbol{F}}^{v} \cdot \underline{n} d A+\iiint_{V(t)} \boldsymbol{Q} d V,
$$

where $\boldsymbol{U}(\underline{x}, t)$ is the column vector with conserved variables, $\underline{\boldsymbol{F}}^{c}(\underline{x}, t)$ is the convective flux vector, $\underline{\boldsymbol{F}}^{v}(\underline{x}, t)$ is the viscous flux vector and $\boldsymbol{Q}(\underline{x}, t)$ is the source term:

$$
\boldsymbol{Q}=\left[\begin{array}{c}
0 \\
-\underline{\Omega} \times(\rho \underline{u}) \\
0
\end{array}\right]
$$

\subsection{Turbulence Modeling}

\subsubsection{Scales of Motion}

The time-dependent, three-dimensional Navier-Stokes equations derived in the previous sections contain all the physics that is needed to obtain the solution of any type of flow (of a calorically perfect gas with Newtonian behavior of the stress tensor). In Computational Fluid Dynamics, approximate numerical solutions of the conservation equations are 
obtained in a discretized flow domain, i.e. on a computational grid or mesh. To assess how fine the computational grid needs to be in order to capture all the relevant scales, an analysis of the scales of motion is needed.

The momentum equations can be non-dimensionalized by introducing the characteristic scales for length $(\mathcal{L})$, velocity $(\mathcal{U})$, density $\left(\rho_{0}\right)$, and viscosity $\left(\mu_{0}\right)$, leading to

$$
\frac{\partial}{\partial \hat{t}}\left(\hat{\rho} \hat{u}_{i}\right)+\frac{\partial}{\partial \hat{x}_{j}}\left(\hat{\rho} \hat{u}_{i} \hat{u}_{j}\right)=-\frac{\partial \hat{p}}{\partial \hat{x}_{i}}+\operatorname{Re}^{-1} \frac{\partial \hat{\tau}_{i j}}{\partial \hat{x}_{j}},
$$

where the hat-sign $(\hat{\bullet})$ indicates a non-dimensional quantity and $\hat{p}=p / \rho_{0} \mathcal{U}^{2}$. Re is the Reynolds number defined as

$$
\operatorname{Re}=\frac{\rho_{0} \mathcal{U} \mathcal{L}}{\mu_{0}}
$$

For small values of Re, the viscous terms in the momentum equations dominate and the non-linear inertial terms can be neglected. However, in most engineering applications Re is large and the inertial terms dominate.

Due to the interaction of the non-linear inertial terms with the viscous terms, instabilities can appear in the flow that lead to highly unsteady, three-dimensional, random irregular motions known as turbulence [68]. It is characterized by the presence of a large range of excited length and time scales.

Richardson [69] introduced the idea of an energy cascade: kinetic energy enters the turbulence (through production) at the largest scales, which is transferred by inviscid processes to smaller and smaller scales until, at the smallest scales, the energy is dissipated by viscous processes. Kolmogorov [70] quantified this idea and introduced three characteristic scales for the smallest motions based on dimensional analysis and by introducing two hypotheses. These hypotheses are repeated below, see Pope [71] for a more detailed discussion.

\section{Kolmogorov's hypothesis of local isotropy}

In general, the large-scale turbulent motions are anisotropic and affected by the boundary conditions of the flow, i.e. they depend on the specific flow problem considered. Kolmogorov argued that this anisotropy is lost when energy is transferred to the smallest motions. So, the first hypothesis states that the small-scale turbulent motions are statistically isotropic.

\section{Kolmogorov's fist similarity hypothesis}

Furthermore, Kolmogorov argued that the statistics of the small-scale motions have a universal form, i.e. geometrically, they are unaffected by the mean flow field and the boundary conditions. This universal form is uniquely determined by the viscous dissipation at these scales (governed by kinematic viscosity $\nu$ ) and the rate of dissipation $\epsilon$, which is 
coupled to the rate at which energy is transferred from the large scales (i.e. the two rates are equal, there is equilibrium; this stems from Kolmogorov's second similarity hypothesis).

With $\nu\left(\left[\right.\right.$ length ${ }^{2} /$ time $\left.]\right)$ and $\epsilon\left(\left[\right.\right.$ length ${ }^{2} /$ time $\left.\left.^{3}\right]\right)$, the following scales for the smallest motions are formed:

- Length scale $\eta=\left(\frac{\nu^{3}}{\epsilon}\right)^{\frac{1}{4}}$;

- Time scale $\tau_{\eta}=\left(\frac{\nu}{\epsilon}\right)^{\frac{1}{2}} ;$ and

- Velocity scale $u_{\eta}=(\epsilon \nu)^{\frac{1}{4}}$.

From experiments on turbulent flows, it is known that the dissipation rate $\epsilon$, which is the reduction in time of the kinetic energy, scales as

$$
\epsilon \sim \mathcal{U}^{2}\left(\frac{\mathcal{U}}{\mathcal{L}}\right)=\frac{\mathcal{U}^{3}}{\mathcal{L}},
$$

where $\mathcal{L} / \mathcal{U}$ is the time scale of the large-scale motions. This means that the the ratio of the smallest to the largest length scale scales as

$$
\frac{\eta}{\mathcal{L}} \sim \operatorname{Re}^{-3 / 4}
$$

In order to accurately resolve all turbulent motions in a three-dimensional domain of size $\mathcal{L}^{3}$, the number of grid points $N_{p}$ that is needed to discretize this domain scales as $(\mathcal{L} / \eta)^{3}$, i.e.

$$
N_{p} \sim \operatorname{Re}^{9 / 4} .
$$

The corresponding time scales that need to be resolved are also very small:

$$
\tau_{\eta} \sim \frac{\mathcal{L}}{\mathcal{U}} \operatorname{Re}^{-1 / 2},
$$

so that the number of time steps $N_{t}$ needed to resolve one time scale of the large-scale motions scales as

$$
N_{t} \sim \operatorname{Re}^{1 / 2}
$$

For flows with Reynolds numbers of $\operatorname{Re}=\mathcal{O}\left(10^{6}\right)$ or higher, the computational and storage requirements for direct numerical simulation (DNS) of turbulence are therefore so huge, that this is not feasible for the available computational resources at the moment or in the foreseeable future. For flow simulations at these high Reynolds numbers, the effects of turbulence on the flow need to be modeled in order to avoid having to resolve all the turbulent structures and fluctuations. That means that coarser grids and larger time steps can be used, which results in reduced computational times and reduced memory requirements. 


\subsubsection{Turbulence Modeling Approach}

In general, turbulence modeling for hydrodynamic problems can be categorized into two approaches.

In the first approach, a spatial filter is applied such that only the large-scale motions are resolved accurately. The effects of the small-scale motions on the resolved ones are modeled. In that it is assumed that the small-scale motions are of a more universal character and not affected by the boundary conditions. This approach is called Large Eddy Simulation (LES) and it gives the most detailed flow solutions but for DNS. However, for the flow cases presented in this thesis, namely wall bounded flows, LES still needs a very fine grid to resolve the large eddies. Furthermore, the corresponding small time scales have to be resolved as well. The computational requirements are still far beyond what is currently feasible for these applications, and therefore this approach will not be pursued here.

The turbulence modeling applied in this work is the second approach, the approach leading to the Unsteady Reynolds-Averaged Navier-Stokes (URANS) equations. In this approach, the flow variables are decomposed in a mean component and a fluctuation. Upon substitution in the Navier-Stokes equations and time-averaging, conservation equations for the mean flow variables are obtained with additional unknown terms. This process is called Reynolds-averaging. The additional terms consist of fluctuation correlations and describe the influence of the fluctuations on the mean flow, mainly an increased (local) diffusivity that leads to increased apparent stresses and increased momentum and energy transfer [68]. These terms have to be modeled in order to close the problem.

The required grid density and the time step size for flow simulations employing the URANS equations are determined by gradients of the mean flow field. These requirements are much less demanding than for LES. Furthermore, if a flow field is statistically stationary, a steady state solution can be computed directly using the RANS approach. A drawback of (U)RANS is the loss of detail of the turbulent motions. Moreover, it is a challenge to construct accurate closure models for (U)RANS, since the modeled fluctuation correlations are less of a universal character than the modeled small-scale motions in LES.

\subsubsection{Reynolds- and Favre Averaging}

The derivation of the URANS equations involves the introduction of flow variables, i.e. $\phi(\underline{x}, t) \in\{\rho(\underline{x}, t), \underline{u}(\underline{x}, t), p(\underline{x}, t), e(\underline{x}, t), h(\underline{x}, t)\}$, that are decomposed in a mean and a fluctuating component, known as the Reynolds-decomposition, as follows:

$$
\phi(\underline{x}, t)=\bar{\phi}(\underline{x}, t)+\phi^{\prime}(\underline{x}, t),
$$

where $\bar{\phi}$ is the mean and $\phi^{\prime}$ the fluctuation. By definition, the mean of the fluctuations is zero: $\overline{\phi^{\prime}} \equiv 0$. Note that the mean is still dependent on time, but its characteristic time scale is much larger than that of the fluctuations.

The mean is found by time averaging over a period $T$, which should be much larger than the period of the small-scale motions and much smaller than the period of large-scale 
motions:

$$
\bar{\phi}(\underline{x}, t)=\frac{1}{T} \int_{t}^{t+T} \phi(\underline{x}, \tau) d \tau .
$$

When this so-called Reynolds-averaging is introduced in the compressible NavierStokes equations, correlations involving density fluctuations appear. Because correlations between $\rho^{\prime}$ and fluctuations of other variables are not readily available, the introduction of density fluctuations is eliminated by using for some variables density-weighed averaging, suggested by Favre [72]:

$$
\widetilde{\phi}(\underline{x}, t)=\frac{\overline{\rho \phi}(\underline{x}, t)}{\bar{\rho}(\underline{x}, t)},
$$

where $\bar{\rho}$ is the Reynolds-averaged density and $\tilde{\phi}$ is the density- or Favre-averaged quantity. The decomposition using Favre-averaging reads

$$
\phi(\underline{x}, t)=\widetilde{\phi}(\underline{x}, t)+\phi^{\prime \prime}(\underline{x}, t),
$$

where $\phi^{\prime \prime}$ is the fluctuation with respect to the Favre-averaged mean value.

For the manipulations using averaged quantities and fluctuations, a set of 'averaging rules' can be formulated:

1. The Reynolds-average of a Reynolds-fluctuation is zero by definition:

$$
\overline{\phi^{\prime}} \equiv 0 \text {. }
$$

2. The Reynolds-average of a Reynolds-averaged quantity is again the Reynolds-averaged quantity:

$$
\overline{(\bar{\phi})}=\bar{\phi}
$$

3. The Reynolds-average of a product of two quantities of which one quantity is already averaged, equals the product of the two Reynolds-averaged quantities:

$$
\overline{(\bar{\phi} \psi)}=\bar{\phi} \bar{\psi}
$$

4. The Reynolds-average of a Favre-averaged quantity equals the Favre-averaged quantity:

$$
\overline{\widetilde{\phi}}=\widetilde{\phi}
$$

because of

$$
\overline{\widetilde{\phi}}=\overline{\left(\overline{\frac{\rho \phi}{\bar{\rho}}}\right)},
$$

and the second rule (equation (3.55) ).

5. Since Reynolds-averaging is a linear operation, the following rule holds:

$$
\overline{\phi+\psi}=\bar{\phi}+\bar{\psi} \text {. }
$$


6. Reynolds-averaging commutes with spatial differentiation, i.e.

$$
\overline{\left(\frac{\partial \phi}{\partial x_{j}}\right)}=\frac{\partial \bar{\phi}}{\partial x_{j}} .
$$

7. Reynolds-averaging also approximately commutes with temporal differentiation, i.e.

$$
\overline{\left(\frac{\partial \phi}{\partial t}\right)} \approx \frac{\partial \bar{\phi}}{\partial t} .
$$

Here, it is assumed that fluctuations associated with turbulence have a time scale much smaller than the period over which time averaging is performed.

8. Finally, the following rule holds:

$$
\overline{\rho \phi^{\prime \prime}}=0,
$$

because of $\phi^{\prime \prime}=\phi-\widetilde{\phi}$, the definition of Favre-averaging, and the previous rules:

$$
\overline{\rho \phi^{\prime \prime}}=\overline{\rho \phi}-\overline{\rho \widetilde{\phi}}=\bar{\rho} \widetilde{\phi}-\bar{\rho} \widetilde{\phi}=0 .
$$

These rules will be used in the next subsection.

\subsubsection{Derivation of URANS Equations}

The unsteady Reynolds-averaged Navier-Stokes equations will be derived in an inertial frame of reference, using the differential formulation in index notation.

\section{Continuity equation}

The continuity equation reads

$$
\frac{\partial \rho}{\partial t}+\frac{\partial}{\partial x_{j}}\left(\rho u_{j}\right)=0 .
$$

The time-averaged continuity equation is obtained by substitution of $u_{i}=\widetilde{u}_{i}+u_{i}^{\prime \prime}$ and subsequently taking the Reynolds-average of equation (3.62). Applying the rules presented in the previous subsection yields

$$
\frac{\partial \bar{\rho}}{\partial t}+\frac{\partial}{\partial x_{j}}\left(\bar{\rho} \widetilde{u}_{j}\right)=0
$$

since $\overline{\rho \widetilde{u}_{j}}=\bar{\rho} \widetilde{u}_{j}$ due to equations (3.56) and (3.57), and $\overline{\rho u_{j}^{\prime \prime}}=0$ due to equation (3.61). Note that equation (3.63) has the same form as the original continuity equation. Due to Favre-averaging, correlations between $\rho^{\prime}$ and $u_{j}^{\prime}$ do not appear. 


\section{Momentum equation}

The momentum equation reads

$$
\frac{\partial}{\partial t}\left(\rho u_{i}\right)+\frac{\partial}{\partial x_{j}}\left(\rho u_{i} u_{j}\right)=-\frac{\partial p}{\partial x_{i}}+\frac{\partial \tau_{i j}}{\partial x_{j}}, i=\{1,2,3\}
$$

Substitution of $u_{i}=\widetilde{u}_{i}+u_{i}^{\prime \prime}$ and subsequently taking the Reynolds-average of equation (3.64) results in

$$
\frac{\partial}{\partial t}\left(\overline{\rho \widetilde{u}_{i}}\right)+\frac{\partial}{\partial t}\left(\overline{\rho u_{i}^{\prime \prime}}\right)+\frac{\partial}{\partial x_{j}}\left(\overline{\rho \widetilde{u}_{i} \widetilde{u}_{j}}+\overline{\rho \widetilde{u}_{i} u_{j}^{\prime \prime}}+\overline{\rho u_{i}^{\prime \prime} \widetilde{u}_{j}}+\overline{\rho u_{i}^{\prime \prime} u_{j}^{\prime \prime}}\right)=-\frac{\partial \bar{p}}{\partial x_{i}}+\frac{\partial \bar{\tau}_{i j}}{\partial x_{j}}
$$

Each term will be considered separately.

- The first term on the left hand side can be rewritten as:

$$
\frac{\partial}{\partial t}\left(\overline{\rho \widetilde{u}_{i}}\right)=\frac{\partial}{\partial t}\left(\bar{\rho}_{i}\right)
$$

due to the averaging rules given in equations (3.56) and (3.57).

- The second term on the left hand side equals zero, since $\overline{\rho u_{i}^{\prime \prime}}=0$, see equation (3.61).

- The third term on the left hand side can be rewritten as:

$$
\frac{\partial}{\partial x_{j}}\left(\overline{\rho \widetilde{u}_{i} \widetilde{u}_{j}}+\overline{\rho \widetilde{u}_{i} u_{j}^{\prime \prime}}+\overline{\rho u_{i}^{\prime \prime} \widetilde{u}_{j}}+\overline{\rho u_{i}^{\prime \prime} u_{j}^{\prime \prime}}\right)=\frac{\partial}{\partial x_{j}}\left(\bar{\rho} \widetilde{u}_{i} \widetilde{u}_{j}+\overline{\rho u_{i}^{\prime \prime} u_{j}^{\prime \prime}}\right)
$$

since

$$
\overline{\rho \widetilde{u}_{i} \widetilde{u}_{j}}=\overline{\overline{\rho u_{i}} \frac{\overline{\rho u_{j}}}{\bar{\rho}}}=\bar{\rho} \widetilde{u}_{i} \widetilde{u}_{j}
$$

and

$$
\overline{\rho u_{i}^{\prime \prime} \widetilde{u}_{j}}=\overline{\rho u_{i}^{\prime \prime} \frac{\overline{\rho u_{j}}}{\bar{\rho}}}=\overline{\rho u_{i}^{\prime \prime}} \widetilde{u}_{j}=0 .
$$

- The first term on the right hand side is the gradient of the Reynolds-averaged pressure. Using the equation of state, equation (3.23), the Reynolds-averaged pressure is coupled to the Favre-averaged internal energy as follows:

$$
\bar{p}=\overline{(\gamma-1) \rho\left(\widetilde{e}+e^{\prime \prime}\right)}=(\gamma-1) \bar{\rho} \widetilde{e},
$$

since $\overline{\rho e^{\prime \prime}}=0$. Note that equation (3.68) has the same form as the original equation of state. 
- Recall that for Newtonian fluids, the shear stress tensor $\tau_{i j}$ is defined as

$$
\tau_{i j}=2 \mu\left(s_{i j}-\frac{1}{3} s_{k k} \delta_{i j}\right)=\mu\left(\left(\frac{\partial u_{i}}{\partial x_{j}}+\frac{\partial u_{j}}{\partial x_{i}}\right)-\frac{2}{3} \frac{\partial u_{k}}{\partial x_{k}} \delta_{i j}\right),
$$

see equation (3.26). In the derivation of the Reynolds-average of the shear stress tensor, which appears in the momentum equation, it is generally assumed that fluctuations of molecular viscosity are negligible. Substitution of the Favre-decomposition for all velocity components and taking the Reynolds-average of the shear stress tensor yields

$$
\bar{\tau}_{i j}=\mu\left(\left(\frac{\partial \widetilde{u}_{i}}{\partial x_{j}}+\frac{\partial \widetilde{u}_{j}}{\partial x_{i}}\right)-\frac{2}{3} \frac{\partial \widetilde{u}_{k}}{\partial x_{k}} \delta_{i j}\right)+\mu\left(\left(\frac{\partial \overline{u_{i}^{\prime \prime}}}{\partial x_{j}}+\frac{\partial \overline{u_{j}^{\prime \prime}}}{\partial x_{i}}\right)-\frac{2}{3} \frac{\partial \overline{u_{k}^{\prime \prime}}}{\partial x_{k}} \delta_{i j}\right) .
$$

This result is problematic because the second part of the result contains gradients of Reynolds-averaged Favre-fluctuations of the velocity components, $\overline{u_{i}^{\prime \prime}}$, which are non-zero. Mathematically, the term $\overline{u_{i}^{\prime \prime}}$ is the difference between the Reynoldsaveraged and Favre-averaged velocity, and thereby a measure of compressibility effects. It is related to the turbulent mass flux $\overline{\rho^{\prime} u_{i}^{\prime}}$ :

$$
\overline{u_{i}^{\prime \prime}}=\overline{u_{i}-\widetilde{u}_{i}}=\bar{u}_{i}-\widetilde{u}_{i}=-\frac{\overline{\rho^{\prime} u_{i}^{\prime}}}{\bar{\rho}} .
$$

Although not exact, see [73], it is assumed that compressibility effects on the shear stress tensor are negligible, i.e.

$$
\bar{\tau}_{i j} \approx \mu\left(\left(\frac{\partial \widetilde{u}_{i}}{\partial x_{j}}+\frac{\partial \widetilde{u}_{j}}{\partial x_{i}}\right)-\frac{2}{3} \frac{\partial \widetilde{u}_{k}}{\partial x_{k}} \delta_{i j}\right) \equiv \widetilde{\tau}_{i j} .
$$

This assumption is valid for the low Mach number flows investigated in the present research.

The end result of averaging the momentum equation can now be written as

$$
\frac{\partial}{\partial t}\left(\bar{\rho} \widetilde{u}_{i}\right)+\frac{\partial}{\partial x_{j}}\left(\bar{\rho} \widetilde{u}_{i} \widetilde{u}_{j}\right)=-\frac{\partial \bar{p}}{\partial x_{i}}+\frac{\partial}{\partial x_{j}}\left(\widetilde{\tau}_{i j}+\bar{\tau}_{\mathrm{t} i j}\right), i=\{1,2,3\} .
$$

where $\bar{\tau}_{\mathrm{t} i j}$ is the so-called Reynolds-stress tensor,

$$
\bar{\tau}_{\mathrm{t} i j} \equiv=-\overline{\rho u_{i}^{\prime \prime} u_{j}^{\prime \prime}}=\bar{\rho}\left(\overline{\frac{\rho u_{i}^{\prime \prime} u_{j}^{\prime \prime}}{\bar{\rho}}}\right)=-\bar{\rho} \widetilde{u_{i}^{\prime \prime} u_{j}^{\prime \prime}},
$$

which is a symmetric tensor. The trace of this tensor is proportional to the so-called Favre-averaged specific turbulence kinetic energy $\widetilde{k}$ (turbulence kinetic energy for short):

$$
\bar{\tau}_{\mathrm{t} i i}=-\bar{\rho} \widetilde{u_{i}^{\prime \prime} u_{i}^{\prime \prime}}=-2 \bar{\rho} \widetilde{k} \Leftrightarrow \widetilde{k}=\frac{1}{2} \widetilde{u_{i}^{\prime \prime} u_{i}^{\prime \prime}} .
$$

Comparing the original and the Reynolds-averaged momentum equation, the Reynoldsstress tensor is a new term with six independent components that has appeared in the momentum equation and thus extra relations are needed to close the equation. 


\section{Energy equation}

For the derivation of the Reynolds-averaged energy equation, the most convenient formulation to start with is the following:

$$
\frac{\partial}{\partial t}\left(\rho e+\frac{1}{2} \rho u_{i} u_{i}\right)+\frac{\partial}{\partial x_{j}}\left(\left(\rho h+\frac{1}{2} \rho u_{i} u_{i}\right) u_{j}\right)=\frac{\partial}{\partial x_{j}}\left(u_{i} \tau_{i j}\right)-\frac{\partial}{\partial x_{j}}\left(q_{j}\right),
$$

with the specific enthalpy $h$ defined as in Equation (3.16), and the heat flux vector $q_{j}$ as in equation (3.31). The derivation of the Reynolds-averaged energy equation starts with substitution of the Favre-decompositions of specific internal energy $e$, specific enthalpy $h$, and the velocity components $u_{i}$. Proceeding in the same way as for the continuity and momentum equations, subsequently the Reynolds-average of the energy equation is taken, leading to the following terms:

- The first term on the left hand side of equation (3.74) yields:

$$
\frac{\partial}{\partial t}\left(\overline{\rho\left(\widetilde{e}+e^{\prime \prime}\right)}+\overline{\frac{1}{2} \rho\left(\widetilde{u}_{i}+u_{i}^{\prime \prime}\right)\left(\widetilde{u}_{i}+u_{i}^{\prime \prime}\right)}\right)=\frac{\partial}{\partial t}\left(\bar{\rho} \widetilde{e}+\frac{1}{2} \bar{\rho} \widetilde{u}_{i} \widetilde{u}_{i}+\frac{1}{2} \overline{\rho u_{i}^{\prime \prime} u_{i}^{\prime \prime}}\right),
$$

where

$$
\frac{1}{2} \overline{\rho u_{i}^{\prime \prime} u_{i}^{\prime \prime}}=\frac{1}{2} \bar{\rho} \widetilde{u_{i}^{\prime \prime} u_{i}^{\prime \prime}}=\bar{\rho} \widetilde{k} .
$$

- The second term on the left hand side of equation (3.74) yields:

$$
\begin{aligned}
& \frac{\partial}{\partial x_{j}}\left(\overline{\left(\rho\left(\widetilde{h}+h^{\prime \prime}\right)+\frac{1}{2} \rho\left(\widetilde{u}_{i}+u_{i}^{\prime \prime}\right)\left(\widetilde{u}_{i}+u_{i}^{\prime \prime}\right)\right)\left(\widetilde{u}_{j}+u_{j}^{\prime \prime}\right)}\right)= \\
& \frac{\partial}{\partial x_{j}}\left(\bar{\rho} \widetilde{h} \widetilde{u}_{j}+\overline{\rho h^{\prime \prime} u_{j}^{\prime \prime}}+\left(\frac{1}{2} \bar{\rho} \widetilde{\rho}_{i} \widetilde{u}_{i}+\frac{1}{2} \overline{\rho u_{i}^{\prime \prime} u_{i}^{\prime \prime}}\right) \widetilde{u}_{j}+\frac{1}{2} \overline{\rho u_{i}^{\prime \prime} u_{i}^{\prime \prime} u_{j}^{\prime \prime}}+\widetilde{u}_{i} \overline{\rho u_{i}^{\prime \prime} u_{j}^{\prime \prime}}\right)= \\
& \frac{\partial}{\partial x_{j}}\left(\bar{\rho} \widetilde{u}_{j}\left(\widetilde{h}+\frac{1}{2} \widetilde{u}_{i} \widetilde{u}_{i}+\widetilde{k}\right)+\overline{\rho h^{\prime \prime} u_{j}^{\prime \prime}}+\frac{1}{2} \overline{\rho u_{i}^{\prime \prime} u_{i}^{\prime \prime} u_{j}^{\prime \prime}}-\widetilde{u}_{i} \bar{\tau}_{\mathrm{t} i j}\right),
\end{aligned}
$$

with $\bar{\tau}_{\text {tij }}$ defined as in Equation (3.72).

- For the first term on the right hand side of equation (3.74) it is again assumed that fluctuations in molecular viscosity are negligible and that compressibility effects on the shear stress tensor are negligible, i.e. $\bar{\tau}_{i j} \approx \widetilde{\tau}_{i j}$. Averaging therefore yields:

$$
\frac{\partial}{\partial x_{j}}\left(\overline{\left(\widetilde{u}_{i}+u_{i}^{\prime \prime}\right) \tau_{i j}}\right) \approx \frac{\partial}{\partial x_{j}}\left(\widetilde{u}_{i} \widetilde{\tau}_{i j}+\overline{u_{i}^{\prime \prime} \tau_{i j}}\right) .
$$

- Finally, for the second term on the right hand side of equation (3.74) it is usually assumed that compressibility effects on the molecular transport of heat is negligible. Averaging therefore yields:

$$
\frac{\partial}{\partial x_{j}}\left(\bar{q}_{j}\right) \approx \frac{\partial}{\partial x_{j}}\left(\widetilde{q}_{j}\right)=\frac{\partial}{\partial x_{j}}\left(-\frac{\gamma \mu}{\operatorname{Pr}} \frac{\partial}{\partial x_{j}} \widetilde{e}\right) .
$$


The final result of Reynolds-averaging the energy equation can be written as:

$$
\begin{aligned}
\frac{\partial}{\partial t}(\bar{\rho} \widetilde{E})+\frac{\partial}{\partial x_{j}}\left(\bar{\rho} \widetilde{H} \widetilde{u}_{j}\right)= & \frac{\partial}{\partial x_{j}}\left(\widetilde{u}_{i}\left(\widetilde{\tau}_{i j}+\bar{\tau}_{\mathrm{t} i j}\right)\right)-\frac{\partial}{\partial x_{j}}\left(\widetilde{q}_{j}+\bar{q}_{\mathrm{t} j}\right) \\
& +\frac{\partial}{\partial x_{j}}\left(\overline{u_{i}^{\prime \prime} \tau_{i j}}-\frac{1}{2} \overline{\rho u_{i}^{\prime \prime} u_{i}^{\prime \prime} u_{j}^{\prime \prime}}\right),
\end{aligned}
$$

with the following remarks:

- the Favre-averaged specific total energy and the Favre-averaged specific total enthalpy now include the Favre-averaged specific turbulence kinetic energy $\widetilde{k}$ :

$$
\begin{gathered}
\widetilde{E}=\widetilde{e}+\frac{1}{2} \widetilde{u}_{i} \widetilde{u}_{i}+\widetilde{k}, \\
\widetilde{H}=\widetilde{h}+\frac{1}{2} \widetilde{u}_{i} \widetilde{u}_{i}+\widetilde{k},
\end{gathered}
$$

- $\bar{\tau}_{\mathrm{t} i j}$ is the Reynolds-stress tensor, defined in Equation (3.72),

- $\bar{q}_{\mathrm{t} j}$ is the turbulent heat flux vector defined as:

$$
\bar{q}_{\mathrm{t} j} \equiv \overline{\rho h^{\prime \prime} u_{j}^{\prime \prime}}=\widetilde{\rho} \widetilde{h^{\prime \prime} u_{j}^{\prime \prime}}
$$

- $\overline{u_{i}^{\prime \prime} \tau_{i j}}$ is a vector corresponding to molecular diffusion of turbulence kinetic energy, and

- $\frac{1}{2} \overline{\rho u_{i}^{\prime \prime} u_{i}^{\prime \prime} u_{j}^{\prime \prime}}$ is a vector corresponding to turbulent transport of turbulence kinetic energy.

Comparing the original energy equation with the new averaged energy equation shows the appearance of several new terms that need to be modeled: besides the Reynolds-stress tensor, which also appeared in the momentum equation, the other new terms are $\bar{q}_{\mathrm{t} j}$, $\overline{u_{i}^{\prime \prime} \tau_{i j}}$ and $\frac{1}{2} \overline{\rho u_{i}^{\prime \prime} u_{i}^{\prime \prime} u_{j}^{\prime \prime}}$. The approximations for all these terms will be presented in the next subsection.

\subsubsection{Closure Approximations}

In order to be able to solve the unsteady Reynolds-averaged Navier-Stokes equations, the new additional terms involving fluctuation correlations have to be modeled. This is known as the closure problem.

\section{Reynolds-Stress Tensor}

The Reynolds-stress tensor appears in the momentum and energy equations. For zero-, one- and two-equation eddy-viscosity models, the Boussinesq approximation is usually 
adopted, which assumes a linear relation between the Reynolds stress and the mean strain rate and reads:

$$
\bar{\tau}_{\mathrm{t} i j}=-\overline{\rho u_{i}^{\prime \prime} u_{j}^{\prime \prime}}=-\bar{\rho} \widetilde{u_{i}^{\prime \prime} u_{j}^{\prime \prime}}=2 \mu_{\mathrm{t}}\left(S_{i j}-\frac{1}{3} S_{k k} \delta_{i j}\right)-\frac{2}{3} \bar{\rho} \tilde{k} \delta_{i j},
$$

where $S_{i j}$ is the mean strain-rate tensor defined as

$$
S_{i j}=\frac{1}{2}\left(\frac{\partial \widetilde{u}_{i}}{\partial x_{j}}+\frac{\partial \widetilde{u}_{j}}{\partial x_{i}}\right)
$$

and $\mu_{\mathrm{t}}(\underline{x}, t)$ is the so-called eddy-viscosity, a coefficient which is dependent on the flow. The actual local value of the eddy-viscosity must be provided by a turbulence model. Note that this approximation yields the correct trace of $\bar{\tau}_{t i j}$, namely $-2 \bar{\rho} \widetilde{k}$, see also Equation (3.73). Most two-equation models have a transport equation for $\tilde{k}$, which will be derived below, so that this quantity can be explicitly calculated. If the transport equation for $\tilde{k}$ is not present, its value is effectively set equal to zero in all equations.

Note that besides using an isotropic eddy-viscosity assumption and a linear relation between the Reynolds stresses and the mean strain rate, the Boussinesq approximation assumes that the Reynolds stresses and the mean strain rate are in equilibrium: the Reynolds stresses adjust instantaneously to a change in mean strain rate, which in reality is not the case. This approximation therefore gives inaccurate results for flows with sudden changes in mean strain rate [68. Section 6.1]. Furthermore, the accuracy of the Boussinesq approximation is known to be lower for flows with 'extra rates of strain' [74], e.g. flows with significant streamline curvature, flows with rotation and flows with boundary-layer separation. Several turbulence models improve predictions for these type of flows by introducing corrections to the eddy-viscosity, see for instance [75, 76, 77. 78], but more general improvements can be expected from a non-linear eddy-viscosity model (e.g. an explicit algebraic stress model [79, 80]), or a second-order closure model. The latter includes the differential equation for each of the 6 independent Reynoldsstress components, however, with additional closure approximations for new unknown correlations (e.g. [81]). These models are more costly in terms of computational-time and-memory requirements. Therefore, in the present work, linear eddy-viscosity models based on the Boussinesq approximation are used, with added corrections to the eddyviscosity.

\section{Turbulent Transport of Heat}

The turbulent transport of heat is modeled in a similar way as the molecular transport of heat, using a turbulent Prandtl number $\mathrm{Pr}_{\mathrm{t}}$ (the ratio between momentum eddy diffusivity and heat-transfer eddy diffusivity):

$$
\bar{q}_{\mathrm{t} j}=\bar{\rho} \widetilde{u_{j}^{\prime \prime} h^{\prime \prime}}=-\frac{\gamma \mu_{\mathrm{t}}}{\operatorname{Pr}_{\mathrm{t}}} \frac{\partial \tilde{e}}{\partial x_{j}}
$$


Usually, a constant value of $\mathrm{Pr}_{t}$ is used. According to Wilcox [68], this is usually satisfactory for shock-free flows up to low supersonic speeds, provided that the heat transfer rate is not too high. In the case of wall bounded flows, a value of 0.9 is usually taken. For free shear flows, values around 0.5 appear to be more appropriate.

\section{Molecular Diffusion and Turbulent Transport of Turbulence Kinetic Energy}

The molecular diffusion, $\overline{u_{i}^{\prime \prime} \tau_{i j}}$, and turbulent transport, $\frac{1}{2} \overline{\rho u_{i}^{\prime \prime} u_{i}^{\prime \prime} u_{j}^{\prime \prime}}$, of turbulence kinetic energy can be left out for flows with Mach numbers up to the supersonic range, since $\bar{\rho} \tilde{k}<<\bar{p}$. However, if $\bar{\rho} \tilde{k}$ does become large, additional closure relations must be provided to ensure conservation of total energy. Usually, the molecular diffusion and turbulent transport of turbulence kinetic energy are modeled as a gradient-transport process:

$$
\overline{u_{i}^{\prime \prime} \tau_{i j}}-\frac{1}{2} \overline{\rho u_{i}^{\prime \prime} u_{i}^{\prime \prime} u_{j}^{\prime \prime}}=\left(\mu+\sigma_{k} \mu_{\mathrm{t}}\right) \frac{\partial \widetilde{k}}{\partial x_{j}}
$$

with $\sigma_{k}$ a (constant) closure coefficient.

\subsubsection{Summary of Closed URANS Equations}

The unsteady Reynolds-averaged Navier-Stokes equations with closure approximations that will actually be solved in the computational method are in a form that is slightly different than the equations presented so far. For numerical stability reasons and without any approximations, the last term in the Boussinesq approximation, equation (3.84), is added to the pressure term, leading to a modified pressure $\bar{p}^{*}$ :

$$
\bar{p}^{*}=\bar{p}+\frac{2}{3} \bar{\rho} \widetilde{k}
$$

This is allowed since

$$
\frac{\partial}{\partial x_{j}}\left(-\frac{2}{3} \bar{\rho} \widetilde{k} \delta_{i j}\right)=\frac{\partial}{\partial x_{i}}\left(-\frac{2}{3} \bar{\rho} \widetilde{k}\right)
$$

and

$$
\frac{\partial}{\partial x_{j}}\left(\widetilde{u}_{i}\left(-\frac{2}{3} \bar{\rho} \widetilde{k} \delta_{i j}\right)\right)=\frac{\partial}{\partial x_{j}}\left(\widetilde{u}_{j}\left(-\frac{2}{3} \bar{\rho} \widetilde{k}\right)\right) .
$$

The conservation equations for the mean flow quantities can now be written as

$$
\frac{\partial}{\partial t} \widetilde{\boldsymbol{U}}+\frac{\partial}{\partial x_{j}}\left(\widetilde{\boldsymbol{F}}_{j}^{c}-\widetilde{\boldsymbol{F}}_{j}^{v}\right)=0
$$

or in integral formulation:

$$
\frac{\partial}{\partial t} \iiint_{V(t)} \tilde{\boldsymbol{U}} d V+\iint_{A(t)}\left(\widetilde{\boldsymbol{F}}_{j}^{c}-\widetilde{\boldsymbol{U}} u_{A j}\right) n_{j} d A-\iint_{A(t)} \widetilde{\boldsymbol{F}}_{j}^{v} n_{j} d A=0,
$$


where $\tilde{\boldsymbol{U}}(\underline{x}, t)$ is the column vector with time-averaged conserved variables

$$
\widetilde{\boldsymbol{U}}=\left[\begin{array}{c}
\bar{\rho} \\
\bar{\rho} \widetilde{u}_{i} \\
\bar{\rho} \widetilde{E}
\end{array}\right]
$$

with the total energy $\widetilde{E}$ defined as

$$
\begin{aligned}
\widetilde{E} & =\widetilde{e}+\frac{1}{2} \widetilde{u}_{i} \widetilde{u}_{i}+\widetilde{k} \\
& =\frac{\bar{p}^{*}}{(\gamma-1) \bar{\rho}}+\frac{1}{2} \widetilde{u}_{i} \widetilde{u}_{i}+\frac{\gamma-\frac{5}{3}}{\gamma-1} \widetilde{k},
\end{aligned}
$$

since

$$
\bar{p}^{*}=(\gamma-1) \bar{\rho} \widetilde{e}+\frac{2}{3} \tilde{\rho} \widetilde{k}
$$

The terms $\underline{\widetilde{\boldsymbol{F}}}^{c}$ and $\widetilde{\boldsymbol{F}}^{v}$ are the convective and viscous flux vectors, respectively, with their spatial components defined as

$$
\begin{aligned}
& \widetilde{\boldsymbol{F}}_{j}^{c}=\left[\begin{array}{c}
\bar{\rho} \widetilde{u}_{j} \\
\bar{\rho} \widetilde{u}_{i} \widetilde{u}_{j}+\bar{p}^{*} \delta_{i j} \\
\left(\bar{\rho} \widetilde{E}+\bar{p}^{*}\right) \widetilde{u}_{j}
\end{array}\right], \\
& \widetilde{\boldsymbol{F}}_{j}^{v}=\left[\begin{array}{c}
0 \\
\widetilde{\tau}_{i j, \text { eff }} \\
\widetilde{u}_{i} \widetilde{\tau}_{i j, \text { eff }}-\widetilde{q}_{j, \text { eff }}+\left(\mu+\sigma_{k} \mu_{\mathrm{t}}\right) \frac{\partial k}{\partial x_{j}}
\end{array}\right] .
\end{aligned}
$$

In this formulation, the Favre-averaged molecular shear stress tensor and the deviatoric part of the Reynolds-stress tensor are combined in an effective shear stress tensor $\widetilde{\tau}_{i j \text {,eff }}$ defined as:

$$
\widetilde{\tau}_{i j, \text { eff }} \equiv 2\left(\mu+\mu_{\mathrm{t}}\right)\left(S_{i j}-\frac{1}{3} S_{k k} \delta_{i j}\right) .
$$

Furthermore, $\widetilde{q}_{j, \text { eff }}$ is the effective heat flux vector defined as:

$$
\widetilde{q}_{j, \mathrm{eff}} \equiv-\left(\frac{\gamma \mu}{\operatorname{Pr}}+\frac{\gamma \mu_{\mathrm{t}}}{\operatorname{Pr}_{\mathrm{t}}}\right) \frac{\partial \tilde{e}}{\partial x_{j}} .
$$

\subsubsection{URANS Equations in a Rotational Frame of Reference}

The URANS equations can also be derived in a rotating frame of reference, starting from the conservation equations derived in subsection 3.2.2. valid for a steadily rotating frame and a non-deformable control volume fixed to this frame. This results in:

$$
\frac{\partial}{\partial t} \iiint_{V(t)} \widetilde{\boldsymbol{U}} d V+\iint_{A(t)}\left(\widetilde{\boldsymbol{F}}_{j}^{c}-\widetilde{\boldsymbol{U}} u_{c . s . j}\right) n_{j} d A=\iint_{A(t)} \widetilde{\boldsymbol{F}}_{j}^{v} n_{j} d A+\iiint_{V(t)} \widetilde{\boldsymbol{Q}} d V,
$$


where $\widetilde{\boldsymbol{Q}}$ is the source vector. This vector is defined as

$$
\widetilde{\boldsymbol{Q}}=\left[\begin{array}{c}
0 \\
-\Omega_{j}\left(\bar{\rho} \widetilde{u}_{k}\right) \epsilon_{i j k} \\
0
\end{array}\right],
$$

where $\epsilon_{i j k}$ is the Levi-Civita symbol.

\subsection{Eddy-Viscosity Turbulence Models}

In the preceding section, the URANS equations have been derived using the Boussinesq approximation. This approximation introduces the eddy-viscosity $\mu_{\mathrm{t}}(\underline{x}, t)$, whose value must be provided by a turbulence model. This section presents some general aspects of widely used eddy-viscosity turbulence models. Based on these aspects, two eddy-viscosity models have been chosen to be used in the present work.

\subsubsection{General Aspects of Eddy-Viscosity Turbulence Models}

Linear eddy-viscosity turbulence models can be categorized in zero-, one-, or two-equation models, indicating the number of transport partial-differential equations used to calculate the eddy-viscosity $\mu_{\mathrm{t}}(\underline{x}, t)$.

Here, we will focus on turbulence models that are able to predict the effects of turbulence including the regions next to solid walls, i.e. the equations can be applied up to a no-slip boundary without the use of empirical wall functions. This is necessary because wall functions are only applicable for attached flows, whereas for the flows encountered in the present work we expect or even force flow separation.

Since the eddy-viscosity has the dimensions of $[\mathrm{kg} / \mathrm{m} \cdot \mathrm{s}]$, it could be modeled using density $\rho$, a velocity scale $v$ and a length scale $l$ :

$$
\mu_{\mathrm{t}} \sim \rho v l,
$$

which is exactly what zero-equation models based on Prandtl's mixing length hypothesis, such as the Baldwin-Lomax model [82], do. Zero-equation models are very simple, but they are incomplete, since prior knowledge of the specific turbulent flow is needed to specify the mixing length for instance. Therefore, these models are limited to flows for which they have been tuned 4 .

One- or two-equation models use one or two transport equations to describe the evolution of some turbulent quantity/quantities. Therefore, the eddy-viscosity derived from these transported quantities becomes dependent on the flow history. Since the turbulence kinetic energy $\widetilde{k}$ appears in the URANS equations, it is advantageous to use a transport equation for $\vec{k}$. This is what most two-equation models choose as one of the two equations. However, one-equation models based on $\widetilde{k}$ are still not complete. Therefore,

\footnotetext{
${ }^{4}$ See Wilcox [68] for a discussion on this subject.
} 
the most popular one-equation models, e.g. the Balwin-Barth [83] and Spalart-Allmaras [84] models, use a transport equation for a variable directly related to the eddy-viscosity and all terms in the URANS equations containing $\widetilde{k}$ are effectively set to zero. The model by Spalart and Allmaras (SA) is one of the two eddy-viscosity models used in this work. It is chosen because, in general, it performs better than the Balwin-Barth model [85], it is a 'local' model, it is robust and converges fast to steady state solutions [86. p. 240]. Furthermore, a one-equation model is computationally less demanding than a two-equation model. A downside of these models is that they are tuned to aerodynamic applications and therefore perform not very well for jet-like free shear regions (they predict too large spreading rates) [68, 87].

Two-equation models commonly use $\widetilde{k}$ as the first transported variable. For compressible flows, the conserved variable is $\bar{\rho} \widetilde{k}$. The transport equation can be derived by multiplying the full momentum equation in non-conservation form by the velocity fluctuation with respect to the Favre-averaged mean velocity and subsequently taking the Reynolds average of the complete equation:

$$
\overline{u_{i}^{\prime \prime}\left(\rho \frac{\partial u_{i}}{\partial t}+\rho u_{j} \frac{\partial u_{i}}{\partial x_{j}}+\frac{\partial p}{\partial x_{i}}+\frac{\partial \tau_{i j}}{\partial x_{j}}\right)}=0 .
$$

After employing the averaging rules presented in equations (3.54)-(3.61), and the continuity equation, the following equation is obtained:

$$
\begin{array}{r}
\frac{\partial}{\partial t}(\bar{\rho} \widetilde{k})+\frac{\partial}{\partial x_{j}}\left(\bar{\rho} \widetilde{k} \widetilde{u}_{j}\right)=-\overline{\rho u_{i}^{\prime \prime} u_{j}^{\prime \prime}} \frac{\partial \widetilde{u}_{i}}{\partial x_{j}}-\overline{\frac{\partial u_{i}^{\prime \prime}}{\partial x_{j}} \tau_{i j}}+\frac{\partial}{\partial x_{j}}\left(\overline{u_{i}^{\prime \prime} \tau_{i j}}-\frac{1}{2} \overline{\rho u_{i}^{\prime \prime} u_{i}^{\prime \prime} u_{j}^{\prime \prime}}\right) \\
-\frac{\partial}{\partial x_{j}}\left(\overline{p^{\prime} u_{j}^{\prime \prime}}\right)-\overline{u_{i}^{\prime \prime}} \frac{\partial \bar{p}}{\partial x_{i}}+\overline{p^{\prime} \frac{\partial u_{i}^{\prime \prime}}{\partial x_{i}}}
\end{array}
$$

The first term on the right hand side of Equation (3.102) represents production of turbulence kinetic energy, hereafter denoted by $P_{k}$. This is the rate at which kinetic energy is transferred from the mean flow to turbulent fluctuations. Note that this production term includes the Reynolds-stress tensor:

$$
P_{k}=-\overline{\rho u_{i}^{\prime \prime} u_{j}^{\prime \prime}} \frac{\partial \widetilde{u}_{i}}{\partial x_{j}}=\bar{\tau}_{\mathrm{t} i j} \frac{\partial \widetilde{u}_{i}}{\partial x_{j}} .
$$

The second term on the right hand side of Equation (3.102) represents dissipation of turbulence kinetic energy to internal energy (heat), hereafter denoted by $D_{k}$. The way this term is modeled depends on the choice of the second transported variable. The third and fourth term on the right hand side of Equation (3.102) represent molecular diffusion and turbulent transport of turbulence kinetic energy, respectively, and are modeled as presented in Equation (3.87). These terms cause spatial transport of turbulence kinetic energy and also appear in the Reynolds-averaged energy equation. The remaining terms on the right hand side of Equation (3.102) represent pressure diffusion, pressure work and 
pressure dilatation, respectively. Little is known about these terms and they are generally ignored. Since pressure work and dilatation vanish for incompressible flows, ignoring them for low Mach number flows is assumed to be a minor source of error. The resulting transport equation for turbulence kinetic energy therefore reads:

$$
\frac{\partial}{\partial t}(\bar{\rho} \widetilde{k})+\frac{\partial}{\partial x_{j}}\left(\bar{\rho} \widetilde{k} \widetilde{u}_{j}\right)=P_{k}-D_{k}+\frac{\partial}{\partial x_{j}}\left(\left(\mu+\sigma_{k} \mu_{\mathrm{t}}\right) \frac{\partial \widetilde{k}}{\partial x_{j}}\right) .
$$

The second transported variable can be chosen based on dimensional analysis. Frequently used choices for the second variable are described below. The transport equation for this variable is usually not derived from the basic conservation equations for mass, momentum and energy. Instead, it is postulated based on physical reasoning and dimensional analysis. Just as the transport equation for turbulence kinetic energy, the equation for the second transported variable usually consists of convection, diffusion, production and destruction terms.

The most widely used two-equation model is the $k-\epsilon$ model. Besides $\widetilde{k}$, it uses a transport equation for turbulent dissipation $\epsilon$. Since $\widetilde{k}$ has the dimensions of $\left[\mathrm{m}^{2} / \mathrm{s}^{2}\right]$ and $\epsilon$ has the dimensions of $\left[\mathrm{m}^{2} / \mathrm{s}^{3}\right]$, the eddy-viscosity can be modeled as:

$$
\mu_{\mathrm{t}} \sim \bar{\rho} \widetilde{k}^{2} / \epsilon
$$

The destruction term in the transport equation for the turbulence kinetic energy (equation (3.104)) for the $k-\epsilon$ model equals

$$
D_{k}=\bar{\rho} \epsilon
$$

There exist a number of different $k-\epsilon$ models, but the model by Jones and Launder [88] with retuned coefficients by Launder and Sharma [89] is considered to be the standard. A disadvantage of the $k-\epsilon$ model is the need of near-wall damping functions, which make the differential equations stiff. Furthermore, it is known to perform poorly for flows with strong adverse pressure gradients. On the other hand, an advantage of this model is that the prediction capabilities of the eddy-viscosity in the outer part of boundary layers is independent of the free-stream values of $\widetilde{k}$ and $\epsilon$.

The second most widely used two-equation model is the $k-\omega$ model, which employs the turbulence frequency $\omega$ as the second transported variable. It has the dimensions $\left[\mathrm{s}^{-1}\right]$. Therefore, the eddy-viscosity is modeled as:

$$
\mu_{\mathrm{t}} \sim \frac{\bar{\rho} \widetilde{k}}{\omega}
$$

Furthermore, the destruction term in the transport equation for the turbulence kinetic energy (equation (3.104)) for the $k-\omega$ model reads

$$
D_{k}=\beta^{*} \bar{\rho} \tilde{k} \omega
$$


where $\beta^{*}$ is a closure coefficient.

The 1988-model by Wilcox [90] is considered to be the standard $k-\omega$ model. Since then, Wilcox has introduced a number of improvements. His 2006-model is the latest version [68]. The $k-\omega$ model has some advantages over the $k-\epsilon$ model: (i) there is no need for near-wall damping functions which stiffen the equations, and (ii) it performs better for flows with strong adverse pressure gradients. However, the prediction capabilities of $k-\omega$ model for the eddy-viscosity in the outer part of boundary layers depends on the free-stream values of $\widetilde{k}$ and $\omega$, an undesired feature that is not present in the $k-\epsilon$ model. Furthermore, the $k-\omega$ model is known to perform less accurate in free shear layers (it predicts larger spreading rates) compared to the $k-\epsilon$ model.

A two-equation model that literally combines the strengths of the $k-\epsilon$ and $k-\omega$ model, is the Shear Stress Transport (SST) model by Menter [91]. It blends both models in such a way that the $k-\omega$ model is used in boundary layer regions, and the $k-\epsilon$ model is used away from boundary layers. It is formulated as a $k-\omega$ model, i.e. the high-Reynolds number version of the $\epsilon$ equation of the $k-\epsilon$ model is transformed to a $\omega$ equation using that $\epsilon \sim \widetilde{k} \omega$. Furthermore, it yields improved results for strong adverse pressure gradients due to a modified eddy-viscosity formulation. Finally, the inclusion of a production limiter removes the unphysical buildup of eddy-viscosity in the stagnation region of airfoils, a problem that has been observed for both the $k-\epsilon$ and the $k-\omega$ model [91]. Therefore, the Shear Stress Transport model by Menter is the second model used in the present work.

In the next subsections, the two chosen eddy-viscosity models will be given in detail: the one-equation eddy-viscosity turbulence model of Spalart and Allmaras, and the twoequation SST model by Menter.

\subsubsection{Spalart-Allmaras One-Equation Turbulence Model}

The one-equation eddy-viscosity model by Spalart and Allmaras (SA) [84] uses a transport equation for a variable directly related to the eddy-viscosity, i.e. $\tilde{\nu}$. This variable has the same behavior as $\nu_{\mathrm{t}}=\mu_{\mathrm{t}} / \bar{\rho}$ except near walls (in the viscous sublayer of a boundary layer), where it behaves linearly with the distance from the wall (as in the log-layer of a boundary layer). Because of this linear behavior, it is easier to resolve and it does not require a very fine mesh near walls. The model was developed based on empiricism, dimensional analysis and Galilean invariance. Although it includes terms that allow for control over the transition location, this option is not used in the present work and the corresponding terms in the governing equations are omitted.

The transport equation, without transition terms, can be written in partial differential equation form as

$\frac{\partial \tilde{\nu}}{\partial t}+\frac{\partial}{\partial x_{j}}\left(\tilde{\nu} \widetilde{u}_{j}\right)=C_{b 1} \tilde{S} \tilde{\nu}+\frac{1}{\sigma}\left[\frac{\partial}{\partial x_{j}}\left((\nu+\tilde{\nu}) \frac{\partial \tilde{\nu}}{\partial x_{j}}\right)+C_{b 2}\left(\frac{\partial \tilde{\nu}}{\partial x_{j}}\right)^{2}\right]-C_{w 1} f_{w}\left(\frac{\tilde{\nu}}{d}\right)^{2}$,

where the molecular kinematic viscosity equals $\nu=\mu / \rho$. The left hand side of equation (3.109) describes convection of $\tilde{\nu}$, whereas the right hand side contains production, 
diffusion and near-wall destruction of $\tilde{\nu}$, respectively. The eddy viscosity is calculated as

$$
\mu_{\mathrm{t}}=\bar{\rho} f_{v 1} \tilde{\nu}
$$

with

$$
\begin{aligned}
f_{v 1} & =\frac{\chi^{3}}{\chi^{3}+C_{v 1}^{3}}, \\
\chi & =\frac{\tilde{\nu}}{\nu}
\end{aligned}
$$

The production term is evaluated using the following relations:

$$
\begin{gathered}
\tilde{S}=\Omega+\frac{\tilde{\nu}}{\kappa^{2} d^{2}} f_{v 2}, \\
f_{v 2}=1-\frac{\chi}{1+\chi f_{v 1}},
\end{gathered}
$$

where $\Omega$ is the magnitude of the mean rotation rate,

$$
\Omega=\sqrt{2 \Omega_{i j} \Omega_{i j}}, \quad \Omega_{i j}=\frac{1}{2}\left(\frac{\partial \widetilde{u}_{i}}{\partial x_{j}}-\frac{\partial \widetilde{u}_{j}}{\partial x_{i}}\right),
$$

$\kappa$ is the Von Kármán constant and $d$ is the distance to the nearest wall. The functions $f_{v 1}$ and $f_{v 2}$ are needed because of the transformation from $\nu_{\mathrm{t}}$ to the working variable $\tilde{\nu}$. The near-wall destruction term is evaluated using the following relations:

$$
\begin{aligned}
f_{w} & =g\left(\frac{1+C_{w 3}^{6}}{g^{6}+C_{w 3}^{6}}\right)^{1 / 6}, \\
g & =r+C_{w 2}\left(r^{6}-r\right), \\
r & =\frac{\tilde{\nu}}{\tilde{S} \kappa^{2} d^{2}} .
\end{aligned}
$$

The constants in the model are defined as

$$
\begin{gathered}
C_{b 1}=0.1355, \quad C_{b 2}=0.622, \\
C_{v 1}=7.1, \quad \sigma=\frac{2}{3}, \quad \kappa=0.41 \\
C_{w 1}=\frac{C_{b 1}}{\kappa^{2}}+\frac{1+C_{b 2}}{\sigma}, \quad C_{w 2}=0.3, \quad C_{w 3}=2 .
\end{gathered}
$$

Note that the diffusion term includes a non-conservative part: $\left(C_{b 2} / \sigma\right)\left(\partial \tilde{\nu} / \partial x_{j}\right)^{2}$. In order to prevent difficulties with the discretization of this term, it is rewritten as:

$$
\frac{C_{b 2}}{\sigma}\left(\frac{\partial \tilde{\nu}}{\partial x_{j}}\right)^{2}=\frac{C_{b 2}}{\sigma}\left(\frac{\partial}{\partial x_{j}}\left(\tilde{\nu} \frac{\partial \tilde{\nu}}{\partial x_{j}}\right)-\tilde{\nu} \frac{\partial}{\partial x_{j}} \frac{\partial \tilde{\nu}}{\partial x_{j}}\right) .
$$


The transport equation as employed in the present work therefore reads

$$
\begin{aligned}
& \frac{\partial \tilde{\nu}}{\partial t}+\frac{\partial}{\partial x_{j}}\left(\tilde{\nu} \widetilde{u}_{j}\right)= \\
& \quad C_{b 1} \tilde{S} \tilde{\nu}+\frac{1}{\sigma}\left(\frac{\partial}{\partial x_{j}}\left(\left(\nu+\left(1+C_{b 2}\right) \tilde{\nu}\right) \frac{\partial \tilde{\nu}}{\partial x_{j}}\right)-C_{b 2} \tilde{\nu} \frac{\partial}{\partial x_{j}} \frac{\partial \tilde{\nu}}{\partial x_{j}}\right)-C_{w 1} f_{w}\left(\frac{\tilde{\nu}}{d}\right)^{2} .
\end{aligned}
$$

The appropriate boundary- and initial conditions will be discussed in section 3.5 .

\subsubsection{Shear Stress Transport Two-Equation Turbulence Model}

The Shear Stress Transport (SST) model blends the $k-\epsilon$ and $k-\omega$ models. It is formulated as a $k-\omega$ model, i.e. the transported conserved variables are

$$
\boldsymbol{U}_{\mathrm{t}}=[\bar{\rho} \widetilde{k}, \bar{\rho} \omega]^{T} .
$$

Here, we introduce the version of the model from 2003 [92] with added sustaining terms that remove non-physical decay of the turbulence variables in the free-stream [93]. This addition is used for external aerodynamic problems only, and simplifies the process of selecting the appropriate values of the turbulence variables at the inlet of the computational domain. The added terms are generally much smaller than the destruction terms inside boundary layers for reasonable free-stream turbulence levels 5 ( $T u \leq 1 \%)$ and do therefore not influence the solution there.

The transport equations read

$$
\begin{gathered}
\frac{\partial}{\partial t}(\bar{\rho} \widetilde{k})+\frac{\partial}{\partial x_{j}}\left(\bar{\rho} \widetilde{k} \widetilde{u}_{j}\right)=P_{k, \lim }-\beta^{*} \bar{\rho}\left(\widetilde{k} \omega-\widetilde{k}_{\mathrm{amb}} \omega_{\mathrm{amb}}\right)+\frac{\partial}{\partial x_{j}}\left(\left(\mu+\sigma_{k} \mu_{\mathrm{t}}\right) \frac{\partial \widetilde{k}}{\partial x_{j}}\right), \\
\frac{\partial}{\partial t}(\bar{\rho} \omega)+\frac{\partial}{\partial x_{j}}\left(\bar{\rho} \omega \widetilde{u}_{j}\right)=\alpha \frac{\bar{\rho}}{\mu_{\mathrm{t}}} P_{k, \lim }-\beta \bar{\rho}\left(\omega^{2}-\omega_{\mathrm{amb}}^{2}\right)+\frac{\partial}{\partial x_{j}}\left(\left(\mu+\sigma_{\omega} \mu_{\mathrm{t}}\right) \frac{\partial \omega}{\partial x_{j}}\right) \\
+2 \bar{\rho}\left(1-F_{1}\right) \frac{\sigma_{\omega 2}}{\omega} \frac{\partial \widetilde{k}}{\partial x_{j}} \frac{\partial \omega}{\partial x_{j}},
\end{gathered}
$$

where $P_{k, \lim }$ is the limited production of turbulent kinetic energy, which prevents the build-up of turbulence in stagnation regions:

$$
P_{k, \lim }=\min \left(P_{k}, 10 \cdot \beta^{*} \bar{\rho} \tilde{k} \omega\right), \quad P_{k}=\widetilde{\tau}_{\mathrm{t} i j} \frac{\partial \widetilde{u}_{i}}{\partial x_{j}} .
$$

\footnotetext{
${ }^{5}$ The free-stream turbulence level in terms of turbulence kinetic energy is defined as $T u=$ $\frac{\sqrt{\frac{1}{3} \widetilde{u}_{i}^{\prime \prime} u_{i}^{\prime \prime}}}{\left|\underline{u}_{\infty}\right|} \cdot 100 \%=\frac{\sqrt{(2 / 3) \widetilde{k}}}{\left|\underline{u}_{\infty}\right|} \cdot 100 \%$.
} 
The eddy viscosity is determined from

$$
\mu_{\mathrm{t}}=\frac{a_{1} \bar{\rho} \widetilde{k}}{\max \left(a_{1} \omega, F_{2} S\right)},
$$

where $S$ is the invariant measure of the mean strain rate: $S=\sqrt{2 S_{i j} S_{i j}}$. This formulation of the eddy viscosity gives improved predictions for flows with adverse pressure gradients, compared to the standard formulation that does not include $S$.

The terms $F_{1}$ and $F_{2}$ are blending functions:

$$
\begin{aligned}
& F_{1}=\tanh \left(\left\{\min \left[\max \left(\frac{\sqrt{\widetilde{k}}}{\beta^{*} \omega d}, \frac{500 \mu}{\bar{\rho} \omega d^{2}}\right)\right], \frac{4 \bar{\rho} \sigma_{\omega 2} \tilde{k}}{C_{D} d^{2}}\right\}^{4}\right), \\
& F_{2}=\tanh \left(\left\{\max \left[\frac{2 \sqrt{\widetilde{k}}}{\beta^{*} \omega d}, \frac{500 \mu}{\bar{\rho} \omega d^{2}}\right]\right\}^{2}\right),
\end{aligned}
$$

where $d$ is the distance to the nearest wall and $C_{D}$ is the positive part of the cross-diffusion term:

$$
C_{D}=\max \left(2 \frac{\bar{\rho} \sigma_{\omega 2}}{\omega} \frac{\partial \tilde{k}}{\partial x_{j}} \frac{\partial \omega}{\partial x_{j}}, 10^{-10}\right) .
$$

Blending function $F_{1}$ is used to blend the model constants of the $k-\epsilon$ and $k-\omega$ models, i.e.

$$
\phi=F_{1} \phi_{1}+\left(1-F_{1}\right) \phi_{2}, \quad \phi=\left\{\sigma_{k}, \sigma_{\omega}, \beta, \alpha\right\},
$$

where subscript 1 refers to the $k-\omega$ set of constants, whereas subscript 2 refers to the $k-\epsilon$ set of constants, which are the converted constants from the model of Launder and Sharma.

Blending function $F_{1}$ equals one over a large part of the boundary layer and tends to zero just before the edge of the boundary layer is reached and is also zero in the freestream. Note that the cross-diffusion term in the transport equation for $\bar{\rho} \omega$ originates from the $k-\epsilon$ model and is only active away from boundary layers.

Blending function $F_{2}$ is used to limit the region where the modified formulation of the eddy viscosity is employed to boundary layers only (and not free shear layers for instance). The layer for which $F_{2}=1$ extends further out into the boundary layer than the layer for which $F_{1}=1$.

Since the blending functions depend on the solution of the turbulence variables, the calculation of the blended model constants must be repeated each time the solution is updated. Furthermore, the resulting blended coefficients are space-dependent and need to be stored locally.

The model constants read:

$$
\begin{array}{cccc}
\sigma_{k 1}=0.85, & \sigma_{\omega 1}=0.5, & \beta_{1}=0.075, & \alpha_{1}=\frac{\beta_{1}}{\beta^{*}}-\frac{\sigma_{\omega 1} \kappa^{2}}{\sqrt{\beta^{*}}} \approx 0.5532 \\
\sigma_{k 2}=1.0, & \sigma_{\omega 2}=0.856, & \beta_{2}=0.0828, & \alpha_{2}=\frac{\beta_{2}}{\beta^{*}}-\frac{\sigma_{\omega 2} \kappa^{2}}{\sqrt{\beta^{*}}} \approx 0.4404 \\
\kappa=0.41 & \beta^{*}=0.09, & a_{1}=0.31
\end{array}
$$


The appropriate boundary conditions for the SST turbulence model, including the ambient values of the turbulence variables present in the sustaining terms, $\widetilde{k}_{\mathrm{amb}}$ and $\omega_{\mathrm{amb}}$, will be presented in section 3.5 .

\subsubsection{Turbulence Model Equations in Integral Conservation Form}

Using Gauß' divergence theorem, the turbulence model equations in partial differential equation form can be rewritten in integral conservation form.

\section{Shear Stress Transport Model}

The resulting integral equations for the SST model can be written in general as

$$
\frac{\partial}{\partial t} \iiint_{V(t)} \boldsymbol{U}_{\mathrm{t}} d V+\iint_{A(t)}\left(\boldsymbol{F}_{\mathrm{t} j}^{c}-\boldsymbol{U}_{\mathrm{t}} u_{A j}\right) n_{j} d A=\iint_{A(t)} \boldsymbol{F}_{\mathrm{t} j}^{v} n_{j} d A+\iiint_{V(t)} \boldsymbol{Q}_{\mathbf{t}} d V,
$$

where $\boldsymbol{U}_{\mathrm{t}}$ is the column vector with conserved turbulence variables, $\boldsymbol{F}_{\mathrm{t} j}^{c}$ and $\boldsymbol{F}_{\mathrm{t} j}^{v}$ are the convective and viscous turbulent flux vector, respectively, defined as

$$
\boldsymbol{F}_{\mathrm{t}_{j}}^{c}=\left[\begin{array}{l}
\bar{\rho} \widetilde{k}_{u_{j}} \\
\bar{\rho} \omega \widetilde{u}_{j}
\end{array}\right], \quad \boldsymbol{F}_{\mathrm{t}_{j}}^{v}=\left[\begin{array}{l}
\left(\mu+\sigma_{k} \mu_{\mathrm{t}}\right) \frac{\partial \widetilde{k}}{\partial x_{j}} \\
\left(\mu+\sigma_{\omega} \mu_{\mathrm{t}}\right) \frac{\partial \omega}{\partial x_{j}}
\end{array}\right] .
$$

Furthermore, $\boldsymbol{Q}_{\mathrm{t}}$ is the source term in the turbulence equations, which includes the production and destruction terms as well as the cross-diffusion term:

$$
\boldsymbol{Q}_{\mathrm{t}}=\left[\begin{array}{c}
P_{k, \lim }-\beta^{*} \bar{\rho}\left(\widetilde{k} \omega-\widetilde{k}_{\mathrm{amb}} \omega_{\mathrm{amb}}\right) \\
\alpha \frac{\bar{\rho}}{\mu_{\mathrm{t}}} P_{k, \lim }-\beta \bar{\rho}\left(\omega^{2}-\omega_{\mathrm{amb}}^{2}\right)+2 \bar{\rho}\left(1-F_{1}\right) \frac{\sigma_{\omega 2}}{\omega} \frac{\partial \widetilde{k}}{\partial x_{j}} \frac{\partial \omega}{\partial x_{j}}
\end{array}\right] .
$$

\section{Spalart-Allmaras Model}

The partial differential equation form of the SA turbulence model can be rewritten in integral conservation form in a similar way as

$$
\frac{\partial}{\partial t} \iiint_{V(t)} U_{\mathrm{t}} d V+\iint_{A(t)}\left(F_{\mathrm{t}_{j}}^{c}-U_{\mathrm{t}} u_{A j}\right) n_{j} d A=\iint_{A(t)} F_{\mathrm{t}_{j}}^{v} n_{j} d A+\iiint_{V(t)} Q_{\mathrm{t}} d V,
$$

with

$$
\begin{gathered}
U_{\mathrm{t}}=\tilde{\nu} \\
F_{\mathrm{t} j}^{c}=\tilde{\nu} \widetilde{u}_{j}, \quad F_{\mathrm{t}_{j}}^{v}=\frac{1}{\sigma}\left(\nu+\left(1+C_{b 2}\right) \tilde{\nu}\right) \frac{\partial \tilde{\nu}}{\partial x_{j}},
\end{gathered}
$$

and

$$
Q_{\mathrm{t}}=C_{b 1} \tilde{S} \tilde{\nu}-\frac{C_{b 2}}{\sigma} \tilde{\nu} \frac{\partial}{\partial x_{j}} \frac{\partial \tilde{\nu}}{\partial x_{j}}-C_{w 1} f_{w}\left(\frac{\tilde{\nu}}{d}\right)^{2}
$$




\subsection{Boundary Conditions}

\subsubsection{Physical Boundary Conditions}

In this subsection, the appropriate physical boundary conditions are presented, i.e. the boundary conditions at a solid wall.

The boundary condition for a viscous flow at a solid wall is the no-slip condition. This condition states that the relative velocity at a no-slip wall is zero:

$$
\underline{\widetilde{u}}-\underline{u}_{\mathrm{wall}}=0,
$$

which simplifies to $\underline{\widetilde{u}}=0$ for a stationary wall.

In this thesis, only adiabatic solid walls are considered, i.e. the normal component of the heat flux vector at the wall is taken equal to zero. Therefore, the component normal to the wall of the gradient of the internal energy is set to zero.

Note that the pressure and density at the wall do not have to be provided from a physical point of view. Section 4.7.1 presents the numerical treatment of the physical boundary conditions. The density and pressure at the wall are discussed there as well.

Furthermore, at a no-slip wall, the eddy-viscosity should be zero. In the SA model, the eddy-viscosity can be controlled directly, so the boundary condition reads

$$
\tilde{\nu}=0 .
$$

The SST turbulence model calculates the eddy-viscosity as presented in equation (3.124). For the eddy-viscosity to be zero on a no-slip wall, the correct boundary condition is that the velocity fluctuations vanish at a no-slip wall, i.e.

$$
\widetilde{k}=0 \text {. }
$$

The value of $\omega$ at the wall follows from the solution of the equations.

\subsubsection{Artificial Boundary Conditions}

Artificial boundaries are needed to obtain a finite computational domain. For aerodynamic problems, these boundaries are commonly located far away from the object in the flow, where the flow is close to uniform and viscous effects are negligible. Unless the outer boundary is a periodic boundary, some boundary conditions have to be specified, depending on the local properties of the flow.

\section{Artificial Boundary Conditions for the URANS equations}

At (locally) planar artificial boundaries, the conservation equations for inviscid flow (the Euler equations) can be simplified to linearized one dimensional equations, with the spatial coordinate normal to the boundary. Since this set of equations is diagonalizable and the flux Jacobian matrix has real eigenvalues, it is a hyperbolic system and the flow is characterized by waves crossing the boundary. The eigenvalues determine the speed of 
the waves. See appendix A for a derivation of the eigenvalues and eigenvectors of the set of inviscid flow equations augmented with turbulence equations. The eigenvalues corresponding to the flow equations read:

$$
\begin{aligned}
\lambda_{1} & =\underline{\widetilde{u}} \cdot \underline{n}-c^{*}, \\
\lambda_{2,3,4} & =\underline{\widetilde{u}} \cdot \underline{n}, \\
\lambda_{5} & =\underline{\widetilde{u}} \cdot \underline{n}+c^{*},
\end{aligned}
$$

where $c^{*}$ is the local (modified) speed of sound and $\underline{n}$ is the unit outward normal vector. According to the theory of Kreiss [94], the number of variables that need to be specified equals the number of waves entering the computational domain, whereas the remaining variables follow from the solution in the computational domain. Thus it depends on the local characteristics of the flow. For subsonic inflow, four variables need to be specified for example. Section 4.7.2 on the numerical treatment of the artificial boundary conditions gives a derivation of some possible approaches.

Besides an inflow/outflow artificial boundary, a symmetry plane is also a possible artificial boundary. For this boundary, the velocity component normal to the boundary and the component normal to the boundary of the gradient of all other variables (tangential velocities, density, pressure) are set equal to zero. The symmetry boundary condition can also be used to model a wind tunnel wall, for which an accurate representation of the boundary layer is of minor interest. The wind tunnel wall is therefore effectively modeled as a slip wall. The required computational grid density normal to such a wall is much less demanding than for a viscous no-slip wall.

\section{Artificial Boundary Conditions for Turbulence Models}

In general, eddy-viscosity turbulence models do not predict transition from laminar to turbulent flow very accurately. Therefore, for external aerodynamic problems it is chosen to let the turbulence models operate in fully-turbulent mode, as recommended by Spalart and Rumsey [93. This is done by specifying the recommended ambient values of the turbulence variables at the (artificial) inflow boundary and adding, if necessary, a source term that prevents eddy-viscosity levels to decay between the inflow boundary and the object of interest. The recommended ambient values are chosen such that transition to turbulence is obtained close to the leading edge of the object in the flow. Furthermore, the resulting free-stream eddy-viscosity does not contaminate the flowfield in non-turbulent flow regions.

Since the SA turbulence model has a near-wall destruction term only, free-stream decay of eddy-viscosity levels is not present. As recommended by Spalart and Rumsey [93], the initial condition and inflow boundary condition for fully turbulent flow behavior reads

$$
\tilde{\nu} \in[3 \nu, 5 \nu],
$$

with $\tilde{\nu}=3 \nu$ being preferable for lower Reynolds numbers because it yields

$$
\left(\frac{\nu_{\mathrm{t}}}{\nu}\right)_{\infty} \approx 0.2
$$


i.e. well below 1 .

The SST model has been presented with sustaining (source) terms included in the transport equation. The appropriate inflow boundary condition for fully turbulent flow behavior reads

$$
\begin{aligned}
& \widetilde{k}_{\infty}=\widetilde{k}_{\mathrm{amb}}=10^{-6}\left|\widetilde{\underline{u}}_{\infty}\right|^{2}, \\
& \omega_{\infty}=\omega_{\mathrm{amb}}=\frac{5\left|\widetilde{\underline{u}}_{\infty}\right|}{L_{\text {charact }}},
\end{aligned}
$$

where $\underline{\widetilde{u}}_{\infty}$ is the free-stream velocity vector and $L_{\text {charact }}$ is a characteristic length scale of the aerodynamic model, such as the chord length of an airfoil. This leads to an eddy-viscosity ratio of

$$
\left(\frac{\mu_{\mathrm{t}}}{\mu}\right)_{\infty}=\left(\frac{\bar{\rho} \widetilde{k}}{\mu \omega}\right)_{\infty}=2 \cdot 10^{-7} \operatorname{Re}_{L} .
$$

At outflow boundaries, the turbulence variables follow from the interior of the computational domain. This is because the corresponding characteristic, $\underline{\widetilde{u}} \cdot \underline{n}$, leaves the computational domain. Furthermore, at symmetry plane boundaries, the variables are specified such that the component of the gradient normal to the boundary of each of these variables is zero.

\subsubsection{Boundary Conditions for Synthetic Jet Actuation}

Since one of the main purposes of this research is to investigate the possibility of using synthetic jet actuation for load/pitch control, a separate subsection is devoted to the way this is incorporated in flow simulations. There are several possible approaches, as observed by Rumsey [95] in a survey of published results of computations for synthetic jet flow control cases from a CFD workshop held in 2004.

The most predictive approach is to include the neck/slot and the cavity of the synthetic jet in the computational domain and employ a moving, no-slip boundary inside the cavity to simulate the movement of an actuator. However, this tends to be a time-consuming option, since it involves reconstructing the computational grid at each time step and including the velocity of the grid in the flow solver.

The second option is to employ surface boundary conditions. This means that the details of the cavity and neck/slot are not included in the computational domain and a time-dependent transpiration boundary condition is applied at the surface where the exit of the synthetic jet is located. There are several options for the prescribed velocity, for instance:

- The most simple option is to prescribe a plug flow in normal direction. In tangential direction, a zero velocity is prescribed during the ejection phase of the actuation cycle. During the ingestion phase, the tangential velocity components cannot be prescribed, they follow from the solution in the computational domain. 


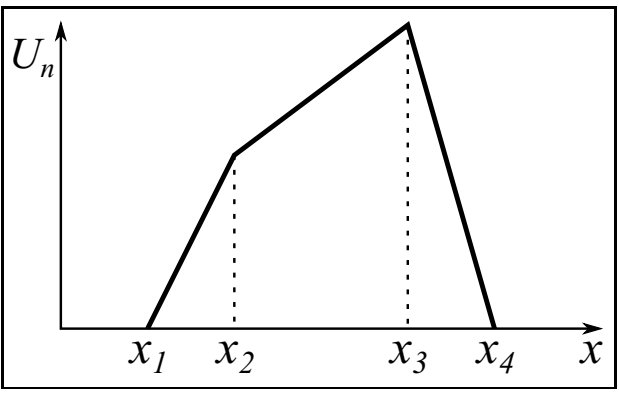

Figure 3.1: Sketch of normal velocity component across the jet-exit-width; reproduced from Ref. [96].

- For the case of a slit, Aram et al. [96] construct a series of simplified, twodimensional velocity boundary conditions. The jet-exit-width $w$ is divided into three parts by introducing four points, $\underline{x}_{1 \ldots 4}$, where $\underline{x}_{1}$ and $\underline{x}_{4}$ are located at the edges (where the relative velocity is always zero due to the no-slip condition), $\underline{x}_{2}$ is located at $25 \%$ and $\underline{x}_{3}$ is located at $75 \%$ of the jet width, see figure 3.1 All the introduced models have zero or uniform tangential velocity during the ejection phase; during ingestion this component cannot be prescribed and follows from the computational domain. At most two spatial degrees of freedom for the normal component of the velocity are employed, which can produce a non-uniform distribution of the normal velocity across the exit of the jet: the profile consists of three piecewise linear parts. They conclude that the following model is a good trade-off between complexity and accuracy:

during ejection, $\mathrm{t} \in\left[0, \frac{1}{2} \mathrm{~T}\right]: \quad$ during ingestion, $\mathrm{t} \in\left[\frac{1}{2} \mathrm{~T}, \mathrm{~T}\right]:$

$$
\begin{array}{cc}
u_{t}(t)=U_{t} & \frac{\partial u_{t}}{\partial n}=0 \\
u_{n}\left(\underline{x}_{2}, t\right)=U_{n}^{(2)} \sin (2 \pi f t) & u_{n}\left(\underline{x}_{2}, t\right)=U_{n}^{(2)} \sin (2 \pi f t) \\
u_{n}\left(\underline{x}_{3}, t\right)=U_{n}^{(3)} \sin (2 \pi f t) & u_{n}\left(\underline{x}_{3}, t\right)=U_{n}^{(3)} \sin (2 \pi f t) \\
U_{n}^{(2)} \neq U_{n}^{(3)} & U_{n}^{(2)}=U_{n}^{(3)}
\end{array}
$$

where $f$ is the actuation frequency and $T$ the period of one actuation cycle, i.e. $T=1 / f$. Since $U_{n}^{(2)}$ and $U_{n}^{(3)}$ can be associated with the spatial-average velocity amplitudes at the left and right half of the jet width, they are coupled through the amplitude of the (sinusoidal) volume flow $Q$, which is constant. Therefore there are only two parameters for this model: $U_{t}$ and $U_{n}^{(2)} / U_{n}^{(3)}$. These parameters have to be matched to results from experiments.

- Balakumar [97] also constructs a two-dimensional boundary condition by applying a spatial $\left(\sin ^{2}\right)$-profile for the velocity component normal to the surface:

$$
u_{n}(s, t)=U_{n} \sin ^{2}(\pi s) \sin (2 \pi f t), \quad s=\frac{\left|\underline{x}-\underline{x}_{\text {start }}\right|}{\left|\underline{x}_{\text {end }}-\underline{x}_{\text {start }}\right|}
$$


where the velocity amplitude $U_{n}$ and the actuation frequency $f$ are matched to results from experiments. The tangential velocity component is constructed as follows: during the ejection phase of the actuation cycle it is assumed that the jet enters the flow domain at a certain (constant) angle with respect to the surface, whereas during the ingestion phase, this velocity component cannot be prescribed.

The option of employing surface boundary conditions is computationally less expensive, since grid movement is not required and less grid points are needed. Furthermore, the computational grids for this option are easier to construct. However, it is difficult to accurately simulate the flow at the exit of the jet in this way, especially for the most simple surface boundary conditions. Additional difficulty is introduced when flow separation occurs at sharp corners inside the geometry of the synthetic jet. Also, specification of turbulence variables (for the ejection phase of the cycle) is difficult for this option, since it is generally unknown how the turbulence variables vary spatially and temporally at the moment the jet exits the actuator.

In the present research a third option is employed to include synthetic jet actuation in flow simulations. It is a hybrid of the first two options, i.e. it involves including the neck/slot and a part of the cavity in the computational domain, and applying a relatively simple time dependent transpiration boundary condition at the bottom of the included synthetic jet geometry. Figure 3.2 shows a sketch of this included geometry. This option is comparable to what some researchers did to simulate synthetic jet actuation for the CFD validation workshop in 2004. For example, Vatsa \& Turkel [98] included only the neck of the synthetic jet in the computational domain and left out the cavity entirely.

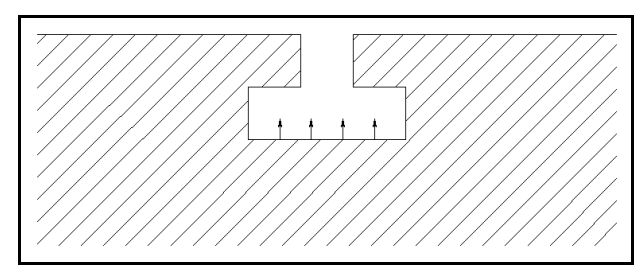

Figure 3.2: Sketch of synthetic jet geometry included in computational domain. Hatched regions indicate solid matter, i.e. regions outside the computational domain.

Although this option increases the complexity of grid generation, the geometry of the synthetic jet does not have to be accurately represented. Furthermore, the flow inside the neck/slit can separate at the inner edges of the neck/slit if part of the cavity is also included in the grid. The flow inside the included geometry can adapt to the cross-flow along the surface. This hybrid option leaves out most of the details of the synthetic jet, but is expected to still lead to a physically accurate situation of the flow near the exit of the jet.

The normal component of the velocity prescribed at the bottom of the included synthetic jet geometry is chosen to be a plug flow varying sinusoidally in time. This can be regarded as a model for a moving piston with a sinusoidal actuation cycle. The location 
of a point on the surface of the piston can be defined by

$$
\underline{x}(t)=\underline{x}(0)+X_{n}(1-\cos (2 \pi f t)) \underline{n},
$$

where $f=\frac{1}{T}$ is the actuation frequency, $X_{n}$ is the amplitude of the displacement of the piston and $\underline{n}$ is the normal vector pointing into the computational domain. Then the velocity of the piston surface equals

$$
u_{n}(t)=\frac{d \underline{x}}{d t} \cdot \underline{n}=U_{n} \sin (2 \pi f t),
$$

where the velocity amplitude $U_{n}$ is given by

$$
U_{n}=2 \pi f X_{n}
$$

The complete set of boundary conditions for this option is defined based on the characteristics of the linearized Euler equations. A distinction is made between the ejection phase (flow entering the domain) and the ingestion phase (flow leaving the domain) of the actuation cycle:

- During the ejection phase, $u_{n}(t) \geq 0, t \in\left[0, \frac{1}{2} T\right]$, four variables need to be specified. Here, we choose to specify the density (equal to the free-stream density) and all the components of the velocity:

$$
\begin{aligned}
\rho(t) & =\rho_{\infty}, \\
u_{n}(t) & =U_{n} \sin (2 \pi f t), \\
u_{t 1}(t) & =0, \\
u_{t 2}(t) & =0,
\end{aligned}
$$

where $u_{n}$ is directed normally to the boundary surface into the computational domain, $u_{t 1}$ and $u_{t 2}$ are directed tangentially to the boundary surface. When needed, the pressure, which is not required for the boundary conditions, is extrapolated from the computational domain. With the inclusion of the turbulence equations, the turbulence variables need to be specified as well. Here it is chosen to apply a zero turbulence level: $\tilde{\nu}=0$ for the SA model or $(\widetilde{k}, \omega)=\left(0, \omega_{\infty}\right)$ for the SST model. Although this is not likely to be accurate, the inclusion of the neck/slit and part of the cavity allows the turbulence variables, and therefore the turbulence level, to develop before the jet exits the actuator.

- During the ingestion phase, $u_{n}(t)<0, t \in\left(\frac{1}{2} T, T\right)$, only one variable can be specified. Here, we choose to specify the normal component of the velocity:

$$
u_{n}(t)=U_{n} \sin (2 \pi f t),
$$

and when needed in the numerical scheme, the remaining variables (tangential components of the velocity, density, pressure and turbulence variables) are extrapolated from the computational domain.

Section 4.7.3 will describe the numerical treatment of these boundary conditions in more detail. 



\section{Chapter 4}

\section{Numerical Solution of THE Mathematical Models}

\subsection{Introduction}

This chapter deals with the numerical solution of the unsteady Reynolds-Averaged NavierStokes equations in combination with the equation(s) of a turbulence model, as derived in chapter 3. This computational procedure consists of the following three steps:

- Pre-processing: the geometry of the flow domain is defined and an appropriate computational domain is derived from the actual flow domain. For external flows, artificial boundaries are located far away from the object in the flow such that they interfere as little as possible with the flow close to the object. The computational domain is then divided into a very large number of non-overlapping cells. The mesh density is chosen such that the geometry is represented accurately and gradients expected in the flow field will be resolved accurately. In other words, the grid density needs to be high in regions with high geometrical curvature and in regions where high gradients of flow variables are expected. Furthermore, it is strived for making the elements as regular as possible with smooth transitions in element size and stretching. This process is called meshing or grid generation.

- Solving: the URANS and turbulence equations are discretized on the mesh, an initial solution is chosen, boundary conditions are imposed and the discretized equations are subsequently solved. This process is repeated for a large number of time steps, marching the solution in time. The discretized equations should be consistent with the continuous equations, and the discretization scheme should be conservative and stable. When consistency and stability requirements are met for a linear or linearized initial value problems, the Lax equivalence theorem gives the sufficient conditions for the numerical solution to converge to the exact solution of the continuous equations. Here, we are dealing with non-linear equations, so that the theorem only gives necessary conditions, not necessarily sufficient conditions, for convergence to the exact solution. Furthermore, the numerical solution should not contain non-physical oscillations near regions with very high gradients of flow variables (such as shocks and contact surfaces). The latter characteristic is also known as monotonicity 
preserving.

- Post-processing: relevant information of the solution is extracted, stored and analyzed.

In the following subsections, a general overview is given of the different aspects associated with solving the conservation equations. Advantages and disadvantages of the different options are discussed and the approach that has been chosen is presented.

\subsubsection{Aspects of Computational Grids}

A computational grid can be structured or unstructured. The method developed in this work employs unstructured grids, since for complicated geometries an unstructured grid is easier to generate. Furthermore, an unstructured grid can have local grid refinement, without changing the grid in the far field, and it can be generated more easily by automatic means. Additionally, unstructured grids can be hybrid grids, which consist of different types of elements. Since the present computational method uses a so-called edge-based or face-based data structure, the elements can be arbitrary, simple polygons (in 2D) or polyhedrons (in 3D). Usually, quadrilaterals (in 2D) and hexahedra or prisms (in 3D) are employed in highly viscous flow regions (such as boundary layers). This reduces the required number of elements and generally improves the accuracy of the solution in these regions, since these types of elements can be aligned with the flow direction.

The choice to use unstructured grids also has potential disadvantages. Since there is no pre-defined ordering in the connectivity of an unstructured grid, the connectivity has to be provided and stored using a more sophisticated data structure than needed for structured grids. This leads to indirect addressing, which could reduce the efficiency of the computational method and also increases the memory requirements. However, the advantage of easier meshing outweighs the possible disadvantages [86].

The process of grid generation is carried out by external programs. Commercial grid generators such as ANSYS ICEM CFD [99] or freely available programs such as GMSH [100] can be used. Preprocessing of the generated grids is done by the program FAVOMESH, developed in the group of Engineering Fluid Dynamics of the University of Twente by P.H. Kelleners and others [101, 102]. The purpose of the preprocessor is to provide the flow solver with the required grid data and connectivity, regardless of the grid generator or original data structure of the grid. FAVOMESH is capable of preprocessing hybrid grids consisting of quadrilaterals and triangles in two dimensions, and hexahedra, prisms, pyramids and tetrahedra in three dimensions.

\subsubsection{Aspects of Spatial Discretization}

The present computational method employs the Finite Volume discretization method, since it is an efficient method that is based directly on the discrete version of the integral conservation equations derived in chapter 3 . The computational space is divided into control volumes and the integral conservation laws are applied to each control volume. 


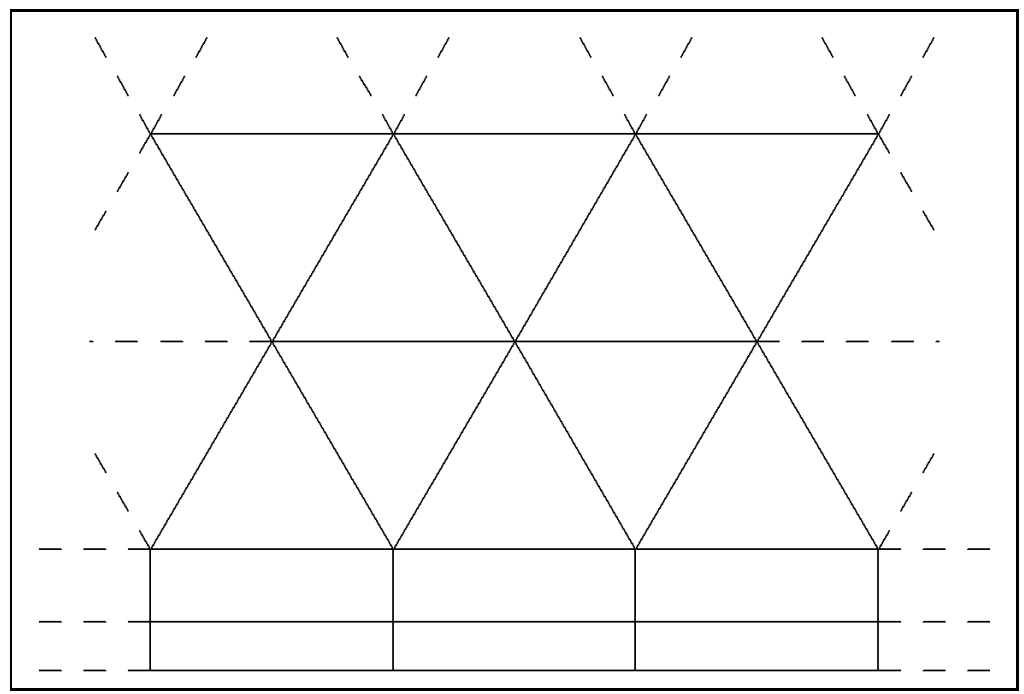

Figure 4.1: Example of a two-dimensional cell-centered computational grid. Lines form control volumes identical to elements.

The surface integrals are approximated by the sum of the fluxes crossing all the faces of each control volume, which automatically makes this method conservative. The sum of the fluxes is associated with the time rate of change of the control-volume-averaged conserved variables.

The order of accuracy of the spatial discretization depends on the order of accuracy of both the integration rule and the approximation of the flux function at the faces of the control volume. For the present computational method, we strive for second order accurate spatial discretizations. Therefore, the midpoint rule is used for the integration together with appropriate second order accurate flux schemes. This will be described in section 4.2. For second-order accurate methods, the control-volume-averaged conserved variables are associated with the centroid of the control volume [103], a fact that is used in the linear reconstruction technique to approximate the solution at both sides of a controlvolume interface. This technique is used to obtain second-order accurate upwind schemes for the convective fluxes. However, in order to preserve monotonicity near flow-solution discontinuities, non-linear limiter functions are needed, such as those by Barth \& Jespersen [104] or by Venkatakrishnan [105. This is due to Godunov's order barrier theorem, which states that a higher-order linear scheme cannot be monotonicity preserving, see [106] for instance.

The two most widely used ways to define the control volumes with respect to the computational grid are:

- The cell-centered approach, for which the control volumes are identical to the elements of the grid. Figure 4.1 shows this approach for a two-dimensional grid. 


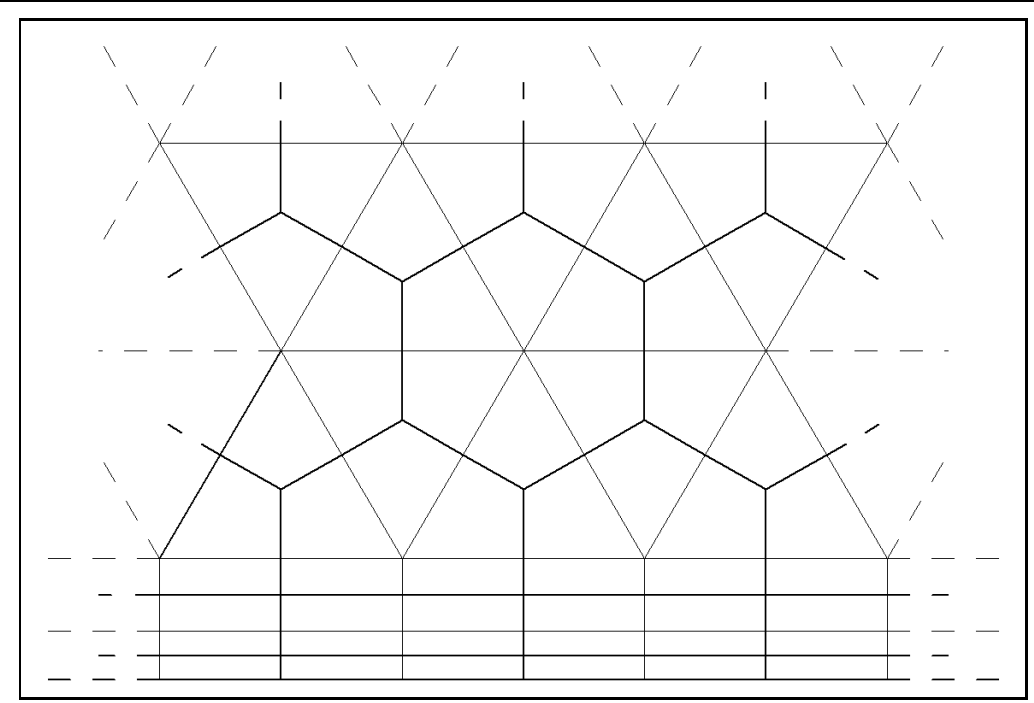

Figure 4.2: Example of a two-dimensional vertex-centered computational grid. Thin lines indicate elements, whereas thick lines indicate median dual control volumes.

- The vertex-centered approach, for which the control volumes are defined around a vertex. There are different options for this approach, but an often used option is the median dual control volume [107]. A three dimensional median dual control volume is constructed by joining the centroids of the surrounding elements to the element-face-centroids and edge mid-points. In two dimensions, it is constructed by joining the centroids of the surrounding elements to the edge mid-points, see figure 4.2

The present computational method uses a data structure based on the interfaces between control volumes, also known as an edge- or face-based data structure. The fluxes are calculated as a loop over these interfaces. Since the boundary conditions are also enforced by a flux crossing the interface between a control volume at the boundary and a "ghost" control volume on the other side of the boundary, the computational method can operate both on cell-centered meshes and vertex-centered median dual meshes. The preprocessor provides the face-based data for a cell-centered mesh or the edge-based data for a vertex-centered mesh.

A disadvantage of the cell-centered method is that the locations of the faces have to be stored, whereas the interface location for median dual meshes is always located in the center between two vertices. However, cell-centered meshes have an important advantage over vertex-centered meshes: the centroid of a cell-centered control volume coincides with the centroid of the element, whereas the centroid of a median-dual control volume does in general not coincide with the vertex location. Moreover, it can even be located outside the control volume. This gives problems for the linear reconstruction technique mentioned 
earlier in this section. Furthermore, this means that for time-accurate calculations on vertex centered meshes a so-called mass matrix must be included in the time derivative term, whereas for cell-centered meshes this matrix is simply an identity matrix (at least for second-order accurate spatial discretizations) [108. Replacing the mass matrix by an identity matrix for vertex-centered meshes compromises the temporal accuracy. Since in the present research we pursue time-accurate solutions and second-order accurate spatial discretizations, the remainder of this thesis describes the computational method for cellcentered meshes only.

\subsubsection{Aspects of Temporal Integration}

The time derivative terms in the conservation equations are discretized separately from the spatial derivative terms. Carrying out the spatial discretization first, results in a system of coupled first-order ordinary differential equations in time. If the computational grid is stationary, it can be written, for a certain control volume $k$, as

$$
V_{\mathrm{k}} \frac{d \boldsymbol{U}_{\mathrm{k}}}{d t}=-\boldsymbol{R}_{\mathrm{k}}
$$

where $V_{\mathrm{k}}$ is the volume of the control volume, $\boldsymbol{U}_{\mathrm{k}}$ is the control-volume-averaged vector with conserved variables and $\boldsymbol{R}_{\mathrm{k}}$ is the so-called residual vector, see section 4.2. The residual vector contains the discretized spatial terms, i.e. the sum of convective and viscous fluxes integrated over the faces of the control volume and, in case of the turbulence equations, a discretized source term. For steady-state solutions, time accuracy is not an issue. For this case, several convergence acceleration techniques have been developed to reduce $\boldsymbol{R}_{\mathrm{k}}$ to "zero"1 1 , such as local time stepping, residual smoothing (only for explicit time stepping methods) and multigrid methods. For time-accurate solutions, these methods can still be applied to a pseudo-time integration with the purpose to reduce the so-called unsteady residual to "zero" for each physical time step. This is called dual-time stepping [109, 110]:

$$
V_{\mathrm{k}} \frac{d \boldsymbol{U}_{\mathrm{k}}}{d \tau}=-\left(V_{\mathrm{k}} \frac{d \boldsymbol{U}_{\mathrm{k}}}{d t}+\boldsymbol{R}_{\mathrm{k}}\right),
$$

where $\tau$ is the pseudo time and the right hand side is the unsteady residual.

There are two options for the discrete (pseudo-) time integration: explicit or implicit. In an explicit method, the control-volume-averaged conserved variables at the next (pseudo) time step are calculated using a residual evaluated at the previous time step. Such a calculation can be carried out using several intermediate stages, such as in RungeKutta schemes [111]. Explicit time integration schemes are relatively easy to implement, require a relatively small amount of operations and do not need much storage. However, it is well known that explicit time integration schemes suffer from a restriction in time step for stability reasons [112]. Compared to a one-stage method, the extra stages in a multistage method can be used to increase the order of accuracy and/or increase the stability region, but this does not really solve the time step restriction.

\footnotetext{
${ }^{1}$ In the present context, the term "zero" means machine accuracy or a sufficiently small value.
} 
Implicit time integration methods evaluate the (unsteady) residual at the new time step. In order to solve the resulting system of algebraic equations, the non-linear residual needs to be linearized about the solution at the previous time step. This results in a linearized system of equations, which includes the Jacobian matrix in the implicit operator, see section 4.4. Although this system of equations can be solved directly, iterative matrix inversion methods are mostly used for these large systems. Examples of iterative methods are the relatively simple Gauß-Seidel iteration method and the more complex Krylov subspace methods.

Implicit time integration methods can utilize much larger time steps, without becoming unstable. For linear problems, they can be constructed such that they are unconditionally stable. In practice, this means that only physical aspects of the simulated flow define the maximum time step size. Furthermore, these methods are known to have better robustness and convergence speed (compared with explicit methods) in the case of stiff equations and/or source terms [86, p. 49], frequently encountered in highly stretched grids (in boundary layer regions) and in the equations from the turbulence model, respectively. However, compared to explicit methods, implicit methods are much more expensive in terms of computational effort per time step and memory requirements. Furthermore, their implementation is more complex.

Since the present work deals with simulating time-accurate turbulent flows, with possible stiff equations and source terms, it has been decided to use an implicit dual-time integration method for the present computational method. The discretization scheme for the physical time derivative determines the accuracy, which in this case is a second-order accurate one-sided finite-difference approximation. The pseudo time steps can be as large as possible, i.e. only restricted by the stability of the iterative method.

To speed up convergence of the iterative solution method for the linearized system of equations, local time stepping is used for the pseudo time steps together with an agglomeration-based algebraic multigrid method, see section 4.6. After a few iteration sweeps, that eliminate high-frequency components of the error efficiently, the multigrid method transfers the remaining low-frequency errors present in a fine-grid solution to coarser grids. There, they effectively become high-frequency errors, which can be reduced efficiently again by the iteration method [113]. Iteration methods are often called relaxation methods or smoothers if they are used to smooth the error present in the solution. The algebraic part of the multigrid method helps to reduce the stiffness in the equations by automatic directional coarsening. An important source of stiffness is high cell stretching in boundary layer regions. The computational grid is only coarsened in the direction of "strong coupling" in the implicit operator, i.e. the direction in which the smoother is effective. In boundary layer regions with highly stretched cells, the direction of "strong coupling" is normal to the wall.

The Jacobian of the flux vector is derived from the first-order accurate version of the convective flux schemes and a thin-layer discretization of the viscous flux term. This reduces the memory requirements, since only the nearest-neighbor control volumes contribute to the flux Jacobian in this case. Furthermore, it increases the diagonal dominance of the implicit operator, which is beneficial for the iteration method [114]. 
The employed iteration method is the Block Symmetric Gauß-Seidel method, see section 4.5. The term "Block" means that the linearized equations associated with the conservation equations applied in a control volume are solved directly. The control volumes are visited and updated sequentially within an iteration sweep. In the Gauß-Seidel method, the variables are updated directly after a correction has been calculated, and not at the end of the iteration sweep, as in the Jacobi iteration method. The term "Symmetric" indicates that each forward iteration sweep is followed by a backward iteration sweep.

Gauß-Seidel iteration in itself does not yield the fastest possible route to convergence. For example, the Krylov subspace method GMRES [115], pre-conditioned by Incomplete LU (ILU) decomposition, achieves substantially faster convergence. However, when used as a smoother in a multigrid method, both methods yield good convergence rates with the Gauß-Seidel method being more efficient than the ILU pre-conditioned GMRES method because of the lower computational cost of the former method [116].

Although the equations corresponding to the turbulence model are coupled to the URANS equations, this coupling is relatively weak [68]. This makes it possible to solve the equations for the turbulence quantities loosely coupled to the URANS equations, i.e. to solve the turbulence model equations with frozen flow variables after having solved the URANS equations with frozen turbulence variables and frozen eddy-viscosity. This strategy is used in the present method. Furthermore, for the moment, the algebraic multigrid method is only applied to the URANS equations.

\subsubsection{Aspects of Computational Time}

Besides the convergence acceleration methods, computational times can be further reduced by parallelization: the simultaneous use of multiple computer processors to solve a certain problem. There are several options to parallelize a computational method [117. First of all, on can parallelize the loops over interfaces or control volumes. Secondly, one can parallelize matrix-vector multiplications. Both of these concepts are only applicable to shared memory computers and are not scalable to a large number of processors. The third option is parallelization by domain decomposition. This option is the most labor-intensive one in terms of implementation, but also the most versatile and scalable approach, applicable to distributed memory computers. Therefore, this approach has been adopted in the present computational method.

The first step of domain decomposition is grid partitioning: dividing the computational grid into a number of partitions with a single layer of overlapping control volumes at the boundaries. This is carried out by METIS routines [118]. Subsequently, the computational method is applied to each grid partition and Message Passing Interface (MPI) routines [119] provide the necessary communication between partitions after each iteration sweep.

This strategy leads to Jacobi-iteration on grid partition boundaries instead of (Symmetric) Gauß-Seidel iteration, since an updated solution is not available until an iteration sweep is completed. Jacobi-iteration is known to converge slower than Gauß-Seidel iteration. For example, for the solution of the Poisson equation, $\nabla^{2} u+f=0$, using a five-point finite-difference stencil, Gauß-Seidel iteration converges twice as fast as Jacobi- 
iteration [114]. Furthermore, no attempt has been made to let the algebraic multigrid method operate across the grid partition boundaries. The effectiveness of the multigrid method is retained as much as possible by favoring grid partitioning normal to walls with highly stretched grids. It is possible that this, in turn, can negatively influence the load balancing and increase the amount of communication between partitions. These choices make the solution method not fully optimal from a mathematical point of view, but since the resulting method is computationally less expensive, it still may be competitive in terms of required total computational time.

\subsubsection{Outlook}

So far, the general aspects of the numerical solution method have been discussed. The approach taken for the present computational method has been presented briefly.

In the remainder of this chapter, the computational method will be described in more detail. First, the spatial discretization will be presented, followed by the time integration method, the iterative solution method and the convergence acceleration techniques. The chapter ends with the numerical treatment of the boundary conditions.

\subsection{Spatial Discretization}

In this section, the Finite-Volume discretization method is introduced. The discretization method will be applied to the URANS equations in an inertial frame of reference, for which the conservation equations in integral formulation (using index notation) are presented in Equation (3.90). The derivation for the turbulence model equations is similar and will be presented briefly in section 4.3. From now on, the tilde and overbar notations of the Favre- and Reynolds averaged variables are dropped, since it is implicitly understood that we are applying the Finite-Volume discretization to the URANS equations.

\subsubsection{Finite Volume Formulation}

The computational domain is formed by multiple non-overlapping, closed cells. In the cell-centered Finite Volume method, these cells are the control volumes and the integral conservation equations are directly applied to each finite control volume present in the computational domain. This means that the time rate of change of the spatially averaged conserved variables in a control volume is balanced by the convective and viscous fluxes crossing the faces surrounding the control volume. As discussed in section 4.1, we are interested in a second-order accurate discretization of these fluxes.

Although the derivation assumes a three-dimensional computational domain, the resulting method is also applicable to two-dimensional computational domains, since it can be thought of as a three-dimensional domain with unit depth in the third coordinate direction. In this direction, the flow will not change and fluxes in this direction will not have to be calculated, since they cancel do not contribute to the overall flux balance. 
The volume of control volume $k$ is denoted by $V_{\mathrm{k}}$. It is enclosed by a set of faces, which are either on the boundary or in the interior of the computational domain. Each face is associated with an outward-pointing face-area vector $\underline{S}_{\mathrm{kl}}$ :

$$
\underline{S}_{\mathrm{kl}}=\underline{n}_{\mathrm{kl}}\left|\underline{S}_{\mathrm{kl}}\right|
$$

where index $l$ is in the set of neighboring interior control volumes and boundary ghost control volumes $2^{2} N_{\mathrm{k}}$, i.e. $l \in N_{\mathrm{k}}$. Furthermore, $\underline{n}_{\mathrm{kl}}$ is the unit outward-pointing normal vector. Note that $\underline{S}_{\mathrm{kl}}=-\underline{S}_{\mathrm{lk}}$.

In this derivation, it is assumed that the computational grid is stationary. The geometry of the control volumes is therefore constant and the velocity of the control-volume surfaces is zero, $\underline{u}_{A}=0$.

The derivation starts with the definition of the control-volume average of $\boldsymbol{U}$ in control volume $k$ :

$$
\boldsymbol{U}_{\mathrm{k}}=\frac{1}{V_{\mathrm{k}}} \iiint_{V_{\mathrm{k}}} \boldsymbol{U} d V
$$

which is associated with the center of gravity of the control volume [103]. Using Equation (4.4), the time derivative term in equation (3.90) can be written for a fixed control volume as

$$
\frac{d}{d t} \iiint_{V_{\mathrm{k}}} \boldsymbol{U} d V=V_{\mathrm{k}} \frac{d \boldsymbol{U}_{\mathrm{k}}}{d t}
$$

The surface integral terms of Equation (3.90) are calculated by summing the contributions from each face of the control volume. For control volume $k$, this can be written as

$$
\iint_{A}\left(\boldsymbol{F}_{j}^{c}-\boldsymbol{F}_{j}^{v}\right) n_{j} d A=\sum_{l \in N_{\mathrm{k}}} \iint_{S_{\mathrm{k} 1}}\left(\boldsymbol{F}_{j}^{c}-\boldsymbol{F}_{j}^{v}\right) n_{j} d A,
$$

where $\boldsymbol{F}_{j}^{c}(\boldsymbol{U})$ is the $j$-component of the convective flux vector, see equation (3.94), and $\boldsymbol{F}_{j}^{v}(\boldsymbol{U}, \underline{\nabla} \boldsymbol{U})$ is the $j$-component of the viscous flux vector, see (3.95).

At each face, the flux is approximated by the midpoint integration rule:

$$
\iint_{S_{\mathrm{k} 1}}\left(\boldsymbol{F}_{j}^{c}-\boldsymbol{F}_{j}^{v}\right) n_{j} d A \approx\left(\left[\underline{\boldsymbol{F}}^{c}\right]_{\mathrm{kl}}-\left[\underline{\boldsymbol{F}}^{v}\right]_{\mathrm{kl}}\right) \cdot \underline{S}_{\mathrm{kl}}
$$

where $[\cdot]_{\mathrm{kl}}$ indicates evaluation at the centroid of face $S_{\mathrm{kl}}$.

The discretization is completed by introducing second-order accurate numerical flux functions $\boldsymbol{\Phi}^{c}$ and $\boldsymbol{\Phi}^{v}$. These functions are approximations of the convective and viscous

\footnotetext{
${ }^{2}$ Ghost control volumes are virtual control volumes located on the boundary, outside of the computational domain. The number of ghost control volumes equals the number of boundary faces. By setting the variables in the ghost control volumes to appropriate values and calculating fluxes over the boundary faces, they can effectively be used to apply the boundary conditions, see section 4.7 .2
} 
flux terms, respectively:

$$
\begin{aligned}
& {\left[\underline{\boldsymbol{F}}^{c}\right]_{\mathrm{kl}} \cdot \underline{S}_{\mathrm{kl}} \approx \boldsymbol{\Phi}_{\mathrm{kl}}^{c}\left(\left[\boldsymbol{U}_{\mathrm{k}}\right]_{\mathrm{kl}},\left[\boldsymbol{U}_{\mathrm{l}}\right]_{\mathrm{kl}}, \underline{S}_{\mathrm{kl}}\right),} \\
& {\left[\underline{\boldsymbol{F}}^{v}\right]_{\mathrm{kl}} \cdot \underline{S}_{\mathrm{kl}} \approx \boldsymbol{\Phi}_{\mathrm{kl}}^{v}\left(\boldsymbol{U}_{\mathrm{k}}, \boldsymbol{U}_{\mathrm{l}},(\underline{\nabla} \boldsymbol{U})_{\mathrm{k}},(\underline{\nabla} \boldsymbol{U})_{1}, \underline{S}_{\mathrm{kl}}\right) .}
\end{aligned}
$$

Here, $\left[\boldsymbol{U}_{\mathrm{k}}\right]_{\mathrm{kl}}$ and $\left[\boldsymbol{U}_{\mathrm{l}}\right]_{\mathrm{kl}}$ are approximations of the conserved variables at the left $(k)$ and right $(l)$ side of the face, respectively. The flux functions must be conservative, i.e.

$$
\begin{aligned}
& \boldsymbol{\Phi}_{\mathrm{kl}}^{c}=-\boldsymbol{\Phi}_{\mathrm{lk}}^{c}, \\
& \boldsymbol{\Phi}_{\mathrm{kl}}^{v}=-\boldsymbol{\Phi}_{\mathrm{lk}}^{v},
\end{aligned}
$$

and they must be consistent, i.e.

$$
\begin{gathered}
\boldsymbol{\Phi}_{\mathrm{kl}}^{c}\left(\boldsymbol{U}, \boldsymbol{U}, \underline{S}_{\mathrm{kl}}\right)=\underline{\boldsymbol{F}}^{c}(\boldsymbol{U}) \cdot \underline{S}_{\mathrm{kl}}, \\
\boldsymbol{\Phi}_{\mathrm{kl}}^{v}\left(\boldsymbol{U}, \boldsymbol{U}, \underline{\nabla} \boldsymbol{U}, \underline{\nabla} \boldsymbol{U}, \underline{S}_{\mathrm{kl} l}\right) \\
=\underline{\boldsymbol{F}}^{v}(\boldsymbol{U}, \underline{\nabla} \boldsymbol{U}) \cdot \underline{S}_{\mathrm{kl}} .
\end{gathered}
$$

For the approximation of the convective flux, there are several second-order accurate flux functions available. A distinction can be made between central and upwind discretizations, see subsections 4.2 .2 and 4.2.3 respectively.

The viscous flux function employed in the present computational method is the secondorder accurate central scheme, which will be discussed in subsection 4.2.6

The semi-discretized conservation equations for control volume $k$ can now be written as

$$
V_{\mathrm{k}} \frac{d \boldsymbol{U}_{\mathrm{k}}}{d t}+\boldsymbol{R}_{\mathrm{k}}=0
$$

where $\boldsymbol{R}_{\mathrm{k}}$ is the residual vector defined as

$$
\boldsymbol{R}_{\mathrm{k}}=\sum_{l \in N_{\mathrm{k}}}\left(\boldsymbol{\Phi}_{\mathrm{kl}}^{c}-\boldsymbol{\Phi}_{\mathrm{kl}}^{v}\right) .
$$

Note that the combined residual vector for all control volumes, denoted by $\boldsymbol{R}$, is not computed by a loop over control volumes. It is more efficient to compute it by a loop over all internal and boundary faces and using the property that the numerical flux function is conservative.

\subsubsection{Central Convective Flux Discretization}

A second-order accurate central scheme for the convective flux can be constructed by averaging the convective fluxes computed from the control-volume averaged conserved variables on both sides of the face:

$$
\boldsymbol{\Phi}_{\mathrm{kl}}^{c}\left(\left[\boldsymbol{U}_{\mathrm{k}}\right]_{\mathrm{kl}},\left[\boldsymbol{U}_{\mathrm{l}}\right]_{\mathrm{kl}}, \underline{S}_{\mathrm{kl}}\right)=\frac{1}{2}\left(\underline{\boldsymbol{F}}^{c}\left(\boldsymbol{U}_{\mathrm{k}}\right)+\underline{\boldsymbol{F}}^{c}\left(\boldsymbol{U}_{\mathrm{l}}\right)\right) \cdot \underline{S}_{\mathrm{kl}}
$$

The central scheme for the convective flux is non-dissipative. Therefore, it needs explicitly added artificial dissipative terms in order to be monotonicity preserving and to prevent so-called odd-even decoupling of the solution. 
Jameson et al. [111] have introduced a central scheme, the JST scheme, with artificial dissipation in the form of a blend of second and fourth differences with coefficients that depend on the local pressure gradient (the pressure switch function). These difference terms are scaled with user-specified constants and with the spectral radius of the convective flux Jacobian, $\left(\lambda^{c}\right)_{\max }$, to form a scalar dissipation scheme. The convective flux function for the JST scheme reads

$$
\boldsymbol{\Phi}_{\mathrm{kl}}^{c}\left(\left[\boldsymbol{U}_{\mathrm{k}}\right]_{\mathrm{kl}},\left[\boldsymbol{U}_{\mathrm{l}}\right]_{\mathrm{kl}}, \underline{S}_{\mathrm{kl}}\right)=\frac{1}{2}\left(\underline{\boldsymbol{F}}^{c}\left(\boldsymbol{U}_{\mathrm{k}}\right)+\underline{\boldsymbol{F}}^{c}\left(\boldsymbol{U}_{\mathrm{l}}\right)\right) \cdot \underline{S}_{\mathrm{kl}}-D_{\mathrm{kl}},
$$

where $D_{\mathrm{kl}}$ is the artificial dissipation flux, which for unstructured grids [120] is given by

$$
\begin{gathered}
D_{\mathrm{kl}}=\left(\lambda_{\mathrm{kl}}^{c}\right)_{\max }\left(\epsilon_{\mathrm{kl}}^{(2)}\left(\boldsymbol{U}_{\mathrm{l}}-\boldsymbol{U}_{\mathrm{k}}\right)-\epsilon_{\mathrm{kl}}^{(4)}\left(L\left(\boldsymbol{U}_{\mathrm{l}}\right)-L\left(\boldsymbol{U}_{\mathrm{k}}\right)\right)\right), \\
L\left(\boldsymbol{U}_{\mathrm{m}}\right)=\sum_{n \in N_{\mathrm{m}}}\left(\boldsymbol{U}_{\mathrm{n}}-\boldsymbol{U}_{\mathrm{m}}\right) .
\end{gathered}
$$

The spectral radius of the convective flux Jacobian at a face is defined as

$$
\left(\lambda_{\mathrm{kl}}^{c}\right)_{\max }=\frac{1}{2}\left(\left|\left(\underline{u}_{\mathrm{k}}+\underline{u}_{\mathrm{l}}\right) \cdot \underline{S}_{\mathrm{kl}}\right|+\left(c_{\mathrm{k}}^{*}+c_{\mathrm{l}}^{*}\right)\left|\underline{S}_{\mathrm{kl}}\right|\right),
$$

and the coefficients $\epsilon^{(2)}$ and $\epsilon^{(4)}$ are given by

$$
\begin{aligned}
& \epsilon_{\mathrm{kl}}^{(2)}=\min \left(\kappa_{2} \bar{\nu}_{\mathrm{kl}}, \frac{1}{2}\right), \\
& \epsilon_{\mathrm{kl}}^{(4)}=\max \left(0, \kappa_{4}-\beta \bar{\nu}_{\mathrm{kl}}\right),
\end{aligned}
$$

with $\kappa_{2}, \kappa_{4}$ and $\beta$ user defined constants. The pressure-switch function $\bar{\nu}_{\mathrm{kl}}$ is used to detect high pressure gradients and is given by

$$
\begin{array}{r}
\bar{\nu}_{\mathrm{kl}}=\max \left\{\nu_{\mathrm{k}}, \nu_{\mathrm{l}}\right\}, \\
\nu_{\mathrm{m}}=\frac{\left|\sum_{n \in N_{\mathrm{m}}}\left(p_{\mathrm{n}}^{*}-p_{\mathrm{m}}^{*}\right)\right|}{\sum_{n \in N_{\mathrm{m}}}\left(p_{\mathrm{n}}^{*}+p_{\mathrm{m}}^{*}\right)} .
\end{array}
$$

Typical values for the constants are $\kappa_{2} \in\left[\frac{1}{4}, \frac{1}{2}\right], \kappa_{4} \in\left[\frac{1}{64}, \frac{1}{32}\right]$ and $\beta=2$.

Note that the artificial dissipation flux contains undivided first and third differences. The sum of these differences for each control volume is therefore equivalent to a finitedifference discretization of a Laplacian operator (second-order differences) multiplied by a term of first order in the grid spacing and a biharmonic operator (fourth-order differences) multiplied by a term of third order in the grid spacing, respectively. The order of accuracy of the complete JST scheme will depend on the behavior of coefficients $\epsilon^{(2)}$ and $\epsilon^{(4)}$. It can be shown that these coefficients are tuned such that in smooth flow, $\epsilon^{(2)}$ is of second 
order whereas $\epsilon^{(4)}$ is of order one. Therefore, the JST scheme remains of second order accuracy in smooth flows. Near shocks, however, it is of first order accuracy, since in that case $\epsilon^{(2)}$ is of order one and $\epsilon^{(4)}$ vanishes.

In subsonic flows shocks will not be present in the computational domain. In that case, the effect of the pressure switch can be switched off completely by choosing $\kappa_{2}=0$ and $\beta=0$. Then, only the (linear) artificial dissipation consisting of fourth-order differences remains.

The artificial dissipation terms can also be scaled with the complete convective flux Jacobian to form a matrix dissipation scheme [121]. This scheme uses concepts employed in the construction of upwind schemes. Its artificial dissipation flux can be written as

$$
D_{\mathrm{kl}}=\left[R|\Lambda| L^{T}\right]_{\mathrm{kl}}\left(\epsilon_{\mathrm{kl}}^{(2)}\left(\boldsymbol{U}_{\mathrm{l}}-\boldsymbol{U}_{\mathrm{k}}\right)-\epsilon_{\mathrm{kl}}^{(4)}\left(L\left(\boldsymbol{U}_{\mathrm{l}}\right)-L\left(\boldsymbol{U}_{\mathrm{k}}\right)\right)\right) \text {, }
$$

where $|\Lambda|$ is a diagonal matrix with the absolute values of the eigenvalues of the convective flux:

$$
|\Lambda|=\operatorname{diag}\left\{\left|\lambda_{1}\right|, \ldots,\left|\lambda_{5}\right|\right\}|\underline{S}|
$$

with

$$
\begin{aligned}
& \lambda_{1}=\underline{u} \cdot \underline{n}-c^{*}, \\
\lambda_{2}=\lambda_{3}= & \lambda_{4}=\underline{u} \cdot \underline{n}, \\
& \lambda_{5}=\underline{u} \cdot \underline{n}+c^{*},
\end{aligned}
$$

and the matrices $R$ and $L^{T}$ contain the right and left eigenvectors, respectively, see appendix A for more information. The matrix $R|\Lambda| L^{T}$ is evaluated at a face by using the arithmetic average of the associated control-volume averaged variables. To prevent zero artificial viscosity for vanishing eigenvalues, i.e. near stagnation points, sonic lines and for grid-aligned flow, the absolute eigenvalues $\left|\lambda_{1}\right|, \ldots,\left|\lambda_{5}\right|$ can be minimized to a fraction of the local speed of sound, for example.

The matrix dissipation scheme is more accurate for the simulation of viscous flows, since the artificially introduced viscosity interferes less with the physical viscous effects on a given computational grid [122]. This means that in comparison with the matrix dissipation scheme, the scalar dissipation scheme needs a more refined grid in highly viscous flow regions in order to obtain grid converged results.

\subsubsection{Upwind Convective Flux Discretization}

Upwind schemes are constructed by considering the wave propagation properties of the convective flux in some way. An analysis of the wave propagation properties of the convective part of the URANS equations augmented with the convective part of the SST turbulence model equations is presented in appendix $\mathrm{A}$

The advantage of upwind schemes is that they are able to capture regions with very high gradients of flow variables (shocks, shear layers) without the need of user-specified, 
flow dependent constants. However, a linear reconstruction of the solution in the control volumes is needed to obtain a second-order accurate upwind scheme, i.e. choosing $\left[\boldsymbol{U}_{\mathrm{k}}\right]_{\mathrm{kl}}=\boldsymbol{U}_{\mathrm{k}}$ and $\left[\boldsymbol{U}_{\mathrm{l}}\right]_{\mathrm{kl}}=\boldsymbol{U}_{\mathrm{l}}$ only yields a first-order accurate scheme. As mentioned before, when very high gradients of the flow variables are present, such as those encountered near shocks, the gradient used in the reconstruction of the solution needs to be limited locally in order to preserve monotonicity. This is carried out by limiter function 3 . The limiting makes higher-order upwind schemes computationally more expensive. In the present work, two upwind schemes are considered that are based on two different approaches: Roe's flux-difference splitting scheme [123] and the AUSM family of flux-vector splitting schemes [124, 125, 126].

Flux-vector splitting schemes only consider the direction of wave propagation and can be written as a central scheme with additional scalar dissipation terms.

On the other hand, flux-difference splitting schemes also consider the actual waves themselves and can be written as a central scheme with an additional matrix dissipation term. Appendix $\mathrm{A}$ shows that when the URANS equations are solved together with a twoequation turbulence model such as the SST turbulence model, this matrix dissipation term is influenced by the convective flux of the turbulence kinetic energy equation. Therefore, Roe's flux-difference splitting scheme is derived for the convective flux of the URANS equations augmented with the convective flux of the SST turbulence model.

\section{Roe's Flux-Difference Splitting Scheme}

Roe's flux-difference splitting scheme is known for its high accuracy in flows with boundary layers [127] and is therefore considered in the present work. At each face of the control volume, a one-dimensional Riemann or shock-tube problem is solved in the direction of the face normal vector, in an approximate (linearized) manner. Therefore, it is a so-called Godunov-type method.

At the face between control volume $k$ and $l$, with $k$ on the left side and $l$ on the right side, we distinguish a left and a right state,

$$
\boldsymbol{U}_{L}=\left(\left[\boldsymbol{U}_{\mathrm{k}}\right]_{\mathrm{kl}},\left(\boldsymbol{U}_{\mathrm{t}}\right)_{\mathrm{k}}\right)^{T}, \quad \boldsymbol{U}_{R}=\left(\left[\boldsymbol{U}_{1}\right]_{\mathrm{kl}},\left(\boldsymbol{U}_{\mathrm{t}}\right)_{\mathrm{l}}\right)^{T},
$$

respectively. The convective flux at this face can be linearized as described in appendix $\mathrm{A}$ Roe's scheme [123] solves this quasi-linear problem exactly, by replacing the convective flux Jacobian matrix by the Roe matrix $\hat{A}_{\text {Roe }}$. This matrix is constructed such that

- it preserves the hyperbolicity of the inviscid flow equations, i.e. $\hat{A}_{\text {Roe }}$ has real eigenvalues and a set of linearly independent eigenvectors;

- it is consistent with the convective flux Jacobian matrix, i.e. if $U_{L}=U_{R}=\boldsymbol{U}$ then

$$
\hat{A}_{\mathrm{Roe}}(\boldsymbol{U})=\frac{\partial}{\partial \boldsymbol{U}}\left(\underline{\boldsymbol{F}}^{c}(\boldsymbol{U}) \cdot \underline{S}_{\mathrm{kl}}\right) ;
$$

\footnotetext{
${ }^{3}$ Note that the pressure switch function in the artificial dissipation for the central scheme can also be interpreted as a limiter: it maximizes the second-difference part of the artificial dissipation near extrema in the solution and switches off the fourth-difference part.
} 
- the convective flux difference

$$
\left(\underline{\boldsymbol{F}}^{c}\left(\boldsymbol{U}_{R}\right)-\underline{\boldsymbol{F}}^{c}\left(\boldsymbol{U}_{L}\right)\right) \cdot \underline{S}_{\mathrm{kl}}=\hat{A}_{\mathrm{Roe}}\left(\boldsymbol{U}_{R}-\boldsymbol{U}_{L}\right),
$$

with

$$
\hat{A}_{\text {Roe }}=\hat{A}_{\text {Roe }}(\hat{\boldsymbol{U}}), \quad \hat{\boldsymbol{U}}=\hat{\boldsymbol{U}}\left(\boldsymbol{U}_{L}, \boldsymbol{U}_{R}\right),
$$

is satisfied for all $\boldsymbol{U}_{L}, \boldsymbol{U}_{R}$.

These conditions ensure that even at shocks and contact discontinuities, where the quasilinear formulation does not hold, the correct propagation of these flow features is still obtained.

It turns out that $\hat{A}_{\text {Roe }}$ equals the convective flux Jacobian matrix, but evaluated using a special state vector $\hat{\boldsymbol{U}}$. Roe [123] shows that $\hat{A}_{\text {Roe, }}$ and thereby $\hat{\boldsymbol{U}}$, can be found by integrating the convective flux Jacobian matrix along a straight line between the left and right states. This line can be defined using a parameter $\theta \in[0,1]$, as $\boldsymbol{U}(\theta)=\boldsymbol{U}_{L}+\theta\left(\boldsymbol{U}_{R}-\boldsymbol{U}_{L}\right)$. The Roe matrix is then found as follows:

$$
\begin{aligned}
\left(\underline{\boldsymbol{F}}^{c}\left(\boldsymbol{U}_{R}\right)-\underline{\boldsymbol{F}}^{c}\left(\boldsymbol{U}_{L}\right)\right) \cdot \underline{S}_{\mathrm{kl}} & =\int_{\theta=0}^{1} \frac{\partial}{\partial \theta}\left(\underline{\boldsymbol{F}}^{c}(\boldsymbol{U}(\theta)) \cdot \underline{S}_{\mathrm{kl}}\right) d \theta \\
& =\int_{\theta=0}^{1} \frac{\partial}{\partial \boldsymbol{U}}\left(\underline{\boldsymbol{F}}^{c}(\boldsymbol{U}(\theta)) \cdot \underline{S}_{\mathrm{kl}}\right) \frac{\partial \boldsymbol{U}(\theta)}{\partial \theta} d \theta \\
& =\left(\int_{\theta=0}^{1} \frac{\partial}{\partial \boldsymbol{U}}\left(\underline{\boldsymbol{F}}^{c}(\boldsymbol{U}(\theta)) \cdot \underline{S}_{\mathrm{kl}}\right) d \theta\right)\left(\boldsymbol{U}_{R}-\boldsymbol{U}_{L}\right)
\end{aligned}
$$

so that

$$
\hat{A}_{\mathrm{Roe}}=\int_{\theta=0}^{1} \frac{\partial}{\partial \boldsymbol{U}}\left(\underline{\boldsymbol{F}}^{c}(\boldsymbol{U}(\theta)) \cdot \underline{S}_{\mathrm{kl}}\right) d \theta .
$$

This process is simplified greatly by introducing the parameter vector $\boldsymbol{W}$,

$$
\boldsymbol{W}=\sqrt{\rho}\left[\begin{array}{c}
1 \\
u \\
v \\
w \\
H \\
k \\
\omega
\end{array}\right],
$$

with $H=E+p^{*} / \rho$. Using $\boldsymbol{W}$, the convective flux vector can be represented by

$$
\underline{\boldsymbol{F}}^{c}(\boldsymbol{U}(\theta))=\left\{\begin{array}{rl}
\underline{\boldsymbol{F}}^{c} & =\underline{\boldsymbol{F}}^{c}(\boldsymbol{W}(\theta)) \\
\boldsymbol{U} & =\overline{\boldsymbol{U}}(\boldsymbol{W}(\theta))
\end{array},\right.
$$


and the Roe matrix can now be found by integrating twice along the straight line connecting $\boldsymbol{W}_{L}$ and $\boldsymbol{W}_{R}$ :

$$
\begin{aligned}
\left(\boldsymbol{U}_{R}-\boldsymbol{U}_{L}\right) & =\left(\int_{\theta=0}^{1} \frac{\partial \boldsymbol{U}(\theta)}{\partial \boldsymbol{W}} d \theta\right)\left(\boldsymbol{W}_{R}-\boldsymbol{W}_{L}\right) \\
& \equiv \hat{B}\left(\boldsymbol{W}_{R}-\boldsymbol{W}_{L}\right) \\
\left(\underline{\boldsymbol{F}}^{c}\left(\boldsymbol{U}_{R}\right)-\underline{\boldsymbol{F}}^{c}\left(\boldsymbol{U}_{L}\right)\right) \cdot \underline{S}_{\mathrm{kl}} & =\left(\int_{\theta=0}^{1} \frac{\partial}{\partial \boldsymbol{W}}\left(\underline{\boldsymbol{F}}^{c}(\boldsymbol{W}(\theta)) \cdot \underline{S}_{\mathrm{kl}}\right) d \theta\right)\left(\boldsymbol{W}_{R}-\boldsymbol{W}_{L}\right) \\
& \equiv \hat{C}\left(\boldsymbol{W}_{R}-\boldsymbol{W}_{L}\right)
\end{aligned}
$$

which leads to

$$
\hat{A}_{R o e}=\hat{C}(\hat{B})^{-1}
$$

Since every component of $\boldsymbol{U}$ and $\left(\underline{\boldsymbol{F}}^{c} \cdot \underline{S}_{\mathrm{kl}}\right)$ is quadratic in the components of $\boldsymbol{W}$, the matrices $\partial \boldsymbol{U} / \partial \boldsymbol{W}$ and $\partial\left(\underline{\boldsymbol{F}}^{c} \cdot \underline{S}_{\mathrm{kl}}\right) / \partial \boldsymbol{W}$ are linear in the components of $\boldsymbol{W}$ and this simplifies the integration. The end result is that the following so-called Roe-averaged variables are needed to construct the state vector $\hat{\boldsymbol{U}}$ :

$$
\begin{gathered}
\hat{u}=\frac{u_{L} \sqrt{\rho_{L}}+u_{R} \sqrt{\rho_{R}}}{\sqrt{\rho_{L}}+\sqrt{\rho_{R}}} \\
\hat{v}=\frac{v_{L} \sqrt{\rho_{L}}+v_{R} \sqrt{\rho_{R}}}{\sqrt{\rho_{L}}+\sqrt{\rho_{R}}} \\
\hat{w}=\frac{w_{L} \sqrt{\rho_{L}}+w_{R} \sqrt{\rho_{R}}}{\sqrt{\rho_{L}}+\sqrt{\rho_{R}}} \\
\hat{H}=\frac{H_{L} \sqrt{\rho_{L}}+H_{R} \sqrt{\rho_{R}}}{\sqrt{\rho_{L}}+\sqrt{\rho_{R}}} \\
\hat{k}=\frac{k_{L} \sqrt{\rho_{L}}+k_{R} \sqrt{\rho_{R}}}{\sqrt{\rho_{L}}+\sqrt{\rho_{R}}} \\
\hat{\omega}=\frac{\omega_{L} \sqrt{\rho_{L}}+\omega_{R} \sqrt{\rho_{R}}}{\sqrt{\rho_{L}}+\sqrt{\rho_{R}}}
\end{gathered}
$$

The Roe-averaged variables given in equations (4.41) to (4.46) completely define the Roe matrix $\hat{A}_{\text {Roe }}$. The average speed of sound is a function of these variables and is given by

$$
\hat{c}^{*}=\sqrt{(\gamma-1)\left(\hat{H}-\frac{1}{2}|\underline{u}|^{2}-\frac{\gamma-\frac{5}{3}}{\gamma-1} \hat{k}\right)} .
$$


The flux function of the convective flux of the URANS equations for Roe's flux difference splitting scheme can now be written as

$$
\begin{aligned}
\boldsymbol{\Phi}_{\mathrm{kl}}^{c}\left(\boldsymbol{U}_{L}, \boldsymbol{U}_{R}, \underline{S}_{\mathrm{kl}}\right)= & \frac{1}{2}\left(\underline{\boldsymbol{F}}^{c}\left(\boldsymbol{U}_{L}\right)+\underline{\boldsymbol{F}}^{c}\left(\boldsymbol{U}_{R}\right)\right) \cdot \underline{S}_{\mathrm{kl}} \\
& -\frac{1}{2}\left|\hat{A}_{R o e, \mathrm{kl}}\right|\left(\boldsymbol{U}_{R}-\boldsymbol{U}_{L}\right),
\end{aligned}
$$

with

$$
\left|\hat{A}_{R o e, \mathrm{kl}}\right|=\left|\hat{A}_{R o e}\left(\hat{\boldsymbol{U}}\left(\boldsymbol{U}_{L}, \boldsymbol{U}_{R}\right)\right)\right|=\hat{R}_{\mathrm{kl}}|\hat{\Lambda}|_{\mathrm{kl}} \hat{L}_{\mathrm{kl}}^{T},
$$

where $\hat{R}_{\mathrm{kl}}$ and $\hat{L}_{\mathrm{kl}}$ are the matrices with right and left eigenvectors of the convective flux Jacobian as defined in equations (A.32) and (A.36), respectively, both evaluated using $\hat{U}$ at the face between control volumes $k$ and $l$. Furthermore, $|\hat{\Lambda}|_{\mathrm{kl}}$ contains the absolute value of the eigenvalues of the convective flux Jacobian on its diagonal, also evaluated using $\hat{U}$ at the face between control volumes $k$ and $l$ :

$$
|\hat{\Lambda}|_{\mathrm{kl}}=\operatorname{diag}\left\{\left|\hat{\lambda}_{1}\right|_{\mathrm{kl}}, \ldots,\left|\hat{\lambda}_{7}\right|_{\mathrm{kl}}\right\}\left|\underline{S}_{\mathrm{kl}}\right|
$$

with

$$
\begin{aligned}
\hat{\lambda}_{1} & =\underline{\hat{u}} \cdot \underline{n}-\hat{c}^{*}, \\
\hat{\lambda}_{2}=\hat{\lambda}_{3}=\hat{\lambda}_{4}=\hat{\lambda}_{6}= & \hat{\lambda}_{7}=\underline{\hat{u}} \cdot \underline{n}, \\
\hat{\lambda}_{5} & =\underline{\hat{u}} \cdot \underline{n}+\hat{c}^{*} .
\end{aligned}
$$

Inspection of $\hat{R}_{\mathrm{kl}}$ and $\hat{L}_{\mathrm{kl}}$, see equations (A.32) and (A.36), shows that we only need part of these matrices to fully evaluate the dissipation term of Roe's flux function for the URANS equations:

$$
\begin{aligned}
& \frac{1}{2}\left(\left|\hat{A}_{R o e, \mathrm{kl}}\right|\left(\boldsymbol{U}_{R}-\boldsymbol{U}_{L}\right)\right)_{[1: 5]}= \\
& \quad \frac{1}{2}\left(\hat{R}_{\mathrm{kl}[1: 5,1: 6]}|\hat{\Lambda}|_{\mathrm{kl}[1: 6,1: 6]} \hat{L}_{\mathrm{kl}[1: 6,1: 6]}\right)\left(\boldsymbol{U}_{R}-\boldsymbol{U}_{L}\right)_{[1: 6]} .
\end{aligned}
$$

As expected, quantities associated with the $\omega$-equation have no influence on this flux function.

When the URANS equations are solved together with the SA turbulence model, the turbulence kinetic energy is not present in the convective flux. In that case, the dissipation term of Roe's flux-difference splitting scheme is obtained as

$$
\begin{aligned}
& \frac{1}{2}\left(\left|\hat{A}_{R o e, \mathrm{kl}}\right|\left(\boldsymbol{U}_{R}-\boldsymbol{U}_{L}\right)\right)_{[1: 5]}= \\
& \quad \frac{1}{2}\left(\hat{R}_{\mathrm{kl}[1: 5,1: 5]}|\hat{\Lambda}|_{\mathrm{kl}[1: 5,1: 5]} \hat{L}_{\mathrm{kl}[1: 5,1: 5]}\right)\left(\boldsymbol{U}_{R}-\boldsymbol{U}_{L}\right)_{[1: 5]},
\end{aligned}
$$

where $\hat{R}_{\mathrm{kl}}$ and $\hat{L}_{\mathrm{kl}}$ are given by equations (A.32) and (A.36), respectively, but with $k$ set to zero. 


\section{Entropy Correction For Roe's Flux-Difference Splitting Scheme}

A downside of Roe's flux difference splitting method is that it is not able to distinguish between stationary compression shocks and non-physical, entropy-condition violating, stationary expansion shocks. In order to solve this problem, some dissipation can be added to spread the expansion shock in an expansion fan by preventing the values of $\lambda_{1}$ and $\lambda_{5}$ to vanish. Here, we use the entropy correction of Harten [128], which replaces $\left|\hat{\lambda}_{i}\right|$ by $\left|\lambda_{i}^{\text {Harten }}\right|$ for $i=\{1,5\}$ :

$$
\left|\hat{\lambda}_{i}^{\text {Harten }}\right|=\left\{\begin{array}{cc}
\left|\hat{\lambda}_{i}\right| & \text { if }\left|\hat{\lambda}_{i}\right|>\delta \\
\frac{\hat{\lambda}_{i}^{2}+\delta^{2}}{2 \delta} & \text { if }\left|\hat{\lambda}_{i}\right| \leq \delta
\end{array} \quad, i=\{1,5\}\right.
$$

where $\delta$ is a small constant, e.g. [129]

$$
\delta=\delta_{0}\left(|\underline{\hat{u}} \cdot \underline{n}|+\hat{c}^{*}\right),
$$

with $\delta_{0} \in(0,0.25]$. To improve the robustness of the computational method, the entropy correction can also be applied to vanishing values of $\hat{\lambda}_{2, \ldots, 4,6,7}$, for instance in the case of grid aligned flow, but this comes with a loss of accuracy in the resolution of shear layers, see for instance Liou [130] for more information. Here, a value of $\delta_{0}=1 \cdot 10^{-4}$ is chosen for these eigenvalues. This value has a minor impact on accuracy, as can be seen in section 5.2 where the correctness of the implementation of the mathematical models is verified.

\section{AUSM Flux-Vector Splitting Scheme}

The second upwind scheme considered in this work is the Advection Upstream Splitting Method (AUSM). The basis of the AUSM flux-vector splitting scheme is a splitting of the convective flux vector in a pure convective part (with convection velocity $\underline{u} \cdot \underline{n}$ ) and a pressure part, which is governed by the acoustic wave speeds, $\underline{u} \cdot \underline{n}+c^{*}$ and $\underline{u} \cdot \underline{n}-c^{*}$ :

$$
\underline{\boldsymbol{F}}^{c} \cdot \underline{S}=(\underline{u} \cdot \underline{S}) \boldsymbol{\Psi}+\boldsymbol{P},
$$

with

$$
\boldsymbol{\Psi} \equiv\left[\begin{array}{c}
\rho \\
\rho \underline{u} \\
\rho E+p^{*}
\end{array}\right], \quad \boldsymbol{P} \equiv\left[\begin{array}{c}
0 \\
p^{*} \underline{S} \\
0
\end{array}\right] .
$$

The original AUSM scheme [124] was developed to combine the strengths of the fluxvector and flux-difference schemes. The flux-vector splitting schemes, such as that by Van Leer, are very robust and efficient but too diffusive for viscous applications whereas the flux-difference splitting schemes are very accurate but have a high computational cost and increased complexity. Since the introduction of the original AUSM scheme, several improvements have been proposed [125, 126] such that a family of AUSM schemes now exists. 
The AUSM family of schemes is considered because one of its versions, "AUSM ${ }^{+}$-up for all speeds", is able to accurately calculate very low Mach-number flows. It can be shown [126], that for the inviscid flow equations in the limit of $\mathrm{M}_{\infty} \rightarrow 0$, the pressure field behaves as

$$
p(\underline{x}, t)=p^{(0)}(t)+\mathrm{M}_{\infty}^{2} p^{(2)}(\underline{x}, t)+\ldots,
$$

and that the zeroth-order velocity and density are coupled to the second-order pressure, $p^{(2)}(\underline{x}, t)$. The "AUSM ${ }^{+}$-up for all speeds"-scheme is constructed such that it has the same behavior as the continuous equations, whereas most convective flux schemes produce first-order pressure fluctuations [131]. This leads to inaccurate behavior of the zeroth-order velocity, density and internal energy. Although the "AUSM ${ }^{+}$-up for all speeds"-scheme has the correct behavior, it still suffers from deteriorating convergence speeds due to the large differences in the eigenvalues of the convective flux for low Mach-number flows.

In what follows, the flux function of the "AUSM+-up for all speeds"-scheme is presented with coefficients that can be chosen such that other versions of the AUSM family of schemes are obtained.

We start by introducing a left state $\boldsymbol{U}_{L}$ and a right state $\boldsymbol{U}_{R}$ at the face between control volume $k$ and $l$, with $k$ on the left side and $l$ on the right side:

$$
\boldsymbol{U}_{L}=\left[\boldsymbol{U}_{\mathrm{k}}\right]_{\mathrm{kl}}, \quad \boldsymbol{U}_{R}=\left[\boldsymbol{U}_{\mathrm{l}}\right]_{\mathrm{kl}} .
$$

The convective flux function can now be written as

$$
\boldsymbol{\Phi}_{\mathrm{kl}}^{c}\left(\boldsymbol{U}_{L}, \boldsymbol{U}_{R}, \underline{S}_{\mathrm{kl}}\right)=\boldsymbol{C}_{\mathrm{kl}}\left(\boldsymbol{U}_{L}, \boldsymbol{U}_{R}, \underline{S}_{\mathrm{kl}}\right)+\boldsymbol{P}_{\mathrm{kl}}\left(\boldsymbol{U}_{L}, \boldsymbol{U}_{R}, \underline{S}_{\mathrm{kl}}\right),
$$

with the pure convective part $\boldsymbol{C}_{\mathrm{kl}}$ and the pressure part $\boldsymbol{P}_{\mathrm{kl}}$.

The pure convective part is defined as

$$
\boldsymbol{C}_{\mathrm{kl}}=c_{\mathrm{kl}}^{*} M_{\mathrm{kl}}\left|\underline{S}_{\mathrm{kl}}\right|\left\{\begin{array}{ll}
\boldsymbol{\Psi}_{L} & \text { if } M_{\mathrm{kl}} \geq 0 \\
\boldsymbol{\Psi}_{R} & \text { if } M_{\mathrm{kl}}<0
\end{array},\right.
$$

where $c_{\mathrm{kl}}^{*}$ is the common speed of sound at the face, $c_{\mathrm{kl}}^{*}=\frac{1}{2}\left(c_{L}^{*}+c_{R}^{*}\right)$, and $M_{\mathrm{kl}}$ is the face Mach number defined as

$$
M_{\mathrm{kl}}=\mathcal{M}_{(4)}^{+}\left(M_{L}\right)+\mathcal{M}_{(4)}^{-}\left(M_{R}\right)+M_{p}
$$

Here the Mach number splitting functions $\mathcal{M}_{(4)}^{ \pm}$are functions of the left and right Mach number, $M_{L}$ and $M_{R}$, defined as

$$
M_{L}=\frac{\underline{u}_{L} \cdot \underline{n}_{\mathrm{kl}}}{c_{\mathrm{kl}}^{*}}, \quad M_{R}=\frac{\underline{u}_{R} \cdot \underline{n}_{\mathrm{kl}}}{c_{\mathrm{kl}}^{*}},
$$

respectively. The term $M_{p}$ is the pressure diffusion, which will be discussed shortly.

The pressure part $\boldsymbol{P}_{\mathrm{kl}}$ is defined as

$$
\boldsymbol{P}_{\mathrm{kl}}=\mathcal{P}_{(5)}^{+}\left(M_{L}\right) p_{L}^{*}+\mathcal{P}_{(5)}^{-}\left(M_{R}\right) p_{R}^{*}+p_{u},
$$


with pressure splitting functions $\mathcal{P}_{(5)}^{ \pm}$and the velocity diffusion term $p_{u}$.

The splitting functions are given by

$$
\begin{aligned}
& \mathcal{M}_{(4)}^{ \pm}(M)=\left\{\begin{array}{ll}
\mathcal{M}_{(1)}^{ \pm}(M) & \text { if }|M| \geq 1 \\
\mathcal{M}_{(2)}^{ \pm}(M)\left(1 \mp 16 \beta \mathcal{M}_{(2)}^{\mp}(M)\right) & \text { if }|M|<1
\end{array},\right. \\
& \mathcal{M}_{(2)}^{ \pm}(M)= \pm \frac{1}{4}(M \pm 1)^{2}, \\
& \mathcal{M}_{(1)}^{ \pm}(M)=\frac{1}{2}(M \pm|M|), \\
& \mathcal{P}_{(5)}^{ \pm}(M)=\left\{\begin{array}{ll}
\frac{1}{M} \mathcal{M}_{(1)}^{ \pm}(M) \\
\mathcal{M}_{(2)}^{ \pm}(M)\left(( \pm 2-M) \mp 16 \alpha M \mathcal{M}_{(2)}^{\mp}(M)\right) & \text { if }|M|<1
\end{array},\right.
\end{aligned}
$$

with coefficients $\alpha$ and $\beta$, which will be defined later. Note that the splitting functions yield a fully upwind scheme for $M \geq 1$.

The pressure diffusion term $M_{p}$ and the velocity diffusion term $p_{u}$ are given by

$$
\begin{aligned}
M_{p} & =-\frac{K_{p}}{f_{a}} \max \left(1-\sigma \bar{M}^{2}, 0\right) \frac{p_{R}^{*}-p_{L}^{*}}{\rho_{\mathrm{kl}} c_{\mathrm{kl}}^{*}}, \\
p_{u} & =-K_{u} \mathcal{P}_{(5)}^{+}\left(M_{L}\right) \mathcal{P}_{(5)}^{-}\left(M_{R}\right)\left(2 \rho_{\mathrm{kl}}\right)\left(f_{a} c_{\mathrm{kl}}^{*}\right)\left(\underline{u}_{R} \cdot \underline{n}_{\mathrm{kl}}-\underline{u}_{L} \cdot \underline{n}_{\mathrm{kl}}\right),
\end{aligned}
$$

with the coefficients $0 \leq K_{p} \leq 1,0 \leq K_{u} \leq 1$ and $\sigma \leq 1$, the face density $\rho_{\mathrm{kl}}=$ $\frac{1}{2}\left(\rho_{L}+\rho_{R}\right)$, the scaling function $f_{a} \in[0,1]$, and the mean local Mach number $\bar{M}^{2}=$ $\frac{1}{2}\left(M_{L}^{2}+M_{R}^{2}\right)$.

Both $M_{p}$ and $p_{u}$ are active for subsonic flow only, if $\sigma=1$. The factor max $\left(1-\sigma \bar{M}^{2}, 0\right)$ has a maximum value of 1 at $\bar{M}=0$ and becomes zero for $\bar{M}^{2} \geq \frac{1}{\sigma}$. Note that the region where $M_{p}$ is active can be extended somewhat above the sonic point if $\sigma<1$, but this is not used in the present work. Furthermore, the product $\mathcal{P}_{(5)}^{+}\left(M_{L}\right) \mathcal{P}_{(5)}^{-}\left(M_{R}\right)$ equals zero for supersonic flow. Therefore, the scheme remains fully upwind for supersonic flow.

The scaling function $f_{a}$ equals one except for the "AUSM+ ${ }^{+}$-up for all speeds"-scheme, for which it reads

$$
f_{a}=M_{0}\left(2-M_{0}\right),
$$

where $M_{0}$ is the reference Mach number, of which its square is defined as

$$
M_{0}^{2}=\min \left(1, \max \left(\bar{M}_{\mathrm{kl}}^{2}, M_{\mathrm{co}}^{2}\right)\right),
$$

with cutoff Mach number $M_{\text {co }}$. The cutoff Mach number can be set equal to the free stream Mach number $M_{\infty}$ but must always be larger than zero. The purpose of $f_{a}$ is to obtain the proper scaling of the pressure fluctuations for $M_{0} \rightarrow 0$, see [126] for proof of this property. Furthermore, the pressure diffusion term will scale with the flow speed instead of the speed of sound for $M_{0} \rightarrow 0$.

The different AUSM schemes are are constructed by setting the coefficients and the function $f_{a}$ as given in table 4.1. The differences between the schemes can be described as follows: 
- The $\mathrm{AUSM}^{+}$scheme is an improvement of the original AUSM schemet in the sense that it gives the exact solution for a stationary contact discontinuity (important for shear layers) and is positivity preserving (important for near-vacuum conditions).

- The $\mathrm{AUSM}^{+}$-up scheme includes the pressure diffusion term (in the pure convective flux) and the velocity diffusion term (in the pressure flux). Both terms are active only for subsonic flow conditions and improve the accuracy and robustness for low speed flows.

- The "AUSM+-up for all speeds"-scheme includes the scaling function $f_{a}$, which leads to the proper scaling of the pressure fluctuations for very low Mach-number flows. This scheme must not be used for low Mach number flows with a leading (zeroth-order) pressure term that is not spatially uniform, such as shock tube problems, for which the order-of-magnitude analysis for $M \rightarrow 0$ is not valid.

\begin{tabular}{|l||c|c|c|c|c|c|}
\hline Scheme & $\alpha$ & $\beta$ & $K_{p}$ & $\sigma$ & $K_{u}$ & $f_{a}$ \\
\hline \hline $\mathrm{AUSM}^{+}$ & $\frac{3}{16}$ & $\frac{1}{8}$ & 0 & - & 0 & - \\
\hline $\mathrm{AUSM}^{+}$-up & $\frac{3}{16}$ & $\frac{1}{8}$ & $\frac{1}{4}$ & 1 & $\frac{3}{4}$ & 1 \\
\hline $\mathrm{AUSM}^{+}$-up for all speeds & $\frac{3}{16}\left(-4+5 f_{a}^{2}\right)$ & $\frac{1}{8}$ & $\frac{1}{4}$ & 1 & $\frac{3}{4}$ & see (4.73) \\
\hline
\end{tabular}

Table 4.1: The coefficients and the function $f_{a}$ for the different AUSM schemes.

\subsubsection{MUSCL-type Solution Reconstruction Technique}

In order to obtain a second-order accurate upwind scheme, a linear reconstruction of the solution in the control volumes is needed. However, this must be done such that no new extrema are introduced in the solution, otherwise monotonicity will not be preserved and spurious wiggles will appear in the solution near very high gradients of the flow variables. Van Leer [132] introduced the MUSCL5 approach, which consists of

- a piece-wise linear reconstruction of the solution in the control volumes, and

- a limiting of the gradient used in the reconstruction such that no new extrema are introduced.

This approach is employed in the present computational method.

The variables used in the reconstruction technique can either be the conserved variables $\boldsymbol{U}$, or an appropriate set of primitive variables, e.g. $\boldsymbol{Q}=\left[\rho, u, v, w, p^{*}\right]^{T}$. In the present

\footnotetext{
${ }^{4}$ The original AUSM scheme [124] is not considered here because it cannot be written using a common speed of sound at the face.

${ }^{5}$ MUSCL stands for Monotone Upstream-centered Scheme for Conservation Laws.
} 
work, the latter option is chosen since it ensures positivity of the pressure $p^{*}$, whereas this may not be the case if the conserved variables are used, e.g. in high speed flows.

No new extrema are introduced if the linearly reconstructed values of the variables at the faces of control volume $k$ lie between the control-volume average values of the nearest neighbor control volumes, including control volume $k$ [132, 104]. So, the gradient can even be limited to zero if the control-volume average value is an extremum with respect to the control-volume average values of the nearest neighbor control volumes.

The limiter functions used to limit the gradient are non-linear functions that effectively reduce the order of accuracy of the local upwind flux function to first-order in the vicinity of high gradients in the flow.

The MUSCL approach actually consists of three separate steps. First, an unlimited gradient is calculated and used to obtain an unlimited reconstructed solution. Then, a gradient limiter function is computed. Finally, the limited reconstructed solution at each side of a face between two control volumes is obtained.

The remainder of this subsection describes the solution reconstruction technique and introduces two limiter functions suitable for multi-dimensional unstructured meshes: the limiter by Barth \& Jespersen [104] and the limiter by Venkatakrishnan [105].

\section{Linear Solution Reconstruction}

A piece-wise linear reconstruction of the primitive variables $\boldsymbol{Q}=\left[\rho, u, v, w, p^{*}\right]^{T}$ in control volume $k$ can be written as

$$
\boldsymbol{Q}(\underline{x}, t)=\boldsymbol{Q}_{\mathrm{k}}(t)+(\underline{\nabla} \boldsymbol{Q}(t))_{\mathrm{k}} \cdot\left(\underline{x}-\underline{x}_{\mathrm{k}}\right) \forall \underline{x} \text { within } V_{\mathrm{k}},
$$

where $(\underline{\nabla} \boldsymbol{Q}(t))_{\mathrm{k}}$ is the gradient of the primitive variables at the centroid of control volume $k$ and $\underline{x}_{\mathrm{k}}$ is the location of the centroid of control volume $k$. The method to compute the gradient of the primitive variables is presented in subsection 4.2.5.

For the upwind schemes presented in this section, the reconstructed values of the conserved variables at the centroid of the faces of the control volumes are needed, i.e. $\left[\boldsymbol{U}_{\mathrm{k}}\right]_{\mathrm{kl}}$ for all $k$ and $l \in N_{\mathrm{k}}$, which can be computed from the primitive variables $\left[\boldsymbol{Q}_{\mathrm{k}}\right]_{\mathrm{kl}}$ at the same location.

\section{Limiter Functions}

With the introduction of a limiter function $\boldsymbol{\Phi}_{\mathrm{k}} \in[0,1]$, the relation to compute $\left[\boldsymbol{Q}_{\mathrm{k}}\right]_{\mathrm{kl}}$ reads

$$
\left[\boldsymbol{Q}_{\mathrm{k}}\right]_{\mathrm{kl}}=\boldsymbol{Q}_{\mathrm{k}}+\boldsymbol{\Phi}_{\mathrm{k}}\left((\underline{\nabla} \boldsymbol{Q})_{\mathrm{k}} \cdot\left(\underline{x}_{\mathrm{kl}}-\underline{x}_{\mathrm{k}}\right)\right),
$$

where the centroid of the face between control volume $k$ and $l$ is denoted by $\underline{x}_{\mathrm{kl}}$.

For a convenient presentation of the limiter functions, the following relations are defined:

$$
\begin{aligned}
\left(\boldsymbol{\Delta}_{2}\right)_{\mathrm{kl}} & =(\underline{\nabla} \boldsymbol{Q})_{\mathrm{k}} \cdot\left(\underline{x}_{\mathrm{kl}}-\underline{x}_{\mathrm{k}}\right) \\
\left(\boldsymbol{\Delta}_{1}\right)_{\mathrm{k}} & = \begin{cases}\boldsymbol{Q}_{\max }-\boldsymbol{Q}_{\mathrm{k}} & \text { if }\left(\boldsymbol{\Delta}_{2}\right)_{\mathrm{kl}}>0 \\
\boldsymbol{Q}_{\min }-\boldsymbol{Q}_{\mathrm{k}} & \text { if }\left(\boldsymbol{\Delta}_{2}\right)_{\mathrm{kl}}<0\end{cases}
\end{aligned}
$$


where

$$
\begin{gathered}
\boldsymbol{Q}_{\max }=\max \left(\boldsymbol{Q}_{\mathrm{k}}, \max _{l \in N_{\mathrm{k}}}\left(\boldsymbol{Q}_{\mathrm{l}}\right)\right), \\
\boldsymbol{Q}_{\min }=\min \left(\boldsymbol{Q}_{\mathrm{k}}, \min _{l \in N_{\mathrm{k}}}\left(\boldsymbol{Q}_{\mathrm{l}}\right)\right)
\end{gathered}
$$

Using these relations, the limiter function of Barth \& Jespersen [104] reads

$$
\begin{aligned}
& \boldsymbol{\Phi}_{\mathrm{k}}=\min _{l \in N_{\mathrm{k}}}\left(\boldsymbol{\Phi}_{\mathrm{kl} l}\right), \\
& \boldsymbol{\Phi}_{\mathrm{kl}}=\left\{\begin{array}{cc}
\min \left(1, \frac{\left(\boldsymbol{\Delta}_{1}\right)_{\mathrm{k}}}{\left(\boldsymbol{\Delta}_{2}\right)_{\mathrm{kl}}}\right) & \text { if }\left(\boldsymbol{\Delta}_{2}\right)_{\mathrm{kl}} \neq 0 \\
1 & \text { if }\left(\boldsymbol{\Delta}_{2}\right)_{\mathrm{kl}}=0
\end{array} .\right.
\end{aligned}
$$

This limiter ensures a monotone solution, but it is quite dissipative and it can inhibit convergence to a steady state solution due to the limiter being non-differentiable and the intermittent activation of the limiter in smooth flow regions [133]. To prevent this problem, Venkatakrishnan [105] introduced the following differentiable limiter function:

$$
\begin{aligned}
& \boldsymbol{\Phi}_{\mathrm{k}}=\min _{l \in N_{\mathrm{k}}}\left(\boldsymbol{\Phi}_{\mathrm{kl}}\right), \\
& \boldsymbol{\Phi}_{\mathrm{kl}}=\left\{\begin{array}{cl}
\frac{1}{\left(\boldsymbol{\Delta}_{2}\right)_{\mathrm{k} l}}\left(\frac{\left(\left(\boldsymbol{\Delta}_{1}\right)_{\mathrm{k}}^{2}+\boldsymbol{\epsilon}_{\mathrm{k}}^{2}\right)\left(\boldsymbol{\Delta}_{2}\right)_{\mathrm{kl}}+2\left(\boldsymbol{\Delta}_{2}\right)_{\mathrm{kl}}^{2}\left(\boldsymbol{\Delta}_{1}\right)_{\mathrm{k}}}{\left(\boldsymbol{\Delta}_{1}\right)_{\mathrm{k}}^{2}+2\left(\boldsymbol{\Delta}_{2}\right)_{\mathrm{kl}}^{2}+\left(\boldsymbol{\Delta}_{1}\right)_{\mathrm{k}}\left(\boldsymbol{\Delta}_{2}\right)_{\mathrm{kl}}+\epsilon_{\mathrm{k}}^{2}}\right) & \text { if }\left(\boldsymbol{\Delta}_{2}\right)_{\mathrm{kl}} \neq 0 \\
1 & \text { if }\left(\boldsymbol{\Delta}_{2}\right)_{\mathrm{kl}}=0
\end{array}\right.
\end{aligned}
$$

with $\epsilon_{\mathrm{k}}^{2}$ defined such that it prevents activation of the limiter in smooth flow regions:

$$
\epsilon_{\mathrm{k}}^{2}=Q_{\mathrm{char}}^{2}\left(K \overline{\Delta x}_{\mathrm{k}}\right)^{3}
$$

Here, $K$ is a user-defined constant, $\boldsymbol{Q}_{\text {char }}$ is a column vector with characteristic values of the primitive variables, e.g. $\boldsymbol{Q}_{\text {char }}=\left(\rho_{\infty},\left|\underline{u}_{\infty}\right|,\left|\underline{u}_{\infty}\right|,\left|\underline{u}_{\infty}\right|, p_{\infty}^{*}\right)^{T}$, and $\overline{\Delta x}_{\mathrm{k}}$ is a characteristic non-dimensional local grid size, in the present work defined as

$$
\overline{\Delta x_{\mathrm{k}}}=\frac{\min _{l \in N_{\mathrm{k}}}\left(2\left|\underline{x}_{\mathrm{kl}}-\underline{x}_{\mathrm{k}}\right|\right)}{L_{\mathrm{char}}},
$$

with $L_{\text {char }}$ a characteristic length scale of the flow problem being considered. This definition of $\epsilon_{\mathrm{k}}^{2}$ ensures that a single value of $K$ can be employed for each primitive variable in the column vector $Q$ to be used in the solution reconstruction technique 6 .

A value of $K=0$ yields a fully limited gradient, whereas the gradient eventually becomes unlimited for very high values of $K$. A value of $K=3$ is usually sufficient to obtain convergence without being too dissipative.

\footnotetext{
${ }^{6}$ Note that the same result would be obtained by using non-dimensional variables during the evaluation of the limiter function in equation (4.82).
} 


\subsubsection{Computation of Gradients}

For the linear solution reconstruction technique as well as the viscous flux function, see subsection 4.2.6, the gradient of the variables $\rho, u, v, w, p^{*}$, and $e$ is needed at the centroid of each control volume.

There are several options available for for the calculation of the gradients. When we only consider options that use the face-based data structure, two options remain. The first option is to employ a discrete version of Gauß' divergence theorem:

$$
(\underline{\nabla} \phi)_{\mathrm{k}}=\frac{1}{V_{\mathrm{k}}} \sum_{l \in N_{\mathrm{k}}}\left([\phi]_{\mathrm{kl}} \underline{S}_{\mathrm{kl}}\right),
$$

where $[\phi]_{\mathrm{kl}}$, the value of $\phi$ at the faces of the control volume, can be found by assuming a linear behavior of $\phi$ between the centroids of the control volumes on either side of each face, which reverts to arithmetic averaging on equidistant grids. The problem with this method is that it yields exact gradients for linear functions only on triangular grids (2D) or tetrahedral grids (3D). It turns out to be particularly inaccurate on hybrid grids, i.e. grids with different element types [134].

The second option for the calculation of gradients is the least-squares method, which yields exact gradients for linear functions on grids consisting of arbitrary element types. Since the standard unweighted least-squares method is inaccurate for highly stretched grids with moderate curvature [135], which are commonly used in boundary layer regions, in the present computational method the weighted least-squares method is employed.

The weighted least-squares method can be derived as follows [101]. Consider control volume $k$ with its direct neighbors $l \in N_{\mathrm{k}}$. The value of $\phi$ at the centroid of each neighboring control volume $l$ can be approximated using a Taylor series expansion as

$$
\phi_{\mathrm{l}}=\phi_{\mathrm{k}}+(\underline{\nabla} \phi)_{\mathrm{k}} \cdot \Delta \underline{x}_{\mathrm{kl}}+\mathcal{O}\left(\left|\Delta \underline{x}_{\mathrm{kl}}\right|^{2}\right)
$$

where $\Delta \underline{x}_{\mathrm{kl}}=\underline{x}_{1}-\underline{x}_{\mathrm{k}}$ and $(\underline{\nabla} \phi)_{\mathrm{k}}$ the gradient we are interested in. Neglecting higherorder terms, the error in the approximation can be written as

$$
\varepsilon_{\mathrm{kl}}=\Delta \phi_{\mathrm{kl}}-(\underline{\nabla} \phi)_{\mathrm{k}} \cdot \Delta \underline{x}_{\mathrm{kl}},
$$

with $\Delta \phi_{\mathrm{kl}}=\phi_{\mathrm{l}}-\phi_{\mathrm{k}}$. Next, a weighted sum of squared errors of all neighboring control volumes $l$ can be defined as

$$
\mathcal{E}_{\mathrm{k}}=\sum_{l \in N_{\mathrm{k}}} \eta_{\mathrm{kl}} \varepsilon_{\mathrm{kl}}^{2},
$$

with the weights defined as

$$
\eta_{\mathrm{kl}}=\left(\left|\Delta \underline{x}_{\mathrm{kl}}\right|^{-N}\right)
$$

As described by Shima et al. [136], a value of $N=2$ gives the most robust gradient computation. The gradient $(\underline{\nabla} \phi)_{\mathrm{k}}$ is found by minimizing $\mathcal{E}_{\mathrm{k}}$. Differentiating $\mathcal{E}_{\mathrm{k}}$ with 
respect to each component of the gradient and setting it equal to zero yields

$$
\begin{aligned}
\frac{\partial \mathcal{E}_{\mathrm{k}}}{\partial(\underline{\nabla} \phi)_{\mathrm{k}}} & =0 \\
\Leftrightarrow & \\
\sum_{l \in N_{\mathrm{k}}} 2 \eta_{\mathrm{kl}} \varepsilon_{\mathrm{kl}} \frac{\partial \varepsilon_{\mathrm{kl}}}{\partial(\underline{\nabla} \phi)_{\mathrm{k}}} & =0 \\
\Leftrightarrow & \\
\sum_{l \in N_{\mathrm{k}}}-2 \eta_{\mathrm{kl}} \Delta \underline{x}_{\mathrm{kl}}\left(\Delta \phi_{\mathrm{kl}}-(\underline{\nabla} \phi)_{\mathrm{k}} \cdot \Delta \underline{x}_{\mathrm{kl}}\right) & =0 .
\end{aligned}
$$

From this result, the gradient is found as

$$
(\underline{\nabla} \phi)_{\mathrm{k}}=\left(\sum_{l \in N_{\mathrm{k}}} \eta_{\mathrm{kl}} \Delta \underline{x}_{\mathrm{kl}} \otimes \Delta \underline{x}_{\mathrm{kl}}\right)^{-1} \sum_{l \in N_{\mathrm{k}}} \eta_{\mathrm{kl}} \Delta \phi_{\mathrm{kl}} \Delta \underline{x}_{\mathrm{kl}}
$$

where $\left(\sum_{l \in N_{\mathrm{k}}} \eta_{\mathrm{kl}} \Delta \underline{x}_{\mathrm{kl}} \otimes \Delta \underline{x}_{\mathrm{kl}}\right)^{-1}$ is a second-order symmetric tensor, which is constant for fixed grids and can be computed and stored during preprocessing. Due to the symmetry, only six elements need to be stored.

For triangular or tetrahedral control volumes, the stencil used in the weighted leastsquares method is augmented with the next-to-nearest neighbor control volumes. This ensures that information on the variation of $\phi$ in sufficient directions is included in the gradient computation, even on highly stretched grids.

\subsubsection{Central Viscous Flux Discretization}

The viscous flux is discretized by a central scheme, which in this case is constructed by using an average of control-volume averaged variables and gradients of variables. It is denoted as

$$
\boldsymbol{\Phi}_{\mathrm{kl}}^{v}\left(\boldsymbol{U}_{\mathrm{k}}, \boldsymbol{U}_{\mathrm{l}},(\underline{\nabla} \boldsymbol{U})_{\mathrm{k}},(\underline{\nabla} \boldsymbol{U})_{\mathrm{l}}, \underline{S}_{\mathrm{kl}}\right)=\underline{\boldsymbol{F}}^{v}\left([\boldsymbol{U}]_{\mathrm{kl}},[\underline{\nabla} \boldsymbol{U}]_{\mathrm{kl}}\right) \cdot \underline{S}_{\mathrm{kl}} \cdot
$$

The definition of the viscous flux is repeated here for convenience:

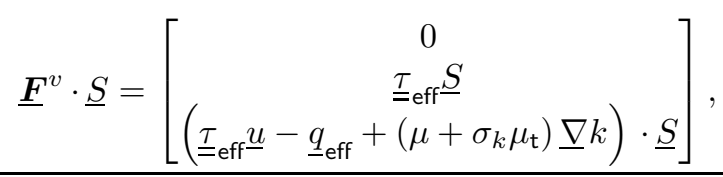


where the effective shear stress tensor is given by

$\underline{\tau}_{\text {eff }}=\left(\mu+\mu_{\mathrm{t}}\right)\left[\begin{array}{ccc}\frac{4}{3} \frac{\partial u_{1}}{\partial x_{1}}-\frac{2}{3} \frac{\partial u_{2}}{\partial x_{2}}-\frac{2}{3} \frac{\partial u_{3}}{\partial x_{3}} & \frac{\partial u_{1}}{\partial x_{2}}+\frac{\partial u_{2}}{\partial x_{1}} & \frac{\partial u_{1}}{\partial x_{3}}+\frac{\partial u_{3}}{\partial x_{1}} \\ \frac{\partial u_{2}}{\partial x_{1}}+\frac{\partial u_{1}}{\partial x_{2}} & \frac{4}{3} \frac{\partial u_{2}}{\partial x_{2}}-\frac{2}{3} \frac{\partial u_{1}}{\partial x_{1}}-\frac{2}{3} \frac{\partial u_{3}}{\partial x_{3}} & \frac{\partial u_{2}}{\partial x_{3}}+\frac{\partial u_{3}}{\partial x_{2}} \\ \frac{\partial u_{3}}{\partial x_{1}}+\frac{\partial u_{1}}{\partial x_{3}} & \frac{\partial u_{3}}{\partial x_{2}}+\frac{\partial u_{2}}{\partial x_{3}} & \frac{4}{3} \frac{\partial u_{3}}{\partial x_{3}}-\frac{2}{3} \frac{\partial u_{1}}{\partial x_{1}}-\frac{2}{3} \frac{\partial u_{2}}{\partial x_{2}}\end{array}\right]$,

and the effective heat flux vector is given by

$$
\underline{q}_{\mathrm{eff}}=-\left(\frac{\gamma \mu}{\mathrm{Pr}}+\frac{\gamma \mu_{\mathrm{t}}}{\operatorname{Pr}_{\mathrm{t}}}\right) \underline{\nabla} e .
$$

The variables for which face-average values are needed are: the velocity vector $\underline{u}$, the molecular viscosity $\mu$, the eddy viscosity $\mu_{\mathrm{t}}$ and, when the SST turbulence model is used, the parameter $\sigma_{k}$. Furthermore, the gradients for which face-average values are needed are: the gradients of all velocity components, i.e. $\underline{\nabla} u_{1}, \underline{\nabla} u_{2}, \underline{\nabla} u_{3}$, the gradient of the specific internal energy $\underline{\nabla} e$ and, when the SST turbulence model is used, the gradient of the turbulence kinetic energy $\underline{\nabla} k$.

In order to improve the accuracy on non-smoothly varying cell-centered grids, a weighted average is used to obtain the value of a variable at the face of a control volume instead of the arithmetic average. The weights consist of distances from the centroid of the face to the centroids of the associated neighboring control volumes and are obtained from a one-dimensional analysis, assuming a linear behavior of the variable. This leads to the following approximation of the value of variable $\phi$ at a face between control volume $k$ and $l$ :

$$
[\phi]_{\mathrm{kl}}=\alpha_{\mathrm{l}, \mathrm{kl}} \phi_{\mathrm{k}}+\alpha_{\mathrm{k}, \mathrm{kl}} \phi_{\mathrm{l}},
$$

with the weights $\alpha_{\mathrm{l}, \mathrm{kl}} \in(0,1)$ and $\alpha_{\mathrm{k}, \mathrm{kl}}=\left(1-\alpha_{\mathrm{l}, \mathrm{kl}}\right) \in(0,1)$ given by

$$
\alpha_{l, \mathrm{kl}}=\frac{\left|\underline{x}_{\mathrm{kl}}-\underline{x}_{\mathrm{l}}\right|}{\left|\underline{x}_{\mathrm{kl}}-\underline{x}_{\mathrm{k}}\right|+\left|\underline{x}_{\mathrm{kl}}-\underline{x}_{1}\right|}, \quad \alpha_{\mathrm{k}, \mathrm{kl}}=\frac{\left|\underline{x}_{\mathrm{kl}}-\underline{x}_{\mathrm{k}}\right|}{\left|\underline{x}_{\mathrm{kl}}-\underline{x}_{\mathrm{k}}\right|+\left|\underline{x}_{\mathrm{kl}}-\underline{x}_{l}\right|},
$$

where $\underline{x}_{\mathrm{kl}}$ is the location of the centroid of the face and $\underline{x}_{\mathrm{k}}$ and $\underline{x}_{1}$ are the locations of the centroid of control volume $k$ and $l$, respectively. The accuracy of this approximation increases if the regularity of the grid improves.

Arithmetic or weighted averaging of gradients can also be applied to approximate the gradient of a variable at a face. However, Haselbacher \& Blazek [134] show that this leads to a wide stencil that allows odd-even decoupling of the solution on quadrilateral (2D) and hexahedral (3D) grids. This can be improved by replacing the component in the direction between the centroids of the associated control volumes by a finite-difference approximation [137], as

$$
\begin{array}{r}
{\overline{[\underline{\nabla} \phi]_{\mathrm{kl}}}}^{=} \alpha_{\mathrm{l}, \mathrm{kl}}(\underline{\nabla} \phi)_{\mathrm{k}}+\alpha_{\mathrm{k}, \mathrm{kl}}(\underline{\nabla} \phi)_{\mathrm{l}}, \\
{[\underline{\nabla} \phi]_{\mathrm{kl}}={\overline{[\bar{\nabla} \phi]_{\mathrm{kl}}}}-\left(\overline{[}_{\bar{\nabla} \phi]_{\mathrm{kl}}} \cdot \frac{\Delta \underline{x}_{\mathrm{kl}}}{\left|\Delta \underline{x}_{\mathrm{kl}}\right|}-\frac{\phi_{\mathrm{l}}-\phi_{\mathrm{k}}}{\left|\Delta \underline{x}_{\mathrm{kl}}\right|}\right) \frac{\Delta \underline{x}_{\mathrm{kl}}}{\left|\Delta \underline{x}_{\mathrm{kl}}\right|},}
\end{array}
$$

with $\Delta \underline{x}_{\mathrm{kl}}=\underline{x}_{1}-\underline{x}_{\mathrm{k}}$. 


\subsection{Spatial Discretization of the Turbulence Model Equa- tions}

This section introduces the discretization of the equations of the turbulence model. As for the URANS equations, it is assumed that the computational grid is stationary. The geometry of the control volumes is therefore constant and the velocity of the controlvolume surfaces is zero, i.e. $\underline{u}_{A}=0$.

The integral conservation equations with additional relations that need to be discretized are given in equations (3.130) to (3.132) for the SST turbulence model and equations (3.133) to (3.136) for the SA turbulence model.

The following subsections present the spatial discretization of the SST turbulence model and the SA turbulence model, respectively.

\subsubsection{Spatial Discretization of the SST Turbulence Model}

The convective flux of the turbulence model equations is a function of the conserved turbulence variables $\boldsymbol{U}_{\mathrm{t}}$ as well as the conserved flow variables $\boldsymbol{U}$. It is chosen to discretize the convective flux vector with a first-order accurate upwind scheme. Experience has shown that this is sufficient, because the turbulence equations are dominated by the source term. The convective flux function $\boldsymbol{\Phi}_{\mathrm{t}, \mathrm{kl}}^{c}$ is given by

$$
\begin{aligned}
\boldsymbol{\Phi}_{\mathrm{t}, \mathrm{kl}}^{c}\left(\left(\boldsymbol{U}_{\mathrm{t}}\right)_{\mathrm{k}},\right. & \left.\left(\boldsymbol{U}_{\mathrm{t}}\right)_{1}, \boldsymbol{U}_{\mathrm{k}}, \boldsymbol{U}_{\mathrm{l}}, \underline{S}_{\mathrm{kl}}\right)= \\
& \frac{1}{2}\left(\left(\boldsymbol{U}_{\mathrm{t}}\right)_{\mathrm{k}}+\left(\boldsymbol{U}_{\mathrm{t}}\right)_{\mathrm{l}}\right)[\underline{u}]_{\mathrm{kl}} \cdot \underline{S}_{\mathrm{kl}}-\frac{1}{2}\left|[\underline{u}]_{\mathrm{kl}} \cdot \underline{S}_{\mathrm{k} l}\right|\left(\left(\boldsymbol{U}_{\mathrm{t}}\right)_{\mathrm{l}}-\left(\boldsymbol{U}_{\mathrm{t}}\right)_{\mathrm{k}}\right),
\end{aligned}
$$

where $[\underline{u}]_{\mathrm{kl}}$ is calculated as defined in equation (4.97).

The viscous flux of the turbulence model equations is a function of the conserved turbulence variables $\boldsymbol{U}_{\mathrm{t}}$, their gradients, and the conserved flow variables $\boldsymbol{U}$. The corresponding viscous flux function, $\boldsymbol{\Phi}_{\mathrm{t}, \mathrm{kl}}^{v}$, employs a second-order accurate central scheme, which is constructed in the same way as for the viscous flux function of the URANS equations, see subsection 4.2.6. The viscous flux function $\boldsymbol{\Phi}_{\mathrm{t}, \mathrm{kl}}^{v}$ can therefore be written as

$$
\begin{aligned}
\boldsymbol{\Phi}_{\mathrm{t}, \mathrm{kl}}^{v}\left(\left(\boldsymbol{U}_{\mathrm{t}}\right)_{\mathrm{k}},\left(\boldsymbol{U}_{\mathrm{t}}\right)_{\mathrm{l}},\left(\underline{\nabla} \boldsymbol{U}_{\mathrm{t}}\right)_{\mathrm{k}},\left(\underline{\nabla} \boldsymbol{U}_{\mathrm{t}}\right)_{1}, \boldsymbol{U}_{\mathrm{k}}, \boldsymbol{U}_{\mathrm{l}}, \underline{S}_{\mathrm{kl}}\right)= & \\
& {\left[\begin{array}{c}
\left([\mu]_{\mathrm{kl}}+\left[\sigma_{k}\right]_{\mathrm{kl}}\left[\mu_{\mathrm{t}}\right]_{\mathrm{kl}}\right)[\underline{\nabla} k]_{\mathrm{kl}} \cdot \underline{S}_{\mathrm{kl}} \\
\left([\mu]_{\mathrm{kl}}+\left[\sigma_{\omega}\right]_{\mathrm{kl}}\left[\mu_{\mathrm{t}}\right]_{\mathrm{kl}}\right)[\underline{\nabla} \omega]_{\mathrm{kl}} \cdot \underline{S}_{\mathrm{kl}}
\end{array}\right], }
\end{aligned}
$$

where the gradients at the face are calculated as defined in equation (4.100), and the other terms as defined in equation (4.97).

The source term of the SST turbulence model equations is a volume integral with integrand $\boldsymbol{Q}_{\mathrm{t}}\left(\boldsymbol{U}_{\mathrm{t}}, \boldsymbol{U}, \underline{\nabla} \boldsymbol{U}_{\mathrm{t}}, \underline{\nabla} \boldsymbol{U}\right)$, as defined in equation (3.132). The discretization of this term is obtained by defining a control-volume average of $\boldsymbol{Q}_{\mathrm{t}}$. For control volume $k$ this reads

$$
\left(\boldsymbol{Q}_{\mathrm{t}}\right)_{\mathrm{k}}=\frac{1}{V_{\mathrm{k}}} \iint_{V_{\mathrm{k}}} \int_{\boldsymbol{Q}_{\mathrm{t}}} d V .
$$


Next, $\left(\boldsymbol{Q}_{\mathrm{t}}\right)_{\mathrm{k}}$ is approximated using the second-order accurate midpoint rule:

$$
\left(\boldsymbol{Q}_{\mathrm{t}}\right)_{\mathrm{k}} \approx \boldsymbol{Q}_{\mathrm{t}}\left(\left(\boldsymbol{U}_{\mathrm{t}}\right)_{\mathrm{k}}, \boldsymbol{U}_{\mathrm{k}},\left(\underline{\nabla} \boldsymbol{U}_{\mathrm{t}}\right)_{\mathrm{k}},(\underline{\nabla} \boldsymbol{U})_{\mathrm{k}}\right),
$$

which leads to the following source function $\boldsymbol{\Phi}_{\mathrm{t}}^{s}$ :

$$
\boldsymbol{\Phi}_{\mathrm{t}, \mathrm{k}}^{s}=V_{\mathrm{k}}\left(\boldsymbol{Q}_{\mathrm{t}}\right)_{\mathrm{k}}=V_{\mathrm{k}} \boldsymbol{Q}_{\mathrm{t}}\left(\left(\boldsymbol{U}_{\mathrm{t}}\right)_{\mathrm{k}}, \boldsymbol{U}_{\mathrm{k}},\left(\underline{\nabla} \boldsymbol{U}_{\mathrm{t}}\right)_{\mathrm{k}},(\underline{\nabla} \boldsymbol{U})_{\mathrm{k}}\right) .
$$

\subsubsection{Spatial Discretization of the SA Turbulence Model}

The spatial discretization of the SA turbulence model is equivalent to that of the SST turbulence model. The convective and viscous flux functions are given by

$$
\begin{aligned}
& \Phi_{\mathrm{t}, \mathrm{kl}}^{c}=\frac{1}{2}\left((\tilde{\nu})_{\mathrm{k}}+(\tilde{\nu})_{1}\right)[\underline{u}]_{\mathrm{kl}} \cdot \underline{S}_{\mathrm{kl}}-\frac{1}{2}\left|[\underline{u}]_{\mathrm{kl}} \cdot \underline{S}_{\mathrm{kl}}\right|\left((\tilde{\nu})_{1}-(\tilde{\nu})_{\mathrm{k}}\right), \\
& \Phi_{\mathrm{t}, \mathrm{kl}}^{v}=\left[\frac{1}{\sigma}\left(\nu+\left(1+C_{b 2}\right) \tilde{\nu}\right)\right]_{\mathrm{kl}}[\underline{\nabla} \tilde{\nu}]_{\mathrm{kl}} \cdot \underline{S}_{\mathrm{kl}},
\end{aligned}
$$

respectively.

The discretization of the source term given by equation (3.136) follows the same lines as for the SST turbulence model, i.e.

$$
\Phi_{\mathrm{t}, \mathrm{k}}^{s}=V_{\mathrm{k}}\left(Q_{\mathrm{t}}\right)_{\mathrm{k}},
$$

where $Q_{\mathrm{t}}=Q_{\mathrm{t}}\left(\left(U_{\mathrm{t}}\right)_{\mathrm{k}}, \boldsymbol{U}_{\mathrm{k}},\left(\underline{\nabla} U_{\mathrm{t}}\right)_{\mathrm{k}},(\underline{\nabla} \boldsymbol{U})_{\mathrm{k}}\right)$. The term given by $\frac{C_{b 2}}{\sigma} \tilde{\nu} \underline{\nabla}^{2} \tilde{\nu}$ is treated as follows:

$$
\left(\frac{C_{b 2}}{\sigma} \tilde{\nu} \underline{\nabla}^{2} \tilde{\nu}\right)_{\mathrm{k}} \approx \frac{C_{b 2}}{\sigma}(\tilde{\nu})_{\mathrm{k}}\left(\underline{\nabla}^{2} \tilde{\nu}\right)_{\mathrm{k}} \approx \frac{C_{b 2}}{\sigma}(\tilde{\nu})_{\mathrm{k}} \frac{1}{V_{\mathrm{k}}} \sum_{l \in N_{\mathrm{k}}}[\underline{\nabla} \tilde{\nu}]_{\mathrm{kl}} \cdot \underline{S}_{\mathrm{kl}},
$$

where the gradient at the faces is defined in equation (4.100).

Calculation of $f_{w}(r)$, see equation (3.116), involves evaluation of amongst others the sixth power of $r$. To prevent numerical difficulties for large $r$, for which $f_{w}$ asymptotes to $\left(1+C_{w 3}^{6}\right)^{1 / 6}$, the dimensionless $r$ is not allowed to increase above 10 .

\subsubsection{Semi-Discretized Turbulence Model Equations}

The semi-discretized conservation equations of the turbulence models for control volume $k$ can now be written as

$$
V_{\mathrm{k}} \frac{d\left(\boldsymbol{U}_{\mathrm{t}}\right)_{\mathrm{k}}}{d t}+\left(\boldsymbol{R}_{\mathrm{t}}\right)_{\mathrm{k}}=0
$$

where $\left(\boldsymbol{R}_{\mathrm{t}}\right)_{\mathrm{k}}$ is the residual given by

$$
\left(\boldsymbol{R}_{\mathrm{t}}\right)_{\mathrm{k}}=\sum_{l \in N_{\mathrm{k}}}\left(\boldsymbol{\Phi}_{\mathrm{t}, \mathrm{kl}}^{c}-\boldsymbol{\Phi}_{\mathrm{t}, \mathrm{kl}}^{v}\right)-\boldsymbol{\Phi}_{\mathrm{t}, \mathrm{k}}^{s} .
$$

For simplicity, these relations are written using the notation for column vectors (boldface). However, it should be understood that these terms are scalars for the one-equation SA turbulence model. 


\subsection{Time Integration}

The spatial discretization of the conservation equations has led to a system of timedependent ordinary differential equations. As mentioned in section 4.1 an implicit dualtime stepping approach is employed to integrate the equations in time. This approach uses a second-order accurate backward discretization scheme in physical time and the resulting discretized equations are integrated to the next physical time step using an implicit backward Euler scheme in so-called pseudo-time.

The dual-time stepping approach is employed for the semi-discretized URANS equations, see equation (4.14), as well as the semi-discretized equations of the turbulence models, see equation (4.110). The derivation in the following subsection is applied to the combined set of all semi-discretized conservation equations written as

$$
V_{\mathrm{k}} \frac{d \boldsymbol{W}_{\mathrm{k}}}{d t}+\boldsymbol{R}_{\mathrm{k}}=0
$$

where $\boldsymbol{W}$ now combines all conserved variables, i.e. $\boldsymbol{W}=\left[\boldsymbol{U}, \boldsymbol{U}_{\mathrm{t}}\right]^{T}$. The non-linear residual vector $\boldsymbol{R}$ combines the residual vector of the URANS equations and that of the equation(s) of a turbulence model when it appears in combination with $\boldsymbol{W}$. Subsequently, approximations to the so-called flux Jacobian matrix will be introduced in subsection 4.4.4 that will effectively decouple the URANS equations from the turbulence equations again.

\subsubsection{Dual-Time Stepping Approach}

The derivation of the dual-time stepping method starts by adding a pseudo-time derivative term to the semi-discretized equations. Choosing $\tau$ as the pseudo-time variable, the result for control volume $k$ is

$$
V_{\mathrm{k}} \frac{d \boldsymbol{W}_{\mathrm{k}}}{d \tau}+V_{\mathrm{k}} \frac{d \boldsymbol{W}_{\mathrm{k}}}{d t}+\boldsymbol{R}_{\mathrm{k}}=0
$$

The introduction of a second-order accurate backward finite-difference scheme for the physical time derivative and a backward Euler scheme for the pseudo-time derivative yields the following set of non-linear equations:

$$
V_{\mathrm{k}} \frac{\Delta \boldsymbol{W}_{\mathrm{k}}^{m}}{\Delta \tau_{\mathrm{k}}}+\left(\boldsymbol{R}^{*}\right)_{\mathrm{k}}^{m+1}=0,
$$

where superscript $m$ denotes the pseudo-time level. Furthermore, the update in pseudotime is given by

$$
\Delta \boldsymbol{W}_{\mathrm{k}}^{m}=\boldsymbol{W}_{\mathrm{k}}^{m+1}-\boldsymbol{W}_{\mathrm{k}}^{m},
$$

the pseudo-time step size is given by

$$
\Delta \tau_{\mathrm{k}}=\tau_{\mathrm{k}}^{m+1}-\tau_{\mathrm{k}}^{m},
$$

and the non-linear unsteady residual at the next pseudo-time level is given by

$$
\left(\boldsymbol{R}^{*}\right)_{\mathrm{k}}^{m+1}=V_{\mathrm{k}} \frac{3 \boldsymbol{W}_{\mathrm{k}}^{m+1}-4 \boldsymbol{W}_{\mathrm{k}}^{n}+\boldsymbol{W}_{\mathrm{k}}^{n-1}}{2 \Delta t}+\boldsymbol{R}_{\mathrm{k}}^{m+1},
$$


where $\Delta t$ is the physical time step size.

Superscript $n$ denotes (evaluation at) the physical time level $t=n \Delta t$, which is the same for the complete computational domain. However, the pseudo-time level can be different for each control volume since pseudo-time accuracy is not required. For $m$ going to infinity we have

$$
\begin{aligned}
\lim _{m \rightarrow \infty}\left(\Delta \boldsymbol{W}_{\mathrm{k}}^{m}\right) & =0 \quad \forall k \\
& \Rightarrow \\
\lim _{m \rightarrow \infty}\left(\left(\boldsymbol{R}^{*}\right)_{\mathrm{k}}^{m+1}\right) & =\left(\boldsymbol{R}^{*}\right)_{\mathrm{k}}^{n+1}=0 \quad \forall k,
\end{aligned}
$$

which means that the solution at the next physical time step has been found, i.e.

$$
\lim _{m \rightarrow \infty}\left(\boldsymbol{W}_{\mathrm{k}}^{m}\right)=\boldsymbol{W}_{\mathrm{k}}^{n+1} \quad \forall k .
$$

In practice, pseudo-time stepping is continued until the $L_{2}$-norm (taken over all control volumes) of each component of the unsteady residual vector has reached a sufficiently small value, e.g. $\left\|\left(\boldsymbol{R}^{*}\right)\right\|_{2}^{m}=10^{-4}\left\|\left(\boldsymbol{R}^{*}\right)\right\|_{2}^{0}$. When this criterion cannot be satisfied within a reasonable number of pseudo-time steps, then there might be some unsteady flow behavior between two physical time steps. This indicates that the physical time step size should be reduced in order to accurately simulate all important unsteady flow phenomena.

In order to solve the system of equations (4.114), the non-linear unsteady residual evaluated at the next pseudo-time level is approximated using a Taylor series expansion around the current pseudo-time level, as

$$
\begin{aligned}
\left(\boldsymbol{R}^{*}\right)_{\mathrm{k}}^{m+1} & \approx\left(\boldsymbol{R}^{*}\right)_{\mathrm{k}}^{m}+\left(\frac{\partial \boldsymbol{R}^{*}}{\partial \tau}\right)_{\mathrm{k}}^{m} \Delta \tau_{\mathrm{k}} \\
& \approx\left(\boldsymbol{R}^{*}\right)_{\mathrm{k}}^{m}+\left[\frac{\partial \boldsymbol{R}^{*}}{\partial \boldsymbol{W}}\right]_{\mathrm{k}}^{m}\left(\frac{\partial \boldsymbol{W}}{\partial \tau}\right)_{\mathrm{k}}^{m} \Delta \tau_{\mathrm{k}} \\
& \approx\left(\boldsymbol{R}^{*}\right)_{\mathrm{k}}^{m}+\left[\frac{\partial \boldsymbol{R}^{*}}{\partial \boldsymbol{W}}\right]_{\mathrm{k}}^{m} \Delta \boldsymbol{W}^{m},
\end{aligned}
$$

where $\left[\frac{\partial \boldsymbol{R}^{*}}{\partial \boldsymbol{W}}\right]_{\mathrm{k}}^{m}$ is a $N^{e} \times\left(N^{e} N^{c}\right)$ matrix with $N^{e}$ the number of conservation equations per control volume and $N^{c}$ the number of control volumes in the computational domain. Furthermore, $\Delta \boldsymbol{W}^{m}$ is a column vector of length $\left(N^{e} N^{c}\right)$, which combines the updates of the control-volume averaged conserved variables in pseudo-time for all control volumes.

Substitution of equation (4.121) in equation (4.117) yields

$$
\left(\boldsymbol{R}^{*}\right)_{\mathrm{k}}^{m+1} \approx V_{\mathrm{k}} \frac{3 \boldsymbol{W}_{\mathrm{k}}^{m}-4 \boldsymbol{W}_{\mathrm{k}}^{n}+\boldsymbol{W}_{\mathrm{k}}^{n-1}}{2 \Delta t}+\boldsymbol{R}_{\mathrm{k}}^{m}+\left(V_{\mathrm{k}} \frac{3}{2 \Delta t} I\right) \Delta \boldsymbol{W}_{\mathrm{k}}^{m}+\left[\frac{\partial \boldsymbol{R}}{\partial \boldsymbol{W}}\right]_{\mathrm{k}}^{m} \Delta \boldsymbol{W}^{m},
$$

where $I$ is the $N^{e} \times N^{e}$ identity matrix. 
Substitution of equation (4.122) in equation (4.114) yields

$$
\left(\frac{V_{\mathrm{k}}}{\Delta \tau_{\mathrm{k}}} I+\frac{3 V_{\mathrm{k}}}{2 \Delta t} I\right) \Delta \boldsymbol{W}_{\mathrm{k}}^{m}+\left[\frac{\partial \boldsymbol{R}}{\partial \boldsymbol{W}}\right]_{\mathrm{k}}^{m} \Delta \boldsymbol{W}^{m}+\left(\boldsymbol{R}^{*}\right)_{\mathrm{k}}^{m}=0,
$$

which can be written for all control volumes combined as the system of linear equations

$$
M^{m} \Delta \boldsymbol{W}^{m}=-\left(\boldsymbol{R}^{*}\right)^{m},
$$

where $M^{m}$ is the system matrix or implicit operator of dimensions $\left(N^{e} N^{c}\right) \times\left(N^{e} N^{c}\right)$, evaluated at the $m$-th pseudo-time level. This matrix is sparse and consists of blocks of dimensions $N^{e} \times N^{e}$. Only the non-zero blocks are calculated and stored in memory. The right hand side is the non-linear unsteady residual or explicit operator.

In general, a large number of control volumes is needed and it is therefore too expensive to solve equation 4.124 directly. As discussed in section 4.1 it is solved using Block Symmetric Gauß-Seidel iteration with additional methods to increase the convergence speed, see sections 4.5 and 4.6 These iteration sweeps, which solve the system of linear equations 4.124 within each pseudo-time step, are called inner iterations, whereas the pseudo-time steps used to let the non-linear residual converge to the next physical time step are the outer iterations.

For infinitely large pseudo-time step size, a Newton iteration method with quadratic convergence of the non-linear residual $\left(\boldsymbol{R}^{*}\right)^{n+1}$ is obtained if [138]

- no approximations to the system matrix are made;

- the system of linear equations 4.124 is solved to sufficient accuracy;

- the initial solution is sufficiently close to the exact solution.

Usually, the initial solution cannot be guaranteed to be close to the exact solution. Furthermore, it is very expensive to exactly solve the system of linear equations and the exact system matrix is quite complex and usually not suited to be combined with Block Symmetric Gauß-Seidel iteration, because of decreased diagonal dominance. Therefore, it is common to use finite pseudo-time step sizes and to approximate the exact system matrix in order to improve the efficiency, reduce the memory requirements and enhance the stability of the computational method [110]. The simplifications to the system matrix should be such that the combination of inner and outer iterations yield a stable and efficient solution method. This is discussed in subsection 4.4.4. These adjustments, however, come at the expense of loosing quadratic convergence speed.

The local pseudo-time step size is set by prescribing a certain CFL number, see subsection 4.5.3 This number, and thereby the pseudo-time step size, can be small during the first pseudo-time steps. This effectively results in under-relaxation and increases the convergence range of the inner iteration method. When the solution is close to the exact solution within the present physical time step, the CFL number can be increased, until the maximum prescribed value has been reached. 


\subsubsection{Steady Flow Solutions}

When the Reynolds-averaged variables are independent of time, the resulting flow field is said to be steady. If a steady flow solution is expected, the use of dual-time stepping is not needed and can be effectively 'switched-off' by prescribing a very large value for the physical time step size $\Delta t$. This means that pseudo-time stepping is used to iterate from the initial flow solution to the final steady flow solution within one physical time step. In the present computational method, the same result is obtained in a more efficient way by leaving out all terms that involve $\Delta t$.

\subsubsection{Initial Conditions}

For steady flow simulations, the conserved flow and turbulence variables in the entire computational domain are initialized at their free-stream value.

The initial conditions for time-accurate simulations using the second-order accurate backward time integration scheme are the flow solutions at two previous instances in time. If only one solution is known, then a first-order accurate Euler backward time integration scheme is used for the first time step instead.

Flow simulations with time-dependent boundary conditions can be started from a converged steady flow solution with steady boundary conditions. For example, simulations with synthetic jet actuation, see subsection 3.5.3 can be started from a steady flow solution with the actuation turned off.

\subsubsection{Approximate Flux Jacobian Matrix}

The system matrix $M$ contains the term $\partial \boldsymbol{R} / \partial \boldsymbol{W}$, which is commonly referred to as the flux Jacobian matrix: the derivative of the sum of convective fluxes, viscous fluxes and source term with respect to the control-volume averaged conserved variables.

To improve the efficiency, reduce the memory requirements and enhance the stability of the computational method, a number of approximations to the flux Jacobian matrix are introduced.

The first approximation to the flux Jacobian matrix employed in the present computational method is to derive it from lower-order or simplified flux schemes, such that only information from nearest-neighbors is used. This approximation reduces the memory requirements, and increases the diagonal dominance of the implicit operator, which is beneficial for the iteration method [114]. Note that the lower-order or simplified flux schemes are used only to calculate the implicit operator, not the explicit operator.

The second approximation employed in the present computational method is to neglect the cross-derivatives of the flux Jacobian matrix, i.e. the derivative of the residual of the URANS equations with respect to the conserved turbulence variables as well as the derivative of the residual of the turbulence model equations with respect to the conserved flow variables. This is allowed since these coupling terms are relatively weak [68]. The result of the second approximation is that the set of discretized URANS equations is decoupled from the set of discretized equations of the turbulence model. This implies 
that each set can be solved separately, i.e. in a loosely coupled way, see subsection 4.5.1. This improves the flexibility of the computational method, since it makes it easier to implement different turbulence model equations. Additionally, both sets of equations can use their own pseudo-time step size, according to their own stability requirements. Furthermore, when the turbulence model equations are converging slowly, it is possible to perform multiple outer iterations (pseudo-time steps) on the turbulence model equations for each outer iteration performed on the URANS equations.

Equation (4.123) can now be rewritten for the URANS equations as

$$
\left(\frac{V_{\mathrm{k}}}{\Delta \tau_{\mathrm{k}}} I+\frac{3 V_{\mathrm{k}}}{2 \Delta t} I+\left(\frac{\partial \boldsymbol{R}_{\mathrm{k}}}{\partial \boldsymbol{U}_{\mathrm{k}}}\right)^{m}\right) \Delta \boldsymbol{U}_{\mathrm{k}}^{m}+\sum_{j \in N_{\mathrm{k}}}\left(\left(\frac{\partial \boldsymbol{R}_{\mathrm{k}}}{\partial \boldsymbol{U}_{\mathrm{l}}}\right)^{m} \Delta \boldsymbol{U}_{\mathrm{l}}^{m}\right)+\left(\boldsymbol{R}^{*}\right)_{\mathrm{k}}^{m}=0
$$

with

$$
\begin{aligned}
& \frac{\partial \boldsymbol{R}_{\mathrm{k}}}{\partial \boldsymbol{U}_{\mathrm{k}}}=\sum_{l \in N_{\mathrm{k}}}\left(\frac{\partial \boldsymbol{\Phi}_{\mathrm{kl}}^{c}}{\partial \boldsymbol{U}_{\mathrm{k}}}-\frac{\partial \boldsymbol{\Phi}_{\mathrm{kl}}^{v}}{\partial \boldsymbol{U}_{\mathrm{k}}}\right), \\
& \frac{\partial \boldsymbol{R}_{\mathrm{k}}}{\partial \boldsymbol{U}_{\mathrm{l}}}=\frac{\partial}{\partial \boldsymbol{U}_{\mathrm{l}}}\left(\sum_{j \in N_{\mathrm{k}}}\left(\boldsymbol{\Phi}_{\mathrm{kj}}^{c}-\boldsymbol{\Phi}_{\mathrm{kj}}^{v}\right)\right)=\frac{\partial \boldsymbol{\Phi}_{\mathrm{kl}}^{c}}{\partial \boldsymbol{U}_{\mathrm{l}}}-\frac{\partial \boldsymbol{\Phi}_{\mathrm{kl}}^{v}}{\partial \boldsymbol{U}_{\mathrm{l}}} .
\end{aligned}
$$

Equation (4.123) can also be rewritten for the turbulence model equations as

$$
\begin{aligned}
\left(\frac{V_{\mathrm{k}}}{\Delta \tau_{\mathrm{k}}} I+\frac{3 V_{\mathrm{k}}}{2 \Delta t} I+\left(\frac{\partial\left(\boldsymbol{R}_{\mathrm{t}}\right)_{\mathrm{k}}}{\partial\left(\boldsymbol{U}_{\mathrm{t}}\right)_{\mathrm{k}}}\right)^{m}\right)\left(\Delta \boldsymbol{U}_{\mathrm{t}}\right)_{\mathrm{k}}^{m} & \\
& +\sum_{j \in N_{\mathrm{k}}}\left(\left(\frac{\partial\left(\boldsymbol{R}_{\mathrm{t}}\right)_{\mathrm{k}}}{\partial\left(\boldsymbol{U}_{\mathrm{t}}\right)_{\mathrm{l}}}\right)^{m}\left(\Delta \boldsymbol{U}_{\mathrm{t}}\right)_{\mathrm{l}}^{m}\right)+\left(\boldsymbol{R}_{\mathrm{t}}^{*}\right)_{\mathrm{k}}^{m}=0
\end{aligned}
$$

with

$$
\begin{aligned}
& \frac{\partial\left(\boldsymbol{R}_{\mathrm{t}}\right)_{\mathrm{k}}}{\partial\left(\boldsymbol{U}_{\mathrm{t}}\right)_{\mathrm{k}}}=\sum_{l \in N_{\mathrm{k}}}\left(\frac{\partial \boldsymbol{\Phi}_{\mathrm{t}, \mathrm{kl}}^{c}}{\partial\left(\boldsymbol{U}_{\mathrm{t}}\right)_{\mathrm{k}}}-\frac{\partial \boldsymbol{\Phi}_{\mathrm{t}, \mathrm{kl}}^{v}}{\partial\left(\boldsymbol{U}_{\mathrm{t}}\right)_{\mathrm{k}}}\right)-\frac{\partial \boldsymbol{\Phi}_{\mathrm{t}, \mathrm{k}}^{s}}{\partial\left(\boldsymbol{U}_{\mathrm{t}}\right)_{\mathrm{k}}}, \\
& \frac{\partial\left(\boldsymbol{R}_{\mathrm{t}}\right)_{\mathrm{k}}}{\partial\left(\boldsymbol{U}_{\mathrm{t}}\right)_{\mathrm{l}}}=\frac{\partial}{\partial\left(\boldsymbol{U}_{\mathrm{t}}\right)_{\mathrm{l}}}\left(\sum_{j \in N_{\mathrm{k}}}\left(\boldsymbol{\Phi}_{\mathrm{t}, \mathrm{kj}}^{c}-\boldsymbol{\Phi}_{\mathrm{t}, \mathrm{kj}}^{v}\right)\right)-\frac{\partial \boldsymbol{\Phi}_{\mathrm{t}, \mathrm{k}}^{s}}{\partial\left(\boldsymbol{U}_{\mathrm{t}}\right)_{\mathrm{l}}}=\frac{\partial \boldsymbol{\Phi}_{\mathrm{t}, \mathrm{kl}}^{c}}{\partial\left(\boldsymbol{U}_{\mathrm{t}}\right)_{l}}-\frac{\partial \boldsymbol{\Phi}_{\mathrm{t}, \mathrm{kl}}^{v}}{\partial\left(\boldsymbol{U}_{\mathrm{t}}\right)_{l}}-\frac{\partial \boldsymbol{\Phi}_{\mathrm{t}, \mathrm{k}}^{s}}{\partial\left(\boldsymbol{U}_{\mathrm{t}}\right)_{l}} .
\end{aligned}
$$

Here we treat the gradients in the source term as being locally constant, such that $\partial \boldsymbol{\Phi}_{\mathrm{t}, \mathrm{k}}^{s} / \partial\left(\boldsymbol{U}_{\mathrm{t}}\right)_{\mathrm{l}}=0$.

The contributions to the approximate flux Jacobian of the URANS or turbulence model equations from the convective and viscous flux functions are calculated by a loop over all internal and boundary faces. During the loop over the internal faces, the property of 
conservativeness of the numerical flux functions is employed to save computational time:

$$
\frac{\partial \boldsymbol{\Phi}_{\mathrm{kl}}}{\partial \boldsymbol{U}_{\mathrm{m}}}=-\frac{\partial \boldsymbol{\Phi}_{\mathrm{lk}}}{\partial \boldsymbol{U}_{\mathrm{m}}}, \quad m=\{k, l\} .
$$

This relation holds for all convective and viscous flux functions of both the URANS and the turbulence model equations.

Since the contribution from the source term of the turbulence model equations is calculated by treating the gradients as being locally constant, this contribution can be determined by a loop over all control volumes.

The following subsections discuss the actual contributions to the approximate flux Jacobian of the URANS and turbulence model equations in more detail. It also introduces possible further approximations, depending on the flux function or source function at hand.

\subsubsection{Flux Jacobian of URANS Equations}

\section{Contribution from convective flux function}

The contributions to the flux Jacobian of the URANS equations from the discretized convective flux are derived from the first-order accurate version of the convective flux function.

The first-order accurate version of the JST scheme, see equation (4.17), reads

$$
\boldsymbol{\Phi}_{\mathrm{kl}}^{c}=\frac{1}{2}\left(\underline{\boldsymbol{F}}^{c}\left(\boldsymbol{U}_{\mathrm{k}}\right)+\underline{\boldsymbol{F}}^{c}\left(\boldsymbol{U}_{\mathrm{l}}\right)\right) \cdot \underline{S}_{\mathrm{kl}}-\frac{1}{2}\left(\lambda_{\mathrm{kl}}^{c}\right)_{\max }\left(\boldsymbol{U}_{\mathrm{l}}-\boldsymbol{U}_{\mathrm{k}}\right) .
$$

This (scalar dissipation) scheme is also employed when the JST scheme with matrix dissipation is used for the explicit operator. For the calculation of the contribution to the flux Jacobian, the spectral radius $\left(\lambda_{\mathrm{kl}}^{c}\right)_{\max }$ is treated as being locally constant, i.e.

$$
\begin{aligned}
& \frac{\partial \boldsymbol{\Phi}_{\mathrm{kl}}^{c}}{\partial \boldsymbol{U}_{\mathrm{k}}}=\frac{1}{2}\left(\frac{\partial \underline{\boldsymbol{F}}^{c}\left(\boldsymbol{U}_{\mathrm{k}}\right) \cdot \underline{S}_{\mathrm{kl}}}{\partial \boldsymbol{U}_{\mathrm{k}}}-\left(\lambda_{\mathrm{kl}}^{c}\right)_{\max }\right), \\
& \frac{\partial \boldsymbol{\Phi}_{\mathrm{kl}}^{c}}{\partial \boldsymbol{U}_{\mathrm{l}}}=\frac{1}{2}\left(\frac{\partial \underline{\boldsymbol{F}}^{c}\left(\boldsymbol{U}_{\mathrm{l}}\right) \cdot \underline{S}_{\mathrm{kl}}}{\partial \boldsymbol{U}_{\mathrm{l}}}+\left(\lambda_{\mathrm{kl}}^{c}\right)_{\max }\right) .
\end{aligned}
$$

First-order accurate upwind convective flux functions are obtained if the left and right state are taken equal to the associated control-volume average states, i.e. $\left[\boldsymbol{U}_{\mathrm{k}}\right]_{\mathrm{kl}}=\boldsymbol{U}_{\mathrm{k}}$ and $\left[\boldsymbol{U}_{1}\right]_{\mathrm{kl}}=\boldsymbol{U}_{\mathrm{l}}$.

The contribution to the flux Jacobian of Roe's flux-difference splitting scheme is calculated by treating the Roe matrix as being locally constant, i.e.

$$
\begin{aligned}
& \frac{\partial \boldsymbol{\Phi}_{\mathrm{kl}}^{c}}{\partial \boldsymbol{U}_{\mathrm{k}}}=\frac{1}{2}\left(\frac{\partial \underline{\boldsymbol{F}}^{c}\left(\boldsymbol{U}_{\mathrm{k}}\right) \cdot \underline{S}_{\mathrm{kl}}}{\partial \boldsymbol{U}_{\mathrm{k}}}-\left|\hat{A}_{R o e, \mathrm{kl}}\right|\right), \\
& \frac{\partial \boldsymbol{\Phi}_{\mathrm{kl}}^{c}}{\partial \boldsymbol{U}_{\mathrm{l}}}=\frac{1}{2}\left(\frac{\partial \underline{\boldsymbol{F}}^{c}\left(\boldsymbol{U}_{\mathrm{l}}\right) \cdot \underline{S}_{\mathrm{kl}}}{\partial \boldsymbol{U}_{\mathrm{l}}}+\left|\hat{A}_{R o e, \mathrm{kl}}\right|\right) .
\end{aligned}
$$


This approximation only slightly diminishes the convergence speed of the resulting implicit time stepping scheme [139].

The term of type $\partial \underline{\boldsymbol{F}}^{c}(\boldsymbol{U}) \cdot \underline{S} / \partial \boldsymbol{U}$ is derived by writing the convective flux vector in terms of conserved variables, and subsequently taking the derivatives with respect to these variables, see appendix B.

The contribution to the flux Jacobian of the AUSM family of flux-vector splitting schemes is rather complicated to derive analytically. Therefore, it is calculated using the 'complex-step derivative approximation' [140, 141], which is generally more accurate than a standard finite-difference approximation. The complex-step method is described in appendix $\mathrm{C}$.

\section{Contribution from viscous flux function}

The contributions from the viscous flux denoted by $-\partial \boldsymbol{\Phi}_{\mathrm{kl}}^{v} / \partial \boldsymbol{U}_{\mathrm{k}}$ and $-\partial \boldsymbol{\Phi}_{\mathrm{kl}}^{v} / \partial \boldsymbol{U}_{\mathrm{l}}$ are derived by approximating the gradients at the faces of each control volume by

$$
[\underline{\nabla} \phi]_{\mathrm{kl}} \approx \frac{\phi_{\mathrm{l}}-\phi_{\mathrm{k}}}{\left|\Delta \underline{x}_{\mathrm{kl}}\right|} \frac{\Delta \underline{x}_{\mathrm{kl}}}{\left|\Delta \underline{x}_{\mathrm{kl}}\right|},
$$

instead of using equation (4.100). The approximate viscous flux function can therefore be written as $\boldsymbol{\Phi}_{\mathrm{kl}}^{v}\left(\boldsymbol{U}_{\mathrm{k}}, \boldsymbol{U}_{\mathrm{l}}\right)$.

The contributions are constructed by differentiating the approximate viscous flux function with respect to the components of the primitive variables $Q=\left[\rho, u_{1}, u_{2}, u_{3}, p^{*}\right]^{T}$ and subsequently using the chain rule of differentiation, i.e.

$$
\begin{aligned}
& \frac{\partial \boldsymbol{\Phi}_{\mathrm{kl}}^{v}}{\partial \boldsymbol{U}_{\mathrm{k}}}=\frac{\partial \boldsymbol{\Phi}_{\mathrm{kl}}^{v}}{\partial \boldsymbol{Q}_{\mathrm{k}}} \frac{\partial \boldsymbol{Q}_{\mathrm{k}}}{\partial \boldsymbol{U}_{\mathrm{k}}} \\
& \frac{\partial \boldsymbol{\Phi}_{\mathrm{kl}}^{v}}{\partial \boldsymbol{U}_{\mathrm{l}}}=\frac{\partial \boldsymbol{\Phi}_{\mathrm{kl}}^{v}}{\partial \boldsymbol{Q}_{\mathrm{l}}} \frac{\partial \boldsymbol{Q}_{\mathrm{l}}}{\partial \boldsymbol{U}_{\mathrm{l}}}
\end{aligned}
$$

where $\partial \boldsymbol{Q} / \partial \boldsymbol{U}$ is given by

$$
\begin{aligned}
& \frac{\partial \boldsymbol{Q}}{\partial \boldsymbol{U}}= \\
& {\left[\begin{array}{ccccc}
1 & 0 & 0 & 0 & 0 \\
-u_{1} / \rho & 1 / \rho & 0 & 0 & 0 \\
-u_{2} / \rho & 0 & 1 / \rho & 0 & 0 \\
-u_{3} / \rho & 0 & 0 & 1 / \rho & 0 \\
(\gamma-1)\left(\frac{1}{2}|\underline{u}|^{2}\right) & -(\gamma-1) u_{1} & -(\gamma-1) u_{2} & -(\gamma-1) u_{3} & (\gamma-1)
\end{array}\right] .}
\end{aligned}
$$

In the explicit operator, the molecular viscosity is a function of the temperature, see equation 3.28 . However, in the derivation of the viscous flux Jacobian, it is treated as being locally constant. The same approach is adopted for the eddy viscosity, $\mu_{\mathrm{t}}$. 
The matrices $\partial \boldsymbol{\Phi}_{\mathrm{kl}}^{v} / \partial \boldsymbol{Q}_{\mathrm{k}}$ and $\partial \boldsymbol{\Phi}_{\mathrm{kl}}^{v} / \partial \boldsymbol{Q}_{\mathrm{l}}$ can be conveniently written using dummy subscript $m=\{k, l\}$ as:

$$
\frac{\partial \boldsymbol{\Phi}_{\mathrm{kl}}^{v}}{\partial \boldsymbol{Q}_{\mathrm{m}}} \equiv\left(\hat{A}^{v}\right)_{\mathrm{m}}=\left[\begin{array}{ccccc}
0 & 0 & 0 & 0 & 0 \\
0 & \left(\hat{A}_{22}^{v}\right)_{\mathrm{m}} & \left(\hat{A}_{23}^{v}\right)_{\mathrm{m}} & \left(\hat{A}_{24}^{v}\right)_{\mathrm{m}} & 0 \\
0 & \left(\hat{A}_{32}^{v}\right)_{\mathrm{m}} & \left(\hat{A}_{33}^{v}\right)_{\mathrm{m}} & \left(\hat{A}_{34}^{v}\right)_{\mathrm{m}} & 0 \\
0 & \left(\hat{A}_{42}^{v}\right)_{\mathrm{m}} & \left(\hat{A}_{43}^{v}\right)_{\mathrm{m}} & \left(\hat{A}_{44}^{v}\right)_{\mathrm{m}} & 0 \\
\left(\hat{A}_{51}^{v}\right)_{\mathrm{m}} & \left(\hat{A}_{52}^{v}\right)_{\mathrm{m}} & \left(\hat{A}_{53}^{v}\right)_{\mathrm{m}} & \left(\hat{A}_{54}^{v}\right)_{\mathrm{m}} & \left(\hat{A}_{55}^{v}\right)_{\mathrm{m}}
\end{array}\right] .
$$

The non-zero elements in the second, third and fourth row are given by

$$
\begin{aligned}
& \left(\hat{A}_{22}^{v}\right)_{\mathrm{m}}=\frac{\beta_{\mathrm{m}}\left[\mu+\mu_{\mathrm{t}}\right]_{\mathrm{kl}}}{\left|\Delta \underline{x}_{\mathrm{kl}}\right|^{2}}\left(\frac{4}{3}\left(\Delta x_{1}\right)_{\mathrm{kl}} \quad, \quad\left(\Delta x_{2}\right)_{\mathrm{kl}} \quad, \quad\left(\Delta x_{3}\right)_{\mathrm{kl}} \quad\right) \quad \underline{S}_{\mathrm{kl}}, \\
& \left(\hat{A}_{23}^{v}\right)_{\mathrm{m}}=\frac{\beta_{\mathrm{m}}\left[\mu+\mu_{\mathrm{t}}\right]_{\mathrm{kl}}}{\left|\Delta \underline{x}_{\mathrm{kl}}\right|^{2}}\left(-\frac{2}{3}\left(\Delta x_{2}\right)_{\mathrm{kl}} \quad, \quad\left(\Delta x_{1}\right)_{\mathrm{kl}} \quad, \quad 0 \quad\right) \cdot \underline{S}_{\mathrm{kl}},
\end{aligned}
$$

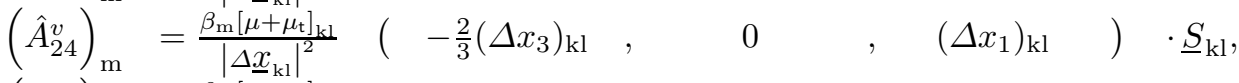

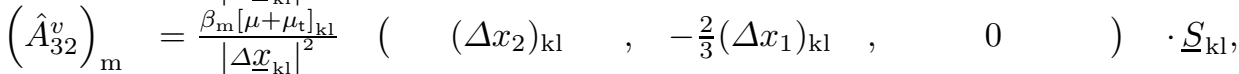

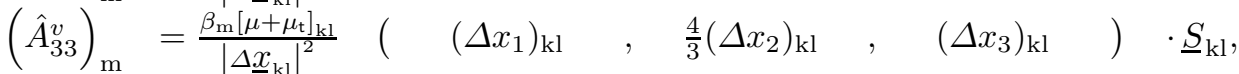

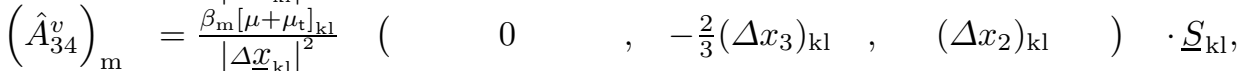

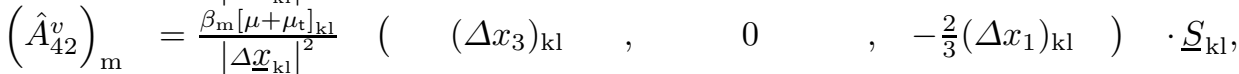

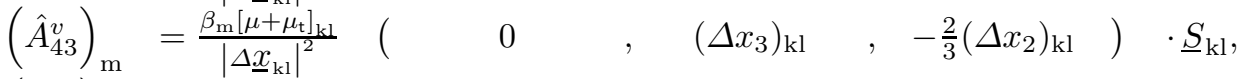

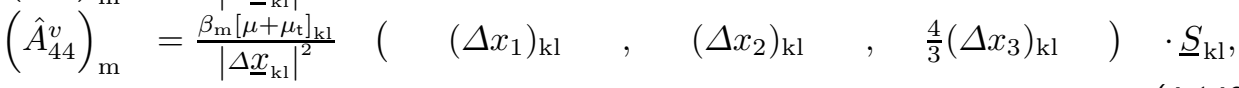

with

$$
\beta_{\mathrm{m}}= \begin{cases}-1, & \text { if } m=k \\ +1, & \text { if } m=l\end{cases}
$$

Furthermore, the elements of the fifth row are given by

$$
\begin{aligned}
& \left(\hat{A}_{51}^{v}\right)_{\mathrm{m}}=\left(\left(\frac{\gamma \mu}{\operatorname{Pr}}+\frac{\gamma \mu_{\mathrm{t}}}{\operatorname{Pr}_{\mathrm{t}}}\right) \frac{-p_{\mathrm{m}}^{*}}{(\gamma-1) \rho_{\mathrm{m}}^{2}} \frac{\beta_{\mathrm{m}} \Delta \underline{x}_{\mathrm{kl}}}{\left|\Delta \underline{x}_{\mathrm{kl}}\right|^{2}}\right) \cdot \underline{S}_{\mathrm{kl}}, \\
& \left(\hat{A}_{52}^{v}\right)_{\mathrm{m}}=\left(\left(\hat{A}_{22}^{v}\right)_{\mathrm{m}},\left(\hat{A}_{32}^{v}\right)_{\mathrm{m}},\left(\hat{A}_{42}^{v}\right)_{\mathrm{m}}\right) \cdot[\underline{u}]_{\mathrm{kl}}+\alpha_{\mathrm{m}}\left[\mu+\mu_{\mathrm{t}}\right]_{\mathrm{kl}}\left[\eta_{1 j}\right]_{\mathrm{kl}}\left(S_{j}\right)_{\mathrm{kl}}, \\
& \left(\hat{A}_{53}^{v}\right)_{\mathrm{m}}=\left(\left(\hat{A}_{23}^{v}\right)_{\mathrm{m}},\left(\hat{A}_{33}^{v}\right)_{\mathrm{m}},\left(\hat{A}_{43}^{v}\right)_{\mathrm{m}}\right) \cdot[\underline{u}]_{\mathrm{kl}}+\alpha_{\mathrm{m}}\left[\mu+\mu_{\mathrm{t}}\right]_{\mathrm{kl}}\left[\eta_{2 j}\right]_{\mathrm{kl}}\left(S_{j}\right)_{\mathrm{kl}}, \\
& \left(\hat{A}_{54}^{v}\right)_{\mathrm{m}}=\left(\left(\hat{A}_{24}^{v}\right)_{\mathrm{m}},\left(\hat{A}_{34}^{v}\right)_{\mathrm{m}},\left(\hat{A}_{44}^{v}\right)_{\mathrm{m}}\right) \cdot[\underline{u}]_{\mathrm{kl}}+\alpha_{\mathrm{m}}\left[\mu+\mu_{\mathrm{t}}\right]_{\mathrm{kl}}\left[\eta_{3 j}\right]_{\mathrm{kl}}\left(S_{j}\right)_{\mathrm{kl}}, \\
& \left(\hat{A}_{55}^{v}\right)_{\mathrm{m}}=\left(\left(\frac{\gamma \mu}{\operatorname{Pr}}+\frac{\gamma \mu_{\mathrm{t}}}{\operatorname{Pr}_{\mathrm{t}}}\right) \frac{1}{(\gamma-1) \rho_{\mathrm{m}}} \frac{\beta_{\mathrm{m}} \Delta \underline{x}_{\mathrm{kl}}}{\left|\Delta \underline{x}_{\mathrm{kl}}\right|^{2}}\right) \cdot \underline{S}_{\mathrm{kl}},
\end{aligned}
$$


with summation over index $j, \underline{\underline{\eta}}=\left(\frac{1}{\mu+\mu_{\mathrm{t}}}\right) \underline{\underline{\tau}}_{\mathrm{eff}}$ and $\left[\eta_{i j}\right]_{\mathrm{kl}}$ indicates that the velocity gradients present in $\eta_{i j}$ are calculated as defined in equation 4.137. Furthermore, $\alpha_{\mathrm{m}}$ is defined as

$$
\alpha_{\mathrm{m}}= \begin{cases}\alpha_{\mathrm{l}, \mathrm{kl}}, & \text { if } m=k, \\ \alpha_{\mathrm{k}, \mathrm{kl}}, & \text { if } m=l .\end{cases}
$$

The coefficients $\alpha_{1, \mathrm{kl}}$ and $\alpha_{\mathrm{k}, \mathrm{kl}}$ are weights used in the central viscous flux discretization, see equation (4.97).

This concludes the construction of the approximate flux Jacobian matrix of the URANS equations. The construction of the contributions to the approximate flux Jacobian of the turbulence model equations is described in the next subsection.

\subsubsection{Flux Jacobian of Turbulence Model Equations}

\section{Contribution from convective flux function}

The contributions from the convective flux function of the two-equation SST turbulence model to the flux Jacobian of the turbulence model equations are given by

$$
\begin{aligned}
& \frac{\partial \Phi_{\mathrm{t}, \mathrm{kl}}^{c}}{\partial\left(\boldsymbol{U}_{\mathrm{t}}\right)_{\mathrm{k}}}=\left[\begin{array}{cc}
\frac{1}{2}\left([\underline{u}]_{\mathrm{kl}} \cdot \underline{S}_{\mathrm{kl}}+\left|[\underline{u}]_{\mathrm{kl}} \cdot \underline{S}_{\mathrm{kl}}\right|\right) & 0 \\
0 & \frac{1}{2}\left([\underline{u}]_{\mathrm{kl}} \cdot \underline{S}_{\mathrm{kl}}+\left|[\underline{u}]_{\mathrm{kl}} \cdot \underline{S}_{\mathrm{kl}}\right|\right)
\end{array}\right] \\
& \frac{\partial \boldsymbol{\Phi}_{\mathrm{t}, \mathrm{kl}}^{c}}{\partial\left(\boldsymbol{U}_{\mathrm{t})_{1}}\right.}=\left[\begin{array}{cc}
\frac{1}{2}\left([\underline{u}]_{\mathrm{kl}} \cdot \underline{S}_{\mathrm{kl}}-\left|[\underline{u}]_{\mathrm{kl}} \cdot \underline{S}_{\mathrm{kl}}\right|\right) & \frac{1}{2}\left([\underline{u}]_{\mathrm{kl}} \cdot \underline{S}_{\mathrm{kl}}-\left|[\underline{u}]_{\mathrm{kl}} \cdot \underline{S}_{\mathrm{kl}}\right|\right)
\end{array}\right]
\end{aligned}
$$

whereas the contributions from the convective flux function of the one-equation SA turbulence model are given by

$$
\begin{aligned}
\frac{\partial \Phi_{\mathrm{t}, \mathrm{kl}}^{c}}{\partial\left(U_{\mathrm{t}}\right)_{\mathrm{k}}} & =\frac{1}{2}\left([\underline{u}]_{\mathrm{kl}} \cdot \underline{S}_{\mathrm{kl}}+\left|[\underline{u}]_{\mathrm{kl}} \cdot \underline{S}_{\mathrm{kl}}\right|\right), \\
\frac{\partial \Phi_{\mathrm{t}, \mathrm{kl}}^{c}}{\partial\left(U_{\mathrm{t}}\right)_{1}} & =\frac{1}{2}\left([\underline{u}]_{\mathrm{kl}} \cdot \underline{S}_{\mathrm{kl}}-\left|[\underline{u}]_{\mathrm{kl}} \cdot \underline{S}_{\mathrm{kl}}\right|\right) .
\end{aligned}
$$

\section{Contribution from viscous flux function}

The contributions from the viscous flux function of the two-equation SST turbulence model to the flux Jacobian of the turbulence model equations are derived by treating the eddy-viscosity as being locally constant and by approximating the gradients at the faces as defined in equation (4.137). With these simplifications it follows that

$$
\frac{\partial \boldsymbol{\Phi}_{\mathrm{t}, \mathrm{kl}}^{v}}{\partial\left(\boldsymbol{U}_{\mathrm{t}}\right)_{\mathrm{m}}}=\left[\begin{array}{cc}
\left([\mu]_{\mathrm{kl}}+\left[\sigma_{k}\right]_{\mathrm{kl}}\left[\mu_{\mathrm{t}}\right]_{\mathrm{kl}}\right) \frac{\beta_{\mathrm{m}} \Delta \underline{x}_{\mathrm{kl}} \cdot \underline{S}_{\mathrm{kl}}}{\rho_{\mathrm{m}}\left|\Delta \underline{x}_{\mathrm{k} 1}\right|^{2}} & 0 \\
0 & \left([\mu]_{\mathrm{kl}}+\left[\sigma_{\omega}\right]_{\mathrm{kl}}\left[\mu_{\mathrm{t}}\right]_{\mathrm{kl}}\right) \frac{\beta_{\mathrm{m}} \Delta \underline{x}_{\mathrm{kl}} \cdot \underline{S}_{\mathrm{kl}}}{\rho_{\mathrm{m}}\left|\Delta \underline{x}_{\mathrm{k} 1}\right|^{2}}
\end{array}\right]
$$

with $m=\{k, l\}$ and $\beta_{\mathrm{m}}$ as defined in equation (4.143). 
Using the same approximations for the the viscous flux function of the SA turbulence model yields the following contributions

$$
\frac{\partial \Phi_{\mathrm{t}, \mathrm{kl}}^{v}}{\partial\left(U_{\mathrm{t}}\right)_{\mathrm{m}}}=\frac{1}{\sigma}\left([\nu]_{\mathrm{kl}}+\left(1+C_{b 2}\right)[\tilde{\nu}]_{\mathrm{kl}}\right) \frac{\beta_{\mathrm{m}} \Delta \underline{x}_{\mathrm{kl}} \cdot \underline{S}_{\mathrm{kl}}}{\left|\Delta \underline{x}_{\mathrm{kl}}\right|^{2}},
$$

with $m=\{k, l\}$ and $\beta_{\mathrm{m}}$ as defined in equation (4.143).

\section{Contribution from source function}

The flux Jacobian of the turbulence model equations includes contributions from the source term, in such a way that the sum of these contributions increases the diagonal dominance of the system matrix. Gradients present in the source term are treated as being locally constant. Therefore, the approximate source term of control volume $k$ used in the derivation of the flux Jacobian does not depend on variables outside of the control volume $k$.

For the contributions from the SST turbulence model, we could follow Menter [91], who constructs a diagonal matrix that includes only the derivatives of the destruction term and (the absolute value of) the cross-diffusion term, i.e.

$$
\frac{\partial \boldsymbol{\Phi}_{\mathrm{t}, \mathrm{k}}^{s}}{\partial\left(\boldsymbol{U}_{\mathrm{t}}\right)_{\mathrm{k}}}=V_{\mathrm{k}}\left[\begin{array}{cc}
-\beta^{*} \omega & 0 \\
0 & -2 \beta \omega-\left|\frac{C D}{\rho \omega}\right|
\end{array}\right]_{\mathrm{k}},
$$

where $C D$ is the cross diffusion term given by

$$
C D=2 \rho\left(1-F_{1}\right) \frac{\sigma_{\omega 2}}{\omega}(\underline{\nabla} k \cdot \underline{\nabla} \omega) .
$$

However, when the production and destruction terms cancel each other in the explicit operator, this approach will increase the diagonal dominance of the system matrix more than necessary and result in slow convergence of the system of linear equations. Therefore, we take a different approach in the present computational method, which is similar to that of Mor-Yossef \& Levy [142] and Spalart \& Allmaras [84]. This approach consists of the following steps:

- The limited production of turbulence kinetic energy is written as

$$
P_{k, \lim }=\left(\frac{P_{k, \lim }}{\rho k}\right) \rho k
$$

and $\left(P_{k, \lim } / \rho k\right)$ is subsequently treated as being locally constant.

- The variable $\omega$ is replaced by $\frac{\rho k}{\mu_{\mathrm{t}}}$ in $\left(\Phi_{\mathrm{t}, \mathrm{k}}^{s}\right)_{1}$ and the variable $k$ is replaced by $\frac{\omega \mu_{\mathrm{t}}}{\rho}$ in $\left(\Phi_{\mathrm{t}, \mathrm{k}}^{s}\right)_{2}$;

- The terms $F_{1}, F_{2}$ and $(\underline{\nabla} k \cdot \underline{\nabla} \omega)$ are treated as being locally constant; 
- The source function is written as

$$
\boldsymbol{\Phi}_{\mathrm{t}, \mathrm{k}}^{s}=V_{\mathrm{k}}\left[\begin{array}{cc}
\frac{\left(\Phi_{\mathrm{t}, \mathrm{k}}^{s}\right)_{1}}{(\rho k)_{\mathrm{k}}} & 0 \\
0 & \frac{\left(\Phi_{\mathrm{t}, \mathrm{k}}^{s}\right)_{2}}{(\rho \omega)_{\mathrm{k}}}
\end{array}\right]\left(\boldsymbol{U}_{\mathrm{t}}\right)_{\mathrm{k}}
$$

- The contributions to the flux Jacobian are constructed by taking the derivatives of equation (4.155) and retaining only negative terms as follows:

$$
\begin{aligned}
\frac{\partial \Phi_{\mathrm{t}, \mathrm{k}}^{s}}{\partial\left(\boldsymbol{U}_{\mathrm{t}}\right)_{\mathrm{k}}}= & \min \left(0, V_{\mathrm{k}}\left[\begin{array}{cc}
\frac{\left(\Phi_{\mathrm{t}, \mathrm{k}}^{s}\right)_{1}}{(\rho k)_{\mathrm{k}}} & 0 \\
0 & \frac{\left(\Phi_{\mathrm{t}, \mathrm{k}}^{s}\right)_{2}}{(\rho \omega)_{\mathrm{k}}}
\end{array}\right]\right) \\
& +\min \left(0, V_{\mathrm{k}}\left[\begin{array}{cc}
\frac{\partial}{\partial(\rho k)_{\mathrm{k}}}\left(\frac{\left(\Phi_{\mathrm{t}, \mathrm{k}}\right)_{1}}{(\rho k)_{\mathrm{k}}}\right) & 0 \\
0 & \frac{\partial}{\partial(\rho \omega)_{\mathrm{k}}}\left(\frac{\left(\Phi_{\mathrm{t}, \mathrm{k}}^{s}\right)_{2}}{(\rho \omega)_{\mathrm{k}}}\right)
\end{array}\right]\right)\left(\boldsymbol{U}_{\mathrm{t}}\right)_{\mathrm{k}} .
\end{aligned}
$$

Additionally, contributions originating from the sustaining terms, see subsection 3.4.3, are neglected. This approach results in the following contributions to the flux Jacobian from the source function of the SST turbulence model:

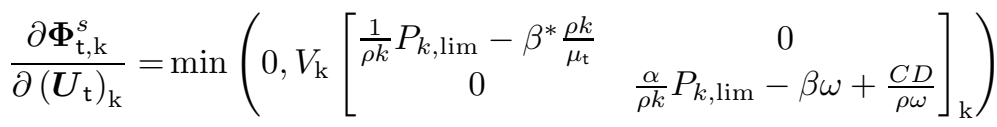

$$
\begin{aligned}
& +\min \left(0, V_{\mathrm{k}}\left[\begin{array}{cc}
-\beta^{* \frac{\rho k}{\mu_{\mathrm{t}}}} & 0 \\
0 & -\beta \omega-2 \frac{C D}{\rho \omega}
\end{array}\right]_{\mathrm{k}}\right) \text {. }
\end{aligned}
$$

The terms $P_{k, \text { lim }}$ and $C D$ are calculated during the evaluation of the explicit operator and can be reused during the construction of the flux Jacobian.

For the contributions from the SA turbulence model, we follow Spalart \& Allmaras [84], and write the source function in a similar way as presented in equation (4.155):

$$
\Phi_{\mathrm{t}, \mathrm{k}}^{s}=\left(\frac{\Phi_{\mathrm{t}, \mathrm{k}}^{s}}{\left(U_{\mathrm{t}}\right)_{\mathrm{k}}}\right)\left(U_{\mathrm{t}}\right)_{\mathrm{k}}
$$

Subsequently, only the negative contributions to the flux Jacobian are retained, i.e.

$$
\frac{\partial \Phi_{\mathrm{t}, \mathrm{k}}^{s}}{\partial\left(U_{\mathrm{t}}\right)_{\mathrm{k}}}=\min \left(0, \frac{\Phi_{\mathrm{t}, \mathrm{k}}^{s}}{\left(U_{\mathrm{t}}\right)_{\mathrm{k}}}\right)+\min \left(0, \frac{\partial \frac{\Phi_{\mathrm{t}, \mathrm{k}}^{s}}{\left(U_{\mathrm{t}}\right)_{\mathrm{k}}}}{\partial\left(U_{\mathrm{t}}\right)_{\mathrm{k}}}\left(U_{\mathrm{t}}\right)_{\mathrm{k}}\right) .
$$


This results in

$$
\begin{aligned}
\frac{\partial \Phi_{\mathrm{t}, \mathrm{k}}^{s}}{\partial\left(U_{\mathrm{t}}\right)_{\mathrm{k}}} & =\min \left(0, V_{\mathrm{k}}\left(C_{b 1} \tilde{S}-\frac{C_{b 2}}{\sigma} \underline{\nabla}^{2} \tilde{\nu}-C_{w 1} f_{w} \frac{\tilde{\nu}}{d^{2}}\right)_{\mathrm{k}}\right) \\
& +\min \left(0, V_{\mathrm{k}}\left(C_{b 1} \tilde{\nu} \frac{\partial \tilde{S}}{\partial \tilde{\nu}}-\frac{C_{w 1}}{d^{2}}\left(f_{w} \tilde{\nu}+\tilde{\nu}^{2} \frac{\partial f_{w}}{\partial \tilde{\nu}}\right)\right)_{\mathrm{k}}\right)
\end{aligned}
$$

with

$$
\begin{aligned}
\frac{\partial \tilde{S}}{\partial \tilde{\nu}} & =\frac{1}{\kappa^{2} d^{2}}\left(f_{v 2}+\tilde{\nu} \frac{\partial f_{v 2}}{\partial \tilde{\nu}}\right) \\
\frac{\partial f_{v 2}}{\partial \tilde{\nu}} & =-\left(\frac{\frac{\partial \chi}{\partial \tilde{\nu}}}{1+\chi f_{v 1}}-\frac{\chi}{\left(1+\chi f_{v 1}\right)^{2}}\left(\frac{\partial \chi}{\partial \tilde{\nu}} f_{v 1}+\chi \frac{\partial f_{v 1}}{\partial \tilde{\nu}}\right)\right) \\
\frac{\partial f_{v 1}}{\partial \tilde{\nu}} & =\left(\frac{3 \chi^{2}}{\chi^{3}+C_{v 1}^{3}}-\frac{3 \chi^{5}}{\left(\chi^{3}+C_{v 1}^{3}\right)^{2}}\right) \frac{\partial \chi}{\partial \tilde{\nu}} \\
\frac{\partial \chi}{\partial \tilde{\nu}} & =\frac{1}{\nu}, \\
\frac{\partial f_{w}}{\partial \tilde{\nu}} & =\frac{\partial f_{w}}{\partial g} \frac{\partial g}{\partial r} \frac{\partial r}{\partial \tilde{\nu}}, \\
\frac{\partial f_{w}}{\partial g} & =\left(\frac{C_{w 3}^{6}}{g^{6}+C_{w 3}^{6}}\right) \frac{f_{w}}{g} \\
\frac{\partial g}{\partial r} & =1+C_{w 2}\left(6 r^{5}-1\right) \\
\frac{\partial r}{\partial \tilde{\nu}} & =\frac{1}{\kappa^{2} d^{2}}\left(\frac{1}{\tilde{S}}-\frac{\tilde{\nu}}{\tilde{S}^{2}} \frac{\partial \tilde{S}}{\partial \tilde{\nu}}\right)
\end{aligned}
$$

As discussed in subsection 4.3.2 $r$ is maximized to a value of 10 during the evaluation of $f_{w}$ in order to prevent numerical difficulties. Since $f_{w}$ asymptotes to $\left(1+C_{w 3}^{6}\right)^{1 / 6}$ for large $r$, we also set $\frac{\partial f_{w}}{\partial \tilde{\nu}}$ to zero for $r>10$ [84].

This concludes the construction of the approximate flux Jacobian of the SST and SA turbulence model equations.

\subsection{Iterative Solution Method}

The employed inner iteration method to solve the system of linear equations given by equation (4.124) is the Block Symmetric Gauß-Seidel iteration method.

\subsubsection{Loosely Coupled Iteration Method}

Due to the approximations to the flux Jacobian matrix, equation (4.124) actually consists of two separate systems of linear equations, i.e. one for the URANS equations and one for 
the turbulence model equations. The inner iteration method is therefore applied twice, i.e. in a loosely-coupled way, as follows:

- The quantities associated with the turbulence variables are frozen and the system of linear equations associated with the URANS equations, see equation (4.125), is solved iteratively. Subsequently, the conserved flow variables of the URANS equations are updated.

- The quantities associated with the flow variables of the URANS equations are frozen and the system of linear equations associated with the turbulence variables, see equation (4.128), is solved iteratively. Subsequently, the conserved turbulence variables are updated and the eddy-viscosity is recalculated.

Since the employed flux Jacobians are approximations of the exact flux Jacobians, it is inefficient to solve both systems of linear equations exactly. Instead, the inner iterations continue until the residuals have been reduced by one order of magnitude. Then, the residuals of the non-linear system of equations are calculated and if these have not converged to a sufficiently small value, the computational method continues with a new outer iteration.

\subsubsection{Block Symmetric Gauß-Seidel Iteration}

The Block Symmetric Gauß-Seidel method consists of a forward iteration sweep followed by a backward iteration sweep. For the system of equations associated with the URANS equations, see equation (4.125), this can be written using inner iteration index $i$ as

$$
\begin{gathered}
M_{\mathrm{kk}}^{m} \Delta \boldsymbol{U}_{\mathrm{k}}^{m, i+\frac{1}{2}}=-\left(\left(\boldsymbol{R}^{*}\right)_{\mathrm{k}}^{m}+\sum_{l<k} M_{\mathrm{kl}}^{m} \Delta \boldsymbol{U}_{\mathrm{k}}^{m, i+\frac{1}{2}}+\sum_{l>k} M_{\mathrm{kl}}^{m} \Delta \boldsymbol{U}_{\mathrm{k}}^{m, i}\right) \\
k=\left\{1,2, \ldots, N^{c}\right\} \text { and } l \in N_{\mathrm{k}} \\
M_{\mathrm{kk}}^{m} \Delta \boldsymbol{U}_{\mathrm{k}}^{m, i+1}=-\left(\left(\boldsymbol{R}^{*}\right)_{\mathrm{k}}^{m}+\sum_{l<k} M_{\mathrm{kl}}^{m} \Delta \boldsymbol{U}_{\mathrm{k}}^{m, i+\frac{1}{2}}+\sum_{l>k} M_{\mathrm{kl}}^{m} \Delta \boldsymbol{U}_{\mathrm{k}}^{m, i+1}\right) \\
k=\left\{N^{c}, N^{c}-1, \ldots, 1\right\} \text { and } l \in N_{\mathrm{k}}
\end{gathered}
$$

where $m$ indicates the pseudo-time step level and $M_{\mathrm{kk}}^{m}$ and $M_{\mathrm{kl}}^{m}$ are the $5 \times 5$ matrices given by

$$
\begin{aligned}
& M_{\mathrm{kk}}^{m}=\frac{V_{\mathrm{k}}}{\Delta \tau_{\mathrm{k}}} I+\frac{3 V_{\mathrm{k}}}{2 \Delta t} I+\left(\frac{\partial \boldsymbol{R}_{\mathrm{k}}}{\partial \boldsymbol{U}_{\mathrm{k}}}\right)^{m}, \\
& M_{\mathrm{kl}}^{m}=\left(\frac{\partial \boldsymbol{R}_{\mathrm{k}}}{\partial \boldsymbol{U}_{\mathrm{l}}}\right)^{m},
\end{aligned}
$$

respectively. The initial solution at each pseudo-time step is taken as $\Delta \boldsymbol{U}=0$. The local system of equations of size $5 \times 5$ is solved directly, using Gaussian elimination with partial pivoting [143]. 
The reason for using forward and backward iteration sweeps is that it is more robust for the type of equations at hand. The convective part of the URANS equations describes the propagation of waves. Iteration in the direction of wave propagation yields faster convergence of the residuals than iteration in the opposite direction [113, Chapter 7]. Since the waves can travel in any direction (depending on the Mach number), Symmetric Gauß-Seidel iteration is more robust than standard Gauß-Seidel iteration.

The method can also be written for the system of equations associated with the turbulence equations in a similar way. However, for both turbulence models, a local matrix inversion using Gaussian elimination is not necessary: the SA turbulence model is a one-equation model, whereas the system matrix for the SST turbulence model contains only block diagonal sub-matrices ('blocks'), for which matrix inversion is simple.

In contrast to Jacobi iteration, the Gauß-Seidel method is characterized by always using the most recent value of $\Delta \boldsymbol{U}^{m}$. This implies that the sequence in which the control volumes are numbered influences the convergence speed of the iteration method. To improve the convergence speed, the present computational method renumbers the control volumes such that the bandwidth of the system matrix is reduced compared to the one for for a random numbering. The employed renumbering method is the ReverseCuthill-McKee algorithm [144, 145].

As mentioned in section 4.1] parallelization by means of domain decomposition is used to accelerate convergence. In this method, communication across grid partitions is performed after each iteration sweep. This strategy leads to Jacobi iteration on gridpartition boundaries, instead of Gauß-Seidel iteration, which can reduce the effectiveness of parallelization to some extent.

\subsubsection{Pseudo-time Step Size}

As mentioned in subsection 4.4.1 a finite pseudo-time step size is used to increase the range of convergence of the implicit time integration method when necessary. Both the URANS equations and turbulence model equation(s) can use their own pseudo-time step size, according to the corresponding stability requirements.

The pseudo-time stepsize for the URANS equations is set by prescribing a certain CFL number, and evaluating [146]

$$
\Delta \tau_{\mathrm{k}}=\mathrm{CFL} \frac{V_{\mathrm{k}}}{\left(\lambda_{\mathrm{k}}^{c}\right)_{\max }+C\left(\lambda_{\mathrm{k}}^{v}\right)_{\max }},
$$

where $C$ is a constant and $\left(\lambda_{\mathrm{k}}^{c}\right)_{\max }$ and $\left(\lambda_{\mathrm{k}}^{v}\right)_{\max }$ are the spectral radii of the convective and viscous flux Jacobian, respectively. They are defined as

$$
\begin{aligned}
& \left(\lambda_{\mathrm{k}}^{c}\right)_{\max }=\sum_{l \in N_{\mathrm{k}}}\left([\underline{u}]_{\mathrm{kl}} \cdot \underline{n}_{\mathrm{kl}}+\left[c^{*}\right]_{\mathrm{kl}}\right)\left|\underline{S}_{\mathrm{kl}}\right|, \\
& \left(\lambda_{\mathrm{k}}^{v}\right)_{\max }=\frac{\gamma}{\rho_{\mathrm{k}}} \sum_{l \in N_{\mathrm{k}}}\left(\frac{[\mu]_{\mathrm{kl}}}{\operatorname{Pr}}+\frac{\left[\mu_{\mathrm{t}}\right]_{\mathrm{kl}}}{\operatorname{Pr} r_{\mathrm{t}}}\right) \frac{\Delta \underline{x}_{\mathrm{kl}} \cdot \underline{S}_{\mathrm{kl}}}{\left|\Delta \underline{x}_{\mathrm{kl}}\right|^{2}}
\end{aligned}
$$


Mavriplis \& Jameson [146] recommend to use $C=4$.

In a similar way, the pseudo-time stepsize for the SST turbulence model equation is set by prescribing a (different) CFL number, and evaluating

$$
\left(\Delta \tau_{\mathrm{k}}\right)_{S S T}=\mathrm{CFL} \frac{V_{\mathrm{k}}}{\left(\left(\lambda_{\mathrm{t}}^{c}\right)_{\mathrm{k}}\right)_{\max }+C\left(\left(\lambda_{\mathrm{t}}^{v}\right)_{\mathrm{k}}\right)_{\max }+\left(\left(\lambda_{\mathrm{t}}^{s}\right)_{\mathrm{k}}\right)_{\max }},
$$

with $C=4$ and the spectral radii of the convective flux Jacobian, viscous flux Jacobian and source Jacobian given by

$$
\begin{aligned}
& \left(\left(\lambda_{\mathrm{t}}^{c}\right)_{\mathrm{k}}\right)_{\max }=\sum_{l \in N_{\mathrm{k}}}\left([\underline{u}]_{\mathrm{kl}} \cdot \underline{n}_{\mathrm{kl}}\right)\left|\underline{S}_{\mathrm{kl}}\right| \\
& \left(\left(\lambda_{\mathrm{t}}^{v}\right)_{\mathrm{k}}\right)_{\max }=\frac{1}{\rho_{\mathrm{k}}} \sum_{l \in N_{\mathrm{k}}}\left([\mu]_{\mathrm{kl}}+\max \left(\left[\sigma_{k}\right]_{\mathrm{kl}},\left[\sigma_{\omega}\right]_{\mathrm{kl}}\right)\left[\mu_{\mathrm{t}}\right]_{\mathrm{kl}}\right) \frac{\Delta \underline{x}_{\mathrm{kl}} \cdot \underline{S}_{\mathrm{kl}}}{\left|\Delta \underline{x}_{\mathrm{kl}}\right|^{2}} \\
& \left(\left(\lambda_{\mathrm{t}}^{s}\right)_{\mathrm{k}}\right)_{\max }=\left(2 \beta \omega_{\mathrm{k}}+\left|\frac{C D}{\rho \omega}\right|_{\mathrm{k}}\right) V_{\mathrm{k}} .
\end{aligned}
$$

See subsection 4.4.6 for definitions of the terms used here.

Since the SA turbulence model consists of a scalar transport equation, its pseudo-time stepsize can be set by evaluating

$$
\left(\Delta \tau_{\mathrm{k}}\right)_{S A}=\mathrm{CFL} \frac{V_{\mathrm{k}}}{\frac{\partial\left(R_{\mathrm{t}}\right)_{\mathrm{k}}}{\partial\left(U_{\mathrm{t}}\right)_{\mathrm{k}}}}
$$

where $\partial\left(R_{\mathrm{t}}\right)_{\mathrm{k}} / \partial\left(U_{\mathrm{t}}\right)_{\mathrm{k}}$ has been derived in subsection 4.4.6.

\subsection{Convergence Acceleration Techniques}

\subsubsection{Agglomeration-Based Algebraic Multigrid Method Introduction}

Although depending on the flow problem, an often encountered behavior of the Block Symmetric Gauß-Seidel method is that the convergence speed of the residuals of the linear system of equations quickly drops. This effect increases with increasingly finer grids and also negatively affects the convergence speed of the solution procedure for the system of non-linear equations. This behavior is caused by the fact that the iterative solution method is efficiently reducing high-frequency errors in the solution, but not the low-frequency errors [113.

Multigrid methods, initially developed to solve elliptic equations [147], are able to deal with this problem. In a multigrid method, low-frequency errors present in a fine-grid solution (after a few iteration sweeps) are transferred to coarser grids where they effectively become high-frequency errors that are reduced efficiently by the iteration method. On the coarser grids, corrections are calculated and transferred to finer grids, where they are used 
to correct the fine-grid solution. Iteration methods are often called relaxation methods or smoothers if they are used to smooth the error present in the solution.

On structured computational grids, the standard geometric multigrid method can be applied. In this method, coarser grids are constructed by doubling the control-volume sizes in all directions, thereby reducing the grid size and computational effort by a factor of four (2D) or eight (3D) when converting to the next coarser grid. However, combined with standard relaxation techniques, this method shows a breakdown in multigrid efficiency for discrete equations that are highly stiff. For the discretized URANS equations, stiffness is mainly encountered in the presence of anisotropic control volumes in boundary layer regions [148]: aspect ratios can be of the order of $10^{4}$. This problem can be resolved, either by choosing a different relaxation technique (e.g. line relaxation) or by directional or semi-coarsening (coarsening in the direction normal to solid walls) [149].

On unstructured computational grids, the construction of coarser grids is more complicated. Besides using nested grids obtained through mesh refinement (see e.g. [150]) and using non-nested grids (see e.g. [151]), a more practical approach is construction of coarse grids by agglomeration. In this method, coarse-grid control volumes are formed by combining a number of neighboring fine-grid control volumes. This was initially developed by Lallemand et al. [152], and subsequently improved for highly stretched meshes through the introduction of directional coarsening by Mavriplis [149].

In the present computational method, an agglomeration-based algebraic multigrid method has been implemented [153]. This method is particularly suited for problems discretized on unstructured grids, since its algebraic part automatically constructs coarser grids by inspection of the system matrix $M$. The grid is only coarsened in the direction of "strong coupling" in the system matrix, i.e. the direction in which the smoother is effective. This is the direction in which the error is smooth and slow to converge. In boundary layer regions with high-aspect-ratio control volumes, the direction of "strong coupling" is normal to the wall. Therefore, this method automatically deals with the problem of stiff equations and standard Block Symmetric Gauß-Seidel iteration is used for relaxation.

\section{Multigrid Formulation}

The multigrid method is applied to the linearized system of discretized URANS equations for a certain pseudo-time step, see equation (4.125), which will be written in this section as

$$
A^{h} \boldsymbol{u}^{h}=\boldsymbol{f}^{h}, \text { with } h=0 .
$$

Here superscript $h=0$ indicates that this system of equations is discretized on the original (finest) computational grid $\Omega^{0}$. The terms present in equation (4.181) are given by

$$
A^{0}=M^{m}, \quad \boldsymbol{u}^{0}=\Delta \boldsymbol{U}^{m}, \quad \boldsymbol{f}^{0}=-\left(\boldsymbol{R}^{*}\right)^{m},
$$

where the system matrix $M$ contains blocks of size $5 \times 5$ as given in equations (4.171) and (4.172), and $m$ indicates the pseudo-time step level. 
A sequence of coarser grids is constructed, in which each coarser grid is indicated by a higher level index $h$, i.e.

$$
\Omega^{h} \in\left\{\Omega^{0}, \Omega^{1}, \ldots, \Omega^{h_{\max }}\right\},
$$

where the coarsest computational grid is denoted by level index $h_{\max }$. The procedure to construct these coarser grids is discussed in one of the following paragraphs.

A certain number of iteration sweeps, say $\nu_{1}$, are performed on grid level $h$. These are called pre-relaxation sweeps and yield an approximate solution denoted by $\hat{\boldsymbol{u}}^{h}$. The iteration method for grid level $h=0$ is given in subsection 4.5.2, and this method is used on coarser levels as well. The error in the solution is defined as

$$
\boldsymbol{v}^{h}=\boldsymbol{u}^{h}-\hat{\boldsymbol{u}}^{h},
$$

whereas the residual of the system of equations is defined as

$$
\boldsymbol{r}^{h}=\boldsymbol{f}^{h}-A^{h} \hat{\boldsymbol{u}}^{h} \text {. }
$$

Since the exact solution $\boldsymbol{u}^{h}$ satisfies equation (4.181), the relation between the residual and the error reads

$$
A^{h} \boldsymbol{v}^{h}=\boldsymbol{r}^{h} .
$$

Equation (4.186) is called the 'defect equation'.

Assuming the iteration method has effectively reduced the high-frequency error of the solution, $\boldsymbol{v}^{h}$ is smooth and can therefore be accurately represented on a coarser grid. The equation to be solved on the coarser grid reads

$$
A^{h+1} \boldsymbol{v}^{h+1}=I_{h}^{h+1} \boldsymbol{r}^{h},
$$

where $A^{h+1}$ and $\boldsymbol{v}^{h+1}$ are coarse grid approximations to $A^{h}$ and $\boldsymbol{v}^{h}$, respectively. Furthermore, $I_{h}^{h+1}$ is a restriction operator that transfers the residual $\boldsymbol{r}^{h}$ from grid level $h$ to grid level $(h+1)$.

After the solution of $\boldsymbol{v}^{h+1}$ is found, it is transferred to grid level $h$ and subsequently used to find a better approximation to $\boldsymbol{u}^{h}$ :

$$
\hat{\boldsymbol{u}}^{h} \leftarrow \hat{\boldsymbol{u}}^{h}+I_{h+1}^{h} \boldsymbol{v}^{h+1},
$$

where $I_{h+1}^{h}$ is a prolongation operator that transfers the correction from grid level $(h+1)$ to grid level $h$. In order to remove possible high-frequency errors introduced by this prolongation, $\nu_{2}$ post-relaxation sweeps are performed on level $h$.

The solution $\boldsymbol{v}^{h+1}$ is found by recursively applying the procedure above, except on grid level $h_{\max }$, for which the iteration method is computationally cheap and can therefore be continued until the residual $\boldsymbol{r}^{h_{\max }}$ is of the order of machine accuracy (or until a pre-defined maximum number of iteration sweeps has been performed). The standard way in which the grid levels are visited leads to a so-called $\mathrm{V}$-cycle, denoted by $\mathrm{V}\left(\nu_{1}, \nu_{2}\right)$. Figure 4.3 shows a flow diagram of a $\mathrm{V}$-cycle on a sequence of four grids. However, other patterns are also possible, such as the $\mathrm{W}\left(\nu_{1}, \nu_{2}\right)$ cycle or $\mathrm{F}\left(\nu_{1}, \nu_{2}\right)$ cycle, see figures 4.4 and 4.5 respectively. 


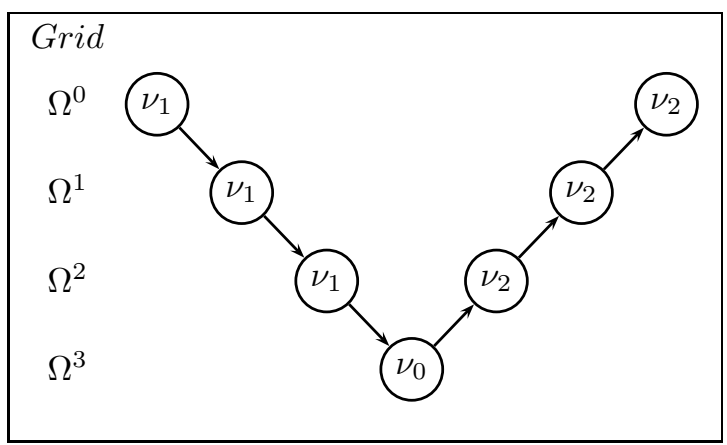

Figure 4.3: $V\left(\nu_{1}, \nu_{2}\right)$ cycle on a sequence of four grids.

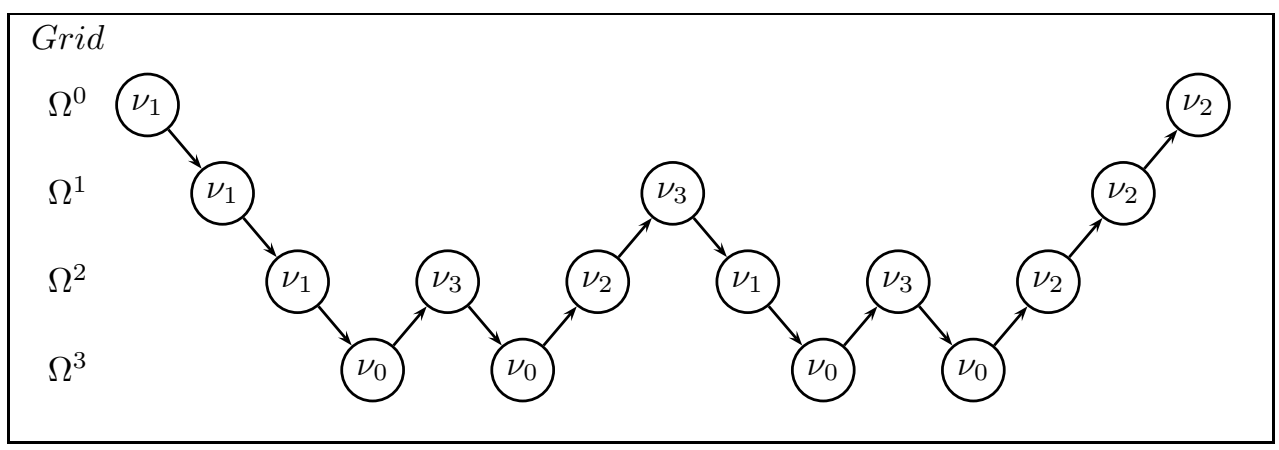

Figure 4.4: $W\left(\nu_{1}, \nu_{2}\right)$ cycle on a sequence of four grids; $\nu_{3}=\nu_{2}+\nu_{1}$.

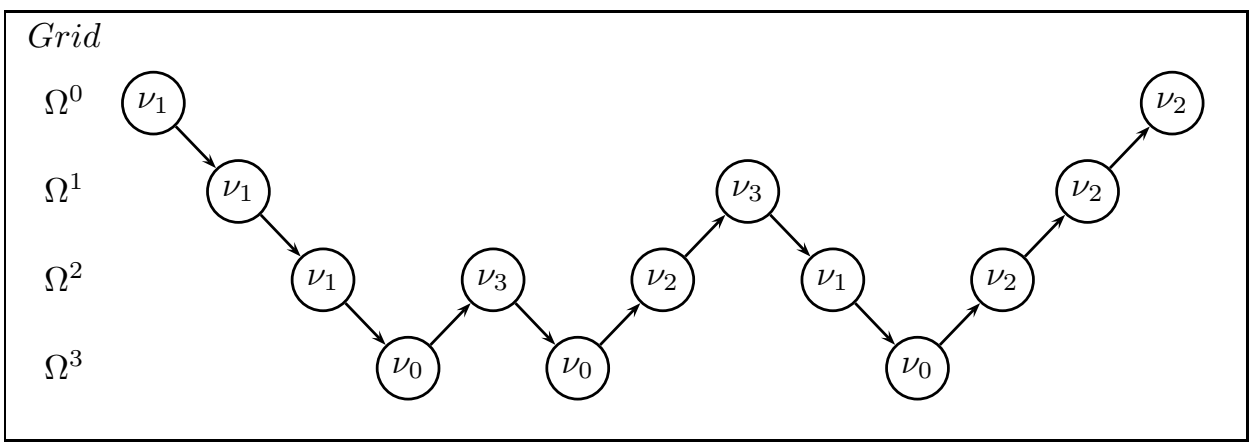

Figure 4.5: $F\left(\nu_{1}, \nu_{2}\right)$ cycle on a sequence of four grids; $\nu_{3}=\nu_{2}+\nu_{1}$. 
The recursive multigrid procedure can be summarized as [113]:

Multigrid Cycle: $\hat{\boldsymbol{u}}^{h} \leftarrow M G C Y C\left(h, c_{t p}, \hat{\boldsymbol{u}}^{h}, A^{h}, \boldsymbol{f}^{h}, \nu_{1}, \nu_{2}\right)$

1. Pre-relaxation: perform $\nu_{1}$ relaxation sweeps

$$
\hat{\boldsymbol{u}}^{h} \leftarrow R E L A X^{\nu_{1}}\left(\hat{\boldsymbol{u}}^{h}, A^{h}, \boldsymbol{f}^{h}\right)
$$

2. Coarse grid correction:

a. Compute the residual $\boldsymbol{r}^{h}=f^{h}-A^{h} \boldsymbol{u}^{h}$

b. Restrict the residual (compute coarse grid r.h.s.) $\boldsymbol{f}^{h+1}=I_{h}^{h+1} \boldsymbol{r}^{h}$

c. Compute an approximate solution of the defect equation on $\Omega^{h+1}$ :

- If $h<h_{\max }$ :

- Recursive call to Multigrid Cycle:

$$
\boldsymbol{v}^{h+1} \leftarrow M G C Y C\left(h+1, c_{t p}, 0, A^{h+1}, \boldsymbol{f}^{h+1}, \nu_{1}, \nu_{2}\right)
$$

- If $\left(c_{t p}=2\right)$ : second call to Multigrid Cycle for W-cycle, i.e.

$$
\boldsymbol{v}^{h+1} \leftarrow M G C Y C\left(h+1, c_{t p}, \boldsymbol{v}^{h+1}, A^{h+1}, \boldsymbol{f}^{h+1}, \nu_{1}, \nu_{2}\right)
$$

- If $\left(c_{t p}=3\right)$ : second call to Multigrid Cycle for F-cycle with $c_{t p}=1$, i.e.

$$
\boldsymbol{v}^{h+1} \leftarrow M G C Y C\left(h+1,1, \boldsymbol{v}^{h+1}, A^{h+1}, \boldsymbol{f}^{h+1}, \nu_{1}, \nu_{2}\right)
$$

- Else: solve $A^{h_{\max }} \boldsymbol{v}^{h_{\max }}=\boldsymbol{f}^{h_{\max }}$ by performing at most $\nu_{\max }$ relaxation sweeps, i.e.

$$
\boldsymbol{v}^{h_{\max }} \leftarrow R E L A X^{\nu_{0} \leq \nu_{\max }}\left(0, A^{h_{\max }}, \boldsymbol{f}^{h_{\max }}\right)
$$

d. Prolongation: $\boldsymbol{v}^{h}=I_{h+1}^{h} \boldsymbol{v}^{h+1}$

e. Smooth $\boldsymbol{v}^{h}$ if necessary

f. Correction: $\hat{\boldsymbol{u}}^{h} \leftarrow \hat{\boldsymbol{u}}^{h}+\boldsymbol{v}^{h}$

3. Post-relaxation: perform $\nu_{2}$ relaxation sweeps

$$
\hat{\boldsymbol{u}}^{h} \leftarrow R E L A X^{\nu_{2}}\left(\hat{\boldsymbol{u}}^{h}, A^{h}, \boldsymbol{f}^{h}\right)
$$

The different cycle types are defined by the value of $c_{t p}$. For a $\mathrm{V}$-cycle: $c_{t p}=1$, for a W-cycle: $c_{t p}=2$, and for an F-cycle: $c_{t p}=3$. 


\section{Coarse Grid Operator}

The coarse-grid operator $A^{h+1}$ is constructed using the Galerkin approach, which prevents the need to re-discretize the URANS equations on coarser grids. In this approach, we require the residual associated with the corrected fine-grid solution to be zero when it is transferred back to a coarser grid [154]:

$$
I_{h}^{h+1}\left(f^{h}-A^{h} \hat{\boldsymbol{u}}^{h}\right)=0,
$$

where $\hat{\boldsymbol{u}}^{h}$ is the corrected fine-grid solution given in equation (4.188). Substitution of equation (4.188) in equation (4.195) yields

$$
\begin{aligned}
I_{h}^{h+1}\left(f^{h}-A^{h}\left(\hat{\boldsymbol{u}}^{h}+I_{h+1}^{h} \boldsymbol{v}^{h+1}\right)\right) & =0 \\
& \Leftrightarrow \\
I_{h}^{h+1} A^{h} I_{h+1}^{h} \boldsymbol{v}^{h+1} & =I_{h}^{h+1}\left(f^{h}-A^{h} \hat{\boldsymbol{u}}^{h}\right) .
\end{aligned}
$$

Since equation (4.196) corresponds to equation (4.187), the coarse-grid operator is defined as

$$
A^{h+1} \equiv I_{h}^{h+1} A^{h} I_{h+1}^{h}, \quad h=\left\{0,1, \ldots, h_{\max }-1\right\} .
$$

This means that the operators on all coarse grid levels depend on the system matrix of the original finest grid.

\section{Restriction and Prolongation Operators}

Since the coarse-grid operators have to be constructed for each pseudo-time step, simple and fast construction of the coarse grid operators is needed. In the present computational method, this is obtained by

- coarsening all five coupled equations per control volume in the same way, i.e. a collective approach;

- using piecewise constant restriction and prolongation operators.

For example, if grid level $h$ consists of 4 control volumes and control volumes $1+2$ and $3+4$ on level $h$ are to be combined into control volumes 1 and 2 on level $(h+1)$, respectively, then

$$
I_{h}^{h+1}=\left[\begin{array}{llll}
I & I & 0 & 0 \\
0 & 0 & I & I
\end{array}\right],
$$

and

$$
I_{h+1}^{h}=\left(I_{h}^{h+1}\right)^{T},
$$

where $I$ is the $5 \times 5$ identity operator. This simple example shows that piecewise constant restriction of the residual to a coarser grid implies summation of the residuals of the 
fine-level control volumes that are joined into a coarse-level control volume. Since the residual on level 0 is given by $\boldsymbol{r}^{0}=-\left(\boldsymbol{R}^{*}\right)^{m}-M^{m} \Delta \boldsymbol{U}^{m}$, this appears to be the natural choice because the residual partly consists of a sum of fluxes over control volume faces. Summing the fine-grid residuals results in a sum of fluxes over faces of the corresponding new coarse grid control volume, since the internal fluxes cancel, see equations (4.10) and (4.11). Furthermore, piecewise constant prolongation yields injection of the correction calculated in a coarse-level control volume into the corresponding agglomerated fine-level control volumes.

The Galerkin coarse-grid operator can now be written as

$$
I_{h}^{h+1} A^{h} I_{h+1}^{h}=\left[A_{\mathrm{kl}}^{h+1}\right],
$$

where $k$ and $l$ denote all coarse grid control volumes on level $(h+1)$ and $A_{\mathrm{kl}}^{h+1}$ is a $5 \times 5$ matrix given by

$$
A_{\mathrm{kl}}^{h+1}=\sum_{i \in G_{k}} \sum_{j \in G_{l}} A_{\mathrm{ij}}^{h},
$$

where $G_{k}$ and $G_{l}$ are the groups of agglomerated control volumes on level $h$ that form the control volumes $k$ and $l$, respectively. Equation (4.201) shows that the matrix $A_{\mathrm{kl}}^{h+1}$ consists of a sum of all the crosscouplings between $G_{k}$ and $G_{l}$.

\section{Improvements to the Basic Approach}

In general, these transfer operators do not lead to very efficient multigrid cycles [155, 113]. The transfer operators do not satisfy the general rule-of-thumb that the order of the restriction of the $\mathrm{i}$-th equation, $m_{i}$, plus the order of the interpolation of the corrections of the j-th unknown, $m^{j}$, should be higher than the highest order of differentiation of the $\mathrm{j}$-th unknown in the i-th equation, $m_{i j}$, [154]:

$$
m_{i}+m^{j}>m_{i j}
$$

In the present case $m_{i}=1$ and $m^{j}=1$, whereas $m_{i j}$ can be equal to $m_{i j}=2$ for the Reynolds-averaged momentum and energy equations. This means that the multigrid cycle can amplify high-frequency errors in regions where viscous flow effects dominate and extra relaxation sweeps are needed to reduce these high frequencies.

Furthermore, it can be shown that for a partial differential equation with second-order derivative terms, the method does not reproduce a coarse-grid operator obtained by rediscretization for the terms that correspond to the second-order derivative terms, see for example [156, 157]. This means that the coarse-grid equations are not entirely consistent with the fine-grid equations.

Instead of increasing the order of restriction and/or prolongation, it is chosen to keep the simple piecewise constant transfer operators. This is because the simple approach preserves the nearest-neighbor stencil and the diagonal dominance of the operator on coarser grids [157], and yields fast construction of the coarse-grid operators, which is important if the residual reduction per pseudo-time step only needs to be one order of 
magnitude. However, some simple improvements can be introduced that do not solve the problems related to the low-order transfer operators but will improve the results without loosing the favorable properties mentioned above.

The first improvement is to smooth the prolongated correction before it is used to correct the fine-grid solution, see item 2(e) of the Multigrid Cycle on page 116. The smoothing consists of averaging the correction with its nearest neighbors [158]. This does not increase the order of prolongation, but is does improve the results [113, Appendix A.6]. However, the smoothing is not incorporated in the coarse-grid operators, as is the case in the "smoothed aggregation" approach [159].

The second improvement that addresses the consistency of the equations on the coarser grids is to multiply those terms in the operator on level $(h+1)$ that correspond to summations of the viscous flux Jacobian matrices from level $h$ by a constant factor. Mavriplis \& Venkatakrishnan [157] use a factor of $\frac{1}{2}$ based on examination of a one-dimensional Poisson problem with a coarsening ratio of two (two neigboring control volumes are agglomerated). However, we use a factor of $\frac{1}{1.8}$, which appears to be more robust for more general problems and non-uniform coarsening [160]. Note that this strategy implies that the contribution from the viscous fluxes to the flux Jacobian matrix must be stored in memory separately from the other contributions.

\section{Agglomeration Procedure}

A control volume on level $h+1$ consists of a number of strongly coupled neighboring control volumes from level $h$. Between strongly coupled control volumes, the error is smooth and slow to converge, and can therefore be accurately represented on the coarser level. For scalar problems, the criterion used to define a strong connection is that the magnitude of the off-diagonal coefficient $a_{\mathrm{ij}}$ is large with respect to the diagonal coefficients $a_{\mathrm{ii}}$ and $a_{\mathrm{jj}}$ :

$$
\frac{a_{\mathrm{ij}}^{2}}{a_{\mathrm{ii}} a_{\mathrm{jj}}}>\text { const. }
$$

However, for systems of equations, we are not dealing with the magnitude of coefficients but with a measure of the 'magnitude' of block matrices. Weiss et al. [153] use the first matrix element of the mass conservation equation as a measure of the magnitude. However, since this equation does not have a viscous flux term, we determine the magnitude based on the fifth matrix element of the energy equation instead. This matrix element contains contributions from a convective flux as well as a viscous flux. The criterion for a strong connection can therefore be written as

$$
\mathcal{S} t_{\mathrm{ij}}=\frac{\left(a_{5,5}\right)_{\mathrm{ij}}^{2}}{\left(a_{5,5}\right)_{\mathrm{ii}}\left(a_{5,5}\right)_{\mathrm{jj}}}>\epsilon_{\mathrm{i}},
$$

where we have chosen to define the threshold $\epsilon_{i}$ as

$$
\epsilon_{\mathrm{i}}=\frac{1}{2} \max _{j}\left(\mathcal{S} t_{\mathrm{ij}}\right)
$$


Note that this criterion always yields at least one strong connection for each control volume.

In the coarsening procedure, a preference is given to agglomerate un-agglomerated control volumes, as in the similar coarsening procedure used by Weiss et al. [153]. The procedure can be summarized as:

Coarsening Procedure: $\left(\Omega^{h+1}, A^{h+1}\right) \leftarrow C P\left(\Omega^{h}, A^{h}\right)$

1. For all control volumes on level $h$, do

- If the current control volume $i$ is not already agglomerated:

- Calculate $\mathcal{S} t_{\mathrm{ij}}$ for all nearest neighbors $j$ of $i$

- If un-agglomerated neighbors for which $\mathcal{S} t_{\mathrm{ij}}>\epsilon_{\mathrm{i}}$ exist:

create a new coarse-grid control volume on level $(h+1)$ by agglomerating $i$ with these neighbors

- Else if agglomerated neighbors for which $\mathcal{S} t_{\mathrm{ij}}>\epsilon_{\mathrm{i}}$ exist:

add $i$ to the existing coarse-grid control volume on level $(h+1)$ that contains the strongest agglomerated neighbor

- Else (no neighbors available7):

- Put $i$ in a new coarse-grid control volume on level $(h+1)$

2. Determine the connectivity on level $(h+1)$

3. Calculate the coarse-grid operator $A^{h+1}$

The coarsening procedure generally yields a coarsening ratio between 2:1 and 3:1 in two-dimensional problems with anisotropic control volumes. The procedure is used repeatedly to construct a sequence of coarser grids until the pre-defined level $h_{\max }$ has been constructed or until the last constructed coarser grid has less than 25 control volumes. If the flow solution does not change dramatically between pseudo-time steps, time can be saved by reusing the known sequence of grids. In the present computational grid, the same sequence of grids is used for a pre-defined interval of pseudo-time steps. However, the coarse-grid operators still have to be recalculated at each pseudo-time step.

\section{Treatment of Boundary Conditions}

On the original finest grid, $\Omega^{0}$, relaxation of the equations in the interior of the computational domain also yields updates of the solution in ghost control volumes outside the computational domain, either implicitly or explicitly, see section 4.7. These updates are used to calculate the residual on level 0 , which is subsequently transferred to level 1 , where it becomes the right-hand side of the coarse-grid equation, see equation 4.187.

\footnotetext{
${ }^{7}$ This possibility can present itself when the multigrid method is applied to non-contiguous grid partitions.
} 
The boundary conditions that are implicitly treated are automatically transferred to coarser grids. However, the boundary conditions that are explicitly treated are not updated on coarser grids. For these boundaries, the error $\boldsymbol{v}_{\text {ghost }}^{h>0}$ for the ghost control volumes is assumed to be zero. Once the multigrid cycle returns to level 0 , the solution in the interior is corrected and relaxed and the boundary conditions are updated again.

\subsubsection{Domain Decomposition}

As outlined in section 4.1 the computational method is parallelized by means of domain decomposition. This involves the following steps:

- Grid partitioning: the grid is divided into a number of partitions with a single layer of control volumes overlapping with neighboring partitions, see figure 4.6. Partitioning is carried out in such a way that the workload per partition is approximately balanced and that the amount of communication between the partitions is minimized as much as possible (multi-constraint partitioning). Minimization of communication is achieved by from minimizing the number of overlapping control volumes. The task of grid partitioning is carried out by METIS routines [118.

- Each grid partition is assigned to a different computer processor and the computational method is applied to each partition. Inter-processor communication is needed to obtain the necessary information in the overlapping control volumes. The communicated information includes for example the update of the conserved variables after each iteration sweep and the gradients of some flow and turbulence variables, which are needed to compute the viscous fluxes. This task is implemented using Message Passing Interface (MPI) routines [119].

It is possible to apply the multigrid method across partition boundaries, and let the coarsest grid problems be solved by only one processor, see for example [161]. However, due to time constraints, it was decided to apply the multigrid method separately to each partition. Therefore, partitioning across strongly coupled control volumes must be avoided. This is accomplished by adding a penalty to the partitioning that involves separating two control volumes of which the cell centers are much closer together than the maximum local distance between cell centers. The smaller the relative distance, the higher the penalty. This results in partitioning in the direction normal to no-slip walls where the control volumes are highly stretched in the direction along the wall. Note that the addition of penalties makes it more difficult to find an optimal load balancing per processor with a minimal amount of necessary inter-processor communication. In general, one must accept a higher degree of imbalance.

Since the update of the solution in the overlapping control volumes is communicated after each iteration sweep, these control volumes are effectively treated by the multigrid method as boundaries with explicitly treated boundary conditions, see subsection 4.6.1. 


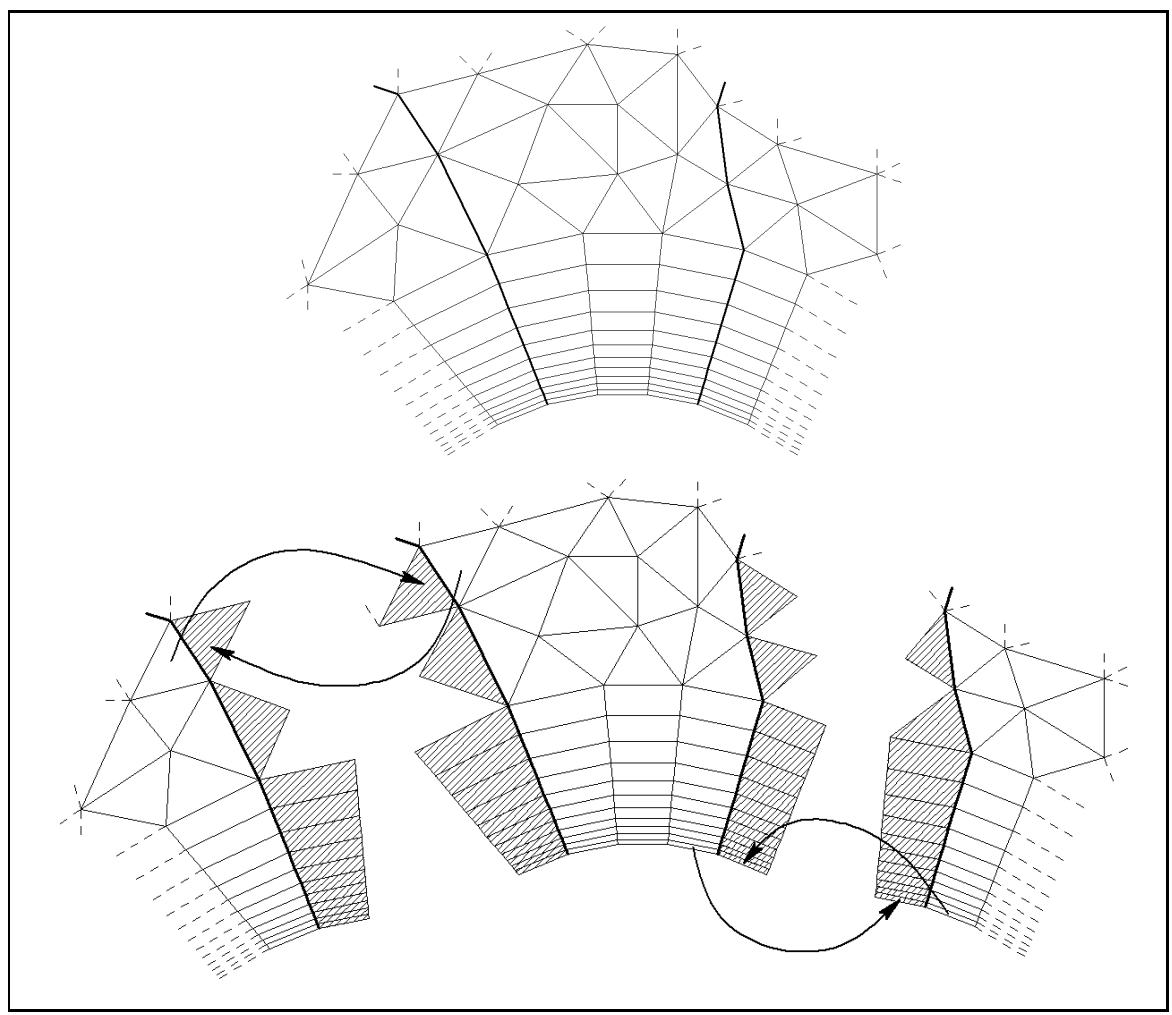

Figure 4.6: Part of a cell-centered, hybrid computational grid with thick lines indicating partition boundaries. Overlapping control volumes for each partition are cross-hatched and arrows indicate examples of communication paths (bottom).

\subsection{Numerical Treatment of Boundary Conditions}

The boundary conditions are satisfied by using one layer of ghost control volumes, i.e. virtual control volumes located outside the computational domain. Each boundary face is therefore associated with a control volume inside the computational domain and a ghost control volume, see figure 4.7. The center of gravity of a ghost control volume is determined by mirroring the center of gravity of the corresponding internal control volume in the boundary face.

The combined convective and viscous flux of the URANS or turbulence equations at a boundary face, say $\boldsymbol{\Phi}_{\mathrm{kl}}$, with internal control volume $k$ and ghost control volume $l$, is implicitly made to satisfy the boundary condition by setting the conserved variables $\boldsymbol{U}_{\mathrm{l}}$ to appropriate values. In the following, these variables are called 'ghost variables', whereas the conserved variables in the corresponding internal control volume, $\boldsymbol{U}_{\mathrm{k}}$, are called 'internal variables'. When a boundary condition prescribes a certain variable at the 


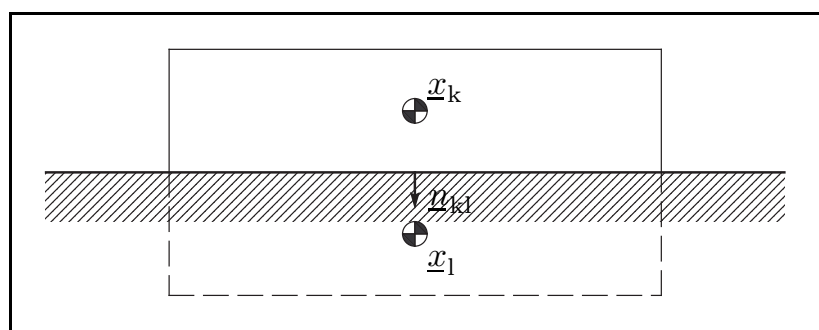

Figure 4.7: Control volume $k$ at a solid boundary together with ghost control volume l, their centroids and the outward normal vector of the face.

boundary, say $\phi_{b}$, the ghost variable $\phi_{1}$ is set to

$$
\phi_{\mathrm{l}}=2 \phi_{b}-\phi_{\mathrm{k}}
$$

Furthermore, when a boundary condition prescribes the gradient of $\phi$ in the direction normal to the boundary, say $\left(\frac{\partial \phi}{\partial n}\right)_{b}$, the ghost variable reads

$$
\phi_{\mathrm{l}}=\left(\frac{\partial \phi}{\partial n}\right)_{b} \frac{\left|\Delta \underline{x}_{\mathrm{kl}}\right|^{2}}{\Delta \underline{x}_{\mathrm{kl}} \cdot \underline{n}_{\mathrm{kl}}}+\phi_{\mathrm{k}},
$$

with $\Delta \underline{x}_{\mathrm{kl}}=\underline{x}_{1}-\underline{x}_{\mathrm{k}}$, see figure 4.7

If a governing equation does not need a certain variable or its gradient to be specified at a boundary, the value of this variable at the boundary follows from the solution of the equation. For the computational method it is necessary to have a value of all variables in the ghost control volumes. Therefore, the unspecified variables are extrapolated from the internal control volumes to the ghost control volumes.

In the implicit solution method, boundary conditions are either treated explicitly or implicitly. In the implicit treatment, the ghost variables are written as an explicit function of the internal variables. The system of linearized equations for the internal control volume contains the product of the flux Jacobians at the boundary with the update of the ghost variables, which can be replaced by an equivalent product given by

$$
\frac{\partial \boldsymbol{\Phi}_{\mathrm{kl}}}{\partial \boldsymbol{U}_{\mathrm{l}}} \Delta \boldsymbol{U}_{\mathrm{l}}=\frac{\partial \boldsymbol{\Phi}_{\mathrm{kl}}}{\partial \boldsymbol{U}_{\mathrm{l}}} \frac{\partial \boldsymbol{U}_{\mathrm{l}}}{\partial \boldsymbol{U}_{\mathrm{k}}} \Delta \boldsymbol{U}_{\mathrm{k}}
$$

where $\partial \boldsymbol{U}_{\mathrm{l}} / \partial \boldsymbol{U}_{\mathrm{k}}$ follows from equation (4.206) and/or equation (4.207). This means that the influence of the ghost control volume is transferred from the off-diagonal block matrix $M_{\mathrm{kl}}$ to the diagonal block matrix $M_{\mathrm{kk}}$ in the system matrix.

However, not all boundary conditions have conserved variables in the ghost control volume that can easily be written as an explicit function of the conserved variables in the corresponding internal control volume. These boundary conditions are treated explicitly, which means that the ghost variables, $\boldsymbol{U}_{1}$, are updated after each iteration sweep of the 
system of linearized equations, yielding a new $\Delta \boldsymbol{U}_{\mathrm{l}}$, which is is subsequently used in the next iteration sweep.

In the present computational method, only the no-slip wall and symmetry boundary conditions for the URANS equations are treated implicitly.

In the next subsections, the numerical treatment of the different boundary conditions is described. Each numerical boundary condition is given in terms of the conserved variables in the ghost control volume for the URANS equations, $\boldsymbol{U}=\left[\rho, \rho u_{1}, \rho u_{2}, \rho u_{3}, \rho E\right]^{T}$, the SA turbulence model, $\left(U_{\mathrm{t}}\right)_{S A}=\tilde{\nu}$, and SST turbulence model, $\left(\boldsymbol{U}_{\mathrm{t}}\right)_{S S T}=[\rho k, \rho \omega]^{T}$. Note that when the SA turbulence model is used, any occurrences of the turbulence kinetic energy in the numerical boundary conditions for the URANS equations should be ignored.

\subsubsection{Physical Boundary: Adiabatic Solid Wall}

The boundary conditions for an adiabatic solid wall needed for the URANS and turbulence model equations have been presented in section 3.5. They only involve specifying the velocity at the wall to be equal to zero, the gradient of the internal energy normal to the wall to be equal to zero and the eddy viscosity at the wall to be equal to zero $(\tilde{\nu}=0$ for the SA turbulence model and $k=0$ for the SST turbulence model). The remaining variables follow from the solution and must be extrapolated to the ghost control volume.

For the extrapolation of the density and thus the thermodynamic pressure, it is allowed to use a zero-gradient normal to the wall, since the resolution of the computational grid in the direction normal to the wall is very high in order to capture the boundary layer. Moreover, from boundary layer theory [162], it is known that the pressure is approximately constant across a boundary layer.

Wilcox [90] notes that in the vicinity very close to a smooth wall, $\omega$ behaves as

$$
\omega \rightarrow \frac{6 \mu}{\rho \beta d^{2}} \text { for } d \rightarrow 0,
$$

where $d$ is the distance normal to the wall. Since this relation has a singularity at the wall, Menter [91] approximates this relation by

$$
\omega_{b}=10 \frac{6 \mu_{\mathrm{k}}}{\rho_{\mathrm{k}} \beta_{\mathrm{k}}\left(d_{\mathrm{k}}\right)^{2}},
$$

where $d_{\mathrm{k}}$ is the distance from the wall to the centroid of the inner control volume. Menter [91] notes that the results are not sensitive to the factor of $10, \omega$ just needs to be sufficiently large near the wall.

The conserved variables in a ghost control volume at a physical boundary can now be derived by employing the relations given in equations (4.206) and (4.207), which yields

$$
\boldsymbol{U}_{\mathrm{l}}=\left[\begin{array}{c}
U_{1} \\
-U_{2} \\
-U_{3} \\
-U_{4} \\
U_{5}-2\left(U_{\mathrm{t} 1}\right)_{S S T}
\end{array}\right]_{\mathrm{k}},
$$


for the URANS equations and

$$
\left[\left(\boldsymbol{U}_{\mathrm{t}}\right)_{S S T}\right]_{\mathrm{l}}=\left[\begin{array}{c}
-\left(U_{\mathrm{t} 1}\right)_{S S T} \\
20 \frac{6 \mu}{\beta d^{2}}-\left(U_{\mathrm{t} 2}\right)_{S S T}
\end{array}\right]_{\mathrm{k}}, \quad\left[\left(U_{\mathrm{t}}\right)_{S A}\right]_{\mathrm{l}}=-\left[\left(U_{\mathrm{t}}\right)_{S A}\right]_{\mathrm{k}},
$$

for the SST and SA turbulence models, respectively.

\subsubsection{Artificial Boundaries}

\section{Symmetry Boundary}

The symmetry boundary is an artificial boundary for which the associated boundary conditions have been presented in section 3.5. It involves specifying the velocity component normal to the boundary to be equal to zero. Also the component of the gradients normal to the boundary of all other variables is specified to be equal to zero. The velocity component normal to the boundary reads

$$
\underline{u}_{b} \cdot \underline{n}_{\mathrm{kl}}=\frac{1}{2}\left(\underline{u}_{1} \cdot \underline{n}_{\mathrm{kl}}+\underline{u}_{\mathrm{k}} \cdot \underline{n}_{\mathrm{kl}}\right)=0
$$

which yields a velocity component normal to the boundary in the ghost control volume equal to

$$
\underline{u}_{\mathrm{l}} \cdot \underline{n}_{\mathrm{kl}}=-\underline{u}_{\mathrm{k}} \cdot \underline{n}_{\mathrm{kl}} \cdot
$$

The gradient normal to the boundary of the remaining velocity components is zero. Application of equation (4.207) then yields

$$
\underline{u}_{1}-\left(\underline{u}_{1} \cdot \underline{n}_{\mathrm{kl}}\right) \underline{n}_{\mathrm{kl}}=\underline{u}_{\mathrm{k}}-\left(\underline{u}_{\mathrm{k}} \cdot \underline{n}_{\mathrm{kl}}\right) \underline{n}_{\mathrm{kl}} \cdot
$$

Combining equations (4.213) and (4.215) yields the following relation for the velocity in the ghost control volume:

$$
\underline{u}_{1}=\underline{u}_{\mathrm{k}}-2\left(\underline{u}_{\mathrm{k}} \cdot \underline{n}_{\mathrm{kl}}\right) \underline{n}_{\mathrm{kl}} \cdot
$$

The conserved variables in a ghost control volume at a symmetry boundary can now be written as

$$
\boldsymbol{U}_{1}=\left[\begin{array}{c}
U_{1} \\
U_{2}-2\left(\left(U_{2}, U_{3}, U_{4}\right) \cdot \underline{n}_{\mathrm{kl}}\right)\left(n_{1}\right)_{\mathrm{kl}} \\
U_{3}-2\left(\left(U_{2}, U_{3}, U_{4}\right) \cdot \underline{n}_{\mathrm{kl}}\right)\left(n_{2}\right)_{\mathrm{kl}} \\
U_{4}-2\left(\left(U_{2}, U_{3}, U_{4}\right) \cdot \underline{n}_{\mathrm{kl}}\right)\left(n_{3}\right)_{\mathrm{kl}} \\
U_{5}
\end{array}\right]_{\mathrm{k}}
$$

for the URANS equations and

$$
\left[\left(\boldsymbol{U}_{\mathrm{t}}\right)_{S S T}\right]_{\mathrm{l}}=\left[\left(\boldsymbol{U}_{\mathrm{t}}\right)_{S S T}\right]_{\mathrm{k}}, \quad\left[\left(U_{\mathrm{t}}\right)_{S A}\right]_{\mathrm{l}}=\left[\left(U_{\mathrm{t}}\right)_{S A}\right]_{\mathrm{k}},
$$

for the SST and SA turbulence models, respectively. 


\section{Subsonic Inlet Boundary}

For an artificial boundary at which the flow is subsonically entering the computational domain, the analysis in appendix A shows that one wave associated with the URANS equations leaves the domain, whereas four waves associated with the URANS equations plus two waves associated with the SST turbulence model (or one wave associated with the SA turbulence model) enter the domain. Therefore, for the URANS equations we can specify four variables and have to extrapolate one variable. For the turbulence model equations, all variables have to be specified, i.e.

$$
\begin{aligned}
{\left[\left(\boldsymbol{U}_{\mathrm{t}}\right)_{S S T}\right]_{\mathrm{l}} } & =2\left[\left(\boldsymbol{U}_{\mathrm{t}}\right)_{S S T}\right]_{b}-\left[\left(\boldsymbol{U}_{\mathrm{t}}\right)_{S S T}\right]_{\mathrm{k}}, \\
{\left[\left(U_{\mathrm{t}}\right)_{S A}\right]_{\mathrm{l}} } & =2\left[\left(U_{\mathrm{t}}\right)_{S A}\right]_{b}-\left[\left(U_{\mathrm{t}}\right)_{S A}\right]_{\mathrm{k}} .
\end{aligned}
$$

In the present computational method, three possible solutions for the specification of the variables of the URANS equations at a subsonic inlet have been implemented. They are:

1. Fixed mass flow with extrapolated pressure

This option specifies the density, $\rho_{b}$ and velocity, $\underline{u}_{b}$, and uses zeroth-order extrapolation of the pressure, $p$. Using equations (4.206) and (4.207) yields the following relation for the conserved variables of the URANS equations in the ghost control volume:

$$
\begin{aligned}
& \boldsymbol{U}_{1}=2\left[\begin{array}{c}
U_{1} \\
U_{2} \\
U_{3} \\
U_{4} \\
\frac{1}{2} \frac{U_{2}^{2}+U_{3}^{2}+U_{4}^{2}}{U_{1}}+\left(U_{\mathrm{t} 1}\right)_{S S T}
\end{array}\right]_{b} \\
& U_{1} \\
& U_{2} \\
& U_{3} \\
& U_{4} \\
&-\left[\begin{array}{c}
\left.\left.U_{\mathrm{t} 1}\right)_{S S T}\right) \\
U_{5}-2\left(\frac{1}{2} \frac{U_{2}^{2}+U_{3}^{2}+U_{4}^{2}}{U_{1}}+\left(U_{\mathrm{k}}\right.\right.
\end{array}\right.
\end{aligned}
$$

2. Fixed total pressure, total temperature and two flow angles with extrapolated

Mach number

This option specifies the total pressure and total temperature, given for a calorically perfect gas by

$$
\begin{aligned}
& p_{t}=p\left(1+\frac{\gamma-1}{2} \mathrm{M}^{2}\right)^{\frac{\gamma}{\gamma-1}}, \\
& T_{t}=T\left(1+\frac{\gamma-1}{2} \mathrm{M}^{2}\right),
\end{aligned}
$$


at the boundary. Furthermore, two flow angles are specified, e.g. by specifying the ratios $\left(u_{2} / u_{1}\right)_{b}$ and $\left(u_{3} / u_{1}\right)_{b}$. The Mach number, $\mathrm{M}=|\underline{u}| / c^{*}$, is extrapolated using zeroth-order extrapolation, i.e.

$$
\mathrm{M}_{b}=\mathrm{M}_{\mathrm{k}}
$$

The pressure at the boundary, $p_{b}$, follows from the definition of total pressure:

$$
p_{b}=\left(p_{t}\right)_{b}\left(1+\frac{\gamma-1}{2} \mathrm{M}_{\mathrm{k}}^{2}\right)^{\frac{-\gamma}{\gamma-1}},
$$

whereas the density at the boundary, $\rho_{b}$, follows from the ideal gas law, see equation (3.18) and the definitions of total pressure $p_{t}$ and total temperature $T_{t}$ :

$$
\rho_{b}=\frac{p_{b}}{R T_{b}}=\frac{\left(p_{t}\right)_{b}}{R\left(T_{t}\right)_{b}}\left(1+\frac{\gamma-1}{2} \mathrm{M}_{\mathrm{k}}^{2}\right)^{\frac{-1}{\gamma-1}} .
$$

From $p_{b}, \rho_{b}$ and the specified turbulence kinetic energy, $k_{b}=\left[\left(U_{\mathrm{t} 1}\right)_{S S T}\right]_{b} / \rho_{b}$, the modified speed of sound is calculated, i.e.

$$
c_{b}^{*}=\sqrt{\frac{p_{b}+\frac{2}{3} \rho_{b} k_{b}}{\gamma \rho_{b}}},
$$

which is subsequently used to calculate the velocity components:

$$
\begin{aligned}
\left(u_{1}\right)_{b} & =\frac{c_{b}^{*} \mathrm{M}_{\mathrm{k}}}{\sqrt{1+\left(\frac{u_{2}}{u_{1}}\right)_{b}^{2}+\left(\frac{u_{3}}{u_{1}}\right)_{b}^{2}}} \\
\left(u_{2}\right)_{b} & =\left(\frac{u_{2}}{u_{1}}\right)_{b}\left(u_{1}\right)_{b} \\
\left(u_{3}\right)_{b} & =\left(\frac{u_{3}}{u_{1}}\right)_{b}\left(u_{1}\right)_{b} .
\end{aligned}
$$

The conserved variables at the boundary follow from the above computed and specified primitive variables:

$$
\boldsymbol{U}_{b}=\left[\begin{array}{c}
\rho_{b} \\
\rho_{b}\left(u_{1}\right)_{b} \\
\rho_{b}\left(u_{2}\right)_{b} \\
\rho_{b}\left(u_{3}\right)_{b} \\
\frac{p_{b}}{\gamma-1}+\frac{1}{2} \rho_{b}\left(\left(u_{1}\right)_{b}^{2}+\left(u_{2}\right)_{b}^{2}+\left(u_{3}\right)_{b}^{2}\right)+\rho_{b} k_{b}
\end{array}\right],
$$

whereas the conserved variables in the ghost control volume now follow from equation (4.206). 
3. Non-reflective subsonic inlet boundary conditions

This option follows from the inviscid characteristic analysis in appendix $\mathrm{A}$ see also [163, 164, leading to the partial differential equation (A.52):

$$
\frac{\partial \boldsymbol{U}}{\partial t}+M \hat{R}_{1} \mathcal{L}=-\boldsymbol{B}
$$

where the augmented set of conserved variables reads $\boldsymbol{W}=[\rho, \rho \underline{u}, \rho E, \rho k, \rho \omega]^{T}$, and $M=\partial \boldsymbol{W} / \partial \boldsymbol{Q}$ for the primitive set of variables $\boldsymbol{Q}=\left[\rho, \underline{u}, p^{*}, k, \omega\right]^{T}$, see equation (A.10). Furthermore, $\hat{R}_{1}$ is the matrix with right eigenvectors given in equation (A.31), $\mathcal{L}$ is a column vector with the components $\mathcal{L}_{i}$ associated with the amplitude of a wave traveling along the characteristics $\frac{d x_{1}}{d t}=\lambda_{i}, \lambda_{i} \in\left\{u_{1}-\right.$ $\left.c^{*}, u_{1}, u_{1}, u_{1}, u_{1}+c^{*}, u_{1}, u_{1}\right\}$, see equation (A.43), and $\boldsymbol{B}$ equal to

$$
\boldsymbol{B}=A_{2} \frac{\partial \boldsymbol{W}}{\partial x_{2}}+A_{3} \frac{\partial \boldsymbol{W}}{\partial x_{3}},
$$

see equation (A.53). Note that the $x_{1}$-direction corresponds to the direction normal to the boundary face, the positive direction being into the computational domain, i.e. $-\underline{n}_{\mathrm{kl}}$.

The non-reflective boundary conditions are implemented as follows:

- The conserved and primitive sets of variables in the internal and ghost control volume are rotated, such that the $x_{1}$-direction corresponds to the direction described above.

- The homogenous form of equation (A.52) is employed, without the partial differential equations corresponding to the SST turbulence model, i.e. the boundary conditions for the turbulence model equations are always prescribed as presented in equation (4.219).

- The components $\mathcal{L}_{2}, \ldots, \mathcal{L}_{5}$ set to zero, whereas $\mathcal{L}_{1}$ corresponds to the outward traveling wave and follows from the solution. The resulting partial differential equation is discretized using finite differences with first-order upwind schemes for the spatial terms and a second-order accurate backward scheme for the physical time derivative term.

- A pseudo-time derivative term is added to the equation, which is discretized using a backward Euler pseudo-time integration, and the resulting discretized equation is written in the delta-formulation, i.e. in terms of $\Delta \boldsymbol{U}_{\mathrm{k}}$ and $\Delta \boldsymbol{U}_{1}$.

- After an iteration sweep of the system of linearized URANS equations over the control volumes within the computational domain, an iteration sweep over the ghost control volumes is performed, which yields a new update of the rotated conserved variables in each ghost control volume.

- The update of the rotated conserved variables in each ghost control volume is rotated back to the original Cartesian coordinate system and subsequently used in an iteration sweep over the control volumes within the computational domain. 
- These steps are repeated until the residuals of the system of linearized equations have been sufficiently reduced, and a new pseudo-time step can be taken.

Note that in the present implementation, the homogenous form of equation (A.52) is used. It is therefore assumed that changes in the solution in the direction tangential to the boundary are small, which is generally the case if the boundary is located far away from the object in the flow.

\section{Subsonic Outlet Boundary}

For an artificial boundary at which the flow is subsonically leaving the computational domain, the analysis in appendix A shows that one wave associated with the URANS equations enters the domain, whereas four waves associated with the URANS equations plus two waves associated with the SST turbulence model (or one wave associated with the SA turbulence model) leave the domain. Therefore, for the URANS equations we can specify one variable and have to extrapolate four variables. For the turbulence model equations, all variables have to be extrapolated as well. In the present computational method, zeroth-order extrapolation is used.

For the turbulence model equations, the following relation is always used for the conserved variables in the ghost control volumes at a subsonic outlet boundary:

$$
\begin{aligned}
{\left[\left(\boldsymbol{U}_{\mathrm{t}}\right)_{S S T}\right]_{\mathrm{l}} } & =\left[\left(\boldsymbol{U}_{\mathrm{t}}\right)_{S S T}\right]_{\mathrm{k}}, \\
{\left[\left(U_{\mathrm{t}}\right)_{S A}\right]_{\mathrm{l}} } & =\left[\left(U_{\mathrm{t}}\right)_{S A}\right]_{\mathrm{k}} .
\end{aligned}
$$

In the present computational method, two possible solutions for the specification of the variables of the URANS equations at a subsonic inlet have been implemented. They are:

1. Fixed static pressure

This option originates from the static pressure at an outflow boundary far away from an object in the flow being constant, i.e. $p_{b}=p_{\infty}$. Using equations (3.92) and (4.206) yields the following relation for the conserved variables of the URANS equations in the ghost control volume:

$$
\boldsymbol{U}_{1}=2\left[\begin{array}{c}
0 \\
0 \\
0 \\
0 \\
\frac{p_{\infty}}{\gamma-1}
\end{array}\right]+\left[\begin{array}{c}
U_{1} \\
U_{2} \\
U_{3} \\
U_{4} \\
-U_{5}+\frac{U_{2}^{2}+U_{3}^{2}+U_{4}^{2}}{U_{1}}+2\left(U_{\mathrm{t} 1}\right)_{S S T}
\end{array}\right]_{\mathrm{k}}
$$

2. Non-reflective subsonic outlet boundary condition

This option follows the same approach as presented for the non-reflective subsonic inlet boundary conditions. However, for the outlet boundary condition, the $x_{1}$-direction is now positive in the outward normal direction, i.e. $+n_{\mathrm{kl}}$. Furthermore, the component $\mathcal{L}_{1}$ is now prescribed equal to zero, whereas the components $\mathcal{L}_{2}, \ldots, \mathcal{L}_{5}$ follow from the solution. 
For steady-state flow simulations, a modification can be introduced that removes the dependency of the steady-state solution on the initial conditions [165, 166]. Instead of specifying the incoming wave amplitude equal to zero, $\mathcal{L}_{1}=0$, a nonzero specification is used:

$$
\mathcal{L}_{1}=K\left(p-p_{\infty}\right), \text { with } K=\sigma \frac{\left(1-\mathcal{M}^{2}\right) c^{*}}{L_{\text {dom }}},
$$

where $\sigma$ is a constant, $\mathcal{M}$ is the maximum Mach number in the computational domain, $c^{*}$ is the local speed of sound and $L_{d o m}$ is a characteristic size of the computational domain. Poinsot \& Lele [166] mention that values of $\sigma$ larger than 0.1 and smaller than 0.7 yield satisfactory results for their test cases, whereas Rudy [165] uses a value of $\sigma=0.58$. In the present computational method, a value of $\sigma=0.5$ is used.

\subsubsection{Synthetic Jet Actuation}

The boundary conditions used for synthetic jet actuation have been presented in subsection 3.5.3. The boundary conditions are split in two time frames: one for the ejection phase, $t \in\left[0, \frac{1}{2} T\right]$, and one for the ingestion phase, $t \in\left(\frac{1}{2} T, T\right)$, with $T=1 / f$ and $f$ the actuation frequency.

1. Ejection Phase

During the ejection phase, the density and velocity are prescribed and the pressure is extrapolated. Furthermore, a zero turbulence level is prescribed, which is obtained using the following relation for the conserved variables for the turbulence equations in the ghost control volume:

$$
\left[\left(\boldsymbol{U}_{\mathrm{t}}\right)_{S S T}\right]_{\mathrm{l}}=\left[\begin{array}{c}
-(\rho k)_{\mathrm{k}} \\
\rho_{\infty} \omega_{\infty}
\end{array}\right], \quad\left[\left(U_{\mathrm{t}}\right)_{S A}\right]_{\mathrm{l}}=-\left[\left(U_{\mathrm{t}}\right)_{S A}\right]_{\mathrm{k}},
$$

where $\omega_{\infty}=\omega_{a m b}$. For the conserved variables of the URANS equations in the ghost control volume, the relation presented in equation (4.220) can be used, which employs zeroth-order extrapolation of the pressure. In the present case, the relation reads

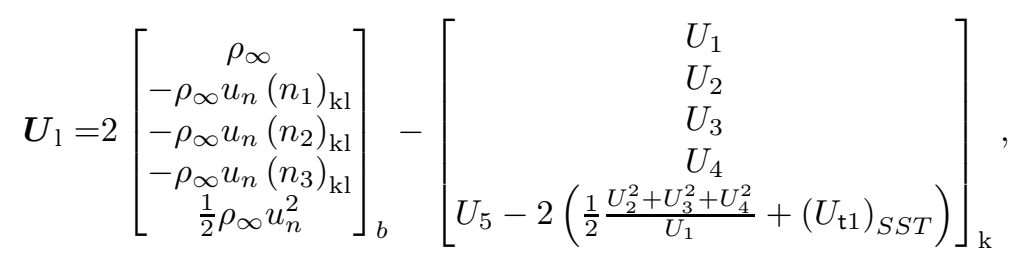

where $u_{n}(t)=U_{n} \sin (2 \pi f t)$, with $U_{n}$ the prescribed velocity amplitude.

2. Ingestion Phase

During the ingestion phase, only the component of the velocity normal to the 
boundary face is prescribed. The remaining variables, including the turbulence variables, are extrapolated using zeroth-order extrapolation. The relation for the conserved turbulence variables in the ghost control volumes is therefore given by equation (4.231), whereas the relation for the conserved flow variables reads

$$
\boldsymbol{U}_{1}=\left[\begin{array}{c}
\rho_{k} \\
\rho_{k}\left(u_{1}\right)_{1} \\
\rho_{k}\left(u_{2}\right)_{1} \\
\rho_{k}\left(u_{3}\right)_{1} \\
\frac{p_{\mathrm{k}}}{\gamma-1}+\frac{1}{2} \rho_{\mathrm{k}}\left|\underline{u}_{1}\right|^{2}+\rho_{\mathrm{k}} k_{\mathrm{k}}
\end{array}\right],
$$

where $\underline{u}_{1}$ reads

$$
\underline{u}_{1}=2\left(-u_{n} \underline{n}_{\mathrm{kl}}+\left(\underline{u}_{\mathrm{k}}-\left(\underline{u}_{\mathrm{k}} \cdot \underline{n}_{\mathrm{kl}}\right) \underline{n}_{\mathrm{kl}}\right)\right)-\underline{u}_{\mathrm{k}},
$$

which yields

$$
\underline{u}_{b}=-u_{n} \underline{n}_{\mathrm{kl}}+\left(\underline{u}_{\mathrm{k}}-\left(\underline{u}_{\mathrm{k}} \cdot \underline{n}_{\mathrm{kl}}\right) \underline{n}_{\mathrm{kl}}\right),
$$

i.e. the component of the velocity normal to the boundary face is prescribed and the other velocity components are extrapolated. 



\section{Chapter 5}

\section{VERIFICATION AND VALIDATION}

\subsection{Introduction}

For any computational method, it is important to assess its accuracy by means of verification and validation [167, 168. Verification is the assessment of the ability to calculate solutions with the expected accuracy to the mathematical models. In other words, verification checks the implementation of the models in terms of mathematics and computer coding. When this has been established, it is important to assess the degree to which the computational method is able to accurately simulate real physics. This is process is known as validation.

This chapter starts with the process of verification, see section 5.2 . Verification involves comparisons of calculated flow solutions with the correct answer, which is provided by 'highly accurate solutions'. A highly accurate solution can be [169]

- a known analytical solution of the mathematical models for a special flow case,

- a benchmark ODE solution, which is a very accurate numerical solution to an ordinary differential equation obtained by simplifying assumptions resulting in a formulation in terms of similarity variables,

- a benchmark PDE solution, which is a very accurate numerical solution for special cases of the partial differential equations or the boundary conditions.

Here solutions are considered benchmark solutions if they have been obtained very carefully by different independent investigators.

An example of a benchmark ODE solution is the Blasius solution of an incompressible laminar flow over a flat plate [162], see subsection 5.2.1. Other verification cases presented in section 5.2 present comparisons with benchmark PDE solutions, obtained from the Turbulence Modeling Resource of the NASA Langley Research Center [170], which has been developed for verification and validation purposes.

One of the most important tasks in verification is checking the spatial and temporal discretization error. For sufficiently fine grids and time step sizes, i.e. in the 'asymptotic region', the lowest-order term in the truncation error dominates. If the computational solution does not have discontinuities, the error in this region should asymptotically approach 
zero as the grid size and time step size approach zero, excluding computer round-off errors and iterative errors [167, 168]. The observed order of convergence should be close to the expected order of convergence. For the present computational method, second-order accuracy in space and time is expected. For iterative errors to be negligible, the computed solutions must be sufficiently converged on each grid and within each time step.

The observed order of spatial convergence, say $p$, can be obtained by calculating a flow solution on three, successively refined, computational grids of the same family and using an expansion of the exact solution in the form of

$$
\phi_{0}=\phi_{h}+C h^{p}+\mathcal{O}\left(h^{p+1}\right)
$$

where $h$ is a measure of the grid size, $\phi_{0}$ is the exact solution on an infinitely refined grid and $C$ a constant independent of $h$. Three solutions $\phi_{h}$ with different $h$ provide enough information to calculate $\phi_{0}, C$ and $p$. This is known as Richardson extrapolation.

The observed order of temporal convergence can also be obtained by means of Richardson extrapolation using three, successively refined time step sizes. In order to minimize the influence of the spatial discretization on the observed order of temporal convergence, computations should be performed on a sufficiently fine computational grid. Also, for grid convergence studies of time-dependent flows, the time step size should be sufficiently small to minimize the influence of the temporal discretization on the observed order of spatial convergence.

When the error of an integral quantity such as the force on an object in the flow is considered, this quantity should be calculated with a method of, at least, the same order of accuracy as the method that solves the flow equations. In the present computational method, integral quantities are calculated with the second-order accurate midpoint integration rule. For two-dimensional problems, such as the flow around an airfoil with chord length $c$, the sectional lift coefficient, $c_{l}$, and drag coefficient, $c_{d}$, are defined as

$$
\begin{array}{cl}
c_{l}=\frac{l}{\frac{1}{2} \rho_{\infty} U_{\infty}^{2} c}, \quad l=\oint\left[(\underline{\underline{\sigma}}(s) \underline{n}(s)) \cdot \underline{k}_{\perp}\right]\left|\frac{d \underline{x}}{d s}\right| d s, \\
c_{d}=\frac{d}{\frac{1}{2} \rho_{\infty} U_{\infty}^{2} c}, \quad d=\oint\left[(\underline{\underline{\sigma}}(s) \underline{n}(s)) \cdot \underline{k}_{\|}\right]\left|\frac{d \underline{x}}{d s}\right| d s,
\end{array}
$$

where $l$ and $d$ are the lift and drag force per unit span, $s$ follows the closed contour of the object, $\underline{\underline{\sigma}}$ is the stress tensor, $\underline{n}$ is the outward pointing normal vector, $\underline{k}_{\perp}$ is the vector perpendicular to the free-stream, $\underline{k}_{\|}$is the vector parallel to the free-stream, and $\rho_{\infty}$ and $U_{\infty}$ are the free-stream density and velocity, respectively.

After the correct implementation of the mathematical models has been verified, it can be assumed that the present computational method is validated for the range of flows that has been previously validated for other verified computational methods of the same type (URANS method using the same turbulence model). This is discussed in subsection 5.3.1. However, a verified and validated computational method does not necessarily give accurate predictions outside the range of validated cases. In this work, the aim is to simulate flows with synthetic jet actuation, which are generally not among the standard 
validated flow cases for URANS methods. In order to be able to assess the accuracy of the computational method for this class of flow problems, subsection 5.3.2 presents a validation study for the flow over a hump model with flow control, aimed at boundary layer separation control. This case has been used in the workshop 'CFD Validation of Synthetic Jets and Turbulent Separation Control' held in Williamsburg, Virginia, USA on March 29-31, 2004, see [171, 172] for more information.

The main topic of this work is the possible use of synthetic jet actuation for pitch control. In the Engineering Fluid Dynamics group of the University of Twente, experiments of synthetic jet actuation for pitch control on an airfoil in a wind tunnel have been performed. The experiments and accompanying flow simulations will be discussed in the next chapter.

\subsection{Verification}

\subsubsection{Laminar Boundary Layer on Flat Plate}

Consider a two-dimensional laminar flow past a semi-infinite flat plate at nearly incompressible flow conditions: $\operatorname{Re}_{L}=1 \cdot 10^{5}, \mathrm{M}=0.1, T_{\text {ref }}=273.15 \mathrm{~K}$. The plate is located in the $\left(x_{1}, x_{3}\right)$-plane, with its leading edge at $x_{1}=0$. The main flow is parallel to this plane.

Here, we will focus on the flow in the boundary layer region around $x_{1} / L=1.9775$, with $L=1 \mathrm{~m}$. The two-dimensional, rectangular computational domain for this problem is shown in figure 5.1] The artificial boundaries are located far enough from the region of interest, such that their influence on the flow solution is negligible. The employed boundary conditions are also indicated in figure 5.1. For the prescribed total pressure and total temperature at the inlet boundary, $\gamma=1.4$ is used.

A steady flow solution is calculated on three successively finer grids. The coarsest grid consists of

- 100 elements in in $x_{2}$-direction, growth factor $g=1.1$ (height of first element $\left.\approx 7.2571 \cdot 10^{-5}\right) \mathrm{m}$;

- 56 elements in negative $x_{1}$-direction along the symmetry boundary, growth factor $g=1.199240158$ (width of first element close to leading edge $\approx 7.5971 \cdot 10^{-5}$ ) $\mathrm{m}$;

- 166 elements in positive $x_{1}$-direction along the wall, growth factor $g=1.05$ (width of first element element close to leading edge $\approx 7.5971 \cdot 10^{-5}$ ) $\mathrm{m}$;

which leads to a total of 22200 elements. The refined grids are constructed by doubling the number of elements in each direction while using a squared growth factor with respect to the previous grid, leading to a total of 88800 and 355200 elements, respectively.

Roe's flux-difference splitting scheme is used for the convective fluxes and during the solution reconstruction technique a gradient limiter is not used. The initial solution for all calculations is the uniform flow solution. The CFL number starts at a value of 1.0 and is increased by a factor of 1.2 each pseudo-time step until it has reached the maximum 


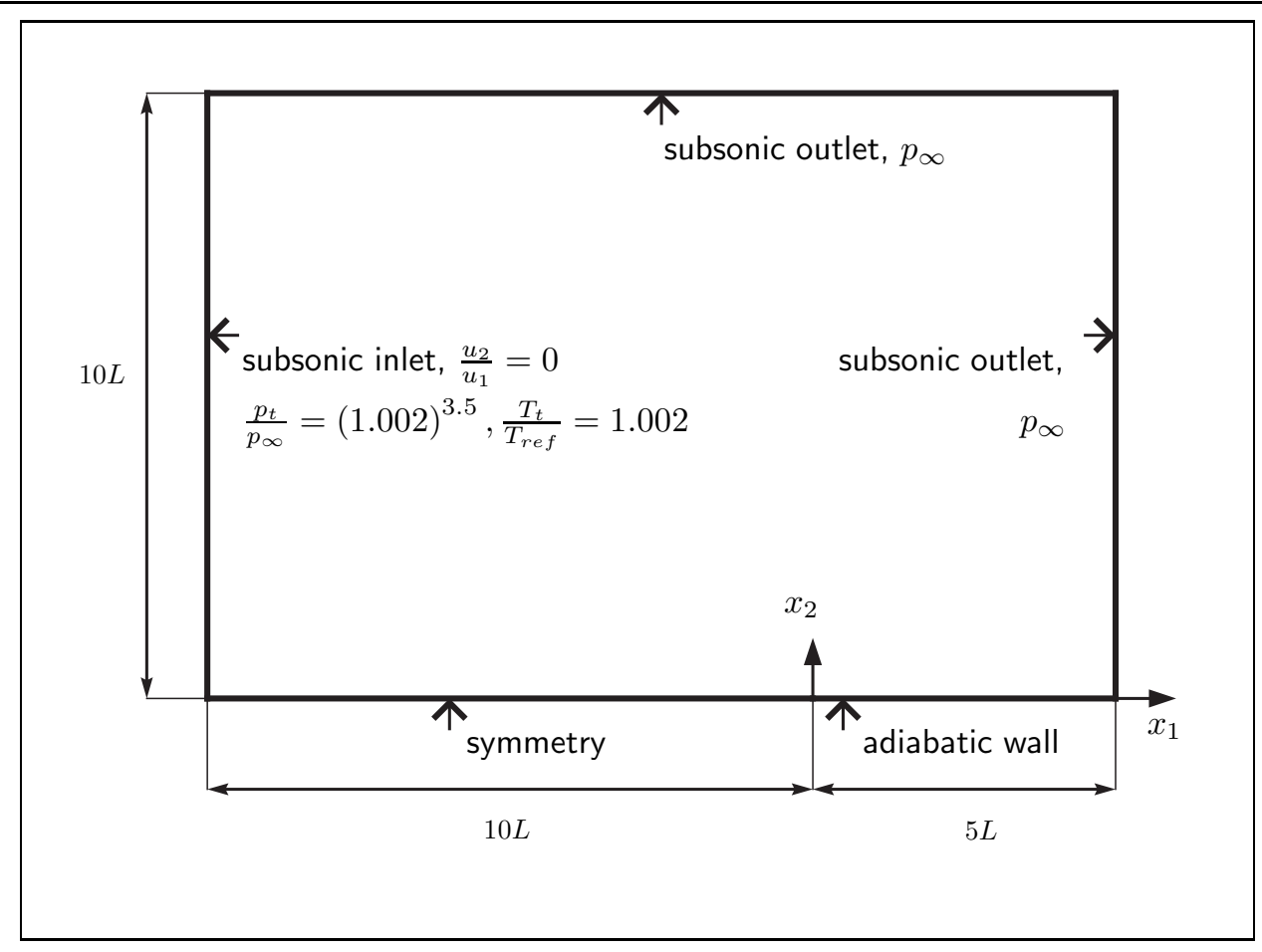

Figure 5.1: Schematic drawing of computational domain for laminar flow past a flat plate together with employed boundary conditions.

value of $10^{5}$. For the grid convergence study, the calculations continue until all non-linear residuals have decreased at least seven orders of magnitude.

To illustrate the effect of the convergence acceleration methods for this case, the simulation on the coarse grid has been performed several times using different options for the iteration method. For this illustration, we let the $\mathrm{L}_{2}$-norm of the residuals of the $x_{1}$-component of the momentum equation decrease at least five orders of magnitude. The standard Gauß-Seidel method using a single grid level is compared with the algebraic multigrid method using $\mathrm{V}(1,1)$ cycles and five grid levels, i.e. $\Omega^{h} \in\left\{\Omega^{0}, \ldots, \Omega^{4}\right\}$. Furthermore, the influence of parallelization is investigated as well, by using up to four grid partitions (CPUs). Figure 5.2 shows the $\mathrm{L}_{2}$-norm of the residuals of the $x_{1}$-component of the momentum equation on the coarse grid as a function of the number of pseudo-time steps (a) and the wall clock time (b). Three iteration sweeps or multigrid cycles are used per pseudo-time step.

The algebraic multigrid method needs between 303 (1 CPU) and 322 (4 CPUs) pseudotime steps to reduce the residuals by five orders of magnitude, whereas the standard iteration method without multigrid needs between 3281 (1 CPU) and 3294 (4 CPUs) pseudo-time steps. Measured in wall clock time, the algebraic multigrid method is ap- 


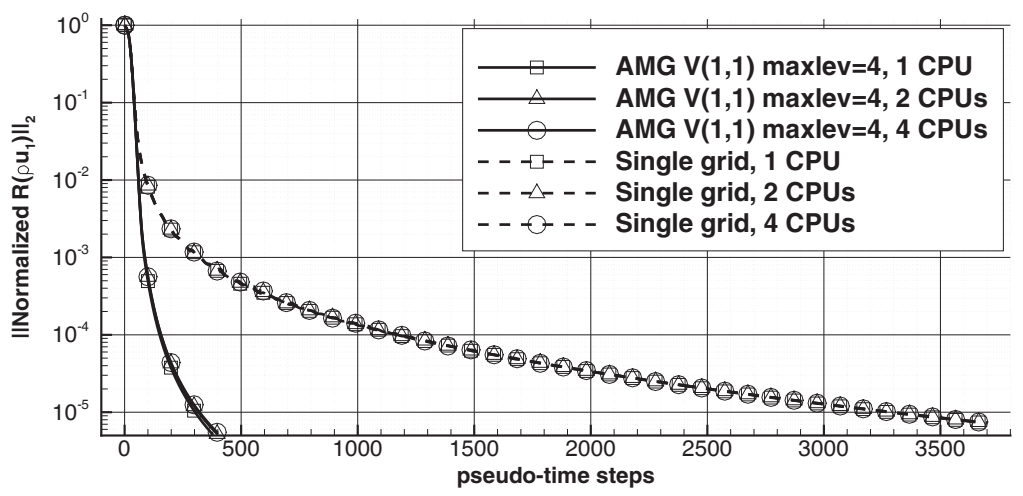

(a) residuals vs. number of pseudo-time steps

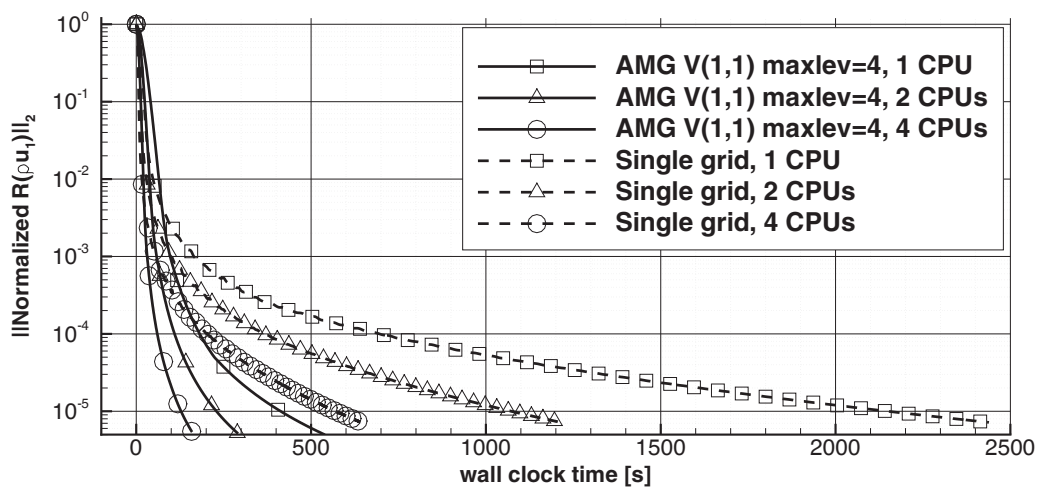

(b) residuals vs. wall clock time

Figure 5.2: $L_{2}$-norm of the residuals of the $x_{1}$-component of the momentum equation on the coarse grid for laminar flow past a flat plate. Three sweeps or cycles per pseudo-time step are used, symbols are shown every 100 pseudo-time steps.

proximately five times faster than the standard iteration method for this flow problem. Furthermore, using 2 or 4 CPUs instead of 1 yields speed-up factors of 2.00 or 3.77 , respectively, without multigrid and approximately 1.80 or 3.25 , respectively, with multigrid. This shows the increasing relative importance of the communication between grid partitions and the use of Jacobi iteration on grid partition boundaries instead of Gauß-Seidel. The present coarse grid does not justify using more than 2 CPUs, i.e. using less than approximately 10000 control volumes per grid partition is not very efficient.

The results of this flow case are compared to the Blasius solution, valid for incom- 


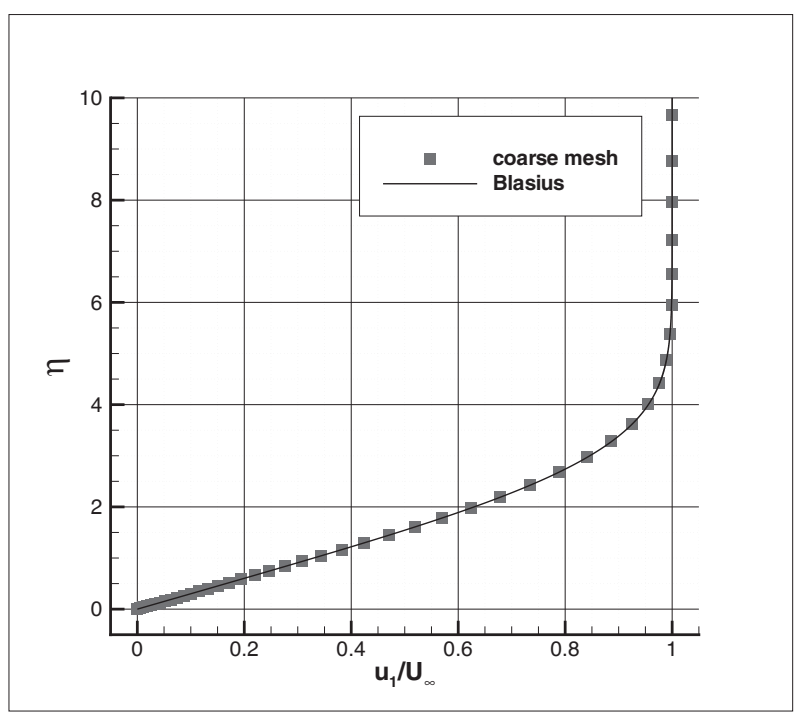

Figure 5.3: Tangential velocity profile in laminar boundary layer above flat plate at $x_{1} / L=$ 1.97775 , for $\operatorname{Re}_{L}=1 \cdot 10^{5}, \mathrm{M}=0.1, T_{\text {ref }}=273.15 \mathrm{~K}$. Computational results on coarse grid and Blasius solution are shown.

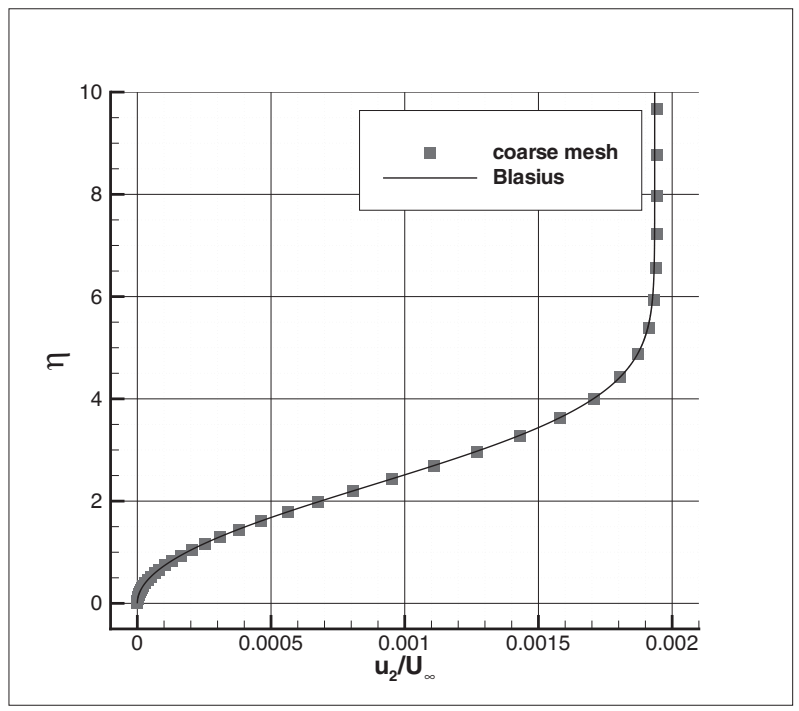

Figure 5.4: Normal velocity profile in laminar boundary layer above flat plate at $x_{1} / L=$ 1.97775 , for $\operatorname{Re}_{L}=1 \cdot 10^{5}, \mathrm{M}=0.1, T_{\text {ref }}=273.15 \mathrm{~K}$. Computational results on coarse grid and Blasius solution are shown. 
pressible flow, see e.g. Schlichting [162. This is the solution to an ordinary differential equation for the stream function, $\Psi(x, y)$, obtained by the introduction of the similarity transformation,

$$
\begin{aligned}
\eta & =x_{2} \sqrt{\frac{U_{\infty}}{\nu x_{1}}}, \\
\Psi & =\sqrt{\nu x_{1} U_{\infty}} f(\eta),
\end{aligned}
$$

to the boundary layer equations. Here, $f(\eta)$ is the dimensionless stream function, $\eta \sim$ $x_{2} / \delta\left(x_{1}\right)$ is the dimensionless coordinate normal to the flat plate, $\delta\left(x_{1}\right)$ is the boundary layer thickness and $x_{1}$ is the coordinate along the plate, with $x_{1}=0$ being the leading edge. The velocity components tangential to the wall, $u_{1}$, and normal to the wall, $u_{2}$ follow from

$$
\begin{aligned}
& u_{1}(x, y)=\frac{\partial \Psi}{\partial x_{2}}=U_{\infty} f^{\prime}(\eta) \\
& u_{2}(x, y)=-\frac{\partial \Psi}{\partial x_{1}}=\frac{1}{2} \sqrt{\frac{\nu U_{\infty}}{x_{1}}}\left(\eta f^{\prime}-f\right)
\end{aligned}
$$

The third-order ordinary differential equation reads

$$
f^{\prime \prime \prime}+\frac{1}{2} f f^{\prime \prime}=0,
$$

which is subject to the following boundary conditions:

$$
\begin{aligned}
\eta=0: & f=0, f^{\prime}=0 \\
\eta \rightarrow \infty: & f^{\prime} \rightarrow 1 .
\end{aligned}
$$

Equation (5.8) can be written as a system of three first-order ordinary differential equations, which can subsequently be integrated with the Runge-Kutta-Fehlberg method [173] from $\eta=0$ to a sufficiently large value, e.g. $\eta_{\max }=10$. A solution of (5.8) is found iteratively, using increasingly more accurate initial guesses for $f^{\prime \prime}(0)$, until $f^{\prime}\left(\eta_{\max }\right)=1$.

Figures 5.3 and 5.4 show the velocity profiles in tangential and normal direction at $x_{1} / L=1.97775$ on the coarse grid together with the Blasius solution, respectively. The computational results on all grids agree quite well with the Blasius solution. However, a close-up of the edge of the boundary layer shows that the results converge towards small overshoots: for $u_{1} / U_{\infty}$ the relative overshoot is approximately $0.029 \%$ and for $u_{2} / U_{\infty}$ the relative overshoot is approximately $0.39 \%$. This can be attributed to the effect of small terms that are present in the Navier-Stokes equations, e.g. $\left(\mu\left(\partial^{2} u_{1} / \partial x_{1}^{2}\right)\right)$, but have been neglected in the derivation of equation (5.8).

Figure 5.5 shows the skin friction coefficient, $c_{f}$, along the wall. The skin friction coefficient is a non-dimensional quantity defined as

$$
c_{f}=\frac{\tau_{w}}{\frac{1}{2} \rho_{\infty} U_{\infty}^{2}},
$$




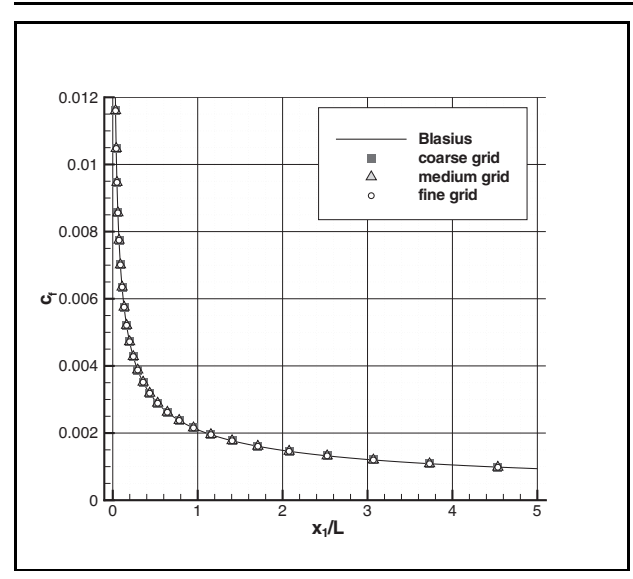

Figure 5.5: Skin friction coefficient $c_{f}$ for laminar flow past a semi-infinite flat plate, $\operatorname{Re}_{L}=1 \cdot 10^{5}, \mathrm{M}=0.1, T_{\text {ref }}=273.15$ $\mathrm{K}$. Computational results use resolution of every fourth point on coarse grid.

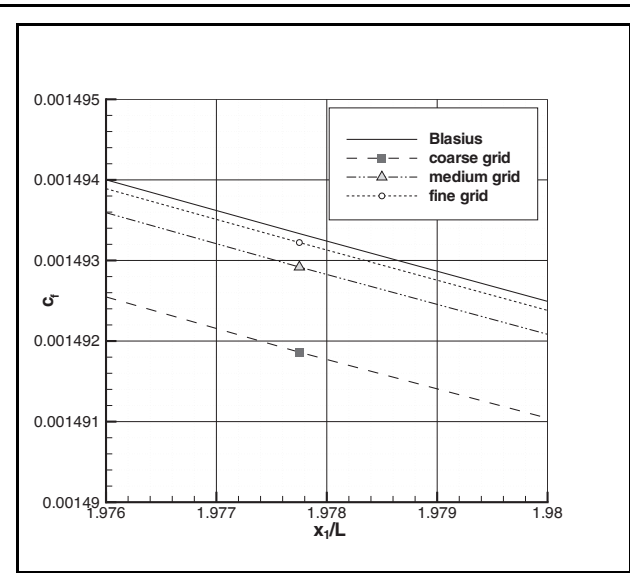

Figure 5.6: Skin friction coefficient $c_{f}$ for laminar flow past a semi-infinite flat plate around $x_{1} / L=1.97775, \operatorname{Re}_{L}=1 \cdot 10^{5}$, $\mathrm{M}=0.1, T_{\text {ref }}=273.15 \mathrm{~K}$.

where $\tau_{w}$ is the wall shear stress. The wall shear stress for the Blasius solution reads

$$
\tau_{w}=\mu\left(\frac{\partial u}{\partial y}\right)_{x_{2}=0}=\mu U_{\infty}\left(\frac{d f^{\prime}}{d \eta} \frac{\partial \eta}{\partial y}\right)_{x_{2}=0}=\mu U_{\infty} f^{\prime \prime}(0) \sqrt{\frac{U_{\infty}}{\nu x_{1}}},
$$

which yields a skin friction coefficient $c_{f}$ given by

$$
c_{f}=\frac{2 f^{\prime \prime}(0)}{\sqrt{\operatorname{Re}_{x_{1}}}} \approx \frac{0.664114}{\sqrt{\operatorname{Re}_{x_{1}}}},
$$

where $\operatorname{Re}_{x_{1}}$ is the local Reynolds number based on the distance from the leading edge. The computed skin friction coefficient on all grids agrees very well with the Blasius solution for the skin friction coefficient. A close-up around $x_{1} / L=1.97775$, see figure 5.6 shows that the computational results converge towards the Blasius solution of $c_{f}$ at this point along the wall. In fact, when we apply Richardson extrapolation to the computational results towards an infinitely refined grid, see table 5.1 the extrapolated value for $c_{f}$ is slightly higher than that of the Blasius solution, the relative error being $7.947 \cdot 10^{-6}$. This difference may also be due to the effect of the aforementioned neglected terms in the derivation of equation (5.8). The observed order of convergence at this location is 1.8, which is slightly lower than the theoretical value of 2 . The reason for this could be the behavior of the flow near the leading edge, where the skin friction tends to infinity. The presence of this singular point might influence the results elsewhere in the computational domain. 


\begin{tabular}{|l|c|}
\hline & $c_{f}$ \\
\hline \hline Coarse grid & 0.001491861354 \\
\hline Medium grid & 0.001492918320 \\
\hline Fine grid & 0.001493222416 \\
\hline Extrapolated value & 0.001493345245 \\
\hline Blasius & 0.001493333378 \\
\hline
\end{tabular}

Table 5.1: Skin friction coefficient $c_{f}$ for laminar flow past a semi-infinite flat plate at $x_{1} / L=1.97775$; Blasius solution and computational results with extrapolated value for $\operatorname{Re}_{L}=1 \cdot 10^{5}, \mathrm{M}=0.1, T_{\text {ref }}=273.15 \mathrm{~K}$.

\subsubsection{Turbulent Boundary Layer on Flat Plate}

The Turbulence Modeling Resource of the NASA Langley Research Center [170] provides a verification case for a two-dimensional turbulent flow past a semi-infinite flat plate at nearly incompressible conditions: $\operatorname{Re}_{L}=5 \cdot 10^{6}, \mathrm{M}=0.2, T_{\text {ref }}=300 \mathrm{~K}$. The plate is located in the $\left(x_{1}, x_{3}\right)$-plane, with its leading edge at $x_{1}=0$. The main flow is parallel to this plane. The two-dimensional, rectangular computational domain for this problem is shown in figure 5.7 together with the employed boundary conditions. As in subsection 5.2.1 $L=1 \mathrm{~m}$ and $\gamma=1.4$ has been used in the specified inlet boundary conditions.

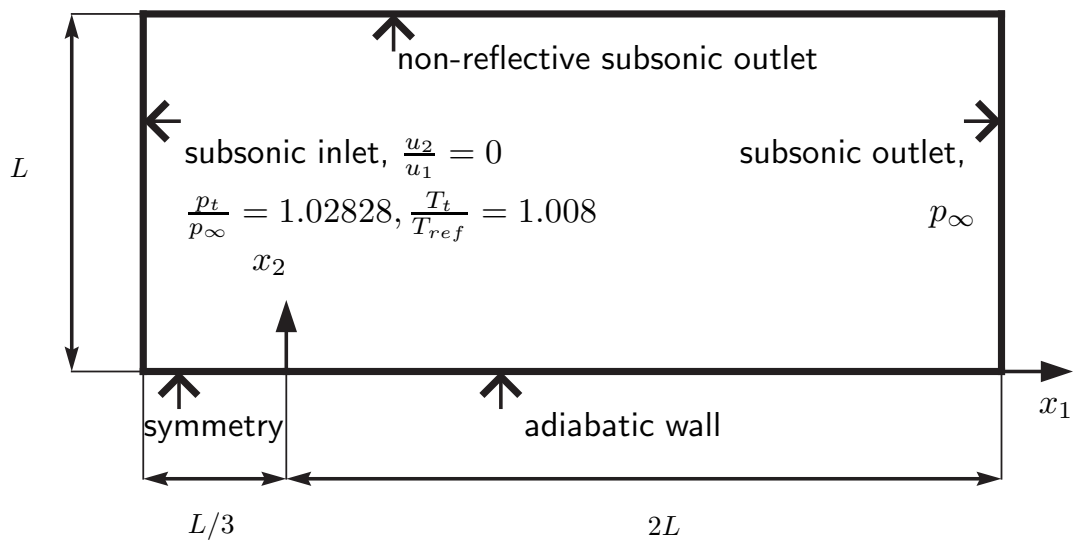

Figure 5.7: Schematic drawing of computational domain for turbulent flow past a flat plate together with employed boundary conditions. 
The computational grids and the benchmark PDE solutions for this case are obtained from the Turbulence Modeling Resource [170]. These benchmark solutions are steady flow solutions using the SA and the SST turbulence models, which have been obtained with the computational methods CFL3D and FUN3D (both developed by NASA). Additionally, the skin friction coefficient $c_{f}$ at $x_{1} / L=0.97008$, as calculated by these and several other independent computational methods (VULCAN, OVERFLOW, TURCOM) on different computational grids, is also provided.

The provided results for the SST turbulence model have been calculated using a different definition of the production term, based on the squared magnitude of the mean rotation rate [91]. This alternative version is known as the SST-V turbulence model and has the advantage that it does not produce turbulence outside boundary layers and wakes, e.g. near stagnation points. Since this definition of the source term is often nearly identical to the exact source term in simple boundary layer flows, comparisons can still be made. Furthermore, the provided solutions have been obtained without the sustaining terms in the equations of the SST turbulence model, see subsection 3.4.3. Therefore, we also do not use these terms here. The employed inlet boundary conditions for the turbulence models are: $\tilde{\nu}_{b}=3 \nu_{\infty}$ for the SA turbulence model and $k_{b}=1.125 \frac{U_{\infty}^{2}}{\operatorname{Re}_{L}}$, $\omega_{b}=125 \frac{U_{\infty}}{L}$ for the SST turbulence model.

The computational grids are a series of four nested grids. Each coarser grid consists of exactly every-other-point of the next finer grid. The number of elements range from $544 \times 384$ on the finest grid with 448 elements along the wall, to $68 \times 48$ on the coarsest grid with 56 elements along the wall. Elements are clustered towards the leading edge in $x_{1}$-direction and towards the wall in $x_{2}$-direction. The height of the first layer of elements at the wall is such that the average non-dimensional grid spacing normal to the wal 1 , $y^{+}$, ranges from approximately 0.1 on the finest grid to approximately 0.8 on the coarsest grid, i.e. below the recommended value of $y^{+}<1$.

The present computational results are calculated using Roe's flux-difference splitting scheme for the convective fluxes of the URANS equations. During the solution reconstruction technique a gradient limiter is not used. All calculations are steady-flow simulations starting from a uniform flow solution. The CFL number for both the URANS and the turbulence model equation(s) starts at a value of 1.0 and is increased by a factor of 1.2 each pseudo-time step until it reaches the maximum value of $10^{5}$. The calculations are continued until all non-linear residuals have decreased at least seven orders of magnitude.

Figures 5.8, 5.9 and 5.10 present results calculated using the SA and SST turbulence models on the finest grid: the skin friction coefficient $c_{f}$ along the plate, the profile of the eddy viscosity ratio $\mu_{\mathrm{t}} / \mu_{\text {ref }}$ at $x_{1} / L=0.97008$ and the profiles of the dimensionless turbulence variables of the SST turbulence model at $x_{1} / L=0.97008$, respectively. Here, the dimensionless turbulence variables are defined as $k / c_{r e f}^{2}$ and $\omega \mu_{\text {ref }} /\left(\rho_{\text {ref }} c_{r e f}^{2}\right)$, where $c_{r e f}$ is the reference speed of sound. The reference variables are derived from $\operatorname{Re}_{L}=$

\footnotetext{
${ }^{1}$ This quantity is defined here as $y^{+}=\frac{\rho u_{\tau} y}{\mu}$, where $y$ is height of the first cell at the wall in the direction normal to the wall and $u_{\tau}$ is the friction velocity, which is defined with the shear stress at the wall, $\tau_{w}$, and the density at the wall, $\rho$, as $u_{\tau}=\sqrt{\frac{\tau_{w}}{\rho}}$.
} 


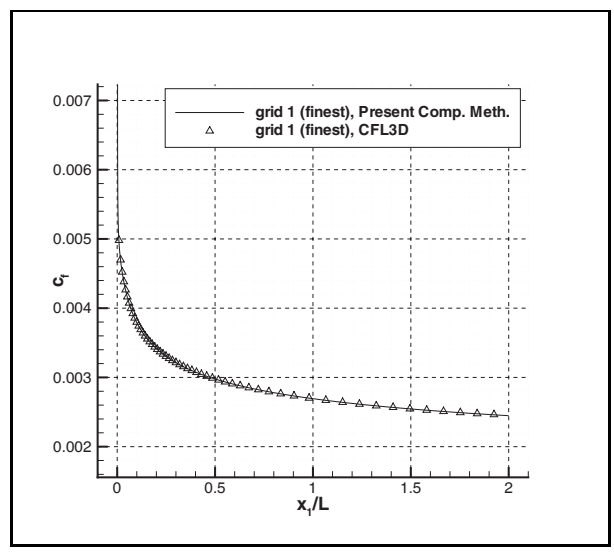

(a) SA turbulence model

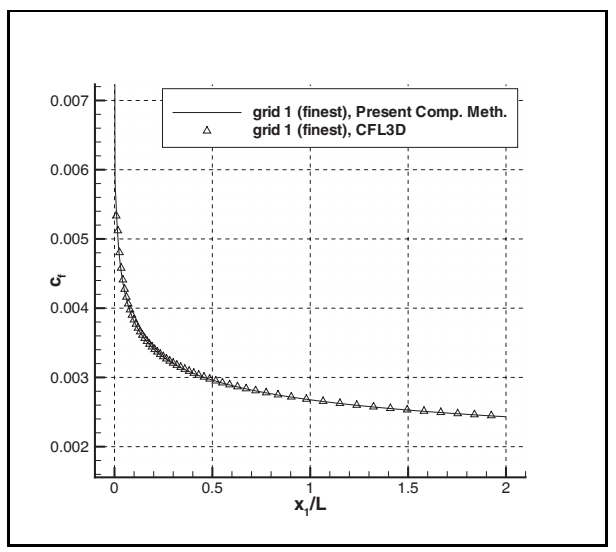

(b) SST turbulence model

Figure 5.8: Skin friction coefficient $c_{f}$ for turbulent flow past a semi-infinite flat plate, for $\operatorname{Re}_{L}=5 \cdot 10^{6}, \mathrm{M}=0.2, T_{\text {ref }}=300 \mathrm{~K}$. Symbols are drawn every eighth point (resolution on the coarsest grid).

$5 \cdot 10^{6}, \mathrm{M}=0.2$ and $T_{\text {ref }}=300 \mathrm{~K}$. The figures show that the results from the present computational method agree well with those from CFL3D and FUN3D.

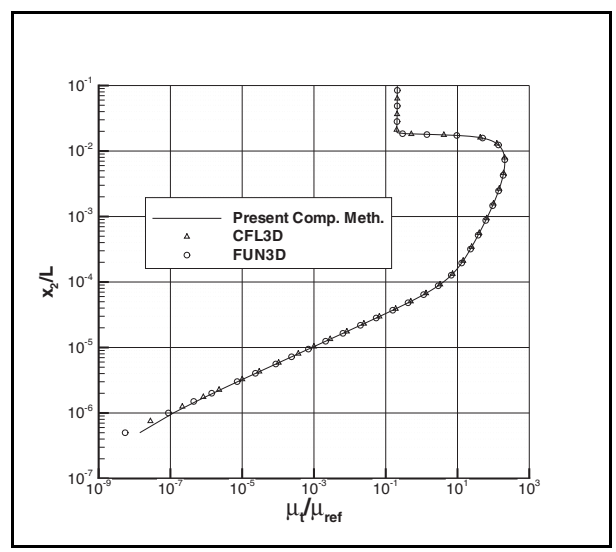

(a) SA turbulence model

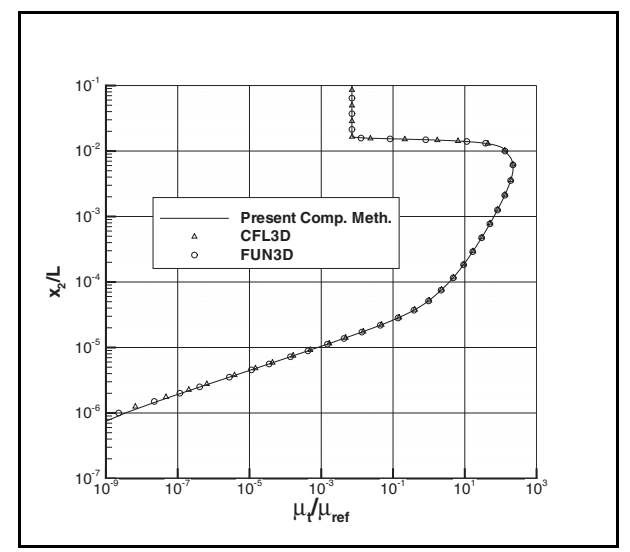

(b) SST turbulence model

Figure 5.9: Eddy viscosity profile in turbulent boundary layer above flat plate at $x_{1} / L=$ 0.97008 , using SA turbulence model (left) and SST turbulence model (right), for $\mathrm{Re}_{L}=$ $5 \cdot 10^{6}, \mathrm{M}=0.2, T_{\text {ref }}=300 \mathrm{~K}$. Computational results on finest grid are shown.

The convergence behavior as a function of the grid resolution is assessed in figures 


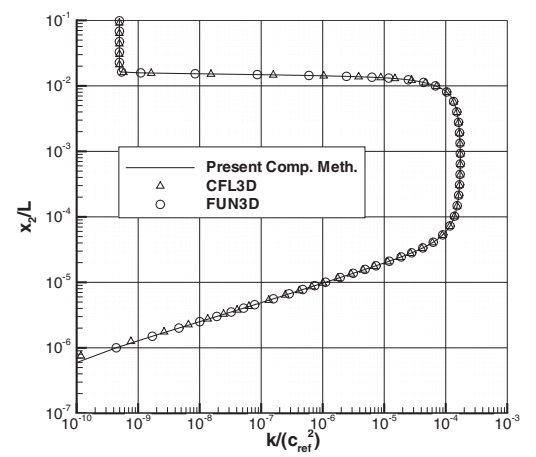

(a) dimensionless turbulence kinetic energy

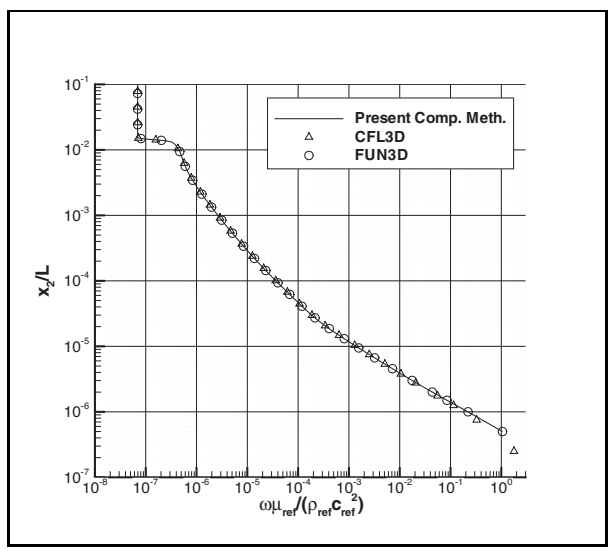

(b) dimensionless turbulence frequency

Figure 5.10: Profiles of turbulence variables of SST turbulence model in turbulent boundary layer above flat plate at $x_{1} / L=0.97008$, for $\operatorname{Re}_{L}=5 \cdot 10^{6}, \mathrm{M}=0.2, T_{\text {ref }}=300 \mathrm{~K}$. Computational results on finest grid are shown.

5.11 and 5.12 These figures show the friction coefficient $c_{f}$ at $x_{1} / L=0.97008$ and the drag coefficient $c_{d}$, respectively, as a function of the grid size $h=\sqrt{1 / N}$, where $N$ is the total number of grid points.

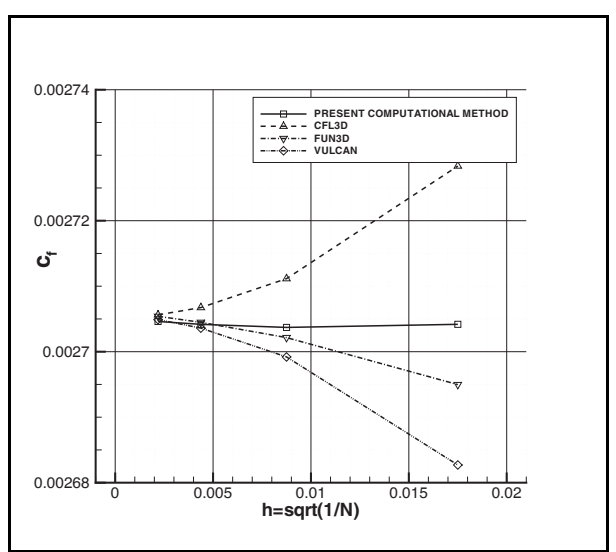

(a) SA turbulence model

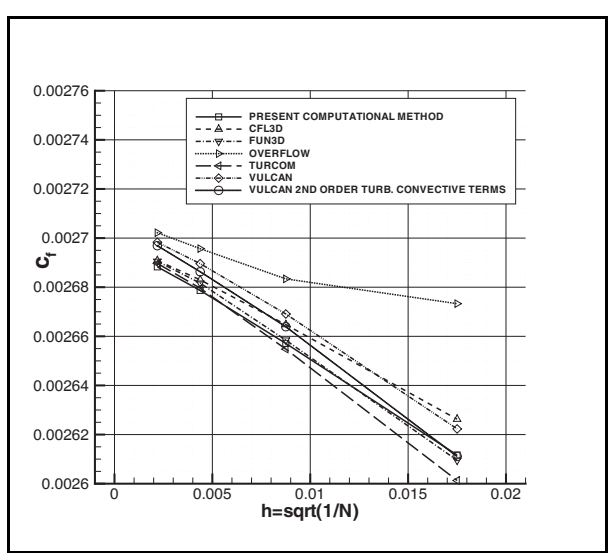

(b) SST turbulence model

Figure 5.11: Convergence of skin friction coefficient $c_{f}$ at $x_{1} / L=0.97008$ with grid size $h=\sqrt{1 / N}, \operatorname{Re}_{L}=5 \cdot 10^{6}, \mathrm{M}=0.2, T_{\text {ref }}=300 \mathrm{~K}$. 


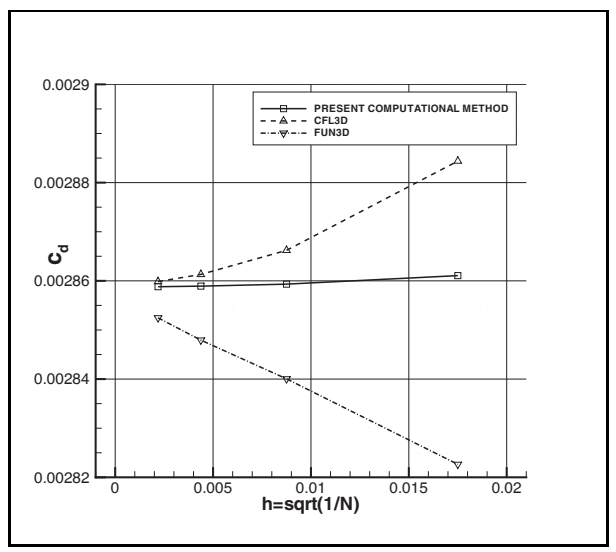

(a) SA turbulence model

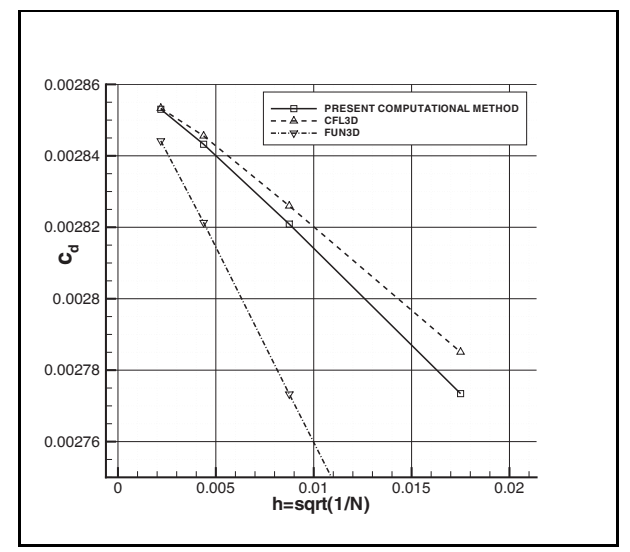

(b) SST turbulence model

Figure 5.12: Convergence of drag coefficient $c_{d}$ with grid size $h=\sqrt{1 / N}$, where $N$ is the number of grid points, for different computational methods, $\operatorname{Re}_{L}=5 \cdot 10^{6}, \mathrm{M}=0.2$, $T_{\text {ref }}=300 \mathrm{~K}$.

For the SA turbulence model, the present computational method yields results for $c_{f}$ at $x_{1} / L=0.97008$ on all grids that are closer to the results on the finest grid than the other computational methods. On the other hand, the convergence is not monotonic, whereas the other methods yield observed orders of convergence of 1.98 (CFL3D) and 1.34 (FUN3D). However, the apparent order of convergence for the drag coefficient $c_{d}$, which is the integrated $c_{f}$ along the plate, is equal to 1.79 for the present computational method. This value is good in comparison with CFL3D and FUN3D, which yield apparent orders of convergence of 1.75 and 0.8 , respectively.

For the SST turbulence model, the present computational model yields results for $c_{f}$ at $x_{1} / L=0.97008$ that are close to the results calculated by CFL3D. The apparent order of convergence for $c_{f}$ at this location is 1.17 for the present computational method, 1.21 for CFL3D and 1.39 for FUN3D. For the drag coefficient $c_{d}$, the apparent orders of convergence are $1.19,1.34$ and 1.07 , respectively.

Except for $c_{f}$ at $x_{1} / L=0.97008$ as calculated by CFL3D with the SA turbulence model, all computational methods show apparent orders of convergence well below the theoretical value of 2 . This might be due to the choice of the computational domain, with a sudden start of the plate. This is not a realistic physical situation and results in a singularity in the flow field. Furthermore, when the grid resolution along the plate changes, the turbulence models likely yield a different location of transition to a turbulent boundary layer. However, due to the presence of the singularity, this effect is not clearly visible.

To show the effect of the singularity at the leading edge, steady-flow simulations have been performed for a different computational domain, which does not have this singularity, 


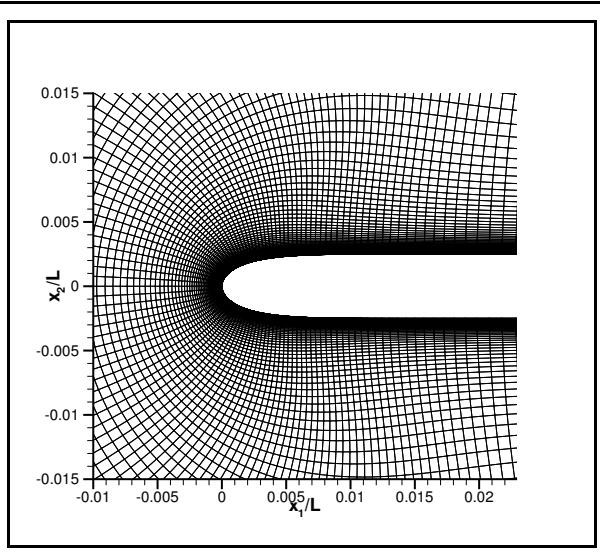

Figure 5.13: Finest grid near leading edge of flat plate with rounded nose.

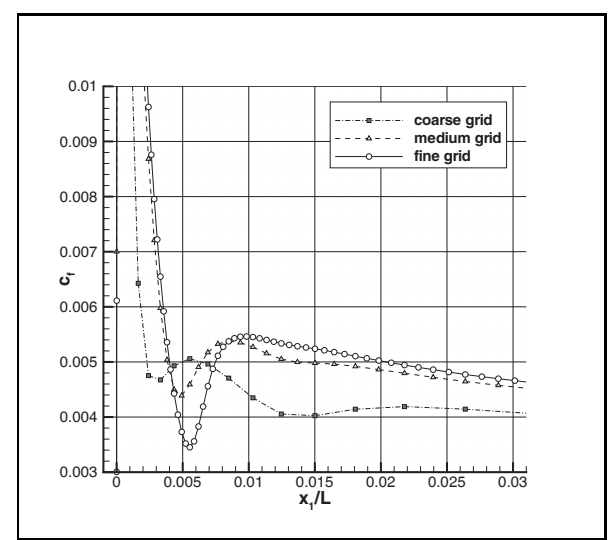

Figure 5.14: Skin friction coefficient $c_{f}$ near leading edge of flat plate with rounded nose, for $\operatorname{Re}_{L}=5 \cdot 10^{6}, \mathrm{M}=0.2$, $T_{\text {ref }}=300 \mathrm{~K}$, and SST turbulence model with sustaining terms activated.

namely the flow around a plate with a thickness of $0.005 \mathrm{~L}$ and a rounded leading edge. A series of three computational grids has been used; figure 5.13 shows a close-up of the finest grid near the leading edge of the plate. Again, each coarser grid consists of exactly every-other-point of the next finer grid and $y^{+}$ranges from approximately 0.1 on the finest grid to approximately 0.4 on the coarsest grid. For the SST turbulence model in fully-turbulent mode with active sustaining terms, the apparent order of convergence for $c_{d}$ is now 1.83 instead of 1.19 for the original flow case, i.e. much closer to the theoretical value of 2 . The effect of the location of transition to a turbulent boundary layer being different on different grids is still present. Figure 5.14 shows the friction factor $c_{f}$ close to the leading edge of the plate. It can be seen that the location of the transition to a turbulent boundary layer, which occurs near the second rise in $c_{f}$, moves downstream with increasing grid resolution. This effect shows close-to first-order convergence behavior. Comparisons of $c_{f}$ at a fixed location on different computational grids may therefore show apparent orders of convergence below the formal order of convergence.

\subsubsection{Turbulent Flow around NACA0012 Airfoil}

Consider the two-dimensional turbulent flow past a NACA0012 airfoil at an angle of attack of $\alpha=0^{\circ}$, for $\mathrm{M}=0.15, \operatorname{Re}_{c}=6 \cdot 10^{6}$, and $T_{\text {ref }}=300 \mathrm{~K}$, with $c$ the chord length of the airfoil. In the Turbulence Modeling Resource of the NASA Langley Research Center [170], this case is presented as a validation case in which computational results are compared to experimentally obtained static surface pressures, and lift and drag measurements. However, since it provides a series of nested grids together with the computational results 
obtained with CFL3D and FUN3D for several turbulence models on one of the grids, it is used here as a verification case instead.

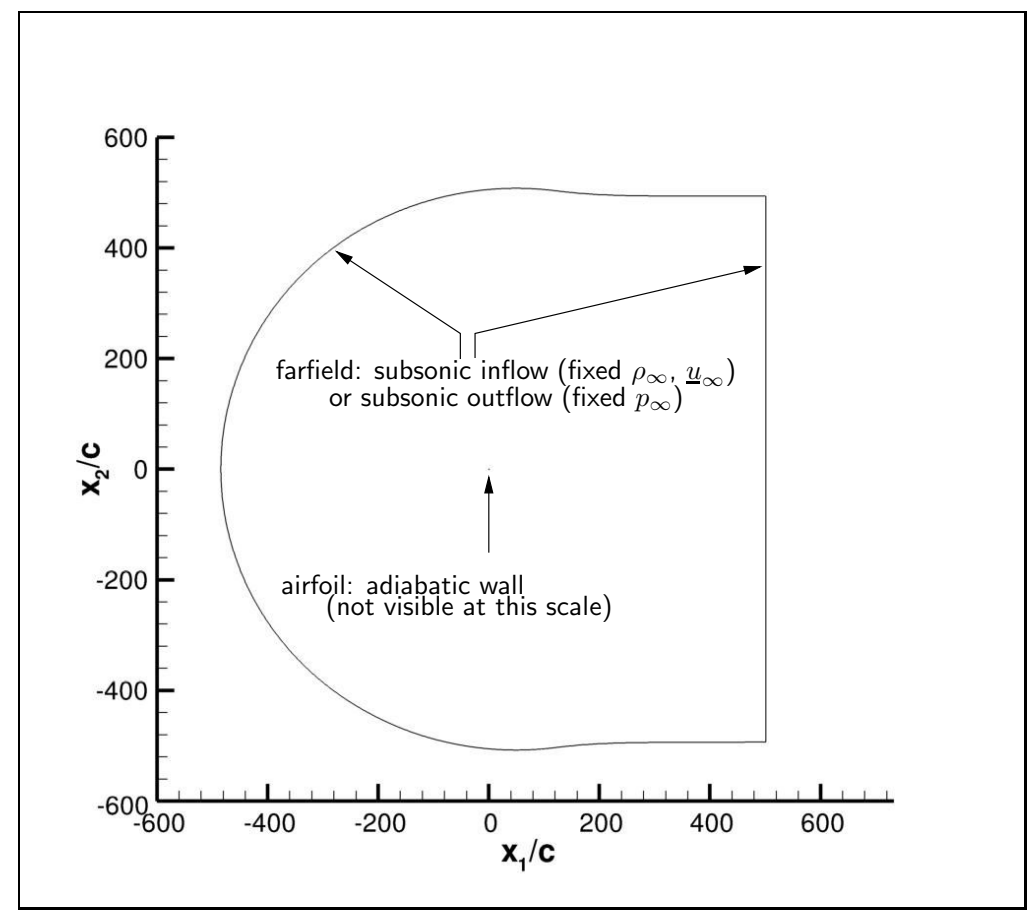

Figure 5.15: Schematic drawing of computational domain for turbulent flow around NACA0012 airfoil, together with employed boundary conditions.

The computational domain is shown in figure 5.15, together with the employed boundary conditions. Note that different farfield boundary conditions, which are based on socalled Riemann invariants, have been used to obtain the provided computational results. However, the farfield boundary is located approximately 500 chord lengths away from the airfoil and differences in the results due to different farfield boundary conditions are assumed to be small.

It is not clear which inlet boundary conditions for the SST turbulence equations have been used to obtain the provided computational results. Therefore, we employ the sustaining terms, see subsection 3.4.3 together with the recommended inflow boundary conditions given in subsection 3.5.2. For the SA model, the employed inlet boundary condition is $\tilde{\nu}=3 \nu_{\infty}$.

The profile of the upper side of the NACA0012 airfoil has been constructed with the 
relation

$$
\begin{aligned}
\frac{x_{2}}{c}=\frac{t}{0.2}\left[0.2969 \sqrt{\frac{x_{1}}{c}}\right. & -0.1260\left(\frac{x_{1}}{c}\right) \\
& \left.-0.3516\left(\frac{x_{1}}{c}\right)^{2}+0.2843\left(\frac{x_{1}}{c}\right)^{3}-0.1015\left(\frac{x_{1}}{c}\right)^{4}\right],
\end{aligned}
$$

with maximum thickness ratio $t=0.12$. The lower side of the airfoil equals the upper side, but mirrored in the $x_{1}$-axis. Since this relation yields a blunt trailing edge, which is not very convenient from a meshing point of view, the end of the airfoil has been extended to $\left(x_{1} / c, x_{2} / c\right)=(1.008930411365,0)$. Subsequently, the airfoil has been scaled down by 1.008930411365 (note that this procedure actually leads to a maximum thickness ratio of approximately 0.11894 instead of 0.12 ). Now, the leading edge of the airfoil is located at $\left(x_{1} / c, x_{2} / c\right)=(0,0)$ and the trailing edge at $\left(x_{1} / c, x_{2} / c\right)=(1,0)$.

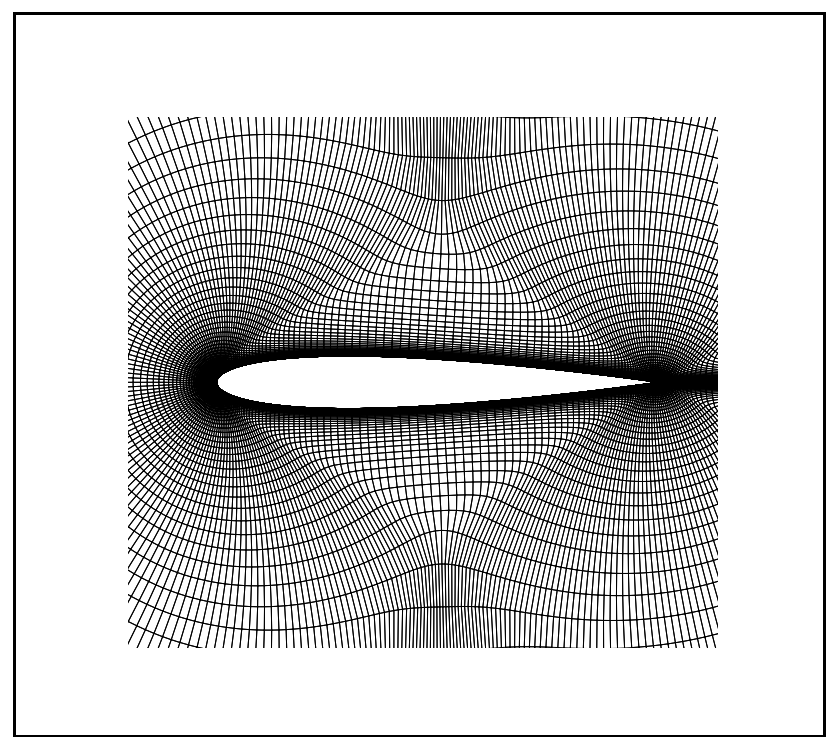

Figure 5.16: Close-up of coarse computational grid near NACA0012 airfoil, provided by the Turbulence Modeling Resource of the NASA Langley Research Center [170].

The computational grids used for this case are a series of three nested C-type grids. Each coarser grid consists of exactly every-other-point of the next finer grid. A close-up of the coarse grid near the airfoil is shown in figure 5.16. The finest grid has 384 elements along the wake line and 1024 elements along the airfoil, with clustering in the regions of the trailing and leading edge. In the perpendicular direction, it has 512 elements with clustering towards the airfoil and wake line. The total number of elements on the grids is 917504,229376 and 57344, respectively. The height of the first layer of elements along the wall is such that the non-dimensional grid spacing normal to the wall, $y^{+}$, is below 1 
on all grids. On the coarse grid, close to the leading edge, $y^{+}$reaches a maximum value of approximately 0.6 .

Two convective flux schemes for the URANS equations have been used for this verification case: Roe's flux-difference splitting scheme with first order reconstruction of the solution, and the JST scheme with scalar dissipation. The solution reconstruction technique is employed without a gradient limiter. The dissipation term of the JST scheme is based on fourth-order differences only: $\kappa_{2}=0, \kappa_{4}=0.015$ and $\beta=0$, see subsection 4.2.2. For the results presented here, the JST scheme is used in combination with the SA turbulence model, whereas Roe's scheme is used in combination with the SA and SST turbulence models.

The calculations are steady-flow simulations, initialized with a uniform flow solution. The CFL number for both the URANS and the turbulence model equation(s) starts at a value of 1.0 and is increased by a factor of 1.2 each pseudo-time step until it reaches the maximum value of $10^{3}$. The calculations are continued until all non-linear residuals have decreased at least seven orders of magnitude.

The steady-flow simulation on the fine grid using Roe's scheme with the SST turbulence model showed oscillatory behavior of the lift and drag coefficients. However, after switching to a time-dependent flow simulation with a time step size of $\Delta t=1 \cdot 10^{-4} \mathrm{~s}$, the calculation did eventually converge to a steady-state situation.

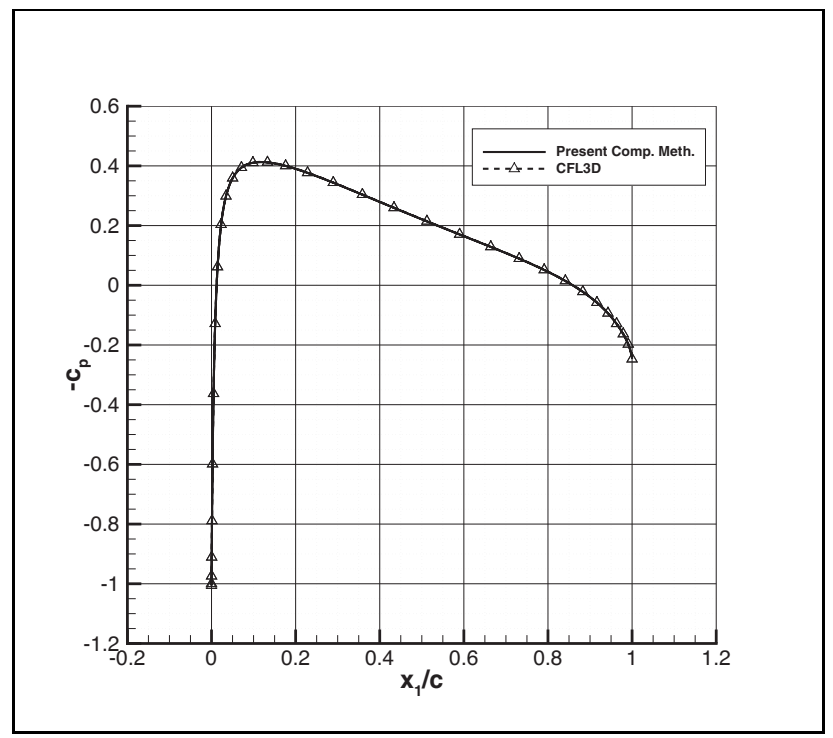

Figure 5.17: Calculated pressure coefficient on medium grid along surface of NACA0012 airfoil using Roe's scheme and the SST turbulence model for $\alpha=0^{\circ}, \mathrm{M}=0.15, \mathrm{Re}_{c}=$ $6 \cdot 10^{6}$ and $T_{\text {ref }}=300 \mathrm{~K}$. Symbols are drawn every eighth point.

Figure 5.17 shows the pressure coefficient, $c_{p}$, along the surface of the airfoil on the 
medium grid using Roe's scheme and the SST turbulence model. The pressure coefficient is a nondimensional quantity defined as

$$
c_{p}=\frac{p-p_{\infty}}{\frac{1}{2} \rho_{\infty} U_{\infty}^{2}},
$$

with $p_{\infty}$ the static free-stream pressure, $\rho_{\infty}$ the free-stream density and $U_{\infty}$ the freestream velocity. As expected for this angle of attack, the pressure distribution is symmetric with respect to the chord line. Very small deviations exist due to iterative errors, but these are not visible on this scale. The same applies to the skin friction coefficient, $c_{f}$ (see equation (5.10) ), which is shown in figure 5.18. The symmetry results in a formally zerolift coefficient, $c_{l}=0$. However, the computed lift coefficient for the SST model on this grid equals $c_{l}=-2.35 \cdot 10^{-6}$, which can be caused by iterative errors or by asymmetry in the provided computational grids. For example, the grid point on the trailing edge of the airfoil is located at $\left(x_{1}, x_{2}\right)=(1.000000000000000 E+00,5.352202629500000 E-08)$ in all grids, whereas the locations of the other points along the airfoil yield an error in the symmetry close to machine accuracy. This gives the airfoil a slightly negative camber, which could therefore also explain the slightly negative lift coefficient.

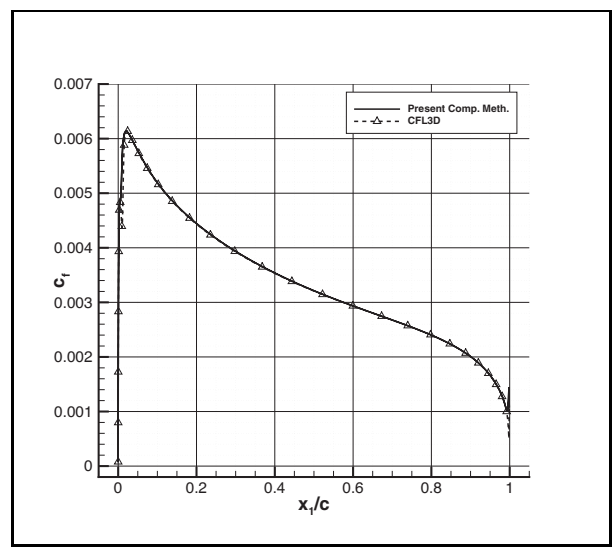

(a) $c_{f}$ along entire surface, symbols drawn every eighth grid point.

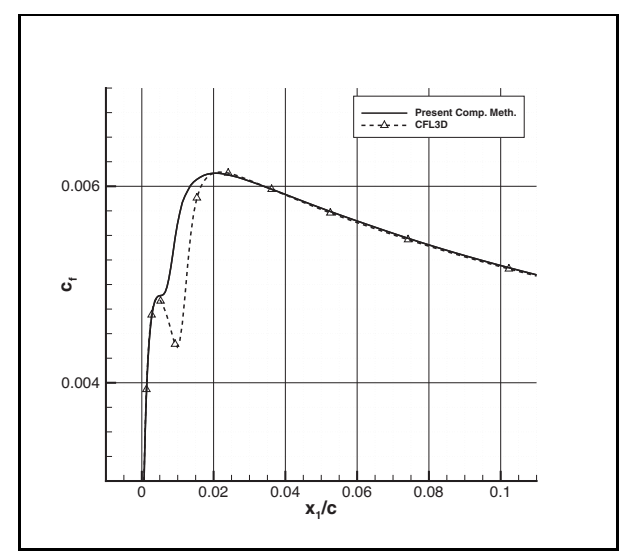

(b) close-up of $c_{f}$ near leading edge, symbols drawn every second grid point.

Figure 5.18: Calculated skin friction coefficient on medium grid along surface of NACA0012 airfoil using Roe's scheme and the SST turbulence model for $\alpha=0^{\circ}$, $\mathrm{M}=0.15, \operatorname{Re}_{c}=6 \cdot 10^{6}$ and $T_{\text {ref }}=300 \mathrm{~K}$.

Figures 5.17 and 5.18 also show that the results obtained with the present computational method compare quite well with the results obtained with CFL3D on the medium grid. There are small differences in the skin friction in the region near the leading edge where transition to a turbulent boundary layer occurs. These differences are likely caused by the use of sustaining terms and different boundary conditions in the present compu- 
tational method. It results in a transition location closer to the leading edge, compared to the results of CFL3D. However, this does not affect the agreement of the skin-friction results downstream of this region.

Tables 5.2(a) and 5.2(b) list the drag coefficient, $c_{d}$, obtained by different computational methods on the medium grid, using the SA turbulence model and the SST turbulence model, respectively. Differences in the drag coefficient produced by the computational methods are small: less than $1 \%$ for both turbulence models. The present results compare well with the results from other computational methods.

\begin{tabular}{|l|c|}
\hline Computational method & $c_{d}$ \\
\hline \hline CFL3D & 0.00819 \\
\hline FUN3D & 0.00812 \\
\hline NTS & 0.00813 \\
\hline SUMB (Stanford U.) & 0.00813 \\
\hline GGNS (Boeing) & 0.00817 \\
\hline Present (Roe's scheme) & 0.00812 \\
\hline Present (JST scheme) & 0.00814 \\
\hline
\end{tabular}

(a) SA turbulence model

\begin{tabular}{|l|c|}
\hline Computational method & $c_{d}$ \\
\hline \hline CFL3D & 0.00809 \\
\hline FUN3D & 0.00816 \\
\hline NTS & 0.00809 \\
\hline Present (Roe's scheme) & 0.00809 \\
\hline
\end{tabular}

(b) SST turbulence model

Table 5.2: Drag coefficient calculated by different computational methods on medium grid for turbulent flow around NACA0012 airfoil at $\alpha=0^{\circ}, \mathrm{M}=0.15, \operatorname{Re}_{c}=6 \cdot 10^{6}$ and $T_{\text {ref }}=300 \mathrm{~K}$.

Tables 5.3 and 5.4 show the results of a grid convergence study for the drag coefficient and skin friction coefficient at $x_{1} / c=0.5121166$, calculated with the present computational method using the SA and SST turbulence models.

The calculations using the SA turbulence model show a non-monotonic convergence behavior for the drag coefficient when Roe's scheme is used, and an apparent order of convergence of 3.34 when the JST scheme is used, i.e. above the nominal order of accuracy of the discretization. The skin friction coefficient at $x_{1} / c=0.5121166$ does show monotonic convergence for both convective flux schemes, but the apparent orders of convergence deviate from the expected value of 2. Therefore, this sequence of grids is probably not entirely in the asymptotic region yet. As expected, the JST scheme yields higher drag (and skin friction) levels in comparison with Roe's scheme, due to the scalar artificial dissipation present in the JST scheme. On the finest grid, however, the results obtained with Roe's scheme and those obtained with the JST scheme are very close, the absolute differences being $\left|\Delta c_{d}\right|=3.2 \cdot 10^{-6}$ and $\left|\Delta c_{f}\right|=5.6 \cdot 10^{-6}$ at $x_{1} / c=0.5121166$.

The calculations using the SST turbulence model show apparent orders of convergence of 1.20 for the drag coefficient and 1.29 for the skin friction coefficient at $x_{1} / c=$ 0.5121166 , again well below the expected value of 2 . It is possible that not all grids are in the the asymptotic region yet for this turbulence model as well. However, it is also possible that the observed convergence behavior is due to conditional statements present 
in the model, in the form of $\max ()$ and $\min ()$ functions (see equations (3.123), (3.124), (3.125) and (3.126) ). The presence of such features in a numerical algorithm is known to hamper grid convergence, as noted by Roache [168] and Eça \& Hoekstra [174] for example.

It can be concluded that the present computational method has difficulty in reaching the formal order of spatial convergence for this flow case, at least for the provided sequence of grids. However, the results compare well with those from other, previously verified, computational methods on the same grid.

\begin{tabular}{|l|c|c|c|}
\cline { 2 - 4 } \multicolumn{1}{c|}{} & $c_{d}$, Roe + SA & $c_{d}$, JST + SA & $c_{d}$, Roe + SST \\
\hline Coarse grid & 0.00813364 & 0.00829061 & 0.00797944 \\
\hline Medium grid & 0.00811915 & 0.00813946 & 0.00809284 \\
\hline Fine grid & 0.00812138 & 0.00812458 & 0.00814232 \\
\hline \hline Apparent order of conv. & - & 3.34 & 1.20 \\
\hline
\end{tabular}

Table 5.3: Drag coefficient calculated on three grids and the apparent order of convergence using the SA and SST turbulence models for turbulent flow around NACA0012 airfoil at $\alpha=0^{\circ}, \mathrm{M}=0.15, \mathrm{Re}_{c}=6 \cdot 10^{6}$ and $T_{\text {ref }}=300 \mathrm{~K}$. Apparent order of convergence based on grid size $h=\sqrt{1 / N}$, with $N$ the number of elements.

\begin{tabular}{|l|c|c|c|}
\cline { 2 - 4 } \multicolumn{1}{c|}{} & $c_{f}$, Roe + SA & $c_{f}$, JST + SA & $c_{f}$, Roe + SST \\
\hline Coarse grid & 0.00320274 & 0.00330930 & 0.00313986 \\
\hline Medium grid & 0.00320682 & 0.00322905 & 0.00317717 \\
\hline Fine grid & 0.00320993 & 0.00321556 & 0.00319241 \\
\hline \hline Apparent order of conv. & 0.39 & 2.57 & 1.29 \\
\hline
\end{tabular}

Table 5.4: Skin friction coefficient at $x_{1} / c=0.5121166$ calculated on three grids and the apparent order of convergence using the SA and SST turbulence models for turbulent flow around NACA0012 airfoil at $\alpha=0^{\circ}, \mathrm{M}=0.15, \mathrm{Re}_{c}=6 \cdot 10^{6}$ and $T_{\text {ref }}=300 \mathrm{~K}$. Apparent order of convergence based on grid size $h=\sqrt{1 / N}$, with $N$ the number of elements.

\subsubsection{Time-dependent flow around circular cylinder}

To verify the correct implementation of the temporal integration scheme for time-accurate calculations, we compute the unsteady, nearly incompressible flow around a circular cylinder for two Reynolds numbers based on the diameter of the cylinder, $\operatorname{Re}_{D}=\frac{\rho_{\infty} U_{\infty} D}{\mu_{\infty}}$. Both cases are characterized by periodic vortex shedding in the wake of the cylinder.

The conditions of the first case are $\operatorname{Re}_{D}=200, \mathrm{M}=0.1, T_{\text {ref }}=273.15 \mathrm{~K}$. This case falls just outside the laminar vortex shedding regime, which ends at approximately $\operatorname{Re}_{D, \text { crit }} \approx 190[175$ ] and is associated with the flow in the wake starting to become 
turbulent and the appearance of spanwise structures. However, many computational results of laminar flow simulations are available for this Reynolds number (e.g. [176, 177, 178, 179, 180]), and therefore we treat this case as a laminar flow and do not use a turbulence model.

The conditions of the second case are $\operatorname{Re}_{D}=5 \cdot 10^{6}, \mathrm{M}=0.2, T_{\text {ref }}=273.15$ $\mathrm{K}$, for which the boundary layer becomes turbulent close to the stagnation point. We choose to employ the SST turbulence model for this case and compare the results with those from Cox et al. [177] (and additional information from the same investigation reported in [181]). Although in reality vortex shedding at higher Reynolds numbers is characterized by three-dimensional shedding modes, both cases are computed as if they were two-dimensional.

A two-dimensional circular computational domain is used, in which the center of the cylinder, with diameter $D$, is located in the origin. The location of the farfield boundary is chosen based on a study by Rumsey et al. [182], which indicates that a farfield grid location of $20 \mathrm{D}$ from the origin results in a shedding-frequency error of approximately $2-3 \%$ compared to an extrapolated result for an infinitely large domain. Based on this study, we locate the farfield boundary at $50 \mathrm{D}$ from the origin, which is assumed to be sufficiently far away. At this farfield boundary, $\rho_{\infty}$ and $\underline{u}_{\infty}$ are fixed for parts of the boundary where the flow is entering the domain, whereas $p_{\infty}$ is fixed where the flow is leaving the domain.

The O-type computational grid used for both cases is shown in figure 5.19. Since we are interested in the verification of the temporal integration scheme, it is a relatively fine grid such that the spatial resolution does not have a large influence on the observed order of temporal convergence. The grid has 256 elements in azimuthal direction: 72 uniformly sized elements in the left half plane and 184 elements in the right half plane, where the elements near $x_{2}=0$ are the smallest and subsequent elements grow in both azimuthal directions with a growth factor $g=1.025744$. In radial direction, the grid has also 256 elements, where the first 100 elements occupy the region up to a diameter of $2 D$ and the remaining 156 elements occupy the region between $2 D$ and $50 D$. The growth factors in the radial direction are $g=1.104602234$ in the first region and $g=1.019885480148$ in the second region.

Franke et al. [176] mention that the distance of the first grid point away from the wall has a strong influence on the results. If this distance is too large, the predicted vortexshedding frequencies are too high. In the present computational grid, the height of the first layer of elements near the wall is $3 \cdot 10^{-6} \mathrm{D}$, roughly one to two orders of magnitude smaller than reported as sufficient for laminar flow cases [176, 177]. For the high-Reynolds number case, the present spacing at the wall is of the same order of magnitude as the one used by Cox et al. [177], who employ $5 \cdot 10^{-6} \mathrm{D}$.

The computational approach for each case is as follows. First, a non-time-accurate (steady state) simulation is carried out to start up the vortex shedding. As soon as the vortex shedding commences, the residuals will show periodic variations and are not reduced any further. Then, a time-accurate simulation is performed for $50 U_{\infty} t / D$ with approximately 20 time steps per shedding cycle. The number of steps per cycle is based 


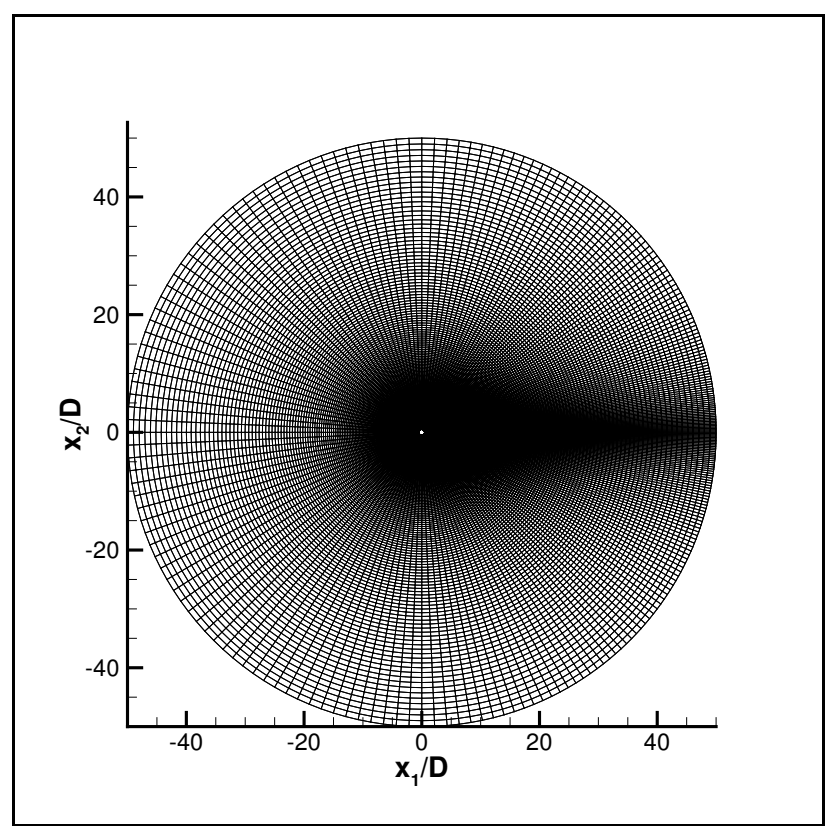

Figure 5.19: Two-dimensional computational grid around circular cylinder.

on the expected approximate result for the dimensionless shedding frequency or Strouhal number, St $=f D / U_{\infty}$, with shedding frequency $f$. Based on results reported in the literature, with which the present results will be compared, we employ an expected shedding frequency of $\mathrm{St}=0.195$ for $\operatorname{Re}_{D}=200$ and $\mathrm{St}=0.3$ for $\mathrm{Re}_{D}=5 \cdot 10^{6}$. Starting from the last known solution of this simulation, several different simulations have been performed with approximately $40,80,160,320$ and 640 time steps per shedding cycle, for $30 U_{\infty} t / D$. The first time step of each simulation uses a first-order accurate Euler-backward scheme, as described in section 4.4.3. Subsequent time steps employ the standard second-order accurate scheme presented in subsection 4.4.1

Roe's flux-difference splitting scheme is employed for the convective fluxes of the URANS equations. A gradient limiter is not used in the solution reconstruction technique. The CFL number is fixed at $10^{5}$ and new time steps are taken after the time-dependent non-linear residuals have decreased four orders of magnitude or as soon as 200 pseudo-time steps have been performed.

Results for $\operatorname{Re}_{D}=200$

Figure 5.20 shows iso-contours of the nondimensional $x_{3}$-component of the vorticity, $\omega_{3} D / U_{\infty}$, in the vicinity of the cylinder, for the simulation with 640 time steps per cycle and a Reynolds number of $\operatorname{Re}_{D}=200$. It can be seen that the periodic vortex shedding leads to the formation of a Von Kármán vortex street in the wake of the cylinder. This 


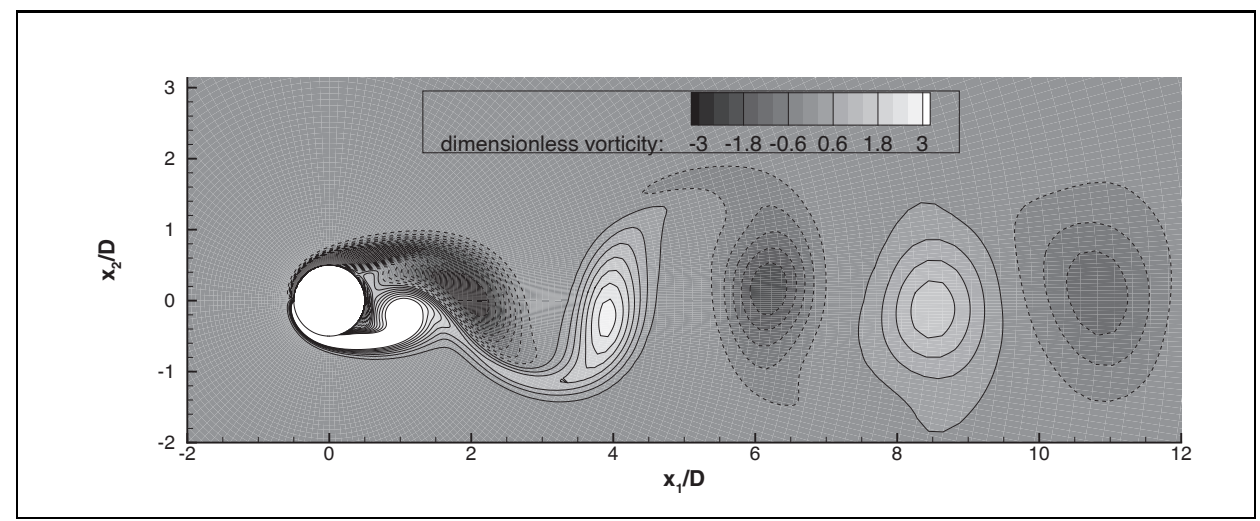

Figure 5.20: Iso-contours of nondimensional $x_{3}$-component of vorticity, $\omega_{3} D / U_{\infty}$, at $U_{\infty} t / D=28.181$ for the simulation at $\operatorname{Re}_{D}=200, \mathrm{M}=0.1, T_{\text {ref }}=273.15 \mathrm{~K}$, with 640 time steps per cycle.

periodic unsteady flow behavior induces a periodic time-dependent lift and drag force on the cylinder, as can be seen in figure 5.21. This figure shows the lift coefficient, $c_{l}$, and drag coefficient, $c_{d}$, during the last computed vortex shedding cycle. The results of all simulations are included, i.e. with approximately 40 time steps per cycle up to approximately 640 time steps per cycle. The results of the simulations with 320 and 640 time steps per cycle are nearly indistinguishable. The Strouhal number can be determined from the period of the lift signal. Note that the frequency of the drag signal is twice that of the lift signal. The drag force is minimal at about the moment the lift is zero, i.e. the case that the wake will be about symmetric and induces a flow towards the cylinder. About the moment the lift is at a maximum or minimum value, the drag is at a maximum value.

Table 5.5 presents the computational results obtained with different computational methods. See table 5.6 for details about the computations and computational methods from the cited literature. The presented results include the amplitude of the lift coefficient, the mean drag coefficient and its amplitude, and the Strouhal number. The results obtained with the present computational method have been determined by fitting a spline (third-order accurate interpolation) through the last cycle of the time-dependent lift and drag coefficients and determining the amplitudes and shedding frequency from the obtained peak values and their occurrence in time. This method circumvents problems due to peak values not coinciding with actual computed instances in time.

Table 5.5 also includes the extrapolated values for $\Delta t \rightarrow 0$ and the apparent order of convergence of all quantities. Note that the amplitude of the lift coefficient does not converge monotonically for all simulations. Therefore, the extrapolated value and apparent order of convergence of this quantity are based on the results obtained with 160 , 320 and 640 time steps per cycle. For the other quantities, they are based on the results obtained with 40, 160 and 640 time steps per cycle. 


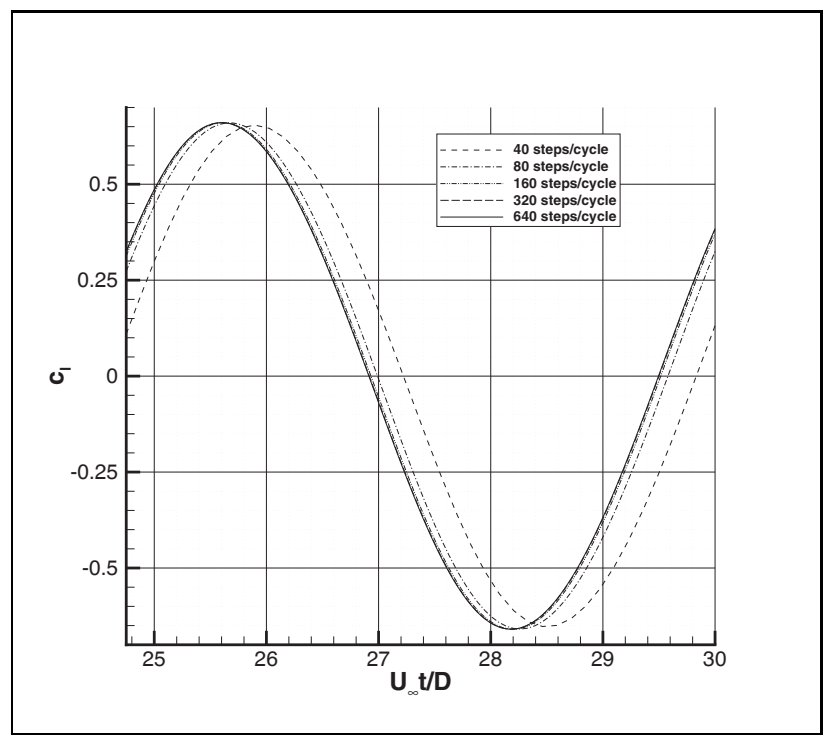

(a) lift coefficient $c_{l}$

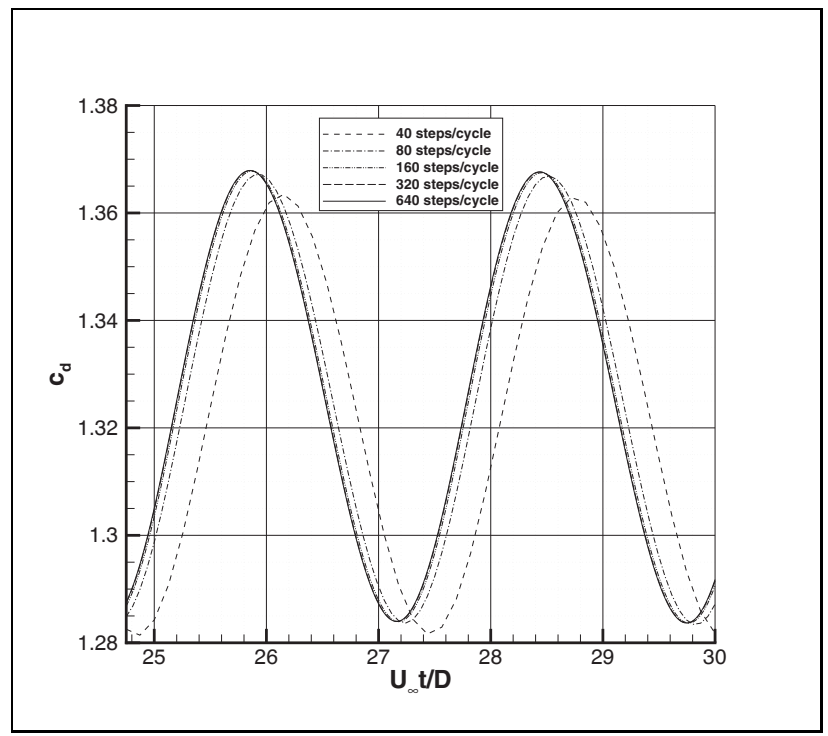

(b) drag coefficient $c_{d}$

Figure 5.21: Calculated force coefficients during one shedding cycle in periodic part of results for unsteady, two-dimensional, laminar flow past a circular cylinder at $\operatorname{Re}_{D}=200$, $\mathrm{M}=0.1$ and $T_{\text {ref }}=273.15 \mathrm{~K}$, using different time step sizes. 


\begin{tabular}{|l|l|l|l|}
\hline Reference & $c_{l}$ & $c_{d}$ & St \\
\hline \hline Cox et al. [177] & not avail. & $\approx 1.36$ & $\approx 0.192$ \\
\hline Franke et al. [176] & \pm 0.65 & 1.31 & 0.194 \\
\hline Liu et al. [178] & \pm 0.69 & $1.31 \pm 0.049$ & 0.192 \\
\hline Rajani et al. [179] & $\pm \approx 0.61$ & $1.3365 \pm \approx 0.042$ & 0.1957 \\
\hline Ding et al. [180] & \pm 0.60 & $1.327 \pm 0.045$ & 0.196 \\
\hline \hline Present Comp. Method & $c_{l}$ & $c_{d}$ & St \\
\hline \hline$\approx 40$ steps/cycle & \pm 0.654039 & $1.32253 \pm 0.04096$ & 0.1915 \\
\hline$\approx 80$ steps/cycle & \pm 0.660043 & $1.32545 \pm 0.04177$ & 0.1932 \\
\hline$\approx 160$ steps/cycle & \pm 0.660439 & $1.32586 \pm 0.04193$ & 0.1936 \\
\hline$\approx 320$ steps/cycle & \pm 0.660407 & $1.32592 \pm 0.04196$ & 0.1937 \\
\hline$\approx 640$ steps/cycle & \pm 0.660398 & $1.32594 \pm 0.04197$ & 0.1937 \\
\hline Extrapolated value & \pm 0.660394 & $1.32594 \pm 0.04197$ & 0.1937 \\
\hline Apparent order of conv. & 1.83 & $2.69,2.30$ & 2.20 \\
\hline
\end{tabular}

Table 5.5: Lift coefficient, drag coefficient and Strouhal number for unsteady, incompressible or nearly incompressible laminar flow past a circular cylinder at $\operatorname{Re}_{D}=200$. Extrapolated values and apparent orders of convergence are based on results obtained with 40, 160 and 640 steps/cycle, except for those of the amplitude of $c_{l}$, which are based on results obtained with 160, 320 and 640 steps/cycle. Approximate values have been extracted from graphs.

The time step refinement study shows that all quantities have an observed order of convergence close to the expected value of 2. The values of the studied quantities for all simulations are very close to the corresponding extrapolated values, even for 40 time steps per cycle. The difference in the Strouhal number is only $1.1 \%$ for example. However, the amplitude of $c_{l}$ needs more time steps per cycle in order to be in the asymptotic region than the other quantities listed in table 5.5.

Since the results from the literature have been obtained with different methods using different spatial and temporal resolutions, exact comparisons are difficult to make. In general, the results from the present computational method are within the bandwidth of the results from the literature. All results for the Strouhal number presented in table 5.5 are approximately within $2 \%$ of each other. For the time-averaged drag coefficient this value is $4 \%$, whereas for the amplitudes of the lift and drag coefficient, this value is $15 \%$.

Based on the present results of the time step refinement study, it might be that not all the results from the literature are time-step converged. For example, the results from Liu et al. [178] have been obtained with a second-order accurate time stepping scheme and 40 time steps per cycle. Furthermore, Franke et al. [176] employ a first-order accurate time integration scheme, but use only 100 time steps per cycle.

Furthermore, probably not all results are grid converged. For example, a grid refinement study by $C o x$ et al. [177] at $\operatorname{Re}_{D}=1000$ (laminar flow) and $\operatorname{Re}_{D}=5 \cdot 10^{6}$ 
Chapter 5. Verification and Validation

\begin{tabular}{|l|l|}
\hline Reference & Details \\
\hline \hline Cox et al. [177] & $\begin{array}{l}\text { compressible URANS }(\mathrm{M}=0.2), \mathcal{O}\left(\Delta x^{2}, \Delta t^{2}\right), \\
197 \times 97 \text { grid points, farfield at } 20 D \text { from center, } \\
500-600 \text { steps/cycle }(6 \text { subiterations per time step) }\end{array}$ \\
\hline Franke et al. [176] & $\begin{array}{l}\text { incompressible URANS, } \mathcal{O}\left(\Delta x^{3}, \Delta t\right), \\
140 \times 144 \text { grid points, farfield at } 20 D \text { from center, } \\
\text { approximately } 100 \text { steps } / \text { cycle }\end{array}$ \\
\hline Liu et al. [178] & $\begin{array}{l}\text { incompressible URANS, } \mathcal{O}\left(\Delta x^{2}, \Delta t^{2}\right), \\
256 \times 256 \text { grid points, unknown farfield location, } \\
\text { approximately 40 steps/cycle }\end{array}$ \\
\hline Rajani et al. [179] & $\begin{array}{l}\text { incompressible URANS, } \mathcal{O}\left(\Delta x^{2}, \Delta t^{2}\right), \\
144 \times 121 \text { grid points, farfield at } 20 D \text { from center, } \\
\text { approximately } 100 \text { steps/cycle }\end{array}$ \\
& $\begin{array}{l}\text { incompressible URANS (stream function-vorticity formulation), } \\
\mathcal{O}\left(\Delta x^{2}, \Delta t^{4}\right), \text { hybrid conventional } / \text { mesh-free FD method, } \\
\text { rectangular domain with farfield at } 30 D \text { (downstream), } \\
8 D(\text { upstream) and } 16 D \text { (cross-stream) from center, } \\
\text { steps/cycle based on stability of RK4 explicit time integration }\end{array}$ \\
\hline
\end{tabular}

Table 5.6: Computational details for simulations by different authors for time-dependent flow around a circular cylinder at $\operatorname{Re}_{D}=200$. Details for Cox et al. [177] also apply to the case of $\operatorname{Re}_{D}=5 \cdot 10^{6}$.

(turbulent flow) shows that the error in the computed Strouhal number on their standard grid with $197 \times 97$ points and the farfield boundary at $20 D$ from the center is within $4 \%$ of the extrapolated value for an infinitely refined grid, whereas a finer grid $289 \times 145$ grid yields an error of $2 \%$. If we assume that these values are also representative for $\operatorname{Re}_{D}=200$, then an error margin of $3 \%$ to their result yields a Strouhal number of $\mathrm{St}=0.192 \pm 0.06$. This error margin is larger than the differences between the results listed in table 5.5 .

Since the purpose of the present investigation is the verification of the temporal integration scheme, a grid refinement study is not considered here. Instead, it can be concluded that the temporal integration scheme of the URANS equations is correctly implemented: second-order accuracy in time is obtained and the results on the present computational grid are within the bandwidth of the results reported in the literature.

Results for $\operatorname{Re}_{D}=5 \cdot 10^{6}$

For the case of $\operatorname{Re}_{D}=5 \cdot 10^{6}$, the URANS equations are solved in combination with the equations of the SST turbulence model. Here, we employ the sustaining terms of the turbulence model, see subsection 3.4 .3 together with the recommended inflow boundary conditions given in subsection 3.5.2. The purpose of this case is to verify whether the addition of a turbulence model still yields the expected temporal accuracy. 


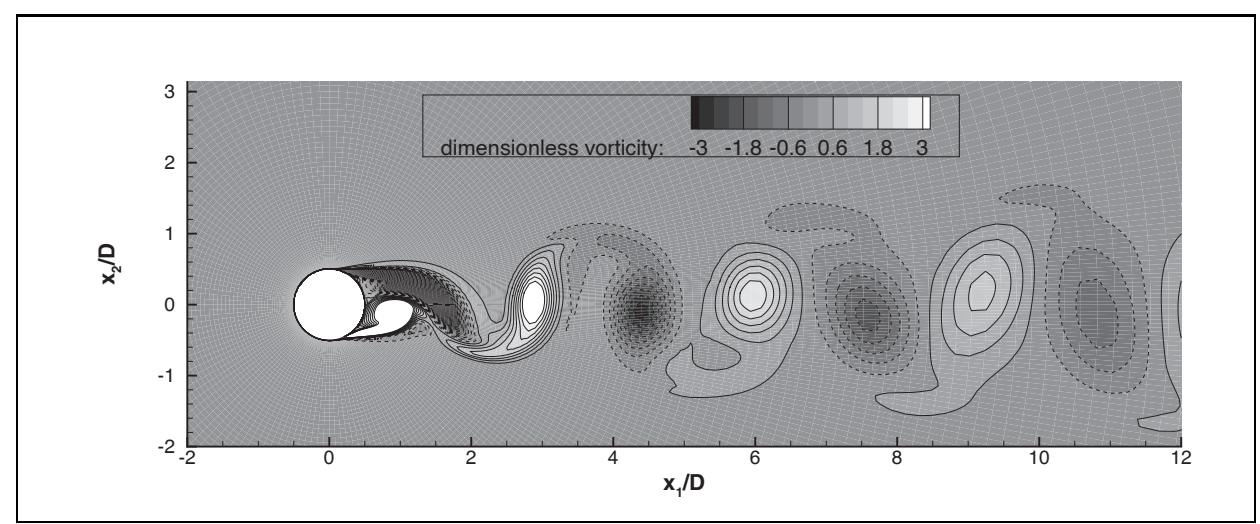

Figure 5.22: Iso-contours of nondimensional $x_{3}$-component of vorticity, $\omega_{3} D / U_{\infty}$, at $U_{\infty} t / D=26.724$ for the simulation at $\operatorname{Re}_{D}=5 \cdot 10^{6}$ with 640 time steps per cycle.

Figure 5.22 shows iso-contours of the nondimensional $x_{3}$-component of the vorticity, $\omega_{3} D / U_{\infty}$, in the vicinity of the cylinder, for the simulation with 640 time steps per cycle and a Reynolds number of $\operatorname{Re}_{D}=5 \cdot 10^{6}$. Again, a Von Kármán vortex street is observed in the wake of the cylinder. In comparison with the case of $\operatorname{Re}_{D}=200$, see figure 5.20 . the shear layer on the surface of the cylinder is much thinner and the wake is narrower for the higher Reynolds number because the turbulent boundary layer separates further downstream. Figure 5.23 shows the lift coefficient, $c_{l}$, and drag coefficient, $c_{d}$, for all employed time step sizes during the last computed vortex shedding cycle. The results of the simulations with 320 and 640 time steps per cycle are nearly indistinguishable.

Table 5.7 presents the computational results obtained with the present computational methods and those from Cox et al. [177, 181]. The details about the computations and computational method used by Cox et al. can be found in table [5.6] As for the case of $\operatorname{Re}_{D}=200$, the results obtained with the present computational method have been determined by fitting a spline (third-order accurate interpolation) through the last cycle of the time-dependent lift and drag coefficients and determining the amplitude and shedding frequency from the obtained peak values and their occurrence in time. The values of the studied quantities for all simulations are very close to the corresponding extrapolated values, even for 40 time steps per cycle. The difference in the Strouhal number is only $1.3 \%$, whereas it is $2.2 \%$ in the amplitude of the lift coefficient. The time step refinement study shows that most quantities have an observed order of convergence close to the expected value of 2 . The observed order of convergence of the amplitude of the drag coefficient equals 1.59 , which is lower than expected but still well above first order accuracy. This quantity also needs more time steps per cycle to be in the asymptotic region than the other quantities.

When we compare the results of Cox et al. [181, 177] with the present results, excellent agreement is observed for the Strouhal number. However, a large difference in the amplitude of the lift coefficient is observed: the value of Cox et al. is approximately twice 


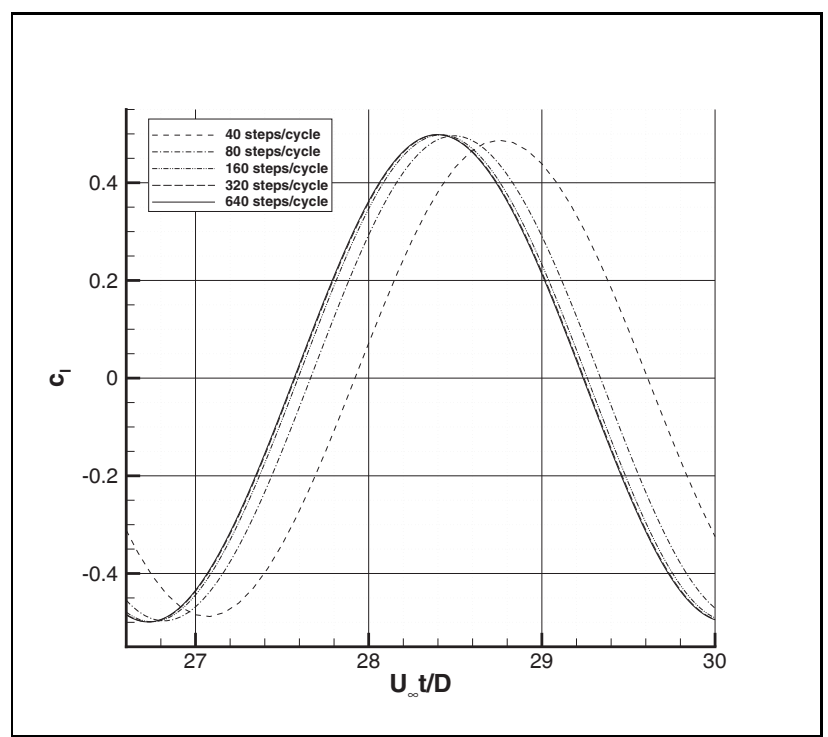

(a) lift coefficient $c_{l}$

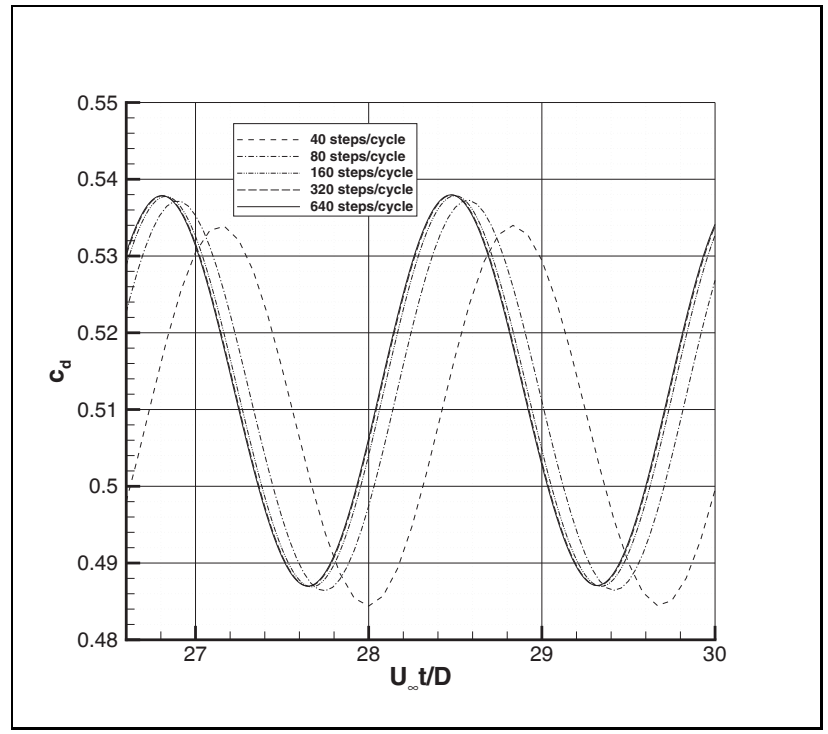

(b) drag coefficient $c_{d}$

Figure 5.23: Calculated force coeff. during one shedding cycle in periodic part of results for unsteady, two-dimensional, turbulent flow past a circular cylinder at $\operatorname{Re}_{D}=5 \cdot 10^{6}$, $\mathrm{M}=0.2, T_{\text {ref }}=273.15 \mathrm{~K}$, using different time step sizes and the SST turbulence model. 


\begin{tabular}{|l|l|l|l|}
\hline Reference & $c_{l}$ & $c_{d}$ & St \\
\hline \hline Cox et al. [177, 181$]$ & $\approx \pm 1.0$ & $\approx 0.687$ & $\approx 0.3$ \\
\hline \hline Present Comp. Method & $c_{l}$ & $c_{d}$ & St \\
\hline \hline$\approx 40$ steps/cycle & \pm 0.487293 & $0.509198 \pm 0.0247915$ & 0.2957 \\
\hline$\approx 80$ steps/cycle & \pm 0.496261 & $0.511860 \pm 0.0254080$ & 0.2985 \\
\hline$\approx 160$ steps/cycle & \pm 0.498131 & $0.512414 \pm 0.0254515$ & 0.2993 \\
\hline$\approx 320$ steps/cycle & \pm 0.498434 & $0.512505 \pm 0.0254455$ & 0.2995 \\
\hline$\approx 640$ steps/cycle & \pm 0.498478 & $0.512521 \pm 0.0254435$ & 0.2995 \\
\hline Extrapolated value & \pm 0.498489 & $0.512525 \pm 0.0254425$ & 0.2995 \\
\hline Apparent order of conv. & 2.48 & $2.45,1.58$ & 2.08 \\
\hline
\end{tabular}

Table 5.7: Lift coefficient, drag coefficient, and Strouhal number for unsteady turbulent flow past a circular cylinder at $\operatorname{Re}_{D}=5 \cdot 10^{6}, \mathrm{M}=0.2$. Extrapolated values and apparent orders of convergence are based on results obtained with 40, 160 and 640 steps/cycle, except for those of the amplitude of $c_{d}$, which are based on results obtained with 160 , 320 and 640 steps/cycle. Approximate values have been extracted from graphs.

as large. Additionally, the average drag coefficient obtained by Cox et al. is approximately $30 \%$ higher than the value obtained with the present method. Differences in, for example, computational domain, grid and boundary conditions can lead to differences in results. Preliminary flow simulations with lower free-stream turbulence levels yield results for the lift and drag coefficient closer to those of Cox et al. The results are therefore sensitive to the inlet boundary conditions for the SST turbulence model, possibly in combination with the sustaining terms. The employed boundary conditions and possible use of sustaining terms by Cox et al. are unknown.

From these results it can be concluded that the combined temporal integration scheme of the URANS equations and the turbulence equations is correctly implemented, since the observed order of convergence is close to the theoretical value of 2 . However, more results together with detailed information about the computational setup from other investigations are needed to verify the absolute values obtained for $\operatorname{Re}_{D}=5 \cdot 10^{6}$ with more certainty.

\subsubsection{Conclusions}

The combined results of the verification cases presented in this section show that the numerical implementation of the mathematical models is correct.

All steady-flow cases show good comparison between the results obtained with the present computational method and those obtained with other, previously verified, computational methods. The verification cases also show that the formal order of spatial convergence can be difficult to obtain in reality, even for previously verified computational methods. It has been shown, for example, that the presence of a singularity in the simulated flow can hamper grid convergence. The removal of this disturbance improves 
the convergence behavior. For a case without such a singularity, turbulent flow around a NACA0012 airfoil, it is also difficult to obtain the formal order of spatial convergence. Here, it it suspected that the provided sequence of computational grids is not in the asymptotic region yet. Furthermore, the presence of conditional statements in the SST turbulence model can also hamper grid convergence [168, 174].

The two cases of unsteady-flow around a circular cylinder both reveal a temporal order of convergence close to the expected value of 2. Good agreement has been obtained for the Strouhal number of vortex shedding with results found in the literature. However, for the high-Reynolds number case there are insufficient results from independent researchers available, with well-documented boundary conditions, to verify the obtained solution.

The effects of multigrid and domain decomposition have been illustrated for a steady laminar flow over a flat plate. The results for this particular case show that

- although the multigrid method is applied separately to each grid partition, the number of pseudo-time steps does not increase dramatically with increasing number of grid partitions,

- measured in terms of wall clock time, the algebraic multigrid method is approximately five times faster than the standard Gauß-Seidel iteration method for a reduction of the residuals by five orders of magnitude,

- the parallelization method shows adequate speed-up factors, for grid partitions with at least 10,000 control volumes. When less than 10,000 control volumes per partition are used, the efficiency deteriorates due to the increasing importance of the communication between grid partitions and the use of Jacobi iteration on the boundaries of the grid partitions.

\subsection{Validation}

\subsubsection{General Remarks}

A verified computational method does not necessarily provide correct physical results for all types of flows. An important reason for this is that the equations that provide closure for the (U)RANS equations, i.e. the linear eddy-viscosity models and the Boussinesq assumption on which they are based, are not general enough to provide real physical behavior in all situations, see also subsection 3.3.5.

The eddy-viscosity turbulence models used in this work are aimed at aerodynamic problems, and the constants in these models are tuned to a pool of these problems. The selected turbulence models, i.e. SA and SST, are among the turbulence models that are better at predicting pressure induced flow separation [92].

Bardina et al. [87] have performed an evaluation of different turbulence models for flows ranging from simple free-shear and zero-pressure-gradient boundary layer flows to complex flows involving flow separation. The SST model is judged to be the best overall model, because of its performance for flows with separation and giving results comparable 
with the best of the other models for simple flows. They rate the SA model as second best. The SA model also needs less elements in the boundary layer regions for accurate solutions compared with other models. For compressible turbulent mixing layers, all tested linear eddy-viscosity models fail to predict the correct decrease of the spreading rate with increasing convective Mach number, $\mathrm{M}=\frac{U_{1}-U_{2}}{c_{1}+c_{2}}$, with $U_{1}$ and $U_{2}$ the free-stream velocities, and $c_{1}$ and $c_{2}$ the speed of sound in the free-streams making up the mixing layer.

Zingg \& Godin [183] have performed an evaluation of different turbulence models for high-lift multi-element airfoil configurations, ranging from one-equations models to algebraic Reynolds-stress models. They conclude that in their experience, the SA turbulence model is the best option for many aerodynamic flows, including attached and mildly separated flows, because of its overall good prediction capabilities combined with simplicity and low computational cost. For flows with large-scale separation, the SST model seems to perform better.

Zingg \& Godin [183] also mention that the prediction of transition from a laminar to a turbulent boundary layer is the most significant factor limiting accurate prediction of aerodynamic flows. Laminar-turbulent transition is a result of complex phenomena, such as the receptivity of the laminar boundary layer to external disturbances, which can lead to natural transition (due to instabilities predicted by linear stability theory), or the more rapid bypass transition (due to direct non-linear phenomena) [184]. Additionally, separation of the laminar boundary layer can also induce transition to turbulence, which can cause the shear layer to reattach [185]. Transition can probably only be more accurately predicted by incorporating additional models, such as the $\gamma-\operatorname{Re}_{\theta}$ model [186], which is a local correlation-based model with transport equations for intermittency, $\gamma$, and transitional momentum thickness Reynolds number, $\operatorname{Re}_{\theta}$. This model is relatively easy to implement in a general-purpose (U)RANS method, but some of the necessary correlations are proprietary information and not in the public domain at the moment.

Besides the already mentioned limitations of all eddy-viscosity turbulence models, specific limitations of the turbulence models used in this work include:

- the overprediction of the extent of separated flow regions, which appears to be caused by underprediction of turbulent stresses in detached shear layers [92],

- the overprediction (mainly by the SA model) of spreading rates of jet-like free shear regions (plane jet: $\mathrm{SA}+36 \%$; round jet: $\mathrm{SA}+180 \%$, SST $+40 \%$ ) [87], see also subsection 3.4 .1

- the underprediction by the SST model of spreading rates of plane wakes $(-30 \%)$ [87],

- the models are not coordinate-invariant since both models need the distance to the nearest no-slip wall [87].

The following subsection presents a validation study of flows with boundary layer separation control, by means of steady suction and synthetic jet actuation through a 
spanwise slit. These types of flow are not among the standard range of validated flow cases.

\subsubsection{Validation of Flows with Boundary Layer Separation Control}

In 2004, the NASA Langley Research Center organized a workshop, CFDVAL2004, with the purpose to assess the capabilities of computational methods for simulation of turbulent flows to predict flow fields induced by synthetic jets and separation control geometries. The workshop included three cases, of which "Case 3: Flow over a Hump Model" is considered here. This case studies the control of boundary layer separation and includes three subcases: a baseline case without flow control, a case with steady suction through a two-dimensional slit and a case with zero mass-flux oscillatory flow control, i.e. synthetic jet actuation, through the same slit. The purpose of the controlled flow cases is to reduce the extent of the separated flow region behind the hump model.

An experimental setup of this problem was tested in a wind tunnel [187, 188, 189]. As in the workshop, the experimental results are used here to validate the computational results. The SA turbulence model is used to obtain the present computational results. Additionally, results obtained with different turbulence models will be described briefly, see for example the summary of all submitted results [172] or the workshop website [171].

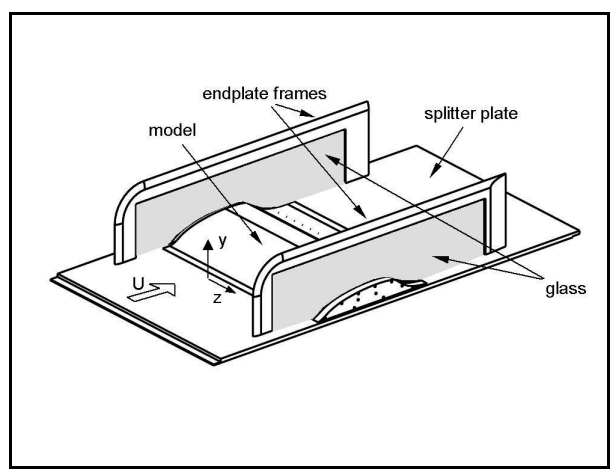

(a) Three-dimensional sketch

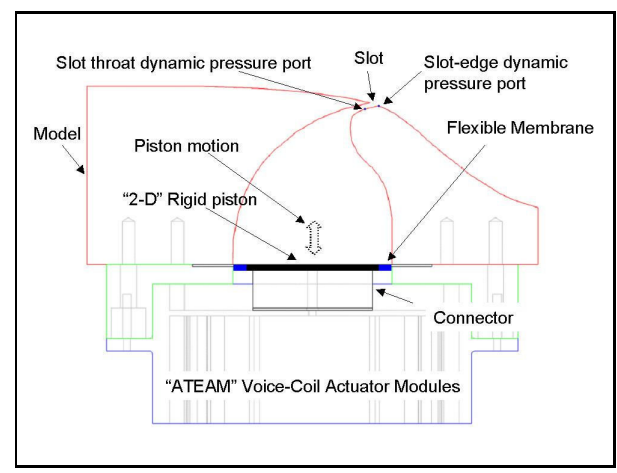

(b) Cross section near slit, with cavity and actuators

Figure 5.24: Wall-mounted hump model used in experiments for CFDVAL2004, from [171].

The wall-mounted hump model used in the experiments is shown in figure 5.24(a). It consists of a relatively long forebody and a relatively short concave ramp forming the aft part. The total chord length of the model is $c=42.00 \mathrm{~cm}$ and its maximum height is $h=5.375 \mathrm{~cm}$. The span of the model is $58.42 \mathrm{~cm}$ and end plates are located at both ends to improve the two-dimensionality of the flow near the centerline of the span of the model. The height of the test section, measured from the upper surface of the 
splitter plate, is $38.18 \mathrm{~cm}$. A two-dimensional slit, with a vertical opening below the lip of $h_{s}=0.7854 \mathrm{~mm}$, is located at $x_{1} / c \approx 0.655$, where the $x_{1}$-direction is the flow direction and $x_{1} / c=0$ corresponds to the leading edge of the hump. The slit is connected to a cavity below the surface of the model. Figure [5.24(b) shows a cross-section of the model near the slit, with the cavity and a synthetic jet actuator included. A row of six of these voice-coil-based actuators drive a rigid spanwise piston, which is secured to the base of the cavity by means of a flexible membrane and flange. The base of the cavity is 5.0801 $\mathrm{cm}$ wide in chordwise direction and covers the full span of the model. This set-up yields maximum jet velocities around $80 \mathrm{~m} / \mathrm{s}$ at frequencies between 60 and $500 \mathrm{~Hz}$. Continuous suction is achieved by removing the piston and connecting a vacuum pump to the base of the cavity. The model is equipped with 153 center-span static pressure ports, and 16 spanwise static pressure ports at $x_{1} / c=0.19$ and $x_{1} / c=0.86$. These ports have a diameter of $0.5 \mathrm{~mm}$. Additionally, 20 pressure transducers capable of measuring unsteady pressures are located in the separated flow region behind the hump. Each transducer is mounted $1 \mathrm{~mm}$ below an orifice with a diameter of $0.25 \mathrm{~mm}$.

Besides pressure measurements on the surface of the hump model and the region behind it, measurements with a Pitot probe and a hot-wire anemometer were performed to determine the velocity profile at $x_{1} / c=-2.14$. Furthermore, two-dimensional particle image velocimetry (PIV) measurements were performed in a plane along the model centerline, normal to the surface. Stereoscopic (three-dimensional) PIV measurements were performed in planes perpendicular to the 2D PIV plane, mainly to assess possible spanwise variations of the flow field. Finally, for the baseline case without flow control, the shear stress on the surface of the model was measured using oil-film interferometry, see Naughton et al. [189] for more information. This technique was also used to determine the extent of the separated flow region behind the hump model.

Experimental and computational results will be compared for a Reynolds number of $\operatorname{Re}_{c}=9.36 \cdot 10^{5}$, a free-stream Mach number of $\mathrm{M}_{\infty}=0.1$, and a reference temperature of $T_{\text {ref }}=298 \mathrm{~K}$, which corresponds to a free-stream velocity of $U_{\infty}=34.6 \mathrm{~m} / \mathrm{s}$. For the baseline case without actuation, the slit is left open. Steady suction through the slit is considered at a mass flow rate of $0.01518 \mathrm{~kg} / \mathrm{s}$, which corresponds to 0.51149095 $\mathrm{kg} / \mathrm{m}^{2} \cdot \mathrm{s}$ at the base of the cavity. Furthermore, synthetic jet actuation is considered at a frequency of $f_{j}=138.5 \mathrm{~Hz}$ with a peak velocity of $\left|\underline{u}_{p}\right|=26.6 \mathrm{~m} / \mathrm{s}$. This peak velocity is measured during the ejection phase in the potential core of the jet, at a location at the exit of the slit, on an imaginary line level with the hump surface. It is interesting to note that both actuated cases do not entirely prevent flow separation.

The numerical flow simulations for all three subcases have been performed on the same two-dimensional computational grid. This grid was constructed and used by Rumsey [190] for his URANS simulations of this workshop case and has been made available through the workshop website ("structured 2d grid \#4") [171].

The computational domain employed here is different from the experimental situation in the sense that the bottom wall of the wind tunnel test section is not included. Instead, the splitter plate is extended upstream to $x_{1} / c=-6.39$, where the entrance of the domain is placed. This extension was found in preliminary simulations to yield a boundary layer 
thickness that approximately matches that of the experiment [171]. The splitter plate is also extended downstream, with the exit of the domain located at $x_{1} / c=4$. Furthermore, the shape of the upper wind tunnel wall has been adjusted, i.e. it has been slightly lowered above the model, which approximately accounts for the effect of side-plate blockage.

Figure 5.25 shows a coarse version of a part of the grid in the region of the hump model (every fourth line is shown). The total number of elements is 208320; the cavity has 120 elements along its bottom and 224 elements in the other direction, whereas the main flow domain has 216 elements in the direction normal to the wall and 840 elements in the flow direction. A grid refinement study showed that the resolution of this grid

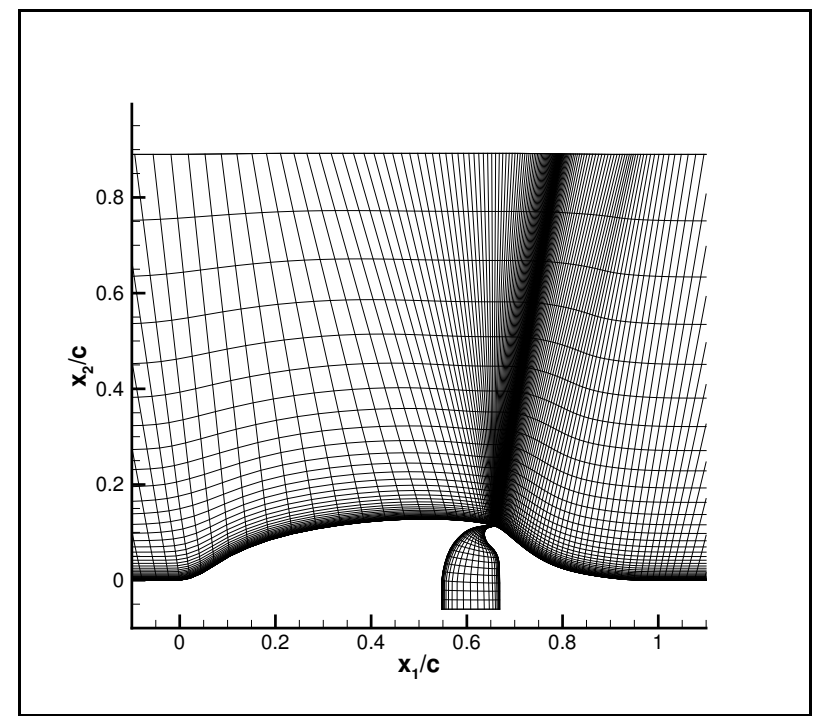

Figure 5.25: Detail of computational grid for the wall-mounted hump model; every fourth line is shown.

is more than sufficient [190]. Although it is a two-dimensional grid, it has one layer of elements in the third dimension, i.e. the grid consists of hexahedral elements. For the present computations, this layer is removed such that the grid consists of quadrilaterals only.

The upper wall is treated as a slip wall (symmetry boundary condition), i.e. the boundary layer is not resolved there. At the entrance of the domain, the density, the two velocity components, and the turbulence variable $\left(\tilde{\nu}=3 \nu_{\infty}\right)$ are prescribed. At the exit of the domain, the pressure is prescribed. The lower wall, including the hump, and the side walls of the cavity are adiabatic no-slip walls. On the bottom of the cavity, a symmetry boundary condition is applied for the case without flow control. For the case with steady suction, the $x_{2}$-component of the velocity is prescribed such that the necessary mass flow rate is obtained (the density is extrapolated), whereas for the case with synthetic jet actuation, the unsteady boundary conditions given in section 3.5 .3 are 
applied (see equations (3.150) and (3.151)). The employed velocity amplitude, $U_{n}$, is chosen as $U_{n}=0.001 c_{\infty}$, with $c_{\infty}$ the free-stream speed of sound. This is based on a suggestion of Rumsey [190].

The present computational results are calculated using Roe's flux-difference splitting scheme for the convective fluxes of the URANS equations. Furthermore, the SA turbulence model is used in all computations. Instead of the weighted least-squares method to calculate the gradients, the discrete version of Gauß' divergence theorem is used, see equation (4.85). Steady-flow simulations using the least-squares gradients showed only three orders of magnitude of residual reduction and yielded erratic behavior of the skin friction coefficient in the region of the top of the hump. The cause of this is not known; it may be due to some small unsteadiness of the flow. However, the present computational results obtained with the alternative gradients show good convergence of the residuals and a smooth behavior of the skin friction coefficient.

The first two flow simulations, the baseline case and the case with steady suction, are steady-flow simulations. Both are initialized with the free-stream conditions, and the non-linear residuals are decreased at least six orders of magnitude. The simulation for the case with synthetic jet actuation is an unsteady flow simulation, which is started from the converged steady-flow solution of the baseline case without actuation. This simulation uses 400 physical time steps per actuation cycle. It turns out that approximately 30 actuation cycles need to be simulated before the solution becomes periodic. Postprocessing is performed on the results from one cycle in the periodic part of the results.

\section{Results for Baseline Case}

First, the results for the baseline case without flow control are presented. Figure 5.29 shows computational and experimental results for the streamwise velocity profile at $x_{1} / c=$ -2.14 . Two sets of experimental results are shown, one with and one without the synthetic jet actuators mounted in the wind tunnel. As can be seen, the presence of the actuators below the hump model and splitter plate causes differences in the boundary layer profile, due to different blockage of the flow in the wind tunnel. The computationally obtained boundary layer thickness equals $\delta_{99} / c=0.0593$, whereas the experimentally obtained boundary layer thicknesses are $\delta_{99} / c=0.0698$ and $\delta_{99} / c=0.0546$ for the case with and without the actuators present in the wind tunnel, respectively. Since the computational results are in between the two experimentally obtained results, they are accepted as sufficiently accurate. Furthermore, this confirms that the combination of the chosen location of the entrance of the computational domain and the applied boundary conditions at the inlet is adequate.

Figures 5.26(a) and 5.26(b) show the computational and experimental results for the distribution of the surface pressure coefficient and the skin friction coefficient, respectively. The computational method using the SA turbulence model predicts the pressure distribution well, except for the region where the flow has separated, behind the location of the slit. Here, the level as well as the shape of the pressure distribution are different. The bump in the pressure coefficient of the experimental results near the suction peak is the result of a joint in the experimental setup, between the forebody and the upper lip 


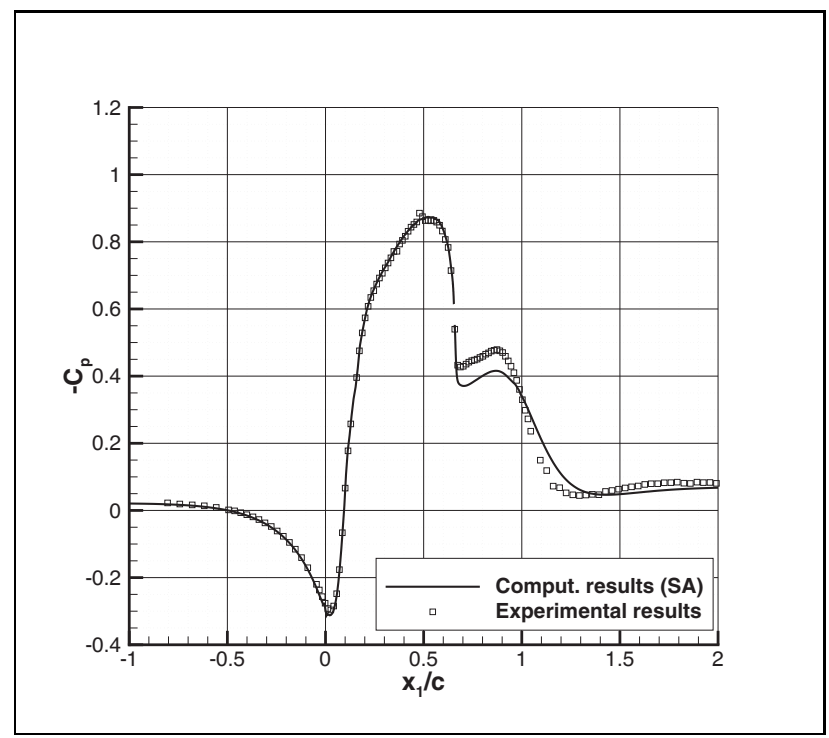

(a) pressure coefficient $c_{p}$

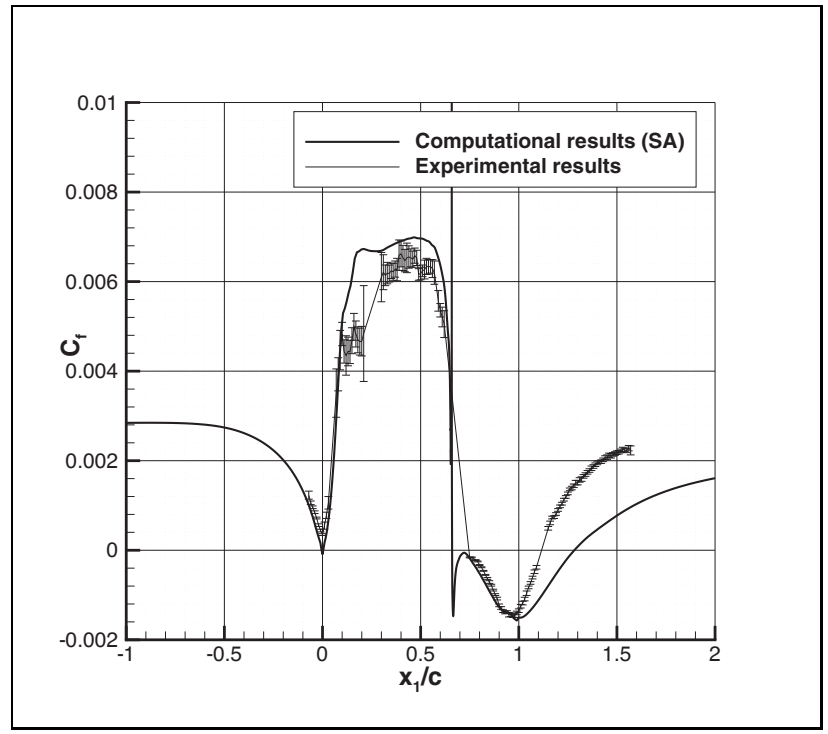

(b) skin friction coefficient $c_{f}$

Figure 5.26: Experimental and computational results for flow over a hump model without actuation (baseline case). $\operatorname{Re}_{c}=9.36 \cdot 10^{5}, \mathrm{M}_{\infty}=0.1$ and $T_{\text {ref }}=298 \mathrm{~K}$. 


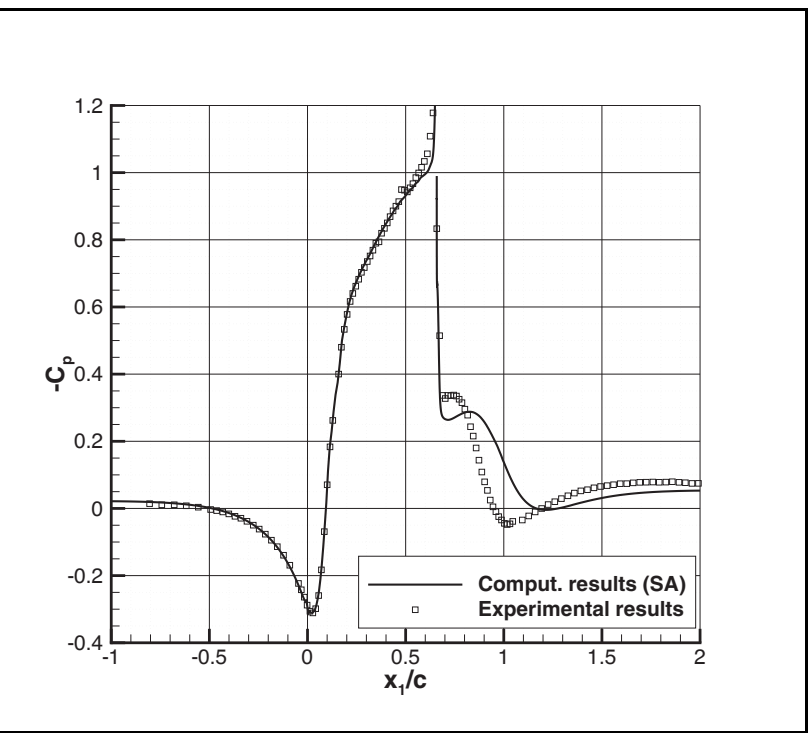

(a) pressure coefficient $c_{p}$

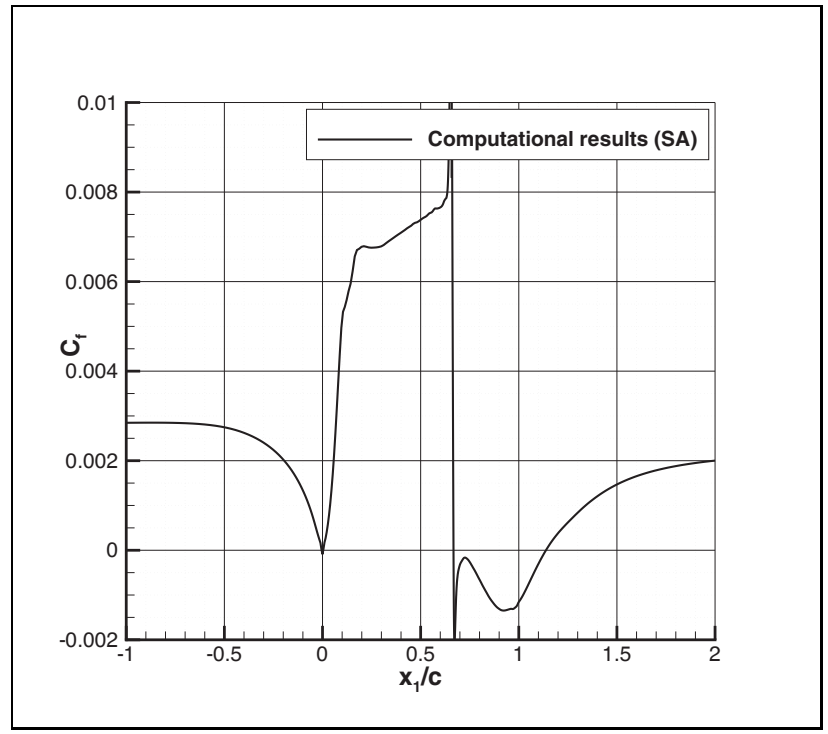

(b) skin friction coefficient $c_{f}$

Figure 5.27: Experimental and computational results for flow over a hump model with steady suction through slit $(0.01518 \mathrm{~kg} / \mathrm{s}) . \mathrm{Re}_{c}=9.36 \cdot 10^{5}, \mathrm{M}_{\infty}=0.1$ and $T_{\text {ref }}=298$ $\mathrm{K}$. 


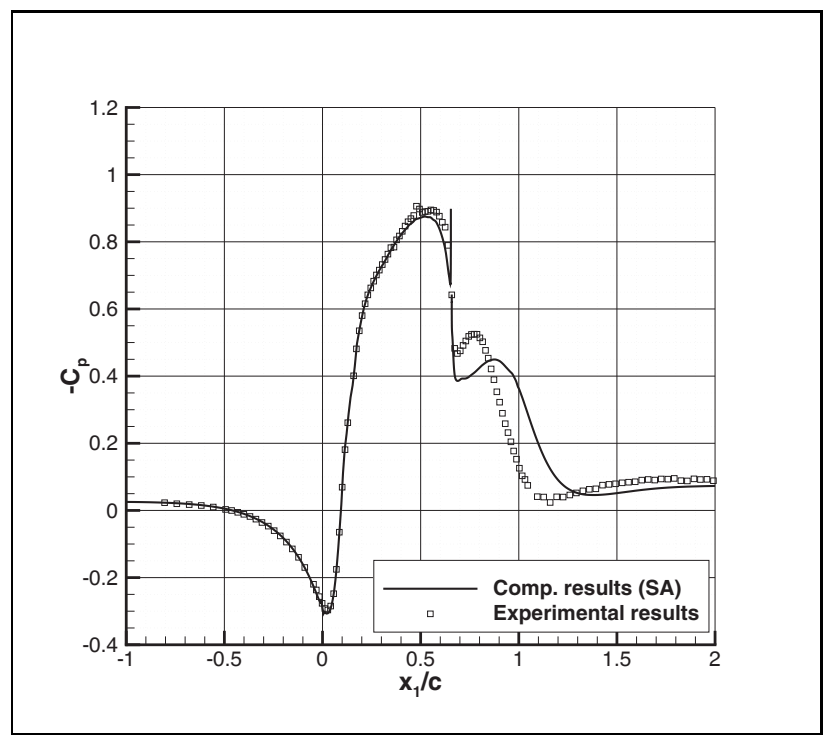

(a) pressure coefficient $c_{p}$

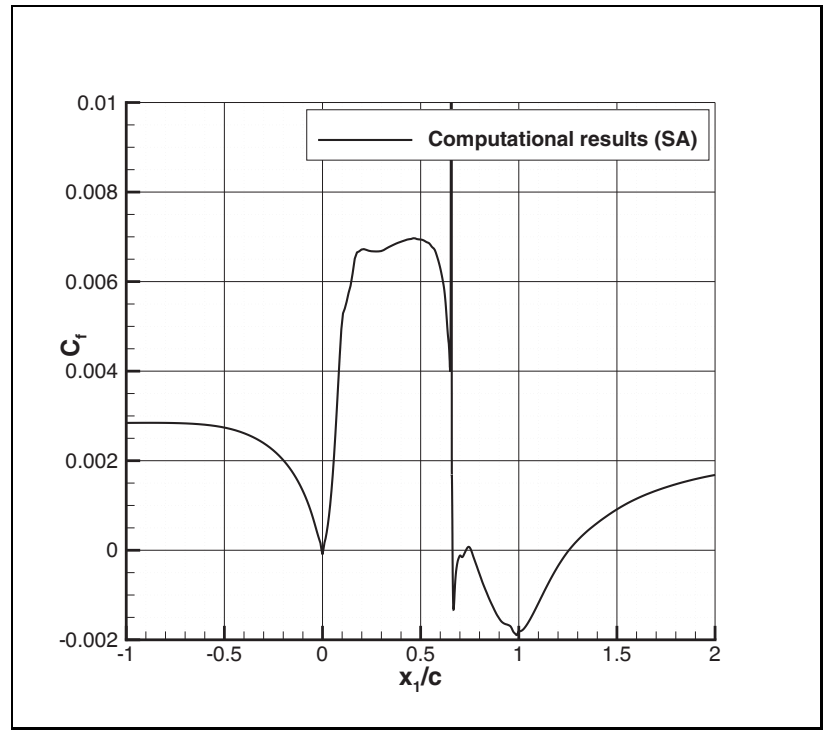

(b) skin friction coefficient $c_{f}$

Figure 5.28: Time-averaged experimental and computational results for flow over a hump model with synthetic jet actuation through slit $\left(f_{j}=138.5 \mathrm{~Hz},\left(U_{n} / c_{\infty}\right)_{\text {cav.bot }}=0.001\right)$. $\operatorname{Re}_{c}=9.36 \cdot 10^{5}, \mathrm{M}_{\infty}=0.1$ and $T_{\text {ref }}=298 \mathrm{~K}$. 


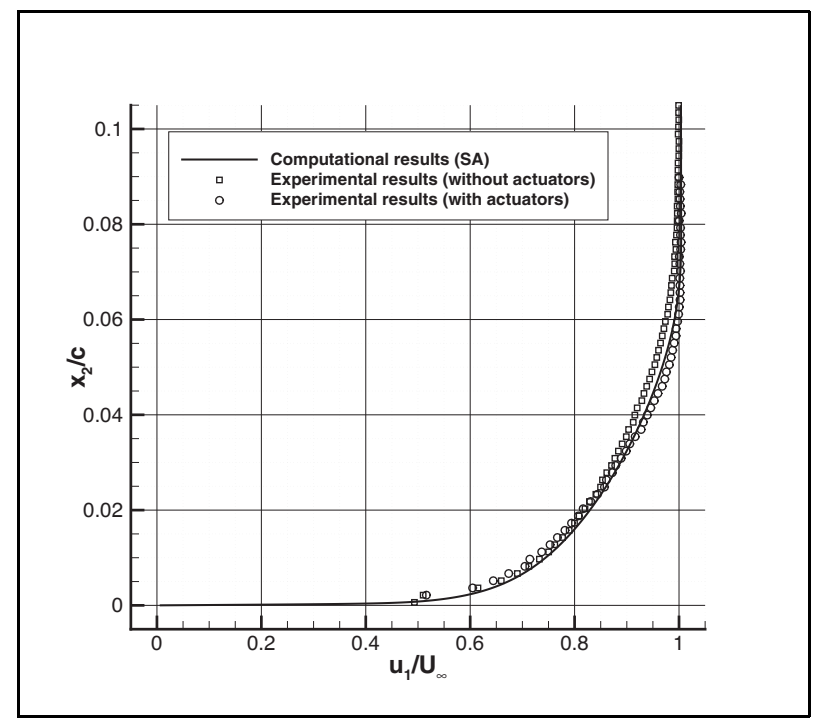

Figure 5.29: Streamwise velocity profile at $x / c=-2.14$ for the baseline case without actuation, $\operatorname{Re}_{c}=9.36 \cdot 10^{5}, \mathrm{M}_{\infty}=0.1$ and $T_{\text {ref }}=298 \mathrm{~K}$. Experimental results are shown for situations with and without synthetic jet actuators present in the wind tunnel.

section [187].

The comparison for the skin friction coefficient is less satisfactory. First, we focus on the region in the middle of the hump. In this region, the predicted skin friction is higher than measured in the experiments. Discrepancies between experimental measurements and numerical simulations were also observed by Naughton et al. [189], although different computational methods and different turbulence models yielded different predictions. Numerical predictions for $c_{f}$ between approximately 0.006 and 0.007 were observed in this region. The present computational results are within this bandwidth. Since the scatter in the experimental results is quite large, as indicated by the error bars, it appears difficult to accurately measure the skin friction in this region. This may be due to three-dimensional effects or due to some small unsteadiness of the flow. As mentioned before, steady-flow simulations using the weighted least-squares method to calculate gradients yielded an erratic behavior of the skin friction coefficient in this region. It is not known whether this is a result of unsteady flow behavior or due to a different reason. A more detailed investigation is needed to confirm this.

When we focus on the region downstream of the location of the slit, it is clear that the extent of the separated flow region is much larger in the simulated flow than in the experiment. Table 5.8 lists the location of separation and reattachment for the different subcases, obtained in the experiments and the flow simulations. For the baseline case without flow control, the flow separation location is well predicted, but the length of the separated flow region is overpredicted by approximately $39 \%$. 
Chapter 5. Verification AND VAlidation

\begin{tabular}{|l|l|l|}
\hline Location $\left(x_{1} / c\right)$ of & Experiment & Simulations (SA turb. model) \\
\hline \hline Separation (no control) & $0.665 \pm 0.005$ & $0.6604 \pm 0.00005$ \\
\hline Reattachment (no control) & $1.11 \pm 0.003$ & $1.278 \pm 0.005$ \\
\hline Separation (suction) & $0.680 \pm 0.005$ & $0.6676 \pm 0.00005$ \\
\hline Reattachment (suction) & $0.94 \pm 0.005$ & $1.135 \pm 0.005$ \\
\hline Separation (SJ actuation) & $0.67 \pm 0.005$ & $0.6625 \pm 0.00005$ \\
\hline Reattachment (SJ actuation) & $0.98 \pm 0.005$ & $1.255 \pm 0.005$ \\
\hline
\end{tabular}

Table 5.8: Locations of separation and reattachment for different subcases, obtained in experiments and numerical simulations. Uncertainty in numerical results is due to local grid resolution.

\section{Results for Steady Suction and Synthetic Jet Actuation}

Before the experimental and numerical results of both controlled flow cases are compared, the employed velocity amplitude in the time-dependent boundary condition for synthetic jet actuation is checked. The peak ejection velocity at the exit of the slit is attained at a phase of 117.9 degrees in the sinusoidal actuation cycle and equals $\left|\underline{u}_{p}\right| \approx 25.7 \mathrm{~m} / \mathrm{s}$, see figure 5.30 This is approximately $3.4 \%$ lower than the value used in the experiments, which is $26.6 \mathrm{~m} / \mathrm{s}$. It is expected that this difference is small enough to not dramatically affect the predicted performance of the synthetic jet in the flow simulation.

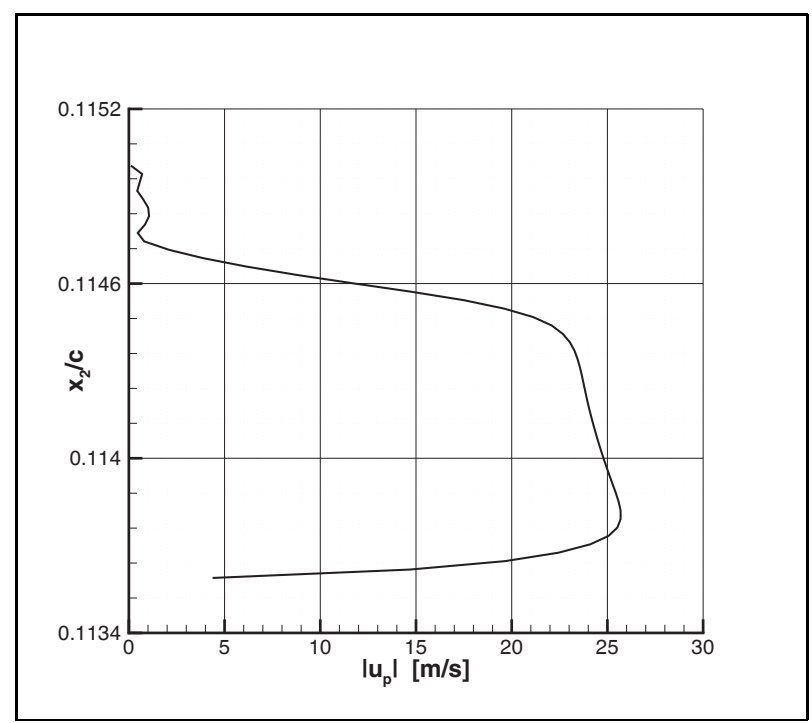

Figure 5.30: Profile of absolute velocity across exit of slit at a phase of 117.9 degrees in the actuation cycle for the case with synthetic jet actuation $\left(f_{j}=138.5 \mathrm{~Hz},\left(U_{n} / c_{\infty}\right)_{\text {cav.bot }}=\right.$ $0.001) . \operatorname{Re}_{c}=9.36 \cdot 10^{5}, \mathrm{M}_{\infty}=0.1$ and $T_{\text {ref }}=298 \mathrm{~K}$. 
The agreement between experimental and computational results for the cases with flow control shows similar behavior as observed for the baseline case. Figure 5.27(a) shows the pressure coefficient for the case with suction, whereas figure 5.28(a) shows the time-averaged pressure coefficient for the case with synthetic jet actuation. In both cases, the pressure distribution is well predicted upstream of the location of flow separation (just after the location of the slit at $\left.x_{1} / c=0.655\right)$, but is not so well predicted downstream of this location. In comparison with the baseline case, the same kind of differences in the shape of the pressure distribution are observed in this region. There are also some small deviations in the region just before the slit, where the predicted pressure is slightly higher than measured in the experiments. When we compare the two cases with each other, it is clear that steady suction has a larger influence on the overall (time-averaged) pressure distribution than synthetic jet actuation. Over the middle of the hump, the pressure continues to decrease towards a strong suction peak just upstream of the slit for the case with steady suction. In the time-averaged pressure distribution for the case with synthetic jet actuation, the suction peak is much lower and confined to a much smaller space upstream of the slit.

Figures 5.27(b) and 5.28(b) show the computationally obtained skin friction coefficient (experimental results are not available) for the case with steady suction and the case with synthetic jet actuation, respectively. Consistent with the results for the pressure distribution, steady suction through the slit yields a strong increase of the skin friction in the region just upstream of the slit. This is not visible in the time-averaged results for the case with synthetic jet actuation. Furthermore, both cases yield a shorter region of separated flow downstream of the slit compared to the baseline case, but this effect is more pronounced in the case with steady suction.

From the listed locations of separation and reattachment in table 5.8, it can be concluded that the flow simulations do predict the trend of delayed flow separation and earlier reattachment. However, the experiments show a more pronounced effect of suction and synthetic jet actuation than the numerical simulations. In comparison with the experiments, the flow simulations yield earlier separation and later reattachment for both actuated cases. In the baseline case, the length of the separated flow region is overpredicted by approximately $39 \%$, whereas in the cases with actuation, the overprediction approximately equals $80 \%$ (suction) and $91 \%$ (synthetic jet). The absolute difference in the reattachment location between the baseline case and the cases with actuation is $0.17 c$ (suction) and $0.13 c$ (synthetic jet) in the experiments, versus $0.143 c$ and $0.023 c$ the flow simulations. This shows that the prediction of the flow with synthetic jet actuation is particularly different from the experiments.

In order to find the reason behind the underprediction of the effect of flow control in the flow simulations, a more detailed view of the solution is needed. This will be done by comparing the computational results from the case with synthetic jet actuation with results from the two-dimensional PIV measurements, focusing on vertical planes located at $x_{1} / c=0.66$ and $x_{1} / c=0.8$. The first location is just downstream of the location of the slit and the second location is in the separated flow region.

Figures 5.31 and 5.32 show the normalized $x_{1}$-component of the velocity and the 


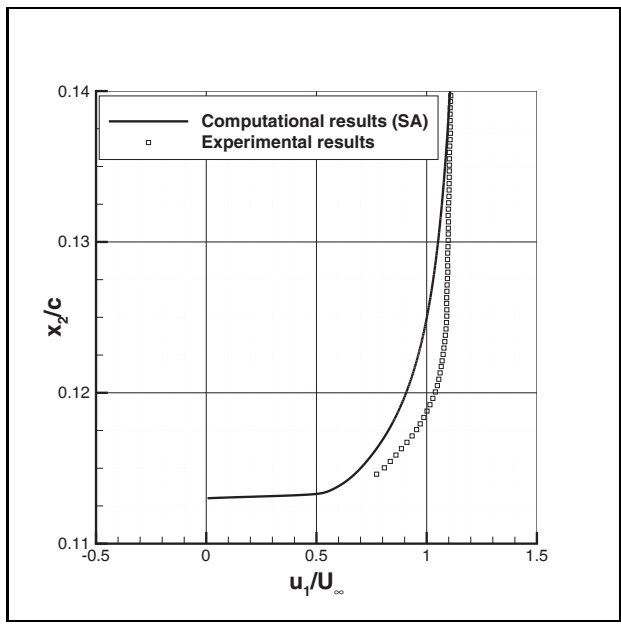

(a) phase $=0^{\circ}$

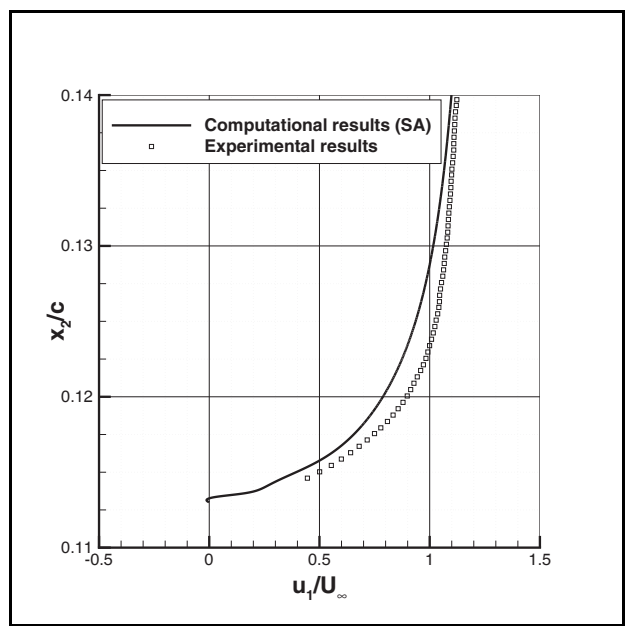

(c) phase $=180^{\circ}$

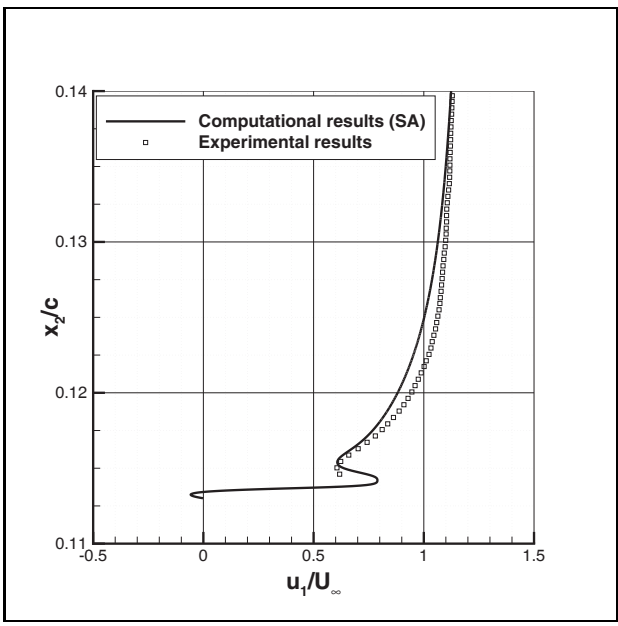

(b) phase $=90^{\circ}$

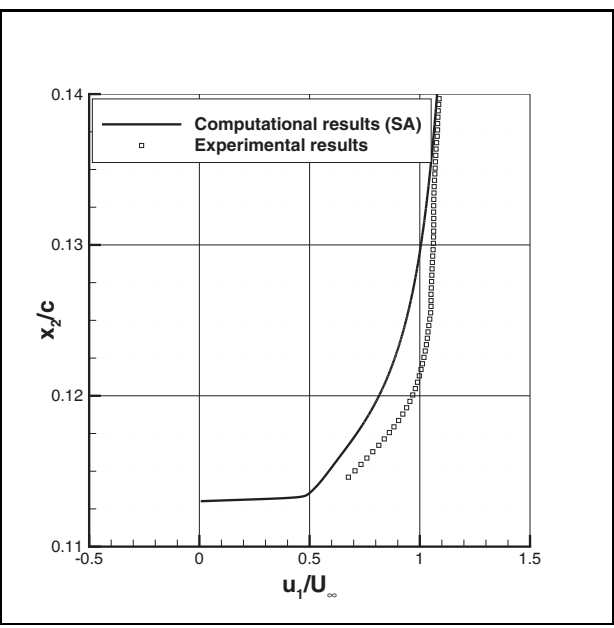

(d) phase $=270^{\circ}$

Figure 5.31: Experimental and computational results for normalized $x_{1}$-component of velocity, $u_{1} / U_{\infty}$, at $x_{1} / c=0.66$ for flow over a hump model with synthetic jet actuation $\left(f_{j}=138.5 \mathrm{~Hz},\left(U_{n} / c_{\infty}\right)_{\text {cav.bot }}=0.001\right)$; maximum ejection at $90^{\circ}$, maximum ingestion at $270^{\circ} \cdot \operatorname{Re}_{c}=9.36 \cdot 10^{5}, \mathrm{M}_{\infty}=0.1$ and $T_{\text {ref }}=298 \mathrm{~K}$. 


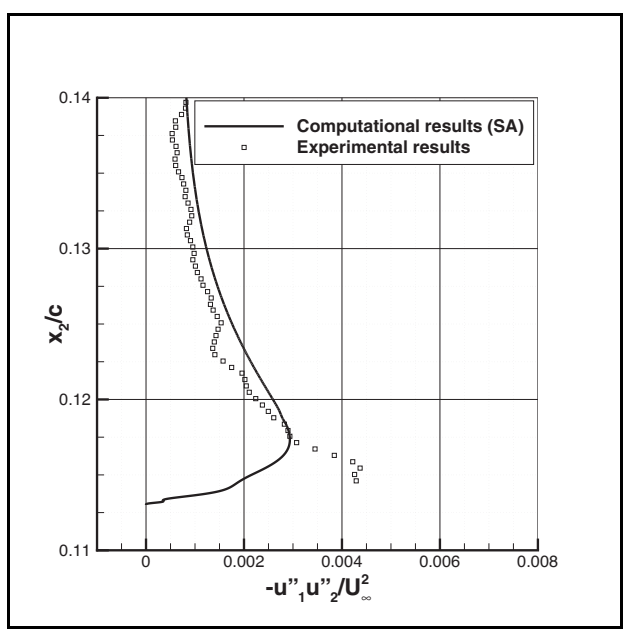

(a) phase $=0^{\circ}$

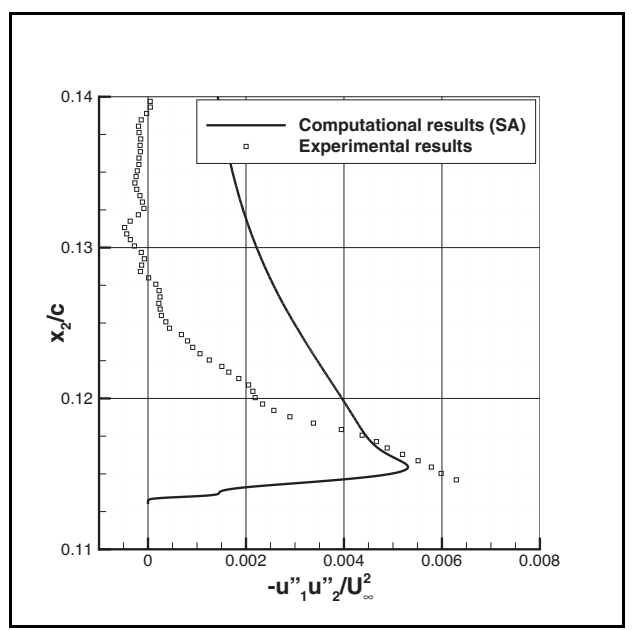

(c) phase $=180^{\circ}$

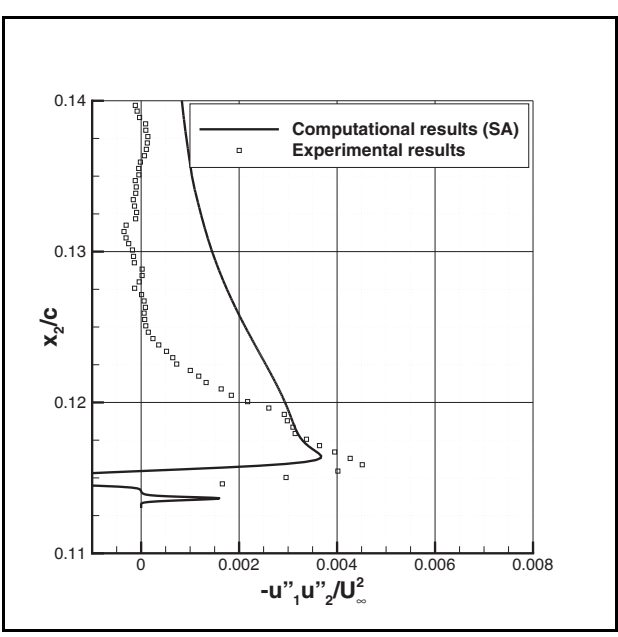

(b) phase $=90^{\circ}$

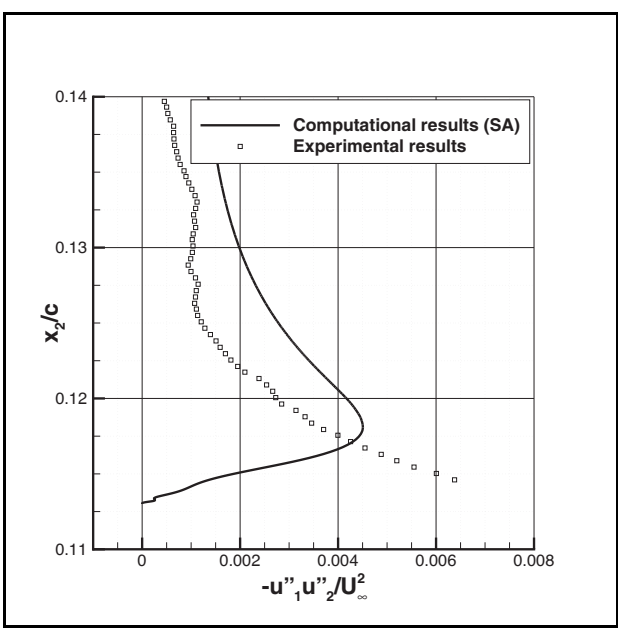

(d) phase $=270^{\circ}$

Figure 5.32: Experimental and computational results for normalized turbulent shear stress, $-\widetilde{u_{1}^{\prime \prime} u_{2}^{\prime \prime}} / U_{\infty}^{2}$, at $x_{1} / c=0.66$ for flow over a hump model with synthetic jet actuation $\left(f_{j}=138.5 \mathrm{~Hz},\left(U_{n} / c_{\infty}\right)_{\text {cav.bot }}=0.001\right)$; maximum ejection at $90^{\circ}$, maximum ingestion at $270^{\circ} . \operatorname{Re}_{c}=9.36 \cdot 10^{5}, \mathrm{M}_{\infty}=0.1$ and $T_{\text {ref }}=298 \mathrm{~K}$. 


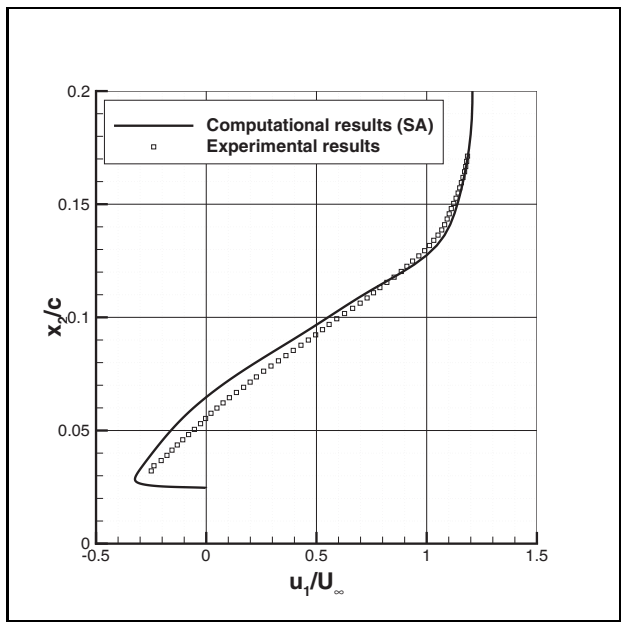

(a) phase $=0^{\circ}$

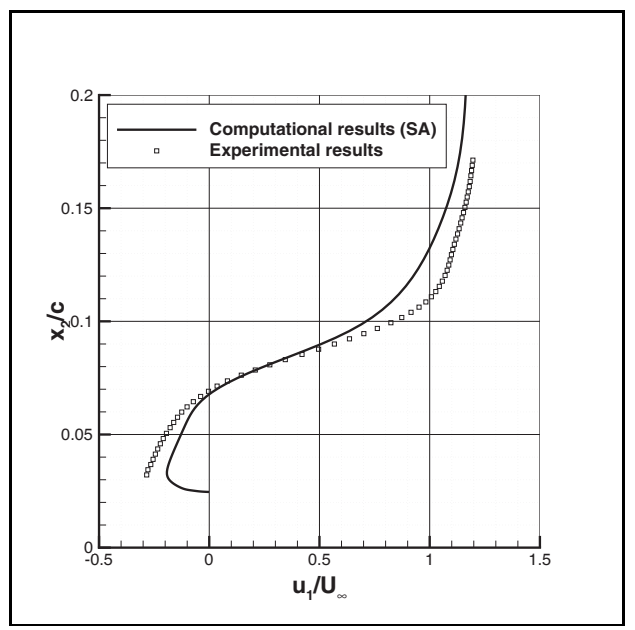

(c) phase $=180^{\circ}$

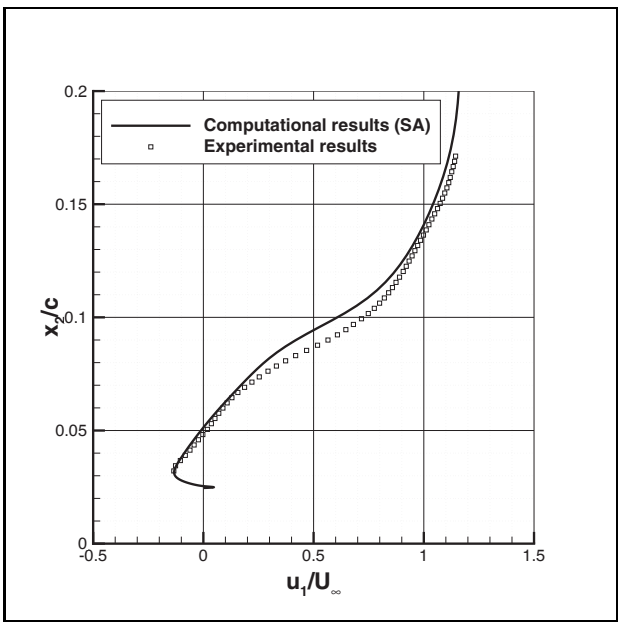

(b) phase $=90^{\circ}$

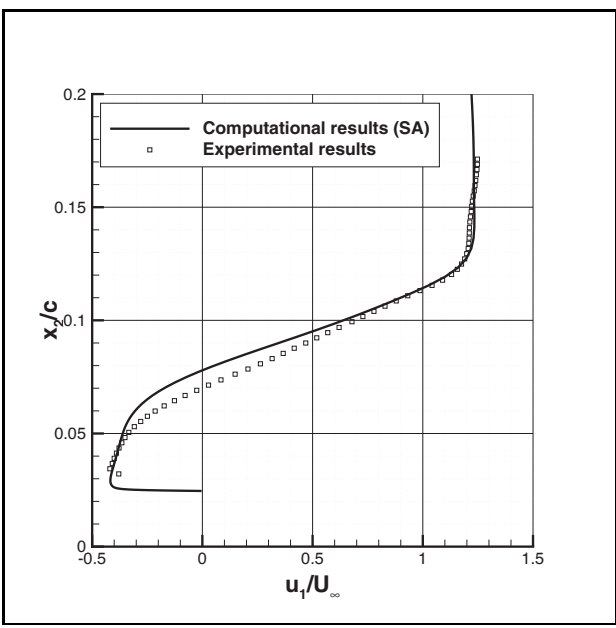

(d) phase $=270^{\circ}$

Figure 5.33: Experimental and computational results for normalized $x_{1}$-component of velocity, $u_{1} / U_{\infty}$, at $x_{1} / c=0.8$ for flow over a hump model with synthetic jet actuation $\left(f_{j}=138.5 \mathrm{~Hz},\left(U_{n} / c_{\infty}\right)_{\text {cav.bot }}=0.001\right)$; maximum ejection at $90^{\circ}$, maximum ingestion at $270^{\circ} \cdot \operatorname{Re}_{c}=9.36 \cdot 10^{5}, \mathrm{M}_{\infty}=0.1$ and $T_{\text {ref }}=298 \mathrm{~K}$. 


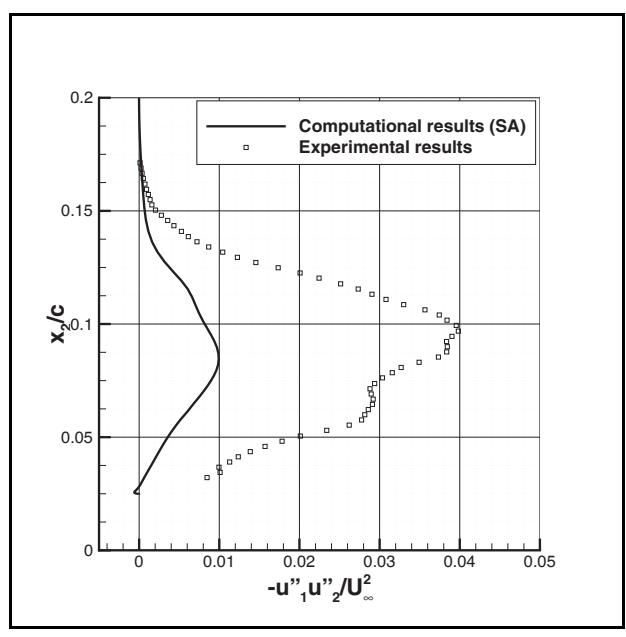

(a) phase $=0^{\circ}$

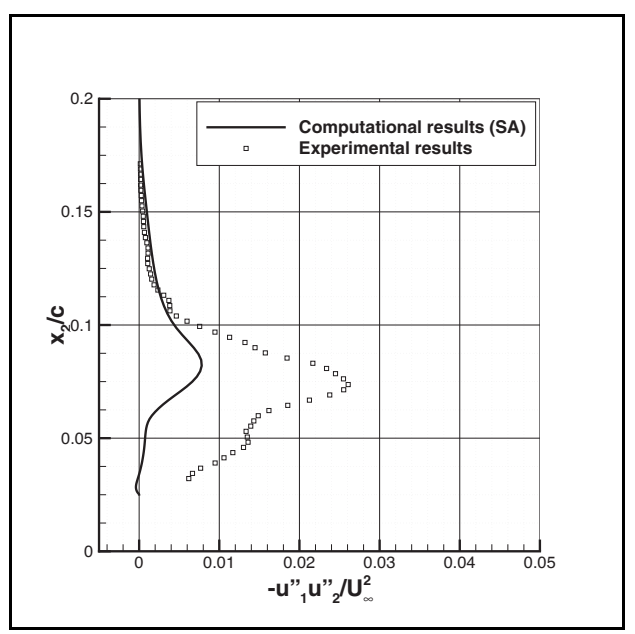

(c) phase $=180^{\circ}$

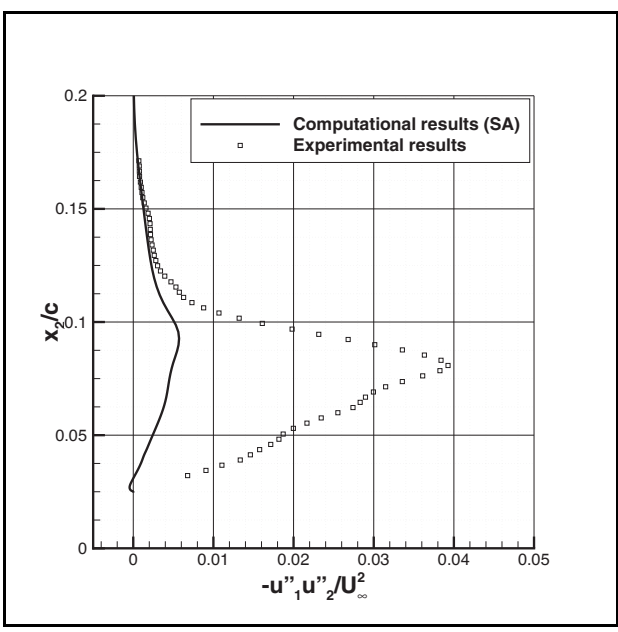

(b) phase $=90^{\circ}$

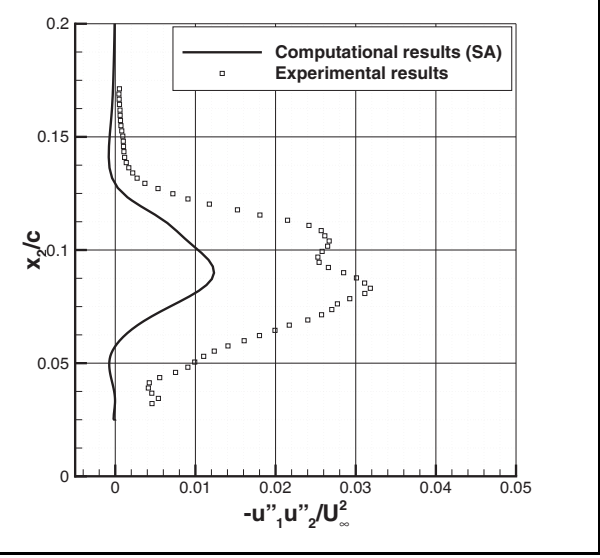

(d) phase $=270^{\circ}$

Figure 5.34: Experimental and computational results for normalized turbulent shear stress, $-\widetilde{u_{1}^{\prime \prime} u_{2}^{\prime \prime}} / U_{\infty}^{2}$, at $x_{1} / c=0.8$ for flow over a hump model with synthetic jet actuation $\left(f_{j}=138.5 \mathrm{~Hz},\left(U_{n} / c_{\infty}\right)_{\text {cav.bot }}=0.001\right)$; maximum ejection at $90^{\circ}$, maximum ingestion at $270^{\circ} . \operatorname{Re}_{c}=9.36 \cdot 10^{5}, \mathrm{M}_{\infty}=0.1$ and $T_{\text {ref }}=298 \mathrm{~K}$. 
turbulent shear stress, respectively, at $x_{1} / c=0.66$ and four phases in the actuation cycle. Note that the phase angles used in these figures are shifted such that a phase of $90^{\circ}$ coincides with maximum ejection at the exit of the slit and $270^{\circ}$ with maximum ingestion at the exit of the slit. In general, the computational method using the SA turbulence model predicts a more gradual velocity profile than measured in the experiments. Furthermore, the turbulent shear stress is underpredicted close to the wall (less turbulent diffusion) and overpredicted further away from the wall (more turbulent diffusion). At $90^{\circ}$, the synthetic jet can clearly be seen in the velocity profile of the computations. The peak value is approximately $27.3 \mathrm{~m} / \mathrm{s}$, which is in the range of expected values. The distinct jet is also seen in the predicted turbulent shear stress; it becomes negative there where $\partial u_{1} / \partial x_{2}$ is negative. In the experimental results, the jet can be seen in the first available data points of the velocity profile, but not in the profile of the turbulent shear stress. Since both $\partial u_{1} / \partial x_{2}$ and $\partial u_{2} / \partial x_{1}$ in the experimental results are negative here 2 , the experimental results do not follow the Boussinesq approximation, see equation (3.84), which predicts a negative value of the turbulent shear stress. In the predicted velocity profiles at $90^{\circ}$ and $180^{\circ}$ one can also see the onset of flow separation in the region very close to the wall. The experimental results in this region have not been included due to large errors in the PIV measurements close to the wall.

Figures 5.33 and 5.34 show the same quantities as the previous two figures, but now in the separated flow region at $x_{1} / c=0.8$. The velocity profiles are generally well predicted here, although the profile at $180^{\circ}$ shows more gradual changes than measured in the experiments. The height of the separated flow region is well predicted at $90^{\circ}$ and $180^{\circ}$, but somewhat overpredicted at $0^{\circ}$ and $270^{\circ}$, i.e. during the ingestion phase. The most dramatic differences between the computational and experimental results can be seen in figure 5.34. Clearly, the turbulent shear stress is underpredicted; the peak values in the experiments are roughly between two times higher (maximum ingestion) and seven times higher (maximum ejection) than in the simulation. Note, however, that the error in the measurements of the turbulent shear stress can be as high as $16 \%$. A more quantitative comparison can therefore not be given.

The underprediction of the turbulent shear stress is also visible in the evolution of the spanwise vorticity, which is shown in figure 5.35 for a phase of $180^{\circ}$ in the actuation cycle. In the flow simulation, the concentrations of negative spanwise vorticity are located at the correct locations, but they persist much longer downstream. The turbulent mixing (diffusion) in the flow simulation is therefore too low and this is likely the cause of the overprediction of the extent of the separated flow region.

Although not presented here, the effects at both locations as described above can also be observed in the results of the baseline case and the case with steady suction. For the baseline case, Rumsey [190] shows that by arbitrarily doubling the eddy-viscosity predicted by the SA model in the separated flow region improves the prediction of the extent of the separated flow region.

\footnotetext{
${ }^{2}$ The sign of $\partial u_{2} / \partial x_{1}$ has been determined by comparing the values of $u_{2}$ at the same level of $x_{2}$ and two different levels of $x_{1}: x_{1} / c=0.66$ and $x_{1} / c=0.67$.
} 


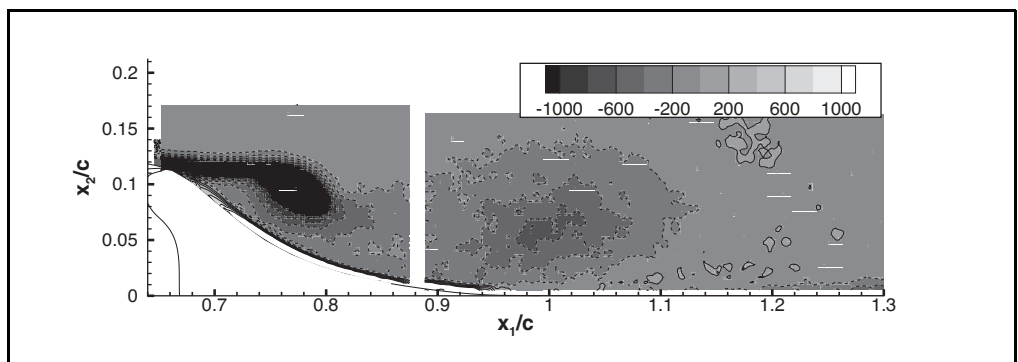

(a) experimental result

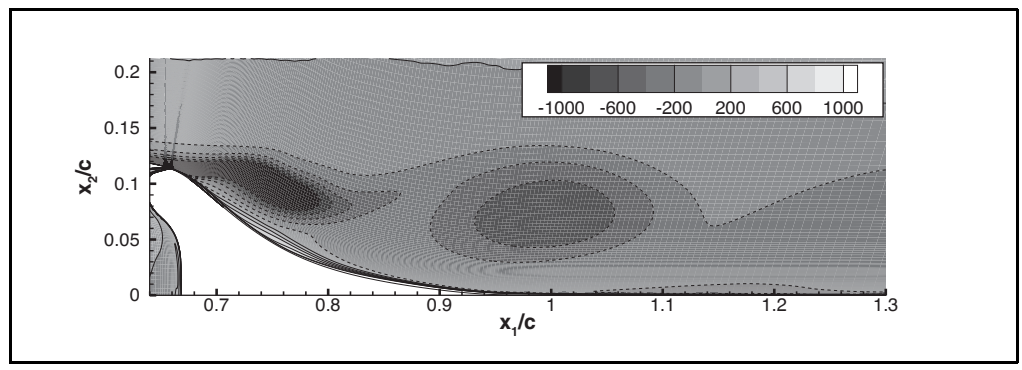

(b) computational result (SA turbulence model)

Figure 5.35: Experimental and computational results for spanwise component of vorticity, $\omega_{3}\left(\mathrm{~s}^{-1}\right)$, for flow over a hump model with synthetic jet actuation at $180^{\circ}\left(f_{j}=138.5\right.$ $\left.\mathrm{Hz},\left(U_{n} / c_{\infty}\right)_{\text {cav.bot }}=0.001\right) . \operatorname{Re}_{c}=9.36 \cdot 10^{5}, \mathrm{M}_{\infty}=0.1$ and $T_{r e f}=298 \mathrm{~K}$.

\section{Discussion}

The present investigation shows that (U)RANS simulations using the SA turbulence model have difficulty in accurately predicting the effect of turbulence in (closed) separated regions. Results obtained with different eddy-viscosity models, such as the SST model and a non-linear explicit algebraic stress model [80], also show the same kind of deficiency, see for example Rumsey [190]. Rumsey also performed different simulations for this hump model, see Rumsey \& Greenblatt [191, 192], to investigate if (U)RANS simulations can predict the effects of variations in the parameters.

Figure 5.36 ([191]) shows the surface pressure coefficient, $c_{p}$, for the case with steady suction, with five different mass flow rates through the slit. The employed mass flow rates are between $0.0053 \mathrm{~kg} / \mathrm{s}$ and $0.0263 \mathrm{~kg} / \mathrm{s}$, with the mass flow rate used in the validation workshop in the middle $(0.01518 \mathrm{~kg} / \mathrm{s})$. Both the experimental results and the computational results using the SA turbulence model are shown. Upstream of the location of the slit, the computational results agree well with the experimental results for all suction mass flow rates. In the region downstream of the slit, the simulations predict a similar trend as observed in the experiments, i.e. with increasing mass flow rate through the slit, the extent of the separated flow region is reduced. However, the simulations also 


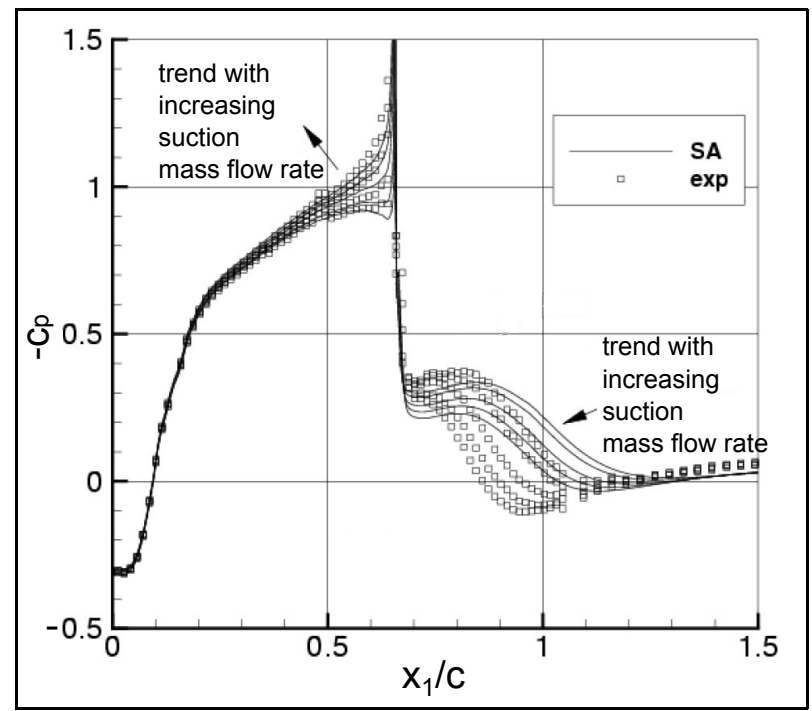

Figure 5.36: Pressure coefficient, $c_{p}$, for flow over a hump model with steady suction through slit using different mass flow rates, between $0.0053 \mathrm{~kg} / \mathrm{s}$ and $0.0263 \mathrm{~kg} / \mathrm{s}$, from [191]. $\operatorname{Re}_{c}=9.36 \cdot 10^{5}, \mathrm{M}_{\infty}=0.1$ and $T_{\text {ref }}=298 \mathrm{~K}$.

predict a pressure increase downstream of the slot with increasing mass flow rate, whereas the experimental results show a steeper pressure recovery instead. Furthermore, the rate at which the separated flow region is reduced in the simulations is lower than observed in the experiments. Results obtained with the SST model and a non-linear explicit algebraic stress model (not shown here) show similar differences in absolute values and similar trends as observed in the results obtained the SA turbulence model [191].

For the case of synthetic jet actuation through the slit, Rumsey \& Greenblatt [191] investigated whether URANS simulations are able to predict the effect of different velocity amplitudes of the jet as well as different actuation frequencies. Figure [5.37] shows the pressure coefficient for the case with synthetic jet actuation, with $f_{j}=138.5 \mathrm{~Hz}$ and different peak ejection velocities, $\left|\underline{u}_{p}\right|$, of the jet. Note that $\left|\underline{u}_{p}\right| \approx 27 \mathrm{~m} / \mathrm{s}$ corresponds to the condition used in the validation workshop. With increasing peak ejection velocity, the experimental results show an increasing suction peak upstream of the slit. In the simulations, the suction peak first decreases for the lower peak ejection velocities, before it increases again for the higher peak ejection velocities. In the region downstream of the slit, the simulations predict a similar trend as observed in the experiments, i.e. with increasing mass flow rate through the slit, the extent of the separated flow region is reduced. However, for the two lowest peak ejection velocities, hardly any difference is observed. The trend is only visible for higher peak ejection velocities. Furthermore, the predicted surface pressure distribution in the separated flow region shows a different trend than observed in the experiments. Although not shown here, the SST model and a 


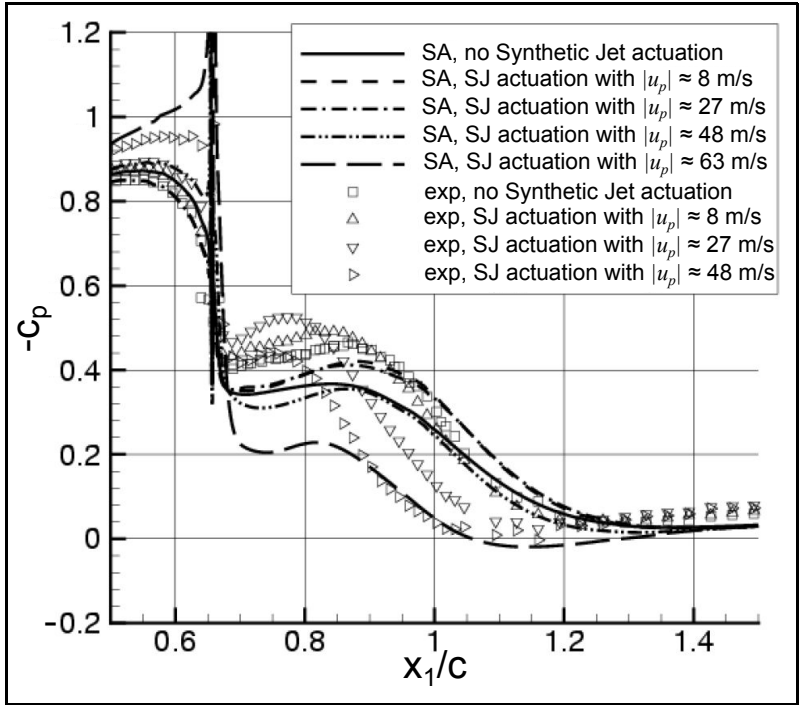

Figure 5.37: Pressure coefficient, $c_{p}$, for flow over a hump model with synthetic jet actuation through slit $\left(f_{j}=138.5 \mathrm{~Hz}\right)$ using different peak ejection velocities of the jet, from [191]. $\operatorname{Re}_{c}=9.36 \cdot 10^{5}, \mathrm{M}_{\infty}=0.1$ and $T_{\text {ref }}=298 \mathrm{~K}$.

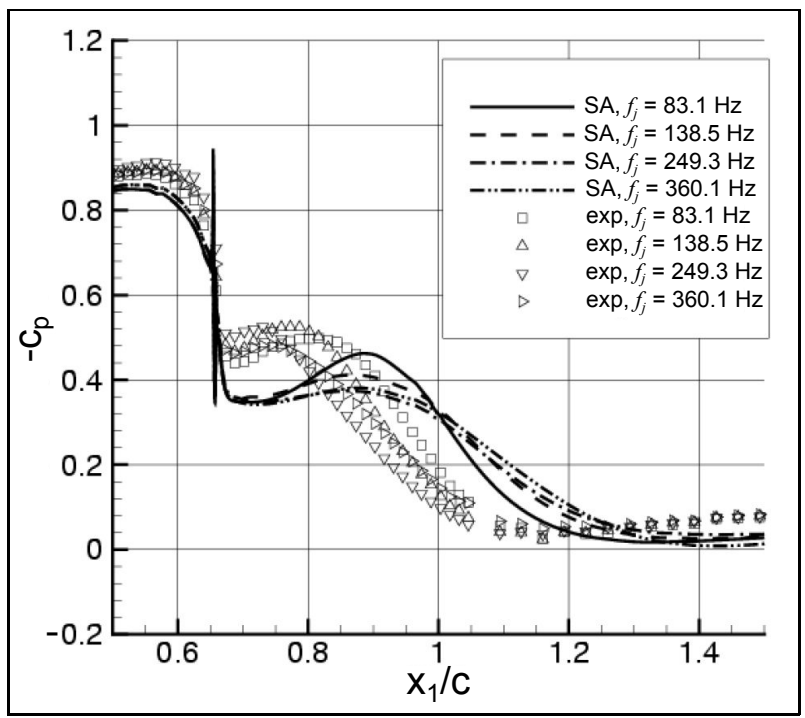

Figure 5.38: Pressure coefficient, $c_{p}$, for flow over a hump model with synthetic jet actuation through slit using a peak ejection velocity of approximately $27 \mathrm{~m} / \mathrm{s}$ and different actuation frequencies, from [191]. $\mathrm{Re}_{c}=9.36 \cdot 10^{5}, \mathrm{M}_{\infty}=0.1$ and $T_{r e f}=298 \mathrm{~K}$. 
non-linear explicit algebraic stress model are slightly better in predicting the trends, both upstream and downstream of the slit [191]. However, similar differences in absolute values are obtained using any of the three employed turbulence models.

Figure 5.38 shows experimental and computational results for the pressure coefficient, $c_{p}$, for $\left|\underline{u}_{p}\right| \approx 27$ and four different actuation frequencies. Note that $f_{j}=138.5$ corresponds to the condition used in the validation workshop. In the experiments, increasing the frequency to $249.3 \mathrm{~Hz}$ yields a slightly smaller separated flow region. For the highest actuation frequency, the extent of the separated flow region is increased again. In the simulations, this trend is not observed. Here, the extent of the separated flow region slightly increases with increasing actuation frequency, but the differences are very small.

For both cases with flow control, Rumsey \& Greenblatt [191] also investigated the effect of the Reynolds number, $\mathrm{Re}_{c}$. For the case with steady suction, the relative momentum through the slit, $\left(\rho_{s} U_{s}^{2} h\right) /\left(\rho_{\infty} U_{\infty}^{2} c\right)$, was kept constant. Here, the subscript ' $s$ ' indicates parameters related to suction through the slit. For the case with synthetic jet actuation, the dimensionless actuation frequency, $f_{j} X / c$, and the relative root-meansquare momentum of the jet, $\left(\rho_{j}\left\langle U_{j}\right\rangle_{\text {r.m.s }}^{2} h\right) /\left(\rho_{\infty} U_{\infty}^{2} c\right)$, were kept constant. Here, the subscript ' $j$ ' refers to parameters related to the synthetic jet, $f_{j}$ is the actuation frequency, $X$ is the distance from the slit to the end of the recirculation zone for the baseline case without actuation and $\left\langle U_{j}\right\rangle_{\text {r.m.s }}$ is the root-mean-square (over the actuation cycle) of the jet peak velocity. Both the experiments and the simulations show a similar trend of increasing effectiveness of suction at higher Reynolds numbers and hardly any effect of the Reynolds number for the case with synthetic jet actuation.

It can be concluded from these results that flow simulations based on the (U)RANS equations can qualitatively predict some of the trends observed in the experiments on the hump model. Using (U)RANS for flow simulations with boundary layer separation control to investigate the feasibility of design changes is therefore justified, but caution needs to be exercised when investigating the effect of the actuation frequency on the effectiveness of boundary layer separation control.

In the next chapter, synthetic jet actuation for pitch control is considered. It will also include comparisons between simulations based on the (U)RANS equations and experiments carried out in a wind tunnel. 


\section{Chapter 6}

\section{Pitch Control using Synthetic Jet ACtuation}

\subsection{Introduction}

\subsubsection{Problem Description}

This chapter deals with the investigation into the use of synthetic jet actuation for pitch control. Unlike boundary layer control, pitch control is associated with control of the aerodynamic performance of a blade or wing over a wide range of angles of attack, see chapter 2 .

The ultimate goal is to apply smart rotor control, or load control, to wind turbine blades. This involves rapid local changes in the aerodynamic performance, i.e. rapid local changes in the effective pitch, such that the magnitude of the fatigue inducing fluctuating forces on the blades is reduced. However, performing experiments and numerical flow simulations on rotating blades is quite demanding in terms of facilities. Additionally, smart rotor control also involves detection of fluctuating aerodynamic forces and a control system that actively regulates the actuation based on the detected forces. As a first step towards the ultimate goal, the present investigation focuses on the potential of synthetic jet actuation to cause rapid changes in the aerodynamic performance of an airfoil. This basic approach involves

- numerical simulations and wind tunnel experiments of synthetic jet actuation on a non-rotating NACA0018 airfoil;

- synthetic jets through long slits (in spanwise direction), located close to the trailing edge with the jet directed perpendicularly to the surface.

First of all, a non-rotating airfoil makes the problem easier to handle: a smaller sized wind tunnel is sufficient and the additional equipment needed for synthetic jet actuation does not necessarily need to be entirely located inside the wind-tunnel model. Furthermore, the use of long spanwise slits allows for two-dimensional flow simulations, which saves computational resources and time.

Secondly, the NACA0018 airfoil has been chosen because it is a basic airfoil that, in theory, could be used for the outboard sections of a wind turbine blade. This is because it 
is thick enough to have good structural strength without loosing too much aerodynamic performance. Furthermore, this airfoil is readily available for the use in wind tunnel experiments in the Silent Wind Tunnel of the University of Twente.

The reason for selecting a slit location close to the trailing edge, directed perpendicularly to the surface is that it is expected that in this way, the jets are able to force flow separation in a time-periodic manner and thereby create a vortical flow region between the slit and the trailing edge. This can modify the direction of the flow at the trailing edge, such that the time-averaged circulation of the airfoil, and thereby the pressure distribution, is altered. This directly affects the aerodynamic force on the airfoil. So, the expectation is that synthetic jet actuation close to the trailing edge can cause changes in the aerodynamic properties of the airfoil, similar to the effect of a steady jet or micro-tab at the same location, see subsection 2.5.2

The purpose of this investigation is to

- identify the parameters involved in synthetic jet actuation through long spanwise slits,

- gain more insight in the effect of these parameters on the aerodynamic performance of the airfoil, i.e. on lift and drag,

- gain more insight in the response time of the lift and drag forces to actuation, i.e. how fast the aerodynamic performance can be changed,

- gain more insight in the flow phenomena associated with synthetic jet actuation,

- determine whether synthetic jet actuation has potential for the purpose of smart rotor control on wind turbine blades.

\subsubsection{Outlook}

In section 6.2 the parameters that govern the present problem are identified and a subset of these parameters is chosen for a parameter study. Subsequently, the experimental setup is presented in section [6.3. This includes the wind-tunnel model, the actuators, the measurement equipment and the wind tunnel facility itself. Section 6.4 describes the approach taken for the numerical computations. This includes the chosen computational domain, the computational grids, the employed convective flux scheme for the URANS equations and the employed turbulence model. In section 6.5 experimental and numerical results are presented and compared for baseline cases without synthetic jet actuation. In the following section, the performance of the actuation system in the experimental setup is investigated. Then, the results of a parameter study of flows with actuation are presented. In this study, the values of the parameters in which we are most interested are varied and the effect of these changes on the aerodynamic performance of the airfoil are monitored. Section 6.7 focuses on low-frequency synthetic jet actuation, for which experimental and numerical results are available, whereas section 6.8 focuses on high-frequency actuation, for which only numerical results are available. 


\subsection{Dimensional Analysis}

A dimensional analysis is a useful tool to obtain the minimal number of independent dimensionless parameters that govern a particular problem. The first step in this process is to identify the set of physically independent variables that influence the dependent variable(s) one is interested in. In the present problem, we are interested in the influence of a set of physically independent variables on the aerodynamic force on the airfoil. This set can be divided into three groups: main flow variables, actuation variables and variables that reflect the design, i.e. geometric variables.

\subsubsection{Physically Independent Variables}

For the actuation variables and geometric variables, it is important to consider that in the numerical simulations the internal workings of the synthetic jet actuator are simplified, i.e. only part of the cavity is included in the computational domain and a time-dependent inflow/outflow boundary condition is applied at the bottom of the included cavity, see section 3.5.3. Therefore, we only include the design of the slit in the geometric variables and the characteristics of the synthetic jet are parameterized at the exit of the slit. The focus therefore lies on the effect that a synthetic jet actuator has on the flow around the airfoil, not on the design and the internal dynamics of synthetic jet actuator.

\section{Main Flow Parameters}

The main flow is a function of five independent variables. Although there are several options to choose the independent main flow variables, we choose those variables that will result in well-known dimensionless parameters, such as the Mach number and the Reynolds number. They are: the magnitude of the free-stream velocity, $U_{\infty}[\mathrm{m} / \mathrm{s}]$, the free-stream speed of sound, $c_{\infty}^{*}[\mathrm{~m} / \mathrm{s}]$, the free-stream density $\rho_{\infty}\left[\mathrm{kg} / \mathrm{m}^{3}\right]$, the freestream viscosity, $\mu_{\infty}[\mathrm{kg} / \mathrm{m} \cdot \mathrm{s}]$, and the angle of attack of the airfoil, $\left.\alpha{ }^{\circ}\right]$.

\section{Geometric Variables}

Since long spanwise slits are employed, we consider the problem to be two-dimensional. Figure 6.1 shows a sketch of the cross-section of the NACA0018 airfoil with a slit located on the lower side, close to the trailing edge. The relevant geometric variables are indicated. They are: the chord length of the NACA0018 airfoil, $c[\mathrm{~m}]$, the chordwise location of the center of the exit of the slit, $x_{j}[\mathrm{~m}]$, the width of the slit, $w_{j}[\mathrm{~m}]$, the depth of the slit, $d_{j}[\mathrm{~m}]$, and the angle of the slit with respect to the (local) surface of the airfoil, $\theta_{j}\left[^{\circ}\right]$.

Depending on the size of the airfoil with respect to the size of the wind tunnel crosssection, the presence of wind tunnel walls affects the flow around the airfoil and thereby its aerodynamic performance. Here, it is assumed that these effects are small, so that the wind tunnel itself is not included in the geometric variables. However, in order to improve the similarity between experiments and numerical simulations, the computational domain is also restricted by wind tunnel walls above and below the airfoil. Since these walls are 


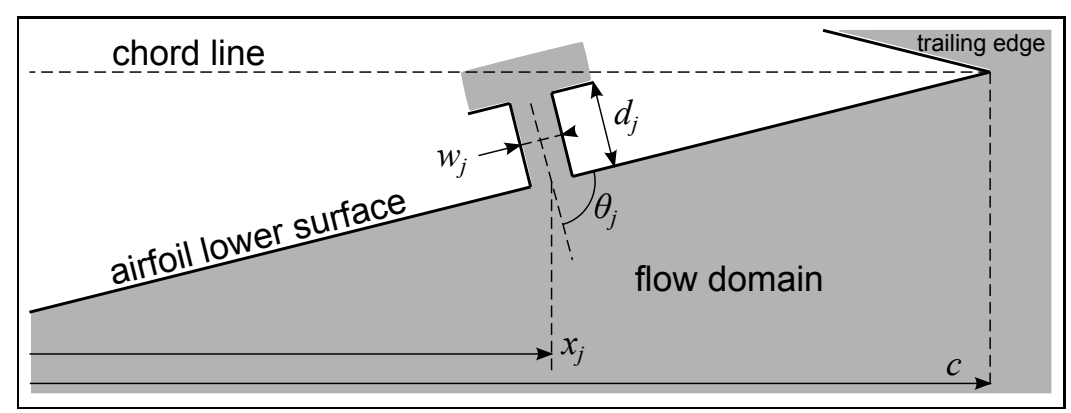

Figure 6.1: Geometric parameters for a two-dimensional spanwise slit located on the lower side of an airfoil.

modeled using symmetry boundary conditions, the effect of confinement is accounted for, but not the effect due to (thickening) boundary layers on all wind tunnel walls. However, this is already (partly) accounted for in the experiments by slightly increasing the crosssectional area of the wind tunnel in the downstream direction, see subsection 6.3.5

\section{Actuation Variables}

For the actuation variables, it is chosen to parameterize the characteristics of the synthetic jet by averages at the exit of the slit. Averaging is performed in space and time. First, spatial averages are computed across the exit of the slit. Next, the spatial averages are averaged in time, but only during the ejection part of the actuation cycle, since the ejection part is considered to be essential for influencing the lift of the airfoil. During the ejection part of the actuation cycle, the boundary condition at the bottom of the included cavity prescribes a time-dependent velocity and density profile, see equation (3.150). Therefore, we parameterize the synthetic jet at the exit of the slit by an average ejection velocity, $\bar{U}_{j}$, and an average ejection density, $\bar{\rho}_{j}$, which are defined as follows

$$
\begin{array}{r}
\bar{U}_{j}=\frac{2}{T_{j} w_{j}} \int_{0}^{T_{j} / 2} \int_{0}^{w_{j}} \underline{u} \cdot \underline{n}_{j} d s d t, \\
\bar{\rho}_{j}=\frac{2}{T_{j} w_{j}} \int_{0}^{T_{j} / 2} \int_{0}^{w_{j}} \rho d s d t .
\end{array}
$$

Here, $\underline{u}(s, t)$ and $\rho(s, t)$ are the time-dependent velocity and density, respectively, with $s$ the coordinate across the slit, i.e. $s \in\left[0, w_{j}\right]$. Furthermore, $\underline{n}_{j}$ is the normal vector into the external flow domain, $w_{j}$ is the width of the slit and $T_{j}=1 / f_{j}$ is the period of actuation, with $f_{j}$ the actuation frequency. In the definitions presented in equations (6.1) and (6.2), we assume that the ejection part corresponds to the first half of the actuation cycle, but time-averaging is actually performed as long as the spatial average of 
the normal velocity at the exit of the slit is positive, which follows from the experiment or simulation. The actuation variables therefore consist of the actuation frequency, $f_{j}\left[\mathrm{~s}^{-1}\right]$, the average ejection velocity, $\bar{U}_{j}[\mathrm{~m} / \mathrm{s}]$, and the average ejection density, $\bar{\rho}_{j}\left[\mathrm{~kg} / \mathrm{m}^{3}\right]$.

\subsubsection{Dimensionless Parameters}

The total number of physically independent variables is 13 . From a dimensional analysis, it follows that the aerodynamic force on the airfoil is a function of 10 dimensionless parameters, since all variables are expressed in the three basic dimensions of mass, length and time. When $c, \rho_{\infty}$ and $U_{\infty}$ are chosen to non-dimensionalize the problem, it follows that the lift and drag coefficients, $c_{l}$ and $c_{d}$, respectively, are governed by the following set of dimensionless parameters:

- main flow parameters: the angle of attack, $\alpha$, the Reynolds number, $\operatorname{Re}_{c}=\frac{\rho_{\infty} U_{\infty} c}{\mu_{\infty}}$, and the Mach number, $\mathrm{M}_{\infty}=\frac{U_{\infty}}{c_{\infty}^{*}}$;

- geometric parameters: the relative chordwise location of the center of the exit of the slit, $x_{j} / c$, the relative slit width, $w_{j} / c$, the relative slit depth, $d_{j} / c$, and the angle of the slit with respect to the airfoil surface, $\theta_{j}$;

- actuation parameters: the dimensionless actuation frequency, $F^{+}=\frac{f_{j} c}{U_{\infty}}$, the momentum coefficient, $c_{\mu}=\frac{\bar{\rho}_{j} \bar{U}_{j}^{2} w_{j}}{\rho_{\infty} U_{\infty}^{2} c}$ and the relative density, $\frac{\bar{\rho}_{j}}{\rho_{\infty}}$.

The momentum coefficient, $c_{\mu}$, is actually a combination of the relative density, $\bar{\rho}_{j} / \rho_{\infty}$, the relative ejection velocity, $\bar{U}_{j} / U_{\infty}$, and the relative slit width, $w_{j} / c$. The momentum coefficient is chosen to replace the relative ejection velocity, since it is frequently used by other authors, see section 2.6. Note however, that there are different definitions of the momentum coefficients in use across the literature.

\subsubsection{Subset of Parameters for Study}

Since it is not feasible to carry out a full parameter study with 10 dimensionless parameters, some parameters are fixed or constrained:

- As already discussed in section 6.1 the slit is directed perpendicularly to the local surface of the airfoil, i.e. $\theta_{j}=90^{\circ}$.

- A chord length of $c=165 \mathrm{~mm}$ together with a slit width of $w_{j}=1 \mathrm{~mm}$ and a depth of $d_{j}=2 \mathrm{~mm}$ is used in the experiments, and will also be used in the numerical simulations. The relative width of the slit is therefore fixed at $w_{j} / c=1 / 165$ and the relative depth at $d_{j} / c=2 / 165$.

- The flow will be kept nearly incompressible for all numerical simulations, i.e. $\mathrm{M}_{\infty}<$ 0.3 and $\bar{\rho}_{j} / \rho_{\infty} \approx 1$. 
- The Reynolds number will not reach values higher than $5.5 \cdot 10^{5}$, which corresponds to $U_{\infty}=50 \mathrm{~m} / \mathrm{s}$. This value is much lower than typical Reynolds numbers for wind turbine blades. To avoid flow features observed in low-Reynolds number flow, e.g. laminar separation bubbles, the airfoil in the experiments has turbulator strips placed close to the leading edge. In the numerical simulations, the turbulence model is used in a fully-turbulent mode such that transition occurs close to the leading edge.

- The location of the slit in the experiments is fixed at $x_{j} / c=0.88$, which is as close to the trailing edge as physically possible in the wind-tunnel model. In the numerical simulations there is not such a restriction and additional locations closer to the trailing edge will be considered.

The parameter study therefore mainly focuses on four dimensionless parameters: the angle of attack, $\alpha$, the dimensionless actuation frequency, $F^{+}$, the momentum coefficient, $c_{\mu}$, and in the numerical simulations the location of the slit, $x_{j} / c$.

Although some different Reynolds numbers will be used, they are quite low due to restrictions of the actuation system and the wind tunnel. By physically and numerically tripping the boundary layer close to the leading edge of the airfoil, i.e. enforcing turbulent boundary layers, we hope to minimize possible strong Reynolds number effects. However, this is not thoroughly investigated but left for future study.

\subsection{Experimental Approach}

\subsubsection{Introduction}

This section describes the experimental setup, including the experimental model, the actuators, the measurement equipment and the wind tunnel. Furthermore, a short description of the measurement procedure is given.

The experimental setup has been developed by ir. E. van Emden, specifically for the present investigation. The experimental results that are presented in this chapter are obtained from measurements carried out by ir. S. M. D. Widjanarko during his Master assignment, see [193].

\subsubsection{Wind-Tunnel Model}

The NACA0018 airfoil is a symmetric airfoil with a maximum thickness of $18 \%$ of the chord length. Its profile is defined by equation (5.13), using $t=0.18$. The wind-tunnel model is a single piece of extruded aluminium with a chord length of $c=165 \mathrm{~mm}$ and a span of $s=0.9 \mathrm{~m}$, equal to the width of test section of the wind tunnel. Boeije [194] compared the shape of the wind-tunnel model with the definition presented in equation (5.13) and found that the maximum deviation is approximately $0.002 c$, which occurs in the area close to the trailing edge. This is probably the result of having a rounded trailing edge instead of a blunt trailing edge as defined by equation (5.13). 
A sketch of the cross section of the wind-tunnel model is shown in figure 6.2 The

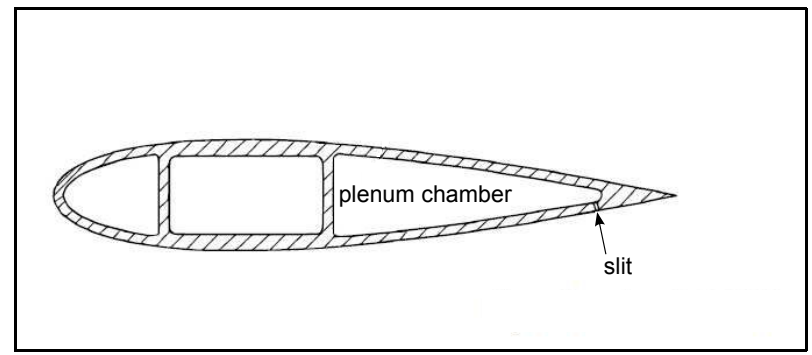

Figure 6.2: Cross section of NACA0018 airfoil used in experiments.

model has three separate internal cavities, of which the aft cavity is used as a plenum chamber for synthetic jet actuation. Four rectangular slits with a width of $w_{j}=1 \mathrm{~mm}$ and a length of $200 \mathrm{~mm}$ are located on the lower side of the airfoil at $x_{j} / c=0.88$, see figures 6.2 and 6.3 . The spanwise distance between successive slits is $10 \mathrm{~mm}$, which is

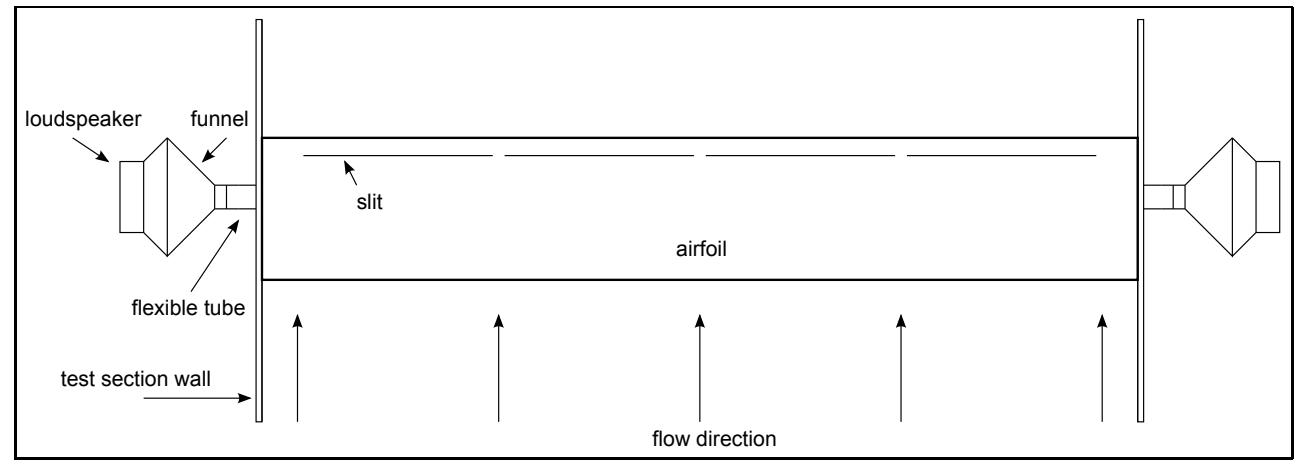

Figure 6.3: Sketch of experimental model (from below)

necessary to guarantee the structural integrity of the model. As will be explained in more detail in section 6.6. the performance of the synthetic jets in terms of peak velocities and spanwise uniformity is too low for the present purposes. To improve the performance, in all experiments with synthetic jet actuation, only the two slits closest to the centerspan location have been in operation. The other two slits have been sealed off with thin adhesive tape on the outer surface of the airfoil.

Based on studies by Bootsma [195] and Widjanarko [193], at $x_{j} / c=0.06$ on both sides of the model, Streifeneder zig-zag tape is applied with a thickness of $0.4 \mathrm{~mm}$, width of $6 \mathrm{~mm}$ and a $70^{\circ}$ zig-zag angle, see figure 6.4. This enforces the boundary layers to become turbulent at that location and eliminates the presence of laminar separation bubbles along the surface of the airfoil for free-stream velocities $U_{\infty} \geq 16 \mathrm{~m} / \mathrm{s}$, which corresponds to $\operatorname{Re}_{c} \geq 1.76 \cdot 10^{5}$. 


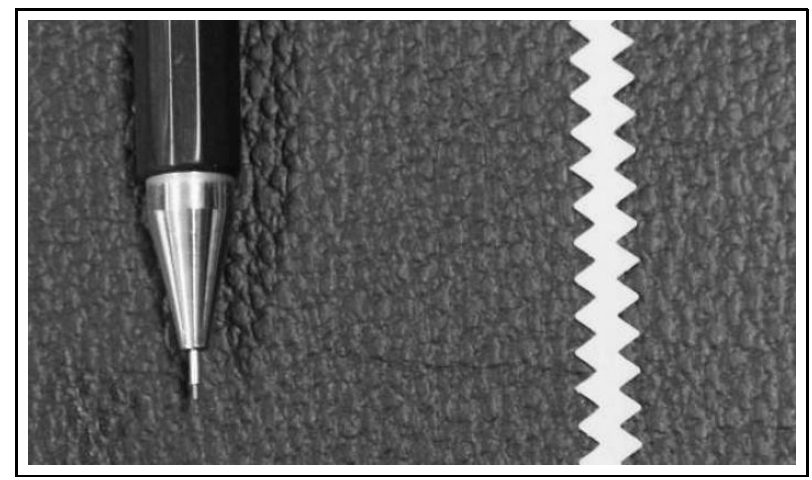

Figure 6.4: Zig-zag tape used for tripping the boundary layer on the airfoil, from [193].

The model is equipped with 29 pressure taps, which are round holes with a diameter of $0.8 \mathrm{~mm}$, distributed over its upper and lower surface. See table 6.1 for detailed information on the location of the pressure taps. Note that they are not all located in the section at center-span, i.e. at $x_{3 \text { center }}$. This is done to reduce the influence the pressure taps have on each other. Each pressure tap is connected to a port of an electronic pressure scanner with a silicon tube, see subsection 6.3.4 for more information. In case a pressure hole would be covered by the zig-zag tape, the tape has been removed locally.

\subsubsection{Actuators}

Two externally mounted, high-quality loudspeakers (JBL $2206 \mathrm{H} / \mathrm{J}$ ) provide the fluctuating pressure inside the plenum, producing a synthetic jet through the slits, see figure 6.3 for a sketch of the configuration. The loudspeakers are rated at nominally $600 \mathrm{~W}$ peak power and they have a good frequency response between 45 to $3500 \mathrm{~Hz}$.

The effective piston diameter is $26.4 \mathrm{~cm}$ and the maximum peak-to-peak excursion before damage is $4.0 \mathrm{~cm}$. In theory, these quantities could be used to calculate average jet velocities. However, due to compressibility effects and viscous losses inside the funnel, connecting tubes, cavity and slits, it is difficult to accurately predict these jet velocities. Furthermore, the cavity is subjected to a fluctuating pressure from two loudspeakers simultaneously, of which the effect is not clear beforehand. Therefore, the performance of the actuation system has been determined experimentally.

\subsubsection{Measurement Equipment}

The static pressure at the 29 pressure taps along the surface of the airfoil are recorded by two electronic pressure scanners (Esterline 9116). One scanner is capable of handling a maximum pressure difference of $7 \mathrm{kPa}$ and is connected to the pressure taps on the pressure side of the airfoil. The other is capable of handling a maximum pressure difference of $17 \mathrm{kPa}$ and is connected to the pressure taps on the suction side of the airfoil. Both 


\begin{tabular}{|c|c|c|c|c|}
\hline no. & $x_{1} / c$ (design) & $x_{1} / c($ realized $)$ & $\left(x_{3 \text { center }}-x_{3}\right) / s$ & side of airfoil \\
\hline 1 & 0.0 & 0.0 & 0.0 & - \\
\hline 2 & 0.0083 & 0.0061 & -0.2342 & $T$ \\
\hline 3 & 0.0167 & 0.0147 & 0.2717 & I \\
\hline 4 & 0.025 & 0.0264 & -0.1161 & I \\
\hline 5 & 0.05 & 0.0475 & -0.3078 & $T$ \\
\hline 6 & 0.075 & 0.0714 & 0.3456 & I \\
\hline 7 & 0.1 & 0.0960 & -0.0433 & $\mathrm{I}$ \\
\hline 8 & 0.194 & 0.1901 & 0.0433 & I \\
\hline 9 & 0.267 & 0.2626 & -0.1533 & I \\
\hline 10 & 0.339 & 0.3354 & 0.1528 & $\mathrm{I}$ \\
\hline 11 & 0.412 & 0.4050 & -0.0794 & I \\
\hline 12 & 0.533 & 0.5318 & 0.0800 & $\mathrm{I}$ \\
\hline 13 & 0.618 & 0.6116 & -0.1900 & $I$ \\
\hline 14 & 0.703 & 0.6972 & 0.3017 & $\mathrm{~T}$ \\
\hline 15 & 0.788 & 0.7750 & 0.1161 & $\mathrm{I}$ \\
\hline 16 & 0.0083 & 0.0070 & 0.2356 & $\mathbf{u}$ \\
\hline 17 & 0.0167 & 0.0147 & -0.2711 & $\mathbf{u}$ \\
\hline 18 & 0.025 & 0.0252 & -0.1156 & $\mathrm{u}$ \\
\hline 19 & 0.05 & 0.0475 & 0.3094 & $\mathbf{u}$ \\
\hline 20 & 0.075 & 0.0714 & -0.3433 & $\mathbf{u}$ \\
\hline 21 & 0.1 & 0.1004 & 0.0433 & $\mathrm{u}$ \\
\hline 22 & 0.194 & 0.1901 & -0.0428 & $\mathrm{u}$ \\
\hline 23 & 0.267 & 0.2687 & 0.1544 & $\mathrm{u}$ \\
\hline 24 & 0.339 & 0.3399 & -0.1517 & $\mathrm{u}$ \\
\hline 25 & 0.412 & 0.4095 & 0.0806 & $\mathrm{u}$ \\
\hline 26 & 0.533 & 0.5273 & -0.0789 & $\mathrm{u}$ \\
\hline 27 & 0.618 & 0.6161 & 0.1911 & $\mathbf{u}$ \\
\hline 28 & 0.703 & 0.6987 & -0.1900 & $\mathbf{u}$ \\
\hline 29 & 0.788 & 0.7855 & -0.1156 & $\mathrm{u}$ \\
\hline
\end{tabular}

Table 6.1: Location of pressure taps on wind-tunnel model, expressed in relative chordwise location, $x_{1} / c$, relative offset from center-span plane, $\left(x_{3 \text { center }}-x_{3}\right) / s$, and the side of the airfoil (l=lower, $u=u p p e r)$. Pressure taps in bold font indicate that these are beyond or close to the outer edges of the two slits closest to the center-span plane. 
scanners have 16 pressure ports, connected to a piezo-resistive pressure sensor, with builtin temperature compensation to reduce thermal errors. The pressures are measured with respect to the common reference-pressure port, which is connected to the atmospheric pressure in the present measurements. The static accuracy given by the manufacturer is $0.15 \%$ of the full-scale pressure, i.e. $10.5 \mathrm{~Pa}$ and $25.5 \mathrm{~Pa}$, respectively. However, the baseline results without actuation show that these figures are rather conservative, as part of these errors are removed by choosing a certain number of samples per measurement, see subsection 6.3.6. Based on these results, see section 6.5. the actual accuracy is extimated to be approximately $3 \mathrm{~Pa}$.

The free-stream velocity is determined by measuring the difference between the total pressure and the static pressure in the free-stream. The total pressure is determined using a Pitot tube, which is placed in the wind tunnel test section, parallel to the flow direction and upstream of the model. The static pressure is an average pressure from several pressure taps in the walls of the test section, near its entrance. The velocity is obtained through Bernoulli's relation, which follows from the assumption of steady, inviscid, incompressible flow:

$$
U_{\infty}=\sqrt{\frac{2\left(p_{\text {total }}-p_{\text {static }}\right)}{\rho_{\infty}}},
$$

where the free-stream density, $\rho_{\infty}$, is calculated with the ideal gas law, equation (3.18). In order to avoid disturbances in the upstream flow, the Pitot tube is removed before recording the static pressures on the surface of the airfoil.

The velocity distributions across the synthetic jets are measured by hot wire anemometry (HWA), using a Dantec StreamLine measuring system operated in constant temperature mode. Constant-temperature anemometry is based on convective heat transfer from a heated wire to the surrounding fluid, whereby the wire is kept at a constant temperature. The heat transfer is primarily related to the fluid velocity. Heating is done by driving an electrical current through the wire, while keeping its electrical resistance constant. The required voltage is related to the heat transfer and is therefore a measure of the velocity. Constant temperature anemometry is known to have a very high frequency response to velocity fluctuations.

The employed hot wire probe (Dantec 55P11, see figure 6.5(a)) has one wire sensor made of tungsten with a thin platinum coating. The wire is directed perpendicularly to the probe axis, which provides the magnitude of the velocity in the direction perpendicular to the wire. The length of the wire is $1.25 \mathrm{~mm}$ and its diameter is $5 \mu \mathrm{m}$. The length of the prongs that hold the wire is $5 \mathrm{~mm}$.

The hot wire system is calibrated by recording the voltage for a probe placed in the potential core of a jet with different, known velocities. Subsequently, the postprocessing software uses a curve fit through these results. Here, King's power law is employed,

$$
E^{2}=A+B U^{n}
$$

where $E$ is the voltage and $U$ is the velocity. In the present case, the constants are equal to $A=7.31, B=3.1$ and $n=0.47$ [193]. To reduce measurement errors due 


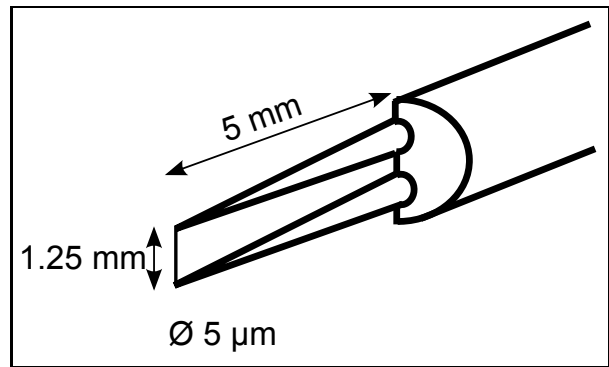

(a) Sketch of Dantec 55P11 hot wire probe, reproduced from [196].

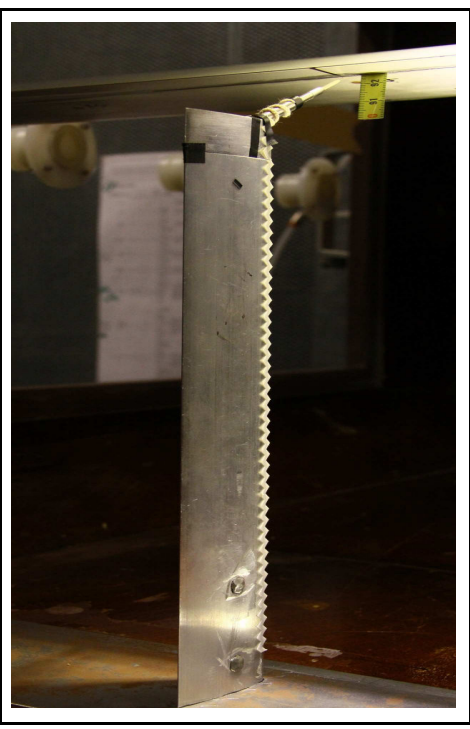

(b) Hot wire mounted in wind tunnel, from [193].

Figure 6.5: Details of hot wire probe and mounting.

to temperature differences during the calibration and the measurements, a temperature signal is recorded and subsequently used to correct the voltages before the conversion to velocities.

In the present measurements, the sampling rate is set at $25 \mathrm{kHz}$ and low-pass filtering is applied at $10 \mathrm{kHz}$. This filter removes high-frequency noise from the signal and prevents signal aliasing.

Figure 6.5(b) shows the way the hot wire is placed in the wind tunnel. The support consists of a vertically mounted airfoil, to which a tube is fitted that holds the hot wire probe. The support is fixed to a traversing mechanism, located outside the test section, which enables the user to manually set the location of the probe. However, the location is set by visual inspection, which can yield errors if an incorrect line of sight is used.

The hot wire support is designed such that the disturbance caused by the support is minimized. Firstly, the probe itself is located upstream and above the support system. Secondly, a helical wire is strung around the tube, preventing the formation of a von Kármán vortex street, which is associated with strong fluctuating aerodynamic forces on the hot wire support. Thirdly, the boundary layer on the vertically mounted airfoil (hot wire support) is tripped, preventing the occurrence of laminar separation bubbles, which are associated with oscillations in the flow. 


\subsubsection{Wind Tunnel}

The experiments are conducted in the closed-loop Silent Wind Tunnel at the University of Twente. Figure 6.6 shows a sketch of the wind tunnel. It has a radial fan, powered by a $130 \mathrm{~kW}$ electric motor, acoustic dampers located at the upstream and downstream side of the fan, a heat exchanger that controls the temperature of the air flowing through the tunnel, a settling chamber with five anti-turbulence screens and a contraction ratio of the nozzle connecting the settling chamber to the test section (not shown in figure 6.6) of $10: 1$. The maximum velocity in the test section is $U_{\infty}=65 \mathrm{~m} / \mathrm{s}$ and the free-stream turbulence level at the entrance of the test section is $0.4 \%$ up to $U_{\infty}=50 \mathrm{~m} / \mathrm{s}$ [197]. After the test section, the flow exhausts in an anechoic chamber of $6 \times 6 \times 4 \mathrm{~m}^{3}(l \times w \times h)$ and is subsequently recaptured by the intake of the diffuser.

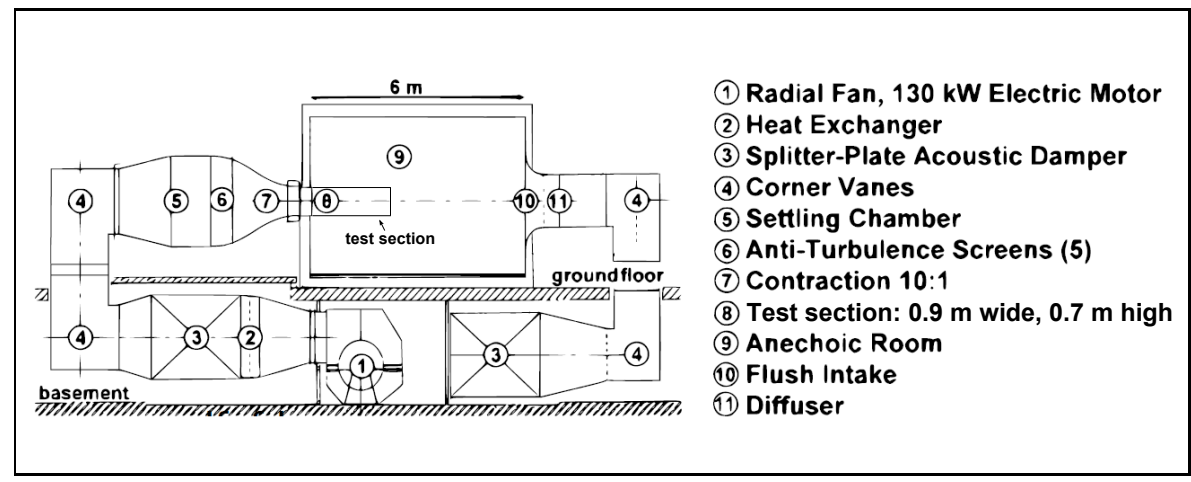

Figure 6.6: Sketch of the closed-loop Silent Wind Tunnel of the University of Twente.

The internal dimensions of the test section are: a width of $0.9 \mathrm{~m}$, a height of 0.7 $\mathrm{m}$, and a length of $2.25 \mathrm{~m}$. The model spans the internal width of the test section, i.e. $s=0.9 \mathrm{~m}$, and is placed in its center. It is bolted to two turning disks mounted flush into the side walls of the wind tunnel. These turning disks are used for setting the angle of attack and they allow for the passage of the silicone tubes that connect the pressure taps to the pressure scanners. The aspect ratio of $s / c \approx 5.45$ is relatively high, which ensures minimal disturbance from the side walls of the wind tunnel. Furthermore, the ratio of the chord length to the height of the test section is approximately 0.236 , which is well below 0.4, above which excessive errors (in maximum lift) due to blocking and constrained streamline curvature are to be expected [198]. The cross-section of the test section is not exactly rectangular, see figure 6.7. The corners are cropped such that the cross-section becomes slightly octagonal. The cropping is reduced towards the exit of the test section, such that the cross-section slightly increases in size (approximately 2.5\% from entrance to exit). This counteracts the disturbance of thickening boundary layers on the test section walls, which would otherwise result in a pressure gradient along the axis of the test section [198]. 


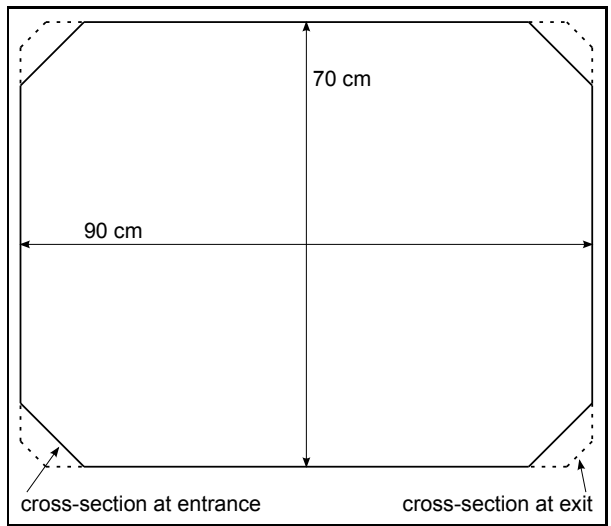

Figure 6.7: Sketch of wind tunnel cross-section at entrance (solid line) and exit (dashed line) of test section.

\subsubsection{Measurement Procedure}

The pressures at the pressure taps along the surface of the airfoil are typically obtained as follows.

- First, the angle of attack is calibrated at $\alpha=0^{\circ}$ by requiring the pressure distribution on the upper and lower side of the airfoil to be equal. Then, different angles of attack are obtained by using a digital level.

- Before each batch of measurements, the ambient pressure is measured using a mercury meter outside the anechoic chamber.

- Before the wind tunnel is switched on, the electronic pressure scanners are zeroed to minimize the offset from the ambient pressure.

- For a certain angle of attack, the required free-stream velocity is set by raising the Pitot tube from the lower wind tunnel wall to a position close to the center of the test section and increasing the wind tunnel motor frequency until the required difference between the total pressure and static free-stream pressure has been obtained. Afterwards, the Pitot tube is lowered again until it rests on the lower wind tunnel wall.

- The temperature in the test section is determined using an electronic thermometer. The temperature is needed together with the static free-stream pressure to calculate the free-stream density.

- The pressures are recorded during a certain time with a certain sampling frequency, and the results are subsequently averaged to remove time-dependent effects. 


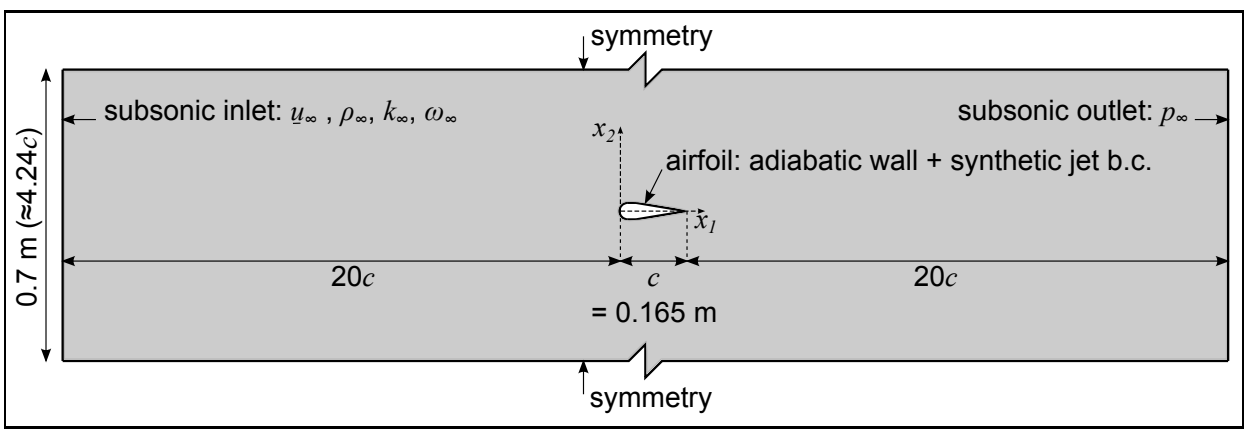

Figure 6.8: Sketch of computational domain for study into pitch control on a NACA0018 airfoil using synthetic jet actuation. Employed boundary conditions are indicated, with the specified variables at the inlet and outlet.

The results presented in this thesis have been obtained by averaging 200 pressure samples per pressure tap, recorded during 20 seconds with a sampling frequency of $10 \mathrm{~Hz}$. The required number of samples per measurement has been determined from analysing different measurements with 1000 samples. Assuming a Gaussian distribution of the samples, it was found that 200 samples approximately yield a confidence probability level of $99 \%$ for an uncertainty value of $15 \mathrm{~Pa}$ [193].

\subsection{Computational Approach}

\subsubsection{Computational Domain and Boundary Conditions}

As mentioned in section 6.2 the computational domain for this study is derived from the wind tunnel test section, see figure 6.8. It is purely two-dimensional, and it has the same height as the test section, i.e. $0.7 \mathrm{~m}$. However, the length of the domain is larger: the domain is extended to 20 chord lengths upstream of the airfoil and 20 chord lengths downstream of the airfoil. This allows for the use of simple inlet and outlet boundary conditions without hampering the accuracy of the flow solution in the vicinity of the airfoil: at the inlet, the free-stream density, free-stream velocity and ambient turbulence levels are prescribed, whereas at the outlet, the free-stream static pressure is prescribed. At the upper and lower boundary of the computational domain, the symmetry boundary condition is applied, which prevents need to resolve the boundary layer there. At the outer surface of the airfoil, the adiabatic no-slip wall boundary condition is employed.

The part of the synthetic jet that is included in the computational domain is shown in figure 6.9. As in the experimental model, the width of the slit equals $w_{j}=1 \mathrm{~mm}$ and its depth equals $d_{j}=2 \mathrm{~mm}$. Furthermore, only a small part of the cavity is included, with a cross-sectional area of $3 \mathrm{~cm}^{2}$ in total. At all internal boundaries, the adiabatic wall boundary condition is applied, except for the bottom boundary of the partial cavity. 
At this boundary, the time-dependent inflow/outflow boundary conditions that model the effect of a moving wall are applied (see subsections 3.5.3 and 4.7.3), as indicated in figure 6.9. For flow simulations without synthetic jet actuation, the adiabatic wall boundary condition is used here as well.

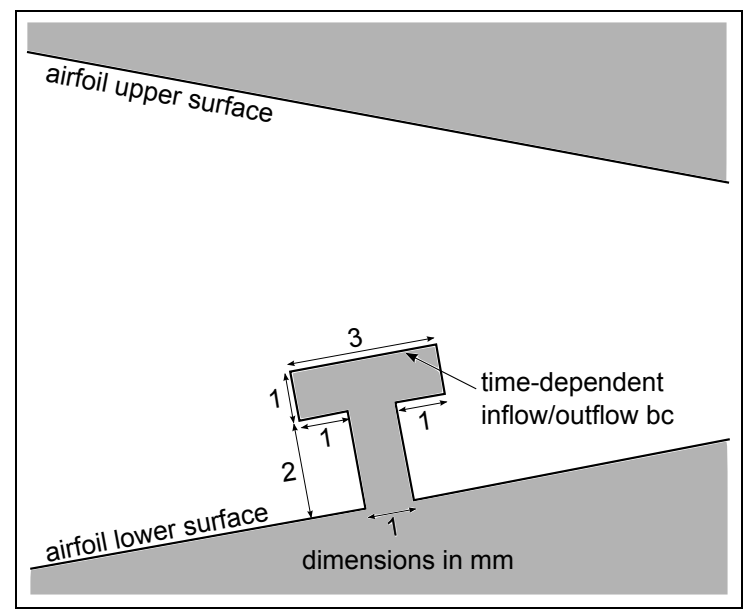

Figure 6.9: Sketch of computational domain in synthetic jet actuator.

\subsubsection{Computational Grids}

For each studied angle of attack, $\alpha$, a computational grid has been constructed using the open-source grid generator GMSH [100. As was done in the verification case presented in subsection 5.2.3 the original contour of the NACA 4-digit airfoil, as defined by equation (5.13), has been extended to zero trailing edge thickness at $\left(x_{1} / c, x_{2} / c\right)=$ $(1.008930411365,0)$. Subsequently, the airfoil has been scaled such that the trailing edge is located at $\left(x_{1} / c, x_{2} / c\right)=(1,0)$ again.

To resolve the boundary layer and the synthetic jet accurately, a layer of thickness $c / 4$ with quadrilaterals has been constructed along the airfoil contour and its wake line, see figure 6.11. The wake line extends one chord length in the downstream direction and its path is defined to be equal to the streamline starting at the trailing edge, as predicted by a potential flow mode 1 .

Based on a grid refinement study for baseline cases without actuation at $\operatorname{Re}_{c}=$ $5.5 \cdot 10^{5}$, the chosen number of elements in the direction normal to the wall and the wake line is 137 elements, with a height of the first element of approximately $2.6 \cdot 10^{-6} \mathrm{~m}$ and a growth factor for subsequent layers of $g=1.05$. The maximum value for $y^{+}$based on the height of the first cell has been found close to the leading edge of the airfoil at

\footnotetext{
${ }^{1}$ Panel method with constant-strength doublet distributions and Kutta-condition at the trailing edge, without influence of wind tunnel walls.
} 
$\operatorname{Re}_{c}=5.5 \cdot 10^{5}$ and $\alpha=9^{\circ}$, and equals approximately $y^{+}=1$. The average value at these conditions is approximately $y^{+}=0.39$.

Along the upper side of the airfoil there are 171 elements, clustered around the leading and trailing edge. Along the lower side of the airfoil there are 330 elements, including 50 elements across the width of the slit. Besides clustering of elements around the leading edge, trailing edge and the edges of the slit, the grid is relatively fine between the slit and the trailing edge, where large gradients in the flow solution are expected. For the case of a slit location of $x_{j} / c=0.88$ and a slit width of $w_{j} / c=1 / 165,103$ elements are located between the slit and the trailing edge. The fine grid is continued in the wake of the airfoil, with 800 elements along the wake line. This will suffice for accurate capturing of vortices that are shed into the wake.

The grid inside the slit and cavity of the synthetic jet also consists of quadrilateral elements. Across the width of the slit there are 50 elements, with clustering at the side edges. Along these side edges of the slit, 170 elements have been placed, with clustering towards each end. In the cavity, there are 50 elements along the top of the cavity on either side of the slit, 50 elements along the sides, and 150 elements along the bottom. Here, the elements have been clustered towards the boundaries and the corners of the slit. The resulting first layer of elements along the internal boundaries has the same height as the first layer of elements along the outer surface of the airfoil, see figure 6.12 .

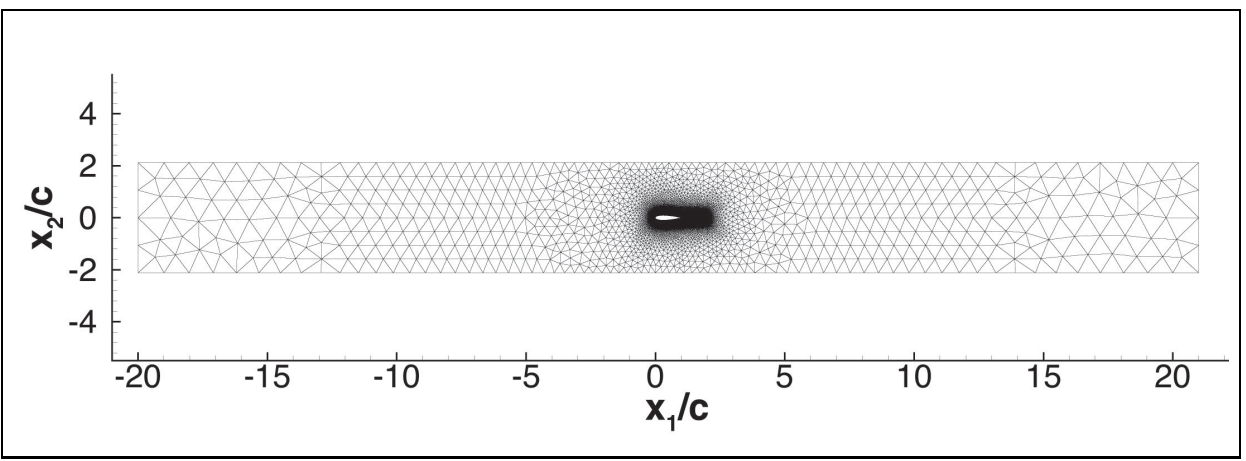

Figure 6.10: Computational grid for $\alpha=0^{\circ}, x_{j} / c=0.88, w_{j} / c=1 / 165, d_{j} / c=2 / 165$ and $\theta_{j}=90^{\circ}$.

The remainder of the computational domain is filled with triangular elements, which gradually increase in size towards the outer boundaries of the domain. Along the upper and lower boundary there are 74 elements, clustered in the region closest to the airfoil. Along the inlet and outlet boundary, 4 elements are located. The grid generator tries to make each triangle as regular (equilateral) as possible, such that each face is (close-to) perpendicular to the line connecting the centroids of the associated neighboring elements and a gradual change in the cell size is obtained. Such elements yield more accurate solutions and a more robust convergence behavior.

The total number of elements in each grid (depending on the angle of attack, $\alpha$ ) is 


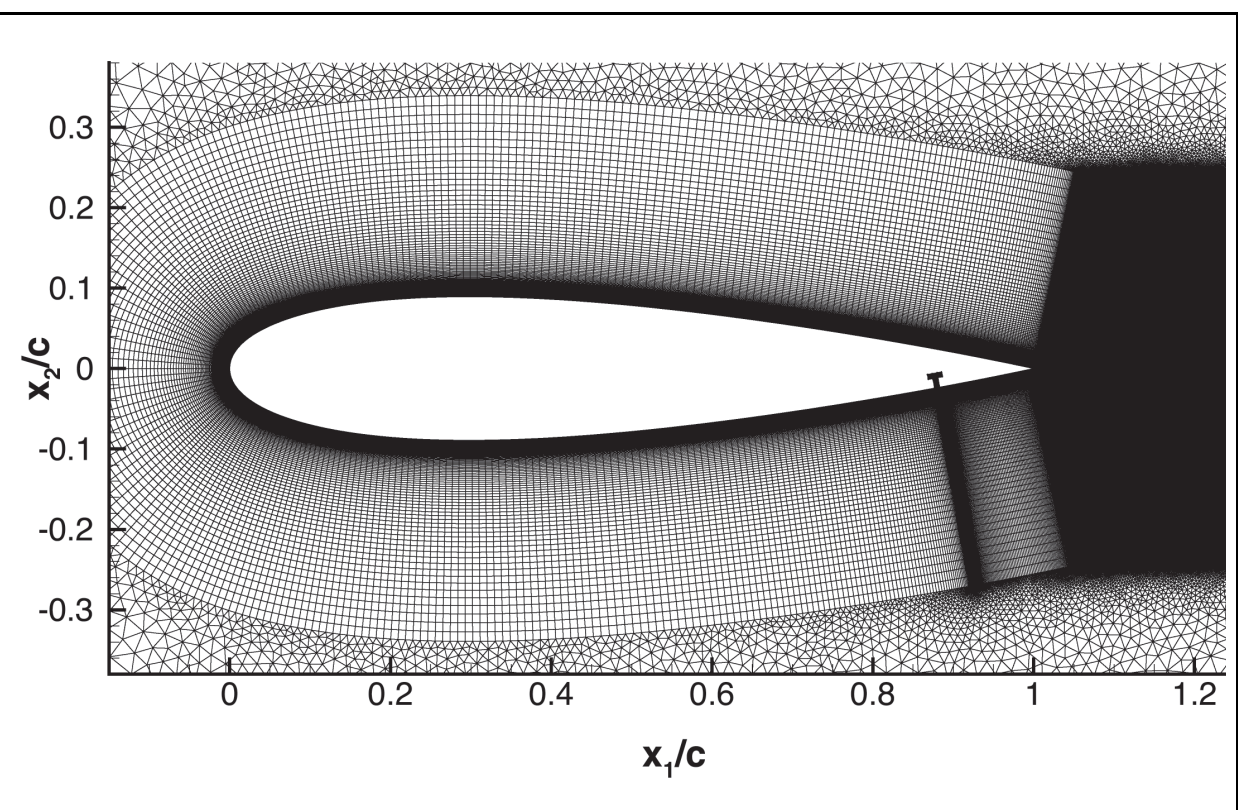

Figure 6.11: Close-up of layer of quadrilateral elements around airfoil for $\alpha=0^{\circ}, x_{j} / c=$ $0.88, w_{j} / c=1 / 165, d_{j} / c=2 / 165$ and $\theta_{j}=90^{\circ}$.

approximately 338000 . The number of quadrilateral elements is 194237 , of which 16000 are located in the slit and cavity of the synthetic jet.

Figures 6.10 6.11 and 6.12 show several images of different aspects of the computational grid for $\alpha=0^{\circ}, x_{j} / c=0.88, w_{j} / c=1 / 165, d_{j} / c=2 / 165$ and $\theta_{j}=90^{\circ}$.

\subsubsection{Discretization and Turbulence Model}

In all flow simulations, Roe's flux-difference splitting scheme is used for the convective fluxes of the URANS equations. Furthermore, the SST turbulence model with active sustaining terms is used in all computations, see section subsection 3.4.3 in combination with the recommended inflow boundary conditions given in subsection 3.5.2 These boundary conditions yield a turbulence level that invokes an early laminar-turbulent boundary layer transition. In other words, the turbulence model operates in fully-turbulent mode.

All flow simulations without synthetic jet actuation, i.e. baseline cases, are steady-flow simulations. These simulations are initiated with the free-stream flow conditions. The CFL number starts at a value of 1.0 and is increased by a factor of 1.2 each pseudo-time step until a maximum CFL number of 500. Above this value, stability problems arise in the regions with large triangles in the computational grid. The calculations continue until all non-linear residuals have decreased at least seven orders of magnitude.

Flow simulations with synthetic jet actuation are time-accurate calculations, initiated 


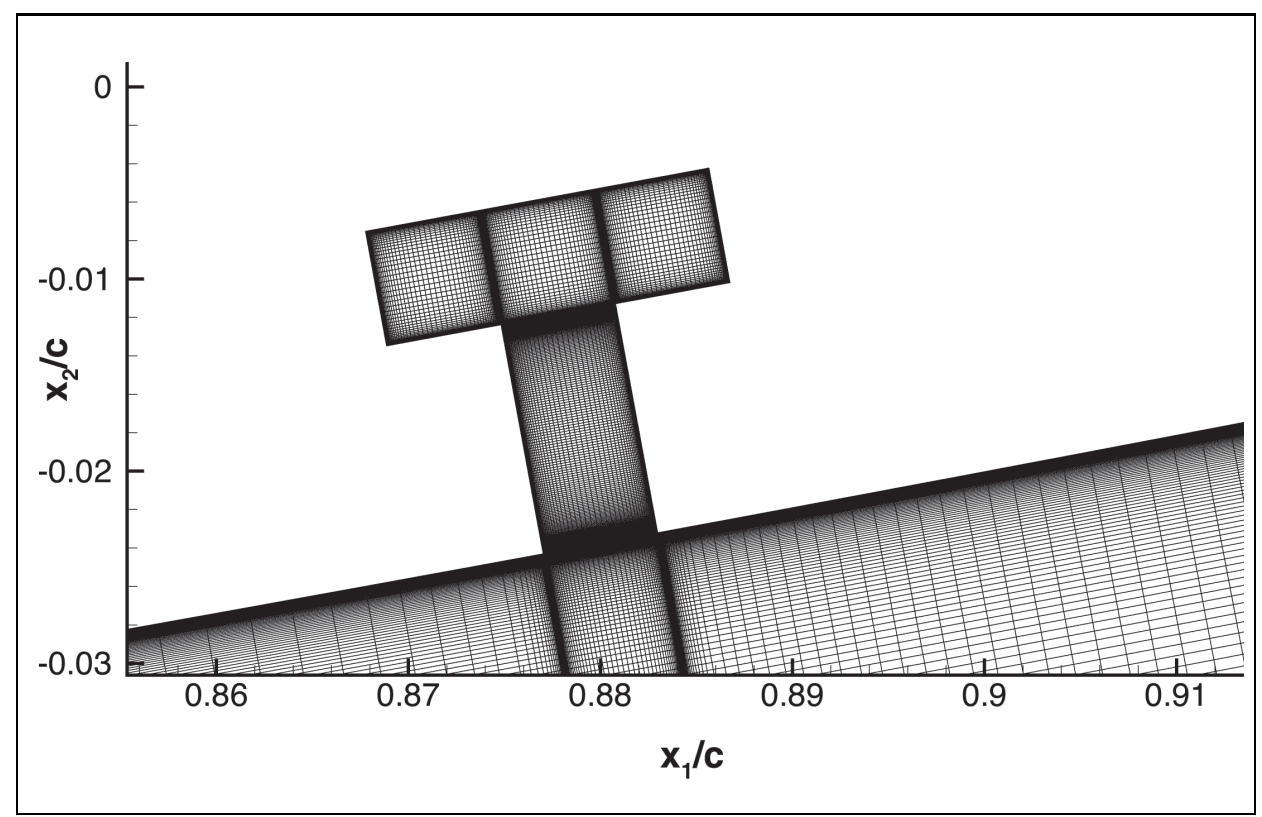

Figure 6.12: Close-up of slit and partial cavity of synthetic jet for $\alpha=0^{\circ}, x_{j} / c=0.88$, $w_{j} / c=1 / 165, d_{j} / c=2 / 165$ and $\theta_{j}=90^{\circ}$.

from a converged baseline case for the same free-stream conditions, with actuation turned off. The number of time steps per actuation cycle varies. It depends on whether the $L_{2^{-}}$ norm of the time-dependent non-linear residuals can be reduced by a certain order of magnitude (usually three), and whether this can be obtained within a reasonable maximum number of pseudo-time steps, e.g. 150. In general, simulations with low-frequency actuation, i.e. $F^{+}<1$, need a relatively high number of time steps per actuation cycle to capture unsteady flow features that have a smaller time scale than the time scale of actuation. For high-frequency actuation cases, the actuation frequency becomes the dominant unsteady flow feature in the flow and fewer time steps per actuation cycle are needed. The number of time steps will be given for each time-accurate flow simulation. In subsection 6.8.1. a time step refinement study is presented for a high-frequency actuation case.

\subsection{Baseline Results}

This section compares the experimental and the computational results for the flow around the NACA0018 airfoil without synthetic jet actuation, i.e. the baseline results.

Besides the computational results obtained with the present method, which solves the RANS equations with the SST turbulence model, results are included that have been 
obtained with Xfoil [199, 200]. Xfoil is a potential flow method that uses panels with linear-vorticity distributions and constant-source distributions. The latter provide for the simulation of viscous shear layers modeled by a two-equation integral boundary layer mode 2 through the concept of displacement thickness. Laminar-turbulent transition is modeled by an envelope version of the $e^{N}$ model, see for example Van Ingen [201] for an extensive overview. The value of $e^{N}$ is the allowed growth factor for instabilities in the boundary layer before the onset of turbulence. The value of $N$ effectively sets the turbulence intensity of the free-stream. Results presented here with free transition use a value of $N=4.85$, which corresponds to a turbulence intensity of $0.4 \%$, equal to the turbulence intensity of the wind tunnel. However, most results presented here employ manual boundary layer tripping at $x_{1} / c=0.06$, as has been done in the experiments with synthetic jet actuation. Note that the influence of the upper and lower wind tunnel walls is not included in the results obtained with $X$ foil.

The baseline results presented here are for two different sets of flow conditions:

- a Reynolds number of $\operatorname{Re}_{c}=1.76 \cdot 10^{5}$ and Mach number of $\mathrm{M}_{\infty}=0.047$, corresponding to a free-stream velocity of $U_{\infty}=16 \mathrm{~m} / \mathrm{s}$,

- a Reynolds number of $\operatorname{Re}_{c}=5.50 \cdot 10^{5}$ and Mach number of $\mathrm{M}_{\infty}=0.146$, corresponding to a free-stream velocity of $U_{\infty}=50 \mathrm{~m} / \mathrm{s}$.

The first set corresponds to the flow conditions used in section 6.7 for the cases with low-frequency synthetic jet actuation. The relatively low Reynolds number is associated with the low performance of the actuation system, which will be explained in section 6.6.

The second set corresponds to the flow conditions used in section 6.8 for the cases with high-frequency actuation. Since the actuation system in the experimental setup cannot reach these high frequencies, there is no reason for using the lower Reynolds number of the first set of flow conditions. So, a higher Reynolds number corresponding to a free-stream velocity closer to the maximum free-stream velocity of the wind tunnel is chosen for this investigation. Although no experiments with high-frequency synthetic jet actuation have been performed at these flow conditions, the baseline results are still included here for the purpose of validation of the RANS model.

We will focus on two angles of attack, namely $\alpha=0^{\circ}$ and $\alpha=8^{\circ}$. These values are also used in section 6.7 for the cases with low-frequency synthetic jet actuation. Note that in the presented figures for $\alpha=8^{\circ}$, the coordinate system has been rotated such that the $x_{1}$-axis is directed along the chord line, and the $x_{2}$-axis is directed perpendicular to this line.

The results computed with the present computational method are grid converged, i.e. it has been checked that further refinement of the computational grid does not yield significant changes in the results.

\footnotetext{
${ }^{2}$ The two-equation integral boundary layer model consists of the Von Kármán's integral momentum equation and the kinetic energy integral equation, supplemented with closure relations for laminar and turbulent boundary layers.
} 
The results for the pressure distribution on the surface of the airfoil is expressed in the pressure coefficient, $c_{p}$, defined as

$$
c_{p}=\frac{p-p_{\infty}}{\frac{1}{2} \rho_{\infty} U_{\infty}^{2}},
$$

with $p_{\infty}$ the static free-stream pressure, $\rho_{\infty}$ the free-stream density and $U_{\infty}$ the freestream velocity. Furthermore, computational results for the skin friction will be shown, expressed in the skin friction coefficient, $c_{f}$, which is defined as

$$
c_{f}=\frac{\tau_{w}}{\frac{1}{2} \rho_{\infty} U_{\infty}^{2}},
$$

with $\tau_{w}$ the shear stress at the wall.

\subsubsection{Effect of Boundary Layer Tripping}

Figures 6.13 and 6.14 show the effect of tripping the boundary layer at $x_{1} / c=0.06$ by means of zig-zag tape, for $\operatorname{Re}_{c}=1.76 \cdot 10^{5}$ and $\operatorname{Re}_{c}=5.50 \cdot 10^{5}$, respectively. Results obtained with Xfoil are also included.

Without tripping, both the experimental results and the results obtained with $\mathrm{X}$ foil show evidence of laminar separation bubbles: a region in which the curve of the pressure coefficient becomes more or less flat, before a sharp rise of the pressure at the location where transition to turbulence and reattachment of the boundary layer occurs. For $\alpha=0^{\circ}$, the extent of the laminar separation bubbles in the experiment is larger than predicted by Xfoil at both Reynolds numbers. Although not shown here, choosing a lower turbulence intensity in Xfoil yields better agreement between the experimental results and the prediction. This suggests that the turbulence intensity of the wind tunnel is lower at this angle of attack. For $\alpha=8^{\circ}$, it appears that the laminar separation bubble on the suction side of the airfoil is approximately of the same size in the experiment and in the prediction by Xfoil at both Reynolds numbers. However, at this angle of attack the spatial resolution of the experimental results in the region of the laminar separation bubble is too low to accurately determine its length.

The results with boundary layer tripping show that the application of zig-zag tape at $x_{1} / c=0.06$ prevents the formation of laminar separation bubbles at both Reynolds numbers and both angles of attack. Comparing the experimental results with the results obtained with $\mathrm{X}$ foil shows that the presence of the zig-zag tape disturbs the pressure distribution in its vicinity. Note that the tape is located between $x_{1} / c \approx 0.04$ and $x_{1} / c \approx 0.08$. This region is indicated in the figures as a grey area. For $\alpha=0^{\circ}$, the disturbance causes a sudden increase in the pressure directly downstream of the tape at $x_{1} / c \approx 0.1$. For $\alpha=8^{\circ}$, the disturbance also affects the suction peak, upstream of the tape. Close to the trailing edge of the airfoil, the experimental results show a somewhat lower pressure level on both sides of the airfoil for $\alpha=0^{\circ}$ and on the suction side of the airfoil for $\alpha=8^{\circ}$. Possibly, the zig-zag tape introduces flow features that cause the boundary layer to develop differently than a naturally developing turbulent boundary layer. 


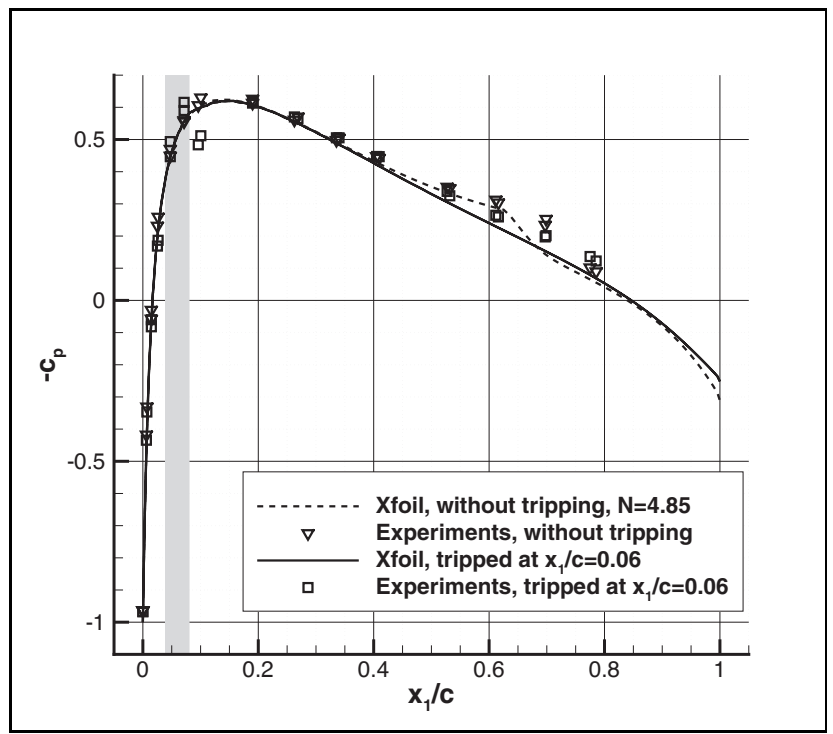

(a) $\alpha=0^{\circ}$

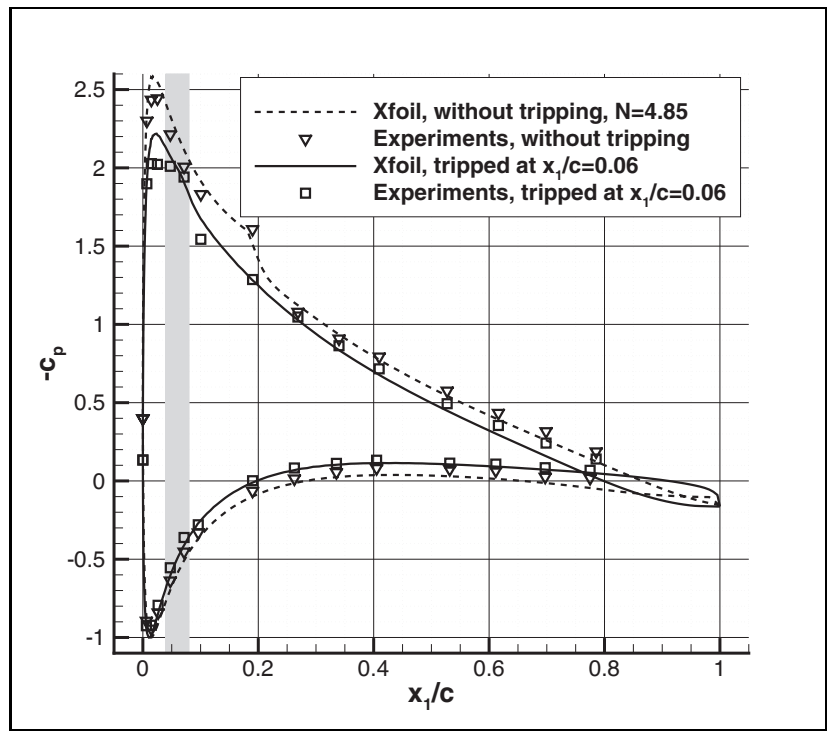

(b) $\alpha=8^{\circ}$

Figure 6.13: Baseline results for pressure coefficient, $c_{p}$, with and without boundary layer tripping, for $\operatorname{Re}_{c}=1.76 \cdot 10^{5}, \mathrm{M}=0.047$. N refers to the parameter of the transition model of Xfoil. Grey area indicates the tripping region in the experiments. 


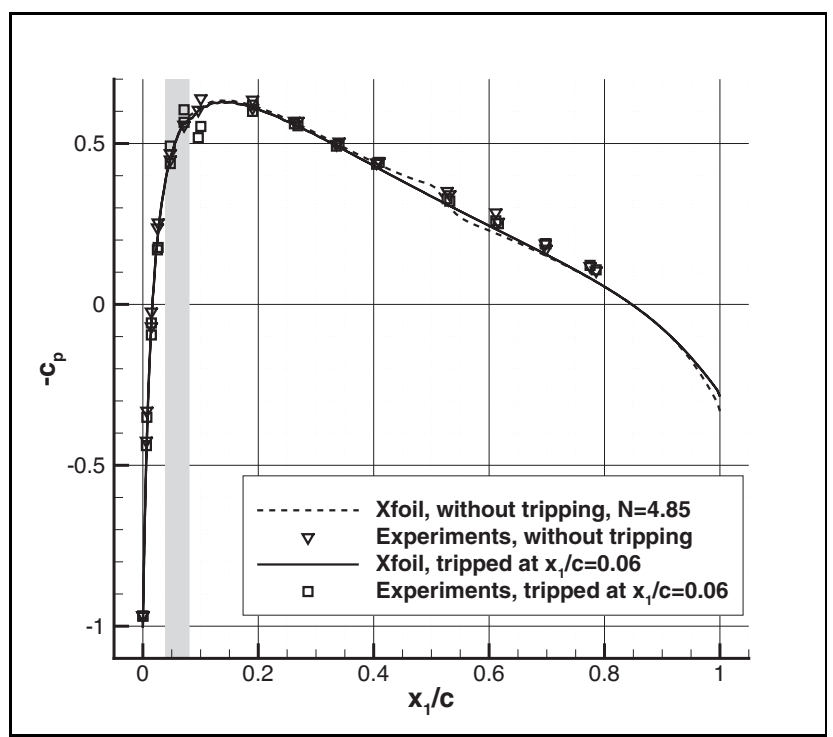

(a) $\alpha=0^{\circ}$

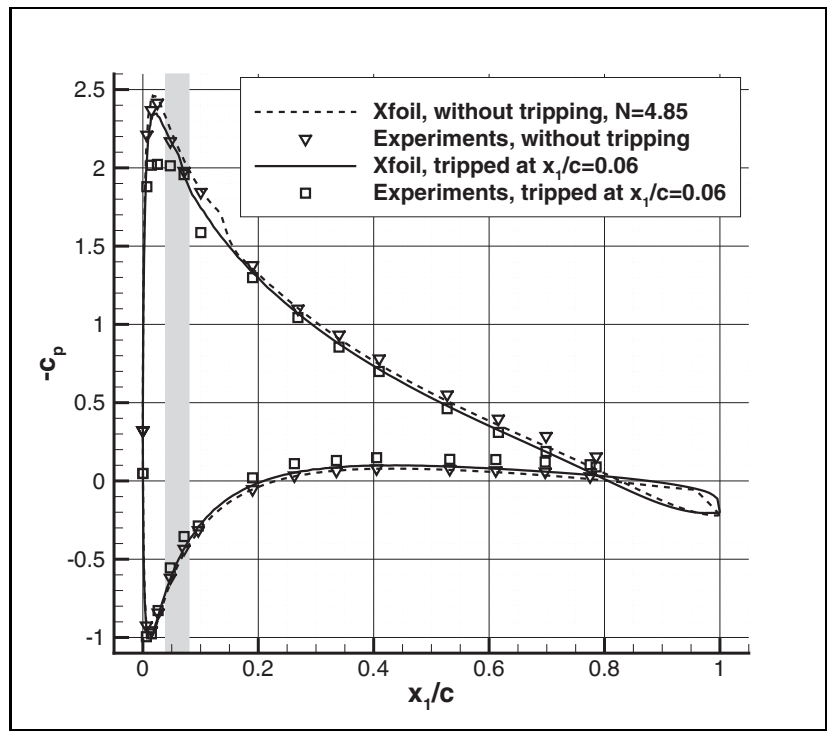

(b) $\alpha=8^{\circ}$

Figure 6.14: Baseline results for pressure coefficient, $c_{p}$, with and without boundary layer tripping, for $\operatorname{Re}_{c}=5.50 \cdot 10^{5}, \mathrm{M}=0.146 . \quad N$ refers to the parameter of the transition model of Xfoil. Grey area indicates the tripping region in the experiments. 


\subsubsection{Validation of RANS Method}

\section{Validation for $\operatorname{Re}_{c}=1.76 \cdot 10^{5}$}

Figure 6.15 shows the results for the pressure coefficient and skin friction coefficient on the surface of the airfoil at $\operatorname{Re}_{c}=1.76 \cdot 10^{5}, \mathrm{M}=0.047$ and $\alpha=0^{\circ}$. Figure 6.16 shows these coefficients at $\alpha=8^{\circ}$ and the same Reynolds and Mach number.

In general, the computational results for the pressure coefficient, $c_{p}$, obtained with the present computational method compare quite well with the experimental results as well as the results obtained with Xfoil. As discussed previously, the experimental results differ from the computational results in the region around the location of the zig-zag tape, which is located between $x_{1} / c \approx 0.04$ and $x_{1} / c \approx 0.08$. Towards the trailing edge, the pressure distributions show some small deviations, with the results of the RANS method in between the experimental results and the results obtained with $\mathrm{X}$ foil.

The results for the skin friction coefficient show that the RANS method using the SST turbulence model generally yields a laminar-turbulent transition further downstream than $x_{1} / c=0.06$ at this Reynolds number, even though the turbulence model operates in fullyturbulent mode. For $\alpha=8^{\circ}$, on the suction (upper) side of the airfoil, transition occurs close to the leading edge, but for $\alpha=0^{\circ}$, transition occurs at approximately $x_{1} / c=0.18$, and for $\alpha=8^{\circ}$ on the pressure (lower) side, transition occurs at approximately $x_{1} / c=$ 0.32 . Note that the presence of the slit on the lower side of the airfoil at $x_{1} / c=0.88$ is clearly visible in the results for the skin friction coefficient.

Furthermore, the results for the skin friction coefficient also show that the RANS method using the SST turbulence model yields earlier boundary layer separation than predicted by Xfoil. For $\alpha=0^{\circ}$, separation is predicted at $x_{1} / c \approx 0.993$ (approximately 11 grid points upstream of the trailing edge), whereas $X$ foil does not predict any boundary layer separation. For $\alpha=8^{\circ}$ on the suction side of the airfoil, separation is predicted at $x_{1} / c \approx 0.835$, whereas $\mathrm{X}$ foil predicts separation at $x_{1} / c \approx 0.97$. Experimental results for the skin friction coefficient are not available. However, it is possible that the boundary layers in the experiments have a higher tendency towards separation, because the experimental results for the pressure coefficient close to the trailing edge in the previously mentioned situations show signs of leveling off to a higher pressure level.

Validation for $\operatorname{Re}_{c}=5.50 \cdot 10^{5}$

Figure 6.17 shows the results for the pressure coefficient and skin friction coefficient at $\operatorname{Re}_{c}=5.50 \cdot 10^{5}, \mathrm{M}_{\infty}=0.146$ and $\alpha=0^{\circ}$. Figure 6.18 shows these coefficients at $\alpha=8$ and the same Reynolds and Mach number.

For $\operatorname{Re}_{c}=5.50 \cdot 10^{5}$ and $\alpha=0^{\circ}$, the results for the pressure coefficient show the same behavior as for $\operatorname{Re}_{c}=1.76 \cdot 10^{5}$ and the same angle of attack: in general, all results compare quite well with each other, except towards the trailing edge, where the pressure distributions start to deviate somewhat. Furthermore, the experimental results differ from the computational results in the region around the zig-zag tape.

For $\operatorname{Re}_{c}=5.50 \cdot 10^{5}$ and $\alpha=8^{\circ}$, the results for the pressure coefficient show a less 


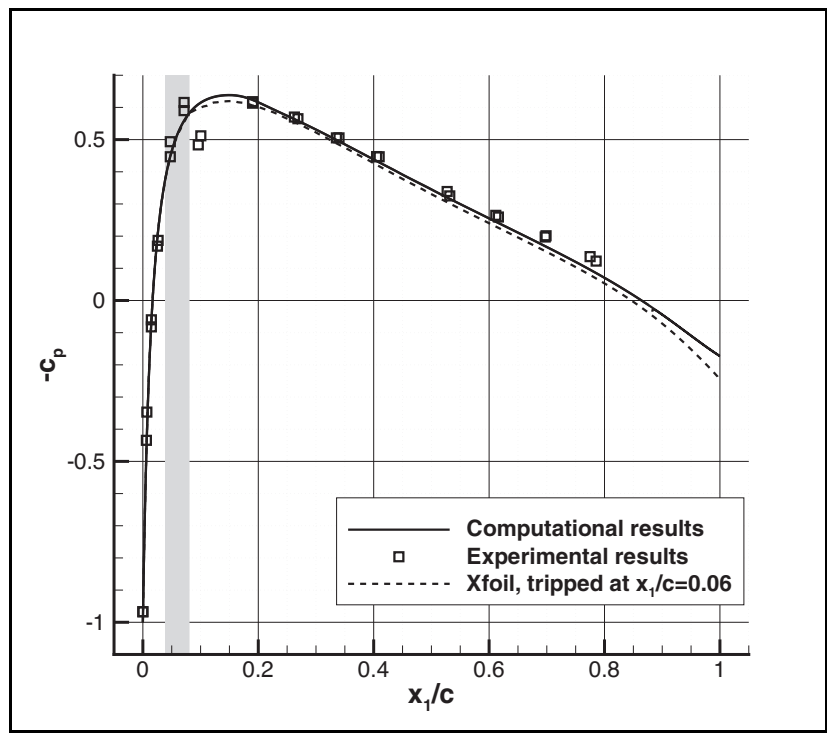

(a) $c_{p}$

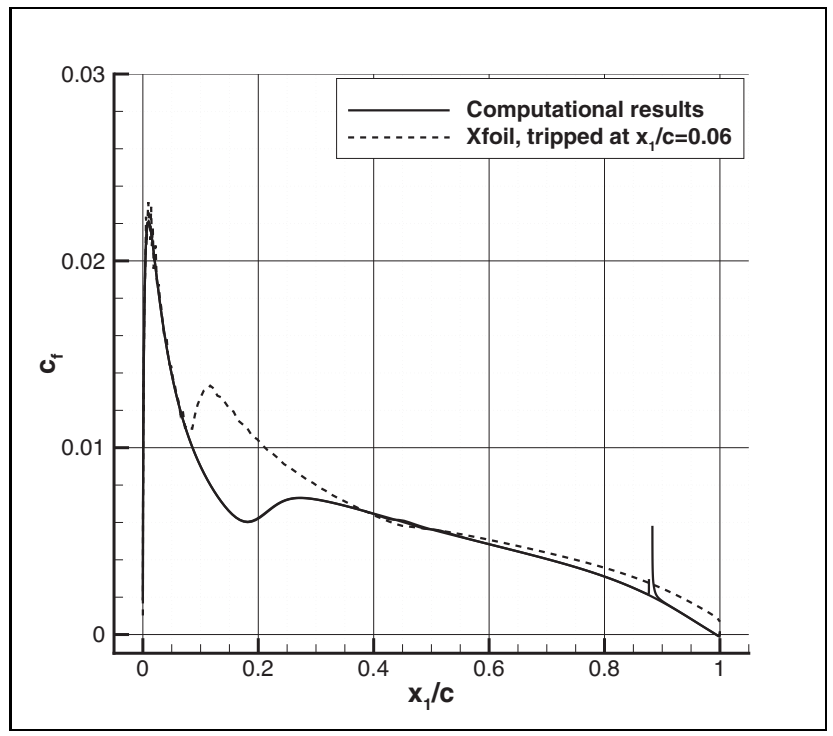

(b) $c_{f}$

Figure 6.15: Baseline results for pressure coefficient, $c_{p}$, and skin friction coefficient, $c_{f}$, for $\alpha=0^{\circ}, \operatorname{Re}_{c}=1.76 \cdot 10^{5}, \mathrm{M}_{\infty}=0.047$. Grey area indicates the tripping region in the experiments. 


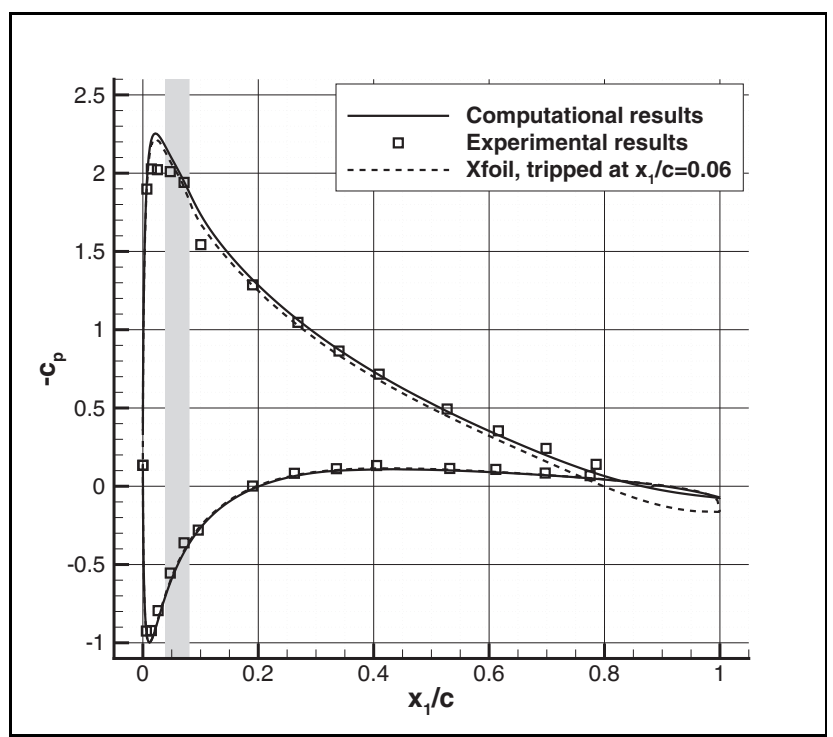

(a) $c_{p}$

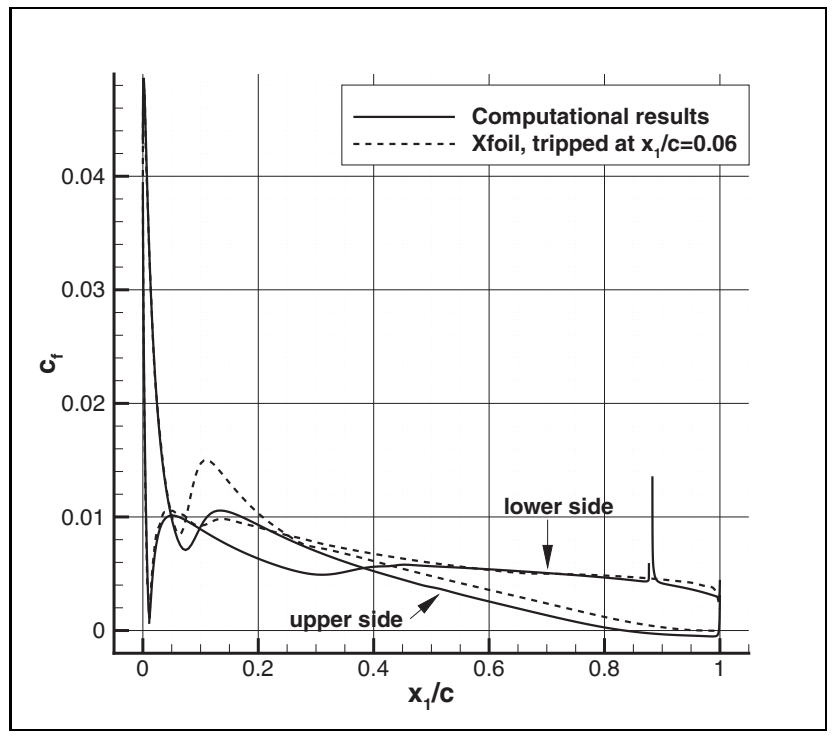

(b) $c_{f}$

Figure 6.16: Baseline results for pressure coefficient, $c_{p}$, and skin friction coefficient, $c_{f}$, for $\alpha=8^{\circ}, \operatorname{Re}_{c}=1.76 \cdot 10^{5}, \mathrm{M}_{\infty}=0.047$. Grey area indicates the tripping region in the experiments. 


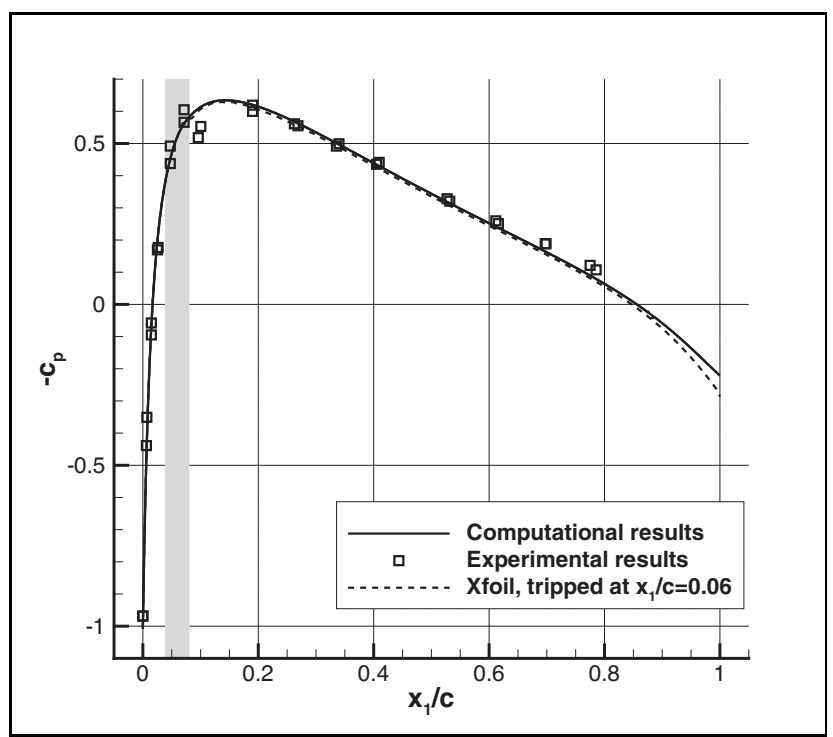

(a) $c_{p}$

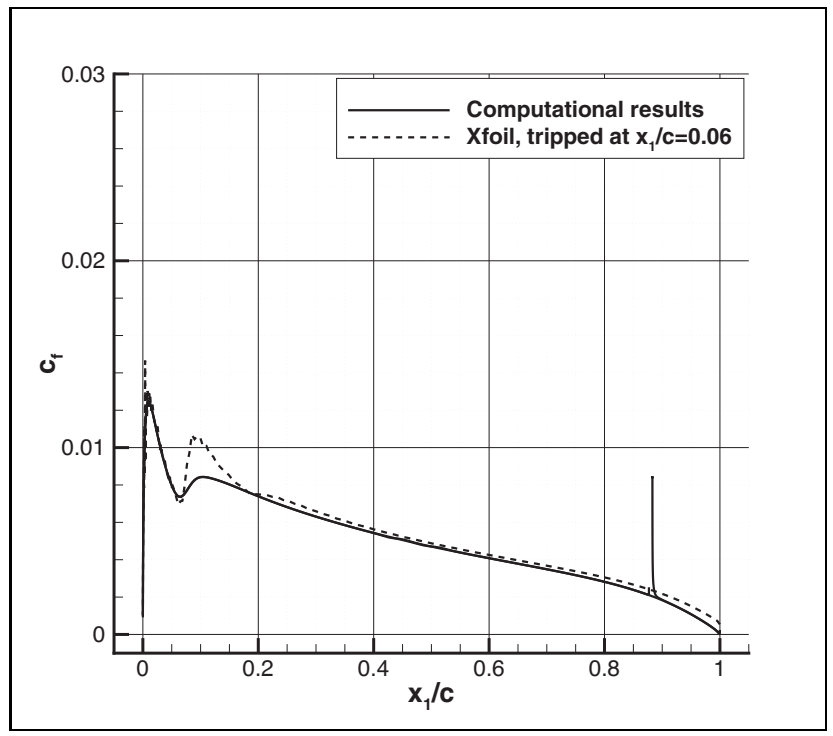

(b) $c_{f}$

Figure 6.17: Baseline results for pressure coefficient, $c_{p}$, and skin friction coefficient, $c_{f}$, for $\alpha=0^{\circ}, \operatorname{Re}_{c}=5.50 \cdot 10^{5}, \mathrm{M}_{\infty}=0.146$. Grey area indicates the tripping region in the experiments. 


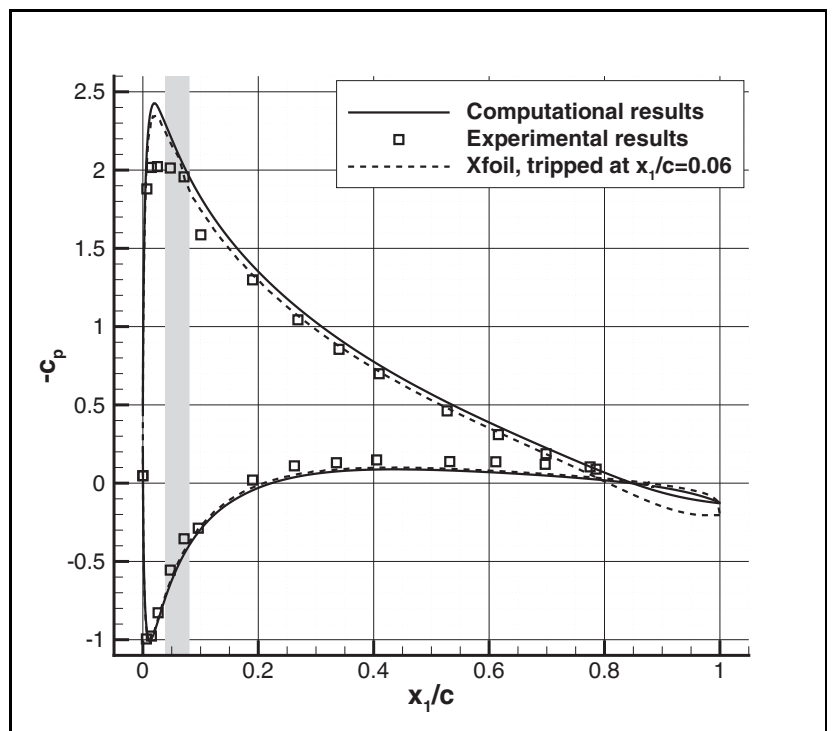

(a) $c_{p}$

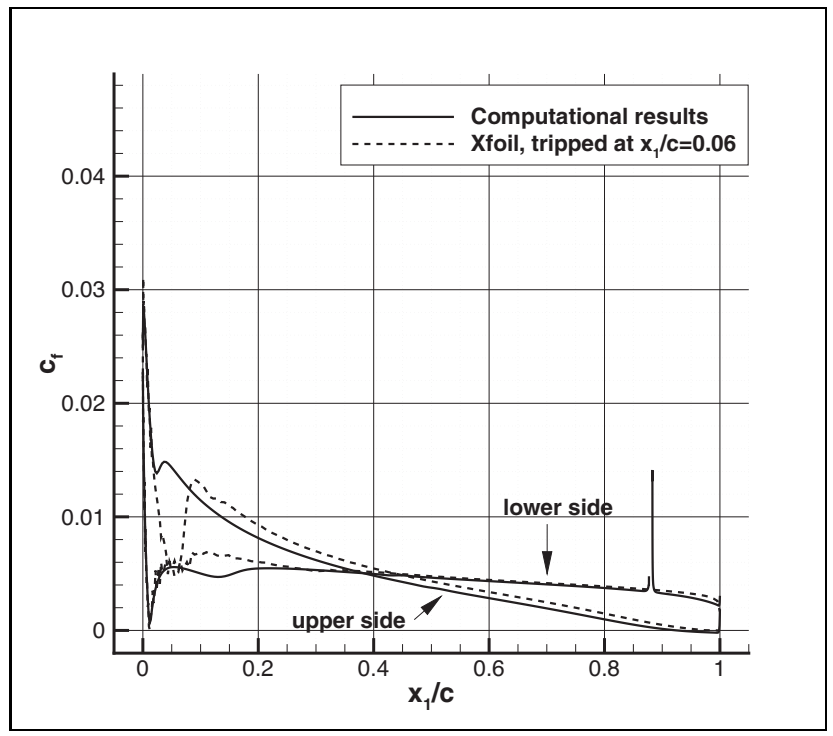

(b) $c_{f}$

Figure 6.18: Baseline results for pressure coefficient, $c_{p}$, and skin friction coefficient, $c_{f}$, for $\alpha=8^{\circ}, \operatorname{Re}_{c}=5.50 \cdot 10^{5}, \mathrm{M}_{\infty}=0.146$. Grey area indicates the tripping region in the experiments. 
satisfactory comparison than for $\operatorname{Re}_{c}=1.76 \cdot 10^{5}$ and the same angle of attack. The experimental results show a lower pressure difference between the upper and lower side of the airfoil than the results from the present computational method and $X$ foil. This might be due to a disturbing effect of the zig-zag tape, which could be more prominent at the higher Reynolds number.

The results for the skin friction coefficient show that the RANS method using the SST turbulence model yields a laminar-turbulent transition location closer to the leading edge than for $\operatorname{Re}_{c}=1.76 \cdot 10^{5}$ at the same angles of attack. For $\operatorname{Re}_{c}=5.50 \cdot 10^{5}$ and $\alpha=0^{\circ}$, the present computational results compare quite well with the results obtained with Xfoil. However, it can be observed that the present computational method generally yields a more gradual transition from a laminar to a turbulent state, with a less pronounced rise in skin friction. For $\operatorname{Re}_{c}=5.50 \cdot 10^{5}$ and $\alpha=8^{\circ}$, the present computational method yields a transition location of $x_{1} / c \approx 0.025$ on the suction side, i.e. upstream of $x_{1} / c=0.06$, and $x_{1} / c \approx 0.14$ on the pressure side, i.e. downstream of $x_{1} / c=0.06$.

Additionally, the results for the skin friction coefficient show that the RANS model using the SST turbulence model yields boundary layers that are closer to separation than predicted by $\mathrm{Xfoil}$. For $\alpha=0^{\circ}$, both methods predict attached boundary layers up to the trailing edge. For $\alpha=8^{\circ}$ on the suction side of the airfoil, the RANS method predicts separation at $x_{1} / c \approx 0.925$, whereas $\mathrm{X}$ foil does not predict boundary layer separation.

\subsubsection{Conclusions}

In the experiments, the application of zig-zag tape at $x_{1} / c=0.06$ is sufficient to trip the boundary layer and remove laminar separation bubbles. However, it also introduces disturbances in the pressure distribution, as can be seen when comparing the experimental results with results obtained with $\mathrm{X}$ foil and the present computational method using the SST turbulence model. The disturbance of the pressure distribution is visible as a lower suction peak at $\alpha=8^{\circ}$. Furthermore, the pressure taps directly downstream of the zig-zag tape at $x_{1} / c \approx 0.1$ register a higher pressure on both sides of the airfoil at $\alpha=0^{\circ}$ and on the suction side of the airfoil at $\alpha=8^{\circ}$. Towards the trailing edge, the pressure distributions in some cases show signs of leveling off to a higher pressure level than predicted by the computational method and $X$ foil, which may also be a result of the presence of the zig-zag tape. This should be taken into account when the experimental results are used to assess the validity of computational results, also for flow cases with synthetic jet actuation.

In general, however, the results of the present computational method using the SST turbulence model compare quite well with the experimental results as well as the results obtained with Xfoil. Besides the differences attributed to the disturbing effect of the zigzag tape in the experiments, another observed difference is the laminar-turbulent transition behavior between the present computational method using the SST turbulence model and $X$ foil using fixed transition. First of all, the present computational method generally yields a more gradual transition from a laminar to a turbulent state, with a less pronounced rise in skin friction. Since experimental results for the skin friction are not available, it is not 
possible to determine which behavior is closer to reality. Secondly, the SST turbulence model operates in fully-turbulent mode, which should yield transition close to the leading edge of the airfoil. However, for the lower Reynolds number, $\operatorname{Re}_{c}=1.76 \cdot 10^{5}$, this is not always the case. For $\operatorname{Re}_{c}=5.50 \cdot 10^{5}$, the agreement is better.

\subsection{Performance of Synthetic Jet Actuation System}

Before the actual experiments in the wind tunnel, the performance of the actuation system of the experimental setup has been tested without a cross-flow. Early measurements of the peak ejection velocity along the four slits in spanwise direction showed that is not possible to obtain uniform spanwise behavior and a reasonable performance along all four slits [193. However, when the two slits closest to the wind tunnel walls are sealed off with thin adhesive tape on the outer surface of the airfoil, the uniformity and performance for the two remaining slits improve, at least for actuation frequencies up to approximately $120 \mathrm{~Hz}$. For higher actuation frequencies, the performance becomes much lower close to the center-span location. This may be an effect of different acoustical behavior inside the cavity at these higher frequencies, due to an increasing ratio of the spanwise length of the cavity to the actuation wavelength. Since we want a close-to two-dimensional situation in the experiments, all results presented in this thesis have been obtained with only the two slits closest to the center-span plane in operation. The pressure taps that are beyond or close to the outer edges of the two operating slits, see table 6.1 will therefore not register much effect of synthetic jet actuation. The measured pressures at these locations are not taken into account when the experimental results are used to assess the effectiveness of synthetic jet actuation and the validity of the accompanying computational results.

Figure 6.19 shows the peak ejection velocity, $\left|\underline{u}_{j}\right|_{\text {peak }}$, for one of the two slits closest to the center-span location. The location of the measurement is in the center of the slit in spanwise direction, in the plane perpendicular to the direction of the jet, approximately one slit width from the exit. This is the closest attainable distance to the slit, without risking to break the delicate hot wire probe. The hot wire is directed parallel to the spanwise direction.

Results have been obtained for a range of actuation frequencies and applied voltages: $f_{j} \in[15,180] \mathrm{Hz}$ and $V_{\mathrm{rms}} \in[15,40] \mathrm{V}$. The results show an approximately linear behavior of the peak velocity with respect to the applied voltage, $V_{\text {rms }}$. Furthermore, the highest peak velocity occurs at the lowest investigated frequency, $f_{j}=15 \mathrm{~Hz}$, and decreases with increasing actuation frequency. For actuation frequencies between 50 and $120 \mathrm{~Hz}$, the performance is approximately constant. A significant drop in the performance is observed at the highest investigated actuation frequency.

Since we want a close-to two-dimensional situation in the experiments, the range of frequencies that can be used in the experimental investigation is on the lower end of the spectrum. Furthermore, to obtain a distinct measurable effect of synthetic jet actuation, the peak ejection velocity needs to be of the same order of magnitude as the free-stream velocity. The only way to meet these criteria and still have a reasonable range of actuation frequencies to investigate is to choose a relatively low free-stream velocity of $U_{\infty}=16$ 


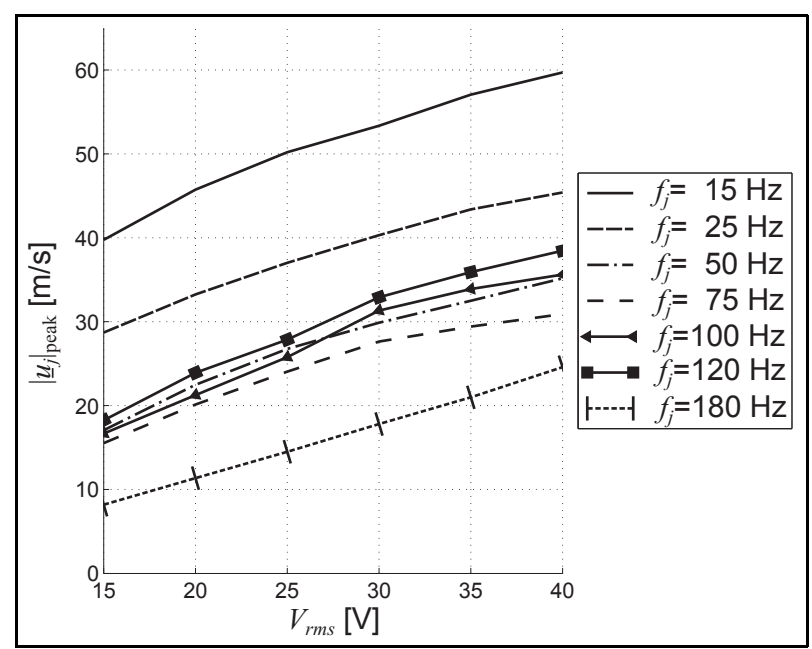

Figure 6.19: Performance of the synthetic jet actuation system in terms of peak ejection velocity, $\left|\underline{u}_{j}\right|_{\text {peak }}$, measured approximately one slit width from exit, as function of the applied r.m.s. voltage, $V_{\mathrm{rms}}$, for different actuation frequencies, from [193].

$\mathrm{m} / \mathrm{s}$. This is associated with a Reynolds number of $\operatorname{Re}_{c}=1.76 \cdot 10^{5}$ and a Mach number of $\mathrm{M}=0.047$. The maximum dimensionless frequency that can be achieved at these flow conditions is $F^{+}=1.238$, i.e. relatively low.

Figure 6.20 shows results of hot wire measurements of the absolute velocity of the jet as a function of time, with and without a cross-flow due to a free-stream velocity of $U_{\infty}=16 \mathrm{~m} / \mathrm{s}$, for $f_{j}=15 \mathrm{~Hz}$ (above) and $f_{j}=120 \mathrm{~Hz}$ (below), $\alpha=0^{\circ}$ (left) and $\alpha=8^{\circ}$ (right). The dimensionless actuation frequencies in the presence of the cross-flow are $F^{+}=0.155$ and $F^{+}=1.238$, respectively. For each case, four actuation cycles are shown. The first part of each actuation cycle corresponds to ejection, the second part corresponds to ingestion. The applied voltage, $V_{\mathrm{rms}}$, is chosen such that the peak ejection velocity is approximately the same for both actuation frequencies. As before, the location for the measurements is in the center of the slit in spanwise direction, in the plane perpendicular to the direction of the jet, approximately one slit width from the exit. Several observations can be made with respect to these results:

- The peak ejection velocity is approximately the same for the cases without and with a cross-flow due to a free-stream velocity of $U_{\infty}=16 \mathrm{~m} / \mathrm{s}$, except for $f_{j}=120 \mathrm{~Hz}$ and $\alpha=8^{\circ}$, for which the performance is reduced in the presence of the cross-flow.

- During the ingestion part of the actuation cycle, differences in the measured velocity exist between the cases without and with a cross-flow. The cross-flow itself is a contributor to the measured data, which is especially clear for $\alpha=8^{\circ}$, for which the measured velocity is of the order of the free-stream velocity. 


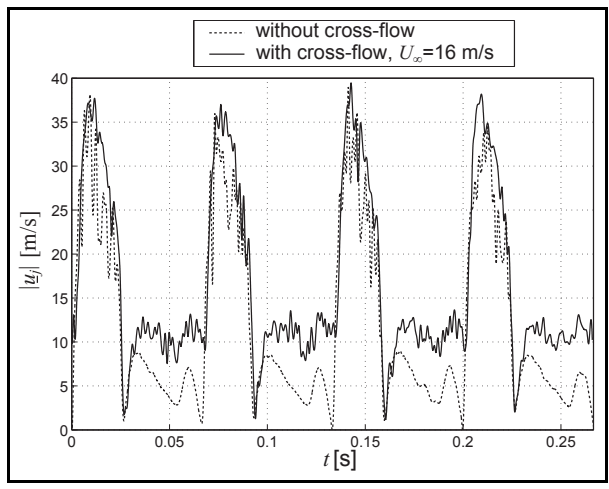

(a) $f_{j}=15 \mathrm{~Hz}, V_{\mathrm{rms}}=20 \mathrm{~V}, \alpha=0^{\circ}$

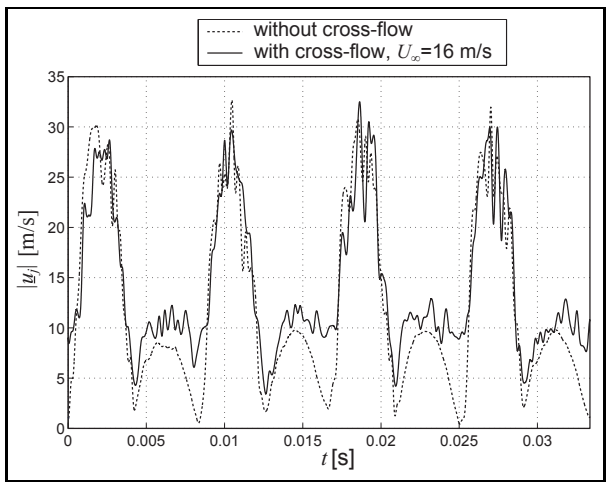

(c) $f_{j}=120 \mathrm{~Hz}, V_{\mathrm{rms}}=40 \mathrm{~V}, \alpha=0^{\circ}$

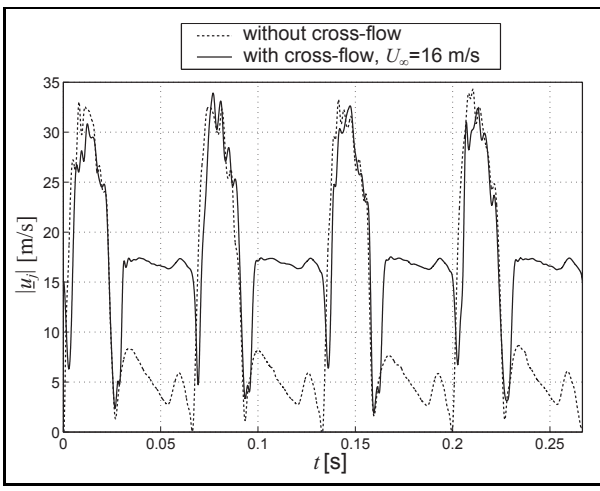

(b) $f_{j}=15 \mathrm{~Hz}, V_{\mathrm{rms}}=20 \mathrm{~V}, \alpha=8^{\circ}$

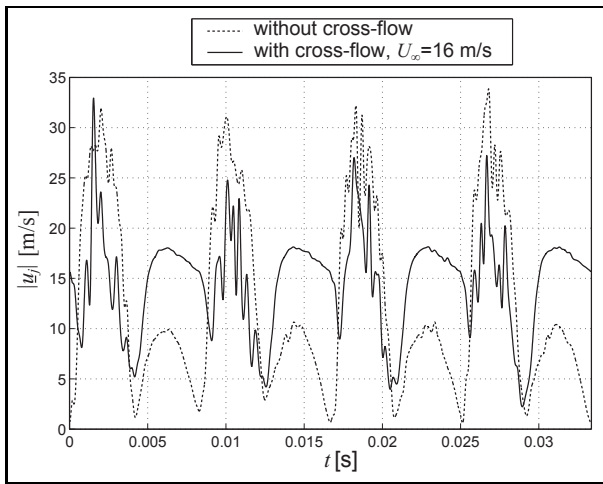

(d) $f_{j}=120 \mathrm{~Hz}, V_{\mathrm{rms}}=40 \mathrm{~V}, \alpha=8^{\circ}$

Figure 6.20: Experimental results for magnitude of jet velocity, $\left|\underline{u}_{j}\right|$, without and with a cross-flow of $U_{\infty}=16 \mathrm{~m} / \mathrm{s}$, for different actuation frequencies and angles of attack, from [193]. Measured at one of the two slits closest to the center-span location, approximately one slit width away from the exit.

- During the ejection part of the actuation cycle, the measurements show rather large fluctuations in the velocity. This is possibly due to the passage of vortices, which arise due to flow separation from the sharp inner edges of the slit.

Widjanarko [193] has performed a frequency analysis of the hot wire data, which shows that above a certain frequency threshold, an energy decay rate of $-5 / 3$ is obtained for each investigated case, indicating turbulent jets. This is to be expected, since the Reynolds number based on the peak ejection velocity and slit width is of the order of $\operatorname{Re}_{j}=2 \cdot 10^{3}$. Furthermore, the spectra exhibit peaks at the actuation frequency and the higher harmonics. 
It can be concluded that only the ejection-part of the hot wire measurements is representative for the performance of the synthetic jet. Since it is difficult to accurately measure the complete velocity profile across the slit, no attempt has been made to compute the average ejection velocity, $\bar{U}_{j}$, as defined by equation (6.1). In the following section, which presents the results of synthetic jet actuation for the low frequency range, only the peak ejection velocities will be reported and subsequently matched in the corresponding flow simulations.

\subsection{Results for Low-Frequency Synthetic Jet Actuation}

Since the highest performance of the synthetic jet actuation is obtained for $f_{j}=15 \mathrm{~Hz}$, this actuation frequency is initially chosen to study the effect of low-frequency actuation on the lift and drag characteristics of the NACA0018 airfoil. As described in the preceding section, this will be done both experimentally and numerically, by matching the peak ejection velocity that has been measured in the experiments.

The free-stream conditions are $\operatorname{Re}_{c}=1.76 \cdot 10^{5}$ and $\mathrm{M}_{\infty}=0.047$. Two angles of attack will be considered: $\alpha=0^{\circ}$ and $\alpha=8^{\circ}$. The geometric parameters of the slit are the relative slit location $x_{j} / c=0.88$, relative slit width $w_{j} / c=1 / 165$, relative slit depth $d_{j} / c=2 / 165$ and the angle with respect to the airfoil surface $\theta_{j}=90^{\circ}$. The actuation parameters are $F^{+}=0.155$ and $\left|\underline{u}_{j}\right|_{\text {peak }} / U_{\infty} \approx 3$.

For the flow simulations with synthetic jet actuation at this low frequency, it turns out that 1280 physical time steps per actuation cycle are needed to obtain good convergence behavior within each time step.

\subsubsection{Peak Ejection Velocity}

The employed velocity amplitude at the bottom boundary of the cavity is $U_{n}=11.622$ $\mathrm{m} / \mathrm{s}$, see subsection 3.5.3 which yields a velocity distribution across the jet at maximum ejection as shown in figure 6.21 for $\alpha=0^{\circ}$ and three different distances from the exit of the slit. The results for $\alpha=8^{\circ}$ are very close to those for $\alpha=0^{\circ}$ and are not shown here.

The results in figure [6.21 suggest that, within reasonable boundaries, a one-point measurement of the peak ejection velocity is not very sensitive to the distance from the exit of the jet; a distance that is difficult to accurately set in the experimental setup. Since the flow is approximately incompressible, the average velocity across the slit at maximum ejection is approximately equal to the velocity amplitude at the bottom boundary of the cavity multiplied by the ratio of the width of the boundary over the width of the jet. At one slit width away from the exit, this ratio is equals $3.00 / 1.375 \approx 2.19$, which yields an average velocity at maximum ejection of approximately $1.6 U_{\infty}$. Furthermore, the velocity profile at maximum ejection has a 'horned' shape. This shape was also observed by Lee \& Goldstein [202] in two-dimensional direct numerical simulations of synthetic jets and by Ugrina [203] in hot wire measurements of crosswise velocity distributions of circular synthetic jets during ejection, particularly at high Reynolds numbers based on the jet velocity and diameter. Lee \& Goldstein [202] mention that the 'horned' velocity profile 


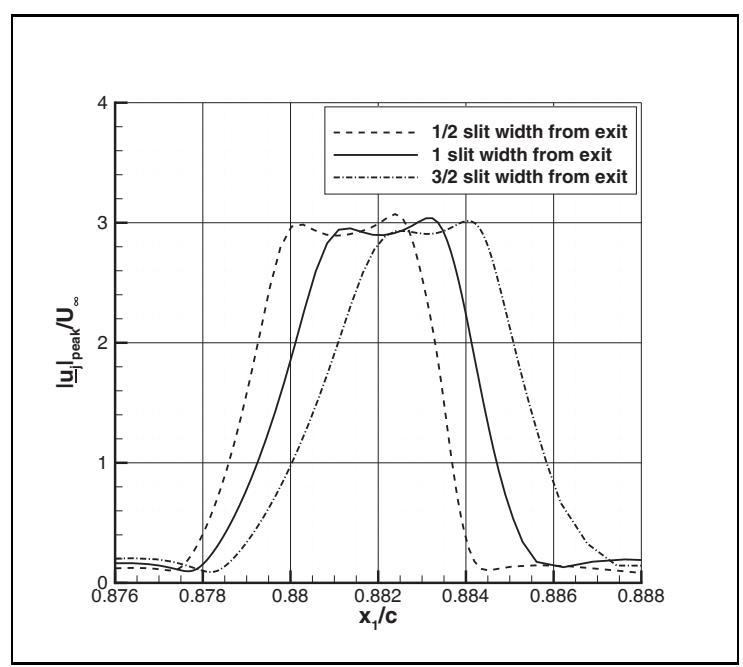

Figure 6.21: Computational results for the relative peak ejection velocity, $\left|\underline{u}_{j}\right|_{\text {peak }} / U_{\infty}$, across the jet, for different distances from the exit of the jet. $\operatorname{Re}_{c}=1.76 \cdot 10^{5}, \mathrm{M}_{\infty}=$ $0.047, x_{j} / c=0.88, w_{j} / c=1 / 165 \approx 0.00606, d_{j} / c=2 / 165, \theta_{j}=90^{\circ}, F^{+}=0.155$.

is common to oscillatory flows as the low-momentum boundary layers along the walls of the slit accelerate more quickly than the bulk flow.

\subsubsection{Time-averaged Pressure Distribution}

Figure 6.22 shows the experimental and computational results for the time-averaged pressure coefficient, $c_{p}$, with and without actuation, at both angles of attack. As in the experiments, a time-averaged $p_{\infty}$ at the inlet of the computational domain has been determined and has subsequently been used to compute the pressure coefficient. In the region between the leading edge of the airfoil and the location of the slit, synthetic jet actuation causes an increase in the pressure difference between the upper and lower side of the airfoil, indicating an increase in lift. The agreement between the experimental and computational pressure distribution is quite good for $\alpha=0^{\circ}$, except for the region around the location of the zig-zag tape. Note that the experimental results from the pressure ports beyond or close to the outer edges of the two slits in operation are not included in the figures. It can be seen that at $\alpha=8^{\circ}$, the effect of synthetic jet actuation is more pronounced in the flow simulations than measured in the experiments.

The computational results show that close to the upstream side of the slit, the timeaveraged pressure along the surface decreases substantially. On the downstream side, the time-averaged pressure first increases, before it decreases again. The largest part of the region between the slit and the trailing edge yields a negative contribution to the lift, since the time-averaged pressure on the lower side is below that of the upper side. 


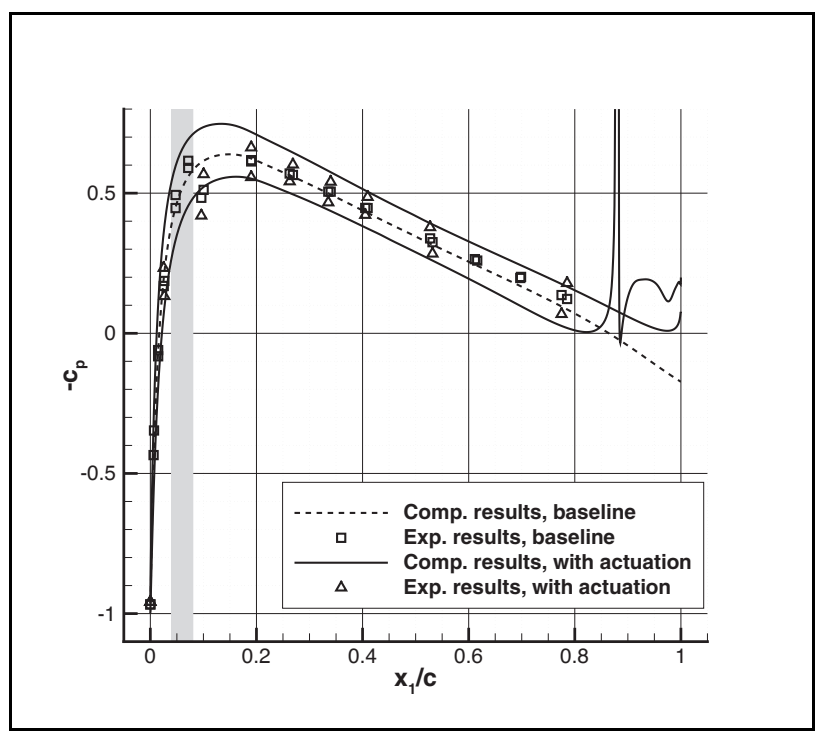

(a) $\alpha=0^{\circ}$

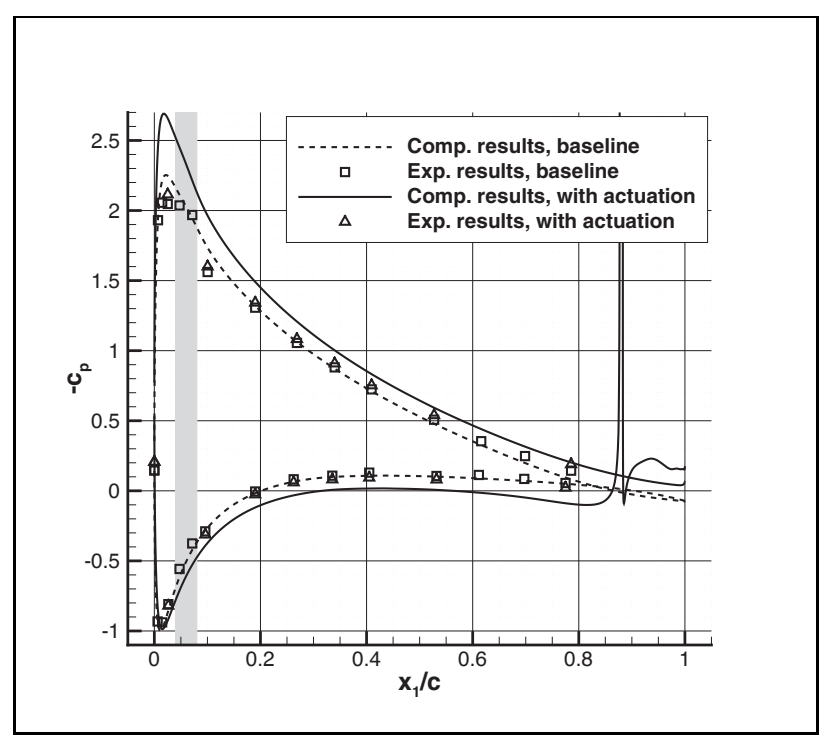

(b) $\alpha=8^{\circ}$

Figure 6.22: Time-averaged pressure coefficient, $c_{p}$, with and without actuation for $\operatorname{Re}_{c}=$ $1.76 \cdot 10^{5}, \mathrm{M}_{\infty}=0.047, x_{j} / c=0.88, w_{j} / c=1 / 165, d_{j} / c=2 / 165, \theta_{j}=90^{\circ}, F^{+}=$ $0.155,\left|\underline{u}_{j}\right|_{\text {peak }} / U_{\infty} \approx 3$. Grey area indicates the tripping region in the experiments. 


\subsubsection{Time-Dependent Lift and Drag Force}

The effect of synthetic jet actuation on the performance of the airfoil in time, as predicted by the computational method, can be seen in figures 6.23 and 6.24 . Figure 6.23 shows the change in the lift coefficient, $\Delta c_{l}$, and figure 6.24 shows the change in the drag coefficient, $\Delta c_{d}$, as a function of the non-dimensional time, $U_{\infty} t / c$, for both investigated angles of attack. Both quantities are changes with respect to the baseline case without actuation. The aerodynamic force on the airfoil is calculated as indicated in appendix D with contributions from the pressure and skin friction along the surface of the airfoil and the internal walls of the slit and cavity, and a possible contribution from a flow of momentum across the bottom boundary of the cavity.

Note that the actuation cycle is defined by the inflow/outflow boundary condition at the bottom boundary of the cavity, where the prescribed velocity component into the computational domain (normal to the boundary) reads $u_{n}=U_{n} \sin \left(2 \pi f_{j} t\right)$, see subsection 3.5.3. Therefore, in the first part of the cycle, corresponding to a phase of $0^{\circ} \leq \phi \leq 180^{\circ}$, air is ejected out of the slit. In the second part, corresponding to $180^{\circ} \leq \phi \leq 360^{\circ}$, air is ingested through the slit into the cavity. Since the nondimensional period of an actuation cycle is $U T_{j} / c \approx 6.465$ for this low actuation frequency, it can be seen that the lift and drag force react in a quasi-steady way to the synthetic jet: the change of the lift and drag coefficients closely follow the actuation cycle and approximately show the same behavior for each cycle.

With respect to the change in the lift coefficient, $\Delta c_{l}$, one can observe the following features (figure 6.23) :

- The change in the lift coefficient becomes negative for a short period in the beginning of the cycle, followed by a steep rise towards positive values.

- The highest positive values coincide with the period between maximum ejection and the end of the ejection part of the cycle. The change in the lift coefficient has a higher maximum for $\alpha=8^{\circ}$ than for $\alpha=0^{\circ}$.

- During the ingestion part of the cycle, the change in the lift coefficient decreases. For $\alpha=0^{\circ}$, it becomes negative, whereas it remains positive for $\alpha=8^{\circ}$.

- The difference between the maximum and minimum change in the lift coefficient within the actuation cycle is quite large with respect to the average over the actuation cycle.

- Additionally, the results are characterized by a high-frequency component with a non-dimensional period of $U_{\infty} T / c \approx 0.37-0.40$, i.e. the frequency is approximately between $242 \mathrm{~Hz}$ and $262 \mathrm{~Hz}$. This is associated with the shedding of vortices from the trailing edge of the airfoil around maximum ejection, which subsequently excitates an acoustic standing wave (resonance mode) between the upper and lower wall of the wind tunnel. This has been corroborated by observing the response of the pressure in vertical planes of the computational domain. The excited mode is the first mode, with anti-nodes at the upper and lower wall of the wind tunnel and 


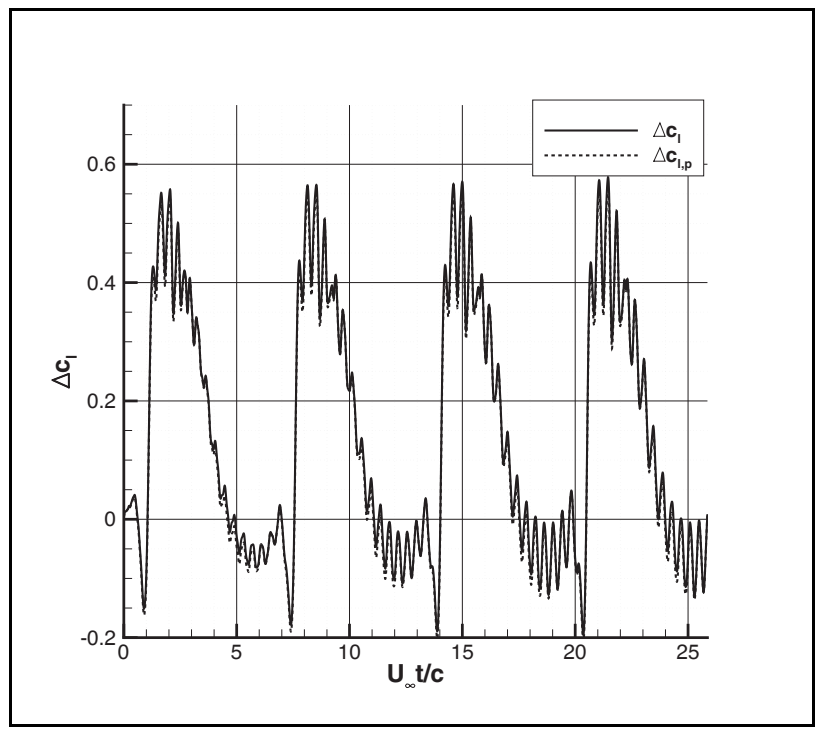

(a) $\alpha=0^{\circ}$

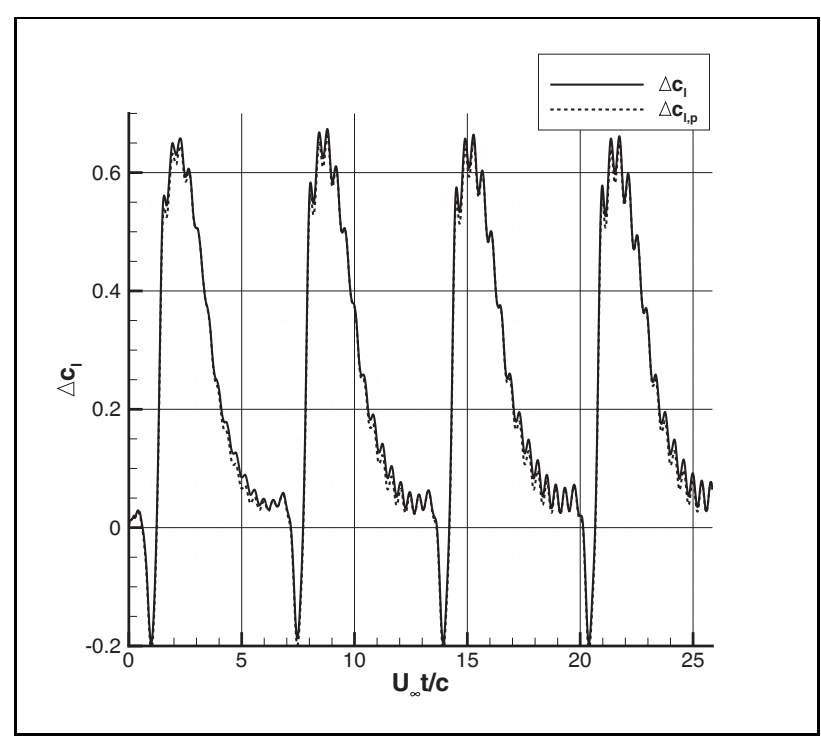

(b) $\alpha=8^{\circ}$

Figure 6.23: Change of lift coefficient, $\Delta c_{l}$, due to synthetic jet actuation for $\mathrm{Re}_{c}=$ $1.76 \cdot 10^{5}, \mathrm{M}_{\infty}=0.047, x_{j} / c=0.88, w_{j} / c=1 / 165, d_{j} / c=2 / 165, \theta_{j}=90^{\circ}, F^{+}=$ $0.155,\left|\underline{u}_{j}\right|_{\text {peak }} / U_{\infty} \approx 3 . \Delta c_{l, p}$ includes only the contribution from the pressure. 


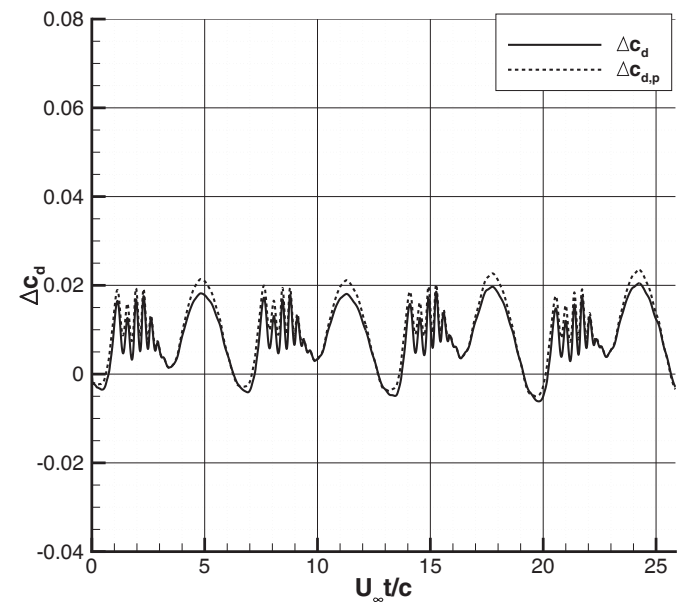

(a) $\alpha=0^{\circ}$

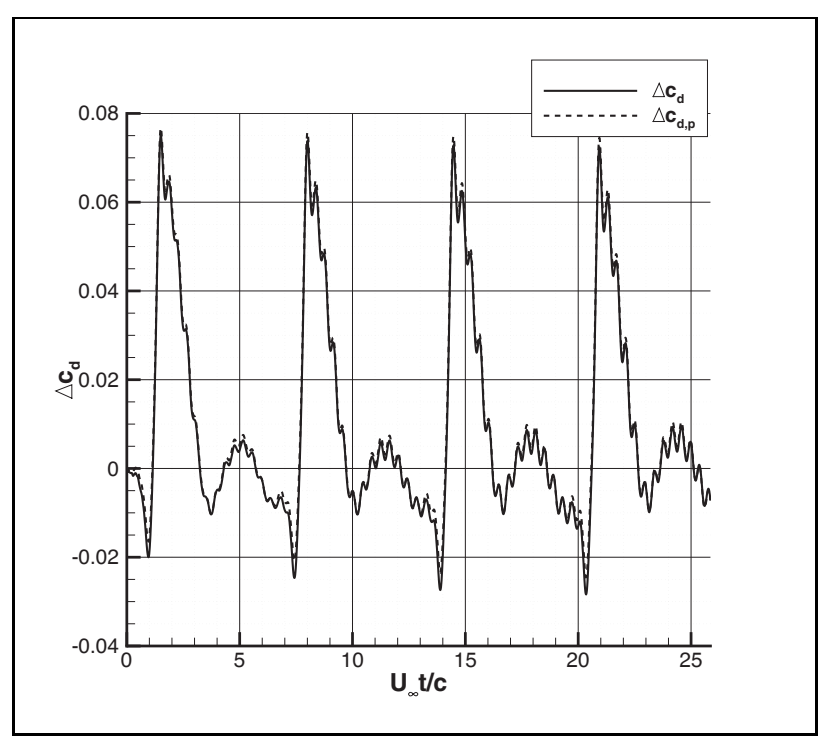

(b) $\alpha=8^{\circ}$

Figure 6.24: Change of drag coefficient, $\Delta c_{d}$, due to synthetic jet actuation for $\operatorname{Re}_{c}=$ $1.76 \cdot 10^{5}, \mathrm{M}_{\infty}=0.047, x_{j} / c=0.88, w_{j} / c=1 / 165, d_{j} / c=2 / 165, \theta_{j}=90^{\circ}, F^{+}=$ $0.155,\left|\underline{u}_{j}\right|_{\text {peak }} / U_{\infty} \approx 3 . \Delta c_{d, p}$ includes only the contribution from the pressure. 
a node at the horizontal plane in the center. The wavelength is therefore $\lambda=2 h$, with $h$ the height of the wind tunnel, so that the frequency is

$$
f=\frac{c}{\lambda} \approx \frac{340 \mathrm{~m} / \mathrm{s}}{1.4 \mathrm{~m}} \approx 243 \mathrm{~Hz}
$$

which almost coincides with the frequency range of vortex shedding. This will be discussed later when we will consider the time-dependence of the flow field around the airfoil. The amplitude of this high-frequency component is higher for $\alpha=0^{\circ}$ than for $\alpha=8^{\circ}$.

The change in the drag coefficient, $\Delta c_{d}$, for $\alpha=0^{\circ}$ and that for $\alpha=8^{\circ}$ is quite different (figure 6.24):

- Firstly, this quantity has higher maximum and minimum values for $\alpha=8^{\circ}$. At this angle of attack, high positive values are observed around maximum ejection. This is not observed for $\alpha=0^{\circ}$. Relatively, i.e. with respect to the baseline, the changes in the drag coefficient are also larger for $\alpha=8^{\circ}$ : the maximum value of the drag coefficient for the case with actuation is roughly 4 times the drag coefficient of the baseline case $\left(c_{d, \text { baseline }}=0.0235\right)$. For $\alpha=0^{\circ}$, this value is approximately 2 $\left(c_{d, \text { baseline }}=0.0171\right)$.

- Secondly, $\Delta c_{d}$ for $\alpha=0^{\circ}$ is mostly positive during the actuation cycle, it is negative only for a short time just after the start of the ejection part of the actuation cycle. For $\alpha=8^{\circ}$, it is also negative at the end of the ejection part and the beginning of the ingestion part of the actuation cycle.

- Thirdly, a high-frequency component can be observed in the change in the drag coefficient, as was also the case for the change in the lift coefficient. Again, this is associated with vortex shedding and the excitation of an acoustic mode between the upper and lower wall of the wind tunnel. For $\alpha=0^{\circ}$, the high-frequency component is only present during the ejection part of the cycle, i.e. when the vortices are being shed. That the effect of the acoustic waves is not registered in the change in the drag coefficient during ingestion is because of the symmetry present at $\alpha=0^{\circ}$ and the fact that the first resonance mode is excited. The upper and lower wind tunnel walls are anti-nodes, whereas the horizontal plane in the center is a node, where the acoustic pressure amplitude vanishes (locally). For $\alpha=8^{\circ}$, the sound pressure waves do show up as a high-frequency component in the change in the drag coefficient during ingestion.

Note that with the employed 1280 time steps per actuation cycle, i.e. $\Delta t \approx 5.21 \cdot 10^{-5}$ $\mathrm{s}$, the high-frequency component in the results is simulated with approximately 80 time steps per period.

Figures 6.23 and 6.24 also show the change of the lift and drag coefficients in which only the pressure distribution has been taken into account, i.e. $\Delta c_{l, p}$ and $\Delta c_{d, p}$, respectively. It is clear that the pressure distribution is the main contributor to the changes in the lift and drag force, for both angles of attack. 
Time-averaging the computational results for the change in the lift coefficient over the last full actuation cycle yields the following results:

$$
\begin{aligned}
& \text { - for } \alpha=0^{\circ}: \overline{\Delta c_{l}}=0.140 \text { and } \overline{\Delta c_{l, p}}=0.130, \\
& \text { - for } \alpha=8^{\circ}: \overline{\Delta c_{l}}=0.238 \text { and } \overline{\Delta c_{l, p}}=0.228 \text {. }
\end{aligned}
$$

These values indicate that the time-averaged performance of synthetic jet actuation as predicted by the simulations is higher at $\alpha=8^{\circ}$ than at $\alpha=0^{\circ}$. Furthermore, they also indicate that an experimentally obtained time-averaged pressure distribution is sufficient to calculate a reasonable approximation of the time-averaged change in the lift coefficient, $\overline{\Delta c_{l}}$. For the experimental results, we estimate the change in the lift coefficient by calculating the average increase in pressure difference between the upper and lower side of the airfoil for the pressure taps 8-12 and 22-26, i.e. in the region $x_{1} / c \in[0.19,0.53]$. This yields the following comparison:

- for $\alpha=0^{\circ}: \overline{\Delta c_{p}} \approx 0.079$ for the experiments and 0.138 for the computations,

- for $\alpha=8^{\circ}: \overline{\Delta c_{p}} \approx 0.057$ for the experiments and 0.226 for the computations.

These values indicate that, contrary to the computations, the experiments show a deterioration of the performance of low-frequency synthetic jet actuation for pitch control at $\alpha=8^{\circ}$. The flow simulations predict an increase in the time-averaged lift coefficient that is between 1.75 and 4 times higher than measured in the experiments. Possible reasons for this effect are discussed in subsection 6.7.5.

\subsubsection{Time-Dependent Flow Field}

Figures 6.25 and 6.26 show the velocity field in the region around the trailing edge of the airfoil at four phases in the actuation cycle, for $\alpha=0^{\circ}$ and $\alpha=8^{\circ}$, respectively.

It is clear that during ejection, i.e. for the phases of $45^{\circ}$ and $90^{\circ}$, the main flow along the lower side of the airfoil is forced downwards by the jet. Between the slit and the trailing edge, a recirculation zone is formed, which is associated with low pressures. This can be seen in figure 6.27 for both angles of attack and a phase of $45^{\circ}$ in the actuation cycle. As long as this region has not reached the trailing edge, the low pressure region on the lower side of the airfoil reduces lift, see figure 6.23. When it reaches the trailing edge, air from the upper side of the airfoil is entrained into this region. The flow leaving the airfoil is thereby directed downwards and this changes the pressure distribution on both sides of the airfoil. It effectively increases the circulation around the airfoil.

At the start of the ingestion phase of the actuation cycle (phase $=180^{\circ}$ ), the flow still leaves the airfoil tangentially to the upper side of the airfoil. At maximum ingestion (phase $=270^{\circ}$ ), the boundary layer upstream of the slit is ingested into the cavity and air with a higher momentum flows towards the surface between the slit and the trailing edge of the airfoil, where a new boundary layer develops. At the trailing edge, the flow is no longer directed downwards but leaves the airfoil tangentially to the lower side. This coincides with a reduced change in the lift coefficient, $\Delta c_{l}$, compared to that at maximum ejection. 


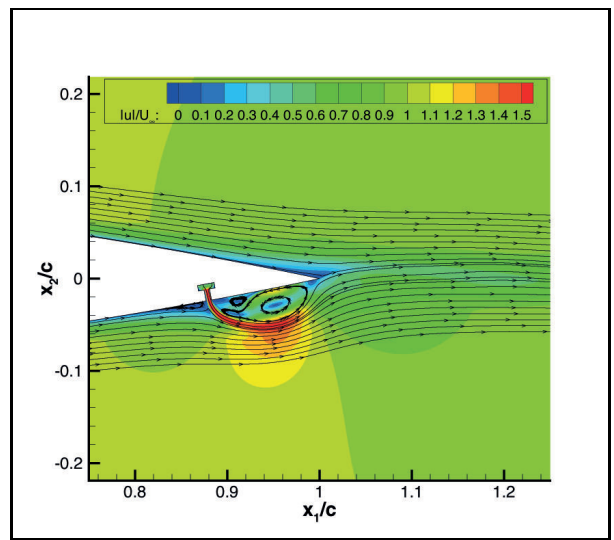

(a) phase $=45^{\circ}$

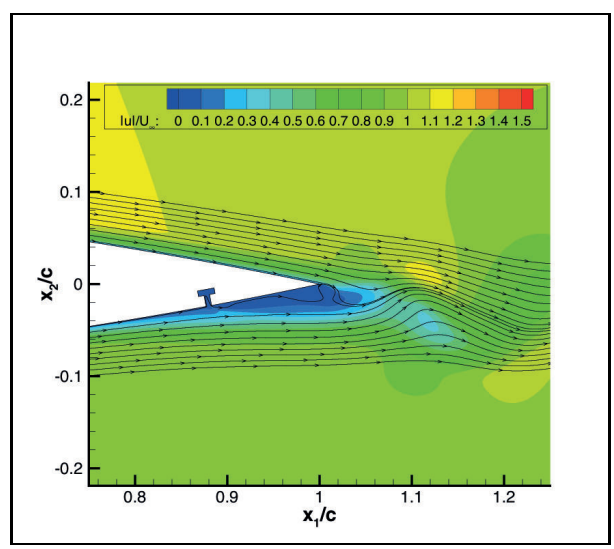

(c) phase $=180^{\circ}$

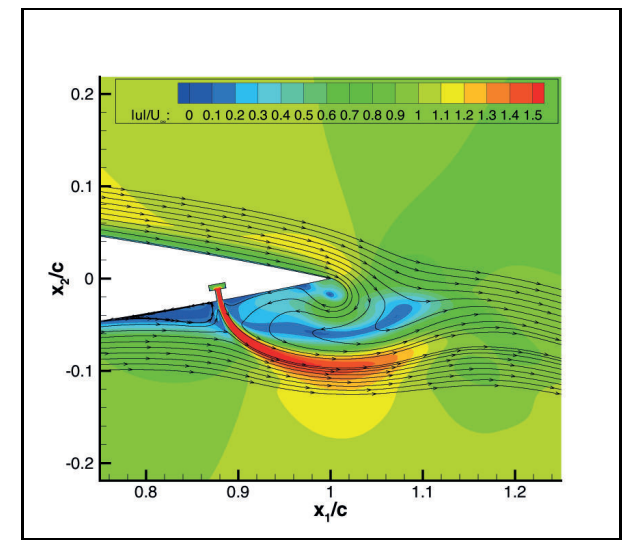

(b) phase $=90^{\circ}$

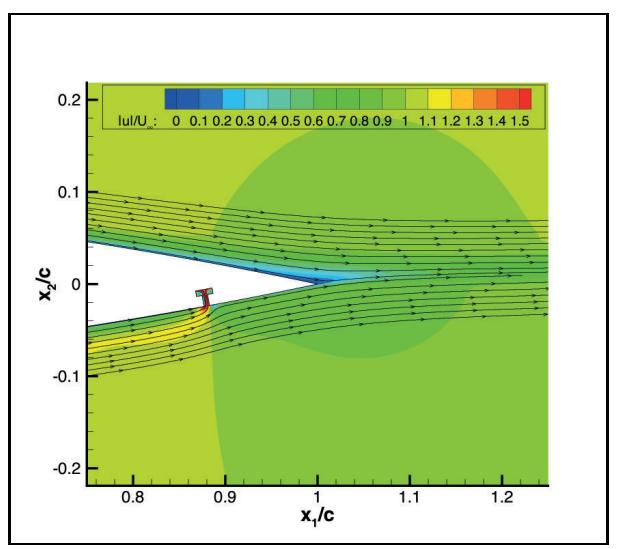

(d) phase $=270^{\circ}$

Figure 6.25: Streamlines and iso-contours of dimensionless velocity magnitude, $|\underline{u}| / U_{\infty}$, at four phases in the actuation cycle, for $\alpha=0^{\circ}, \operatorname{Re}_{c}=1.76 \cdot 10^{5}, \mathrm{M}_{\infty}=0.047$, $x_{j} / c=0.88, w_{j} / c=1 / 165, d_{j} / c=2 / 165, \theta_{j}=90^{\circ}, F^{+}=0.155,\left|\underline{u}_{j}\right|_{\text {peak }} / U_{\infty} \approx 3$.

For $\alpha=0^{\circ}$, the change in the lift coefficient even becomes negative during maximum ingestion. For $\alpha=8^{\circ}$, the separated flow region on the upper side of the airfoil, which was completely removed at maximum ejection, has reappeared.

Figures 6.28 and 6.29 show iso-contours of the dimensionless spanwise vorticity, $\omega_{z} c / U_{\infty}$, in the region around the trailing edge of the airfoil at four phases in the actuation cycle, for $\alpha=0^{\circ}$ and $\alpha=8^{\circ}$, respectively.

At maximum ejection ( phase $=90^{\circ}$ ), the vorticity with a positive sign associated with the jet, interacts with the vorticity with a negative sign from the upper side of the airfoil 


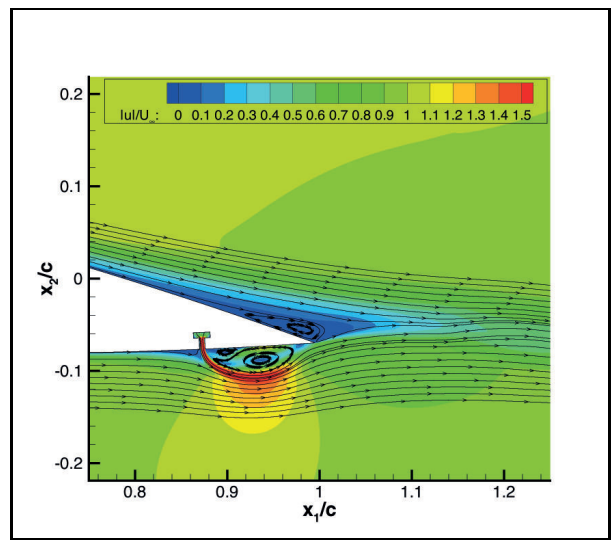

(a) phase $=45^{\circ}$

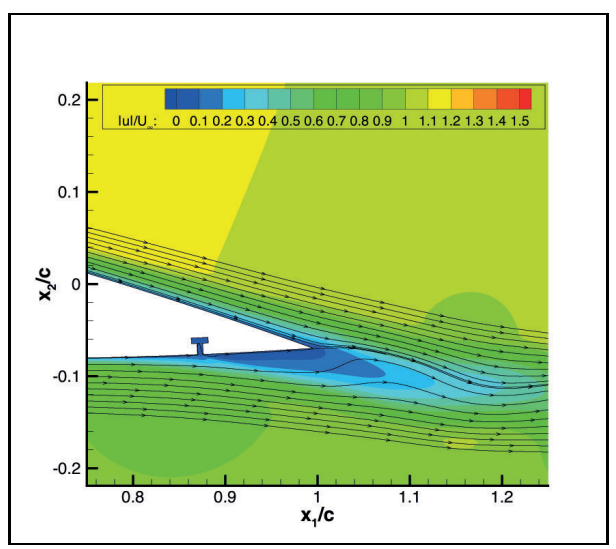

(c) phase $=180^{\circ}$

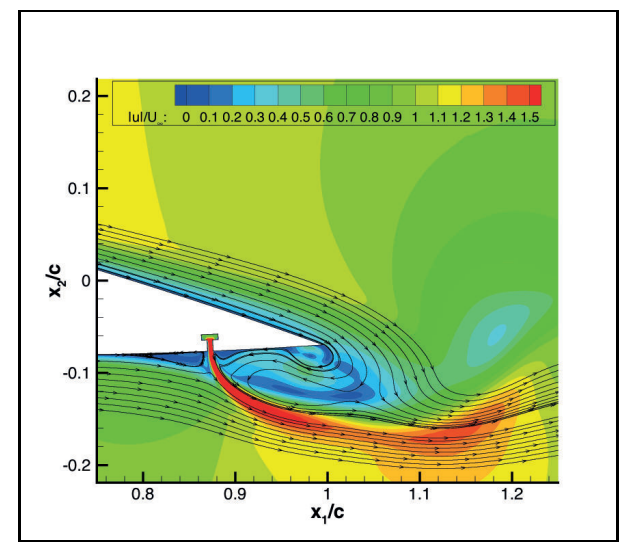

(b) phase $=90^{\circ}$

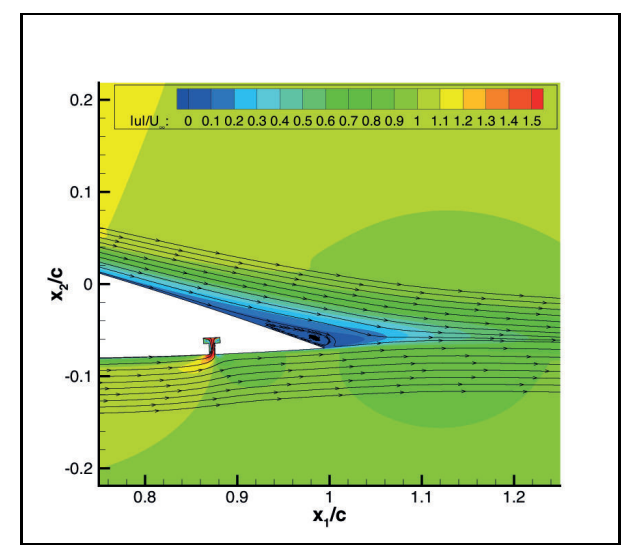

(d) phase $=270^{\circ}$

Figure 6.26: Streamlines and iso-contours of dimensionless velocity magnitude, $|\underline{u}| / U_{\infty}$, at four phases in the actuation cycle, for $\alpha=8^{\circ}, \operatorname{Re}_{c}=1.76 \cdot 10^{5}, \mathrm{M}_{\infty}=0.047$, $x_{j} / c=0.88, w_{j} / c=1 / 165, d_{j} / c=2 / 165, \theta_{j}=90^{\circ}, F^{+}=0.155,\left|\underline{u}_{j}\right|_{\text {peak }} / U_{\infty} \approx 3$.

and vortex shedding begins. At a phase of $180^{\circ}$, a vortex street is clearly visible in the wake of the airfoil for both angles of attack. At maximum ingestion (phase $=270^{\circ}$ ), vortex shedding no longer occurs. The vortices have been convected downstream, far away from the airfoil. The high-frequency oscillation present in the change in the lift coefficient, $\Delta c_{l}$, and in the change in the drag coefficient, $\Delta c_{d}$, during the ingestion part of the actuation cycle is thus the result of the resonating sound waves. 


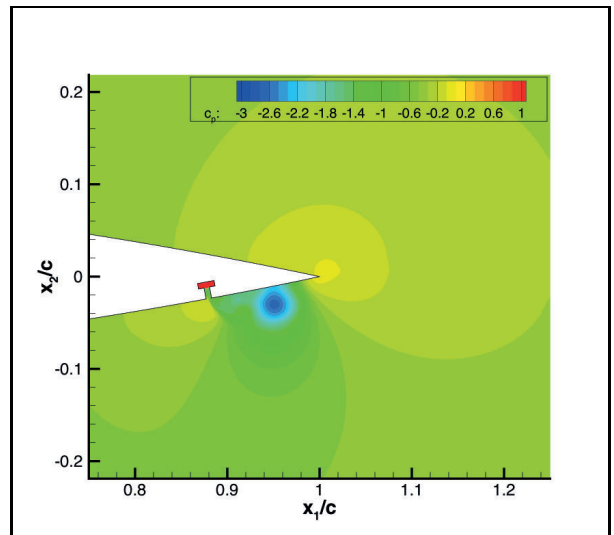

(a) $\alpha=0^{\circ}$

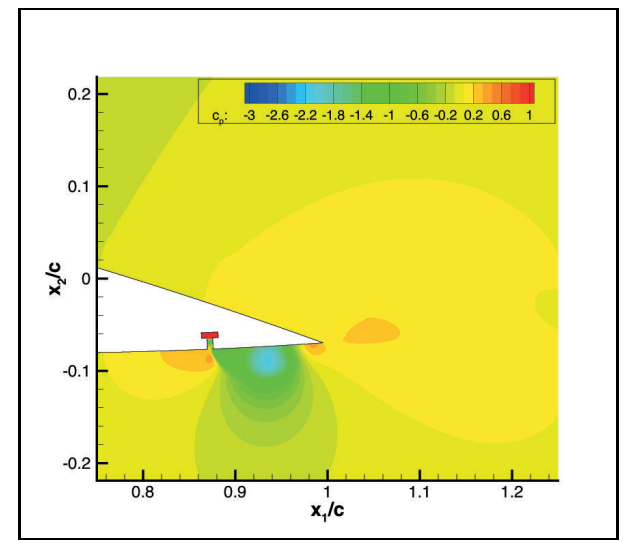

(b) $\alpha=8^{\circ}$

Figure 6.27: Iso-contours of pressure coefficient, $c_{p}$, at a phase of $45^{\circ}$ in the actuation cycle, for $\operatorname{Re}_{c}=1.76 \cdot 10^{5}, \mathrm{M}_{\infty}=0.047, x_{j} / c=0.88, w_{j} / c=1 / 165, d_{j} / c=2 / 165$, $\theta_{j}=90^{\circ}, F^{+}=0.155,\left|\underline{u}_{j}\right|_{\text {peak }} / U_{\infty} \approx 3$.

\subsubsection{Discussion}

It has been shown that, in general, the computational method quite well predicts the effect of low-frequency synthetic jet actuation on the time-averaged pressure distributions on the upper and lower surface of the airfoil. However, the effects are overpredicted in comparison with the experimental results, especially at the higher angle of attack. Since only two of the four slits are in operation, we expect three-dimensional flow effects in the experiments that cannot be respresented in the two-dimensional flow configuration considered in the simulations.

In the flow simulations for $\alpha=8^{\circ}$, the synthetic jet is able to remove the separated flow region on the upper (suction) side of the airfoil during a long period in the actuation cycle, such that the flow leaves the trailing edge along the upper side. This yields a substantial increase in lift. It is possible that due to three-dimensional flow effects in the experiments, the synthetic jets are not able to completely remove a separated flow region on the upper side of the airfoil.

Hot wire measurements of the jet velocity show strong oscillations in the data, especially around maximum ejection. It is thought to be the effect of passing vortices due to flow separation inside the slit. The two-dimensional flow simulations also predict flow separation inside the slit, but in a quasi-steady way, without the generation of vortices. This difference may be caused by the two-dimensional flow configuration considered in the simulations and the use of an eddy-viscosity turbulence model. Although it is not feasible in the present experimental setup, it would be most useful to be able to determine the momentum coefficients in the experiments and compare these to the momentum 


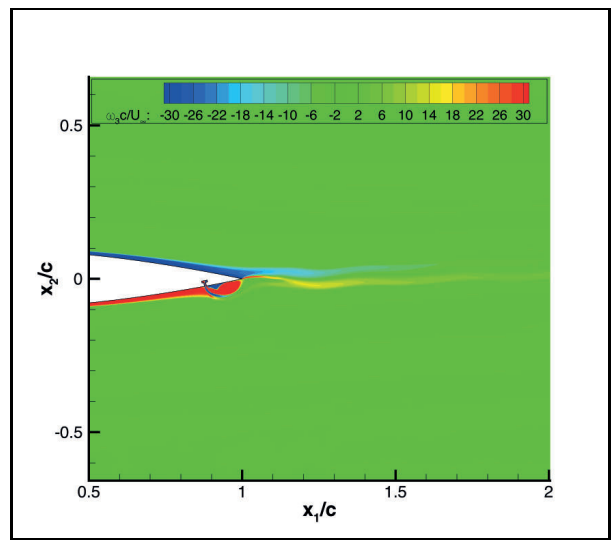

(a) phase $=45^{\circ}$

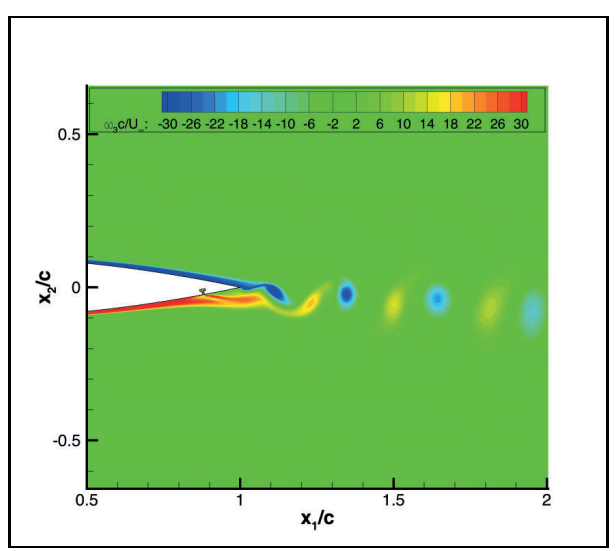

(c) phase $=180^{\circ}$

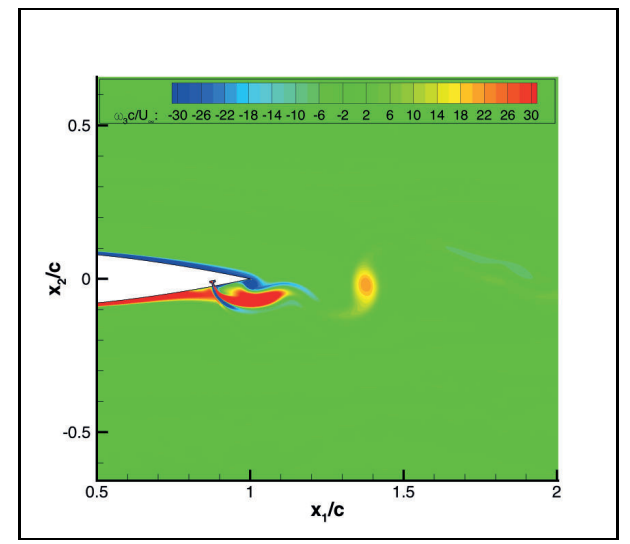

(b) phase $=90^{\circ}$

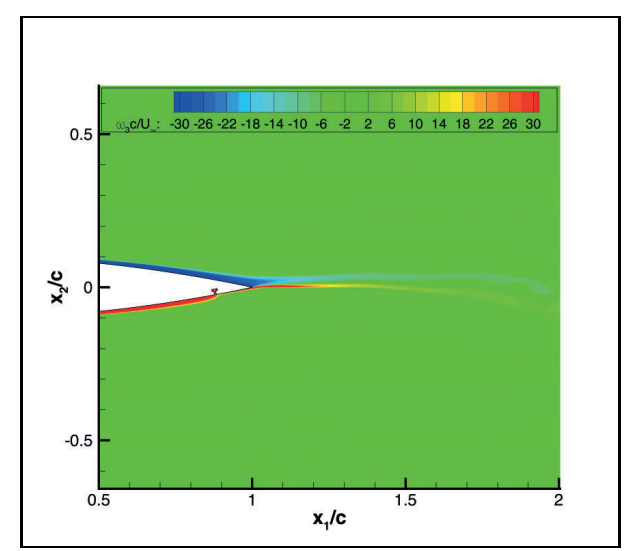

(d) phase $=270^{\circ}$

Figure 6.28: Iso-contours of dimensionless spanwise vorticity, $\omega_{3} c / U_{\infty}$, at four phases in the actuation cycle, for $\alpha=0^{\circ}, \mathrm{Re}_{c}=1.76 \cdot 10^{5}, \mathrm{M}_{\infty}=0.047, x_{j} / c=0.88$, $w_{j} / c=1 / 165, d_{j} / c=2 / 165, \theta_{j}=90^{\circ}, F^{+}=0.155,\left|\underline{u}_{j}\right|_{\text {peak }} / U_{\infty} \approx 3$.

coefficients in the numerical flow simulations.

The computational results show that for the employed low actuation frequency, large fluctuations on top of the time-averaged change in the lift coefficient occur, with a frequency equal to the actuation frequency. Since this frequency is low, these large fluctuations could lead to a structural behavior in which the fatigue loads are increased, rather than reduced.

It can be concluded that low frequency synthetic jet actuation is not a feasible option for controlling loads on wind turbine blades. Therefore, in the next section, the potential 


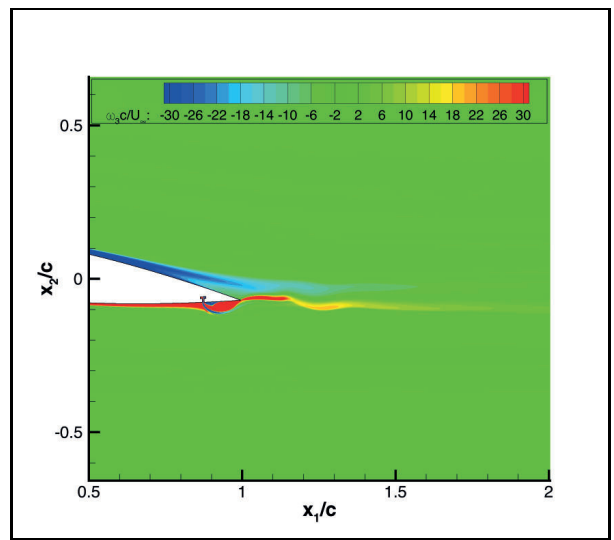

(a) phase $=45^{\circ}$

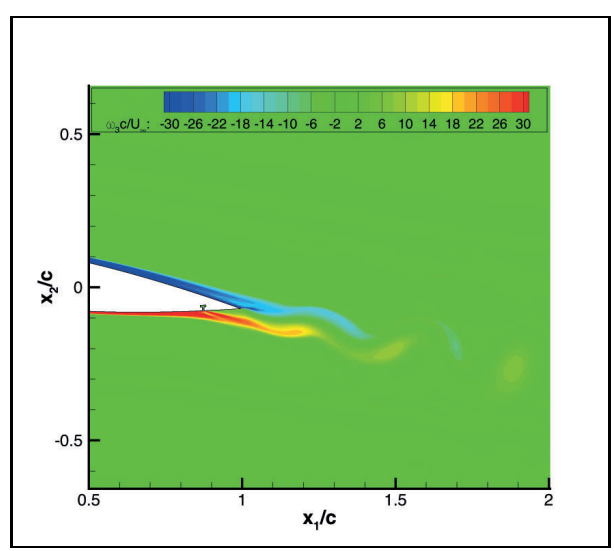

(c) phase $=180^{\circ}$

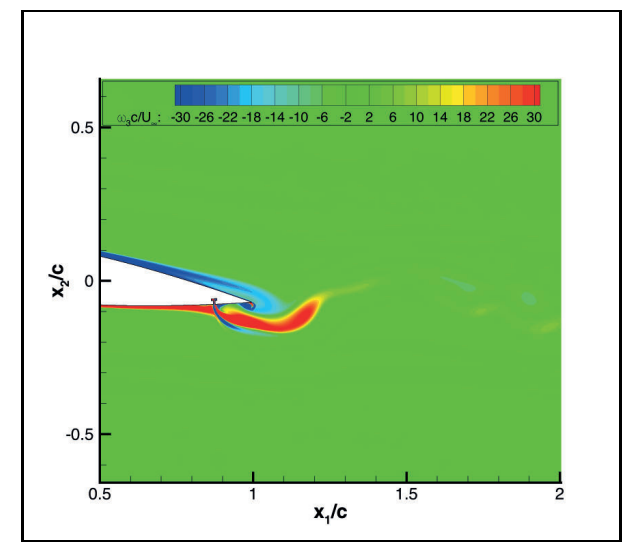

(b) phase $=90^{\circ}$

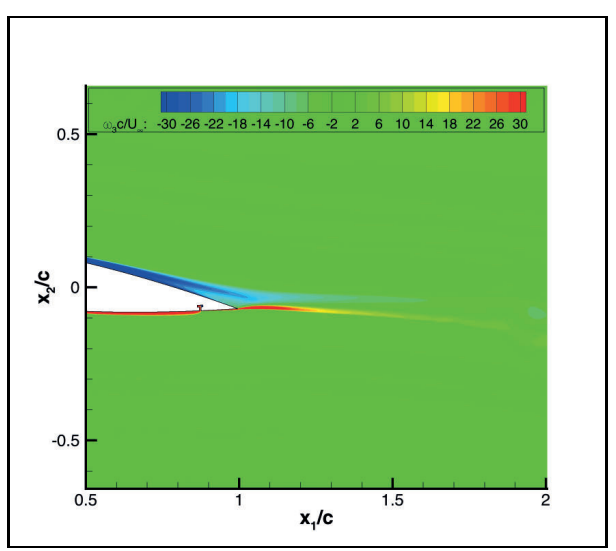

(d) phase $=270^{\circ}$

Figure 6.29: Iso-contours of dimensionless spanwise vorticity, $\omega_{3} c / U_{\infty}$, at four phases in the actuation cycle, for $\alpha=8^{\circ}, \mathrm{Re}_{c}=1.76 \cdot 10^{5}, \mathrm{M}_{\infty}=0.047, x_{j} / c=0.88$, $w_{j} / c=1 / 165, d_{j} / c=2 / 165, \theta_{j}=90^{\circ}, F^{+}=0.155,\left|\underline{u}_{j}\right|_{\text {peak }} / U_{\infty} \approx 3$.

of high-frequency synthetic jet actuation is investigated.

\subsection{Results for High-Frequency Synthetic Jet Actuation}

This section presents the results of a parameter study of flows with high-frequency synthetic jet actuation. It focuses on four dimensionless parameters: the angle of attack, $\alpha$, the dimensionless actuation frequency, $F^{+}$, the momentum coefficient, $c_{\mu}$, and the 
location of the slit, $x_{j} / c$. See subsection 6.2.2 for the definition of these parameters.

In order to be able to compare the results of the numerical simulations to possible future experimental measurements of high-frequency actuation, the flow in all simulations is kept nearly incompressible, $M_{\infty}=0.146$, and the Reynolds number is $\operatorname{Re}_{c}=5.5 \cdot 10^{5}$. This corresponds to a free-stream velocity of $U_{\infty}=50 \mathrm{~m} / \mathrm{s}$. Furthermore, the geometry of the slit is fixed, as discussed in subsection 6.2.3 $w_{j} / c=1 / 165, d_{j} / c=2 / 165$ and $\theta_{j}=90^{\circ}$. In the remainder of this section, the fixed parameters are not mentioned again, except in the captions of the figures.

The investigated dimensionless actuation frequencies are between $F^{+}=1$ and $F^{+}=$ 30 , with the main focus on $F^{+}=10$ and $F^{+}=20$. The velocity amplitude applied at the bottom of the cavity is varied such that a range of momentum coefficients is obtained, but the relative density in the jets is always kept close to one, $\bar{\rho}_{j} / \rho \approx 1$. Three locations of the slit are investigated: the original location of $x_{j} / c=0.88$, and the additional locations of $x_{j} / c=0.95$ and $x_{j} / c=0.985$. Two angles of attack have been considered, namely $\alpha=0^{\circ}$ and $\alpha=9^{\circ}$. The latter angle of attack is chosen because it is close to the angle of attack at which the lift over drag ratio is maximal, i.e. the angle of attack at which a wind turbine blade section would operate in ideal circumstances.

\subsubsection{Timestep Refinement}

A time step refinement study has been performed for $\alpha=0, x_{j} / c=0.88, F^{+}=20$ and $c_{\mu} \approx 0.006$. In this study, the applied velocity amplitude at the bottom of the cavity, $U_{n}$, see subsection 3.5.3, has been fixed such that

$$
\frac{U_{n} w_{c a v}}{U_{\infty} w_{j}}=1.2 .
$$

Here, the ratio of the width of the cavity to the width of the slit equals $w_{c a v} / w_{j}=3$, see figure 6.9 in section 6.4 . The meaning of equation 6.8 is that without compressibility effects, the spatial-averaged velocity across the exit of the slit at maximum ejection, will be $1.2 U_{\infty}$. Since the momentum coefficient is a spatial- and time-averaged result of each simulation, it cannot be linked directly to the velocity amplitude at the bottom of the cavity and its value differs somewhat for the different time step sizes. In subsection 6.8.2 the relation between the applied velocity amplitude and the resulting momentum coefficient is discussed in more detail.

The employed computational grid is the grid presented in subsection 6.4. Starting from a converged baseline steady-flow simulation without synthetic jet actuation, three successive simulations have been performed with synthetic jet actuation. Each simulation has been run until the lift and the drag coefficients have become periodic, which turns out to be at approximately $12 c / U_{\infty}$. For the first of these time-accurate simulations, 40 time steps per cycle have been used, and in the two subsequent simulations the number of time steps is doubled, i.e. 80 and 160 time steps per cycle.

The results are presented in table 6.2. which successively lists the relative density of the jet, $\bar{\rho}_{j} / \rho_{\infty}$, the relative ejection velocity, $\bar{U}_{j} / U_{\infty}$, the momentum coefficient, $c_{\mu}$, the 


\begin{tabular}{|c|c|c|c|c|c|}
\hline time steps per cycle & $\bar{\rho}_{j} / \rho_{\infty}$ & $\bar{U}_{j} / U_{\infty}$ & $c_{\mu}$ & $\overline{\Delta c_{l}}$ & $\overline{\Delta c_{d}}$ \\
\hline \hline $40\left(\Delta t=4.12500 \cdot 10^{-6} \mathrm{~s}\right)$ & 0.98410 & 1.02052 & 0.00621 & 0.11801 & 0.00138 \\
\hline $80\left(\Delta t=2.06250 \cdot 10^{-6} \mathrm{~s}\right)$ & 0.98359 & 1.00920 & 0.00607 & 0.09226 & 0.00121 \\
\hline $160\left(\Delta t=1.03125 \cdot 10^{-6} \mathrm{~s}\right)$ & 0.98305 & 1.00393 & 0.00600 & 0.09598 & 0.00115 \\
\hline \hline apparent order of conv. & - & 1.1 & 1.0 & - & 1.5 \\
\hline
\end{tabular}

Table 6.2: Performance of synthetic jet actuation in periodic part of results, including time-averaged change in lift coefficient, $\overline{\Delta c_{l}}$, and time-averaged change in drag coefficient, $\overline{\Delta c_{d}}$, for different number of time steps per actuation cycle, $\operatorname{Re}_{c}=5.5 \cdot 10^{5}, \mathrm{M}_{\infty}=0.146$, $\alpha=0^{\circ}, x_{j} / c=0.88, w_{j} / c=1 / 165, d_{j} / c=2 / 165, \theta_{j}=90^{\circ}, F^{+}=20, c_{\mu} \approx 0.006$, $\bar{\rho}_{j} / \rho_{\infty} \approx 1$.

time-averaged change in the lift coefficient, $\overline{\Delta c_{l}}$, and finally the time-averaged change in the drag coefficient, $\overline{\Delta c_{d}}$. Time averaging has been performed over a number of actuation cycles in the periodic part of the simulations.

The results show that the values of all investigated coefficients calculated with 80 and 160 time steps per cycle are quite close to each other. For example, the values for the time-averaged changes in lift and drag are within $5 \%$ of each other. However, not all of the coefficients in table 6.2 have a monotonic convergence behavior. Those that do converge have an apparent order of convergence between 1.0 and 1.5. The momentum coefficient and the time-averaged change in drag coefficient decrease with decreasing time step size, whereas the time-averaged change in lift coefficient first decreases for 80 time steps per cycle and then increases for 160 steps. The latter increase is relatively small with respect to the former decrease $(\approx 14 \%)$.

Although not all of the investigated parameters are in the asymptotic range yet, the smallest investigated time step sizes yield only small differences in the values of the parameters. It was decided to use 80 time steps per cycle for most of the simulations in the parameter study of high-frequency actuation, to keep computational times within reasonable bounds. A minimum simulated time of $12 c / U_{\infty}$ and a dimensionless actuation frequency of $F^{+}=20$ corresponds to a minimum of 240 simulated actuation cycles. Using 80 steps per cycle leads to a total of 19200 time steps per simulation at this frequency. Even two minutes of computational time per time step, which is not unreasonable for the available computational resources, leads to almost a month of computational time for each simulation.

Additionally, a simulation for the frequency of $F^{+}=1$ has been performed using 400 time steps per actuation cycle. The simulated flows for $F^{+} \geq 5$ show unsteady behavior with the largest amplitude at the actuation frequency. However, this is not the case for $F^{+}=1$. At this actuation frequency, flow features occur with frequencies above the actuation frequency. It turned out that using 400 time steps per cycle for $F^{+}=1$ leads to good convergence behavior within each time step. 


\subsubsection{Performance of Synthetic Jets}

Before the effect of high-frequency synthetic jet actuation on the cross-flow and the aerodynamic performance of the airfoil is presented, the performance of the synthetic jet at the exit of the slit is discussed. The performance for the periodic part of the results and in the presence of the cross-flow will be shown for an angle of attack of $\alpha=0^{\circ}$ and a slit location of $x_{j} / c=0.88$. For the other slit locations and the other angle of attack, very similar results have been obtained which are not shown here.

Figure 6.30 shows the momentum coefficient, $c_{\mu}$, as function of the applied velocity amplitude at the bottom of the cavity, expressed as $\left(U_{n} w_{c a v}\right) /\left(U_{\infty} w_{j}\right)$, as discussed in the preceding subsection. The different curves correspond to different dimensionless actuation frequencies. Additionally, three curve fits are included. It can be seen that higher actuation frequencies yield a higher performance. The relation between the velocity amplitude at the bottom of the cavity and the momentum coefficient, which is determined at the exit of the slit, is close to quadratic. So, apart from small compressibility effects $\left(\bar{\rho}_{j} / \rho_{\infty} \approx 1\right), \bar{U}_{j}$ behaves approximately linearly with the imposed velocity amplitude at the bottom of the cavity, $U_{n}$. This can be seen in figure 6.31 which shows the performance in terms of the relative ejection velocity, $\bar{U}_{j} / U_{\infty}$.

Normal velocity profiles across the slit, at different phases during the actuation cycle, are shown in figure 6.32 for a velocity amplitude at the bottom of the cavity equal to $\left(U_{n} w_{\text {cav }}\right) /\left(U_{\infty} w_{j}\right)=1.2$ and different actuation frequencies corresponding to $F^{+}=\{1,5,10,20,30\}$, respectively. Note that the actuation cycle is defined by the inflow/outflow boundary condition at the bottom boundary of the cavity, where the prescribed velocity component into the computational domain (normal to the boundary) reads $u_{n}=U_{n} \sin \left(2 \pi f_{j} t\right)$, see subsection 3.5.3. Therefore, in the first part of the cycle, corresponding to a phase of $0^{\circ} \leq \phi \leq 180^{\circ}$, air is ejected out of the slit. In the second part, corresponding to $180^{\circ} \leq \phi \leq 360^{\circ}$, air is ingested into the slit and cavity.

The following observations can be made:

- The normal velocity profiles across the exit of the slit do not resemble a simple spatially uniform or $\left(\sin ^{2}\right)$-profile during ejection, as proposed and used by Balakumar [97] for example. Furthermore, the behavior in time cannot be approximated accurately by a sine function. Also, the non-uniform velocity distributions given by equation 3.145, which were proposed by Aram et al. [96] and consist of three piecewise linear parts across the slit with a sinusoidal behavior in time, are unable to accurately represent the normal velocity profiles observed in figure 6.32. Including the slit and at least some part of the cavity below the slit in the computational domain is therefore necessary to let the synthetic jet adapt to the cross-flow along the surface of the airfoil.

- Differences in the normal velocity profiles between the actuation frequencies are relatively small during ingestion. Especially at a phase of $270^{\circ}$, which corresponds to maximum ingestion, the velocity profiles agree very well. An exception is the case of $F^{+}=30$, for which larger negative normal velocities are observed during ingestion. 


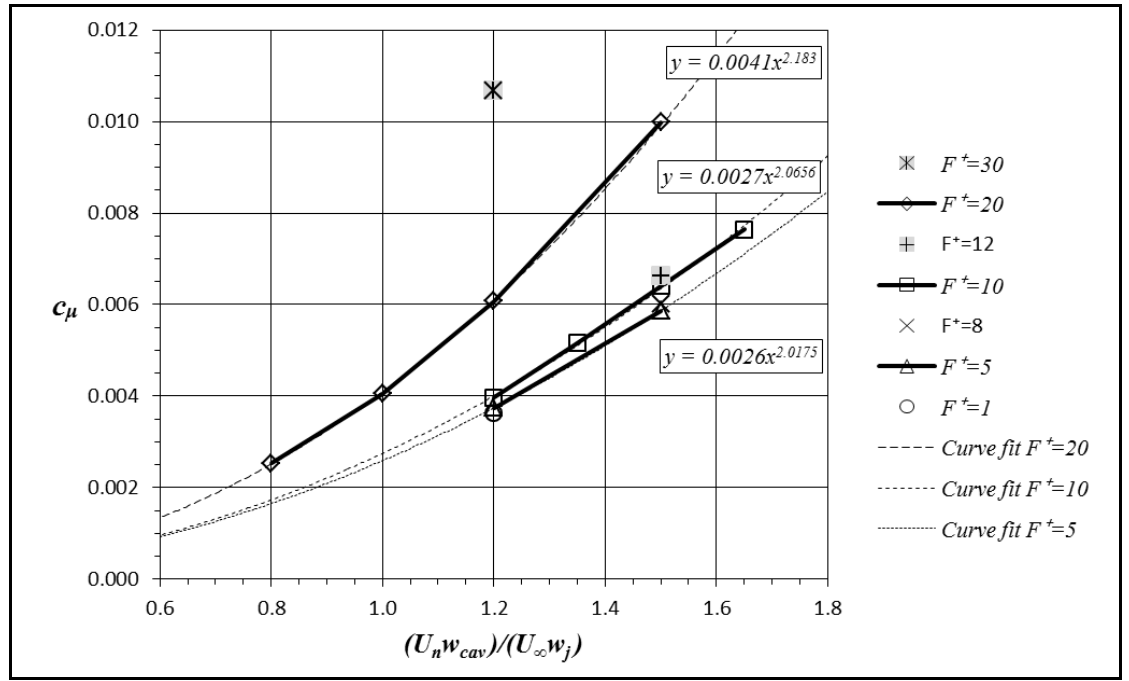

Figure 6.30: Momentum coefficient, $c_{\mu}$, as function of the applied velocity amplitude at the bottom of the cavity, expressed as $\left(U_{n} w_{\text {cav }}\right) /\left(U_{\infty} w_{j}\right)$, for $\operatorname{Re}_{c}=5.5 \cdot 10^{5}, \mathrm{M}_{\infty}=$ $0.146, \alpha=0^{\circ}, x_{j} / c=0.88, w_{j} / c=1 / 165, d_{j} / c=2 / 165, \theta_{j}=90^{\circ}, \bar{\rho}_{j} / \rho_{\infty} \approx 1$.

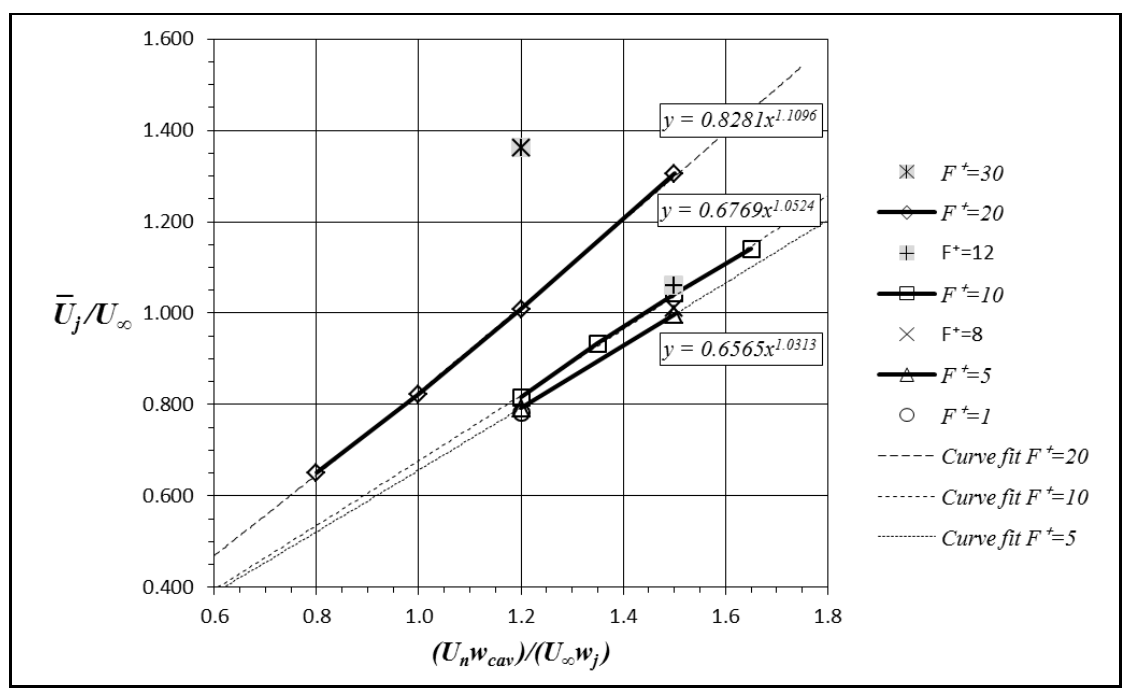

Figure 6.31: Relative ejection velocity, $\bar{U}_{j} / U_{\infty}$, as function of the applied velocity amplitude at the bottom of the cavity, expressed as $\left(U_{n} w_{\text {cav }}\right) /\left(U_{\infty} w_{j}\right)$, for $\operatorname{Re}_{c}=5.5 \cdot 10^{5}$, $\mathrm{M}_{\infty}=0.146, \alpha=0^{\circ}, x_{j} / c=0.88, w_{j} / c=1 / 165, d_{j} / c=2 / 165, \theta_{j}=90^{\circ}, \bar{\rho}_{j} / \rho_{\infty} \approx 1$. 


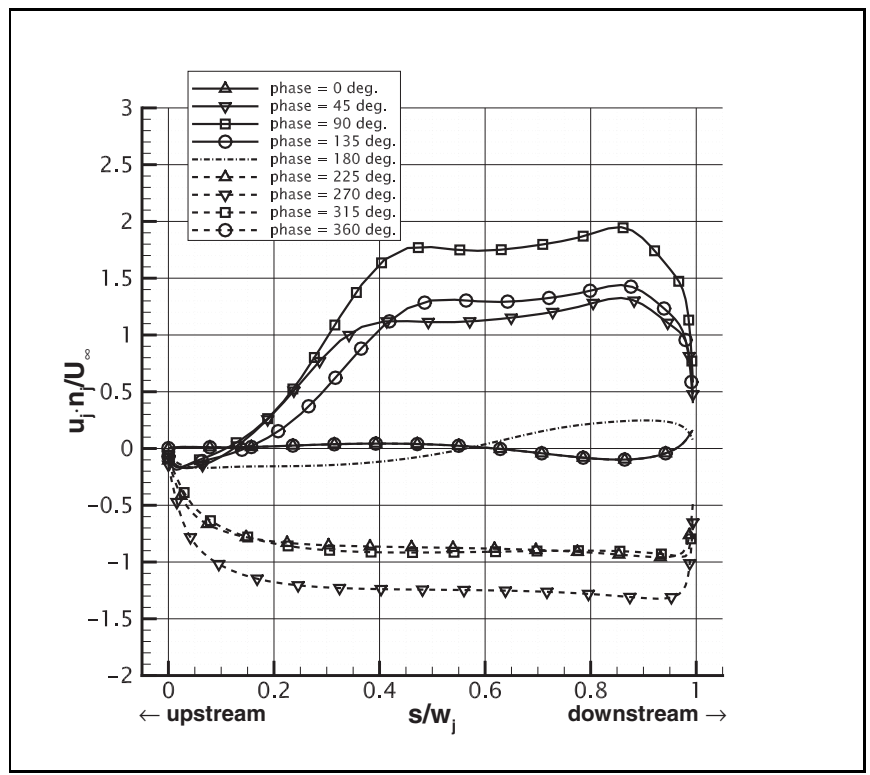

(a) $F^{+}=1$

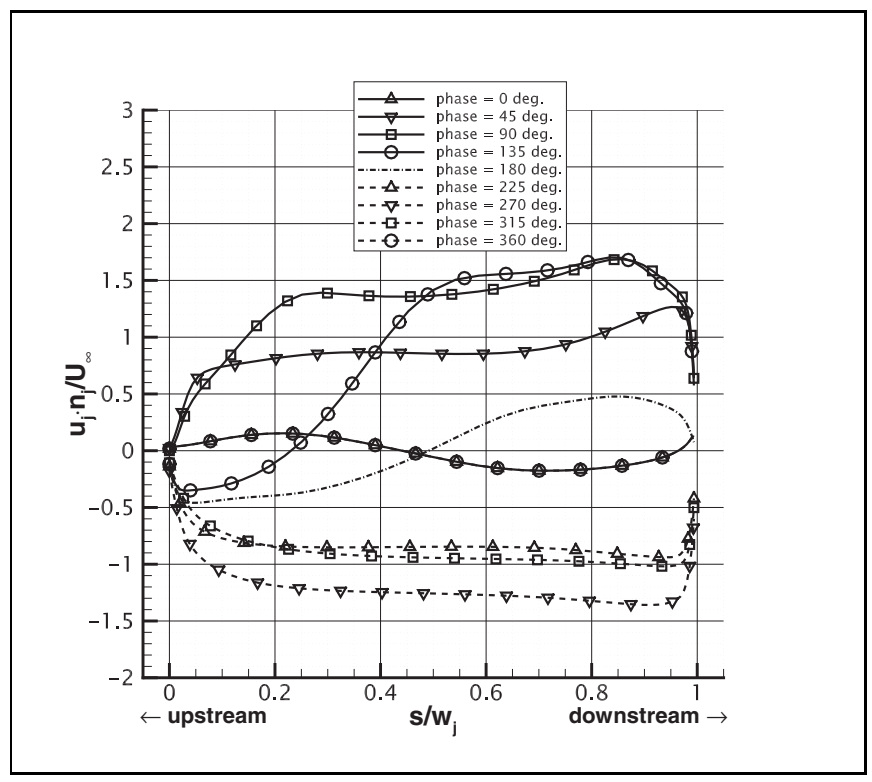

(b) $F^{+}=5$

Figure 6.32: continues on next page. . 


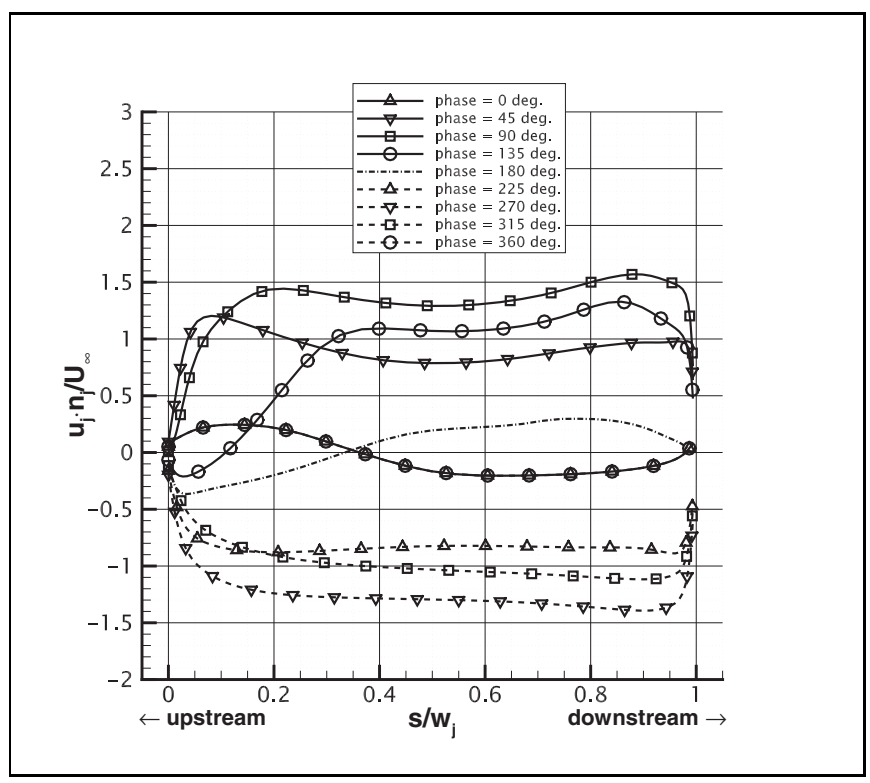

(c) $F^{+}=10$

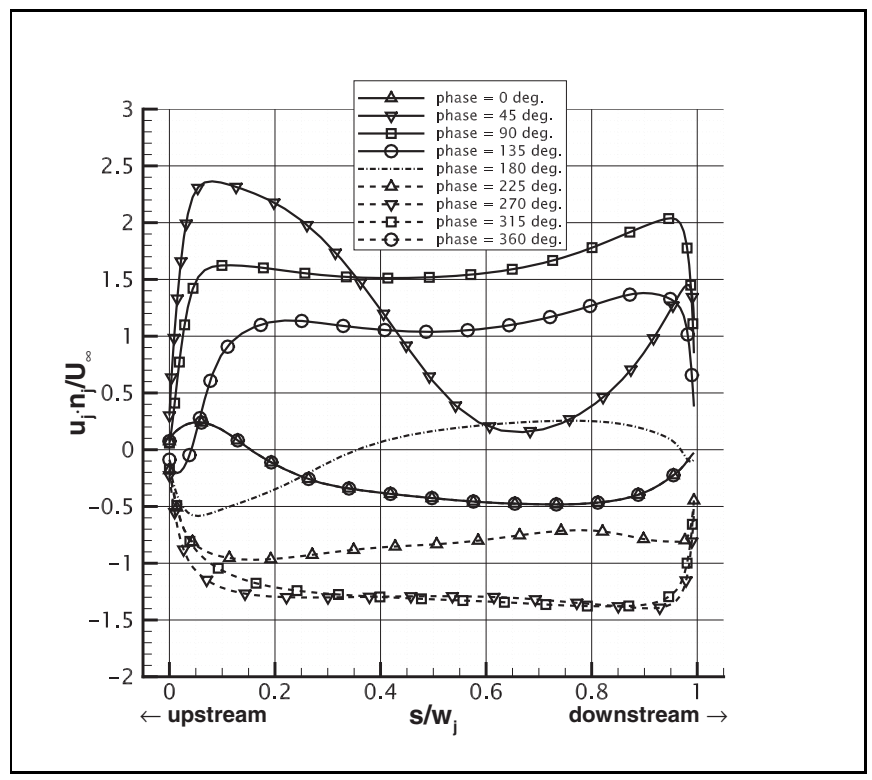

(d) $F^{+}=20$

Figure 6.32: continues on next page... 


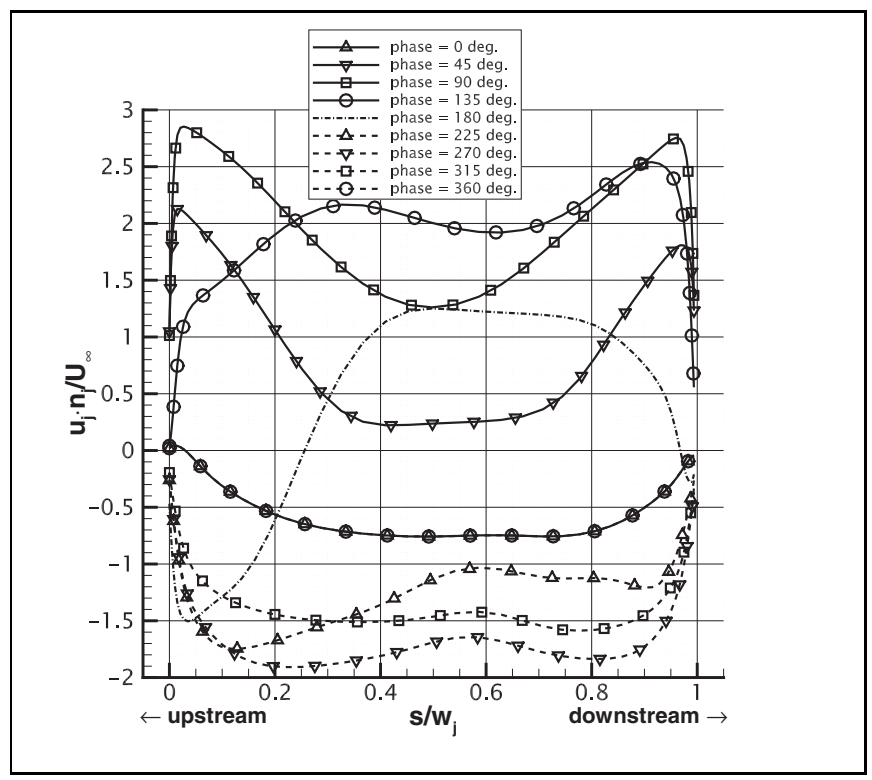

(e) $F^{+}=30$

Figure 6.32: Distribution of relative normal velocity across slit, $\underline{u}_{j} \cdot \underline{n}_{j} / U_{\infty}$, at different phases during actuation cycle, for $\operatorname{Re}_{c}=5.5 \cdot 10^{5}, \mathrm{M}_{\infty}=0.146, \alpha=0^{\circ}, x_{j} / c=0.88$, $w_{j} / c=1 / 165, d_{j} / c=2 / 165, \theta_{j}=90^{\circ}, \bar{\rho}_{j} / \rho_{\infty} \approx 1,\left(U_{n} w_{c a v}\right) /\left(U_{\infty} w_{j}\right)=1.2$. Maximum ejection: phase $=90^{\circ}$, maximum ingestion: phase $=270^{\circ}$

- During the ejection part of the cycle, i.e. between $0^{\circ}$ and $180^{\circ}$, the velocity profiles become increasingly fuller (filling the width of the slit) for higher actuation frequencies, with higher velocity gradients near the sides of the slit. The velocity profiles have a 'horned' shape, which was also observed in figure 6.21 for low-frequency actuation. Towards higher actuation frequencies, these 'horns' are located closer to the sides of the slit.

- At the beginning of the cycle, i.e. at a phase of $0^{\circ}$, the normal velocity is not zero across the slit. Air is already being ejected close to the upstream side of the slit, whereas it is still being ingested close to the downstream side of the slit. With increasing actuation frequency, the lagging part of the normal velocity profile increases in size and the normal velocity becomes more negative there.

- At a phase of $180^{\circ}$, i.e. in the middle of the cycle, a similar lagging effect is visible: air is already being ingested close to the upstream side of the slit, whereas it is still being ejected close to the downstream side of the slit. Again, the lagging part of the normal velocity profile increases in size with increasing actuation frequency. 


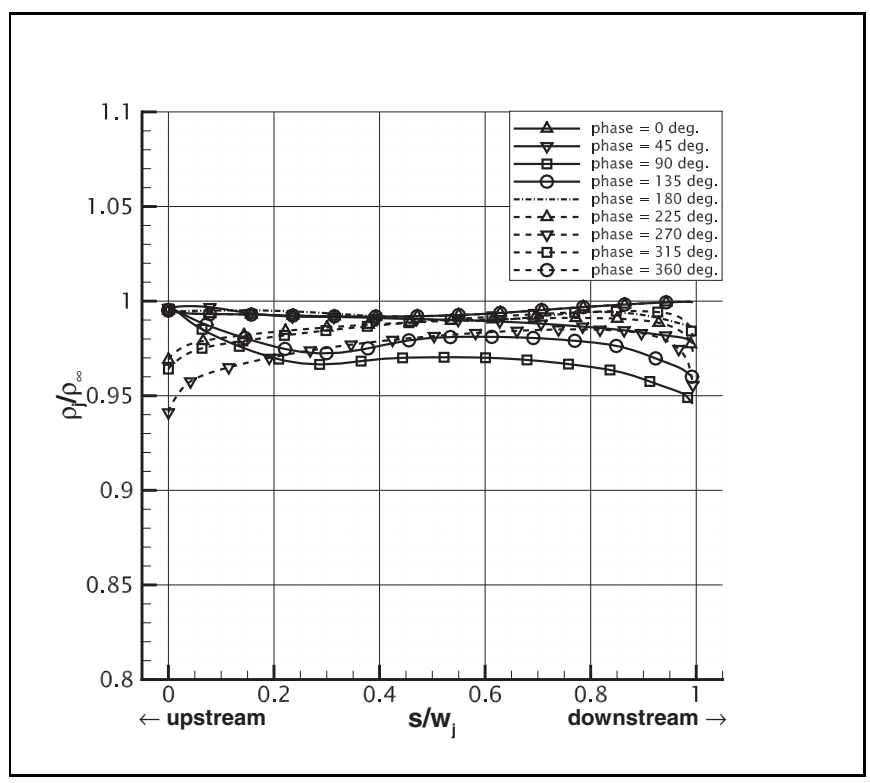

(a) $F^{+}=1, c_{\mu}=0.0036, \bar{\rho}_{j} / \rho_{\infty}=0.98$

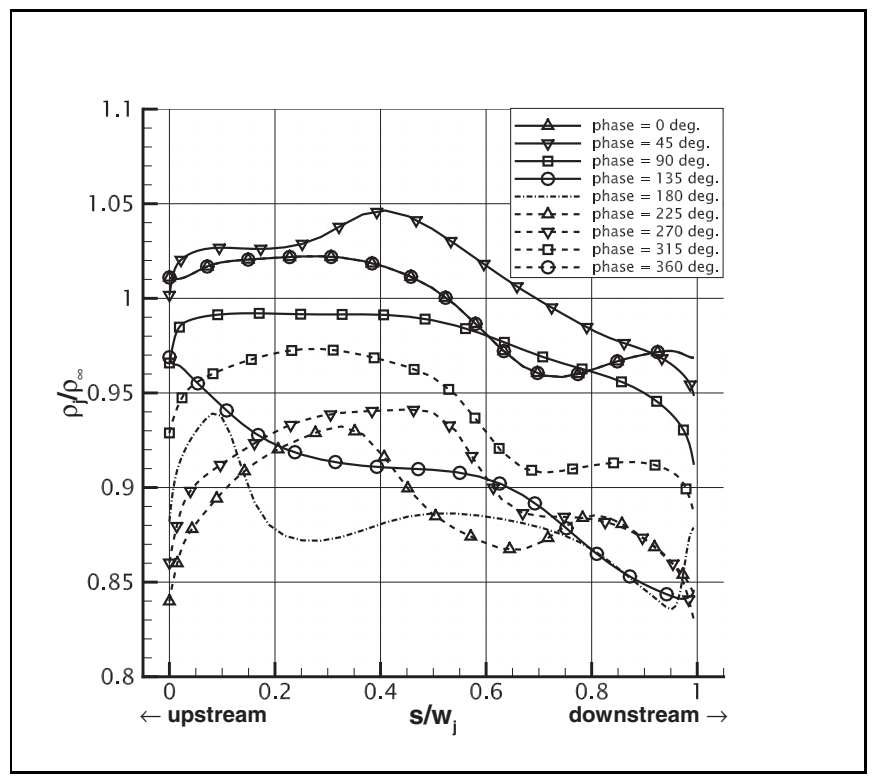

(b) $F^{+}=30, c_{\mu}=0.011, \bar{\rho}_{j} / \rho_{\infty}=0.95$

Figure 6.33: Distribution of relative density across slit, $\rho_{j} / \rho_{\infty}$, at different phases during actuation cycle, for $\operatorname{Re}_{c}=5.5 \cdot 10^{5}, \mathrm{M}_{\infty}=0.146, \alpha=0^{\circ}, x_{j} / c=0.88, w_{j} / c=1 / 165$, $d_{j} / c=2 / 165, \theta_{j}=90^{\circ},\left(U_{n} w_{\text {cav }}\right) /\left(U_{\infty} w_{j}\right)=1.2$. 
With increasing actuation frequency and amplitude, compressibility effects become more important. This can be seen in figure 6.33 which shows the density ratio across the slit at different phases during the actuation cycle for $F^{+}=1$ and $F^{+}=30$. For both cases the velocity amplitude at the bottom of the cavity equals $\left(U_{n} w_{c a v}\right) /\left(U_{\infty} w_{j}\right)=1.2$. Although the spatial- and time-averaged density during the ejection part of the cycle is close to the free-stream density, i.e. $\bar{\rho}_{j} / \rho_{\infty} \in[0.95,0.99]$ for all investigated cases, figure 6.33 shows that for $F^{+}=30$, the density across the slit can deviate as much as $15 \%$ from the free-stream value. Furthermore, the maximum observed Mach number across the slit is approximately $\mathrm{M}=0.43$ for this case. This shows that compressibility effects can become important for high-frequency synthetic jet actuation and confirms the importance of solving the governing equations for compressible flow.

\subsubsection{Characteristic Flow Phenomena and Aerodynamic Perfor- mance}

Characteristic aspects of high-frequency synthetic jet actuation will now be illustrated for the case of $\alpha=0^{\circ}, x_{j} / c=0.88, F^{+}=20$ and $c_{\mu}=0.0061$, which corresponds to $\left(U_{n} w_{\text {cav }}\right) /\left(U_{\infty} w_{j}\right)=1.2$. This case is the starting point for the parameter study, which will be presented in the next subsections.

\section{Velocity Field in Trailing Edge Region}

Figure 6.34 shows streamlines and iso-contours of the dimensionless velocity magnitude, $|\underline{u}| / U_{\infty}$, in the region around the trailing edge, at four phases in the actuation cycle. Again, a phase of $90^{\circ}$ corresponds to maximum ejection (velocity) and $270^{\circ}$ corresponds to maximum ingestion (velocity). The results are within the the periodic part of the simulation.

A region with low velocities can be observed between the slit and the trailing edge at all phases shown in figure 6.34. The streamlines that initially follow the lower surface of the airfoil towards the trailing edge are deflected downwards during the ejection part of the actuation cycle. During ingestion, air is being ingested into the slit, mostly from the upstream boundary layer, but also from the low-velocity region downstream of the slit. Downstream of the slit, the main flow is not directed towards the wall during ingestion, as was observed for low-frequency actuation. This is due to the presence of counterclockwise spanwise vortices that have been generated by the synthetic jet during the first part of the actuation cycle. The induced velocities by these vortices effectively cause an obstruction to the main flow.

Figure 6.35 shows the time-averaged velocity field, for which time averaging has been performed over a single actuation cycle in the periodic part of the simulation. In a time-averaged sense, the low-velocity region between the slit and the trailing edge is a stationary recirculation zone. This displaces the main flow, which effectively adds (aft) camber to the airfoil. The largest displacement of the flow along the lower side of the airfoil occurs at $x_{1} / c=0.92$ and is approximately $0.01 c$. Downstream of this location, 
Chapter 6. Pitch Control using SJ Actuation

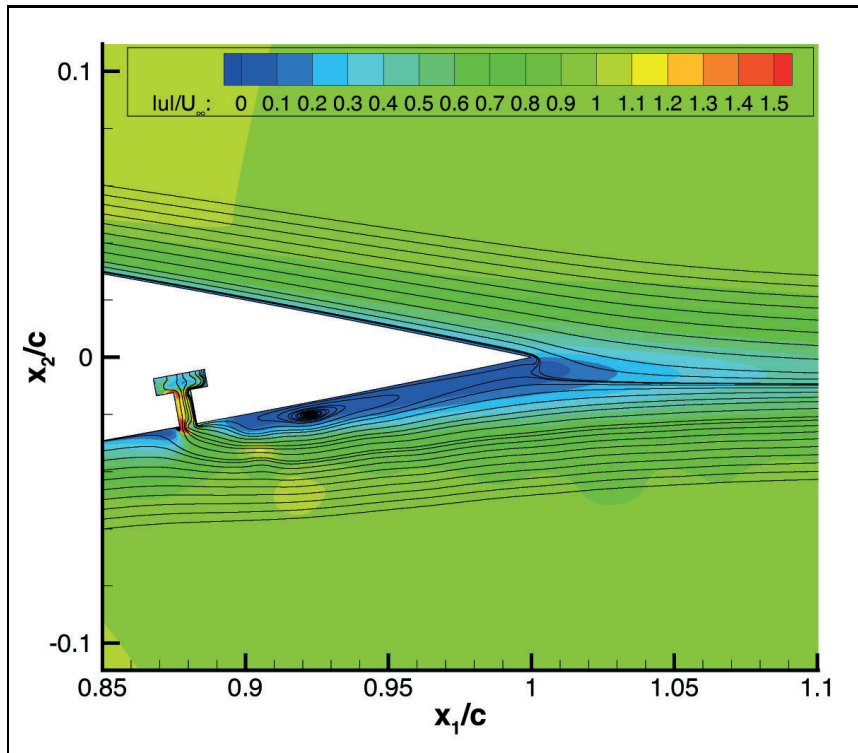

(a) phase $=45^{\circ}$

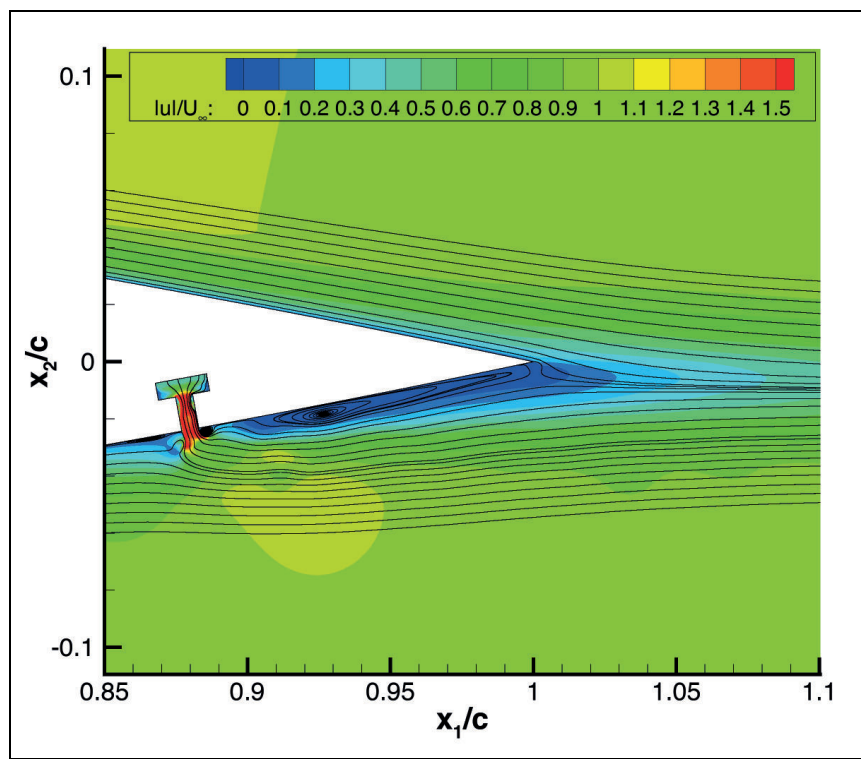

(b) phase $=90^{\circ}$

Figure 6.34: continues on next page... 
6.8 Results for High-Frequency Synthetic Jet ACtuation

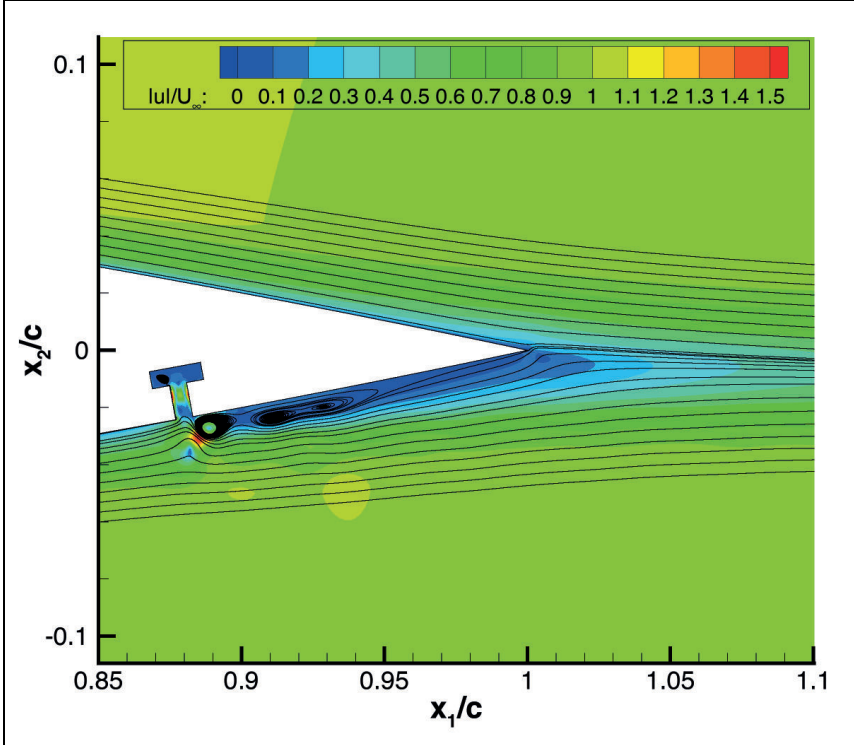

(c) phase $=180^{\circ}$

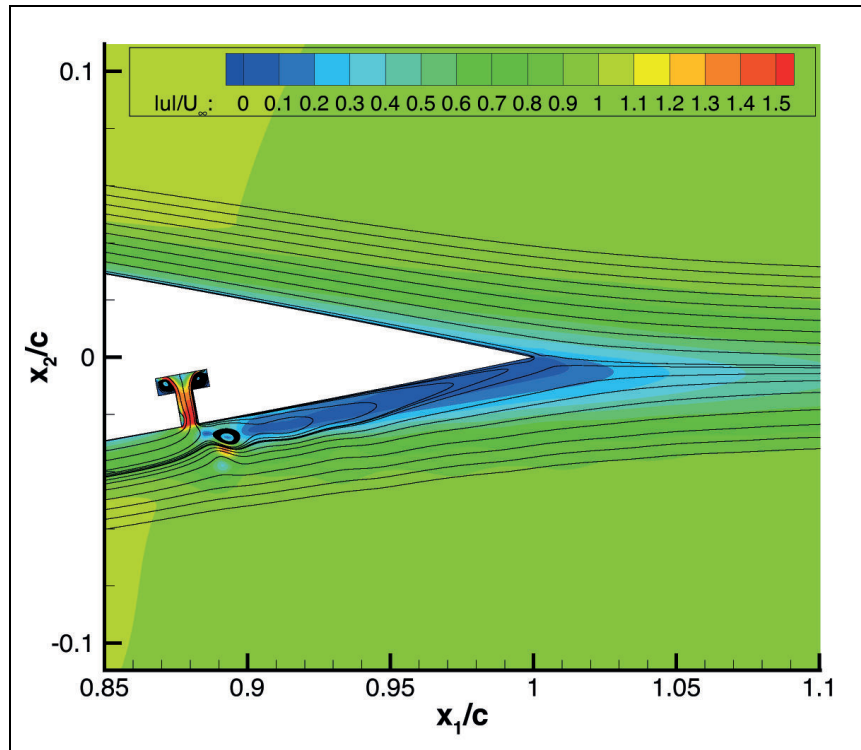

(d) phase $=270^{\circ}$

Figure 6.34: Streamlines and iso-contours of dimensionless velocity magnitude, $|\underline{u}| / U_{\infty}$, at four phases in the actuation cycle, for $\operatorname{Re}_{c}=5.5 \cdot 10^{5}, \mathrm{M}_{\infty}=0.146, \alpha=0^{\circ}, x_{j} / c=$ $0.88, w_{j} / c=1 / 165, d_{j} / c=2 / 165, \theta_{j}=90^{\circ}, F^{+}=20, c_{\mu}=0.0061, \bar{\rho}_{j} / \rho_{\infty} \approx 1$. 


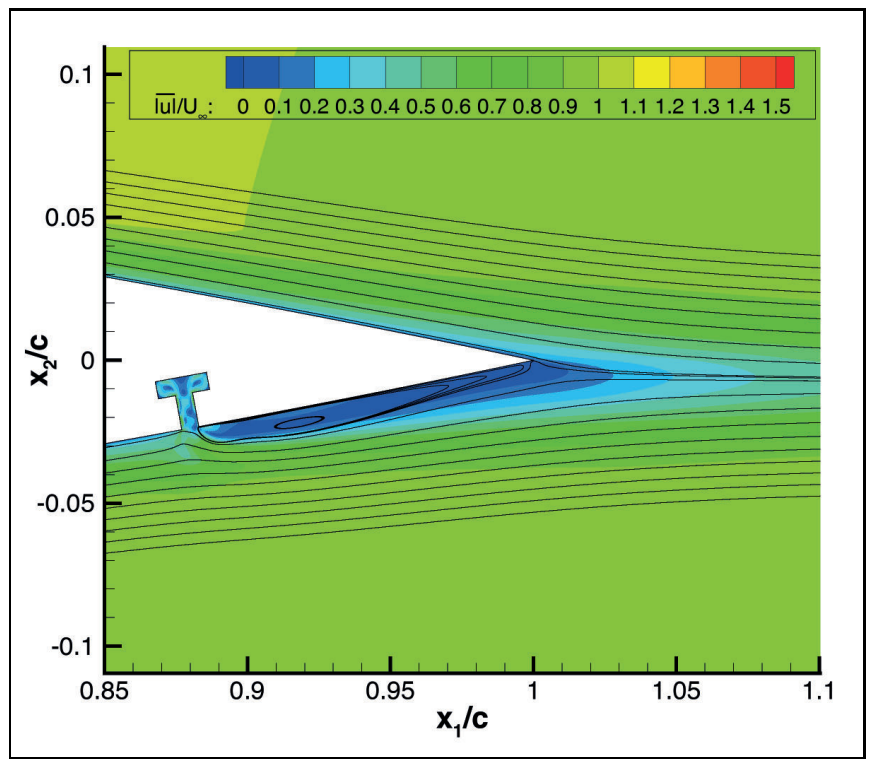

Figure 6.35: Streamlines and iso-contours of time-averaged dimensionless velocity magnitude, $\overline{|\underline{u}|} / U_{\infty}$, for $\operatorname{Re}_{c}=5.5 \cdot 10^{5}, \mathrm{M}_{\infty}=0.146, \alpha=0^{\circ}, x_{j} / c=0.88, w_{j} / c=1 / 165$, $d_{j} / c=2 / 165, \theta_{j}=90^{\circ}, F^{+}=20, c_{\mu}=0.0061, \bar{\rho}_{j} / \rho_{\infty} \approx 1$.

the displacement is reduced again. Note that there is no entrainment of low-momentum fluid from the upper side of the airfoil in the present case.

\section{Spanwise Vorticity Field in Trailing Edge Region}

The obstruction to the main flow can be observed more closely in figure 6.36, which shows streamlines and iso-contours of the spanwise vorticity field. The vortices generated by the synthetic jet are convected downstream with the local flow velocity. Vortices with a negative sign (clockwise) are rapidly dissipated, whereas a train of vortices with a positive sign (counter-clockwise) remains visible for a certain distance. The flow that has been directed downwards during the ejection part of the cycle is kept there due to the induced velocities by the counter-clockwise vortices.

The nine streamlines shown in figure 6.36 have been constructed backwards, starting from the straight line beginning at $\left(x_{1} / c, x_{2} / c\right)=(1.15,0.03)$ and ending at $\left(x_{1} / c, x_{2} / c\right)=$ $(1.15,-0.03)$, such that the asymmetry in the flow field can be observed easily. It is clear that most of the fluid directly behind the trailing edge originates from above the airfoil. This is associated with an increase of the circulation, i.e. an increase in lift, since the airfoil is geometrically symmetric and the angle of attack is $\alpha=0^{\circ}$. 


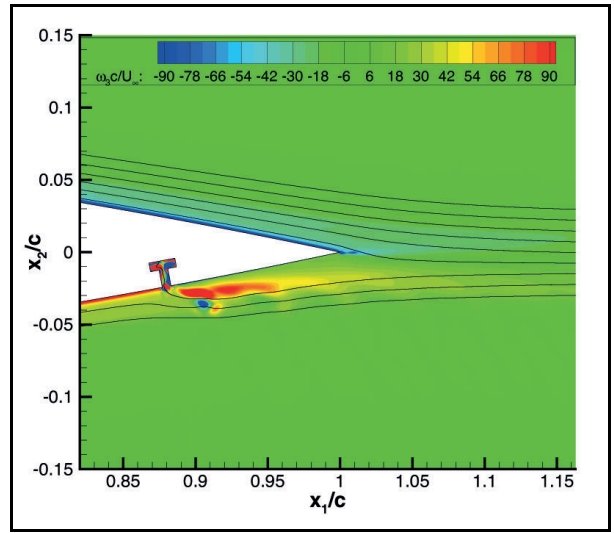

(a) phase $=45^{\circ}$

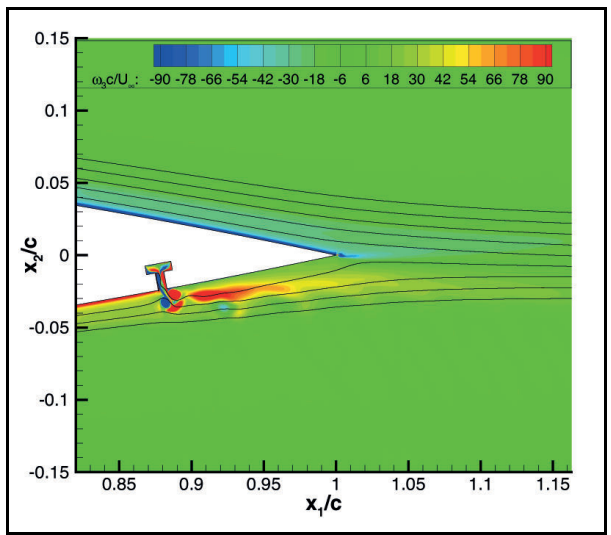

(c) phase $=180^{\circ}$

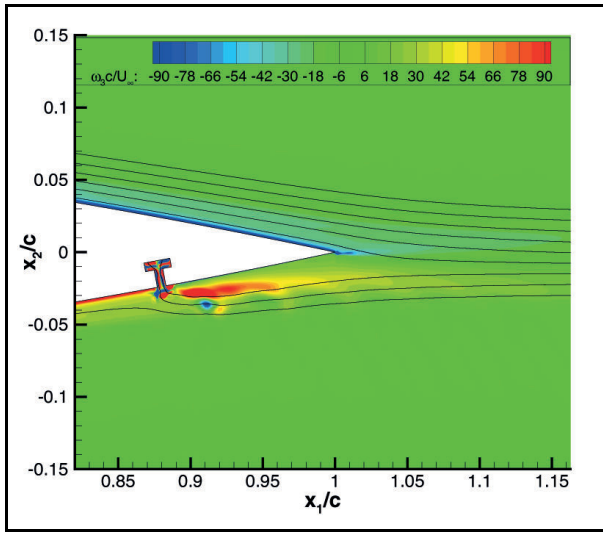

(b) phase $=90^{\circ}$

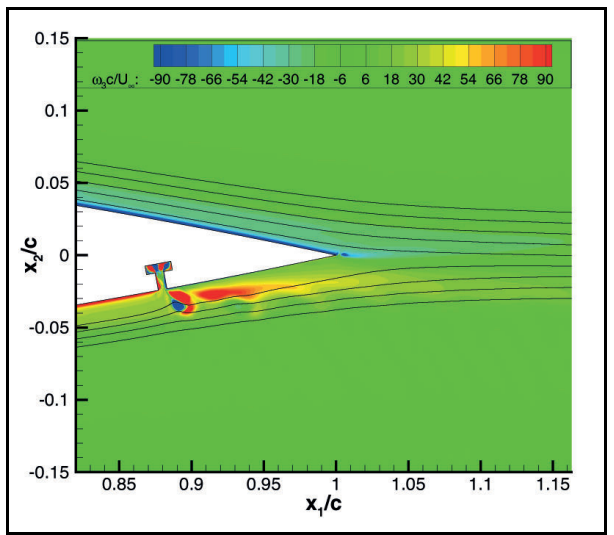

(d) phase $=270^{\circ}$

Figure 6.36: Streamlines and iso-contours of dimensionless spanwise vorticity, $\omega_{3} c / U_{\infty}$, at four phases in the actuation cycle, for $\operatorname{Re}_{c}=5.5 \cdot 10^{5}, \mathrm{M}_{\infty}=0.146, \alpha=0^{\circ}, x_{j} / c=0.88$, $w_{j} / c=1 / 165, d_{j} / c=2 / 165, \theta_{j}=90^{\circ}, F^{+}=20, c_{\mu}=0.0061, \bar{\rho}_{j} / \rho_{\infty} \approx 1$. 


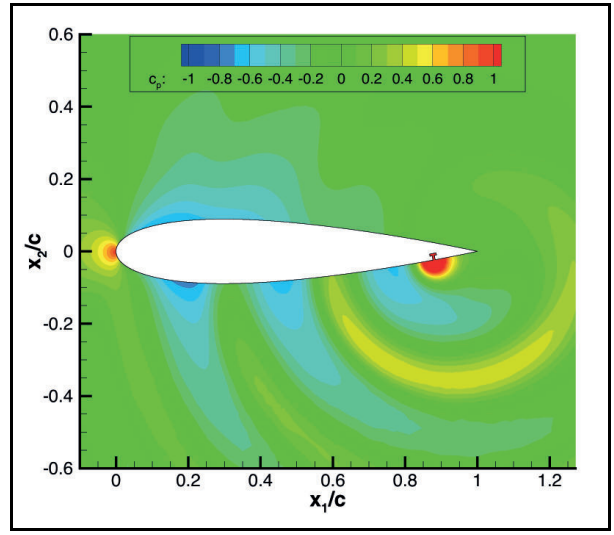

(a) phase $=45^{\circ}$

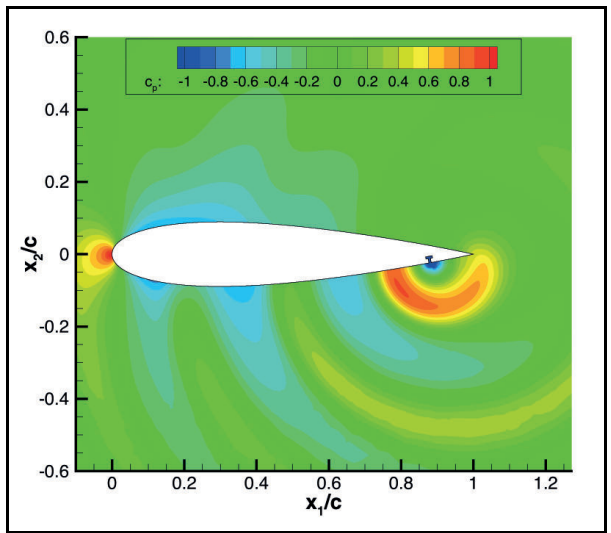

(c) phase $=180^{\circ}$

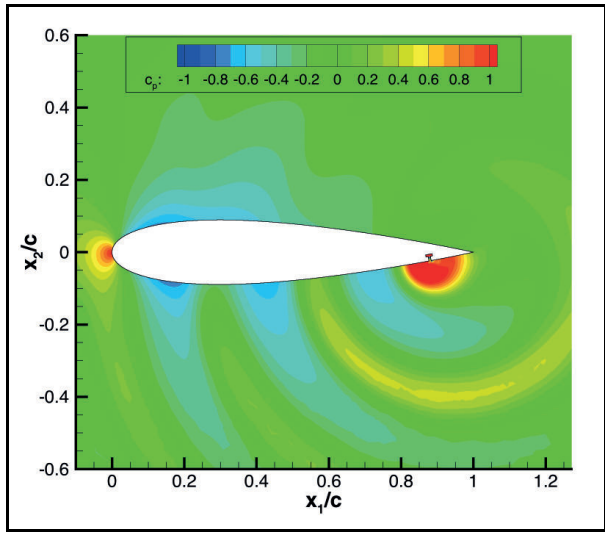

(b) phase $=90^{\circ}$

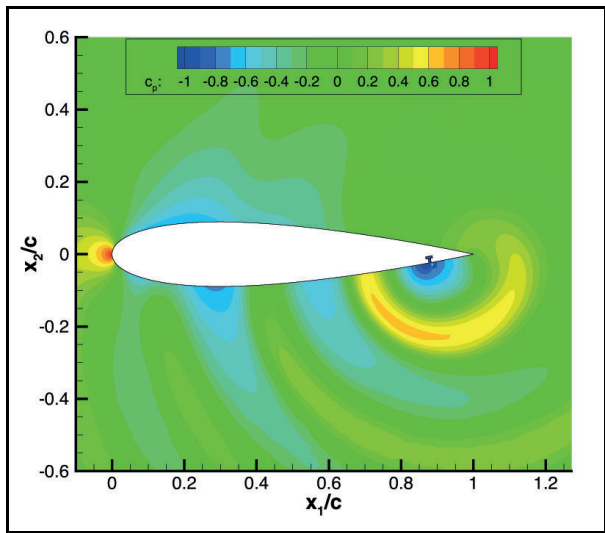

(d) phase $=270^{\circ}$

Figure 6.37: Iso-contours of pressure coefficient, $c_{p}$, at four phases in the actuation cycle, for $\operatorname{Re}_{c}=5.5 \cdot 10^{5}, \mathrm{M}_{\infty}=0.146, \alpha=0^{\circ}, x_{j} / c=0.88, w_{j} / c=1 / 165, d_{j} / c=2 / 165$, $\theta_{j}=90^{\circ}, F^{+}=20, c_{\mu}=0.0061, \bar{\rho}_{j} / \rho_{\infty} \approx 1$. 


\section{Pressure Field Around Airfoil}

The synthetic jet and its associated vortices are created by sinusoidal oscillatory displacements of an actuator surface inside the cavity, which is modeled here by means of an inflow/outflow boundary condition. Due to the action of the synthetic jet, pressure fluctuations (acoustic waves) are generated that are radiated into the flow field, as can be seen in figure 6.37. As expected, high pressures are associated with ejection, whereas low pressures are associated with ingestion. On the surface of the airfoil, where the (relative) flow velocity is zero, pressure waves travel with the speed of sound, which approximately equals $1 / \mathrm{M}_{\infty}=c_{\infty}^{*} / U_{\infty}$ in dimensionless quantities. The dimensionless wavelength for the present actuation frequency therefore equals $1 /\left(\mathrm{M}_{\infty} F^{+}\right) \approx 0.342$ per unit chord. This agrees with the results. For example, four adjacent negative pressure peaks can be seen along the lower surface of the airfoil in figure 6.37(d).

Note that the acoustic pressure waves also travel along the upper side of the airfoil, but there the amplitude is much lower. This can be seen more clearly in figure 6.38 which shows the pressure distribution on both sides of the airfoil at a phase of $180^{\circ}$ in the actuation cycle.

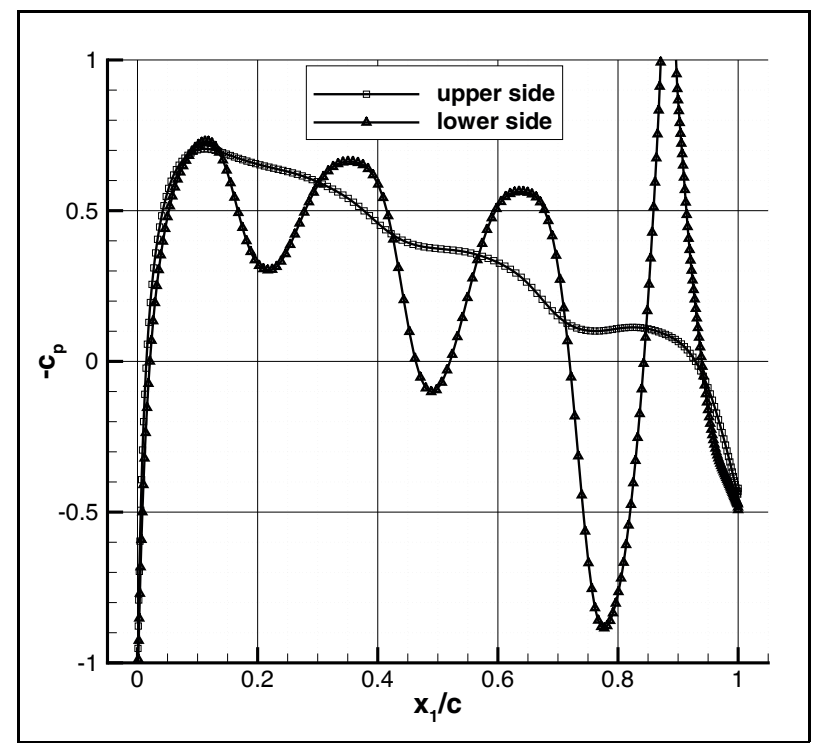

Figure 6.38: Pressure coefficient, $c_{p}$, along the upper and lower surface of the airfoil, at a phase of $180^{\circ}$ in the actuation cycle, for $\operatorname{Re}_{c}=5.5 \cdot 10^{5}, \mathrm{M}_{\infty}=0.146, \alpha=0^{\circ}, x_{j} / c=$ $0.88, w_{j} / c=1 / 165, d_{j} / c=2 / 165, \theta_{j}=90^{\circ}, F^{+}=20, c_{\mu}=0.0061, \bar{\rho}_{j} / \rho_{\infty} \approx 1$. Each symbol represents a grid point.

Figure 6.39 shows the time-averaged pressure distribution on the surface of the airfoil. Here, time averaging has been performed over one actuation cycle in the periodic part of the simulation. Due to this averaging, the acoustic pressure waves traveling along both 
sides of the airfoil filter out of the results. Figure 6.39 also includes the baseline results without synthetic jet actuation. It can be seen that on the average, the obtained pressure difference upstream of the slit causes lift, i.e. $p_{\text {upper }}\left(x_{1} / c\right)-p_{\text {lower }}\left(x_{1} / c\right)<0$. On the other hand, part of the region between the slit and the trailing edge yields a negative contribution to the lift, since the time-averaged pressure on the lower side is below that of the upper side. This was also observed for the low-frequency actuation cases, see subsection 6.7.2 It makes the option of synthetic jet actuation different from the option of a trailing edge flap, since in the latter case the flap itself does contribute to the overall increase in lift.

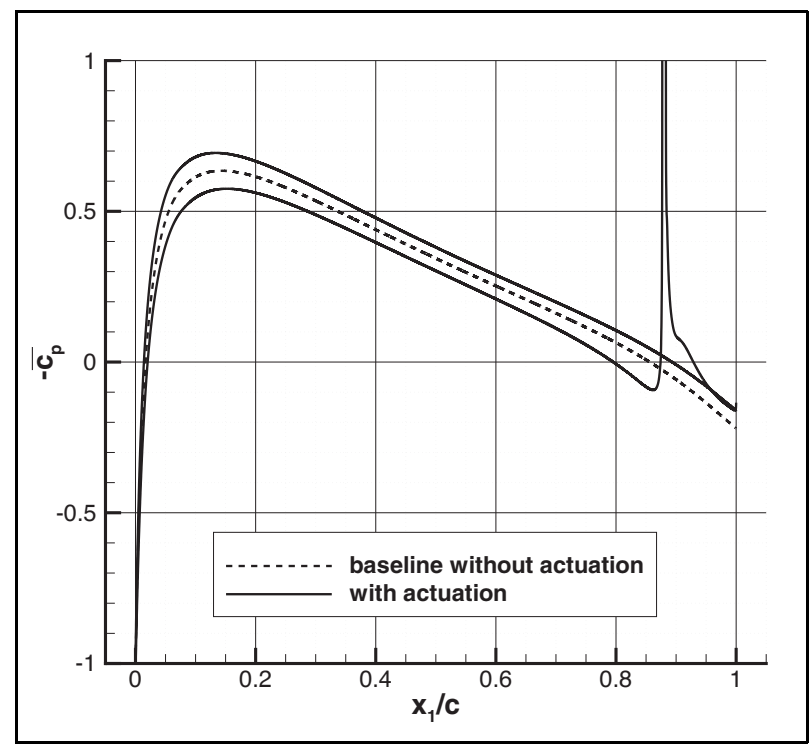

Figure 6.39: Time-averaged pressure coefficient, $\overline{c_{p}}$, along the upper and lower surface of the airfoil, for $\operatorname{Re}_{c}=5.5 \cdot 10^{5}, \mathrm{M}_{\infty}=0.146, \alpha=0^{\circ}, x_{j} / c=0.88, w_{j} / c=1 / 165$, $d_{j} / c=2 / 165, \theta_{j}=90^{\circ}$. Dashed line: baseline case without actuation. Solid line: synthetic jet actuation with $F^{+}=20, c_{\mu}=0.0061, \bar{\rho}_{j} / \rho_{\infty} \approx 1$.

\section{Aerodynamic Performance}

Figure 6.40 shows the change in the lift coefficient, $\Delta c_{l}$, and figure 6.41 shows the change in the drag coefficient, $\Delta c_{d}$, as a function of the non-dimensional time, $U_{\infty} t / c$. Both figures show the entire signal, as well as a filtered signal that shows an average value for each actuation cycle.

Due to the acoustic pressure waves caused by the action of the high-frequency synthetic jet, the response of $\Delta c_{l}$ is not smooth. On top of the average increase of $\overline{\Delta c_{l}}=0.092$ in the periodic part of the simulation, a high-frequency component is 


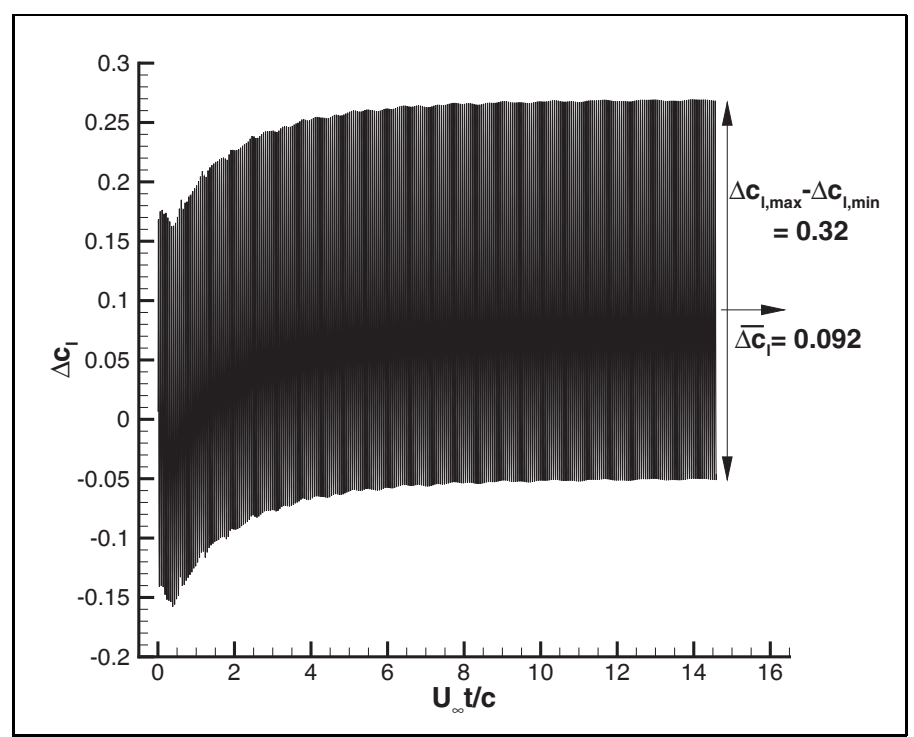

(a) $\Delta c_{l}$

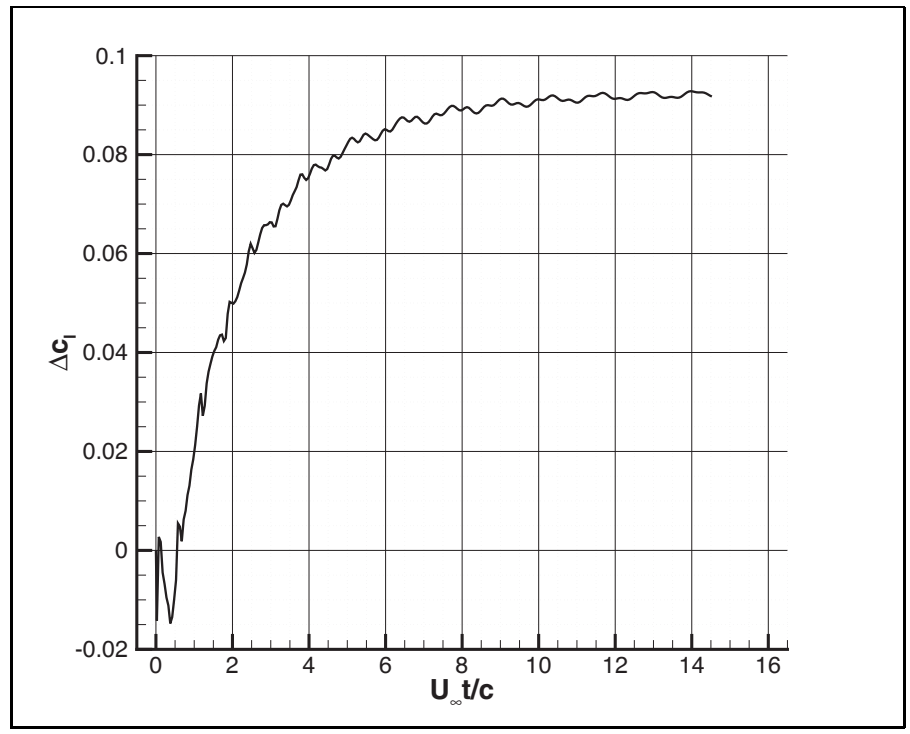

(b) filtered $\Delta c_{l}$

Figure 6.40: Change of lift coefficient, $\Delta c_{l}$, as a function of dimensionless time, $U_{\infty} t / c$, for $\operatorname{Re}_{c}=5.5 \cdot 10^{5}, \mathrm{M}_{\infty}=0.146, \alpha=0^{\circ}, x_{j} / c=0.88, w_{j} / c=1 / 165, d_{j} / c=2 / 165$, $\theta_{j}=90^{\circ}, F^{+}=20, c_{\mu}=0.0061, \bar{\rho}_{j} / \rho_{\infty} \approx 1$. 


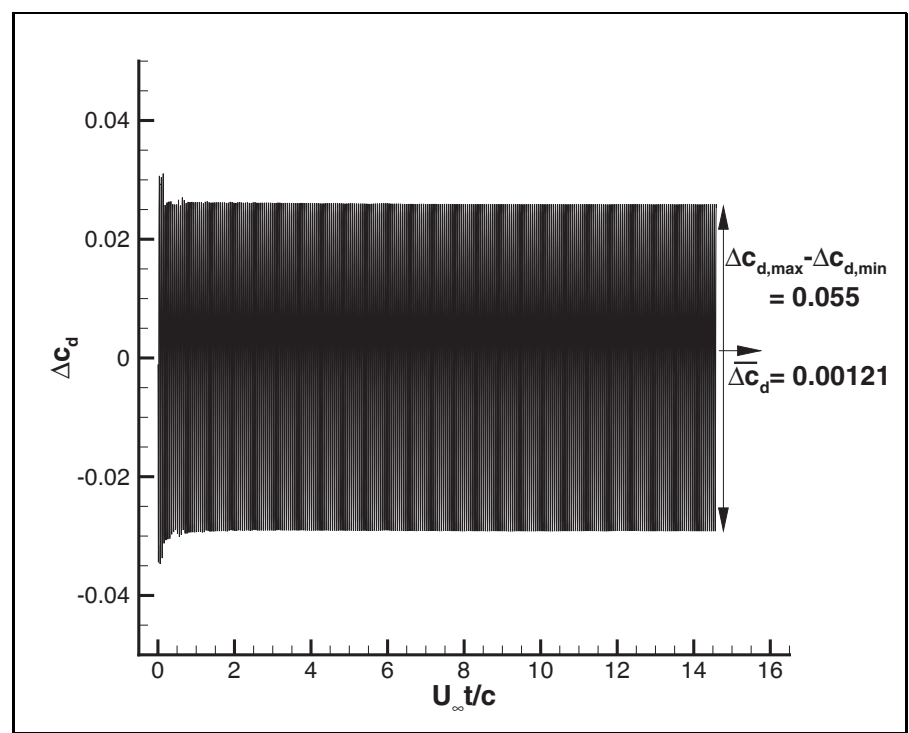

(a) $\Delta c_{d}$

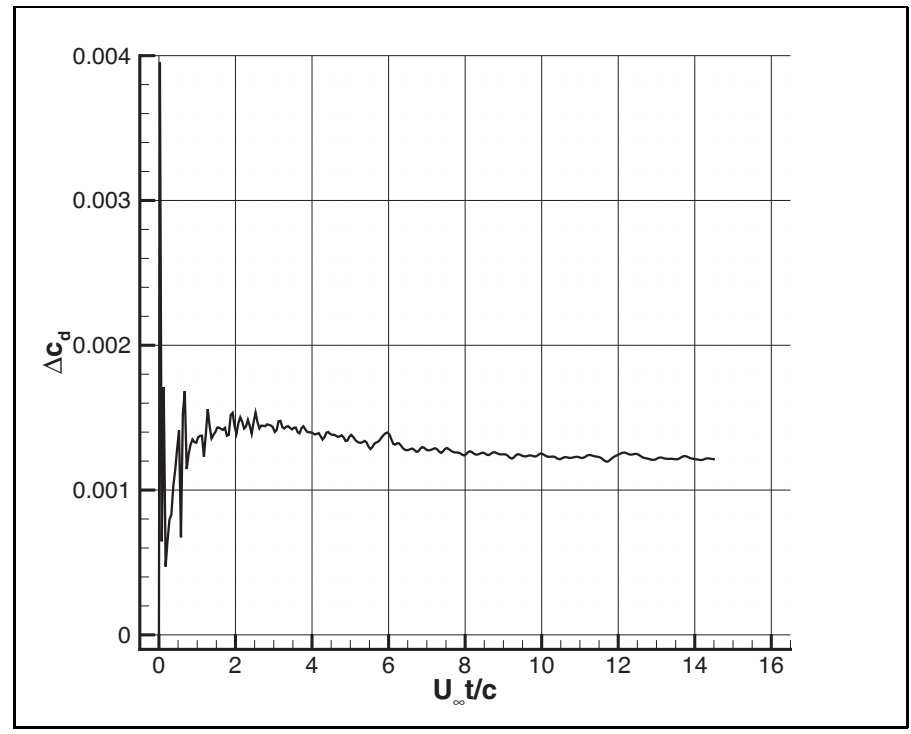

(b) filtered $\Delta c_{d}$

Figure 6.41: Change of drag coefficient, $\Delta c_{d}$, as a function of dimensionless time, $U_{\infty} t / c$, for $\operatorname{Re}_{c}=5.5 \cdot 10^{5}, \mathrm{M}_{\infty}=0.146, \alpha=0^{\circ}, x_{j} / c=0.88, w_{j} / c=1 / 165, d_{j} / c=2 / 165$, $\theta_{j}=90^{\circ}, F^{+}=20, c_{\mu}=0.0061, \bar{\rho}_{j} / \rho_{\infty} \approx 1$. 
present, with a large amplitude: the difference between the maximum and minimum value is $\Delta c_{l, \max }-\Delta c_{l, \min }=0.32$. The amplitude of this high-frequency component is approximately constant for the whole simulated time.

A similar response can be observed for $\Delta c_{d}$. For the baseline case, the computed drag coefficient is $c_{d}=0.0139$. Due to synthetic jet actuation, the average change in the drag coefficient is $\overline{\Delta c_{d}}=0.00121$, whereas the difference between the maximum and minimum value is $\Delta c_{d, \max }-\Delta c_{d, \min }=0.055$. Except for a short period at the start of the simulation, the amplitude of the high-frequency component in the drag coefficient is also approximately constant.

In the filtered signals, it can be seen that between the onset of actuation and $U_{\infty} t / c \approx$ 0.55 , the change in lift has a negative peak. Subsequently, the change in lift quickly increases. Approximately $50 \%$ of the final change in the lift coefficient is obtained within $2 c / U_{\infty}$. The filtered signal for the change in drag shows some sharp peaks directly following the start of the simulation. After $U_{\infty} t / c=2$, the peaks have reduced in magnitude and the change in the drag coefficient gradually reduces towards the the final change in the drag coefficient.

Although not shown here, it is interesting to note that the pressure distribution is the main contributor to the change in lift and drag. This was also observed for lowfrequency synthetic jet actuation. For possible future wind tunnel experiments, it is therefore sufficient to measure the surface pressure distribution to obtain a reasonable approximation of the time-averaged change in the lift coefficient.

\section{Power Consumption}

The average actuator power can be expressed in terms of a jet power coefficient, $c_{p w r}$, defined as

$$
c_{p w r}=\frac{\bar{P}}{\frac{1}{2} \rho_{\infty} U_{\infty}^{3} c},
$$

where $\bar{P}$ is the time-averaged power per unit span, given by

$$
P=\frac{1}{T_{j}} \int_{0}^{T_{j}} \int_{0}^{w_{c a v}} \Delta p(s, t) U_{n} \sin \left(2 \pi f_{j} t\right) d s d t,
$$

with $\Delta p(s, t)$ the time-dependent local pressure difference across the actuator. Here, we assume a constant backpressure. Furthermore, the period of actuation is $T_{j}=1 / f_{j}$, and $s$ is the coordinate across the bottom of the cavity, i.e. $s \in\left[0, w_{\text {cav }}\right]$.

For the present case, the power consumption is $\bar{P}=175 \mathrm{~W} / \mathrm{m}$, such that the jet power coefficient is $c_{p w r}=0.0141$.

\section{Discussion}

The dominant frequency in the lift and drag response is the actuation frequency, i.e. $F^{+}=20$, which is very high. For the NREL $5 \mathrm{MW}$ reference offshore wind turbine [25], 
operating at the rated wind speed of $11.4 \mathrm{~m} / \mathrm{s}$ and a tip ratio of $\lambda=7$, a dimensionless actuation frequency of $F^{+} \approx f c / \Omega r=20$ corresponds to

$$
\begin{aligned}
& \text { - } f_{j} \approx 411 \mathrm{~Hz} \text { at } r / R=0.77, \text { where } c \approx 3 \mathrm{~m}, \\
& \text { - } f_{j} \approx 1128 \mathrm{~Hz} \text { at } r / R=1, \text { where } c=1.419 \mathrm{~m} .
\end{aligned}
$$

Jonkman et al. [25] list the most important natural frequencies of the blades and tower of a land-based version of the NREL reference wind turbine, for the stationary condition with the high-speed shaft brake engaged. The listed structural natural frequencies (first and second tower bending modes, first drivetrain torsion mode, first and second flapwise blade modes, first edgewise blade mode) are all below $3 \mathrm{~Hz}$. Higher-order modes are less important, since they are associated with small displacements. The oscillation in the lift and drag coefficients for the outboard blade sections is at a frequency of at least a factor 100 higher than the most important natural frequencies. Therefore, it is expected that the high-frequency oscillation is virtually 'invisible' to the structure, i.e. the response of the structure to these high-frequency oscillations will be negligible.

For the present case, the obtained time-averaged change in lift is $\overline{\Delta c_{l}}=0.092$, which corresponds to an effective change in the angle of attack of $\overline{\Delta \alpha} \approx 0.84^{\circ}$. The following remarks with respect to this aerodynamic performance can be made:

- In comparison with the option of micro-tabs, with a height of $0.01 c$, located at $0.95 c$, the performance of the synthetic jet for the present set of parameters is at least a factor 2 lower [44], see subsection 2.5.2. However, the synthetic jet is located further upstream than the micro-tabs. Furthermore, due to the presence of the the micro-tab, low-momentum fluid from the upper side of the airfoil is entrained into the recirculation zone along the lower side of the airfoil, see figure 2.10 In the present case, the synthetic jet is not able to accomplish this.

- The performance of the synthetic jet is also much lower than that of a steady jet of the same width at the same location, with a steady average jet velocity of $1.2 U_{\infty}$, for which a change in lift of $\Delta c_{l} \approx 0.4$ was obtained [46]. For the present synthetic jet, the space- and time-averaged ejection velocity is $\bar{U}_{j}=1.01 U_{\infty}$, i.e. lower than for the steady jet. Furthermore, in comparison with the steady jet, the synthetic jet only 'works' half of the time. So, the momentum coefficient of the steady jet is much larger than that of the synthetic jet. The displacement of the flow along the lower side of the airfoil is approximately $0.01 c$ for the synthetic jet, whereas it is approximately $0.06 \mathrm{c}$ for the steady jet, see figure 2.13 Furthermore, the steady jet is able to entrain low-momentum fluid from the upper side of the airfoil, whereas the synthetic jet (in a time-averaged sense) is unable to do so in this case.

For smart rotor control, a minimal effective change in the angle of attack of $\Delta \alpha=3^{\circ}$ is deemed necessary, , see subsection 2.5.1] This is more than a factor 3 higher than achieved with synthetic jet actuation in the case studied in this subsection. In the following subsections, the effects of the momentum coefficient $c_{\mu}$, the actuation frequency, $\mathrm{F}^{+}$, and slit location, $x_{j} / c$, are investigated to see whether a larger effective change in the angle of attack can be generated. 


\subsubsection{Effect of Momentum Coefficient}

To investigate the effect of the momentum coefficient on the aerodynamic performance, we have varied the velocity amplitude at the bottom of the cavity. The employed values are $\left(U_{n} w_{\text {cav }}\right) /\left(U_{\infty} w_{j}\right)=\{0.8,1.0,1.2,1.5\}$. The other investigated parameters are kept constant at $\alpha=0^{\circ}, F^{+}=20$ and $x_{j} / c=0.88$.

Figure 6.42 shows the time-averaged change of lift coefficient, $\overline{\Delta c_{l}}$, and time-averaged change of drag coefficient, $\overline{\Delta c_{d}}$, in the periodic part of the simulations. In the range of investigated momentum coefficients, the changes in lift and drag increase with increasing momentum coefficient. However, the rate of change for $\overline{\Delta c_{d}}$ decreases with increasing momentum coefficient, probably due to the thrust effect of the synthetic jet during ejection. For $\overline{\Delta c_{l}}$, the rate of change increases with increasing momentum coefficient. The lowest investigated momentum coefficient corresponds to an average ejection velocity of $\bar{U}_{j} \approx 0.65 U_{\infty}$. The highest investigated momentum coefficient corresponds to an average ejection velocity of $\bar{U}_{j} \approx 1.31 U_{\infty}$, which is associated with local maximum velocities across the jet close to $3 U_{\infty}$, see figure 6.43. It is expected that still higher values of $c_{\mu}$ are not realistic. Figure 6.42 also shows the jet power coefficient, $c_{p w r}$, which behaves approximately linearly with $c_{\mu}$. In figure 6.30 it was shown that $c_{\mu}$ behaves approximately quadratic with the velocity amplitude at the bottom of the cavity. Therefore, the jet power coefficient scales as $c_{\text {pwr }} \sim U_{n}{ }^{3}$, which means that power consumption quickly increases with increasing jet velocities.

Higher momentum coefficients cause the vorticity generated by the synthetic jet to increase in magnitude and to be ejected further into the flow along the lower side of the airfoil. This can be seen in figure 6.44 which shows the time-averaged dimensionless spanwise vorticity, $\bar{\omega}_{3} c / U_{\infty}$, for the cases of $c_{\mu} \approx 0.006$ and $c_{\mu} \approx 0.01$. The former is associated with $\left(U_{n} w_{\text {cav }}\right) /\left(U_{\infty} w_{j}\right)=1.2$, whereas the latter is associated with $\left(U_{n} w_{\text {cav }}\right) /\left(U_{\infty} w_{j}\right)=1.5$. Time averaging has been performed over a single actuation cycle in the periodic part of the simulation.

The displacement of the flow along the lower side of the airfoil for $c_{\mu} \approx 0.006$ and $c_{\mu} \approx 0.01$ can be seen in figure, 6.45. This figure shows streamlines and iso-contours of the time-averaged dimensionless velocity magnitude, $\overline{\mid \underline{u}} \mid / U_{\infty}$. In a time-averaged sense, the low-velocity region between the slit and the trailing edge is larger for the higher momentum coefficient. The displacement of the flow along the lower side of the airfoil is maintained for a longer distance, with a maximum displacement of $0.016 \mathrm{c}$ occurring between $x_{1} / c=0.95$ and the trailing edge. For the lower momentum coefficient, the maximum displacement is $0.01 c$ and occurs at one specific location: $\left(x_{1} / c=0.92\right)$. Furthermore, for the higher momentum coefficient, there is some entrainment of lowmomentum fluid from the upper side of the airfoil, in a time-averaged sense. This is not the case for the lower momentum coefficient.

Increasing the momentum coefficient also increases the amplitude of the pressure waves that are radiated from the slit, which is associated with an increase in noise generation. Furthermore, the amplitudes of the high-frequency oscillation of $\Delta c_{l}$ and $\Delta c_{d}$ are also increased. For example, $\Delta c_{l, \max }-\Delta c_{l, \min }=0.32$ and $\Delta c_{d, \max }-c_{d, \min }=0.055$ for $\Delta c_{\mu} \approx 0.006$, whereas $\Delta c_{l, \max }-\Delta c_{l, \min }=0.40$ and $\Delta c_{d, \max }-\Delta c_{d, \min }=0.071$ for 


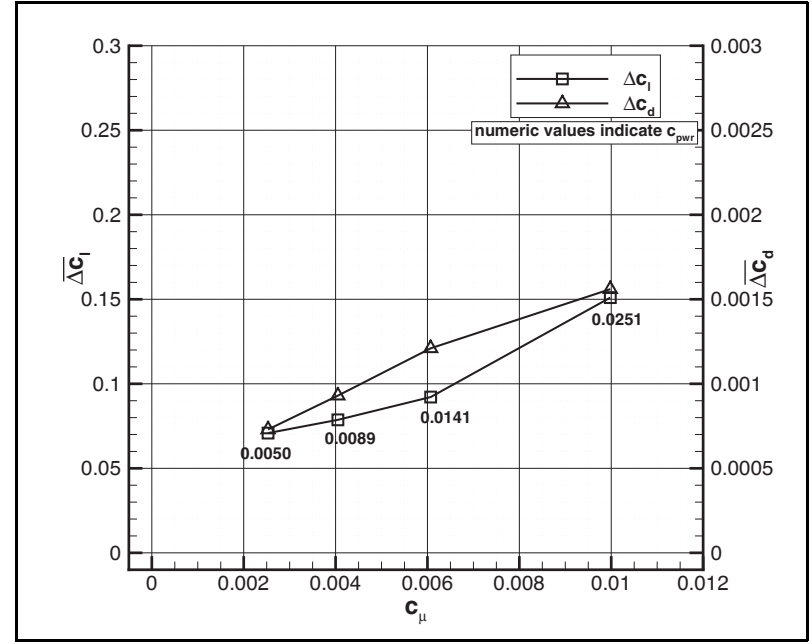

Figure 6.42: Time-averaged change of lift coefficient, $\overline{\Delta c_{l}}$, and time-averaged change of drag coefficient, $\overline{\Delta c_{d}}$, as a function of momentum coefficient, $c_{\mu}$, for $\operatorname{Re}_{c}=5.5 \cdot 10^{5}$, $\mathrm{M}_{\infty}=0.146, \alpha=0^{\circ}, x_{j} / c=0.88, w_{j} / c=1 / 165, d_{j} / c=2 / 165, \theta_{j}=90^{\circ}, F^{+}=20$, $\bar{\rho}_{j} / \rho_{\infty} \approx 1$. For each simulation, the jet power coefficient, $c_{\text {pwr }}$, is indicated.

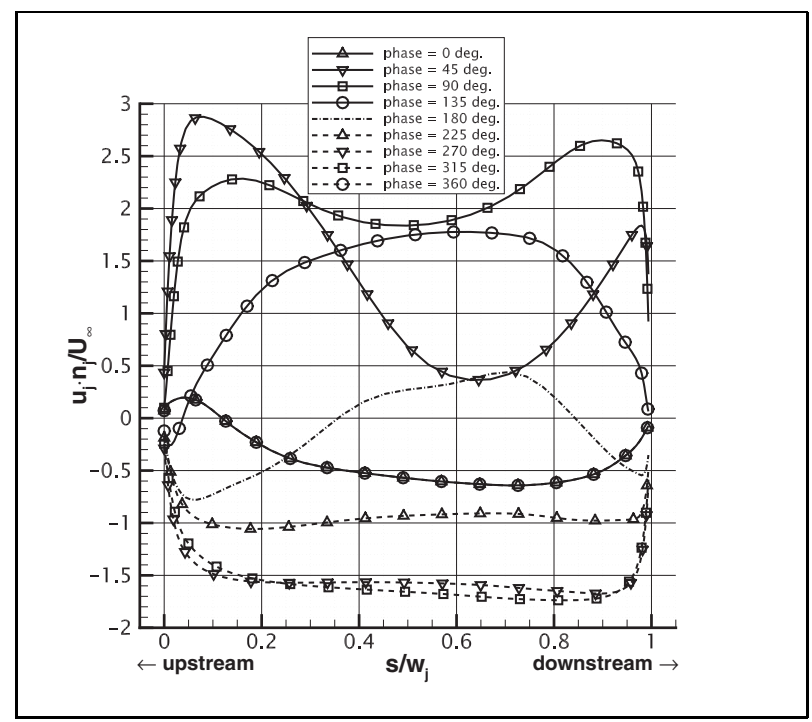

Figure 6.43: Distribution of relative normal velocity across slit, $\underline{u}_{j} \cdot \underline{n}_{j} / U_{\infty}$, at different phases during actuation cycle, for $\operatorname{Re}_{c}=5.5 \cdot 10^{5}, \mathrm{M}_{\infty}=0.146, \alpha=0^{\circ}, x_{j} / c=$ $0.88, w_{j} / c=1 / 165, d_{j} / c=2 / 165, \theta_{j}=90^{\circ}, F^{+}=20, c_{\mu} \approx 0.01, \bar{\rho}_{j} / \rho_{\infty} \approx 1$, $\left(\left(U_{n} w_{\text {cav }}\right) /\left(U_{\infty} w_{j}\right)=1.5\right)$. 


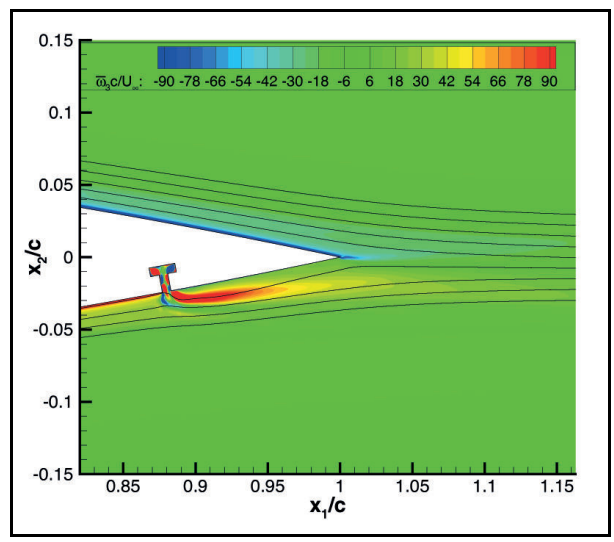

(a) $c_{\mu} \approx 0.006$

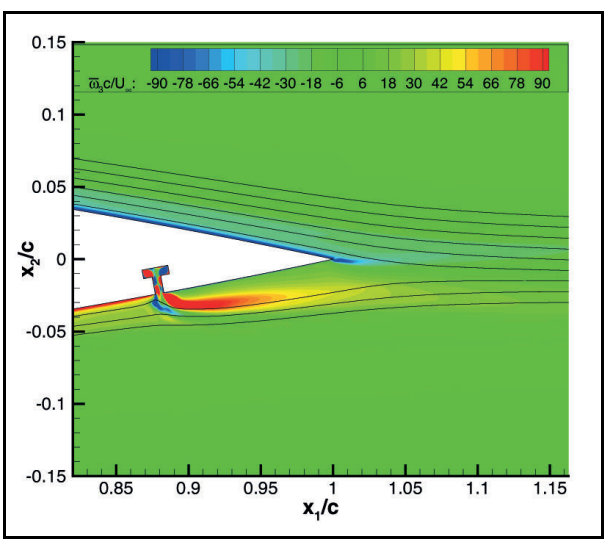

(b) $c_{\mu} \approx 0.01$

Figure 6.44: Streamlines and iso-contours of time-averaged dimensionless spanwise vorticity, $\bar{\omega}_{3} c / U_{\infty}$, for two values of the momentum coefficient, $c_{\mu}, \operatorname{Re}_{c}=5.5 \cdot 10^{5}$, $\mathrm{M}_{\infty}=0.146, \alpha=0^{\circ}, x_{j} / c=0.88, w_{j} / c=1 / 165, d_{j} / c=2 / 165, \theta_{j}=90^{\circ}, F^{+}=20$, $\bar{\rho}_{j} / \rho_{\infty} \approx 1$.

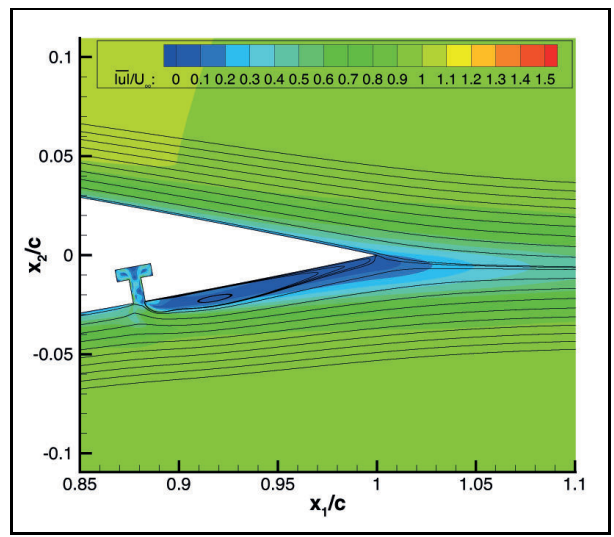

(a) $c_{\mu} \approx 0.006$

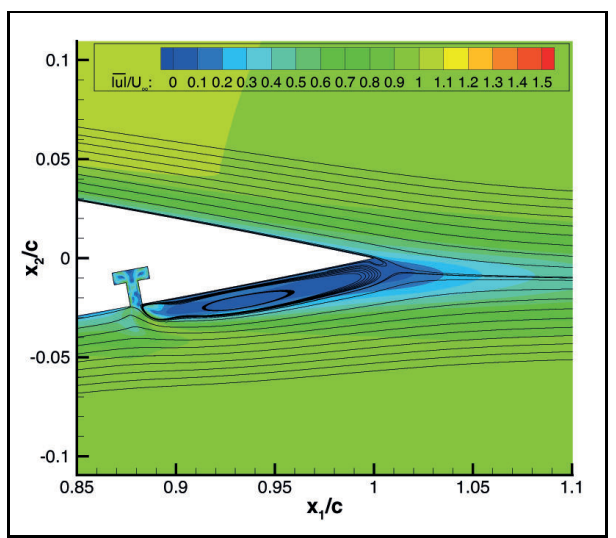

(b) $c_{\mu} \approx 0.01$

Figure 6.45: Streamlines and iso-contours of time-averaged dimensionless velocity magnitude, $\overline{|\underline{u}|} / U_{\infty}$, for two values of the momentum coefficient, $c_{\mu}, \operatorname{Re}_{c}=5.5 \cdot 10^{5}$, $\mathrm{M}_{\infty}=0.146, \alpha=0^{\circ}, x_{j} / c=0.88, w_{j} / c=1 / 165, d_{j} / c=2 / 165, \theta_{j}=90^{\circ}, F^{+}=20$, $\bar{\rho}_{j} / \rho_{\infty} \approx 1$. 
$c_{\mu} \approx 0.01$.

The change in the lift coefficient for the highest investigated momentum coefficient is still quite low: $\overline{\Delta c_{l}} \approx 0.15$. The associated change in angle of attack is approximately $\overline{\Delta \alpha} \approx 1.4^{\circ}$.

\subsubsection{Effect of Dimensionless Actuation Frequency}

This subsection presents the results regarding the effect of the dimensionless actuation frequency, $F^{+}$, on the aerodynamic performance. For this purpose, the applied actuation frequency for the inflow/outflow boundary condition at the bottom of the cavity has been varied. The employed values are $F^{+}=\{1,5,10,20,30\}$. Several different values for $c_{\mu}$ have been used. The other investigated parameters are kept constant at $\alpha=0^{\circ}$, $x_{j} / c=0.88$.

Figure 6.46(a) shows the results of the time-averaged change in the lift coefficient, $\overline{\Delta c_{l}}$ as a function of $c_{\mu}$, for different values of $F^{+}$. Figure 6.46 (b) shows this dependency for $\overline{\Delta c_{d}}$. The following observations can be made with respect to these results:

- For $F^{+}=1$ and $c_{\mu} \approx 0.004$, there is a small negative change in the lift coefficient. In contrast to $\overline{\Delta c_{l}}$, this case has the highest value of $\overline{\Delta c_{d}}$ of all investigated cases.

- For $F^{+}=5, \overline{\Delta c_{l}}$ is positive but very small. Furthermore, for the higher value of $c_{\mu}$, $\overline{\Delta c_{l}}$ is somewhat lower than for the lower value of $c_{\mu}$. However, $\overline{\Delta c_{d}}$ does increase for the higher momentum coefficient, although the overall level is lower than for $F^{+}=1$.

- For $F^{+}=10, \overline{\Delta c_{l}}$ and $\overline{\Delta c_{d}}$ increase at a much higher rate than for $F^{+}=20$. Around $c_{\mu} \approx 0.006$, the change in the lift coefficient for $F^{+}=10$ is a factor 1.6 higher than that for $F^{+}=20$.

- For $F^{+}=30$, the jet power coefficient, $c_{p w r}$, has increased considerably, without the expected associated increase of $c_{\mu}$. Furthermore, the performance in terms of $\overline{\Delta c_{l}}$ is low, even for the present value of $c_{\mu}$. Thus, this case is associated with a high energy consumption, without an appreciable increase in performance.

- The maximum time-averaged change in the lift coefficient of the investigated cases shown in figure 6.46 is $\overline{\Delta c_{l}} \approx 0.192$, which is obtained for $F^{+}=10$ and $c_{\mu}=$ $0.0077\left(\left(U_{n} w_{\text {cav }}\right) /\left(U_{\infty} w_{j}\right)=1.65\right)$. The associated change in angle of attack is approximately $\overline{\Delta \alpha} \approx 1.75^{\circ}$.

The response of the change in the lift coefficient in time, for different actuation frequencies and a momentum coefficient of $c_{\mu} \approx 0.004$ is shown in figure 6.47. The response is plotted as a function of dimensionless time, $U_{\infty} t / c$, during one dimensionless time unit in the periodic part of the simulations. For each case shown here, the difference between the maximum and minimum value, i.e. $\Delta c_{l, \max }-\Delta c_{l, \min }$, is large with respect to the time-averaged change in the lift coefficient, $\overline{\Delta c_{l}}$. For $F^{+} \geq 5$, the dominant frequency component in the response is the actuation frequency, whereas for $F^{+}=1$ 


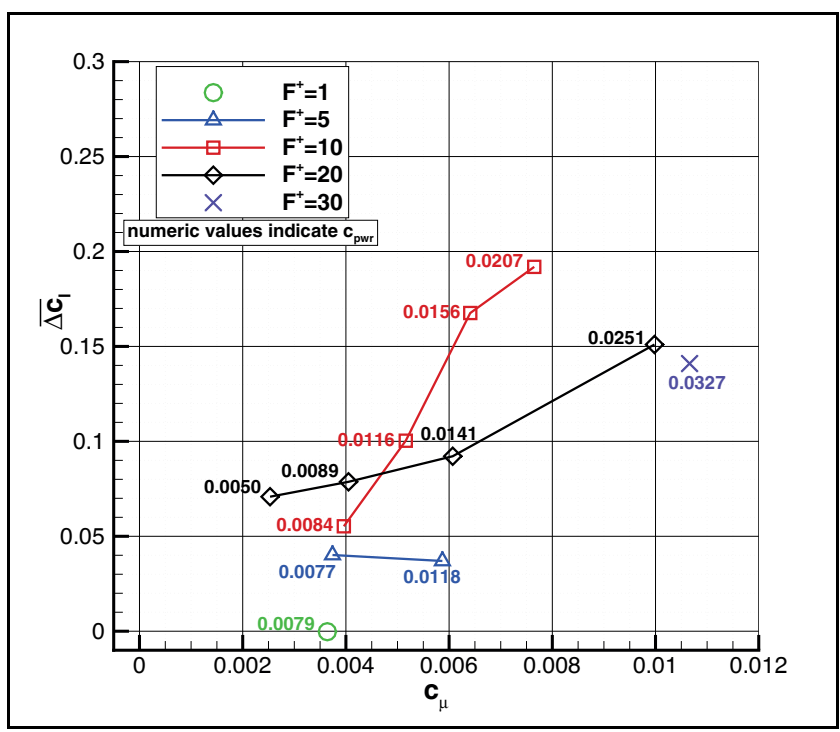

(a) $\overline{\Delta c_{l}}$

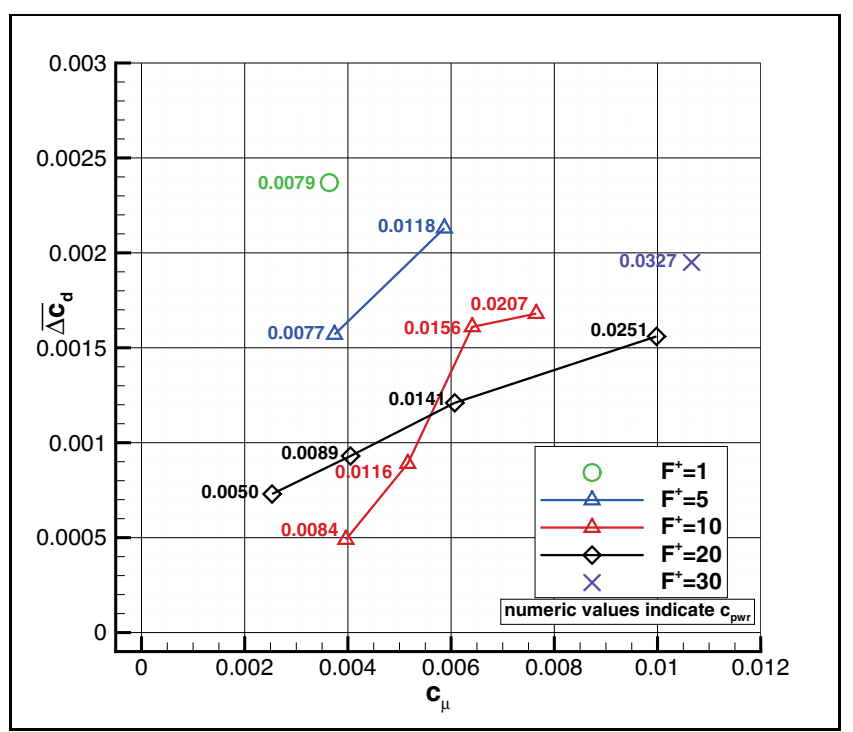

(b) $\overline{\Delta c_{d}}$

Figure 6.46: Time-averaged change of lift coefficient, $\overline{\Delta c_{l}}$, and time-averaged change of drag coefficient, $\overline{\Delta c_{d}}$, as a function of momentum coefficient, $c_{\mu}$, for different dimensionless actuation frequencies, $F^{+}, \operatorname{Re}_{c}=5.5 \cdot 10^{5}, \mathrm{M}_{\infty}=0.146, \alpha=0^{\circ}, x_{j} / c=0.88$, $w_{j} / c=1 / 165, d_{j} / c=2 / 165, \theta_{j}=90^{\circ}, \bar{\rho}_{j} / \rho_{\infty} \approx 1$. For each simulation, the jet power coefficient, $c_{\text {pwr }}$, is indicated. 
there are more dominant frequencies involved. This explains the reason for using 400 time steps per actuation cycle for the case of $F^{+}=1$. For the other cases, 80 time steps per cycle have been used. The highest amplitude for the high-frequency oscillation is obtained for $F^{+}=10$, also at higher momentum coefficients (not shown here).

Figure 6.48 shows streamlines and iso-contours of the dimensionless streamwise vorticity, $\omega_{3} c / U_{\infty}$, for four different dimensionless actuation frequencies at a phase of $180^{\circ}$ in the actuation cycle. The momentum coefficient is $c_{\mu} \approx 0.004$ for all cases. The results show that with increasing $F^{+}$, the spanwise vortices are more closely spaced and become smaller in size. For $F^{+}=1$ it appears that there are two vortices with a positive sign that have been ejected from the slit. However, these two vortices start out as one vortex, which later breaks up in two parts due to the effect of the vorticity with a negative sign above it. For the two lower dimensionless actuation frequencies, the vortices are too far apart to effectively form a quasi-steady obstruction to the flow along the lower side of the airfoil. For $F^{+}=10$, the vortices are still separated, but nevertheless are able to form an obstruction at this phase in the actuation cycle. For $F^{+}=20$, the vortices follow each other so closely that they are effectively joined. At this momentum coefficient, the change in the lift coefficient is higher for $F^{+}=20$ than for $F^{+}=10$.

For a momentum coefficient of $c_{\mu} \approx 0.006$, the performance for $F^{+}=10$ has increased above the performance for $F^{+}=20$, see figure 6.46. This can also be observed in figure 6.49 which shows streamlines and iso-contours of the dimensionless streamwise vorticity, $\omega_{3} c / U_{\infty}$, for a phase of $180^{\circ}$, but now at the higher momentum coefficient of $c_{\mu} \approx 0.006$. The results for $F^{+}=10$ and $F^{+}=20$ are shown. It can be seen that for $F^{+}=10$, the vortices are being ejected further into the flow field along the lower side of the airfoil. The obstruction to the cross-flow is therefore larger, as is the associated increase in circulation around the airfoil. When comparing the case of $F^{+}=10$ and $c_{\mu} \approx 0.006$ with the case of $F^{+}=10$ and $c_{\mu} \approx 0.004$, shown in figure $6.48(\mathrm{c})$, the differences in the shape of the ejected vortices and their distance to the surface of the airfoil are clearly visible. Figure 6.50 shows the differences in the time-averaged velocity field in the trailing edge region for these cases. With increasing momentum coefficient, the recirculation zone between the slit and the trailing edge becomes larger, in a time-averaged sense. A remarkable change in the size of the recirculation zone is obtained for $F^{+}=10$ :

- For the lower momentum coefficient the extent of the recirculation zone is only $0.03 c$ along the surface and the maximum displacement of the flow along the surface is approximately $0.007 c$.

- For the higher momentum coefficient, the recirculation zone extends all the way to the trailing edge and the maximum displacement of the flow along the surface is approximately $0.02 c$, from $x_{1} / c=0.95$ to the trailing edge.

The increase in the size of the recirculation zone with increasing momentum coefficient is much more gradual for $F^{+}=20$, which explains the lower rate of change of $\overline{\Delta c_{l}}$ with respect to $c_{\mu}$ at this actuation frequency.

Concluding, a dimensionless frequency of at least $F^{+}=10$ is necessary to generate positive-signed vortices that are close enough to each other to effectively form a 


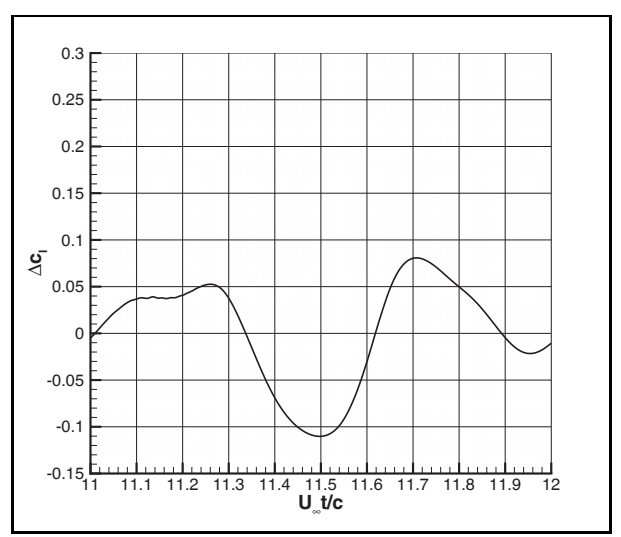

(a) $F^{+}=1$

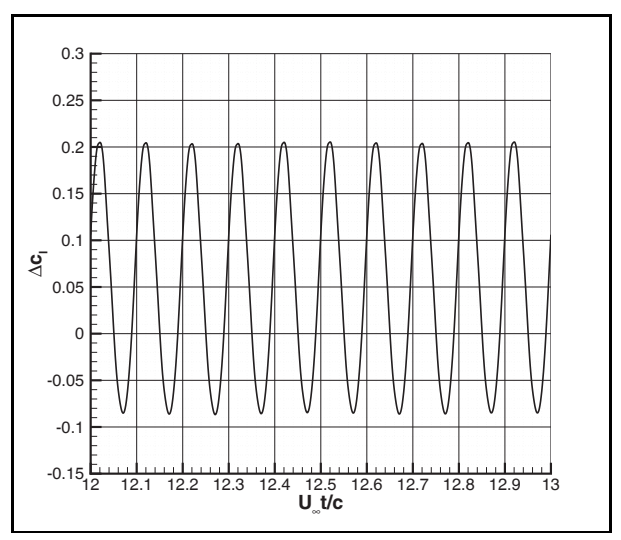

(c) $F^{+}=10$

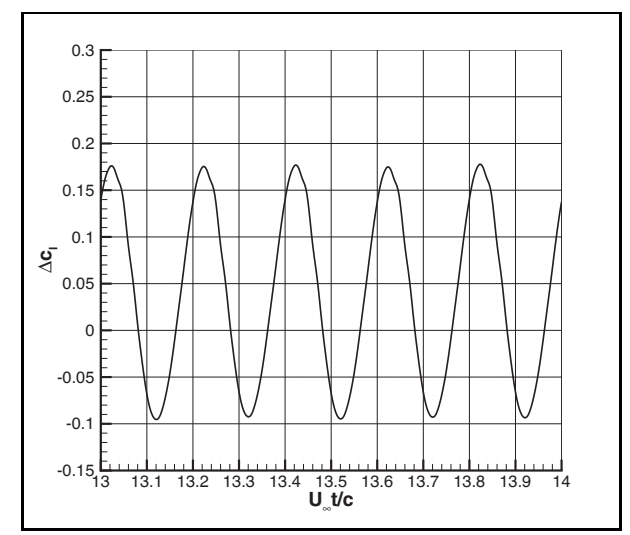

(b) $F^{+}=5$

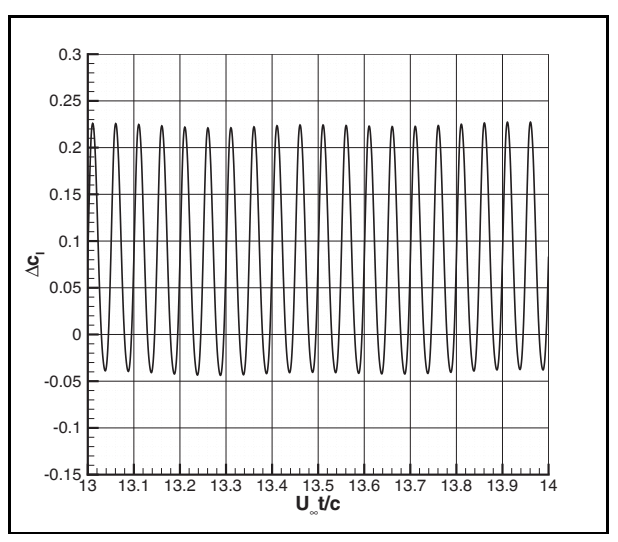

(d) $F^{+}=20$

Figure 6.47: Change of lift coefficient, $\Delta c_{l}$, as a function of dimensionless time, $U_{\infty} t / c$, for different dimensionless actuation frequencies, $F^{+}, \operatorname{Re}_{c}=5.5 \cdot 10^{5}, \mathrm{M}_{\infty}=0.146$, $\alpha=0^{\circ}, x_{j} / c=0.88, w_{j} / c=1 / 165, d_{j} / c=2 / 165, \theta_{j}=90^{\circ}, c_{\mu} \approx 0.004, \bar{\rho}_{j} / \rho_{\infty} \approx 1$. 


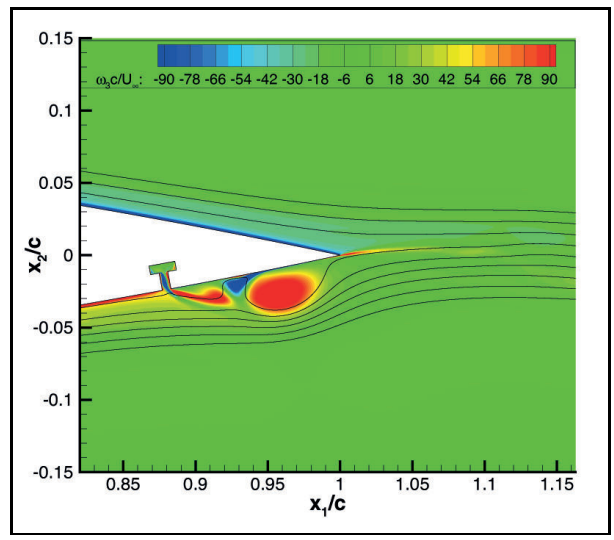

(a) $F^{+}=1$

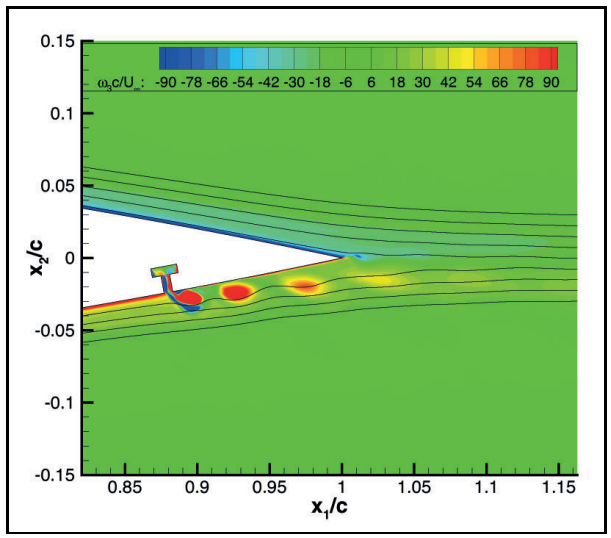

(c) $F^{+}=10$

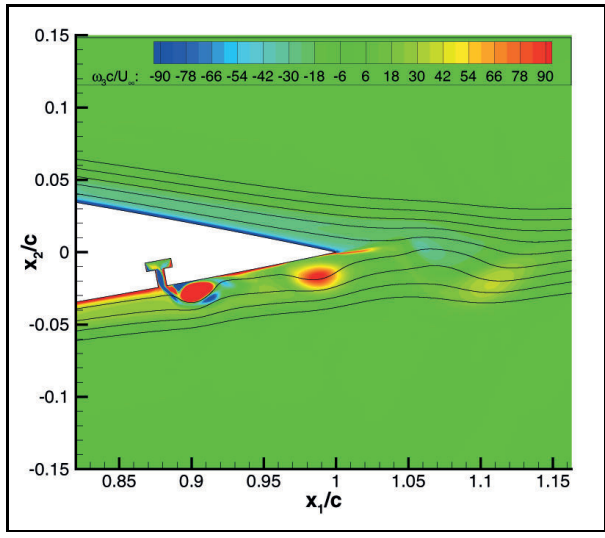

(b) $F^{+}=5$

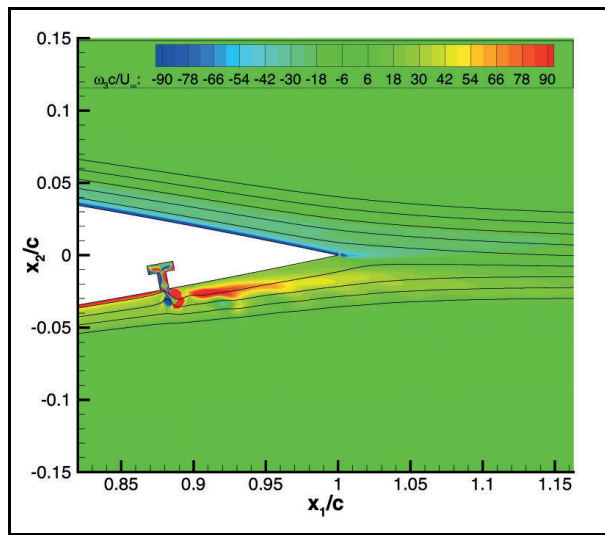

(d) $F^{+}=20$

Figure 6.48: Streamlines and iso-contours of dimensionless spanwise vorticity, $\omega_{3} c / U_{\infty}$, at a phase of $180^{\circ}$, for different dimensionless actuation frequencies, $F^{+}, \operatorname{Re}_{c}=5.5 \cdot 10^{5}$, $\mathrm{M}_{\infty}=0.146, \alpha=0^{\circ}, x_{j} / c=0.88, w_{j} / c=1 / 165, d_{j} / c=2 / 165, \theta_{j}=90^{\circ}, c_{\mu} \approx 0.004$, $\bar{\rho}_{j} / \rho_{\infty} \approx 1$. 


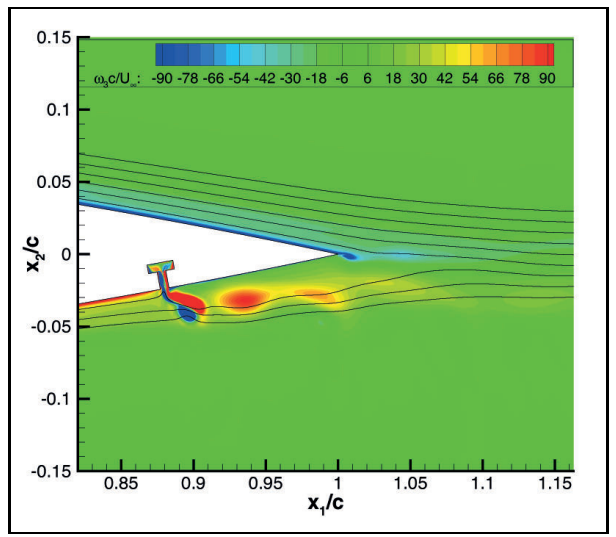

(a) $F^{+}=10$

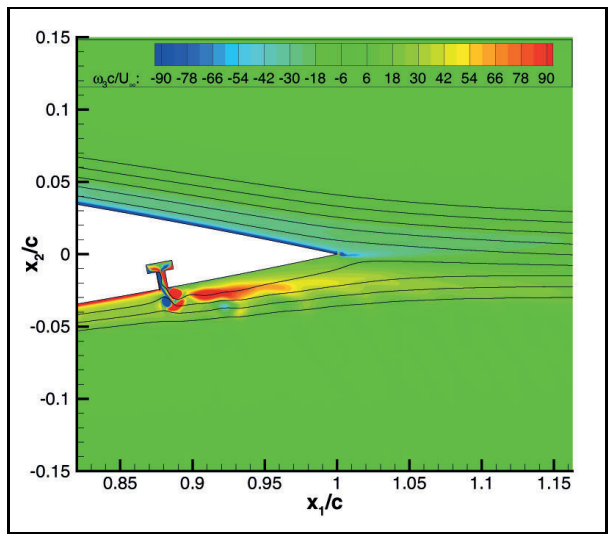

(b) $F^{+}=20$

Figure 6.49: Streamlines and iso-contours of dimensionless spanwise vorticity, $\omega_{3} c / U_{\infty}$, at a phase of $180^{\circ}$, for different dimensionless actuation frequencies, $F^{+}, \operatorname{Re}_{c}=5.5 \cdot 10^{5}$, $\mathrm{M}_{\infty}=0.146, \alpha=0^{\circ}, x_{j} / c=0.88, w_{j} / c=1 / 165, d_{j} / c=2 / 165, \theta_{j}=90^{\circ}, c_{\mu} \approx 0.006$, $\bar{\rho}_{j} / \rho_{\infty} \approx 1$.

quasi-steady obstruction to the flow along the lower side of the airfoil. However, for a dimensionless frequency of $F^{+}=30$, much energy is consumed, without an appreciable increase in performance. Based on the present investigation, dimensionless frequencies in the range of $10 \leq F^{+}<30$ are optimal.

\subsubsection{Effect of Slit Location}

The effect of locating the slit further downstream, i.e. closer to the trailing edge, can be seen in figure 6.51. This figure shows the time-averaged change of lift coefficient, $\overline{\Delta c_{l}}$, and time-averaged change of drag coefficient, $\overline{\Delta c_{d}}$, as a function of momentum coefficient, $c_{\mu}$, for $F^{+}=\{10,20\}$ and two different slit locations, $x_{j} / c=0.88$ and $x_{j} / c=0.95$. It is observed that for $x_{j} / c=0.95$, the performance increases considerably in comparison with that for the location of $x_{j} / c=0.88$ and the same momentum coefficient. Maximum changes of the lift coefficient of $\overline{\Delta c_{l}} \approx 0.22$ can be obtained, which corresponds to an effective change of the angle of attack of $\overline{\Delta \alpha} \approx 2^{\circ}$. Furthermore, the accompanying changes in the drag coefficient are lower for $x_{j} / c=0.95$.

Figure 6.52 shows different distributions of the time-averaged pressure coefficient, $\overline{c_{p}}$, along the upper and lower surface of the airfoil for $F^{+}=20$. Time averaging has been performed over one actuation cycle in the periodic part of the simulations. The case for $x_{j} / c=0.88$ and $c_{\mu}=0.0061$, which was discussed in subsection 6.8.3, is shown here with a solid line. The two other results differ from this result with respect to the location of the slit and the value of the momentum coefficient: 


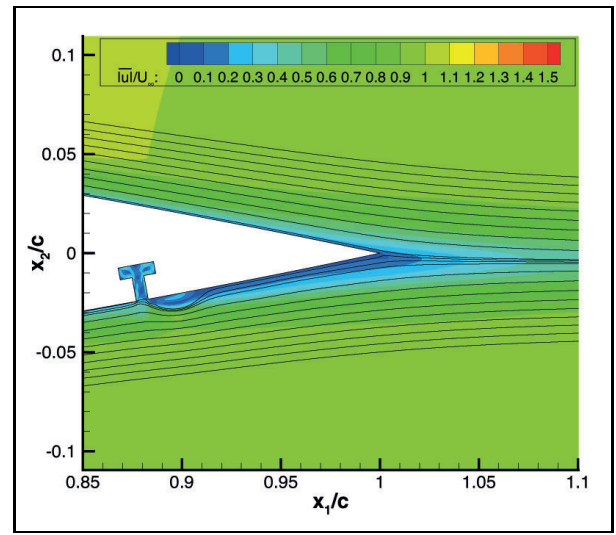

(a) $F^{+}=10, c_{\mu} \approx 0.004$

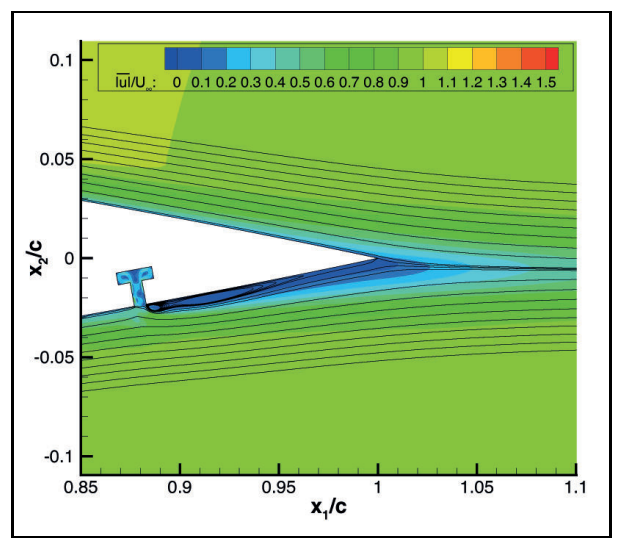

(c) $F^{+}=20, c_{\mu} \approx 0.004$

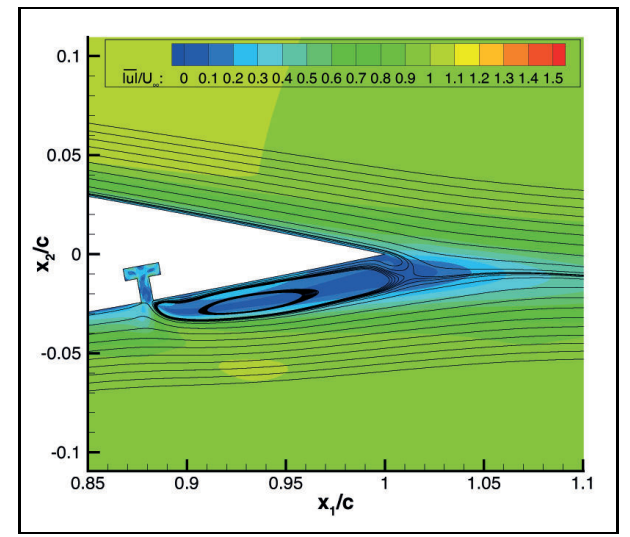

(b) $F^{+}=10, c_{\mu} \approx 0.006$

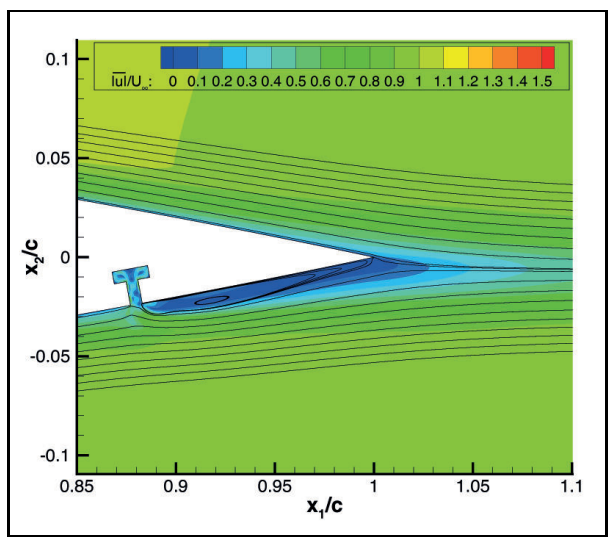

(d) $F^{+}=20, c_{\mu} \approx 0.006$

Figure 6.50: Streamlines and iso-contours of time-averaged dimensionless absolute velocity, $\overline{|\underline{u}|} / U_{\infty}$, for $F^{+}=\{10,20\}$ and two values of the momentum coefficient, $c_{\mu}$, $\operatorname{Re}_{c}=5.5 \cdot 10^{5}, \mathrm{M}_{\infty}=0.146, \alpha=0^{\circ}, x_{j} / c=0.88, w_{j} / c=1 / 165, d_{j} / c=2 / 165$, $\theta_{j}=90^{\circ}, \bar{\rho}_{j} / \rho_{\infty} \approx 1$.

- The dashed line shows the effect of locating the slit closer to the trailing edge, in this case $x_{j} / c=0.95$, while keeping the momentum coefficient approximately constant. The change in the lift coefficient is $\overline{\Delta c_{l}} \approx 0.159$ for this location of the slit, which is a factor 1.77 higher than that for $x_{j} / c=0.88$. It can be seen that the pressure difference between the upper and the lower surface of the airfoil increases for almost the entire range of chordwise locations. Furthermore, since the distance between the slit and the trailing edge is smaller, the negative contribution to the 


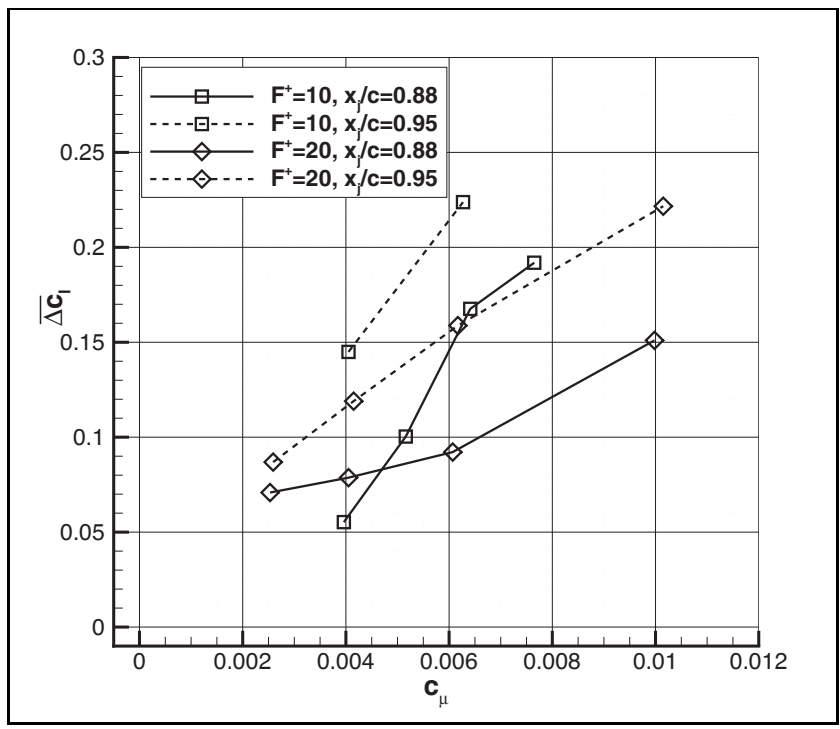

(a) $\overline{\Delta c_{l}}$

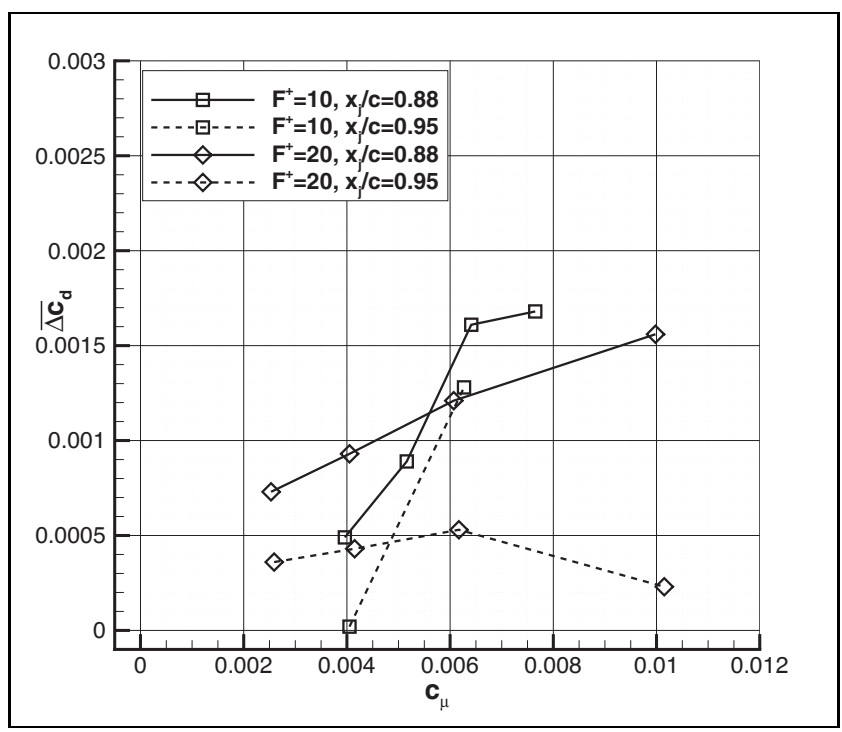

(b) $\overline{\Delta c_{d}}$

Figure 6.51: Time-averaged change of lift coefficient, $\overline{\Delta c_{l}}$, and time-averaged change of drag coefficient, $\overline{\Delta c_{d}}$, as a function of momentum coefficient, $c_{\mu}$, for different dimensionless actuation frequencies, $F^{+}$, and different slit locations, $x_{j} / c, \operatorname{Re}_{c}=5.5 \cdot 10^{5}$, $\mathrm{M}_{\infty}=0.146, \alpha=0^{\circ}, x_{j} / c=0.88, w_{j} / c=1 / 165, d_{j} / c=2 / 165, \theta_{j}=90^{\circ}, \bar{\rho}_{j} / \rho_{\infty} \approx 1$. 


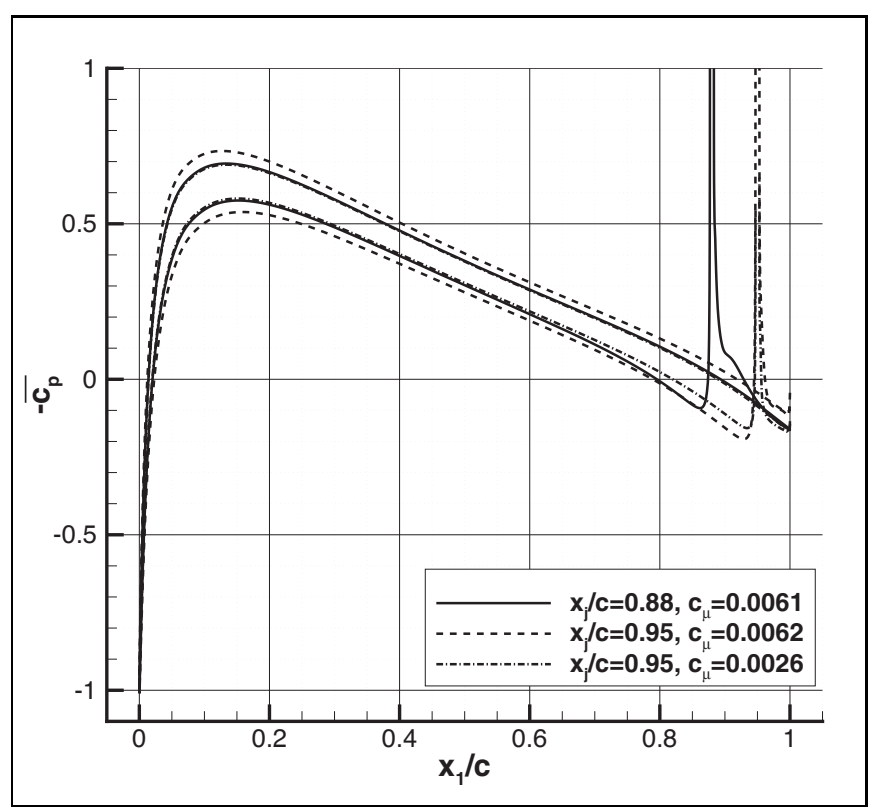

Figure 6.52: Time-averaged pressure coefficient, $\overline{c_{p}}$, along the upper and lower side of the airfoil for different locations of the slit, $x_{j} / c$, and different momentum coefficients, $c_{\mu}, \operatorname{Re}_{c}=5.5 \cdot 10^{5}, \mathrm{M}_{\infty}=0.146, \alpha=0^{\circ}, w_{j} / c=1 / 165, d_{j} / c=2 / 165, \theta_{j}=90^{\circ}$, $F^{+}=20, \bar{\rho}_{j} / \rho_{\infty} \approx 1$.

overall change in lift, with which this region is associated, is also smaller. Also note that the pressure level at the trailing edge is lower on both sides of the airfoil for the case of $x_{j} / c=0.95$.

- The dash-dotted line shows the result for $x_{j} / c=0.95$ and $c_{\mu}=0.0026$, which is associated with approximately the same change in the lift coefficient as the case for $x_{j} / c=0.88$ and $c_{\mu}=0.0061$, see figure [6.46] The pressure distribution on the upper and lower surface is only slightly different from that for the case of $x_{j} / c=0.88$ and $c_{\mu}=0.0061$, except in the region where the slits are located. At the trailing edge, the pressure level is approximately equal for both cases. The power coefficient for $x_{j} / c=0.95$ and $c_{\mu}=0.0026$ is $c_{p w r}=0.0050$, a reduction of $64.5 \%$ with respect to the case for $x_{j} / c=0.88$ and $c_{\mu}=0.0061$.

The flow field for the two locations of the slit, $x_{j} / c=0.88$ and $x_{j} / c=0.95$, are compared in figures 6.53 and 6.54 Both cases have the same dimensionless actuation frequency and momentum coefficient: $F^{+}=20$ and $c_{\mu} \approx 0.006$.

Figure 6.53 shows the dimensionless spanwise vorticity, $\omega_{3} c / U_{\infty}$, at a phase of $180^{\circ}$. At this phase, the vortices that have just been ejected out of the slit essentially have the same shape and size for both locations of the slit. However, the direction in which 


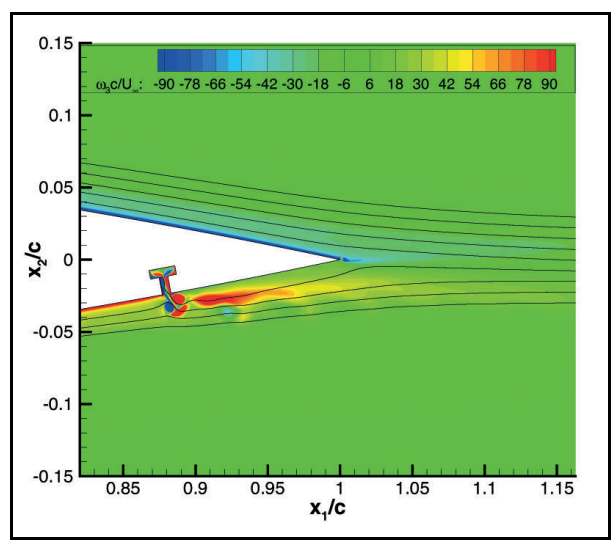

(a) $x_{j} / c=0.88$

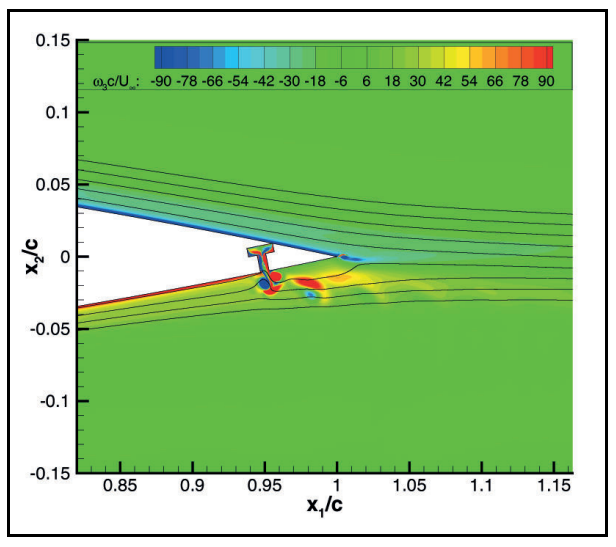

(b) $x_{j} / c=0.95$

Figure 6.53: Streamlines and iso-contours of dimensionless spanwise vorticity, $\omega_{3} c / U_{\infty}$, at a phase of $180^{\circ}$, for different locations of the slit, $x_{j} / c, \operatorname{Re}_{c}=5.5 \cdot 10^{5}, \mathrm{M}_{\infty}=0.146$, $\alpha=0^{\circ}, w_{j} / c=1 / 165, d_{j} / c=2 / 165, \theta_{j}=90^{\circ}, F^{+}=20, c_{\mu} \approx 0.006, \bar{\rho}_{j} / \rho_{\infty} \approx 1$.

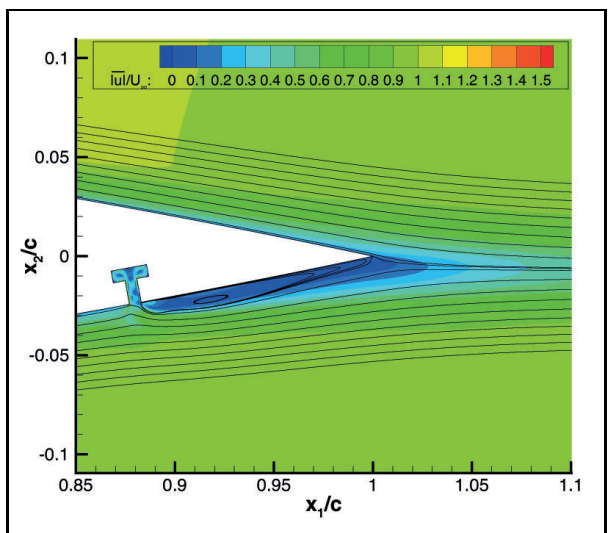

(a) $x_{j} / c=0.88$

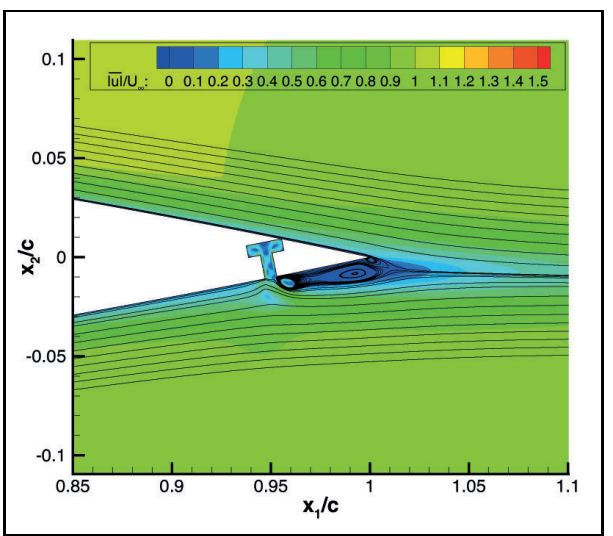

(b) $x_{j} / c=0.95$

Figure 6.54: Streamlines and iso-contours of time-averaged dimensionless velocity magnitude, $\overline{|\underline{u}|} / U_{\infty}$, for different locations of the slit, $x_{j} / c, \operatorname{Re}_{c}=5.5 \cdot 10^{5}, \mathrm{M}_{\infty}=0.146$, $\alpha=0^{\circ}, w_{j} / c=1 / 165, d_{j} / c=2 / 165, \theta_{j}=90^{\circ}, F^{+}=20, c_{\mu} \approx 0.006, \bar{\rho}_{j} / \rho_{\infty} \approx 1$. 


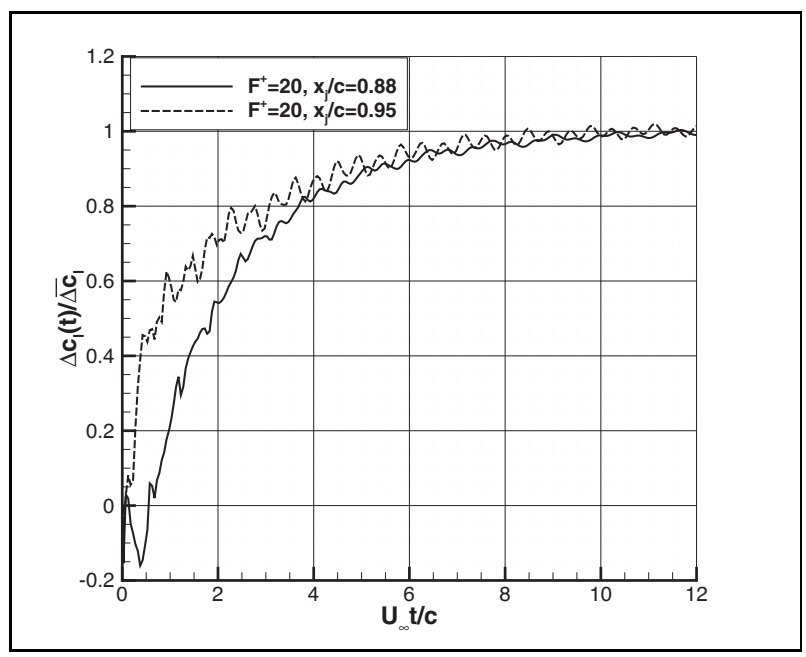

(a) $x_{j} / c=0.88$ and $x_{j} / c=0.95$ for $F^{+}=20$

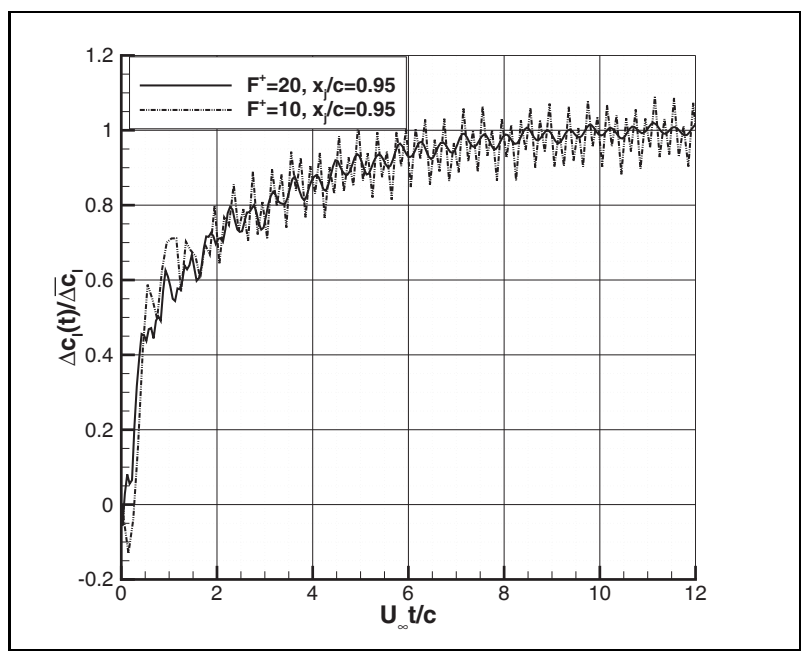

(b) $F^{+}=10$ and $F^{+}=20$ for $x_{j} / c=0.95$

Figure 6.55: Filtered relative change of lift coefficient, $\Delta c_{l} / \overline{\Delta c_{l}}$, as a function of dimensionless time, $U_{\infty} t / c$, for two different locations of the slit, $x_{j} / c$, and two different dimensionless actuation frequencies, $F^{+}, \operatorname{Re}_{c}=5.5 \cdot 10^{5}, \mathrm{M}_{\infty}=0.146, \alpha=0^{\circ}, w_{j} / c=1 / 165$, $d_{j} / c=2 / 165, \theta_{j}=90^{\circ}, c_{\mu} \approx 0.006, \bar{\rho}_{j} / \rho_{\infty} \approx 1$. 
they are moving is different: for $x_{j} / c=0.88$ they are tilted more in the direction of the cross-flow than for the case of $x_{j} / c=0.95$.

Figure 6.54 shows streamlines and iso-contours of the time-averaged dimensionless velocity magnitude, $\overline{|\underline{u}|} / U_{\infty}$. As before, time-averaging has been performed over one actuation cycle in the periodic part of the simulations. It is clear that for $x_{j} / c=0.95$, the synthetic jet is better able to invoke a displacement of the flow along the lower side of the airfoil, in a time-averaged sense. Furthermore, the synthetic jet is also able to entrain low-momentum fluid from the upper side of the airfoil into the recirculation region on the lower side. The maximum displacement occurs at the trailing edge and equals $0.013 c$. Note that this displacement is smaller than the displacement obtained for $F^{+}=10, x_{j} / c=0.88$ and the same momentum coefficient (see figure 6.50(b)). However, the change in the lift coefficient is approximately the same for both cases (see figure 6.51). This is because the recirculation region is longer for $F^{+}=10, x_{j} / c=0.88$ and $c_{\mu} \approx 0.006$, such that the main flow along the lower side is already moving more or less parallel to the lower side of the airfoil by the time it has reached the trailing edge, see figure 6.50 (b). This is not the case for $F^{+}=20, x_{j} / c=0.95$ and $c_{\mu} \approx 0.006$. Here, the main flow along the lower side is directed more parallel to the chord line when it has reached the trailing edge, in a time-averaged sense. Therefore, the displacement obtained for $x_{j} / c=0.95$ is more effective in generating additional circulation around the airfoil.

For a dimensionless frequency of $F^{+}=20$, momentum coefficient of $c_{\mu} \approx 0.006$ and slit location of $x_{j} / c=0.88$, an undesirable initial negative peak in the time-dependent response of the change in the lift coefficient has been observed. This behavior is plotted again in figure 6.55(a), which shows the filtered relative change in the lift coefficient, $\Delta c_{l} / \overline{\Delta c_{l}}$, as a function of $U_{\infty} t / c$. Note that $\overline{\Delta c_{l}}$ is the time-averaged change in the periodic part of the simulation. As before, the filtered signals show an average value for each actuation cycle. The response for the same actuation frequency and momentum coefficient, but for a slit location of $x_{j} / c=0.95$ is shown as a dashed line. At this location, the initial reverse peak in $\Delta c_{l}$ is not present and $50 \%$ of the final change in the lift coefficient is already obtained within $c / U_{\infty}$ instead of $2 c / U_{\infty}$ for $x_{j} / c=0.88$.

In figure 6.55(b) the filtered relative change in the lift coefficient for $F^{+}=20$, $c_{\mu} \approx 0.006$ and $x_{j} / c=0.95$ is compared with that for $F^{+}=10, c_{\mu} \approx 0.006$ and $x_{j} / c=0.95$. Thus, the only difference is the dimensionless actuation frequency. It can be seen that the response for both actuation frequencies is equally fast. However, the response for $F^{+}=10$ does have a small initial negative peak in the filtered change in the lift coefficient, and the signal has stronger oscillations.

Summarizing, a slit location closer to the trailing edge is preferred, due to the increased performance for the same momentum coefficient and the shorter aerodynamic response time. Effective changes in the angle of attack of $\overline{\Delta \alpha} \approx 2^{\circ}$ can be obtained for a slit location of $x_{j} / c=0.95$.

The next subsection, which discusses the effect of the angle of attack on the performance, also includes one simulation with synthetic jet actuation even closer to the trailing edge: $x_{j} / c=0.985$. It will be shown that the trend of increasing performance for slit locations closer to the trailing edge continues. 


\subsubsection{Effect of Angle of Attack}

For pitch control, synthetic jet actuation must be able to generate changes of the lift coefficient over a range of angles of attack. So far, results have been presented for $\alpha=0$ degrees. Due to the long turnaround times of the flow simulations with actuation, it has not been possible to include a range of angles of attack in the parameter study. Instead, one other angle of attack has been selected, $\alpha=9^{\circ}$, which is close to the angle of attack at which the lift-to-drag ratio is maximal. This can be observed in figure 6.56 which shows computational results of the lift coefficient, $c_{l}$, drag coefficient, $c_{d}$, and the liftto-drag ratio, $l / d$, for a NACA0018 airfoil at different angles of attack, $\operatorname{Re}_{c}=5.5 \cdot 10^{5}$, $\mathrm{M}_{\infty}=0.146$, but without the presence of wind tunnel walls. The inclusion of $\alpha=9^{\circ}$ will provide some insight on whether the changes of the lift coefficient that are observed at $\alpha=0^{\circ}$ can also be expected at other angles of attack.

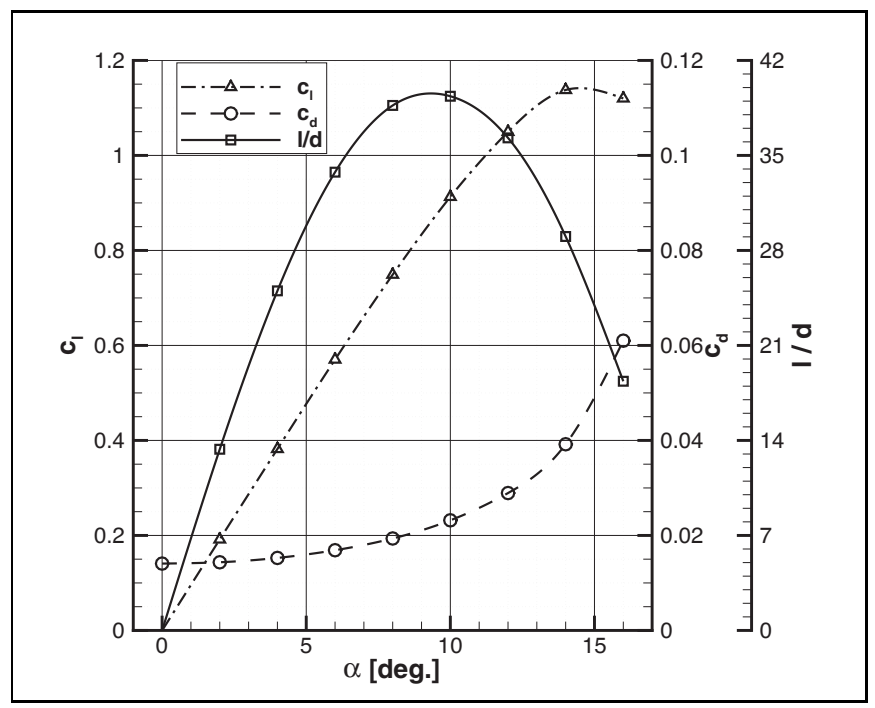

Figure 6.56: Lift coefficient, $c_{l}$, drag coefficient, $c_{d}$, and the lift-to-drag ratio, $l / d$, for a NACA0018 airfoil at different angles of attack, $\alpha, \operatorname{Re}_{c}=5.5 \cdot 10^{5}, \mathrm{M}_{\infty}=0.146$. Each symbol represents a computational result, calculated using SST turbulence model with active sustaining terms, without wind tunnel walls.

The simulations for $\alpha=9^{\circ}$ include two dimensionless actuation frequencies, $F^{+}=10$ and $F^{+}=20$. Three locations of the slit are investigated: $x_{j} / c=0.88, x_{j} / c=0.95$ and finally $x_{j} / c=0.985$. Furthermore, we have focussed on momentum coefficients in the region $c_{\mu} \in[0.006,0.01]$.

Before presenting the results for the changes in the lift and drag coefficients, the results for the dimensionless actuation frequency of $F^{+}=10$ are discussed. All simulations with synthetic jet actuation at $\alpha=9^{\circ}$ and a dimensionless actuation frequency of $F^{+}=10$ show no significant changes of the lift coefficient for all considered locations of the slit, i.e. 


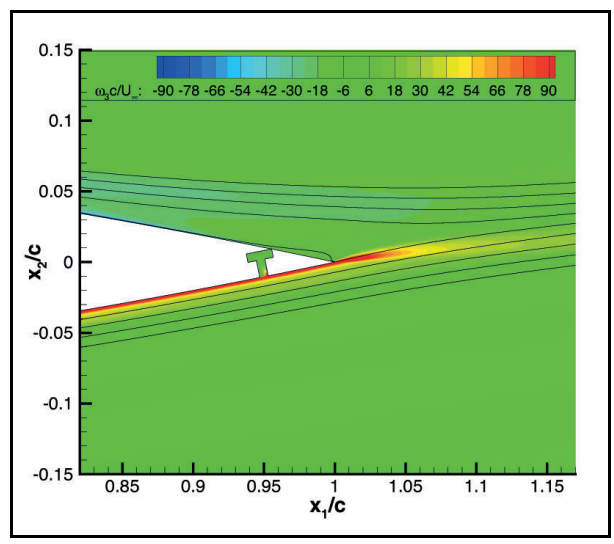

(a) baseline without actuation

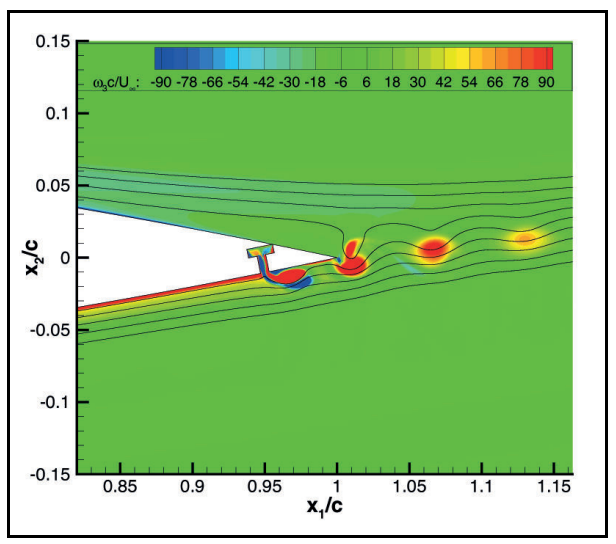

(b) $F^{+}=10, c_{\mu} \approx 0.006, \bar{\rho}_{j} / \rho_{\infty} \approx 1$

Figure 6.57: Streamlines and iso-contours of dimensionless spanwise vorticity, $\omega_{3} c / U_{\infty}$, without and with actuation at a phase of $180^{\circ}$, for $\operatorname{Re}_{c}=5.5 \cdot 10^{5}, \mathrm{M}_{\infty}=0.146, \alpha=9^{\circ}$, $x_{j} / c=0.95, w_{j} / c=1 / 165, d_{j} / c=2 / 165, \theta_{j}=90^{\circ}$.

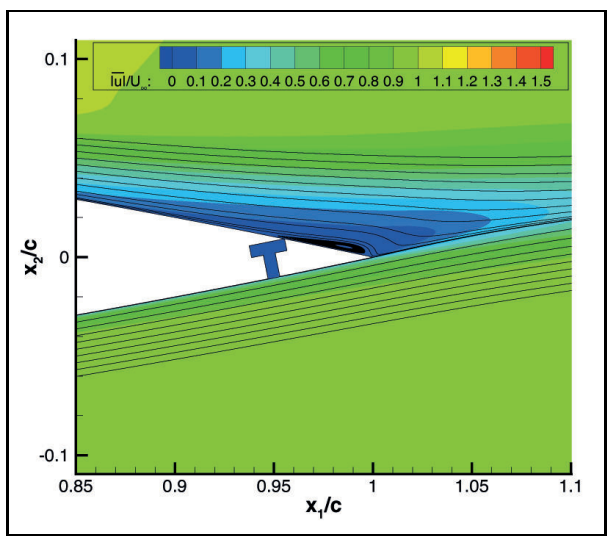

(a) baseline without actuation

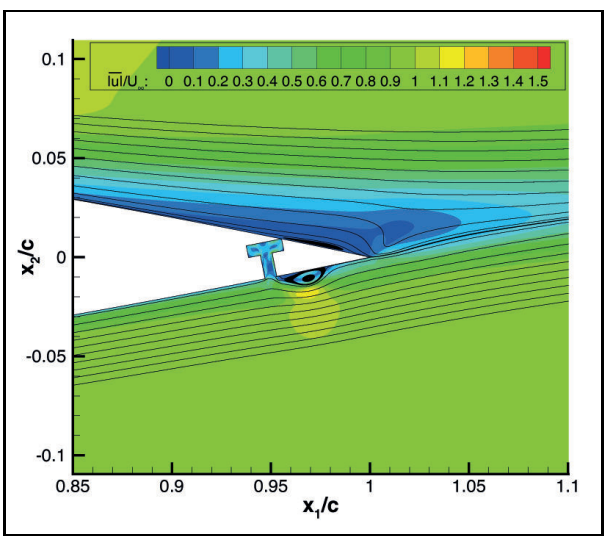

(b) $F^{+}=10, c_{\mu} \approx 0.006, \bar{\rho}_{j} / \rho_{\infty} \approx 1$

Figure 6.58: Streamlines and iso-contours of time-averaged dimensionless velocity magnitude, $\overline{|\underline{u}|} / U_{\infty}$, with and without actuation, for $\operatorname{Re}_{c}=5.5 \cdot 10^{5}, \mathrm{M}_{\infty}=0.146, \alpha=9^{\circ}$, $x_{j} / c=0.95, w_{j} / c=1 / 165, d_{j} / c=2 / 165, \theta_{j}=90^{\circ}$. 
$x_{j} / c=0.88, x_{j} / c=0.95$ and $x_{j} / c=0.985$. This is surprising, since the performance at $\alpha=0^{\circ}$ is quite good for the same actuation frequency and momentum coefficient. In figures 6.57 and 6.58 it can be observed 3 that for $F^{+}=10, c_{\mu} \approx 0.006$ and $x_{j} / c=0.95$, the generated vortices are not ejected far into the flow along the lower side of the airfoil. In a time-averaged sense, the synthetic jet is not able to generate a substantial recirculation region that extends to the trailing edge. The associated time- and space-averaged ejection velocity of the jet is $\bar{U}_{j}=1.05 U_{\infty}$, and the power coefficient is $c_{p w r}=0.015(\bar{P}=185$ $\mathrm{W} / \mathrm{m}$ on this scale).

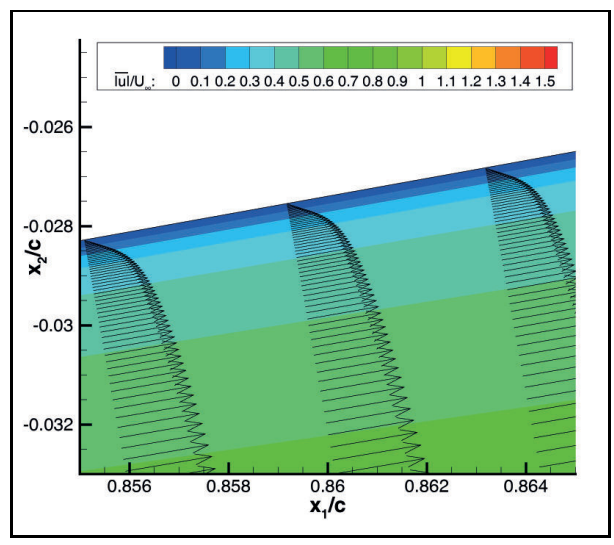

(a) $\alpha=0^{\circ}$

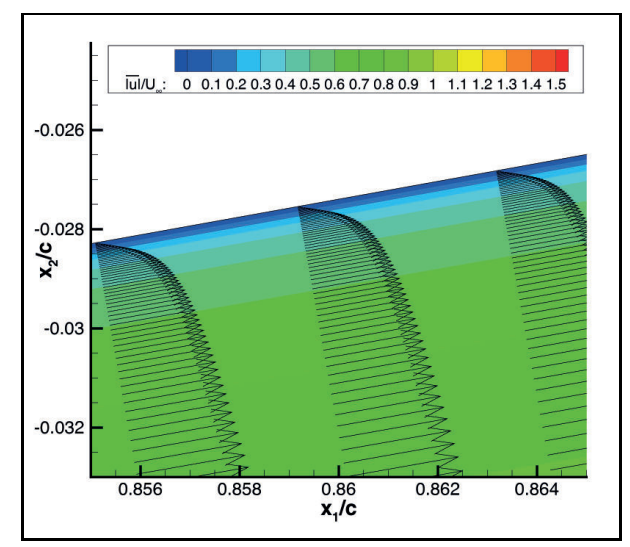

(b) $\alpha=9^{\circ}$

Figure 6.59: Velocity vectors and iso-contours of dimensionless absolute velocity, $|\underline{u}| / U_{\infty}$, without actuation, for $\operatorname{Re}_{c}=5.5 \cdot 10^{5}, \mathrm{M}_{\infty}=0.146, x_{j} / c=0.95, w_{j} / c=1 / 165$, $d_{j} / c=2 / 165, \theta_{j}=90^{\circ}$.

The reason for the lower performance at this angle of attack is the difference in the boundary layer of the cross-flow encountered by the synthetic jet. This can be observed in figure 6.59, which shows velocity vectors and iso-contours of dimensionless velocity magnitude, $|\underline{u}| / U_{\infty}$, in the boundary layer region upstream of the location of the slit $\left(x_{j} / c=0.95\right)$. Results are shown for $\alpha=0^{\circ}$ and $\alpha=9^{\circ}$, both without actuation. It is clear that the boundary layer thickness along the lower side of the airfoil is smaller for $\alpha=9^{\circ}$. Thus, there is more momentum in the cross-flow close to the wall. This means that when the momentum coefficient of the synthetic jet is kept constant, the displacement of the flow along the lower side of the airfoil is smaller for $\alpha=9^{\circ}$ than for $\alpha=0^{\circ}$. It is expected that the performance will increase for higher momentum coefficients. However, a simulation for $c_{\mu}=0.0116$, which corresponds to $\bar{U}_{j}=1.42 U_{\infty}$ and $c_{p w r}=0.037(\bar{P}=458 \mathrm{~W} / \mathrm{m}$ on this scale), does not show any improvement yet. As discussed previously, it is expected that still higher values of $c_{\mu}$ are not realistic.

\footnotetext{
${ }^{3}$ In the figures for $\alpha=9^{\circ}$, the coordinate system has been rotated such that the $x_{1}$-axis is directed along the chord line, and the $x_{2}$-axis is directed perpendicular to this line.
} 


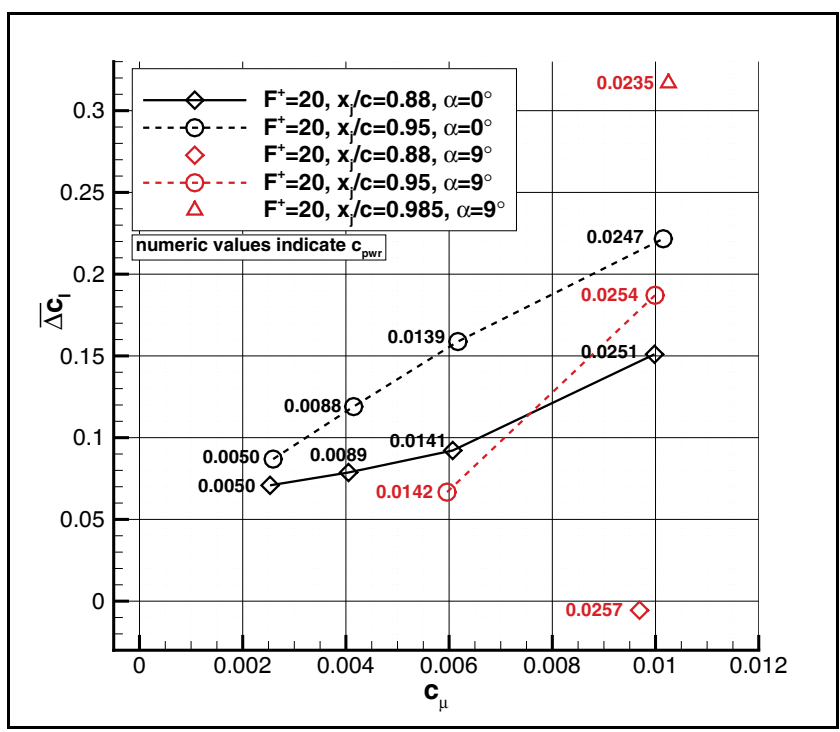

(a) $\overline{\Delta c_{l}}$

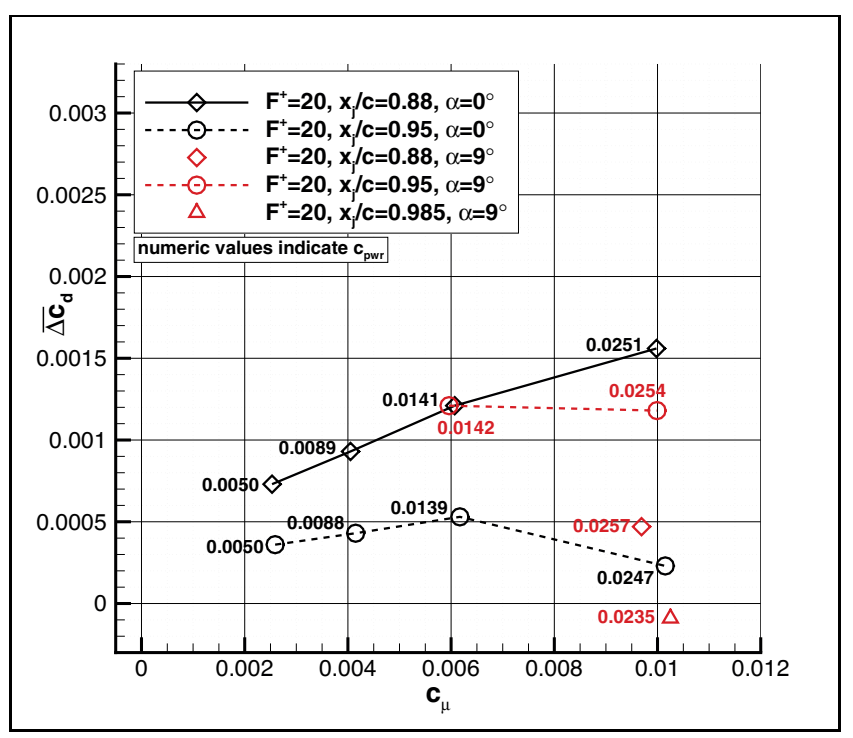

(b) $\overline{\Delta c_{d}}$

Figure 6.60: Time-averaged change of lift coefficient, $\overline{\Delta c_{l}}$, and time-averaged change of drag coefficient, $\overline{\Delta c_{d}}$, as a function of momentum coefficient, $c_{\mu}$, for different angles of attack, $\alpha$, and different locations of the slit, $x_{j} / c, \operatorname{Re}_{c}=5.5 \cdot 10^{5}, \mathrm{M}_{\infty}=0.146$, $w_{j} / c=1 / 165, d_{j} / c=2 / 165, \theta_{j}=90^{\circ}, F^{+}=20, \bar{\rho}_{j} / \rho_{\infty} \approx 1$. For each simulation, the jet power coefficient, $c_{\text {pwr }}$, is indicated. 
In the remainder of this subsection, we will focus on a dimensionless actuation frequency of $F^{+}=20$, at which the synthetic jet is able to generate changes in the lift coefficient. This can be seen in figure, 6.60, which presents the results of the timeaveraged changes in the lift and drag coefficients in the periodic part of the results of the simulations. The following observations can be made with respect to these results:

- For a constant momentum coefficient and constant location of the slit, the change in the lift coefficient for $\alpha=9^{\circ}$ is lower than that for $\alpha=0^{\circ}$. This is expected, as outlined above.

* For a slit location of $x_{j} / c=0.88$ and a momentum coefficient of $c_{\mu} \approx 0.01$, a small negative change in the lift coefficient is obtained.

* For a slit location of $x_{j} / c=0.95$ and a momentum coefficient of $c_{\mu} \approx$ 0.01 , the change in the lift coefficient is $\overline{\Delta c_{l}}=0.187$. This corresponds to an effective change in the angle of attack of $\overline{\Delta \alpha} \approx 1.7^{\circ}$, which is $15.6 \%$ lower than the value obtained for $\alpha=0^{\circ}$ and the same actuation parameters. Furthermore, based on the two simulations performed for $x_{j} / c=0.95$, the rate of change of $\overline{\Delta c_{l}}$ with respect to the momentum coefficient has increased, compared to the rate of change obtained for $\alpha=0^{\circ}$ and the same actuation parameters. The accompanying change in the drag coefficient is slightly higher for $\alpha=9^{\circ}$.

- As already observed in subsection 6.8.6, the performance increases for slit locations closer to the trailing edge of the airfoil. For a slit location of $x_{j} / c=0.985$ and a momentum coefficient of $c_{\mu} \approx 0.01$, associated with a space- and time-averaged ejection velocity of $\bar{U}_{j}=1.32 U_{\infty}$, the change in the lift coefficient is $\overline{\Delta c_{l}}=0.317$. This corresponds to an effective change in the angle of attack of $\overline{\Delta \alpha} \approx 2.9^{\circ}$, which is close to the minimum performance deemed necessary for smart rotor control on wind turbine blades. Note that the accompanying change in the drag coefficient is very small (and has a negative sign, i.e. a favorable effect).

Figure 6.61 shows the time-averaged pressure coefficient, $\overline{c_{p}}$, for $\alpha=9, F^{+}=20$, $c_{\mu} \approx 0.01$ and the three locations of the slit considered here. The baseline result without actuation is also included. For $x_{j} / c=0.88$, the pressure distribution for the case with actuation differs from the baseline case only in the region around the slit. The small negative change in the lift coefficient and the small positive change in the drag coefficient result from the low pressure region downstream of the slit. For the two other locations of the slit, the pressure difference between the upper and the lower surface of the airfoil increases for almost the entire range of chordwise locations. Clearly, actuation closer to the trailing edge directly affects the circulation of the airfoil. This was also observed previously for $\alpha=0^{\circ}$.

The associated flow fields in the region around the trailing edge are shown in figures 6.62 and 6.63. It is clear that synthetic jet actuation for $x_{j} / c=0.88$ generates a recirculation region, but it does not extend to the trailing edge. For $x_{j} / c=0.95$, the recirculation region does extend to the trailing edge, but the displacement of the flow along 


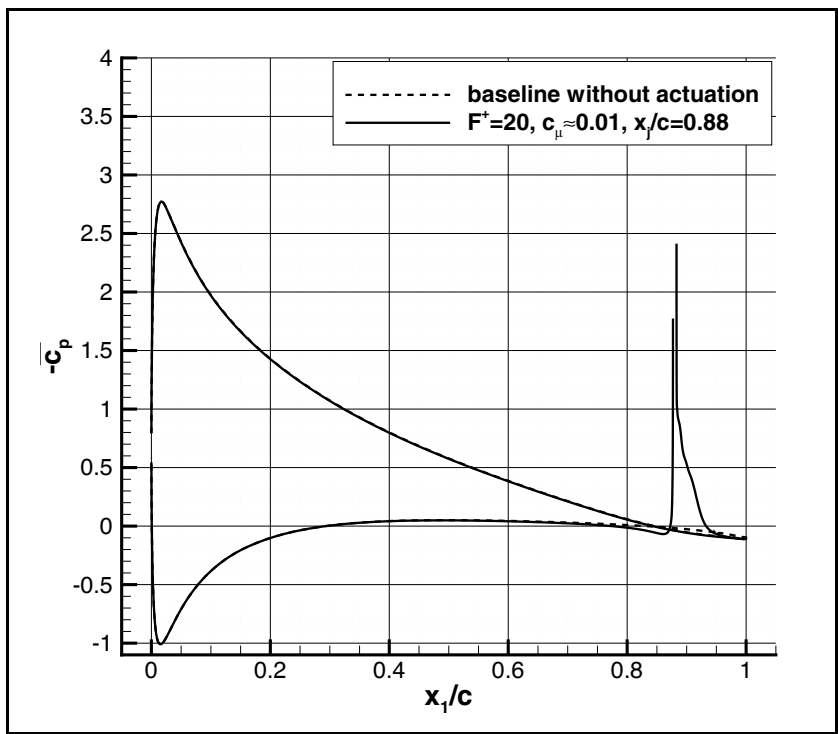

(a) $x_{j} / c=0.88$

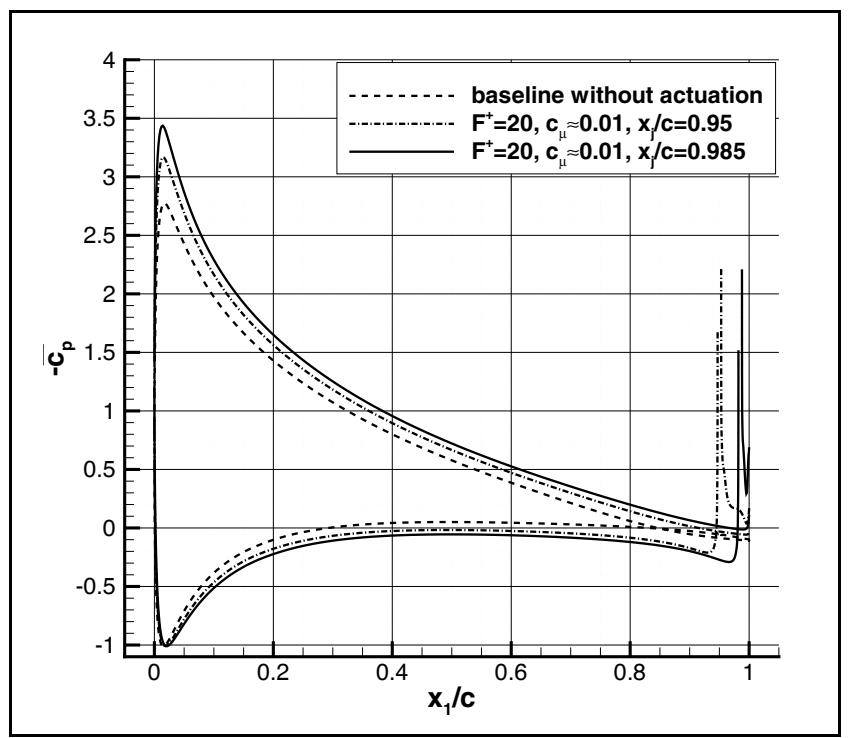

(b) $x_{j} / c=0.95$ and $x_{j} / c=0.985$

Figure 6.61: Time-averaged pressure coefficient, $\overline{c_{p}}$, along the upper and lower side of the airfoil, for different locations of the slit, $x_{j} / c, \operatorname{Re}_{c}=5.5 \cdot 10^{5}, \mathrm{M}_{\infty}=0.146, \alpha=9^{\circ}$, $w_{j} / c=1 / 165, d_{j} / c=2 / 165, \theta_{j}=90^{\circ}, F^{+}=20, c_{\mu} \approx 0.01, \bar{\rho}_{j} / \rho_{\infty} \approx 1$. 


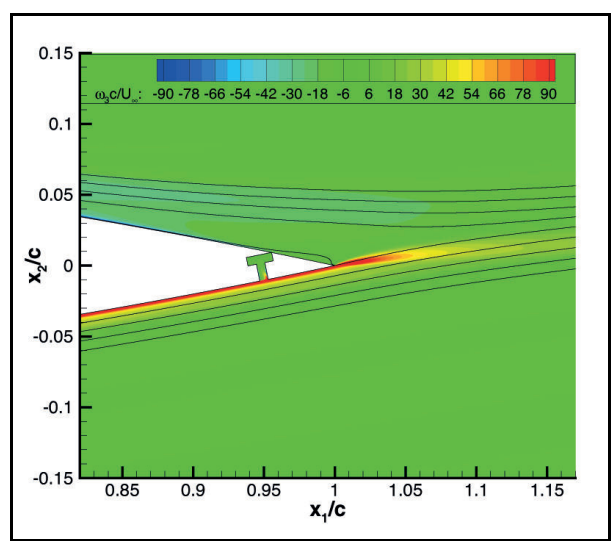

(a) baseline without actuation $\left(x_{j} / c=0.95\right)$

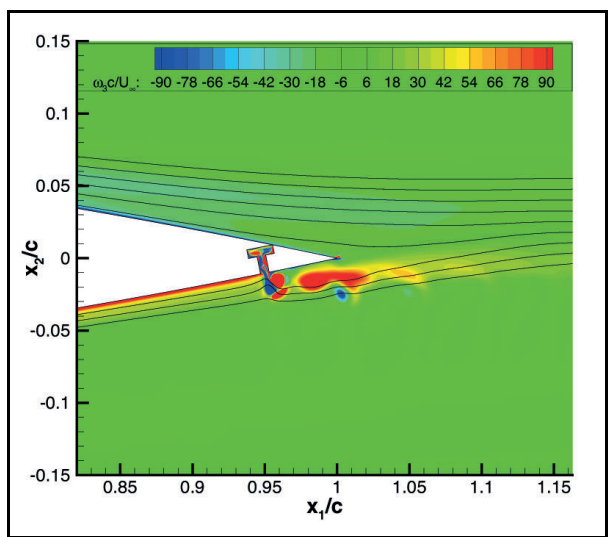

(c) $x_{j} / c=0.95$

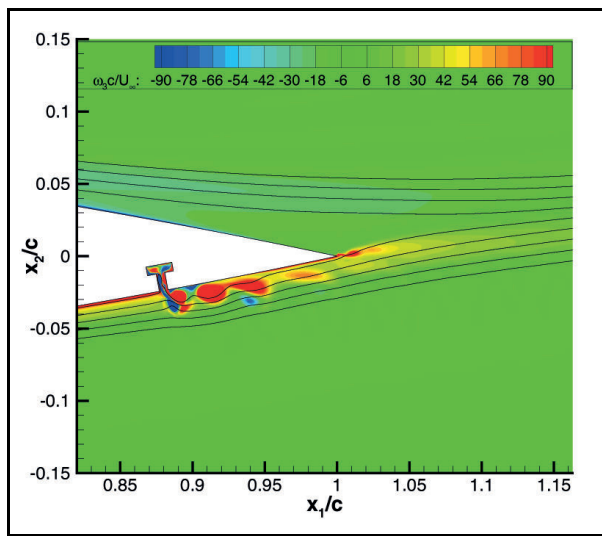

(b) $x_{j} / c=0.88$

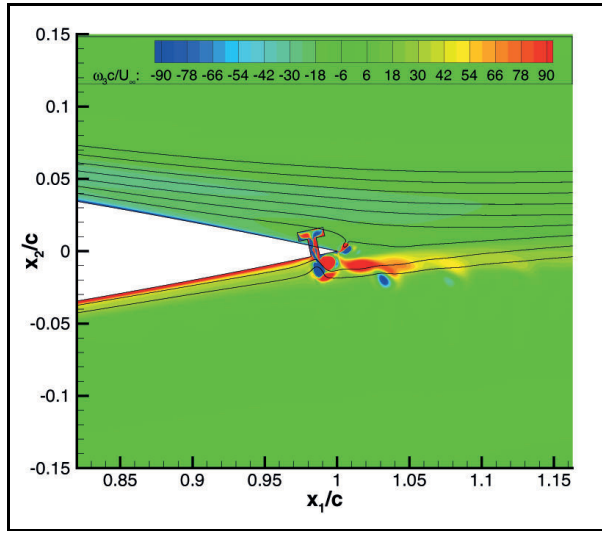

(d) $x_{j} / c=0.985$

Figure 6.62: Streamlines and iso-contours of dimensionless spanwise vorticity, $\omega_{3} c / U_{\infty}$, without and with actuation at a phase of $180^{\circ}$, for different locations of the slit, $x_{j} / c$, $\operatorname{Re}_{c}=5.5 \cdot 10^{5}, \mathrm{M}_{\infty}=0.146, \alpha=9^{\circ}, w_{j} / c=1 / 165, d_{j} / c=2 / 165, \theta_{j}=90^{\circ}$, $F^{+}=20, c_{\mu} \approx 0.01, \bar{\rho}_{j} / \rho_{\infty} \approx 1$. 


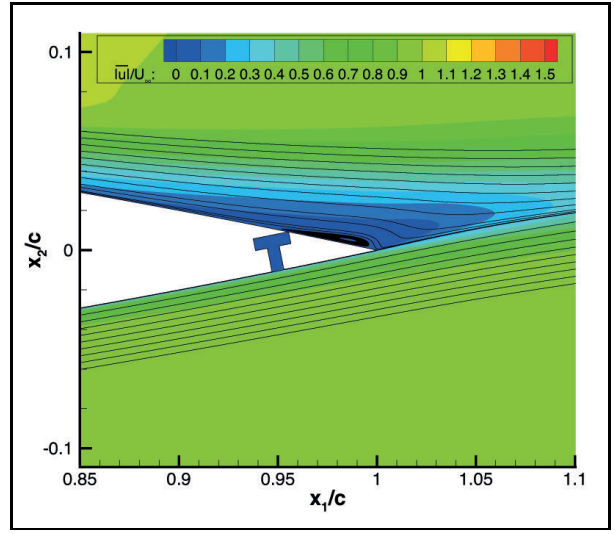

(a) baseline without actuation $\left(x_{j} / c=0.95\right)$

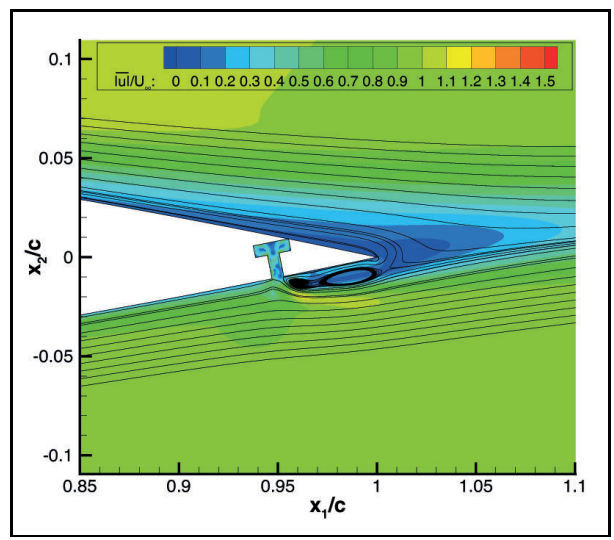

(c) $x_{j} / c=0.95$

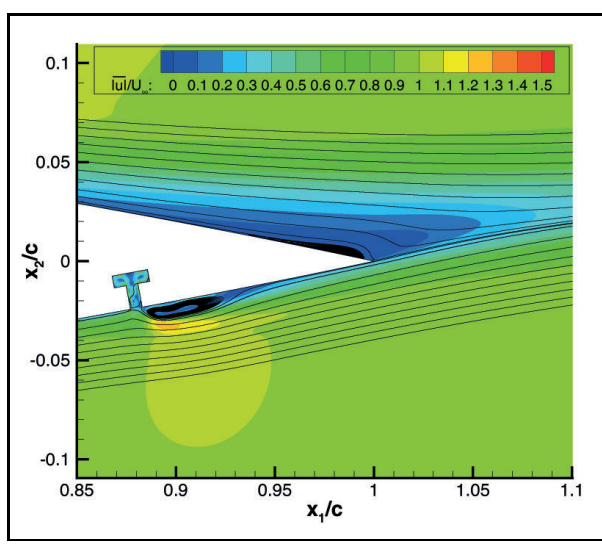

(b) $x_{j} / c=0.88$

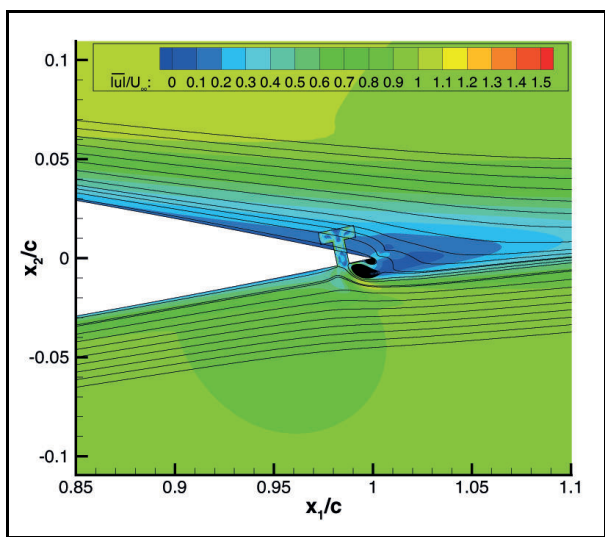

(d) $x_{j} / c=0.985$

Figure 6.63: Streamlines and iso-contours of time-averaged dimensionless velocity magnitude, $\overline{|\underline{u}|} / U_{\infty}$, without and with actuation for different locations of the slit, $x_{j} / c$, $\operatorname{Re}_{c}=5.5 \cdot 10^{5}, \mathrm{M}_{\infty}=0.146, \alpha=9^{\circ}, w_{j} / c=1 / 165, d_{j} / c=2 / 165, \theta_{j}=90^{\circ}$, $F^{+}=20, c_{\mu} \approx 0.01, \bar{\rho}_{j} / \rho_{\infty} \approx 1$. 
the lower side of the airfoil is not as large as for $\alpha=0^{\circ}$ and the same slit location and actuation parameters. Note that the slit and the cavity for $x_{j} / c=0.985$ partly overlap with the flow domain above the upper side of the airfoil. In the numerical simulations this does not cause interference. However, it is clear that for the actual application of a synthetic jet at this location, a different design of the geometry of the trailing edge is needed.

\subsubsection{Discussion}

It has been shown in the preceding subsections that high-frequency synthetic jet actuation through a long spanwise slit near the trailing edge of an airfoil is associated with relatively large fluctuations in the response of the lift and drag coefficients superposed on the time-averaged changes of these coefficients. This is a result of the pressure fluctuations caused by the action of the synthetic jet and is associated with noise emission. For the application of smart rotor control on wind turbine blades, the important structural resonance modes of the blades and other components of the wind turbine should not be excited. Therefore, higher dimensionless frequencies of the order of $F^{+}=10$ or higher are more desirable. This frequency also is the minimum frequency necessary to generate positive-signed vortices that are close enough to each other to effectively form a quasisteady obstruction to the flow along the lower side of the airfoil and therewith provide extra (aft) camber. However, for a dimensionless frequency of $F^{+}=30$, much energy is consumed, without an appreciable increase in performance.

The performance is higher for slit locations closer to the trailing edge, in terms of the achieved change in the lift coefficient and in terms of the aerodynamic response time. Furthermore, the accompanying change in the drag coefficient is smaller for slit locations closer to the trailing edge.

The results for $\alpha=9^{\circ}$ show that the performance decreases for higher angles of attack, due to the smaller thickness of the boundary layer along the pressure side of the airfoil, just upstream of the location of the slit. This is associated with a higher momentum in the cross-flow close to the wall, which reduces the ability of the synthetic jet to form an obstruction to the main flow along the surface of the airfoil.

The results of the flow simulations for $\alpha=0^{\circ}$ show that actuation at a dimensionless frequency of $F^{+}=10$ yields the highest performance in terms of changes in the lift coefficient, $\Delta c_{l}$. However, for $\alpha=9^{\circ}$, hardly any change in the lift coefficient is observed for a reasonable range of momentum coefficients. This is also the case for $F^{+}=20$ and $x_{j} / c=0.88$ at $\alpha=9^{\circ}$.

The case that shows the best performance of all investigated cases in terms of the change in the lift coefficient corresponds to $F^{+}=20, c_{\mu} \approx 0.01$ and $x_{j} / c=0.985$. This case yields a change in the lift coefficient of $\overline{\Delta c_{l}}=0.317$ at $\alpha=9^{\circ}$, which corresponds to an effective change of the angle of attack of $\overline{\Delta \alpha} \approx 2.9^{\circ}$. The achieved performance is very close to the minimum performance deemed necessary for smart rotor control on wind turbine blades. The accompanying change in the drag coefficient is very small (and is favorable). This is an advantage of (synthetic) jets in comparison with the potential 
options of a trailing edge flap or a micro-tab, which cause larger increases in drag.

The power consumption coefficient associated with the case that yields the best aerodynamic performance is $c_{p w r}=0.0235$. To assess the implications of this power consumption for a large wind turbine, we will again consider the NREL $5 \mathrm{MW}$ reference offshore wind turbine [25]. This wind turbine has a blade radius of $R=63 \mathrm{~m}$ and a rated rotor speed of 12.1 rotations per minute, i.e. $\Omega=1.27 \mathrm{rad} / \mathrm{s}$ and the tip speed is $80 \mathrm{~m} / \mathrm{s}$. Using the definition of $c_{p w r}$, see equation 6.9, and $U_{\infty}=U_{\text {eff }} \approx \Omega r$, the average power consumption per blade can be approximated as

- $\bar{P} \approx 9.89 \mathrm{~kW}$ per meter span at $r / R=0.77$, where $c \approx 3 \mathrm{~m}$,

- $\bar{P} \approx 10.2 \mathrm{~kW}$ per meter span at $r / R=1$, where $c=1.419 \mathrm{~m}$,

i.e. $\bar{P} \approx 10 \mathrm{~kW} / \mathrm{m}$ per blade at the outboard blade sections.

Applying synthetic jet actuation along $25 \%$ of the span of each blade simultaneously, leads to a very high power consumption of $9.45 \%$ of the nominal power production of the wind turbine, which is quite substantial. This power consumption appears to be prohibitive. Therefore, before this technology can actually be applied to a wind turbine blade, further study is needed:

- to determine which part of a rotating wind turbine blade has to be provided with synthetic jet actuation in order to achieve the required change in lift,

- to find a less power intensive combination of the parameters involved in highfrequency synthetic jet actuation,

- to determine the effect of distributed actuation, i.e. operating the synthetic jets not simultaneously all the time. 



\section{Chapter 7}

\section{Conclusions And Recommendations}

The technology of smart rotor control is expected to allow wind turbines to increase in size, due to its potential to reduce fatigue inducing load variations on the blades and other components. The aerodynamic effect needed for smart rotor control is 'local pitch control', which can also be employed for other turbomachinery or in wing aerodynamics in the field of aeronautics. In the present research, synthetic jets have been investigated as a potential option for local pitch control, by means of flow simulations complemented with experiments. The next sections present the conclusions of this investigation and the recommendations for future research.

\subsection{Conclusions}

\section{General Aspects of Synthetic Jet Actuation}

The aerodynamic flow around a blade (or blade section) with synthetic jet actuation is characterized by:

- a high Reynolds number based on the chord length, which implies turbulent flow,

- a large range of length and time scales, from very small turbulence scales, to the scales relevant for the wind turbine:

* length scales include scales of turbulent eddies to length scales of the order of the jet width, to length scales of the order of the chord length of the lift-generating object,

* time scales include very small turbulence scales, to time scales of the order of the actuation period, to time scales of the order of $c / U_{\infty}$, i.e. the convective time scale,

- the formation of vortical structures by the synthetic jet, which interact with each other and with the main flow along the blade (or blade section).

A computational method for the simulation of the effects of synthetic jet actuation should account for these aspects. 


\section{Aspects of the Computational Method}

The computational method developed in the present research solves the unsteady Reynoldsaveraged Navier-Stokes (URANS) equations for compressible viscous flow together with the equations of a linear eddy-viscosity turbulence model. With respect to this method, the following conclusions can be drawn.

- In principle, the computational method is able to deal with the aforementioned characteristic flow effects, while keeping computational costs within reasonable bounds, such that a parameter study of synthetic jet actuation for a two-dimensional configuration can be carried out.

- The mathematical models have been correctly implemented, as demonstrated by several verification cases. Although the formal second order convergence with grid size can be difficult to achieve in some cases, all steady-flow cases show good comparison between the present computational method and other, previously verified, computational methods. The temporal discretization has also been verified and it showed a temporal order of convergence close to the expected value of 2 .

- An agglomeration-based algebraic multigrid method and a parallelization method by means of domain decomposition have been implemented, which reduce computational times. Their influence has been illustrated for a steady laminar flow over a flat plate. The results for this particular case show that

* although the multigrid method is applied separately to each grid partition, the number of pseudo-time steps does not increase dramatically with increasing number of grid partitions,

* measured in terms of wall clock time, the algebraic multigrid method is approximately five times faster than the standard Gauß-Seidel iteration method for a reduction of the residuals by five orders of magnitude,

* the parallelization method shows adequate speed-up factors, for grid partitions with at least 10,000 control volumes. When less than 10,000 control volumes per partition are used, the efficiency deteriorates due to the increasing importance of the communication between grid partitions and the use of Jacobi iteration on the boundaries of the grid partitions.

\section{Aspects of Validation}

The degree to which the computational method is able to accurately predict the effect of synthetic jet actuation has been investigated by comparing computational results with experimental results. For boundary layer separation control, a validation study has been performed on the case of the flow over a wall-mounted hump. It can be concluded that:

- the flow simulations predict the correct trend of delayed separation and earlier reattachment of the flow, 
- the effect of flow control is underpredicted in comparison with the experimental results.

The underprediction of the effect of synthetic jet actuation on boundary layer separation control is attibuted to the underprediction of the turbulent shear stress by the employed eddy-viscosity turbulence model of Spalart \& Allmaras (SA). Results from other studies show that the Shear Stress Transport (SST) turbulence model and a non-linear explicit algebraic stress model have the same kind of deficiency, although these models are slightly better in predicting the trends for the effects of actuation frequency and amplitude in the case of synthetic jet actuation.

For pitch control, we have been able to compare results from flow simulations to experimentally obtained surface pressure measurements. The experiments have been carried out in the University of Twente's silent wind tunnel. Due to the low performance (in terms of jet peak velocities) of the actuation system in the experimental setup, actuation could only be performed across half of the total span of the airfoil. Furthermore, comparisons between experiments and simulations have only been possible at relatively low actuation frequencies. It can be concluded that the developed computational method:

- generally adequately predicts the effect of low-frequency synthetic jet actuation on the time-averaged pressure distributions on the upper and lower surface of the airfoil,

- overpredicts the effect of synthetic jet actuation in comparison with the experimental results, especially at the higher investigated angle of attack. It is suspected that due to the actuation being performed over only a part of the span in the experiments, three-dimensional flow effects occur that are not represented in the two-dimensional flow configuration considered in the numerical simulations.

\section{Aspects of Synthetic Jet Modeling}

With respect to the modeling of synthetic jet actuation in the present computational method, it can be concluded that the inclusion of the slit and (part of) the cavity in the computational domain is essential. Without the inclusion of the slit and cavity, it is a challenge to accurately prescribe all the observed time-dependent characteristics of a synthetic jet at the exit of the slit by means of a surface boundary condition. It has been shown that the profile of the normal component of the velocity across the exit of the slit

- has a 'horned' shape during part of the actuation cycle, as has also been observed by other authors, both in experiments and in numerical simulations,

- does not resemble a simple spatially uniform or $\left(\sin ^{2}\right)$-profile, as used in the literature,

- has a time-dependent behavior that cannot be approximated by a sine function.

Furthermore, it can be concluded that compressibility effects cannot be neglected. It has been shown that compressibility effects become more important with increasing 
actuation frequencies and amplitudes. Maximum deviations from the free-stream density of $15 \%$ at the exit of the slit are observed for the present configuration with $F^{+}=30$ and $c_{\mu} \approx 0.01$. Additionally, associated with synthetic jet actuation at high frequencies and with high amplitudes is the generation of strong pressure fluctuations that propagate through the flow field. Solving the equations for compressible flow yields the correct wave speed of these pressure waves.

\section{Parameter Study of Synthetic Jet Actuation for Pitch Control}

Pitch control aims for changes in the aerodynamic characteristics, mainly the lift coefficient, over the range of angles of attack corresponding to the linear $c_{l}(\alpha)$-regime. Based on a parameter study of synthetic jet actuation through long spanwise slits near the trailing edge of a non-rotating airfoil configuration, it can be concluded that synthetic jet actuation is able to generate changes of the lift coefficient in this regime.

It has been shown that for reasonable values of the momentum coefficient, $c_{\mu}$, the response of the lift and drag coefficients in time is associated with relatively large fluctuations superposed on the time-averaged changes of these coefficients.

For very low actuation frequencies, i.e. $F^{+} \ll 1$, the fluctuations of the lift and drag coefficients are a result of a quasi-steady reaction of the flow field to the action of the synthetic jet. For high actuation frequencies, i.e. $F^{+}>1$, the fluctuations of the lift and drag coefficients are a result of pressure fluctuations, i.e. acoustic waves, that are generated by the action of the synthetic jet and propagate through the flow field.

Only high-frequency synthetic jet actuation $F^{+} \geq 10$ is a feasible option for smart rotor control. This is because it is expected that the frequency of the fluctuations of the lift and drag coefficients is then high enough to prevent the excitation of important structural resonance modes of the blades and other components of a large wind turbine. However, at very high actuation frequencies, e.g. $F^{+}=30$, the power consumption increases without an appreciable increase in aerodynamic performance.

For synthetic jet actuation at high frequencies, $F^{+} \geq 1$, vortices are generated, which are subsequently convected with the flow along the surface of the airfoil. When these vortices are generated in rapid succession $\left(F^{+} \geq 10\right)$, they can effectively form an obstruction to the main flow. In a time-averaged sense, this results in a recirculation region along the surface of the airfoil, downstream of the slit, which displaces the main flow along the surface. This displacement can influence the direction of the flow at the trailing edge, i.e. the circulation around the airfoil, which is associated with a change in the lift coefficient.

The time-averaged change in the lift coefficient for high-frequency synthetic jet actuation increases with increasing momentum coefficient, $c_{\mu}$, a location of the slit closer to 
the trailing edge, i.e. higher $x_{j} / c$, and decreasing angle of attack, $\alpha$ (for a slit located on the lower side of the airfoil). This is explained as follows:

- Higher momentum coefficients cause the generated vortices to be ejected a larger distance from the surface into the cross-flow. Furthermore, the magnitude of the generated vorticity is increased. In a time-averaged sense, the recirculation region increases in size and the displacement of the flow along the surface of the airfoil (between the slit and the trailing edge) becomes larger, i.e. the virtual aft camber increases. Additionally, the ability of the synthetic jet to entrain low-momentum fluid from the opposite side of the airfoil into the recirculation zone increases.

- For a location of the slit closer to the trailing edge, the effectiveness of the displacement of the flow along the surface increases, since the direction of the displaced flow at the trailing edge becomes less parallel to the surface and more parallel to the chord line. This increases the change of the circulation around the airfoil.

- Changes in the angle of attack causes changes in the thickness of the boundary layer along the surface of the airfoil, just upstream of the location of the slit. For a slit location on the lower side of the airfoil, decreasing the angle of attack increases the thickness of the boundary layer and therefore decreases the momentum of the cross-flow close to the wall. The synthetic jet is therefore better able to cause a displacement of this cross-flow, for a certain value of the momentum coefficient.

The aerodynamic response time reduces with increasing relative location of the slit, $x_{j} / c$. Increasing the relative location from $x_{j} / c=0.88$ to $x_{j} / c=0.95$, reduced the response time at which $50 \%$ of the final increase in lift is obtained from $2 c / U_{\infty}$ to $c / U_{\infty}$. Short response times are important, since it increases the effectiveness of smart rotor control for load variations that occur at higher frequencies.

A disadvantage of high-frequency synthetic jet actuation, in particular at higher momentum coefficients, is the noise emission associated with the pressure waves that are generated by the action of the synthetic jet. However, this may be less important for offshore wind turbines.

The time-averaged change in the drag coefficient is generally lower than for a microtab or trailing edge flap, which can also be used for pitch control. Furthermore, the drag decreases for slit locations closer to the trailing edge.

In the present computational parameter study, the maximum obtained time-averaged change in the lift coefficient is $\overline{\Delta c_{l}}=0.317$, which corresponds to an effective change of the angle of attack of $\overline{\Delta \alpha} \approx 2.9^{\circ}$. This is very close to the performance deemed necessary for smart rotor control. The power consumption coefficient associated with this aerodynamic performance is $c_{p w r}=0.0235$. For the NREL $5 \mathrm{MW}$ reference offshore wind turbine [25], with a blade radius of $63 \mathrm{~m}$ and a rated rotor speed of 12.1 rotations per 
minute, this would amount to an average power consumption per blade of approximately $\bar{P}=10 \mathrm{~kW}$ per meter span at the outboard blade sections. Due to the large distance to the blade root, generating changes in the lift force at the outboard blade sections will have the largest impact on the fatigue loads. However, applying synthetic jet actuation along $25 \%$ of the span of the three blades simultaneously, leads to a substantial power consumption of $9.45 \%$ of the nominal power production of the wind turbine. This power consumption appears to be prohibitive. Additionally, locating a slit very close to the trailing edge at $x_{j} / c=0.985$ may require a different design of the trailing edge.

\subsection{Recommendations}

The computational method based on the unsteady Reynolds-averaged Navier-Stokes equations is able to deal with all the flow aspects associated with the present problem. However, the effect of the linear eddy-viscosity turbulence model on the predicted performance has not been thoroughly investigated yet. It is therefore recommended to

- improve the present experimental setup by designing and testing synthetic jet actuators that are capable of reaching the actuation frequencies and momentum coefficients that have been identified as most promising, i.e. $F^{+}=20$ and $c_{\mu} \approx 0.01$,

- repeat the simulations with a computational method that is based on a more advanced turbulence model, for the configuration that yields the best performance in terms of time-averaged change in the lift coefficient.

More advanced turbulence models are nonlinear eddy-viscosity models, or Reynolds stress models, for example. Also, Large Eddy Simulation may be considered.

In the present parameter study, not all parameters have been investigated. It would be interesting to also identify the effect of the Reynolds number, $\operatorname{Re}_{c}$, the relative depth of the slit, $d_{j} / c$, the relative width of the slit, $w_{j} / c$ and the angle of the jet with respect the the surface of the airfoil, $\theta_{j}$. It would be most useful to find a configuration that has the necessary performance but consumes less power. Furthermore, the effect of synthetic jet actuation for other angles of attack should be investigated, including a range of negative angles of attack.

So far, a two-dimensional non-rotating airfoil configuration has been studied. This study should be extended to a complete rotating wind turbine blade, on which synthetic jet actuation is applied along part of the span. Although not discussed in this thesis, the option of three-dimensional flow simulations in a rotating frame of reference has been implemented in the computational method. However, this option has not been thoroughly verified yet.

For the actual implementation of smart rotor control on a wind turbine blade, the advantages and disadvantages of all the potential options should be considered, including 
those of synthetic jets. At present, the power consumption of synthetic jet actuation appears to be prohibitive. Possibly, a less power intensive configuration can be found. Also, a combination of synthetic jet actuation with other control methods might be considered. Furthermore, the required low maintenance and high reliability of smart rotor control is still an issue for all potential options. 



\section{BIBLIOGRAPHY}

[1] Manwell, J. F., McGowan, J. G., and Rogers, A. L., Wind Energy Explained Theory, Design and Application, Wiley, 2nd ed., 2009.

[2] Sutherland, H. J., "On the Fatigue Analysis of Wind Turbines," Sandia National Laboratories, Technical Report SAND99-0089, 1999.

[3] IEC, "Wind Turbines - Part 1: Design Requirements," IEC 61400-1, 3rd ed., 2005.

[4] Hansen, M. H., "Aeroelastic Instability Problems for Wind Turbines," Wind Energy, Vol. 10, 2007, pp. $551-557$.

[5] Snel, H., "Review of Aerodynamics for Wind Turbines," Wind Energy, Vol. 6, No. 3, 2003, pp. $203-211$.

[6] Tegen, S., Hand, M., Maples, B., Lantz, E., Schwabe, P., and Smith, A., "2010 Cost of Wind Energy Review," NREL Technical Report TP-5000-52920, 2012.

[7] Lantz, E., Hand, M., and Wiser, R., "The Past and Future Cost of Wind Energy," NREL conference paper CP-6A20-54526, presented at the 2012 World Renewable Energy Forum in Denver, Colorado, 2012.

[8] Sieros, G., Chaviaropoulos, P., Sørensen, J. D., Bulder, B. H., and Jamieson, P., "Upscaling Wind Turbines: Theoretical and Practical Aspects and their Impact on the Cost of Energy," Wind Energy, Vol. 15, 2012, pp. 3 - 17.

[9] Barlas, T. K. and Van Kuik, G. A. M., "State of the Art and Prospectives of Smart Rotor Control for Wind Turbines," The Science of Making Torque from Wind, Journal of Physics: Conference Series, Vol. 75: 012080, 2007.

[10] Sørensen, J. N., "Aerodynamic Aspects of Wind Energy Conversion," Annual Review of Fluid Mechanics, Vol. 43, 2011, pp. $427-448$.

[11] Oerlemans, S., Detection of Aeroacoustic Sound Sources on Aircraft and Wind Turbines, Ph.D. thesis, University of Twente, 2009.

[12] Betz, A., "Das Maximum der theoretisch möglichen Ausnützung des Windes durch Windmotoren," Gesamte Turbinenwesen, Vol. 26, 1920, pp. $307-309$.

[13] Snel, H., "Review of the Present Status of Rotor Aerodynamics," Wind Energy, Vol. 1, 1998, pp. $46-69$. 
[14] Van Kuik, G. A. M., On the Limitations of Froude's Actuator Disc Concept, Ph.D. thesis, Eindhoven University of Technology, 1991.

[15] Glauert, H., "Windmills and Fans," Aerodynamic Theory, edited by W. F. Durand, Vol. 14, Dover Publications Inc., 1963, pp. 324 - 340.

[16] Schepers, J. G., Heijdra, J., Thomsen, K., Larsen, T., Foussekis, D., Rawlinson Smith, R., Kraan, I., Visser, B., Oye, S., Ganander, H., Carlen, I., Voutsinas, S., Belessis, M., and Drost, L., "Verification of European Wind Turbine Design Codes," VEWTDC, final report, ECN-C-01-055, 2002.

[17] Somers, D. M., "Design and Experimental Results for the S809 Airfoil," NREL/SR440-6918, 1997.

[18] Anderson, J. D., Fundamentals of Aerodynamics, McGraw-Hill, 3rd ed., 2001.

[19] Wagner, H., "Über die Enstehung des dynamischen Auftriebes von Tragflügeln," Zeitschrift für angewandte Mathematik und Mechanik, VDI Verlag G.M.B.H., Band 5 Heft 1, 17-35, 1925.

[20] Leishman, J. G., "Challenges in Modeling the Unsteady Aerodynamics of Wind Turbines," AIAA paper 2002-0037, 2002.

[21] Jones, R. T., "The Unsteady Lift of a Wing of Finite Aspect Ratio," NACA Report No. 681, 1939.

[22] Chow, C. Y. and Huang, M. K., "The Initial Lift and Drag of an Impulsively Started Airfoil of Finite Thickness," Journal of Fluid Mechanics, Vol. 118, 1982, pp. 393 409.

[23] Gaunaa, M., Unsteady Aerodynamic Forces on NACA 0015 Airfoil in Harmonic Translatory Motion, Ph.D. thesis, Technical University of Denmark, 2002.

[24] Gaunaa, M., Bergami, L., and Heinz, J. C., "Indicial Response Function for FiniteThickness Airfoils - A Semi-Empirical Approach," AIAA Paper 2011-542, 2011.

[25] Jonkman, J., Butterfield, S., Musial, W., and Scott, G., "Definition of a 5-MW Reference Wind Turbine for Offshore System Development," NREL Technical Report TP-500-38060, 2009.

[26] Snel, H. and Schepers, J. G., "Engineering Models for Dynamic Inflow Phenomena," ECN Technical Report, ECN-RX-91-064, 1991.

[27] Schepers, J. G., "IEA Annex XX: Dynamic Inflow Effects at Fast Pitching Steps on a Wind Turbine Placed in the NASA-Ames Wind Tunnel," ECN technical report, ECN-E-07-085, 2007.

[28] Bossanyi, A. E., "Wind Turbine Control for Load Reduction," Wind Energy, Vol. 6, No. 3, 2003, pp. $229-244$. 
[29] Larsen, T., Madsen, H., and Thomsen, K., "Active Load Reduction using Individual Pitch, based on Local Blade Flow Measurements," Wind Energy, Vol. 8, 2004, pp. $67-80$.

[30] De Goeij, W. C., Van Tooren, M. J. L., and Beukers, A., "Implementation of Bending-Torsion Coupling in the Design of a Wind-Turbine Rotor-Blade," Applied Energy, Vol. 63, No. 3, 1999, pp. $191-207$.

[31] Lobitz, D. W. and Veers, P. S., "Load Mitigation with Bending/Twist-Coupled Blades on Rotors Using Modern Control Strategies," Wind Energy, Vol. 6, 2003, pp. $105-117$.

[32] Collis, S. S., Joslin, R. D., Seifert, A., and Theofilis, V., "Issues in Active Flow Control: Theory, Control, Simulation, and Experiment," Progress in Aerospace Sciences, Vol. 40, 2004, pp. $237-289$.

[33] Van Kuik, G. A. M. and Berg, D. E., editors, Wind Energy, Smart Blades Special, Vol. 13: 2 - 3, 2010.

[34] Maldonado, V., Farnsworth, J., and Gressick, W. Amitay, M., "Active Control of Flow Separation and Structural Vibrations of Wind Turbine Blades," Wind Energy, Vol. 13, 2010, pp. $221-237$.

[35] Barlas, T. K., Active Aerodynamic Load Control on Wind Turbines - Aeroservoelastic Modeling and Wind Tunnel Experiments, Ph.D. thesis, Delft University of Technology, 2011.

[36] Harris, M., Hand, M., and Wright, A., "LIDAR for Turbine Control," NREL Technical Report TP-500-39154, 2006.

[37] Dunne, F., Simley, E., and Pao, L. Y., "LIDAR Wind Speed Measurement Analysis and Feed-Forward Blade Pitch Control for Load Mitigation in Wind Turbines," NREL Technical Report SR-5000-52098, 2011.

[38] Barlas, T. K. and Van Kuik, G. A. M., "Review of State of the Art in Smart Rotor Control Research for Wind Turbines," Progress in Aerospace Sciences, Vol. 46, 2010, pp. $1-27$.

[39] Johnson, S. J., Van Dam, C. P., and Berg, D. E., "Active Load Control Techniques for Wind Turbines," Sandia National Laboratories, Technical Report SAND20084809, 2008.

[40] Johnson, S. J., Baker, J. P., Van Dam, C. P., and Berg, D. E., "An overview of active load control techniques for wind turbines with an emphasis on microtabs," Wind Energy, Vol. 13, 2010, pp. 239 - 253.

[41] Pechlivanoglou, G., Passive and Active Flow Control Solutions for Wind Turbine Blades, Ph.D. thesis, Technischen Universitt Berlin, 2013. 
[42] Blaylock, M., Chow, R., and Van Dam, C. P., "Comparison of Microjets with Microtabs for Active Aerodynamic Load Control," AIAA Paper 2010-4409, 2010.

[43] Troldborg, N., "Computational study of the Risö-B1-18 airfoil with a hinged flap providing variable trailing edge geometry," Wind Engineering, Vol. 29, No. 2, 2005, pp. $89-113$.

[44] Bæk, P., Gaunaa, M., Srensen, N. N., and Fuglsang, P., "A Comparison of Two Devices for Distributed Active Load Control of Wind Turbine Blades," Torque 2010: The Science of Making Torque from Wind, EWEA, 2010, pp. $611-617$.

[45] Chow, R. and Van Dam, C. P., "On the Temporal Response of Active Load Control Devices," Wind Energy, Vol. 13, 2010, pp. 135 - 149.

[46] Boeije, C. S., de Vries, H., Cleine, I., Van Emden, E., Zwart, G. G. M., Stobbe, H., Hirschberg, A., and Hoeijmakers, H. W. M., "Fluidic Load Control for Wind Turbine Blades," AIAA Paper 2009-0684, 2009.

[47] Glezer, A. and Amitay, M., "Synthetic Jets," Annual Review of Fluid Mechanics, Vol. 34, No. 1, 2002, pp. $503-529$.

[48] Smith, B. L. and Swift, G. W., "A comparison between synthetic jets and continuous jets," Experiments in Fluids, Vol. 34, 2003, pp. $467-472$.

[49] Quinn, W. R., "Turbulent Free Jet Flows Issuing from Sharp-Edged Rectangular Slots: The Influence of Slot Aspect Ratio," Experimental Thermal and Fluid Science, Vol. 5, 1992, pp. $203-215$.

[50] Brozozowski, D. and Glezer, A., "Transient separation control using pulsecombustion actuation," AIAA Paper 2006-3024, 2006.

[51] Gilarranz, J. L., Traub, L. W., and Rediniotis, O. K., "A New Class of Synthetic Jet ActuatorsPart I: Design, Fabrication and Bench Top Characterization," Journal of Fluids Engineering, Vol. 127, 2005, pp. $367-376$.

[52] Seifert, A. and Pack, L., "Oscillatory Excitation of Unsteady Compressible Flows Over Airfoils at Flight Reynolds Number," AIAA Paper 99-0925, 1999.

[53] Amitay, M. and Glezer, A., "Role of Actuation Frequency in Controlled Flow Reattachment over Stalled Airfoil," AIAA Journal, Vol. 40, No. 2, 2002, pp. 209 216.

[54] Greenblatt, D. and Wygnanski, I. J., "The control of flow separation by periodic excitation," Progress in Aerospace Sciences, Vol. 36, 2000, pp. 487 - 545.

[55] Seifert, A., Bachar, T., Koss, D., Shepshelovits, M., and Wygnanski, I., "Oscillatory Blowing, a Tool to Delay Boundary Layer Separation," AIAA Journal, Vol. 31, No. 11, 1993, pp. $2052-2060$. 
[56] Glezer, A., Amitay, M., and Honohan, A. M., "Aspects of Low- and High-Frequency Actuation for Aerodynamic Flow Control," AIAA Journal, Vol. 43, No. 7, 2005, pp. $1501-1511$.

[57] DeSalvo, M. E., Amitay, M., and Glezer, A., "Modification of the aerodynamic performance of airfoil at low angle of attack: Trailing edge trapped vortices," AIAA Paper 2002-3165, 2002.

[58] DeSalvo, M. E. and Glezer, A., "Aerodynamic performance modification at low angles of attack by trailing edge vortices," AIAA Paper 2004-2118, 2004.

[59] DeSalvo, M. E. and Glezer, A., "Airfoil aerodynamic performance modification using hybrid surface actuators," AIAA Paper 2005-0874, 2005.

[60] Shea, P. R. and Smith, D. R., "Aerodynamic control of a rectangular wing using gurney flaps and synthetic jets," AIAA Paper 2009-886, 2009.

[61] Lopez, O. D., Moser, R. D., Brzozowski, D. P., and Glezer, A., "Aerodynamic performance of airfoils with tangential synthetic jet actuators close to the trailing edge," AIAA Paper 2009-3674, 2009.

[62] Petz, R. and Nitsche, W., "Designing Actuators for Active Separation Control Experiments on High-Lift Configurations," Active Flow Control, Vol. 95, 2007, pp. 69 $-84$.

[63] Cattafesta, L. N. and Sheplak, M., "Actuators for Active Flow Control," Annual Review of Fluid Mechanics, Vol. 43, 2011, pp. $247-272$.

[64] Papila, M., Sheplak, M., and Cattafesta, L. N., "Optimization of Clamped Circular Piezoelectric Composite Actuators," Sens. Actuators A Phys., Vol. 147, 2008, pp. $310-323$.

[65] Shavit, A. and Gutfinger, C., Thermodynamics: from Concepts to Applications, Prentice Hall Europe, 1995.

[66] Gad-el Hak, M., "Stokes' Hypothesis for a Newtonian, Isotropic Fluid," Journal of Fluids Engineering, Vol. 117, No. 1, 1995, pp. $3-5$.

[67] Vincenti, W. G. and Kruger, C. H., Introduction to Physical Gas Dynamics, Wiley, 1965.

[68] Wilcox, D. C., Turbulence Modeling for CFD, DCW Industries, Inc., 3rd ed., 2006.

[69] Richardson, L. F., Weather Prediction By Numerical Process, Cambridge University Press, 1922. 
[70] Kolmogorov, A. N., "The local structure of turbulence in incompressible viscous fluid for very large Reynolds numbers," Dokl. Akad. Nauk SSSR, Vol. 30, 1941, pp. 299 - 303, [In Russian; English reprint available in: Proceedings of the Royal Society of London, Series A: Mathematical and Physical Sciences, Vol. 434, 1991, pp. 9 - 13.].

[71] Pope, S. B., Turbulent Flows, Cambridge University Press, 2000.

[72] Favre, A., "Équations des Gaz Turbulents Compressibles," Journal de Mécanique, Vol. 4, No. 3, 1965, pp. $361-390,391-421$.

[73] Ristorcelli, J. R., "Carrying the mass flux terms exactly in the first and second moment equations of compressible turbulence," ICASE Report No. 93-87, 1993.

[74] Bradhaw, P., "Effects of Streamline Curvature on Turbulent Flow," AGARD-AG169, 1973.

[75] Spalart, P. R. and Shur, M. L., "On the Sensitization of Turbulence Models to Rotation and Curvature," Aerosp. Sci. Technol., Vol. 1, No. 5, 1997, pp. 297 302.

[76] Shur, M. L., Strelets, M. K., Travin, A. K., and Spalart, P. R., "Turbulence Modeling in Rotating and Curved Channels: Assessing the Spalart-Shur Correction," AIAA Journal, Vol. 38, No. 5, 2000, pp. $784-792$.

[77] Mani, M., Ladd, J. A., and Bower, W. W., "Rotation and Curvature Correction Assessment for One- and Two-Equation Turbulence Models," Journal of Aircraft, Vol. 41, No. 2, 2004, pp. $268-273$.

[78] Cazalbou, J.-B., Chassaing, P., Dufour, G., and Carbonneau, X., "Two-equation modeling of turbulent rotating flows," Physics of Fluids, Vol. 17, 055110, 2005.

[79] Wallin, S. and Johansson, A. V., "An Explicit Algebraic Reynolds Stress Model for Incompressible and Compressible Turbulent Flows," Journal of Fluid Mechanics, Vol. 403, 2000, pp. $89-132$.

[80] Rumsey, C. L. and Gatski, T. B., "Summary of EASM Turbulence Models in CFL3D with Validation Test Cases," NASA TM 212431, 2003.

[81] Launder, B. E., Reece, G. J., and Rodi, W., "Progress in the Development of a Reynolds-Stress Turbulent Closure," Journal of Fluid Mechanics, Vol. 68, No. 3, 1975 , pp. $537-566$.

[82] Baldwin, B. S. and Lomax, H., "Thin Layer Approximation and Algebraic Model for Separated Turbulent Flows," AIAA Paper 78-257, 1978.

[83] Baldwin, B. S. and Barth, T. J., "A One-Equation Turbulence Transport Model for High Reynolds Number Wall-Bounded Flows," NASA TM 102847, 1990. 
[84] Spalart, P. R. and Allmaras, S. R., "A One-Equation Turbulence Model for Aerodynamic Flows," Recherche Aerospatiale, Vol. 1, 1994, pp. 5 - 21.

[85] Sai, V. A. and Lutfy, F. M., "Analysis of the Baldwin-Barth and Spalart-Allmaras One-Equation Turbulence Models," AIAA Journal, Vol. 33, No. 10, 1995, pp. 1971 $-1974$.

[86] Blazek, J., Computational Fluid Dynamics: Principles and Applications, Elsevier, 2nd ed., 2005.

[87] Bardina, J. E., Huang, P. G., and Coakley, T. J., “Turbulence Modeling Validation, Testing, and Development," NASA TM 110446, 1997.

[88] Jones, W. P. and Launder, B. E., "The Prediction of Laminarization with a TwoEquation Model of Turbulence," International Journal of Heat and Mass Transfer, Vol. 15, 1972, pp. $301-314$.

[89] Launder, B. E. and Sharma, B. I., "Application of the Energy-Dissipation Model of Turbulence to the Calculation of Flow Near a Spinning Disc," Letters in Heat and Mass Transfer, Vol. 1, No. 2, 1974, pp. 131 - 138.

[90] Wilcox, D. C., "Reassessment of the Scale-Determining Equation for Advanced Turbulence Models," AIAA Journal, Vol. 26, No. 11, 1988, pp. 1299 - 1310.

[91] Menter, F. R., "Improved Two-Equation k- $\omega$ Turbulence Models for Aerodynamic Flows," NASA TM 103975, 1992.

[92] Menter, F. R., Kuntz, M., and Langtry, R., "Ten Years of Industrial Experience with the SST Turbulence Model," Turbulence, Heat and Mass Transfer 4, edited by K. Hanjalic, Y. Nagano, and M. Tummers, Begell House, Inc., 2003, pp. 625 632.

[93] Spalart, P. R. and Rumsey, C. L., "Effective Inflow Conditions for Turbulence Models in Aerodynamic Calculations," AIAA Journal, Vol. 45, No. 10, 2007, pp. 2544 2553.

[94] Kreiss, H. O., "Initial Boundary Value Problems for Hyperbolic Systems," Communications on Pure and Applied Mathematics, Vol. 23, 1970, pp. 277 - 298.

[95] Rumsey, C. L., "Successes and Challenges for Flow Control Simulations," AIAA Paper 2008-4311, 2008.

[96] Aram, E., Mittal, R., and Cattafesta, L., "Toward Simple Boundary Condition Representations of Zero-Net Mass-Flux Actuators in Grazing Flow," AIAA Paper 2009-4018, 2009.

[97] Balakumar, P., "Computations of Flow over a Hump Model Using Higher Order Method with Turbulence Modeling," AIAA Paper 2005-1270, 2005. 
[98] Vatsa, V. N. and Turkel, E., "Simulation of Synthetic Jets Using Unsteady ReynoldsAveraged Navier-Stokes Equations," AIAA Journal, Vol. 44, No. 2, 2006, pp. 217 $-224$.

[99] ANSYS ICEM CFD 12.1 Manual, ANSYS, Inc., November 2009.

[100] Geuzaine, C. and Remacle, J.-F., "Gmsh: a three-dimensional finite element mesh generator with built-in pre- and post-processing facilities," International Journal for Numerical Methods in Engineering, Vol. 79, No. 11, 2009, pp. 1309 - 1331.

[101] Kelleners, P. H., An Edge-based Finite Volume Method for Inviscid Compressible Flow with Condensation, Ph.D. thesis, University of Twente, 2007.

[102] Hospers, J. M., Development and Application of Hybrid Grids for Finite Volume Methods, Master's thesis, University of Twente, 2007.

[103] Kelleners, P. H., "Simulation of inviscid compressible multi-phase flow with condensation," Annual research briefs, Center for Turbulence Research, NASA Ames, Stanford University, 2003, pp. 49-67.

[104] Barth, T. J. and Jespersen, D. C., "The Design and Application of Upwind Schemes on Unstructured Meshes," AIAA Paper 89-0366, 1989.

[105] Vankatakrishnan, V., "Convergence to Steady State Solutions of the Euler Equations on Unstructured Grids with Limiters," Journal of Computational Physics, Vol. 118, 1995, pp. $120-130$.

[106] Wesseling, P., Principles of Computational Fluid Dynamics, Springer Series in Computational Mathematics, Springer, 2001.

[107] Barth, T. J., "Aspects of Unstructured Grids and Finite-Volume Solvers for the Euler and Navier-Stokes Equations," VKI Lecture Series 1994-05, 1994, Revised 1995.

[108] Venkatakrishnan, V. and Mavriplis, D. J., "Implicit Method for the Compuation of Unsteady Flows on Unstructured Grids," ICASE Report No. 95-60, 1995.

[109] Jameson, A., "Time Dependent Calculations Using Multigrid, with Applications to Unsteady Flows Past Airfoils and Wings," AIAA Paper 911596, 1991.

[110] Pulliam, T. H., "Time Accuracy and the Use of Implicit Methods," AIAA Paper 93-3360, 1993.

[111] Jameson, A., Schmidt, W., and Turkel, E., "Numerical Solutions of the Euler Equations by Finite Volume Methods Using Runge-Kutta Time-Stepping Schemes," AIAA Paper 81-1259, 1981.

[112] Wesseling, P., "Von Neumann stability conditions for the convection-diffusion equation," IMA Journal of Numerical Analysis, Vol. 16, 1996, pp. 583 - 598. 
[113] Trottenberg, U., Oosterlee, C. W., and Schüller, A., Multigrid, Elsevier Academic Press, 2001.

[114] Morton, K. W. and Mayers, D. F., Numerical Solution of Partial Differential Equations, Cambridge University Press, 1994.

[115] Saad, Y. and Schultz, M. H., "GMRES: A generalized minimal residual algorithm for solving nonsymmetric linear systems," SIAM Journal on Scientific and Statistical Computing, Vol. 7, 1986, pp. $856-869$.

[116] Mavriplis, D. J., "On Convergence Acceleration Techniques for Unstructured Meshes," ICASE Report No. 98-44, 1998.

[117] Winkelmann, R., Häuser, J., and Williams, R. D., "Strategies for Parallel and Numerical Scalability of CFD Codes," Comput. Methods Appl. Mech. Engrg., Vol. 174, 1999, pp. $433-456$.

[118] Karypis, G. and Kumar, V., "A Fast and Highly Quality Multilevel Scheme for Partitioning Irregular Graphs," SIAM Journal on Scientific Computing, Vol. 20, No. 1, 1999, pp. $359-392$.

[119] Gropp, W., Lusk, E., and Skjellum, A., Using MPI: Portable Parallel Programming with the Message Passing Interface, Scientific and Engineering Computation, MIT Press, 2nd ed., 1999.

[120] Jameson, A., Baker, T. J., and Weatherill, N. P., "Calculation of Inviscid Transonic Flow over a Complete Aircraft," AIAA Paper 86 - 0103, 1986.

[121] Swanson, R. C. and Turkel, E., "On Central-Difference and Upwind Schemes," Journal of Computational Physics, Vol. 101, 1992, pp. 292 - 306.

[122] Swanson, R. C., Radespiel, R., and Turkel, E., "Comparison of Several Dissipation Algorithms for Central Difference Schemes," ICASE Report No. 97-40, 1997.

[123] Roe, P. L., "Approximate Riemann Solvers, Parameter Vectors, and Difference Schemes," Journal of Computational Physics, Vol. 43, 1981, pp. 357 - 372.

[124] Liou, M.-S. and Steffen, C. J., "A New Flux Splitting Scheme," Journal of Computational Physics, Vol. 107, 1993, pp. $23-39$.

[125] Liou, M.-S., "A Sequel to AUSM: AUSM+," Journal of Computational Physics, Vol. 129, 1996, pp. $364-382$.

[126] Liou, M.-S., "A Sequel to AUSM, Part II: AUSM+'-up for all speeds," Journal of Computational Physics, Vol. 214, 2006, pp. $137-170$.

[127] van Leer, B., Thomas, J. L., Roe, P. L., and Newsome, R. W., "A Comparison of Numerical Flux Formulas for the Euler and Navier-Stokes Equations," AIAA Paper 87-1104, 1987. 
[128] Harten, A., "High Resolution Schemes for Hyperbolic Conservation Laws," Journal of Computational Physics, Vol. 49, 1983, pp. 357 - 393.

[129] Yee, H. C., Klopfer, G. H., and Montagné, J. L., "High-Resolution Shock-Capturing Schemes for Inviscid and Viscous Hypersonic Flows," Journal of Computational Physics, Vol. 88, 1990, pp. $31-61$.

[130] Liou, M.-S., "Mass Flux Schemes and Connection to Shock Instability," Journal of Computational Physics, Vol. 160, 2000, pp. $623-648$.

[131] Guillard, H. and Viozat, C., "On the behavior of upwind schemes in the low Mach number limit," Computers and Fluids, Vol. 28, 1999, pp. $63-86$.

[132] van Leer, B., "Towards the Ultimate Conservative Difference Scheme, V. A Second Order Sequel to Godunov's Method," Journal of Computational Physics, Vol. 32, 1979, pp. $101-136$.

[133] Michalak, C. and Ollivier-Gooch, C., "Accuracy preserving limiter for the highorder accurate solution of the Euler equations," Journal of Computational Physics, Vol. 228, 2009, pp. $8693-8711$.

[134] Haselbacher, A. and Blazek, J., "Accurate and Efficient Discretization of NavierStokes Equations on Mixed Grids," AIAA Journal, Vol. 38, No. 11, 2000, pp. 2094 $-2102$.

[135] Mavriplis, D. J., "Revisiting the Least-squares Procedure for Gradient Reconstruction on Unstructured Meshes," AIAA Paper 2003-3986, 2003.

[136] Shima, E., Kitamura, K., and Fujimoto, K., "New Gradient Calculation Method for MUSCL Type CFD Schemes in Arbitrary Polyhedra," AIAA Paper 2010-1081, 2010.

[137] Crumpton, P., Moiner, P., and Giles, M., "An Unstructured Algorithm for High Reynolds Number Flows on Highly-Stretched Grids," 10th Int. Conf. Num. Meth. for Laminar and Turbulent Flows, Swansea, England, July 21 - 25, 1997.

[138] Dwight, R. P., Efficienty Improvements of RANS-based Analysis and Optimization Using Implicit and Adjoint Methods on Unstructured Grids, Ph.D. thesis, School of Mathematics, University of Manchester, 2006.

[139] Barth, T. J., "Analysis of Implicit Local Linearization Techniques for Upwind and TVD Algorithms," AIAA Paper 87-0595, 1987.

[140] Martins, J. R. R. A., Kroo, I. M., and Alonso, J. J., "An Automated Method for Sensitivity Analysis using Complex Variables," AIAA Paper 2000-0689, 2000.

[141] Anderson, W. K., Newman, J. C., Whitfield, D. L., and Nielsen, E. J., "Sensitivity Analysis for Navier-Stokes Equations on Unstructured Meshes Using Complex Variables," AIAA Journal, Vol. 39, No. 1, 2001, pp. $56-63$. 
[142] Mor-Yossef, Y. and Levy, Y., "The Unconditionally Positive-Convergent Implicit Time Integration Scheme for Two-Equation Turbulence Models: Revisited," Computers a, Vol. 38, 2009, pp. $1984-1994$.

[143] Pang, T., An Introduction to Computational Physics, Cambridge University Press, 1997.

[144] Cuthill, E. and McKee, J., "Reducing the Bandwidth of Sparse Symmetric Matrices," Proc. ACM 24th National Conference, 1969, pp. 157 - 161.

[145] Gibbs, N. E., Poole, W. G., and Stockmeyer, P. K., "An Algorithm for Reducing the Bandwidth and Profile of a Sparse Matrix," SIAM Journal on Numerical Analysis, Vol. 13, 1976, pp. $236-250$.

[146] Mavriplis, D. J. and Jameson, A., "Multigrid Solutions of the Navier-Stokes Equations on Triangular Grids," AIAA Journal, Vol. 28, No. 8, 1990, pp. 1415 - 1425.

[147] Fedorenko, R. P., "A relaxation method for solving elliptic difference equations," USSR Computational Mathematics and Mathematical Physics, Vol. 1, No. 4, 1962, pp. $1092-1096$.

[148] Pierce, N. A., Giles, M. B., Jameson, A., and Martinelli, L., "Accelerating ThreeDimensional Navier-Stokes Calculations," AIAA Paper 97-1953, 1997.

[149] Mavriplis, D. J., "Multigrid Strategies for Viscous Flow Solvers on Anisotropic Unstructured Meshes," Journal of Computational Physics, Vol. 145, 1998, pp. 141 $-165$.

[150] Perez, E., "Finite Element and Multigrid Solution of the Two-Dimensional Euler Equations on a Non-Structured Mesh," INRIA Report No. 442, 1985.

[151] Mavriplis, D. J., "Multigrid Solution of the Two-Dimensional Euler Equations on Unstructured Triangular Meshes," AIAA Journal, Vol. 26, No. 7, 1988, pp. 824 831.

[152] Lallemand, M. H., Steve, H., and Dervieux, A., "Unstructured multigridding by volume agglomeration: Current status," Computers \& Fluids, Vol. 21, 1992, pp. 397 $-433$.

[153] Weiss, J. M., Maruszewski, J. P., and Smith, W. A., "Implicit Solution of Preconditioned NavierStokes Equations Using Algebraic Multigrid," AIAA Journal, Vol. 37, No. 1, 1999, pp. $29-36$.

[154] Brand, A., Multigrid Techniques: 1984 Guide with Applications to Fluid Dynamics, Vol. 85 of GMD-Studien, Gesellschaft für Mathematik und Datenverarbeitung, 1984.

[155] Stüben, K., "A Review of Algebraic Multigrid," Journal of Compuational and Applied Mathematics, Vol. 128, 2001, pp. $281-309$. 
[156] Koobus, B., Lallemand, M. H., and Dervieux, A., "Unstructured VolumeAgglomeration MG : solution of the Poisson equation," Rapport de Recherche INRA No. 1946, 1993.

[157] Mavriplis, D. J. and Venkatakrishnan, V., "Agglomeration Multigrid for TwoDimensional Viscous Flows," Computers \& Fluids, Vol. 24, No. 5, 1995, pp. $553-$ 570 .

[158] Carré, G., "An Implicit Multigrid Method by Agglomeration Applied to Turbulent Flows," Computers \& Fluids, Vol. 26, No. 3, 1997, pp. $299-320$.

[159] Vanek, P., Mandel, J., and Brezina, M., "Algebraic Multigrid on Unstructured Meshes," University of Colorado at Denver, UCD/CCM Report No. 34, 1994.

[160] Braess, D., "Towards Algebraic Multigrid for Elliptic Problems of Second Order," Computing, Vol. 55, No. 4, 1995, pp. $379-393$.

[161] Krechel, A. and Stüben, K., "Parallel algebraic multigrid based on subdomain blocking," GMD-Report 71, German National Research Center for Information Technology, 1999.

[162] Schlichting, H., Boundary Layer Theory, McGraw-Hill, 7th ed., 1979.

[163] Thompson, K. W., "Time-Dependent Boundary Conditions for Hyperbolic Systems," Journal of Computational Physics, Vol. 68, 1987, pp. 1- 24.

[164] Thompson, K. W., "Time-Dependent Boundary Conditions for Hyperbolic Systems II," Journal of Computational Physics, Vol. 89, 1990, pp. 439 - 461.

[165] Rudy, D. H. and Strikwerda, J. C., "A Nonreflecting Outflow Boundary Condition for Subsonic Navier-Stokes Calculations," Journal of Computational Physics, Vol. 36, 1980, pp. $55-70$.

[166] Poinsot, T. J. and Lele, S. K., "Boundary Conditions for Direct Simulations of Compressible Viscous Flows," Journal of Computational Physics, Vol. 101, 1992, pp. $104-129$.

[167] Oberkampf, W. L. and Trucano, T. G., "Verification and validation in computational fluid dynamics," Progress in Aerospace Sciences, Vol. 38, 2002, pp. 209 - 272.

[168] Roache, P. J., "Quantification of Uncertainty in Computational Fluid Dynamics," Annual Review of Fluid Mechanics, Vol. 29, 1997, pp. 123 - 160.

[169] AIAA, "Guide for the Verification and Validation of Computational Fluid Dynamics Simulations," G-077-1998, American Institute of Aeronautics and Astronautics, 1998.

[170] Rumsey, C. L., "Turbulence Modeling Resource," NASA Langley Research Center, URL: http://turbmodels.larc.nasa.gov/, accessed April 12, 2012. 
[171] Rumsey, C. L., "Workshop CFD Validation of Synthetic Jets and Turbulent Separation Control," NASA Langley Research Center, URL: http://cfdval2004.larc.nasa.gov/, accessed April 12, 2012.

[172] Rumsey, C. L., Gatski, T. B., Sellers, W. L., Vatsa, V. N., and Viken, S. A., "Summary of the 2004 Computational Fluid Dynamics Validation Workshop on Synthetic Jets," AIAA Journal, Vol. 44, No. 2, 2006, pp. $194-207$.

[173] Fehlberg, E., "Low-order classical Runge-Kutta formulas with step size control and their application to some heat transfer problems," NASA Technical Report R-315, 1969.

[174] Eça, L. and Hoekstra, M., "A Verification Excercise for Two 2-D Steady Incompressible Turbulent Flows," European Congress on Computational Methods in Applied Sciences and Engineering (ECCOMAS), edited by P. Neittaanmäki, T. Rossi, K. Majava, and O. Pironneau, 2004.

[175] Williamson, C. H. K., "Vortex Dynamics in the Cylinder Wake," Annual Review of Fluid Mechanics, Vol. 28, 1996, pp. $477-539$.

[176] Franke, R., Rodi, W., and Schönung, B., "Numerical Calculation of Laminar VortexShedding Flow Past Cylinders," Journal of Wind Engineering and Industrial Aerodynamics, Vol. 35, 1990, pp. $273-257$.

[177] Cox, J. S., Brentner, K. S., and Rumsey, C. L., "Computation of Vortex Shedding and Radiated Sound for a Circular Cylinder: Subcritical to Transcritical Reynolds Numbers," Theoretical and Compuational Fluid Dynamics, Vol. 12, 1998, pp. 233 $-253$.

[178] Liu, C., Zheng, X., and Sung, C. H., "Preconditioned Multigrid Methods for Unsteady Incompressible Flows," Journal of Computational Physics, Vol. 139, 1998, pp. $35-57$.

[179] Rajani, B. N., Kandasamy, A., and Majumdar, S., "Numerical Simulation of Laminar Flow past a Circular Cylinder," Applied Mathematical Modelling, Vol. 33, 2009, pp. $1228-1247$.

[180] Ding, H., Shu, C., Yeo, K. S., and Xu, D., "Simulation of incompressible viscous flow past a circular cylinder by hybrid FD scheme and meshless least square-based finite difference method," Comput. Methods Appl. Mech. Engrg., Vol. 193, 2004, pp. $727-744$.

[181] Cox, J. S., Brentner, K. S., Rumsey, C. L., and Younis, B. A., "Computation of Vortex Shedding and Radiated Sound for a Circular Cylinder," 4th International Symposium on Fluids-Structures Interactions, Aeroelasticity, Flow-Induced Vibration and Noise, Vol. 1, 1997, pp. $447-454$. 
[182] Rumsey, C. L., Thomas, J. L., Warren, G. P., and Liu, G. C., "Upwind NavierStokes Solutions for Separated Periodic Flows," AIAA Journal, Vol. 25, No. 4, 1987, pp. $535-541$.

[183] Zingg, D. W. and Godin, P., "A perspective on turbulence models for aerodynamic flows," International Journal of Computational Fluid Dynamics, Vol. 23, No. 4, 2009, pp. $327-335$.

[184] Arnal, D. and Casalis, G., "Laminar-turbulent transition prediction in threedimensional flows," Progress in Aerospace Sciences, Vol. 36, 2000, pp. 173 - 191.

[185] Malkiel, E. and Mayle, R. E., "Transition in a Separation Bubble," Journal of Turbomachinery, Vol. 118, No. 4, 1996, pp. $752-760$.

[186] Menter, F. R., Langtry, R., and Völker, S., "Transition Modelling for General Purpose CFD Codes," Flow, Turbulence and Combustion, Vol. 77, 2006, pp. 277 -303.

[187] Greenblatt, D., Paschal, K. B., Yao, C.-S., Harris, J., Schaeffler, N. W., and Washburn, A. E., "A Separation Control CFD Validation Test Case, Part 1: Baseline and Steady Suction," AIAA Journal, Vol. 44, No. 12, 2006, pp. 2820 - 2830.

[188] Greenblatt, D., Paschal, K. B., Yao, C.-S., and Harris, J., "A Separation Control CFD Validation Test Case, Part 2: Zero Efflux Oscillatory Blowing," AIAA Journal, Vol. 44, No. 12, 2006, pp. $2831-2845$.

[189] Naughton, J. W., Viken, S. A., and Greenblatt, D., "Skin-Friction Measurements on the NASA Hump Model," AIAA Journal, Vol. 12, No. 6, 2006, pp. $1255-1265$.

[190] Rumsey, C. L., "Reynolds-Averaged NavierStokes Analysis of Zero Efflux Flow Control over a Hump Model," Journal of Aircraft, Vol. 44, No. 2, 2007, pp. $444-452$.

[191] Rumsey, C. L. and Greenblatt, D., "Parametric Study of Flow Control Over a Hump Model Using an Unsteady Reynolds-Averaged Navier-Stokes Code," NASA TM 214897, 2007.

[192] Rumsey, C. L. and Greenblatt, D., "Flow Control Predictions Using Unsteady Reynolds-Averaged Navier-Stokes Modeling: A Parametric Study," Journal of Aircraft, Vol. 47, No. 9, 2009, pp. $2259-2262$.

[193] Widjanarko, S. M. D., Active Aero-Fluidic Load Control on Wind Turbine Blades, Master's thesis, University of Twente, 2011.

[194] Boeije, C. S., Exploratory Study of Fluidic Control of Pitch Angle and Flow Separation for a Wind Turbine Airfoil, Master's thesis, University of Twente, 2008.

[195] Bootsma, K., "De invloed van turbulatoren op de grenslaag van een profiel van een windturbine blad (The influence of turbulators on the boundary layer of a wind turbine blade section)," BSc thesis University of Twente, 2011. 
[196] Dantec Dynamics, URL: http://www.dantecdynamics.com/, accessed September 13, 2012.

[197] Zwart, G. G. M., "Turbulence Measurements 130 kW Wind Tunnel," Technical report, Engineering Fluid Dynamics, University of Twente, 2008.

[198] Pope, A. and Harper, J. J., Low-Speed Wind Tunnel Testing, Wiley, 1966.

[199] Drela, M., "XFOIL: An Analysis and Design System for Low Reynolds Number Airfoils," Low Reynolds Number Aerodynamics, edited by T. J. Müller, Vol. 54 of Lecture Notes in Engineering, Springer Verlag, 1989, pp. $1-12$.

[200] Drela, M. and Giles, M. B., "Viscous-Inviscid Analysis of Transonic and Low Reynolds Number Airfoils," AIAA Journal, Vol. 25, No. 10, 1987, pp. $1347-1355$.

[201] van Ingen, J. L., "The $e^{N}$ method for transition prediction: historical review of work at TU Delft," AIAA Paper 2008-3830, 2008.

[202] Lee, C. Y. and Goldstein, D. B., "Two-Dimensional Synthetic Jet Simulation," AIAA Journal, Vol. 40, No. 3, 2002, pp. $510-516$.

[203] Ugrina, S., Experimental Analysis and Analytical Modeling of Synthetic Jet-Cross Flow Interactions, Ph.D. thesis, University of Maryland, 2007. 



\section{Appendix A}

\section{Characteristics of Augmented SEt of Inviscid Flow Equations}

This appendix presents the characteristic analysis of the convective part of the URANS equations augmented with the convective part of the SST turbulence model equations. The analysis will show that in the inviscid limit, i.e. $\operatorname{Re} \rightarrow \infty$, this set of equations is hyperbolic. Hyperbolic equations describe the propagation of waves. The knowledge of what kind of waves are propagated and the associated propagation velocities is important for understanding the physical behavior of flows at high Reynolds numbers. This knowledge is necessary for the construction of numerical flux functions for the convective fluxes. Furthermore, it is used to construct physically correct boundary conditions.

In this analysis, the tilde and overbar notations of the Favre- and Reynolds averaged variables are dropped for readability reasons.

In the limit of $\operatorname{Re} \rightarrow \infty$, the viscous fluxes of the URANS and turbulence equations become negligible and the equations in partial differential form can be written as

$$
\frac{\partial \boldsymbol{W}}{\partial t}+\underline{\nabla} \cdot \underline{\boldsymbol{F}}^{c}=0
$$

where $\boldsymbol{W}(\underline{x}, t)=[\rho, \rho \underline{u}, \rho E, \rho k, \rho \omega]^{T}$ is the column vector with time-averaged conserved variables. Furthermore, $\underline{\boldsymbol{F}}^{c}$ is the (non-linear) convective flux vector given in equation (3.94), augmented with the convective flux vector of the SST turbulence model given in equation (3.131):

which can be rewritten as

$$
\underline{\boldsymbol{F}}^{c}=\left[\begin{array}{c}
\rho \underline{u} \\
\rho \underline{u} \underline{u}+p^{*} \underline{\underline{I}} \\
\left(\rho E+p^{*}\right) \underline{u} \\
\rho k \underline{u} \\
\rho \omega \underline{u}
\end{array}\right]
$$

$$
\underline{\boldsymbol{F}}^{c}=\left[\begin{array}{c}
\rho \underline{u} \\
\rho \underline{u} \underline{u}+p^{*} \underline{\underline{I}} \\
\left(\frac{\gamma p^{*}}{\gamma-1}+\frac{1}{2} \rho|\underline{u}|^{2}+\frac{\bar{\gamma}-\frac{5}{3}}{\gamma-1} \rho k\right) \underline{u} \\
\rho k \underline{u} \\
\rho \omega \underline{u}
\end{array}\right],
$$


due to equations (3.92) and (3.93).

\section{A.1 Eigenvalues and Eigenvectors}

\section{A.1.1 Problem Definition}

The characteristic analysis starts with writing equation (A.1) in quasi-linear form, as

$$
\frac{\partial \boldsymbol{W}}{\partial t}+A_{1} \frac{\partial \boldsymbol{W}}{\partial x_{1}}+A_{2} \frac{\partial \boldsymbol{W}}{\partial x_{2}}+A_{3} \frac{\partial \boldsymbol{W}}{\partial x_{3}}=0,
$$

with

$$
A_{1}=\frac{\partial \boldsymbol{F}_{1}^{c}}{\partial \boldsymbol{W}}, \quad A_{2}=\frac{\partial \boldsymbol{F}_{2}^{c}}{\partial \boldsymbol{W}}, \quad A_{3}=\frac{\partial \boldsymbol{F}_{3}^{c}}{\partial \boldsymbol{W}}
$$

being the Jacobian matrices in $x_{1^{-}}, x_{2^{-}}$and $x_{3}$-direction, respectively.

We seek the eigenvalues and the eigenvectors of a linear combination of $A_{1}, A_{2}$ and $A_{3}$, say $K$, corresponding with a propagation direction $\underline{s}$, e.g. a direction normal to a face:

$$
K=A_{1} s_{1}+A_{2} s_{2}+A_{3} s_{3} .
$$

If there is a vector $\boldsymbol{r}^{(i)}$ such that

$$
K \boldsymbol{r}^{(i)}=\lambda_{i} \boldsymbol{r}^{(i)} \Leftrightarrow\left(K-\lambda_{i} I\right) \boldsymbol{r}^{(i)}=0, \quad i=\{1, \ldots, 5\}
$$

for a scalar $\lambda_{i}$, then $\lambda_{i}$ is called an eigenvalue of $K$ with corresponding right eigenvector $\boldsymbol{r}^{(i)}$. On the other hand, if there is a vector $\boldsymbol{l}^{(i)}$ such that

$$
\boldsymbol{l}^{(i)^{T}}\left(K-\lambda_{i} I\right)=0, \quad i=\{1, \ldots, 5\},
$$

then $\boldsymbol{l}^{(i)}$ is the corresponding left eigenvector of $K$. The eigenvalues can be found by the fact that Equation (A.6) or (A.7) has nontrivial solutions if

$$
\left|K-\lambda_{i} I\right|=0
$$

where $|$.$| indicates the matrix determinant.$

Let the set of left and right eigenvectors be scaled such that $\boldsymbol{l}^{(i)^{T}} \boldsymbol{r}^{(j)}=\delta_{i j}$. Furthermore, let $L^{T}$ be the matrix whose rows are $\left\{\boldsymbol{l}^{(1)^{T}}, \ldots, \boldsymbol{l}^{(5)^{T}}\right\}$ and let $R$ be the matrix whose columns are $\left\{\boldsymbol{r}^{(1)}, \ldots, \boldsymbol{r}^{(5)}\right\}$, then

$$
L^{T} K R=\Lambda,
$$

where $\Lambda=\operatorname{diag}\left\{\lambda_{1}, \ldots, \lambda_{5}\right\}$. Note that $L^{T} R=I$, due to the scaling of the left and right eigenvectors. This means that $L^{T}$ is the inverse of $R$, i.e. $L^{T}=R^{-1}$.

The matrices $L^{T}$ and $R$ together form a similarity transformation that diagonalizes the Jacobian matrix $K$. 


\section{A.1.2 Transformation to Primitive Variables}

The problem of finding the eigenvalues and eigenvectors is simplified by rewriting equation (A.3) in terms of the primitive variables, $\boldsymbol{Q}=\left[\rho, \underline{u}, p^{*}, k, \omega\right]^{T}$, by means of a similarity transformation $M$ :

$$
M=\frac{\partial \boldsymbol{W}}{\partial \boldsymbol{Q}}=\left[\begin{array}{ccccccc}
1 & 0 & 0 & 0 & 0 & 0 & 0 \\
u_{1} & \rho & 0 & 0 & 0 & 0 & 0 \\
u_{2} & 0 & \rho & 0 & 0 & 0 & 0 \\
u_{3} & 0 & 0 & \rho & 0 & 0 & 0 \\
\frac{1}{2}|\underline{u}|^{2}+\frac{\gamma-\frac{5}{3}}{\gamma-1} k & \rho u_{1} & \rho u_{2} & \rho u_{3} & \frac{1}{\gamma-1} & \frac{\gamma-\frac{5}{3}}{\gamma-1} \rho & 0 \\
k & 0 & 0 & 0 & 0 & \rho & 0 \\
\omega & 0 & 0 & 0 & 0 & 0 & \rho
\end{array}\right] .
$$

Substitution of $\boldsymbol{W}=\boldsymbol{W}(\boldsymbol{Q}(\underline{x}, t))$ in equation (A.3) and multiplying by the inverse of $M$, given by

$$
\begin{aligned}
& M^{-1}= \\
& {\left[\begin{array}{ccccccc}
1 & 0 & 0 & 0 & 0 & 0 & 0 \\
-u_{1} / \rho & 1 / \rho & 0 & 0 & 0 & 0 & 0 \\
-u_{2} / \rho & 0 & 1 / \rho & 0 & 0 & 0 & 0 \\
-u_{3} / \rho & 0 & 0 & 1 / \rho & 0 & 0 & 0 \\
(\gamma-1)\left(\frac{1}{2}|\underline{u}|^{2}\right) & -(\gamma-1) u_{1} & -(\gamma-1) u_{2} & -(\gamma-1) u_{3} & (\gamma-1) & -\left(\gamma-\frac{5}{3}\right) & 0 \\
-k / \rho & 0 & 0 & 0 & 0 & 1 / \rho & 0 \\
-\omega / \rho & 0 & 0 & 0 & 0 & 0 & 1 / \rho
\end{array}\right],}
\end{aligned}
$$

yields a transformed equation in terms of the primitive variables:

$$
\frac{\partial \boldsymbol{Q}}{\partial t}+\hat{A}_{1} \frac{\partial \boldsymbol{Q}}{\partial x_{1}}+\hat{A}_{2} \frac{\partial \boldsymbol{Q}}{\partial x_{2}}+\hat{A}_{3} \frac{\partial \boldsymbol{Q}}{\partial x_{3}}=0
$$

The matrices $\hat{A}_{1}, \hat{A}_{2}$ and $\hat{A}_{3}$ are the Jacobian matrices of the transformed equation, given by

$$
\begin{aligned}
& \hat{A}_{1}=M^{-1} A_{1} M=M^{-1} \frac{\partial \boldsymbol{F}_{1}^{c}}{\partial \boldsymbol{Q}} \\
& \hat{A}_{2}=M^{-1} A_{2} M=M^{-1} \frac{\partial \boldsymbol{F}_{2}^{c}}{\partial \boldsymbol{Q}} \\
& \hat{A}_{3}=M^{-1} A_{3} M=M^{-1} \frac{\partial \boldsymbol{F}_{3}^{c}}{\partial \boldsymbol{Q}} .
\end{aligned}
$$

Again, we seek the eigenvalues of a linear combination of the Jacobian matrices:

$$
\hat{K}=\hat{A}_{1} s_{1}+\hat{A}_{2} s_{2}+\hat{A}_{3} s_{3}=M^{-1} \frac{\partial}{\partial \boldsymbol{Q}}\left(\underline{\boldsymbol{F}}^{c} \cdot \underline{s}\right) .
$$


The eigenvalues of $\hat{K}$ are easier to find than those of $K$. Furthermore, they are equal to the eigenvalues of $K$, due to the similarity transformation:

$$
\begin{aligned}
|\hat{K}-\lambda I| & =\left|M^{-1} K M-\lambda I\right| \\
& =\left|M^{-1} K M-M^{-1} \lambda I M\right| \\
& =\left|M^{-1}(K-\lambda I) M\right| \\
& =\left|M^{-1}\right||K-\lambda I||M| \\
& =|K-\lambda I| .
\end{aligned}
$$

The eigenvectors of $\hat{K}$, combined in $\hat{L}^{T}$ and $\hat{R}$, are also easier to find than those of $K$. However, they are not the same as those of $K$, but are related to them through

$$
\begin{aligned}
& \Lambda=\hat{L}^{T} \hat{K} \hat{R}=\hat{L}^{T} M^{-1} K M \hat{R} \\
& \quad \Rightarrow L^{T}=\hat{L}^{T} M^{-1}, \quad R=M \hat{R}
\end{aligned}
$$

Therefore, the eigenvectors of $\hat{K}$ are constructed first and equation (A.18) is employed to find the eigenvectors of the convective flux Jacobian in terms of conserved variables.

\section{A.1.3 Eigenvalues of Convective Flux Jacobian}

Evaluating equation (A.16) yields

$$
\hat{K}=\left[\begin{array}{ccccccc}
\underline{u} \cdot \underline{s} & \rho s_{1} & \rho s_{2} & \rho s_{3} & 0 & 0 & 0 \\
0 & \underline{u} \cdot \underline{s} & 0 & 0 & s_{1} / \rho & 0 & 0 \\
0 & 0 & \underline{u} \cdot \underline{s} & 0 & s_{2} / \rho & 0 & 0 \\
0 & 0 & 0 & \underline{u} \cdot \underline{s} & s_{3} / \rho & 0 & 0 \\
0 & \gamma p^{*} s_{1} & \gamma p^{*} s_{2} & \gamma p^{*} s_{3} & \underline{u} \cdot \underline{s} & 0 & 0 \\
0 & 0 & 0 & 0 & 0 & \underline{u} \cdot \underline{s} & 0 \\
0 & 0 & 0 & 0 & 0 & 0 & \underline{u} \cdot \underline{s}
\end{array}\right] .
$$

Application of equation (A.8) yields the following eigenvalues:

$$
\begin{aligned}
\lambda_{1} & =\underline{u} \cdot \underline{s}-c^{*}|\underline{s}|, \\
\lambda_{2}=\lambda_{3}= & \lambda_{4}=\underline{u} \cdot \underline{s}, \\
\lambda_{5} & =\underline{u} \cdot \underline{s}+c^{*}|\underline{s}|, \\
\lambda_{6}= & \lambda_{7}=\underline{u} \cdot \underline{s},
\end{aligned}
$$

where $c^{*}$ is the modified speed of sound defined as

$$
c^{*}=\sqrt{\frac{\gamma p^{*}}{\rho}} .
$$




\section{A.1.4 Eigenvectors of the Convective Flux Jacobian}

The left eigenvectors of $\hat{K}$ are found by solving

$$
\boldsymbol{l}^{(i)^{T}}\left(\hat{K}-\lambda_{i} I\right)=0, \quad i=\{1, \ldots, 7\}
$$

This set of equations has several degrees of freedom left. For $\lambda=\underline{u} \cdot \underline{s}$, the components of the left eigenvectors must satisfy

$$
\begin{array}{r}
l_{1} \text { arbitrary, } \\
l_{2}+c^{* 2} l_{5}=0, \\
l_{3}+c^{* 2} l_{5}=0, \\
l_{4}+c^{* 2} l_{5}=0, \\
l_{2} s_{1}+l_{3} s_{2}+l_{4} s_{3}=0, \\
l_{6} \text { arbitrary, } \\
l_{7} \text { arbitrary, }
\end{array}
$$

whereas for $\lambda=\underline{u} \cdot \underline{n} \pm c^{*}|\underline{s}|$, the components must satisfy

$$
\begin{array}{r}
l_{1}=0, \\
l_{2}= \pm \rho c^{*} n_{1} l_{5}, \\
l_{3}= \pm \rho c^{*} n_{2} l_{5}, \\
l_{4}= \pm \rho c^{*} n_{3} l_{5}, \\
l_{5} \text { arbitrary, } \\
l_{6}=0, \\
l_{7}=0,
\end{array}
$$

where $\underline{n}=\left(n_{1}, n_{2}, n_{3}\right)$ is the unit vector along $\underline{s}$, i.e. $\underline{n}=\frac{\underline{s}}{|\underline{s}|}$.

The following linearly independent set of left eigenvectors can now be constructed:

$$
\hat{L}^{T}=\left[\begin{array}{ccccccc}
0 & -n_{1} \alpha_{1}^{(1)} & -n_{2} \alpha^{(1)} & -n_{3} \alpha_{1}^{(1)} & \frac{1}{\rho c^{*}} \alpha_{1}^{(1)} & 0 & 0 \\
\alpha_{1}^{(2)} & 0 & s_{3} \alpha_{2}^{(2)} & -s_{2} \alpha_{2}^{(2)} & -\frac{\alpha_{1}^{(2)}}{c^{* 2}} & \alpha_{3}^{(2)} & \alpha_{4}^{(2)} \\
\alpha_{1}^{(3)} & -s_{3} \alpha_{2}^{(3)} & 0 & s_{1} \alpha_{2}^{(3)} & -\frac{\alpha_{1}^{(3)}}{c^{* 2}} & \alpha_{3}^{(3)} & \alpha_{4}^{(3)} \\
\alpha_{1}^{(4)} & s_{2} \alpha_{2}^{(4)} & -s_{1} \alpha_{2}^{(4)} & 0 & -\frac{\alpha_{1}^{(4)}}{c^{* 2}} & \alpha_{3}^{(4)} & \alpha_{4}^{(4)} \\
0 & n_{1} \alpha_{1}^{(5)} & n_{2} \alpha_{1}^{(5)} & n_{3} \alpha_{1}^{(5)} & \frac{1}{\rho c^{*}} \alpha_{1}^{(5)} & 0 & 0 \\
\alpha_{1}^{(6)} & 0 & s_{3} \alpha_{2}^{(6)} & -s_{2} \alpha_{2}^{(6)} & -\frac{\alpha_{1}^{(6)}}{c^{* 2}} & \alpha_{3}^{(6)} & \alpha_{4}^{(6)} \\
\alpha_{1}^{(7)} & -s_{3} \alpha_{2}^{(7)} & 0 & s_{1} \alpha_{2}^{(7)} & -\frac{\alpha_{1}^{(7)}}{c^{* 2}} & \alpha_{3}^{(7)} & \alpha_{4}^{(7)}
\end{array}\right],
$$


where $\alpha_{j}^{(i)}$ are arbitrary constants, for which we make the following convenient choices:

$$
\begin{array}{r}
\alpha_{1}^{(1)}=\alpha_{1}^{(5)}=1, \\
\alpha_{1}^{(2)}=n_{1}, \quad \alpha_{1}^{(3)}=n_{2}, \quad \alpha_{1}^{(4)}=n_{3}, \\
\alpha_{1}^{(6)}=\alpha_{1}^{(7)}=0, \\
\alpha_{2}^{(2)}=\alpha_{2}^{(3)}=\alpha_{2}^{(4)}=\frac{1}{|\underline{s}|}, \quad \alpha_{6}^{(2)}=\alpha_{7}^{(2)}=0, \\
\alpha_{3}^{(2)}=\alpha_{3}^{(3)}=\alpha_{3}^{(4)}=0, \quad \alpha_{3}^{(6)}=1, \quad \alpha_{3}^{(7)}=0, \\
\alpha_{4}^{(2)}=\alpha_{4}^{(3)}=\alpha_{4}^{(4)}=0, \quad \alpha_{4}^{(6)}=0, \quad \alpha_{4}^{(7)}=1 .
\end{array}
$$

This leads to the following set of left eigenvectors of $\hat{K}$ :

$$
\hat{L}^{T}=\left[\begin{array}{ccccccc}
0 & -n_{1} & -n_{2} & -n_{3} & 1 / \rho c^{*} & 0 & 0 \\
n_{1} & 0 & n_{3} & -n_{2} & -n_{1} / c^{* 2} & 0 & 0 \\
n_{2} & -n_{3} & 0 & n_{1} & -n_{2} / c^{* 2} & 0 & 0 \\
n_{3} & n_{2} & -n_{1} & 0 & -n_{3} / c^{* 2} & 0 & 0 \\
0 & n_{1} & n_{2} & n_{3} & 1 / \rho c^{*} & 0 & 0 \\
0 & 0 & 0 & 0 & 0 & 1 & 0 \\
0 & 0 & 0 & 0 & 0 & 0 & 1
\end{array}\right]
$$

The set of corresponding right eigenvectors of $\hat{K}$ reads

$$
\hat{R}=\left(\hat{L}^{T}\right)^{-1}=\left[\begin{array}{ccccccc}
\rho / 2 c^{*} & n_{1} & n_{2} & n_{3} & \rho / 2 c^{*} & 0 & 0 \\
-n_{1} / 2 & 0 & -n_{3} & n_{2} & n_{1} / 2 & 0 & 0 \\
-n_{2} / 2 & n_{3} & 0 & -n_{1} & n_{2} / 2 & 0 & 0 \\
-n_{3} / 2 & -n_{2} & n_{1} & 0 & n_{3} / 2 & 0 & 0 \\
\rho c^{*} / 2 & 0 & 0 & 0 & \rho c^{*} / 2 & 0 & 0 \\
0 & 0 & 0 & 0 & 0 & 1 & 0 \\
0 & 0 & 0 & 0 & 0 & 0 & 1
\end{array}\right] .
$$

Next, the matrices with left and right eigenvectors of the convective flux Jacobian in terms of conserved variables are constructed. The matrix $R$ reads

$$
\begin{aligned}
& R=M \hat{R}= \\
& {\left[\begin{array}{ccccccc}
\alpha & n_{1} & n_{2} & n_{3} & \alpha & 0 & 0 \\
\alpha\left(u_{1}-c^{*} n_{1}\right) & u_{1} n_{1} & u_{1} n_{2}-\rho n_{3} & u_{1} n_{3}+\rho n_{2} & \alpha\left(u_{1}+c^{*} n_{1}\right) & 0 & 0 \\
\alpha\left(u_{2}-c^{*} n_{2}\right) & u_{2} n_{1}+\rho n_{3} & u_{2} n_{2} & u_{2} n_{3}-\rho n_{1} & \alpha\left(u_{2}+c^{*} n_{2}\right) & 0 & 0 \\
\alpha\left(u_{3}-c^{*} n_{3}\right) & u_{3} n_{1}-\rho n_{2} & u_{3} n_{2}+\rho n_{1} & u_{3} n_{3} & \alpha\left(u_{3}+c^{*} n_{3}\right) & 0 & 0 \\
\alpha\left(H^{*}-c^{*} \underline{u} \cdot \underline{n}\right) & \underline{a} \cdot \underline{e}_{1} & \underline{a} \cdot \underline{e}_{2} & \underline{a} \cdot \underline{e}_{3} & \alpha\left(H^{*}+c^{*} \underline{u} \cdot \underline{n}\right) & \beta \rho & 0 \\
\alpha k & k n_{1} & k n_{2} & k n_{3} & \alpha k & \rho & 0 \\
\alpha \omega & \omega n_{1} & \omega n_{2} & \omega n_{3} & \alpha \omega & 0 & \rho
\end{array}\right],}
\end{aligned}
$$


with the abbreviations

$$
\alpha=\frac{\rho}{2 c^{*}}, \quad \beta=\frac{\gamma-\frac{5}{3}}{\gamma-1},
$$

and the modified specific total enthalpy

$$
H^{*}=E+p^{*} / \rho=\frac{c^{* 2}}{\gamma-1}+\frac{1}{2}|\underline{u}|^{2}+\frac{\gamma-\frac{5}{3}}{\gamma-1} k .
$$

Furthermore, $\underline{e}_{i}$ is the unit vector in $i$-direction and vector $\underline{a}$ is defined as

$$
\underline{a}=\left(\frac{1}{2}|\underline{u}|^{2}+\frac{\gamma-\frac{5}{3}}{\gamma-1} k\right) \underline{n}+\rho(\underline{u} \times \underline{n}) .
$$

The matrix $L^{T}$ reads

$$
\begin{aligned}
& L^{T}=\hat{L}^{T} M^{-1}=
\end{aligned}
$$

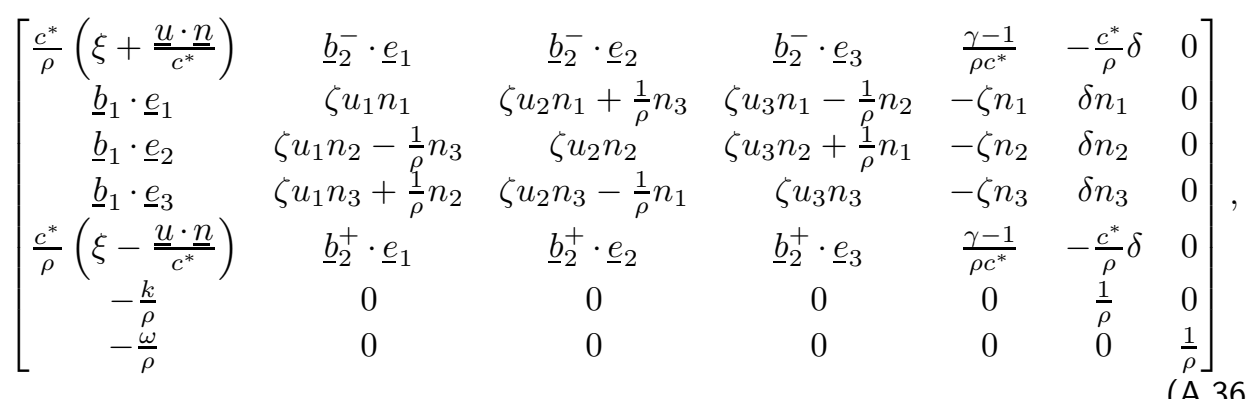

with the abbreviations

$$
\begin{aligned}
& \zeta=\frac{\gamma-1}{c^{* 2}}, \quad \xi=\zeta\left(\frac{1}{2}|\underline{u}|^{2}\right), \quad \delta=\frac{\gamma-\frac{5}{3}}{c^{* 2}}, \\
& \underline{b}_{1}=(1-\xi) \underline{n}-\frac{1}{\rho}(\underline{u} \times \underline{n}), \quad \underline{b}_{2}^{ \pm}= \pm \frac{1}{\rho} \underline{n}-\frac{\gamma-1}{\rho c^{*}} \underline{u} .
\end{aligned}
$$

This completes the construction of the left and right eigenvector matrices of the convective flux Jacobian.

\section{A.2 Wave Propagation Along Characteristics}

Since a complete set of eigenvectors has been found and all eigenvalues are real, the system of equations (A.3) is hyperbolic. This means that the system of equations describes the propagation of waves. This can be explained in a simple way if we consider the propagation direction $\underline{s}=(1,0,0)$, i.e. in the $x_{1}$-direction. Since the URANS equations are rotationally invariant, the following analysis is valid for any direction by introducing an appropriate 
rotation matrix that rotates the coordinate system such that the new $x_{1}$-axis corresponds to the new propagation direction. The inverse rotation matrix should then be used to transform the results back to the original Cartesian coordinate system.

Equation (A.12) can be rewritten as

$$
\frac{\partial \boldsymbol{Q}}{\partial t}+\left(\hat{R}_{1} \Lambda \hat{L}_{1}^{T}\right) \frac{\partial \boldsymbol{Q}}{\partial x_{1}}+\hat{\boldsymbol{B}}=0
$$

with $\hat{\boldsymbol{B}}$ containing terms with derivatives normal to the propagation direction,

$$
\hat{\boldsymbol{B}}=\hat{A}_{2} \frac{\partial \boldsymbol{Q}}{\partial x_{2}}+\hat{A}_{3} \frac{\partial \boldsymbol{Q}}{\partial x_{3}},
$$

and $\Lambda=\operatorname{diag}\left(u_{1}-c^{*}, u_{1}, u_{1}, u_{1}, u_{1}+c^{*}, u_{1}, u_{1}\right)$. Furthermore, $\hat{L}_{1}^{T}$ and $\hat{R}_{1}$ are linearly independent sets of left and right eigenvectors of $\hat{A}_{1}$, respectively, given by

$$
\begin{aligned}
\hat{L}_{1}^{T}= & {\left[\begin{array}{ccccccc}
0 & -\rho c^{*} & 0 & 0 & 1 & 0 & 0 \\
c^{* 2} & 0 & 0 & 0 & -1 & 0 & 0 \\
0 & 0 & 1 & 0 & 0 & 0 & 0 \\
0 & 0 & 0 & 1 & 0 & 0 & 0 \\
0 & \rho c^{*} & 0 & 0 & 1 & 0 & 0 \\
0 & 0 & 0 & 0 & 0 & 1 & 0 \\
0 & 0 & 0 & 0 & 0 & 0 & 1
\end{array}\right], } \\
\hat{R}_{1}= & {\left[\begin{array}{ccccccc}
\frac{1}{2 c^{* 2}} & \frac{1}{c^{* 2}} & 0 & 0 & \frac{1}{2 c^{* 2}} & 0 & 0 \\
-\frac{1}{2 \rho c^{*}} & 0 & 0 & 0 & \frac{1}{2 \rho c^{*}} & 0 & 0 \\
0 & 0 & 1 & 0 & 0 & 0 & 0 \\
0 & 0 & 0 & 1 & 0 & 0 & 0 \\
\frac{1}{2} & 0 & 0 & 0 & \frac{1}{2} & 0 & 0 \\
0 & 0 & 0 & 0 & 0 & 1 & 0 \\
0 & 0 & 0 & 0 & 0 & 0 & 1
\end{array}\right] . }
\end{aligned}
$$

Note that these matrices are slightly different sets of eigenvectors than those given in equations (A.30) and (A.31) with $\underline{s}=(1,0,0)$. However, this will not affect the results and the matrices used here are more convenient in the present analysis.

Multiplication of equation (A.38) by $\hat{L}_{1}^{T}$, yields the so-called compatibility equations

$$
\hat{L}_{1}^{T} \frac{\partial \boldsymbol{Q}}{\partial t}+\mathcal{L}=-\hat{L}_{1}^{T} \hat{\boldsymbol{B}}
$$


where $\mathcal{L}$ is a column vector defined as

$$
\mathcal{L}=\Lambda \hat{L}_{1}^{T} \frac{\partial \boldsymbol{Q}}{\partial x_{1}}=\left[\begin{array}{c}
\left(u_{1}-c^{*}\right)\left(\frac{\partial p^{*}}{\partial x_{1}}-\rho c^{*} \frac{\partial u_{1}}{\partial x_{1}}\right) \\
u_{1}\left(c^{* 2} \frac{\partial \rho}{\partial x_{1}}-\frac{\partial p^{*}}{\partial x_{1}}\right) \\
u_{1}\left(\frac{\partial u_{2}}{\partial x_{1}}\right) \\
u_{1}\left(\frac{\partial u_{3}}{\partial x_{1}}\right) \\
\left(u_{1}+c^{*}\right)\left(\frac{\partial p^{*}}{\partial x_{1}}+\rho c^{*} \frac{\partial u_{1}}{\partial x_{1}}\right) \\
u_{1}\left(\frac{\partial k}{\partial x_{1}}\right) \\
u_{1}\left(\frac{\partial \omega}{\partial x_{1}}\right)
\end{array}\right] .
$$

The homogenous form of equation (A.42) is a decoupled system of five advection equations describing the conservation of each of the so-called characteristic variables $V_{i}$ along the corresponding characteristics $\frac{d x_{1}}{d t}=\lambda_{i}$. Consequently, each component $\mathcal{L}_{i}$ is associated with a temporal change of the amplitude of the wave traveling along $\frac{d x_{1}}{d t}=\lambda_{i}$. The waves corresponding to $\lambda_{1}$ and $\lambda_{5}$ are acoustic waves, the wave corresponding to $\lambda_{2}$ is an entropy wave, the waves corresponding to $\lambda_{3}$ and $\lambda_{4}$ are shear waves and the waves corresponding to $\lambda_{6}$ and $\lambda_{7}$ are waves associated with the turbulence quantities. Changes in characteristic variables are related to changes in the primitive variables according to $\delta \boldsymbol{V}=\hat{L}_{1}^{T} \delta \boldsymbol{Q}$ :

$$
\begin{aligned}
& \delta V_{1}=\delta p^{*}-\rho c^{*} \delta u_{1} \quad \text { constant along } \frac{d x_{1}}{d t}=u_{1}-c^{*}, \\
& \delta V_{2}=c^{* 2} \delta \rho-\delta p^{*} \quad \text { constant along } \frac{d x_{1}}{d t}=u_{1}, \\
& \delta V_{3}=\delta u_{2} \quad \text { constant along } \frac{d x_{1}}{d t}=u_{1} \\
& \delta V_{4}=\delta u_{3} \quad \text { constant along } \frac{d x_{1}}{d t}=u_{1} \\
& \delta V_{5}=\delta p^{*}+\rho c^{*} \delta u_{1} \quad \text { constant along } \frac{d x_{1}}{d t}=u_{1}+c^{*} \\
& \delta V_{6}=\delta k \quad \text { constant along } \frac{d x_{1}}{d t}=u_{1} \\
& \delta V_{7}=\delta \omega \quad \text { constant along } \frac{d x_{1}}{d t}=u_{1} .
\end{aligned}
$$

The result of the present analysis is useful for the construction of artificial boundary conditions. The number of boundary conditions to be imposed at a boundary equals the number of waves traveling into the computational domain. The property of $\mathcal{L}$ being associated with the wave amplitudes can be used in the construction of non-reflective boundary conditions, for which the wave amplitudes corresponding to waves traveling into the computational domain can be set to zero. 
Multiplying equation (A.42) by $\hat{R}_{1}$ yields

$$
\begin{gathered}
\frac{\partial \boldsymbol{Q}}{\partial t}+\hat{R}_{1} \mathcal{L}=-\hat{\boldsymbol{B}} \\
\frac{\partial}{\partial t}\left[\begin{array}{c}
\rho \\
u_{1} \\
u_{2} \\
u_{3} \\
p^{*} \\
k \\
\omega
\end{array}\right]+\left[\begin{array}{c}
\frac{1}{c^{* 2}}\left(\mathcal{L}_{2}+\frac{1}{2}\left(\mathcal{L}_{1}+\mathcal{L}_{5}\right)\right) \\
\frac{1}{2 \rho c^{*}}\left(\mathcal{L}_{5}-\mathcal{L}_{1}\right) \\
\mathcal{L}_{3} \\
\mathcal{L}_{4} \\
\frac{1}{2}\left(\mathcal{L}_{1}+\mathcal{L}_{5}\right) \\
\mathcal{L}_{6} \\
\mathcal{L}_{7}
\end{array}\right]=-\hat{\boldsymbol{B}} .
\end{gathered}
$$

This result can also be written in terms of the conserved variables by substituting $Q=$ $\boldsymbol{Q}(\boldsymbol{W}(\underline{x}, t))$ in the time-derivative term of equation (A.51) and multiplying the equation by transformation matrix $M$, see equation (A.10), which yields

$$
\frac{\partial \boldsymbol{W}}{\partial t}+M \hat{R}_{1} \mathcal{L}=-\boldsymbol{B} .
$$

with

$$
\boldsymbol{B}=A_{2} \frac{\partial \boldsymbol{W}}{\partial x_{2}}+A_{3} \frac{\partial \boldsymbol{W}}{\partial x_{3}} .
$$

Equation (A.52) directly relates the temporal change in the conserved variables to the wave amplitudes of waves traveling along the characteristics. 


\section{Appendix B}

\section{Convective Flux Jacobian Matrix of the Augmented System of EQUATIONS}

This appendix presents the derivation of the flux Jacobian matrix of the convective flux of the URANS equations (given in equation (3.94)) augmented with the convective flux of the SST turbulence model equations (given in equation (3.131)). Part of this convective flux Jacobian is used in the time integration method presented in section 4.4

In this analysis, the tilde and overbar notations of the Favre- and Reynolds averaged variables are dropped for readability reasons.

The convective flux vector of the augmented system of equations reads

$$
\underline{\boldsymbol{F}}^{c}=\left[\begin{array}{c}
\rho \underline{u} \\
\rho \underline{u} \underline{u}+p^{*} \underline{\underline{I}} \\
\left(\rho E+p^{*}\right) \underline{u} \\
\rho k \underline{u} \\
\rho \omega \underline{u}
\end{array}\right],
$$

Multiplying equation (B.1) by the face-area vector $\underline{S}=\underline{n}|\underline{S}|$, and rewriting the result in terms of the conserved flow variables $\boldsymbol{W}=\left[\rho, \rho u_{1}, \rho u_{2}, \rho u_{3}, \rho E, \rho k, \rho \omega\right]^{T}$ yields

$$
\underline{\boldsymbol{F}}^{c}(\boldsymbol{W}) \cdot \underline{S}=\left[\begin{array}{c}
W_{2} n_{1}+W_{3} n_{2}+W_{4} n_{3} \\
\frac{W_{2}}{W_{1}}\left(W_{2} n_{1}+W_{3} n_{2}+W_{4} n_{3}\right)+p^{*}(\boldsymbol{W}) n_{1} \\
\frac{W_{3}}{W_{1}}\left(W_{2} n_{1}+W_{3} n_{2}+W_{4} n_{3}\right)+p^{*}(\boldsymbol{W}) n_{2} \\
\frac{W_{4}}{W_{1}}\left(W_{2} n_{1}+W_{3} n_{2}+W_{4} n_{3}\right)+p^{*}(\boldsymbol{W}) n_{3} \\
\left(W_{5}+p^{*}(\boldsymbol{W})\right) \frac{1}{W_{1}}\left(W_{2} n_{1}+W_{3} n_{2}+W_{4} n_{3}\right) \\
\frac{W_{5}}{W_{1}}\left(W_{2} n_{1}+W_{3} n_{2}+W_{4} n_{3}\right) \\
\frac{W_{6}}{W_{1}}\left(W_{2} n_{1}+W_{3} n_{2}+W_{4} n_{3}\right)
\end{array}\right]|\underline{S}|,
$$

where the modified pressure as function of the conserved variables is given by

$$
p^{*}(\boldsymbol{W})=(\gamma-1)\left(W_{5}-\frac{1}{2} \frac{W_{2}^{2}+W_{3}^{2}+W_{4}^{2}}{W_{1}}-\frac{\gamma-\frac{5}{3}}{\gamma-1} W_{6}\right) .
$$


After taking the derivative with respect to each component of the conserved flow variables, the convective flux Jacobian matrix $\partial \underline{\boldsymbol{F}}^{c}(\boldsymbol{W}) \cdot \underline{S} / \partial \boldsymbol{W}$ is constructed as follows:

$\frac{\partial \underline{\boldsymbol{F}}^{c}(\boldsymbol{W}) \cdot \underline{S}}{\partial \boldsymbol{W}}=$

$|\underline{S}| \times$

$\left[\begin{array}{ccccccc}0 & n_{1} & n_{2} & n_{3} & 0 & 0 & 0 \\ \phi n_{1}-u_{1} u_{n} & u_{n}-\gamma_{2} u_{1} n_{1} & u_{1} n_{2}-\gamma_{1} u_{2} n_{1} & u_{1} n_{3}-\gamma_{1} u_{3} n_{1} & \gamma_{1} n_{1} & -\gamma_{\frac{5}{3}} n_{1} & 0 \\ \phi n_{2}-u_{2} u_{n} & u_{2} n_{1}-\gamma_{1} u_{1} n_{2} & u_{n}-\gamma_{2} u_{2} n_{2} & u_{2} n_{3}-\gamma_{1} u_{3} n_{2} & \gamma_{1} n_{2} & -\gamma_{\frac{5}{3}} n_{2} & 0 \\ \phi n_{3}-u_{3} u_{n} & u_{3} n_{1}-\gamma_{1} u_{1} n_{3} & u_{3} n_{2}-\gamma_{1} u_{2} n_{3} & u_{n}-\gamma_{2} u_{3} n_{3} & \gamma_{1} n_{3} & -\gamma_{\frac{5}{3}} n_{3} & 0 \\ u_{n}\left(\phi-H^{*}\right) & H^{*} n_{1}-\gamma_{1} u_{1} u_{n} & H^{*} n_{2}-\gamma_{1} u_{2} u_{n} & H^{*} n_{3}-\gamma_{1} u_{3} u_{n} & \gamma u_{n} & -\gamma_{\frac{5}{3}} u_{n} & 0 \\ -k u_{n} & k n_{1} & k n_{2} & k n_{3} & 0 & u_{n} & 0 \\ -\omega u_{n} & \omega n_{1} & \omega n_{2} & \omega n_{3} & 0 & 0 & u_{n}\end{array}\right]$,

with the abbreviations

$$
\begin{aligned}
\gamma_{1} & =\gamma-1, \quad \gamma_{2}=\gamma-2, \quad \gamma_{\frac{5}{3}}=\gamma-\frac{5}{3}, \\
u_{n} & =\underline{u} \cdot \underline{n}, \\
\phi & =\frac{\partial}{\partial W_{1}}\left(p^{*}(\boldsymbol{W})\right)=(\gamma-1)\left(\frac{1}{2}|\underline{u}|^{2}\right),
\end{aligned}
$$

and the modified specific total enthalpy given by

$$
H^{*}=\frac{\gamma}{\gamma-1} \frac{p^{*}}{\rho}+\frac{1}{2}|\underline{u}|^{2}+\frac{\gamma-\frac{5}{3}}{\gamma-1} k .
$$




\section{Appendix C}

\section{Complex-Step Gradient Approximation}

This appendix describes the "complex-step" method to obtain the contribution of a certain flux scheme to the flux Jacobian matrix, without the need to derive it analytically. This method is similar to a finite-difference approximation, in the sense that it is also derived from a Taylor series approximation of a perturbed flux function. However, instead of a real-valued perturbation, the flux function is given a complex-valued perturbation in the complex-step method. The derivation follows Martins et al. [140].

The contribution to the flux Jacobian matrix follows from a complex-valued perturbation on each of the conserved variables. For example, for the calculation of $\partial \Phi_{\mathrm{kl}}^{c} / \partial U_{\mathrm{k}, 1}$, i.e. the column vector associated with the derivative of $\boldsymbol{\Phi}_{\mathrm{kl}}^{c}$ with respect to the first component of column vector $\boldsymbol{U}_{k}$, a complex-valued perturbation i $\boldsymbol{h}_{1}=\mathrm{i}\left(h_{1}, 0,0,0,0\right)^{T}$ is introduced, where $\mathrm{i}=\sqrt{-1}$ is the imaginary unit. A Taylor series expansion around the unperturbed value reads

$$
\boldsymbol{\Phi}_{\mathrm{kl}}^{c}\left(\boldsymbol{U}_{\mathrm{k}}+\mathrm{i} \boldsymbol{h}_{1}, \boldsymbol{U}_{\mathrm{l}}\right)=\boldsymbol{\Phi}_{\mathrm{kl}}^{c}\left(\boldsymbol{U}_{\mathrm{k}}, \boldsymbol{U}_{1}\right)+\mathrm{i} h_{1} \frac{\partial \boldsymbol{\Phi}_{\mathrm{kl}}^{c}}{\partial U_{\mathrm{k}, 1}}+\mathcal{O}\left(h_{1}^{2}+\mathrm{i} h_{1}^{3}\right) .
$$

From this result, a second-order accurate approximation (with respect to $h_{1}$ ) of $\partial \boldsymbol{\Phi}_{\mathrm{kl}}^{c} / \partial U_{\mathrm{k}, 1}$ is obtained as

$$
\frac{\partial \boldsymbol{\Phi}_{\mathrm{kl}}^{c}}{\partial U_{\mathrm{k}, 1}} \approx \operatorname{Im}\left(\frac{\boldsymbol{\Phi}_{\mathrm{kl}}^{c}\left(\boldsymbol{U}_{\mathrm{k}}+\mathrm{i} \boldsymbol{h}_{1}, \boldsymbol{U}_{\mathrm{l}}\right)}{h_{1}}\right) .
$$

In order to obtain the complete derivative $\partial \Phi_{\mathrm{kl}}^{c} / \partial \boldsymbol{U}_{\mathrm{k}}$, five convective flux evaluations are needed, instead of the double amount for the standard finite-difference approximation. Additionaly, the complex-step method it is not prone to subtractive cancellation errors since it does not involve the substraction of two nearly equal-valued flux evaluations. Therefore, the perturbation $h_{i}$ can be given a very small value, such that the truncation error will be of the order of machine precision. In the present computational method, a value of

$$
h_{i}=\left(10^{-20}\right)\left(\operatorname{sign}\left(U_{\mathrm{k}, i}\right) \max \left(1,\left|U_{\mathrm{k}, i}\right|\right)\right)
$$

is used.

A drawback of the complex-step method is the increased memory requirement of the use of complex valued variables, but the accuracy of the calculated derivative outweighs 
this drawback. Furthermore, the algorithm to evaluate the convective flux has to be altered somewhat if it is to work with complex valued variables. The alterations are as follows:

- Relational operators, such as "<" or ">", are not defined for complex variables. In order to let the new algorithm follow the same path as the original algorithm that works with real valued variables, only the real parts of the complex variables must be compared. Functions that are based on relational operators, such as $\min ()$ or $\max ()$, should return a complex value based on comparing the real parts of the complex valued arguments, e.g.

$$
\max \left(a_{r}+\mathrm{i} a_{i}, b_{r}+\mathrm{i} b_{i}\right)=\left\{\begin{array}{ll}
a_{r}+\mathrm{i} a_{i} & \text { if } a_{r} \geq b_{r} \\
b_{r}+\mathrm{i} b_{i} & \text { otherwise }
\end{array} .\right.
$$

- Most arithmetic operators have a correct analytic complex definition. However, the "absolute value" function does not. This should be altered as follows:

$$
\operatorname{abs}\left(a_{r}+\mathrm{i} a_{i}\right)=\left\{\begin{array}{ll}
-\left(a_{r}+\mathrm{i} a_{i}\right) & \text { if } a_{r}<0 \\
a_{r}+\mathrm{i} a_{i} & \text { otherwise }
\end{array} .\right.
$$

Again, this alteration ensures that the new algorithm follows the same path as the original algorithm. 


\section{Appendix D}

\section{Computation of Aerodynamic ForCES}

This appendix describes the approach taken to calculate the aerodynamic force, $\underline{F}$ on an airfoil with unit spanwise length, on which synthetic jet actuation is applied. The aerodynamic force follows from the conservation equation for momentum, see section 3.1. within a specific, fixed, control volume, $V$. The control volume is bounded by an external surface, $A$. Here, we choose a convenient control volume that coincides with the contour of the airfoil, follows the geometry of the slit and cavity of the synthetic jet, and has a unit length in spanwise direction. At the spanwise ends, the control volume cuts the airfoil, such that at both ends a force, $\frac{1}{2} \underline{R}$, and a moment must be applied to keep the airfoil in it's place in the flow. The sum of the forces is the opposite of the aerodynamic force, i.e. $\underline{R}=-\underline{F}$.

The conservation equation for momentum states that the rate of change of momentum equal is to the sum of forces applied to the control volume, i.e.

$$
\iiint_{V} \frac{\partial}{\partial t}(\rho \underline{u}) d V+\iint_{A} \rho \underline{u} \underline{u} \cdot \underline{n} d A=\iint_{A}(-p \underline{I}+\underline{\tau}) \underline{n} d A+\underline{R},
$$

where $\underline{n}$ is the outward unit normal vector, i.e. $\underline{n}$ is directed out of the airfoil. Furthermore, $\rho$ is the density of the flow, $\underline{u}$ is the flow velocity, $p$ is the static pressure, and $\underline{\underline{\tau}}$ is the shear stress tensor.

Note that only the part of the surface $A$ that corresponds to the bottom boundary of the cavity, i.e. $A_{c a v}$, has nonzero flow velocities. When we assume that any flow that crosses this boundary does not have a major influence on the momentum of the medium contained in $V$ (i.e. $V$ is a plenum), the first term in equation D.1 can be neglected. Furthermore, the part of $A$ corresponding to the surfaces at the spanwise ends that cut the airfoil do also not contribute to the aerodynamic force, due to the two-dimensional flow configuration. What remains is $A_{\text {surface, }}$ which is the airfoil surface, minus the bottom boundary of the cavity, $A_{\text {cav }}$. The aerodynamic force can now be determined as

$$
\underline{F}=\iint_{A_{\text {surface }}+A_{\text {cav }}}(-p \underline{\underline{I}}+\underline{\underline{\tau}}) \underline{n} d A-\iint_{A_{\text {cav }}} \rho \underline{u} \underline{u} \cdot \underline{n} d A .
$$

where $\underline{n}$ is the outward unit normal vector, i.e. $\underline{n}$ is directed out of the airfoil. 



\section{ACKNOWLEDGEMENTS}

The work presented in this thesis could not have been completed without the support of many people, whom I would like to sincerely thank.

First of all, I would like to express my gratitude to Harry Hoeijmakers for his guidance and supervision during a great many years, starting with the period when I joined the group of Engineering Fluid Dynamics as a Master student. Harry, thank you for giving me the opportunity to work as a researcher in your group and for providing me with an interesting $\mathrm{PhD}$ project. And thank you for your contributions and feed-back to this thesis.

Furthermore, I would like to thank Mico Hirschberg and Edwin van der Weide for sharing their extensive knowledge about experimental research and computational fluid dynamics, respectively.

The Energy Research Centre of the Netherlands (ECN) deserves my gratitude for initiating and funding this research. Especially, I would like to thank Herman Snel, Arne van Garrel, Hüseyin Özdemir, Ozlem Ceyhan and Peter Eecen for sharing their expertise and for the pleasant atmosphere during our meetings.

I am indebted to Ivor Cleine and Chris Boeije for their experimental work on continuous jet actuation. Furthermore, I would like to thank Ellen van Emden, Stephanus Widjanarko and Immanuel Geesing for the development of the current experimental setup and for performing the experiments with synthetic jet actuation. Also thanks to Klaas Bootsma for his work on boundary-layer tripping.

I would like to thank Arjen Koop for introducing me to his version of the Euler code EFD-Flow, Philip Kelleners for providing the pre-processor FAVOMESH and Jacco Hospers for extending the pre-processor to hybrid meshes. Furthermore, I would like to thank Sietse Jongsma for paving the way on the subject of eddy-viscosity turbulence models.

Many thanks to the former and present staff members and the students (PhD or MSc) of the Engineering Fluid Dynamics group for creating such a pleasant environment to work in. Furthermore, I would like to acknowledge the support from Herman Stobbe for manufacturing the wind tunnel model, from Wouter den Breeijen for all his help on technical and computer-related issues, and from Anjenet Mettivier-Meijer for her secretarial work.

I would also like to thank my parents, Ben and Hilly, for putting me on this track.

Finally, I would like to express my deepest gratitude to my partner Ellen van Emden and our son Jelle for bringing me so much happiness and for your support, love, patience and understanding!

Hein de Vries, June 2013 



\section{About The Author}

Hein de Vries was born on 27 February 1980 in the municipality of Lemsterland (Friesland), the Netherlands. He grew up in the villages of Noordwijk (Groningen) and Pesse (Drenthe).

In 1998, he completed his pre-university secondary education (VWO) at O.S.G. De Groene Driehoek in Hoogeveen. He moved to Enschede to study Mechanical Engineering at the University of Twente, with a specialization in Fluid Dynamics in the group of professor Harry Hoeijmakers.

At the Global Research Center of General Electric in Niskayuna, New York, he performed an internship. During this internship, he performed computations in the field of thermal engineering and studied heat recovery systems for a hybrid, multi-MW, solid-oxide fuel-cell power plant.

In September 2005, he obtained his ir.-degree (MSc) following the defence of his Master thesis "Optimized Schemes for Wave Propagation", which was the result of a graduation project supervised by Rob Hagmeijer.

Directly following his graduation, he worked for 8 months as a researcher within the group of Engineering Fluid Dynamics of the University of Twente on a project for Shell Global Solutions. In this project, he studied the flow in the annulus of a sealless gas compressor.

In June 2006, he started his PhD project "On Synthetic Jet Actuation for Aerodynamic Load Control", also at the University of Twente. This work was supervised by Harry Hoeijmakers, Mico Hirschberg and Edwin van der Weide, and monitored by Herman Snel from the Energy Research Centre of the Netherlands (ECN). The results of this work are presented in the present thesis.

He currently lives with his partner Ellen van Emden and their son Jelle in Boekelo, the Netherlands. 
